Florida International University

FIU Digital Commons

FIU Electronic Theses and Dissertations

University Graduate School

$11-2-2012$

\title{
Development and Verification of Control and Protection Strategies in Hybrid AC/DC Power Systems for Smart Grid Applications
}

Vahid Salehi Pour Mehr

Florida International University, vsale001@fiu.edu

DOI: $10.25148 /$ etd.FI12120404

Follow this and additional works at: https://digitalcommons.fiu.edu/etd

\section{Recommended Citation}

Salehi Pour Mehr, Vahid, "Development and Verification of Control and Protection Strategies in Hybrid AC/DC Power Systems for Smart Grid Applications" (2012). FIU Electronic Theses and Dissertations. 804.

https://digitalcommons.fiu.edu/etd/804

This work is brought to you for free and open access by the University Graduate School at FIU Digital Commons. It has been accepted for inclusion in FIU Electronic Theses and Dissertations by an authorized administrator of FIU Digital Commons. For more information, please contact dcc@fiu.edu. 


\section{FLORIDA INTERNATIONAL UNIVERSITY \\ Miami, Florida}

\section{DEVELOPMENT AND VERIFICATION OF CONTROL AND PROTECTION STRATEGIES IN HYBRID AC/DC POWER SYSTEMS FOR SMART GRID APPLICATIONS}

A dissertation submitted in partial fulfillment of the requirements for the degree of DOCTOR OF PHILOSOPHY in ELECTRICAL ENGINEERING by

Vahid Salehi Pour Mehr 
To: Dean Amir Mirmiran

College of Engineering and Computing

This dissertation, written by Vahid Salehi Pour Mehr, and entitled Development and Verification of Control and Protection Strategies in Hybrid AC/DC Power Systems for Smart Grid Applications, having been approved in respect to style and intellectual content, is referred to you for judgment.

We have read this dissertation and recommend that it be approved.

$\begin{array}{r}\hline \text { Nezih Pala } \\ \hline \text { Jean Andrian } \\ \hline \text { BerrinTansel } \\ \hline \text { Stavros Georgakopoulos } \\ \hline \text { Osama Mohammed, Major Professor }\end{array}$

Date of Defense: November 2, 2012

The dissertation of Vahid Salehi Pour Mehr.

Dean Amir Mirmiran

College of Engineering and Computing

Dean Lakshmi N. Reddi

University Graduate School

Florida International University, 2012 
(C) Copyright 2012 by Vahid Salehi Pour Mehr

All rights reserved. 


\section{DEDICATION}

I would like to dedicate this dissertation to my wife, Maryam, who brought love into my life and my parents who always encourage and support me in all of my educational life. 


\section{ACKNOWLEDGMENTS}

I would like to express my gratitude to my advisor, Professor Osama Mohammed, whose expertise, understanding, and patience, added considerably to my graduate experience. I appreciate his vast knowledge, significant contribution and technical skills in many electrical engineering areas (e.g., power systems, electric machinery, magnetics,

power electronics and signal processing). I also appreciate his excellent ability and assistance in writing reports (including journal and conference papers, proposals, scholarship applications and this dissertation). These have made my work more technically and highly prestigious. He provided support at all levels of research including technical guidance and discussions, laboratory facilities and financial support for developing the smart grid test-bed at Florida International University.

I would like to thank the members of my committee, Dr. Jean Andrian, Dr. Nezih Pala, Dr. Berrin Tansil and Dr Stavros Georgakopoulos for the assistance they provided. Also, I would like to thank the external dissertation committee member, Dr. Mark Roberts from Naval Surface Warfare Center for the many technical discussions and for his practical insights.

I'd like to acknowledge the university graduate school for awarding the Doctoral Evidence Acquisition Fellowship (DEA) to me in 2012. It significantly enhanced my ability to collect the evidence needed for my doctoral research.

Finally, I'd like to acknowledge my doctoral student colleague, Ahmed Mohamed for his significant contribution and cooperation in my research and other doctoral students who put efforts in implementing and testing required equipment used for my dissertation. Also, I'm grateful for the help of undergraduate student interns who are 
involved in developing smart grid test-bed at Energy System Research Laboratory of FIU.

In addition, I recognize that this research would not have been possible without the partial financial support from the Office of Naval Research (ONR), and the US Department of Energy (DOE) as well as other sources brought to the ECE Department through my major research advisor. 


\author{
ABSTRACT OF THE DISSERTATION \\ DEVELOPMENT AND VERIFICATION OF CONTROL AND \\ PROTECTION STRATEGIES IN HYBRID AC/DC POWER \\ SYSTEMS FOR SMART GRID APPLICATIONS \\ by \\ Vahid Salehi Pour Mehr \\ Florida International University, 2012 \\ Miami, Florida \\ Professor Osama Mohammed, Major Professors
}

Modern power networks incorporate communications and information technology infrastructure into the electrical power system to create a smart grid in terms of control and operation. The smart grid enables real-time communication and control between consumers and utility companies allowing suppliers to optimize energy usage based on price preference and system technical issues. The smart grid design aims to provide overall power system monitoring, create protection and control strategies to maintain system performance, stability and security.

This dissertation contributed to the development of a unique and novel smart grid test-bed laboratory with integrated monitoring, protection and control systems. This testbed was used as a platform to test the smart grid operational ideas developed here. The implementation of this system in the real-time software creates an environment for studying, implementing and verifying novel control and protection schemes developed in this dissertation. Phasor measurement techniques were developed using the available Data Acquisition (DAQ) devices in order to monitor all points in the power system in real 
time. This provides a practical view of system parameter changes, system abnormal conditions and its stability and security information system. These developments provide valuable measurements for technical power system operators in the energy control centers. Phasor Measurement technology is an excellent solution for improving system planning, operation and energy trading in addition to enabling advanced applications in Wide Area Monitoring, Protection and Control (WAMPAC).

Moreover, a virtual protection system was developed and implemented in the smart grid laboratory with integrated functionality for wide area applications. Experiments and procedures were developed in the system in order to detect the system abnormal conditions and apply proper remedies to heal the system.

A design for DC microgrid was developed to integrate it to the AC system with appropriate control capability. This system represents realistic hybrid AC/DC microgrids connectivity to the $\mathrm{AC}$ side to study the use of such architecture in system operation to help remedy system abnormal conditions.

In addition, this dissertation explored the challenges and feasibility of the implementation of real-time system analysis features in order to monitor the system security and stability measures. These indices are measured experimentally during the operation of the developed hybrid AC/DC microgrids. Furthermore, a real-time optimal power flow system was implemented to optimally manage the power sharing between $\mathrm{AC}$ generators and DC side resources.

A study relating to real-time energy management algorithm in hybrid microgrids was performed to evaluate the effects of using energy storage resources and their use in mitigating heavy load impacts on system stability and operational security. 


\section{TABLE OF CONTENTS}

CHAPTER

PAGE

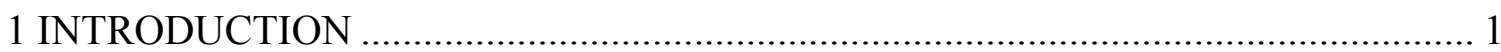

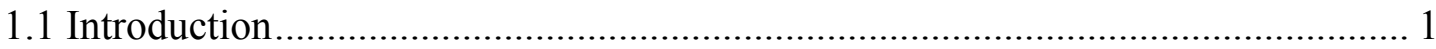

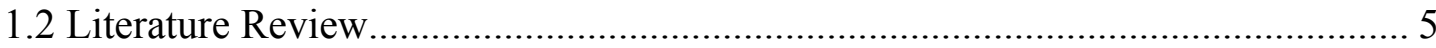

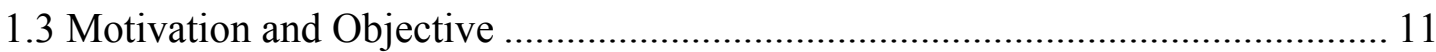

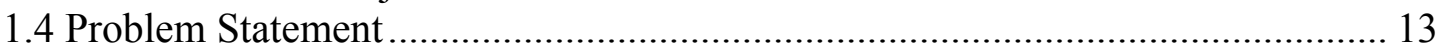

1.5 Original Contributions of the Dissertation.......................................................... 17

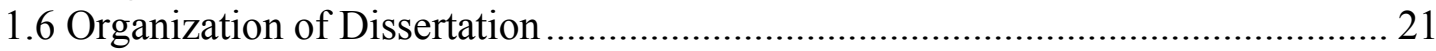

2 REVIEW OF POWER SYSTEM OPERATION AND CONTROL ............................... 25

2.1 Modern Hybrid AC/DC Power System ............................................................ 25

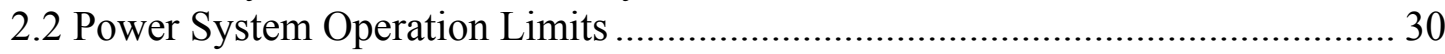

2.3 Cascading Events and System Blackout ............................................................ 33

2.4 Power System Monitoring with SCADA............................................................. 37

2.5 Wide Area Monitoring, Control and Protection .................................................. 39

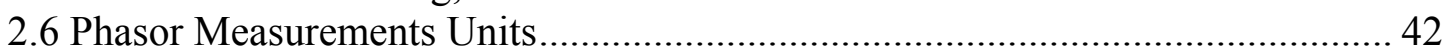

2.7 Applications of PMUs in Power System ……………….................................... 47

3 DEVELOPMENT OF SMART POWER SYSTEM TEST-BED ………………….....53

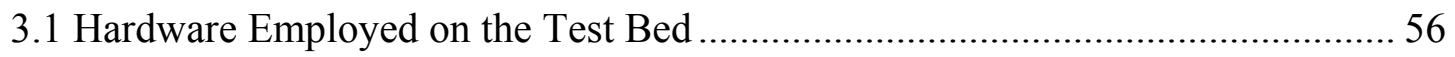

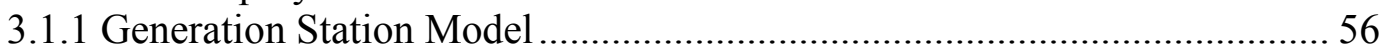

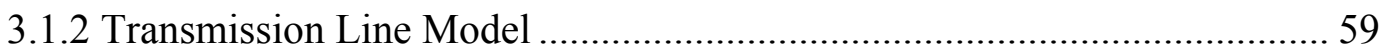

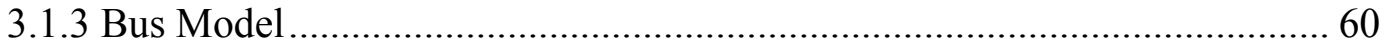

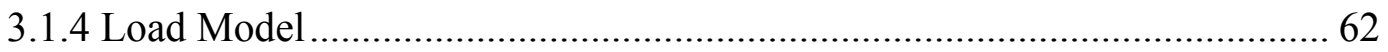

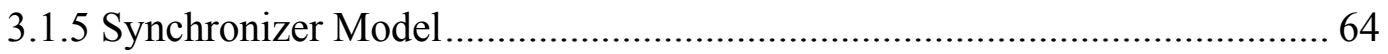

3.2 Real-Time System Monitoring and Control Platform ............................................ 65

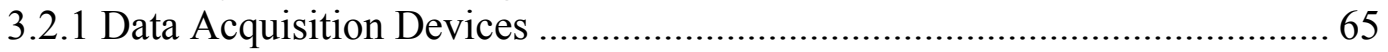

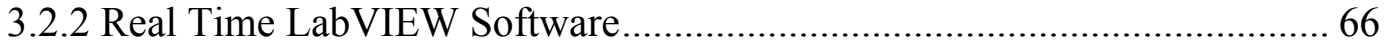

3.3 Test and Verification of Developed Techniques .................................................... 67

4 PHASOR MEASUREMENT UNITS AND THEIR APPLICATIONS IN SMART

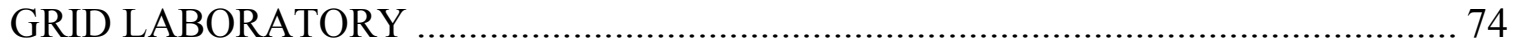

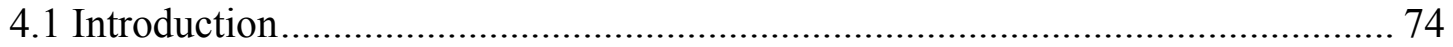

4.2 Measuring Synchronous Phasors ………………………………………….... 76

4.2.1 Phasor Representation of Sinusoids .......................................................... 76

4.2.2 Techniques for Signal Analysis ................................................................ 77

4.2.3 Phasor of Nominal Frequency Signals..................................................... 78

4.2.4 Nonrecursive Updates of Phasors .............................................................. 79

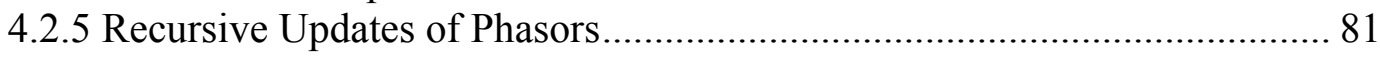

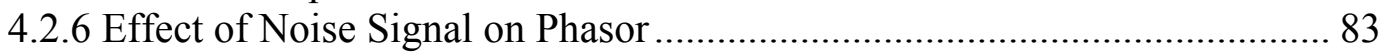

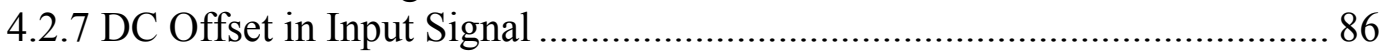

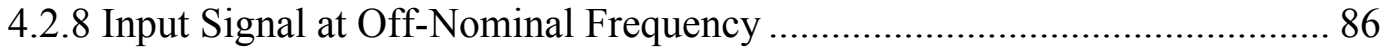


4.2.9 Frequency Estimated from Balance Three-Phase Inputs ........................... 88

4.3 Description of Laboratory Implementation of PMUs Setup .............................. 90

4.4 Real-Time Results of Developed PMUs ............................................................... 92

4.5 Description of Developed Industrial PMUs Setup............................................ 96

4.5.1 SEL-421 Protection, Automation and Control System.............................. 96

4.5.2 SEL-451 Protection, Automation and Control System.............................. 98

4.5.3 SEL-734 Meter................................................................................ 100

4.5.4 SEL-3530 Real-Time Automation Controller (RTAC) .......................... 101

4.5.5 SEL-2407 Satellite-Synchronized Clock .............................................. 102

5 DESIGN AND IMPLEMENTATION OF AN INTEGRATED WIDE AREA

MONITORING, CONTROL AND PROTECTION SYSTEM................................... 104

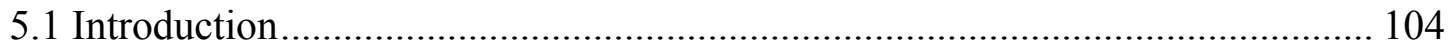

5.2 Developed Wide Area Monitoring Systems .................................................. 107

5.2.1 Real-Time Networked Model Parameters Measurement.......................... 109

5.2.2 Real-Time Voltage Stability Monitoring ............................................... 112

5.3 Developed Generation Control Scheme in Smart Grid Test-Bed ....................... 114

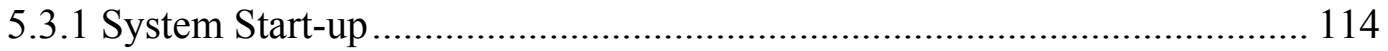

5.3.2 System Continuous Operation ............................................................ 118

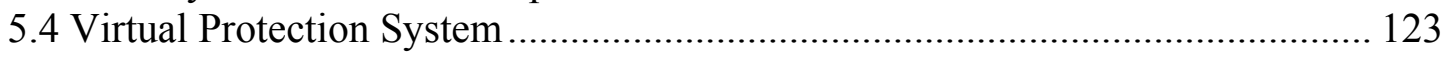

5.5 Design and Developing of Virtual Protection System .................................... 126

5.5.1 Over/Under Voltage and ROCOV Protection........................................... 127

5.5.2 Over/Under Frequency and ROCOF Protection ..................................... 128

5.5.3 Over-current Function....................................................................... 130

5.5.4 Distance Protection, MHO Characteristic............................................... 134

5.5.5 Distance Protection, Polygonal Characteristic........................................ 136

5.5.6 Voltage Unbalance Function................................................................... 139

5.5.7 Current Unbalance Function............................................................... 140

5.5.8 Inverse Power Flow Function ............................................................. 142

5.6 Test and Verification of Virtual Protection System in Test-bed........................ 143

5.7 Experiments for test of Wide Area Protection System in Test-bed .................... 147

6 DESIGN AND CONTROL OF HYBRID AC/DC SYSTEMS ............................... 151

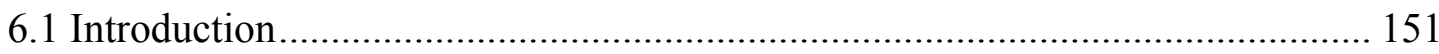

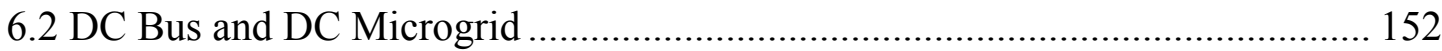

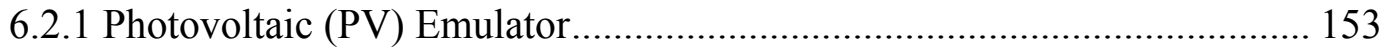

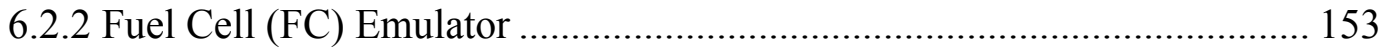

6.2.3 Controlled DC-DC Boost Converter...................................................... 154

6.2.4 Bi-directional DC-DC battery charger/discharger ................................. 155

6.2.5 Three-phase controlled AC-DC rectifier and bi-directional AC-DC......... 157

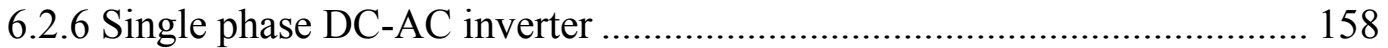

6.2.7 Dynamic DC Load emulator.............................................................. 159

6.3 Hybrid AC/DC System Architecture in Smart Grid Test-bed ........................... 160

6.4 DC Microgrid Operation; Experimental Results .......................................... 161

6.5 Integrated Hybrid AC/DC Operation; Experimental Results ........................... 165 


\section{IMPLEMENTATION OF REAL-TIME POWER SYSTEM SECURITY AND}

VOLTAGE STABILITY MONITORING SYSTEM ………………….................. 170

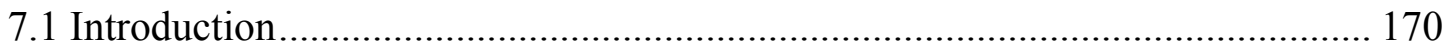

7.2 Modern Monitoring and Control Systems in Power Systems............................. 172

7.3 Developed Online Security Monitoring System; Experimental Results.............. 177

7.4 Voltage Stability Monitoring System on Smart Grid ......................................... 182

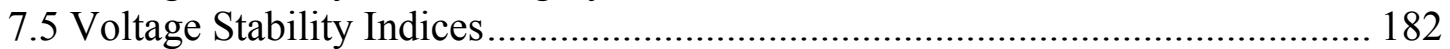

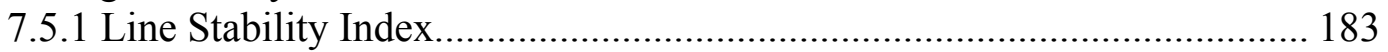

7.5.2 Fast Voltage Stability Index..................................................................... 184

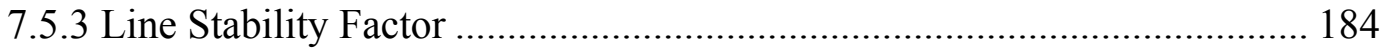

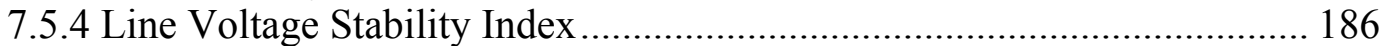

7.5.5 Linear Voltage Stability Indicator .......................................................... 186

7.5.6 The dS/dY Voltage Stability Sensitivity Factor........................................ 187

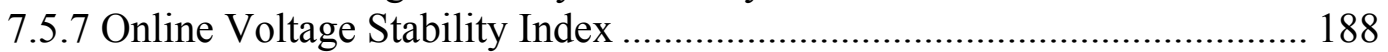

7.5.8 Transmission Line Marginal Loading..................................................... 189

7.6 Comparing Voltage Stability Indices By Simulation and Experiment ................ 192

7.6.1 Voltage Stability Indices in Steady State ................................................... 192

7.6.2 Voltage Stability Indices during Dynamic Load Change ........................... 195

8 ENERGY MANAGEMENT SCHEME FOR STABILITY AND SECURITY

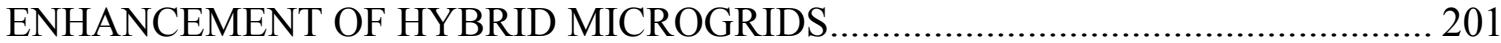

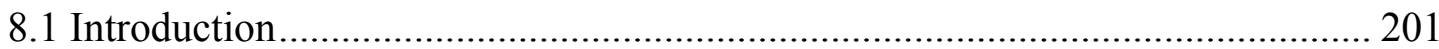

8.2 Stability Transient Simulation of Microgrids; Electric Ship Integrated Power

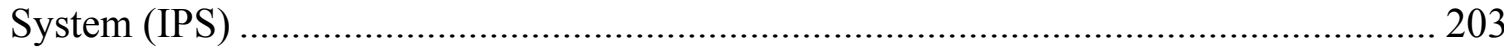

8.2.1 Single Pulse-load Simulation.................................................................... 205

8.2.2 Repeated Pulses (Train of Pulsed-load) Simulation ................................. 212

8.3 Dynamic Security Analysis of the Various Shipboard IPS Architectures Involving

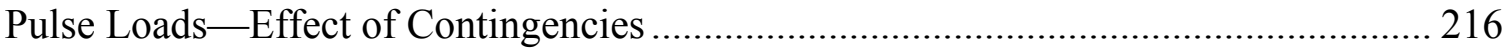

8.3.1 Outage of Main Components during Pulse Load...................................... 220

8.3.2 Effects of Storages Distribution during Pulse Load ................................. 222

8.4 Energy Management in Hybrid Microgrids ................................................... 235

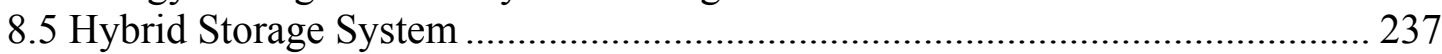

8.6 Real-Time Energy Management Algorithm ................................................... 238

8.6.1 Fully Charged Super Capacitors .............................................................. 239

8.6.2 Li-ion Batteries Support Super Capacitors …………………………….... 239

8.6.3 Supplying Normal Loads by PVs ........................................................ 240

8.6.4 Supply Loads with Battery during Peak Period........................................... 241

8.7 Testing the Real-Time Energy Management Algorithm .................................. 242

8.8 Power Sharing Control of Hybrid System during Generation Outage ................ 246

8.9 Real-time Optimal Power Flow Management System......................................... 249

8.10 Optimal Power Flow Techniques................................................................. 251

8.10.1 AC Optimization (Interior Point Method) ………..................................... 251

8.10.2 DC Optimization (Linear Programming) .................................................. 252

8.11 Real-time Control and Monitoring System of Hybrid AC/DC Microgrid........ 254 
8.12 Implementation of Real-time Optimal Power Flow.... 2588

8.13 Experimental Results of Real-Time OPF Management System....................... 260

9 CONCLUSIONS AND RECOMMENDATIONS FOR FUTURE RESEARCH ....... 266

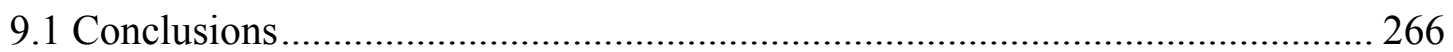

9.2 Recommendations for Future Work............................................................ 270

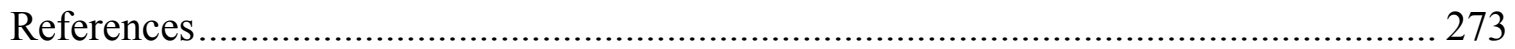

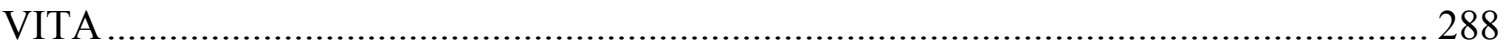




\section{LIST OF TABLES}

TABLE

PAGE

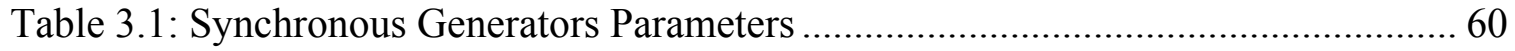

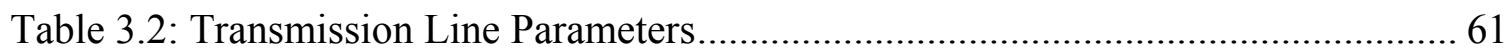

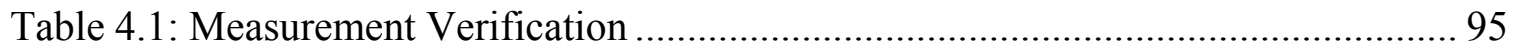

Table 5.1: Real-time Voltage Stability Indices........................................................ 113

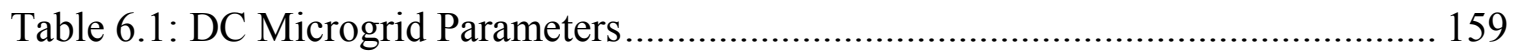

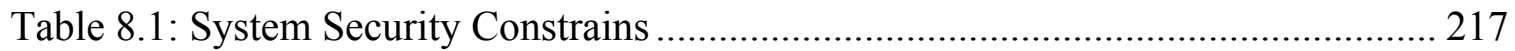

Table 8.2: Event List for Contingency Analysis.................................................... 220

Table 8.3: System security and stability assessment for studied cases........................ 235

Table 8.4: Different OPF Results for Real-time System Snapshot data ........................ 262 


\section{LIST OF FIGURES}

FIGURE

PAGE

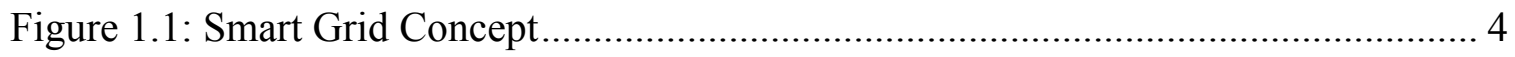

Figure 2.1: Typical Hybrid AC/DC Microgrid ......................................................... 27

Figure 2.2: Comparison between AC and DC distribution systems ............................. 30

Figure 2.3: Wide Area Monitoring, Protection and Control System (WAMPAC) ......... 42

Figure 2.4: Major elements of the modern PMU [90] …......................................... 43

Figure 2.5: Phasor measurement systems and levels of phasor data concentrators......... 45

Figure 2.6: Conceptual diagram of a power system with PMUs ................................. 48

Figure 2.7: Two bus system with PMUs.......................................................... 48

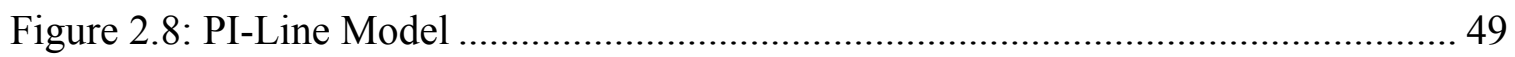

Figure 2.9: Time-Area Diagram of Different Protection Scheme ................................ 52

Figure 3.1: Generation station and its components.............................................. 58

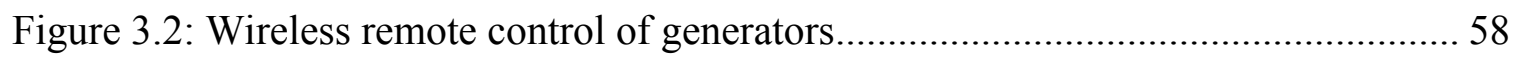

Figure 3.3: Overall schematic of a generator station and its measurements,

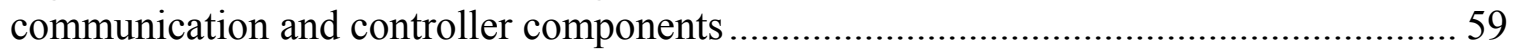

Figure 3.4: Single line diagram and developed box for power transmission line $\pi$-model

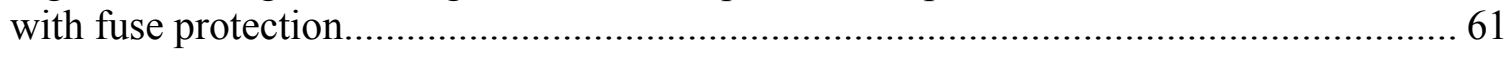

Figure 3.5: Implemented Bus Model with communication capability .............................. 61

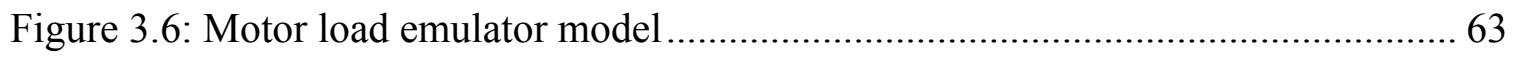

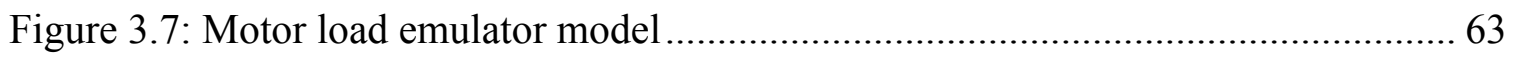

Figure 3.8: Synchronizer bus model with both side measurements............................ 65

Figure 3.9: Flowchart algorithm of the synchronizer developed in real-time software.... 65

Figure 3.10: Different types of DAQs and their connection to the Ethernet/LAN.......... 66 
Figure 3.11: Overall power system schematic and single line. 69

Figure 3.12: Test-bed power system communication infrastructure 69

Figure 3.13: Block diagram of a phasor and sequences calculation sub VI in the LabVIEW. 70

Figure 3.14: Front Panel of measurement sub VI in the LabVIEW ............................. 70

Figure 3.15: Implemented test-bed setup in power system lab................................. 71

Figure 3.16: Real-Time voltages of generator G1 in LabVIEW ................................. 72

Figure 3.17: Real-Time currents of generator G1 in LabVIEW ................................... 72

Figure 3.18: Voltages, currents and phasor diagram of generator G1 in measurement

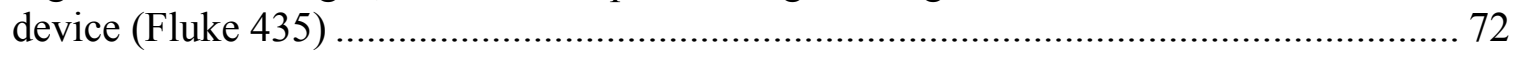

Figure 4.1: Different phasor measurement units (PMUs) .......................................... 75

Figure 4.2: A sinusoid and its representation as a phasor........................................... 76

Figure 4.3: Update of phasor estimates with $\mathrm{N}$ sample windows ............................... 81

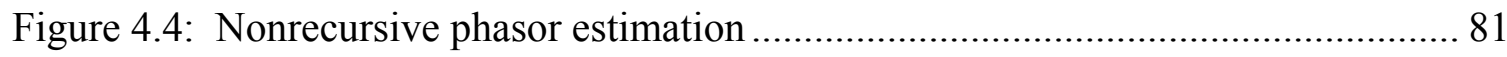

Figure 4.5: Recursive phasor estimation............................................................ 83

Figure 4.6: Single Line Diagram of Laboratory Test Setup in LabVIEW..................... 91

Figure 4.7: Overall View of Laboratory Test Setup ............................................. 91

Figure 4.8: Block Diagram of Implemented PMU ................................................ 92

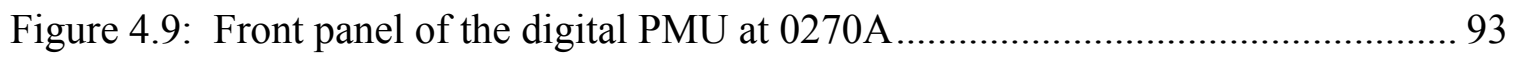

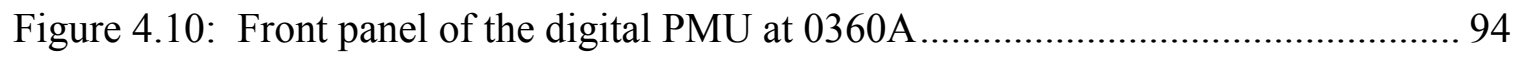

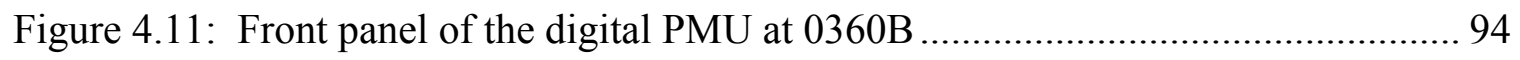

Figure 4.12: Result verifying by Fluke 43 ..................................................... 95

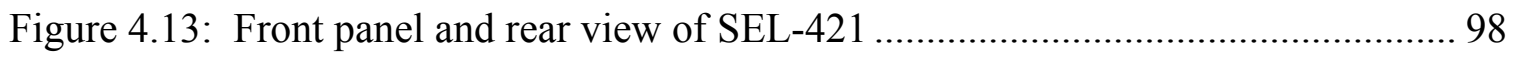

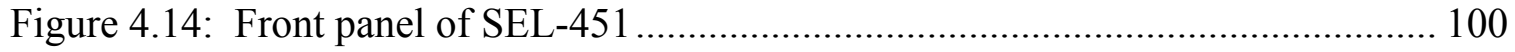


Figure 4.15: System Control and Synchrophasor Data Concentration 102

Figure 4.16: PMUs hardware architecture for implemented setup 103

Figure 5.1: Overall implemented power system and developed wide area monitoring, protection and control system 106

Figure 5.2: Synchronized phasor measurement system developed by real-time software 108

Figure 5.3: PMU placement for a transmission line and related pi-model 109

Figure 5.4: Voltages and Current magnitude of receiving and sending of the line during load increase. 111

Figure 5.5: Active and reactive power of the load at receiving end side..... 111

Figure 5.6: Measured line pi-model parameters during the experiment

Figure 5.7: Experimental results of online voltage stability indices monitoring..... 114

Figure 5.8: Overall view of real-time generation monitoring, communication and controller VI.

Figure 5.9: Synchronizers controller actions in order to connect generators to grid..... 117

Figure 5.10: Front panel of dynamic load to match the frequency of the generator side to Grid side.

Figure 5.11: (a) Active power and (b) Reactive power of generators during load increase in manual control

Figure 5.12: (a) Active power and (b) Reactive power of generators during load increase in automatic control

Figure 5.13: (a) Voltage and (b) frequency of generators during load increase in manual (upper plot) and automatic (lower plot) control mode.... 121 Figure 5.14: Overall implemented real-time system for integrated wide area monitoring, control and protection systems. 122

Figure 5.15: Conventional power system real-time monitoring, analysis and control procedure. 
Figure 5.16: Modern power system real-time monitoring, analysis and control procedure

Figure 5.17: Over/Under Voltage Function in LabVIEW real-time software ................ 128

Figure 5.18: Over/Under Frequency Function in LabVIEW real-time software............ 130

Figure 5.19: ANSI/IEEE characteristics for $\mathrm{Tp}=1$ and time inverse overcurrent

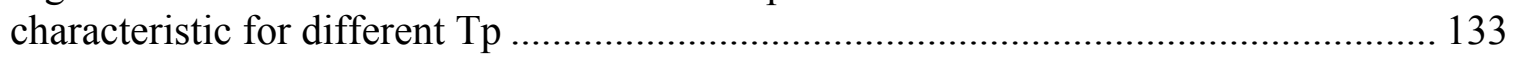

Figure 5.20: Over-current Function in LabVIEW real-time software .......................... 133

Figure 5.21: Over-current relay setting in LabVIEW real-time software ...................... 133

Figure 5.22: MHO impedance relay characteristic .............................................. 135

Figure 5.23: MHO zone definition and settings parameters ................................... 136

Figure 5.24: MHO Distance relay in LabVIEW real-time software ............................ 136

Figure 5.25: Polygonal zone definition and settings parameters ................................ 138

Figure 5.26: Polygonal Distance relay in LabVIEW real-time software....................... 138

Figure 5.27: Distance relay setting in LabVIEW real-time software ........................... 139

Figure 5.28: Voltage Unbalance Function in LabVIEW real-time software ................. 140

Figure 5.29: Current Unbalance Function in LabVIEW real-time software ................. 142

Figure 5.30: Inverse Power Flow Function in LabVIEW real-time software................ 142

Figure 5.31: Digital generic relay model implemented at real-time software ............... 143

Figure 5.32: Single-phase fault at motor terminal and relay tripping action ................ 144

Figure 5.33: Generic relay tripping action for single phase to ground fault................ 145

Figure 5.34: Single-phase outage for motor and relay tripping action ....................... 146

Figure 5.35: Using generic relay for system wide area monitoring, protection and control 147

Figure 5.36: (a) Relays trip signal and (b) breaker status of all generators during G1 outage. 
Figure 5.37: (a) Active power and (b) frequency of all generators during G1 outage .. 149

Figure 6.1: DC network schematic interconnected to AC side and DC source and load emulators 152

Figure 6.2: Output voltage from the fuel cell emulator 154

Figure 6.3: A block diagram of the DC-DC boost converter controller 155

Figure 6.4: A block diagram of the Bi-directional battery charger controller ..... 157

Figure 6.5: Operation of the bi-directional converter as a reactive power compensator 158

Figure 6.6: Output power of the dynamic load emulator 160

Figure 6.7: Overall power system schematic and single line diagram of implemented hybrid AC/DC setup 162

Figure 6.8: A flow chart of the real-time algorithm to manage the charge/discharge process of batteries. 163

Figure 6.9: Performance of the various components of the DC microgrid corresponding to step change in the power reference. 164

Figure 6.10: Performance of the integrated hybrid AC/DC microgrid corresponding to step change in the load demand reference.

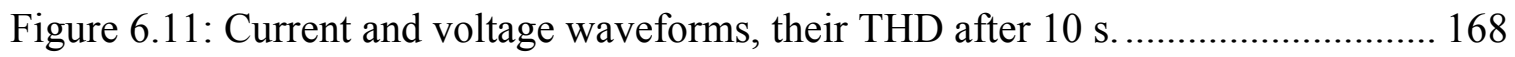

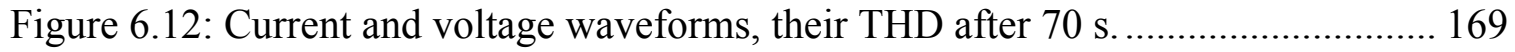

Figure 7.1: Power system time frame for phenomena, control actions and studies....... 172

Figure 7.2: single line of the setup for implementing online security assessment ........ 174

Figure 7.3: Front panel of measurement VI with all system parameters ..................... 176

Figure 7.4: Implemented fast real-time SCADA system with WAMPAC capability in

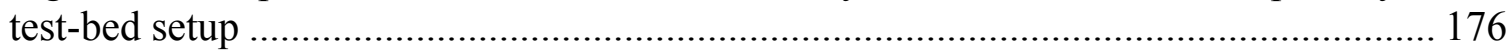

Figure 7.5: General sequence for contingency assessment and provided results for both

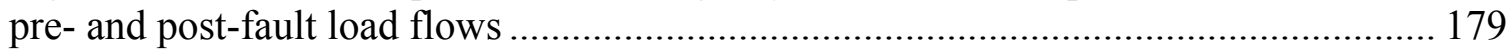

Figure 7.6: Contingency analysis typical reports............................................... 180 
Figure 7.8: Reciving end active and reactive load power

Figure 7.7: Single Line Diagram and the test Setup for implementing Voltage Stability

Indices

Figure 7.9: Voltage stability indices in steady state 194

Figure 7.10: Experimental results of load active and reactive power 195

Figure 7.11: Experimental results of two sides voltage and current amplitude. 196

Figure 7.12: Experimental results of line parameters 196

Figure 7.13: Experimental results of monitored voltage stability indices . 198

Figure 7.14: Simulation results of voltage stability indices 199

Figure 8.1: Ship power system single line 205

Figure 8.2: Pulsed Load power and generator active power behavior 207

Figure 8.3: Main AC buses and DC buses voltage during pulse-load 208

Figure 8.4: Power-Delta curve behavior during Pulsed Load. 208

Figure 8.5: Power-Delta Curve for main generators during Pulsed Load 209

Figure 8.6: Main buses voltages and frequencies during pulsed Load ........................ 210

Figure 8.7: Frequency and voltage variation for 1 second Pulsed load..... 211

Figure 8.8: Voltage and active power of Induction generator for 1 second pulsed Load212 Figure 8.9: Pulsed train load and generators active power. 213

Figure 8.10: Main buses frequency and voltage behavior for train pulse-load 214

Figure 8.11: Power-Delta curve for train pulse-load for two main generators 214

Figure 8.12: Main buses frequencies and voltages during fast pulse train load 215

Figure 8.13: The IPS system with zonal load distribution in Normal Condition and Pulse Loadccurance 218 
Figure 8.14: Contingency events, Cable 1-2 outage (an open ring, close to radial, architecture)

Figure 8.15: Contingency events, Cable 1-4outage (an open ring, close to radial, architecture)

Figure 8.16: Contingency events of MTG2 outage 225

Figure 8.17: Contingency events of MTG2 outage with distributed storages in all zones

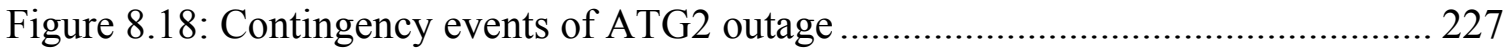

Figure 8.19: Contingency events of PMSM outage................................................ 228

Figure 8.20: Pulse Load and Storage at Zone 1 ..................................................... 230

Figure 8.21: Pulse Load at Zone 1 and Storage at zone 1 ........................................ 231

Figure 8.22: Pulse Load at Zone 1 and storage distributed between Zones 1,4 ........... 232

Figure 8.23: Pulse Load at Zone 1 and Storage distributed between Zones 1,2,3\&4.... 233

Figure 8.24: Pulse Load at Zone 1 without any storage ......................................... 234

Figure 8.25: Battery/SC hybrid storage system: (a) Passive hybrid, (b) Active hybrid. 239

Figure 8.26: Operation of the system for a 24 hours interval, while applying Algorithms 1

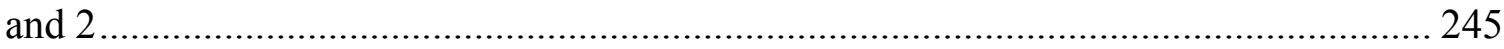

Figure 8.27: Generators active and reactive power during G3 outage and DC side power

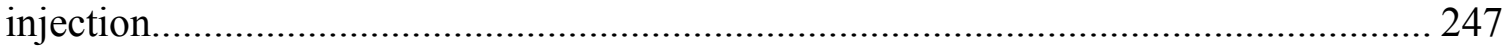

Figure 8.28: Generators voltage and frequency during G3 outage and DC side power

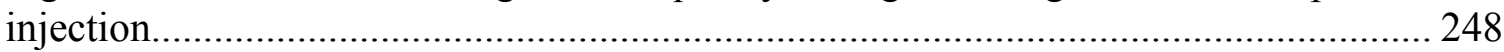

Figure 8.29: Overall power system single line diagram of implemented setup in the real-

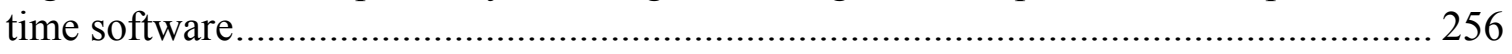

Figure 8.30: Experimental input torque-output power curves for generators................ 256

Figure 8.31: Implemented overall real-time SCADA system with WAMPAC capability in

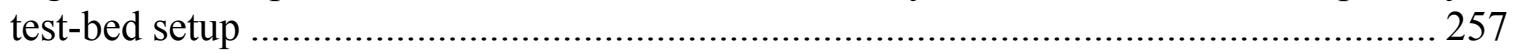

Figure 8.32: Overall power system single line diagram of implemented setup............. 259 
Figure 8.33: Real-time system load flow results for a specific system snapshot

Figure 8.34: Real-time system optimal power results for a same system snapshot using

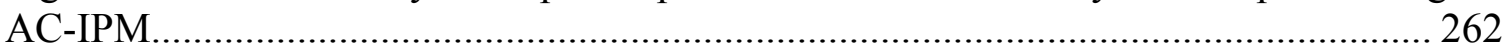

Figure 8.35: Real-time Monitoring and Control System of AC-Side........................... 263

Figure 8.36: DC Side Equipments and Real-time Controller ...................................... 263 


\section{CHAPTER 1}

\section{INTRODUCTION}

\subsection{Introduction}

Power system planners need to operate the power system under increasingly complex conditions. The high penetration of renewable generation and demand impose an immense challenge to the operation of the electric grid. With these challenging conditions being introduced into the power system, the overall system operation requires new and innovative methodologies in order to enhance the power system performance [1], [2]. Modern power networks incorporate communications and information technology infrastructures into the electrical power system to create a smart grid in terms of operation. The smart grid is a power system that enables real-time communication and control between the consumer and utility allowing suppliers to optimize energy usage based on environmental, price preferences and system technical issues. The smart grid will utilize digital information technology to create more efficient, reliable and responsive networks that are flexible [3]-[5]. The communication capability is one of the potential benefits for digital relays, which communicate not only with a control center, but also with each other in a two-way manner. This in turn will facilitate the overall system-wide area protection and control philosophy. The smart grid designs aims are to provide overall power system monitoring, create control strategies to maintain system performance and security and reduce the cost of operation, maintenance and system availability planning. The smart grid control gives us capabilities such as: predicting system behavior, anticipatory operation and adaptation to new environment, handling 
distributed resource, stochastic demand and optimal response to smart appliances [6]. The smart grid is considered to have observability with all interconnected nodes with data integration and analysis to support advances in system operation and control. The selfmanaging and reliable smart grid is seen as the future of protection and control systems [7]. This philosophy requires finding a way to implement it in the laboratory scale and then in the real power system.

While many smart grid topics were identified in the literature, there are some illustrations of the topics involved in developing new smart grid ideas and a test-bed platform to test these ideas [8]-[20]. These include:

- Phasor measurement units and their application in power systems for improved monitoring, protection and control.

- Integration of distributed energy sources including renewable resources (wind, solar, fuel cell, etc) in power generation and their fully controlled structure.

- Intelligent protection schemes and their application in detecting, mitigating and preventing cascading outages, islanding situation and total grid blackout occurrences.

- Integration of Hybrid AC-DC systems creating new micro grid solutions for residential and industrial applications.

- Real-time or dynamic energy management and demand side management based on renewable uncertainty.

- Reactive power support and distributed voltage control, remote control of FACTS and HVDC devices to enhance overall system performance and 
islanding detection of a local and wide-area power system and develop proper separation actions.

Due to the power fluctuation of renewable energy sources, voltage and frequency deviations are occurred in the end user side and islanded power systems whose ability to maintain stable supply-demand balance is low. Therefore, it is necessary to control the system frequency and voltage at the supply side despite the control limitations. Therefore, mutual cooperation control between the generation and the demand sides is required because it is difficult to maintain the power system quality and security by only the generation side control. Technically, a smart grid, which maintains generation-load balance by monitoring system key parameters and load side information, is necessary for future electric power systems. It is expected that smart technology will lead to reduced investment in equipment and an increase higher availability of power generation. Figure 1.1 shows the smart concept of power grids which uses two-way communication between control center and all power system general components.

Power and energy systems researchers need to develop a platform for smart grid technology to study and identify the issues involved in their operation. This approach should include objectives such as visualization enhancement of the power system, realtime analysis for wide-area network, model validation, developing strategies for widearea monitoring, protection and control system (WAMPAC). Nowadays, modern technologies in the communication infrastructure lead to their high accuracy, security and data transfer speed. Hence, the power system uses these new and updated facilities 


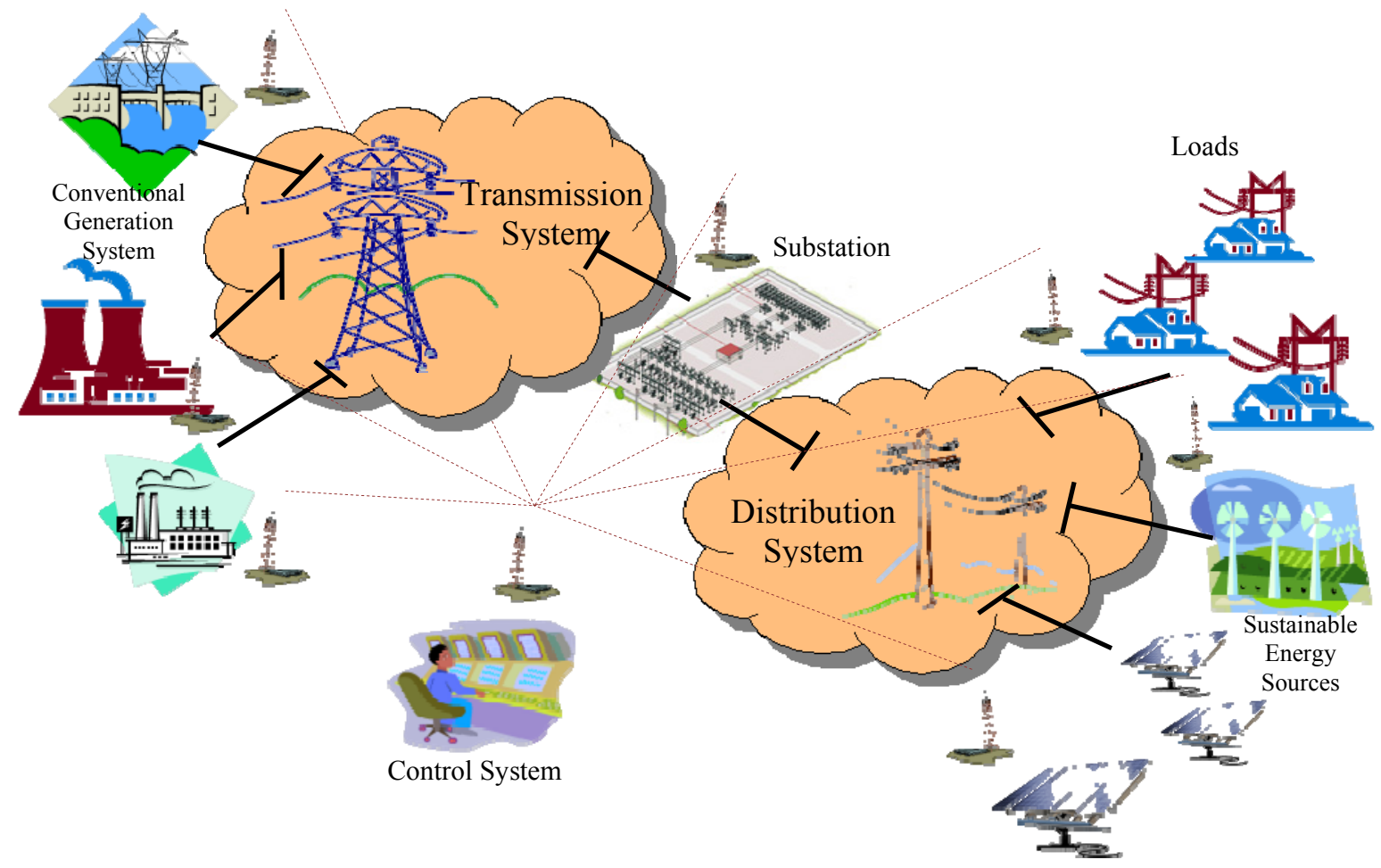

Figure 1.1: Smart Grid Concept

to create a communication infrastructure for wide-area power system in order to improve grid observability and control flexibility. This infrastructure is responsible for fast data exchange among the system components and the control centers [2] and [5]. Phasor Measurement Units (PMUs) are the most recent technology to measure power system parameters accurately and in time-synchronized format. They measure the system main parameters such as voltage and current phasors and frequency information which are synchronized to a common time reference with a high level of precision. Furthermore, protection and control solutions based on synchrophasors for wide-area applications are being introduced as a preventive or self-healed system for frequency, voltage and rotor angle instabilities. The system data are collected by the PMUs and are sent to a phasor data concentrator (PDC). The PDCs, in turn, send the system data to a control center [24]. The PMU-based state measurement is expected to be more efficient than the present state 
estimation since synchronized phasor signals provide the state variables, in particular, the voltage angles. A successful protection and control application requires a carefully designed WAMPAC architecture, including thoroughly considered latency (Communication Delay) [25]. The concept of wide-area monitoring, control and protection may be used as a platform for more flexible or adaptive protection and control strategies, having a better management of disturbances and higher power transfers and operating economies. On the basis of a modern wide-area monitoring system, advanced protection and control strategies can be applied through development and implementation of new analytical tools and extensive study procedures. Clear understanding of the requirements for large-scale WAMPAC systems and designing a system to meet the requirements are essential for achieving the cost/benefits objectives and the deployments success [25]. It is expected that WAMPAC system will, in the near future, reduce the number of cascaded events (failure or blackouts) and will improve the reliability and security of energy production, especially in power systems which has operational uncertainties.

\subsection{Literature Review}

In recent years, trends toward diversify the electricity market and the increased importance of environmental issues have led to diversification of power system configurations. This is occurring through the installation of distributed power sources based on photovoltaic and wind power generation technologies in addition to other resources. Under these circumstances, there is concern regarding the effect on the stability and reliability of power networks with respect to issues such as overload, voltage 
and frequency variation and harmonic distortion. Against this background, there has been an increased focus on the concept of Smart Grid, which has the capability of collecting wide-area power system information on a real-time basis from intelligent power grids using information and communication technology [26]. Power and energy systems researchers need to develop a platform for smart grid technology to study and identify the issues involved in their operation. Such approaches should include objectives including visualization enhancement of the power system, real-time analysis for wide area network, model validation, developing strategies for wide area monitoring, protection and control system (WAMPAC) [52]-[54]. Moreover, concerns related to the use of renewable generation, energy storage, demand response and electric vehicles introduce further complexity to system operation and control [1]. Recent technologies enhanced the communication structure of power system by its high accuracy, security and data transfer speed. Hence, the smart grid uses modern communication infrastructure at wide area power system to improve grid reliability, reduce the price of electricity, improve operational efficiency, security and safety and promote environmental quality [2],[27][28]. The communication infrastructure is responsible for fast data exchange among the system components and control centers [5], [23]. Currently, Phasor Measurement Units (PMUs) are the most accurate and advanced time-synchronized technology available. They provide voltage and current phasors and frequency information, synchronized to a common time reference with high precision. Therefore, wide area measurement system based on synchrophasors for wide area applications are being introduced as a preventive or self-healed system for frequency, voltage and rotor angle instabilities [69]-[74]. Hence references [56]-[68] present innovative and novel methods for control and protection of 
electrical grids in wide area scales. The system data is collected by the PMUs and is sent to a phasor data concentrator (PDC). The PDCs, in turn, send the system data to a control center [24]. The PMU-based state measurement is expected to be more efficient than the present state estimation since synchronized phasor signal provide the state variables, in particular, the voltage angles. Designing an integrated protection and control system with the modern and fast communication layer is a new challenge of power system engineering. These systems will reduce the system outages due to the cascaded events which may cause an overall outage or the system blackout. Furthermore, these systems will improve the reliability and security of energy production, especially in power grids integrated with operational uncertainties.

The smart grid was first introduced in past decade and in generally it refers to a class of technology people are using to enhance and modernize utility electricity delivery systems, using computer-based remote control and automation. These systems are made possible by two-way communication technology and computer processing that has been used for decades in other industries. [30] Recently, federal government investment on this subject cause the concept has now moved from pilot projects to the full-scale deployments [29]. Energy research organizations have made considerable progress in formulation and promoting a vision for the future smart power grids [31]-[39]. References [34] and [35] present smart grids for future power delivery. Reference [36] discusses the integration issues in smart grid. Reference [37] presents interesting and promising concepts in smart grid architecture. Specific technologies such as smart metering infrastructure were presented in [38]. The design of such grid will be based on cross boundaries of knowledge in communication theory, optimization, control, social 
and environmental constraints as well as dynamic optimization techniques [10]. Most of the referenced literatures attempted to create a roadmap for studying smart grid issues in terms of implementation and operation. However, the great concern is that they works are presenting wide and diverse topics which are general and not clear enough. Such clarity is needed for engineers and researchers to approach the smart grid aims and see the advantages of making the conventional power system as an intelligent grid. Therefore, in this dissertation we will implement several smart grid concepts and test the related studies in a realistic manner. Also, explanation of practical insights of the developed procedures will help us to present the feasibility of the methods covered in the literatures.

Technically, international standards such as IEC 61850 and 61968, define the overall architecture of a Smart Grid and its characteristics can be summarized as follows [11]:

- Self-healing by detecting and instantly responding to system problems and restoring

- Provision of high power quality to all consumers and industrial customers

- Accommodating a wide variety of generation options in local and regional scale

- Empowerment of the customer by allowing energy management

- Tolerance of attack by standing resilient to physical and cyber attacks

- Optimizing assets and operating efficiency

Many countries have been trying to develop the Smart Grid technologies by performing some projects as test-beds with different points of view. In the US, there are some projects such as GridWise, GridWorks [40] with plans to expand the results to 
focus on improving the efficiency of its legacy transmission and distribution networks. This project aims to give consumers more information about energy use and cost to enable them to identify the benefits. It will also test and promote technologies designed to make the power system more reliable.

The European Union has Smart Grid projects [41], while focusing mainly on suitably adopting renewable energy resources. In this perspective, the main goal of this study is to prepare a comprehensive inventory of Smart Grid projects in Europe and use project data to support the analysis of trends and developments. This report looks into several aspects of the Smart Grids landscape to describe the state of the art of their implementation, the emerging hallmarks of the new electricity system and some foreseeable developments.

Canada is running integration of decentralized Energy Program [42]. In addition, "Smart Grid CANADA" which is a national organization dedicated to promoting a more modern and efficient electricity grid for the benefit of all Canadians, creates Canada's smart Grid Repository. This reference identify that by exploring Canada's smart grid projects, this system defines the principal gateway connecting the national smart grid community to relevant sources of information in one centralized location.

In Korea, a project called K-Grid has been launched to design a highly integrated Korean Smart Grid System [43]. This project aims to explore projects on the development of technology that encompasses the convergence of electric power and IT.

Hybrid power systems are combinations of AC and DC systems. Such a system has two or more energy conversion devices (e.g., electricity resources generators or storage devices), that when integrated, could be used to overcome limitations that may be 
inherent in either. System efficiencies are typically higher than the case of utilizing single resource. Higher reliability can be accomplished with redundant technologies and/or energy storage systems used in conjunction with $\mathrm{AC} / \mathrm{DC}$ infrastructures. Some hybrid AC/DC systems include both, which can simultaneously improve the quality and availability of power. Since energy management, control and operation of a hybrid grid are more complicated than those of an individual AC or DC grid, various operating modes of a hybrid AC/DC grid were investigated in [75]-[83]. These references investigate microgrids coordinated control between $\mathrm{AC}$ and DC sides and their active load-generation control by intelligent methods. The microgrid and system coupling in addition to interconnection issues are studied as well. Defining protection principals and strategies in microgrids are also discussed in these references.

The coordination control schemes among various converters are proposed to harness maximum power from renewable power sources. It is also used to minimize power transfer between $\mathrm{AC}$ and $\mathrm{DC}$ networks as well as maintain the stable operation of both AC and DC grids under variable supply and demand conditions when the hybrid grid operates in both grid-tied and islanding modes. Advanced power electronics and control technologies used will make the future power grid much smarter. A power management strategy is necessary for the hybrid $\mathrm{AC} / \mathrm{DC}$ power system to manage the power flow in order to satisfy the load requirements [44]. Hybrid power systems require a much more complex control and protection scheme that must ensure efficient and robust power transfer from the sources without risks of their rapidly degraded reliability due to prolonged over-current and/or under-voltage conditions. Therefore, rather than achieving a single voltage/current regulation goal at the output, the control system must regulate 
these power converters to balance the power flow of these energy sources so as to satisfy the load requirements while ensuring the various limitations of electrochemical components such as the battery overcharge limit, limited PV output power and etc. [45]. A hybrid smart AC/DC power system and its control strategies were proposed in [46] and the impact of power quality is taken into consideration from the view point of generator side, a grid side and a demand side management in [47] and [48] and power quality management for smart grids is proposed in [49]. However, these types of controls are not real time and comprehensive for overall power system.

In addition intelligent protection relays for smart grid were also presented in [50] and [51]. These works proposed the concepts of intelligent protection relay systems for Smart Grid and have developed a prototype relay based on these concepts. The results of the field trial of this system demonstrate that such a system has the capability of evaluating the power system characteristics in detail on a real-time basis and contributing toward supervision, coordination check and optimization of setting values. Moreover, these works did show that an agent system can be applied under practical conditions. The main conclusion in these references identify that the development of real-time protection for smart grid applications is necessary and it should be as an integrated protectioncontrol system talking into consideration all economic and operational issue in real-time.

\subsection{Motivation and Objective}

The motivation of this research is to develop the ideas for smart grid operation and create a platform to implement and verify these ideas. This hardware/software based system includes implementation of control strategies for generating stations and power 
transfer to programmable loads in a laboratory scale of up to $35 \mathrm{~kW}$ in AC power and 36 $\mathrm{kW}$ in renewable sources and energy storages. Appropriate software need to be developed to monitor all system parameters as well as operate and control the various interconnected components in varying connectivity architectures. The interconnection of alternating energy such as wind emulators, PV arrays and Fuel cell emulators also need to be implemented and integrated into this system. Implementation of the real-time operation and analysis capability provides a platform for investigation of many challenging aspects of a real smart power system. In addition there is a big concern about the implementation of the monitoring, control and protection of the system in real-time environment which will be addressed by this research.

Therefore, the aim of this research is to apply the concept of real-time analysis of a smart grid and implement it on a smart grid test-bed. The implemented system in this dissertation will have many capabilities to perform experimental research and studies. The test-bed uses laboratory scale components in addition to commercial hardware to create a microgrid setup for modeling the realistic behavior of a large power system.

As mentioned before, the main objective of this dissertation is to use real-time measurements in smart grids to identify the power system states. Online power system analysis feature are developed to deploy real-time data in order to monitor system state with online optimal load flow and security assessment results. The developed software environment to monitor real-time data as a SCADA center will be discussed. This realtime software has the capability to run analysis software in engine mode and hence the system online analysis can be implemented in real-time data. In this setup, the monitoring system updates the SCADA system approximately every one second and then the 
analysis software can update the data immediately after and analyze the system situation with the help of monitoring and alarm systems. Consequently, this system can analyze a snapshot of the power systems in less than three seconds and return the real-time calculation results. Studies related to the time range of phenomena and control actions has also been implemented and an online system power flow and security assessment was developed. In addition a real-time optimal power flow management system has been developed for smart microgrid. The commercial Power Factory Package was used to communicate with real-time LabVIEW software in order to receive system data and utilize real-time data in online mode to calculate the Optimal Power Flow (OPF) and return the optimal schedule of generators and reactive power demand from DC side by the available communication link.

\subsection{Problem Statement}

The increased complexities in operating the power grid have emphasized the need for grid revitalization. Advanced applications in wide area monitoring, protection and control (WAMPAC) systems offer solutions to improve system planning, operation, maintenance and energy trading. Synchronized measurement technology and applications are important element and is enabler of WAMPAC.

Time synchronization is not a new concept or a new application in power systems. As power system and telecommunication technologies have advanced, the time frame of measured and communicated synchronized information has steadily been reduced from minutes, to seconds, milliseconds and now microseconds. At present, phasor measurement units (PMUs) are the most accurate and advanced time-synchronized 
technology available to power engineers and system operators for WAMPAC applications. This technology was made possible by advancements in computer and processing technologies and the availability of GPS signals. We are approaching a time where all metering devices will be time synchronized with high precision and accurate time tags as part of any measurement. To achieve the potential benefits, advancements in time synchronization must be matched by advancements in other areas. One example is data communications, where communication channels have become faster and more reliable in streaming PMU data from remote sites to a central facility.

While a number of applications based on GPS-synchronized data have already been developed, there is a need for researchers to continue improving existing and developing applications using the latest visualization techniques, intelligent operational tools, advanced algorithms and information semantics to improve grid reliability under complicated power system conditions. This analysis also helps identify policy, economic and technology barriers to commercial deployment.

Implementing a WAMPAC system presents some unique challenges. These challenges are technical, such as system data transmission, process, analyze and store vast amounts of data. The ideal situation for using fast PMUs data and designing realtime control centers for power system should properly address the following issues:

- As the number of installed PMUs is increasing, the system architecture must be designed in a way to keep the system operation more advanced and flexible to change quickly the strategies toward system performance enhancement. 
- As many of the system components will be installed, operated and maintained by different utilities, the system architecture should be flexible to accommodate the diverse requirements of the power system. Therefore, the designed system should be compatible with any operating system.

- The modern and novel communications should be applied to the power system in order to increase the data communication bandwidth and monitoring latency. Reducing the bandwidth requirements will help to reduce the ongoing cost of PMUs applications. It will also help to reduce the latency of the PMU data transferring. For real-time applications, reducing the communication latency is an essential factor.

- A roadmap should be developed to include applications that guide PMU installations and system architecture to enhance system observability and controllability locally and regionally.

- Data exchange between customers and utilities and developing of the overall system infrastructures are based on system requirements. All customers connecting to the overall system would need to fulfill key integration requirements (hardware, software and communication interoperability, data quality, etc). It is also beneficial to prioritize applications from the grid perspective.

- The consistent and accurate performance of all PMUs is the key to the overall performance of the system. Developing uniform requirements and protocols for data collection, communications, interoperability and security through standards (NERC, IEEE) and testing procedures. Assure 
ease of PMU upgrades (particularly considering the novelty of the IEEE C37.118 standard) and calibration.

- Developing and promoting the new applications for integrated real-time monitoring and control systems in order to enhance system stability, security and reliability.

- Compared to current EMS monitoring tools that use information from state estimation and SCADA over several-second intervals, WAMPAC introduce the possibility of directly measuring the system state instead of estimating it based on system models and telemetry data. With proper implementation, the new technology also allows us providing data integrity validation without added cost.

- WAMPAC should be used for early warning systems, system integrity protection scheme, detecting and analyzing system stability and enabling faster system restoration.

- Faster and more accurate analysis of a vast number of data during transient events by validation and development of power system models should be addressed by WAMPAC.

The above issues should be studied through comprehensive analysis of various applications and related advantages of WAMPAC. Based on implementing WAMPAC, we need to apply novel and innovative controller and protection strategies for power system which maintains system normal operation under different scenarios which may occur. The use of intelligent and integrated smart controllers are necessary to detect network change in topology and load and generation variations which are all related to 
the normal operation of power system. In other word, we need to find a solution for controlling power system in a proper manner to avoid exceeding production cost, loosing stability, voltage and frequency drop and so on. As a technical example, in case of adding sustainable energy resource in any place of grid, the fast monitoring system should detect this new power source and then the smart control and protection system should change the production level as well as network topology in order to achieve all power system requirements. In this case, it may comply removing costly production sources, changing production control settings to maintain frequency and voltage considerations. The control options in smart control method will be accomplished through switching strategies and by changing the energy production level to keep the voltage and frequency to secure whole power system or in a microgrid.

\subsection{Original Contributions of the Dissertation}

In this research a unique and novel hardware/software based Smart Grid test-bed facility was developed in a laboratory environment as a testing environment to verify the concepts developed in this dissertation. The developed system was the product of a number of researchers at the Energy Systems Research Laboratory, Florida International University. This hardware test-bed is capable of studying and developing real-time automated power system network operation and control strategies which is the focus of this dissertation. In addition the developed test-bed system was used as a platform to study, test and implement grid connectivity issues of alternate energy sources and validate power sharing and energy storage ideas. The developed system was achieved by integrating of different hardware devices and emulators. The communication interfaces 
between these devices and their operation in a wide area network infrastructure. The size of the presented system is significant enough reaching a $72 \mathrm{~kW}$ level of combined $\mathrm{AC}$, alternate generation and energy storage assets on the AC and DC sides of the system. The real-time analysis capability of the developed system provides a platform for investigation of the most challenging aspects of operating a real power system. This is one of the main focuses of this dissertation.

In addition, an integrated wide area system in the laboratory-scale Smart Grid test-bed, with capabilities for WAMS, WACS and WAPS, was developed for this dissertation. The implementation of this system in the real-time software creates an environment for studying and verifying the control and protection schemes for the $\mathrm{AC}$ side of this power system. Verification experiments were developed and implemented on the test-bed to verify the system performance and its capabilities.

This system was used for studying integrated wide area control and protection system to monitor the system status for abnormalities such as over/under voltage, overloads of equipment and any other conditions. In the implemented example, when the loadings on a power system line increases, the relay would indicate that the system is in moving condition to a fault situation prior to actual condition. This might be by disconnecting that part of the system by available protection devices. Hence by setting the protection relay under the settings of the physical relay, the control scheme can retrieve the normal status by a proper control action. This case may be the changing of system topology or power dispatching in a control center. Self healing strategies can maintain the system continuous operation with appropriate control scheme. A 
contribution of this research is the development of a real-time platform to monitor, control and protect the system by integrated design and implementation of WAMPAC.

The developed WAMPAC real-time system was used to monitor the real-time system stability and security margin. This system investigates the appropriate indices, formulate them and define their implementation on wide area monitoring and the test-bed control center. These developments are presented in this dissertation to provide a practical view of system stability and security margin using PMUs in wide area networks. Furthermore, voltage stability indices were formulated and developed as well as measured during the operation of the power system when load changes are taking place. Testing these indices practically in real-time and in different circumstances provides the new insight of stability issues which are accomplished in this research. A wide area monitoring system with high data resolution rate is achieved so far in this thesis. This system is designed to improve capabilities such as maintaining system normal operation and take a proper remedial action when encountered by unexpected circumstances by monitoring critical states in wide area system. Several examples are developed in this dissertation to verify this aspect as a tool to be used by the system operator. As a result, we created a novel infrastructure for the system operator to have proper knowledge and visualization about the power system's current situation and the level of stability margins.

In addition a Virtual Protection System was developed and tested in the real-time system control platform. Tools and procedures were developed in the system so that it can be used for failures detection and apply the proper solutions on the power system. Following a disturbance, one or more components overload and hence fail. The equilibrium of the load flow will consequently change and the power will then be 
redistributed to other normal components and this makes additional load transfer to other resources in the system automatically. Thus, a cascading failure is triggered by the overload failures and it could cause network collapse resulting in a blackout. The PMUs data can be used to follow the phase angle of each bus to detect the system failures which may cause cascaded events. In this process, the developed procedures detect upcoming faults by network data and analyze the network reaction to this outage. This is possible by algorithms including state estimation, N-1 contingency, fault calculation, OPF, etc. Therefore, in this dissertation the online calculation software was used to estimate system states following any circumstances. An example was implemented on the test-bed and the results were used to explain these new ideas.

The dissertation developed a new virtual protection system idea to use the realtime wide area protection system for applying adaptive online settings of protection relays. The protection coordination settings in a large area network are completely dependent on the topology and the system status which are varying frequently. For example the application of distributed generation, whose generation depends on the energy availability such as photovoltaic and wind power, may cause different operational settings for relays connected to the same network. This system is capable to detect the grid status and will run the protection coordination software to achieve proper settings for the relays. Finally, the developed procedures will apply the new settings using the realtime software and communication networks to the selected system protection devices. 
In summary the contributions achieved in this dissertation are:

- Developing a unique and novel hardware/software based Smart Grid testbed facility in a laboratory environment as a testing environment to verify the concepts developed in this dissertation.

- Developed real-time software environment for studying and verifying the control and protection schemes for the AC side of the power system.

- Developed and verified an integrated wide area system in the laboratoryscale Smart Grid test-bed, including capabilities for WAMS, WACS and WAPS.

- Tested and verified the system voltage stability and security margin using PMUs in wide area networks.

- Implemented and modified online system analysis including online load flow, contingency (security) analysis and Optimal Power Flow in real-time format

- Developed a novel real-time energy management algorithm for mitigation of heavy loads in hybrid microgrids

\subsection{Organization of Dissertation}

This dissertation is organized in nine chapters including this chapter which introduces the general contribution of this dissertation.

Second chapter will review the power system operation and control in modern networks and microgrids including hybrid $\mathrm{AC}$ and DC systems. It also discusses 
monitoring, control and protection aspects. Finally PMUs and their applications will be introduced.

Chapter 3 is presents the smart power system laboratory and its components by modeling, design parameters and procedures. This chapter also presents some test results and verification for real-time system monitoring and control of this smart microgrid.

Chapter 4 of this thesis will focus on developing and implementing of PMUs in a laboratory scale power system test-bed in digital form by using real-time analysis software. At first the measurement techniques for phasors is presented. Then, a simplified power system was implemented in order to test the real-time application of PMUs. This system uses different DAQs to gather the voltage and current data and measure the power system parameters such as the full voltage and current phasors, positive, negative and zero sequence data as well as power and frequency of each component. In addition, the industrial level setup of PMUs is introduced to apply same concept in real power system.

Chapter 5 explains the developed wide area monitoring and control strategies in the smart grid test-bed. This chapter demonstrates the results of experiments for system real-time monitoring technically and the control structure for system start-up and continuous operation. In addition this chapter introduces the implemented virtual protection system in the smart grid lab with detailed functions description and experiments results for system protection in wide area scale. Also the applications of this protection system are also discussed in this chapter.

Chapter 6 will introduce the architecture and design procedure of DC parts in details and control of this hybrid AC/DC system in terms of operation and control with experimental results. 
In chapter 7 of this dissertation explores the challenges and opportunities to implement online system analysis capability in order to monitor the system security indices. Therefore, the implemented hardware/software setup for monitoring and analyzing system in real-time format is detailed in this dissertation using the online results from the real-time software. Online load flow and contingency analysis results aim to identify the proper security monitoring of the power system in a wide area which is useful for most applications including system remedial actions. In addition this chapter focuses on evaluating online voltage stability indices for an interconnected power system. The voltage stability indices were measured during the operation of the power system when the dynamic changes take places. The evaluation of the voltage stability was performed by simulation and measurement of real-time data using Phasor Measurement Units (PMUs). The evaluation of the dynamic voltage stability indices were performed by creating dynamic load step change in both simulation and implementation at the laboratory-scale power system. The developed simulation involved the modeling of transmission line emulators with a pi network structure. The monitoring software was developed and implemented for wide area control and monitoring system on the laboratory power system test-bed.

Chapter 9 is presents the case study of system stability and security assessment in presence of pulse loads which may affects the system stability. The storages distribution is also studied by the simulation results for the test case. In addition, this chapter presents a real-time energy management algorithm for mitigating of pulse loads in hybrid microgrids and the effects of using battery storages in proper time in order to minimize the cost and to mitigate the pulse loads adverse effects. In addition, in this chapter details 
of the developed real-time Optimal Power Flow (OPF) management system which is implemented in the smart grid test-bed are presented. The online power system analysis feature was developed to deploy real-time data in order to monitor system state with the online optimal power flow. The developed software environment was made to monitor the real-time data as a SCADA center. This real-time software has the capability to run analysis software in engine mode and hence the system online analysis can be implemented by real-time data monitoring speed. In this setup, the monitoring system updates the SCADA system approximately every one second and then the analysis software can update the data immediately and calculates the generations optimal power sharing. Consequently, the developed procedures in this dissertation can analyze the power system continuously in less than three seconds snapshots and return the real-time calculation results.

Chapter 9 provides general discussion of the results and outcomes of this dissertation as well as presenting some suggestion and recommendations for further research and activities related to its topic. 


\section{CHAPTER 2}

\section{REVIEW OF POWER SYSTEM OPERATION AND CONTROL}

\subsection{Modern Hybrid AC/DC Power System}

Ideas related to smart grids operation and control are gaining significant interest in the research community. So far the majority of this interest has been related to implementation issues on the AC side. Fewer publications has concentrated issues related to the implementation of the $\mathrm{DC}$ microgrid or Hybrid $\mathrm{AC} / \mathrm{DC}$ microgrid. In this dissertation, we concentrated on the whole AC/DC hybrid smart power system as well as the building of a platform to implement many of the developed models for the future power system.

Recent increased trends in the consumption of electric power clearly indicate that there is a renewed demand for renewable and hence there will be an increased use of DC power in the end-user equipment. Computers, TVs and other electronic-based apparatus use low-voltage DC obtained by means of a single-phase rectifier followed by a DC voltage regulator. In factories, the same input stage is used for process-control equipment, while directly-fed AC machines are replaced by $\mathrm{AC}$ drives that include a twostage conversion process. Hence, DC distribution was proposed to address some of these challenges to have highly reliable supply of power. These new DC systems utilize power electronic based converters to convert AC sources to DC power and distribute the power using DC lines. The converters convert DC power back to AC power at the load, when it is needed. For motor type loads driven by adjustable speed drives, this conversion may even not be needed, as the motor drives then can directly be interfaced with the DC grid. 
The power converters are the main components of these systems. The Hybrid system proposed has advantages of being utilized for both $\mathrm{DC}$ and $\mathrm{AC}$ grids. This system consists of DC generations (implemented on the testbed) as emulators for renewable energy sources.

Renewable power resources are also safe, clean and abundant in nature. However, due to the power fluctuation of renewable energy sources, voltage and frequency deviations occur in islanded or remote area power systems whose ability to maintain stable supply-demand balance to low levels. Therefore, it is necessary to control the system frequency and voltage not only at the AC supply side but also on the DC generation sides and interconnected points. Hence, in case of using the renewable energy plants connected to the power system, the supply-side control has limitations. Therefore, mutual cooperation of control with the demand side is required. This is because it is difficult to maintain the power quality by only the supply-side control. From this viewpoint, a smart grid, which maintains stable supply-demand balance by monitoring the power information of the demand side, is necessary.

The electrical energy production from renewable sources is at DC (as is the case for photovoltaic systems and fuel cells) or requires a similar two-stage conversion as case of $\mathrm{AC}$ drives, e.g. variable-speed wind turbines and natural-gas micro-turbines. By using DC for distribution systems it would thus be possible to skip one stage in the conversion in all these cases, with consequent savings and higher reliability due to a decreased number of components.

Moreover, the energy delivery at the DC side is characterized by lower losses and voltage drops in the lines or cables. 


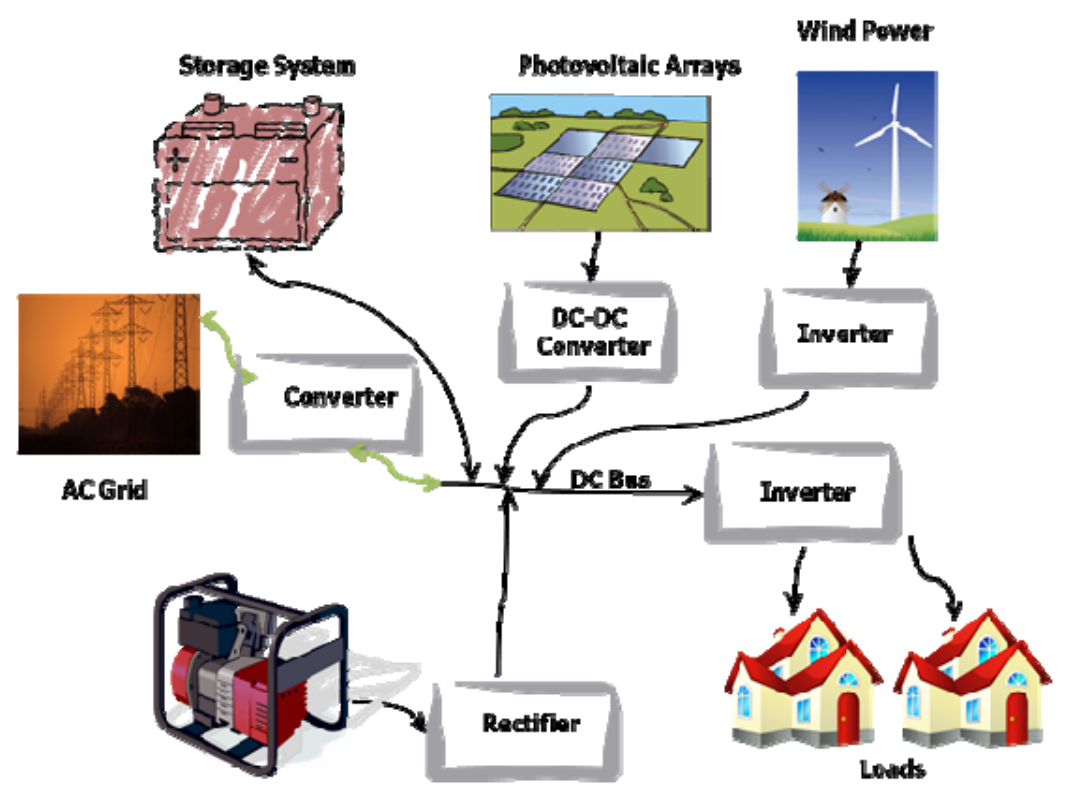

AC Generator

Figure 2.1: Typical Hybrid AC/DC Microgrid

A DC distribution system also allows direct connection of battery blocks for backup energy storage. The latter is often needed for avoiding supply interruptions in hospitals or big office buildings or in industrial complexes with high power quality demands and is presently implemented with Uninterruptible Power Supply (UPS) systems using two conversions (from AC to DC and back). A direct connection to a dc network would thus save two conversions in this case increasing system efficiency.

The smart grid provides an excellent opportunity to manage the power quality and security. The impact on power quality is taken into consideration from the viewpoint of the generator side and a grid side and the demand side management. Various load management and control strategies have been developed to make the power system smarter. It is expected that smart technologies will lead to reduced investment in primary equipment and it will increase the availability of power supply.

Currently, the smart AC grid is implemented by practical examples at the industrial level. However, the DC smart grid is not implemented or proposed seriously 
except in the case of HVDC systems at the transmission level. The DC grid has the following advantages compared to an $\mathrm{AC}$ grid:

- Each power generator connected to the DC grid can easily be incorporated because it controls only the DC bus voltage.

- When the AC grid experiences an abnormal or fault conditions, the DC grid is disconnected from the $\mathrm{AC}$ grid and then it is switched on for stand-alone operation in which the generated power is supplied to the loads connected to the DC grid.

- The cost and loss of DC system can be reduced because only a single AC-gridconnected inverter unit is needed.

- Distributed generators usually supply DC power. Therefore, the phase detection like AC grid is not needed. Therefore, the cost and loss of the system can be reduced.

- Although the DC distribution line is required, the cost performance of DC houses, hospitals and information centers are satisfactory.

However the DC grid has the following disadvantages compared to an AC grid.

- Difficult to scale up voltage to reduce current.

- It uses thick cables and short distances to reduce voltage losses.

- The DC distribution is limited to a small short line length $(\mathrm{km})$.

- There are limited protection devices and coordination problem exist in the DC systems.

Both DC and AC grids have advantages and disadvantages. For example, an AC grid has advantages such as the cost of short and medium voltage level transmission 
lines. Therefore, the next-generation hybrid power system comprising both grids is required.

Figure 2.1 shows the idea of having a DC bus in a DC distribution system. As it is obvious, this kind of topology for each microgrid could be implemented. The advantages of such DC bus are:

- Islanded operation

- Reduction of power converters number and their complexity

- Increase in power quality for public network's end users

- Better integration of DG

Considering both configuration, the DC bus is the only connection common to all converters and hence, the control system is based on the DC voltage value. The presence of different supply sources makes it possible to integrate and optimize system management with field bus systems. The control system must be robust and should keep the DC voltage within the acceptable range. Renewable energy generators must supply all the available power, if loads, network or storage systems absorb it.

Storage systems have to be recharged as soon as possible and need to be discharged if necessary. For maintaining system load-generation balance, the AC/DC interface converter has to absorb or deliver power from or to the network.

In this research, a smart microgrid, which has both $\mathrm{AC}$ and DC sides and is integrated together, is proposed as an $\mathrm{AC} / \mathrm{DC}$ hybrid power system. The proposed smart grid consists of several sources with a generator-side converter, a grid-side inverter and controllable loads. 


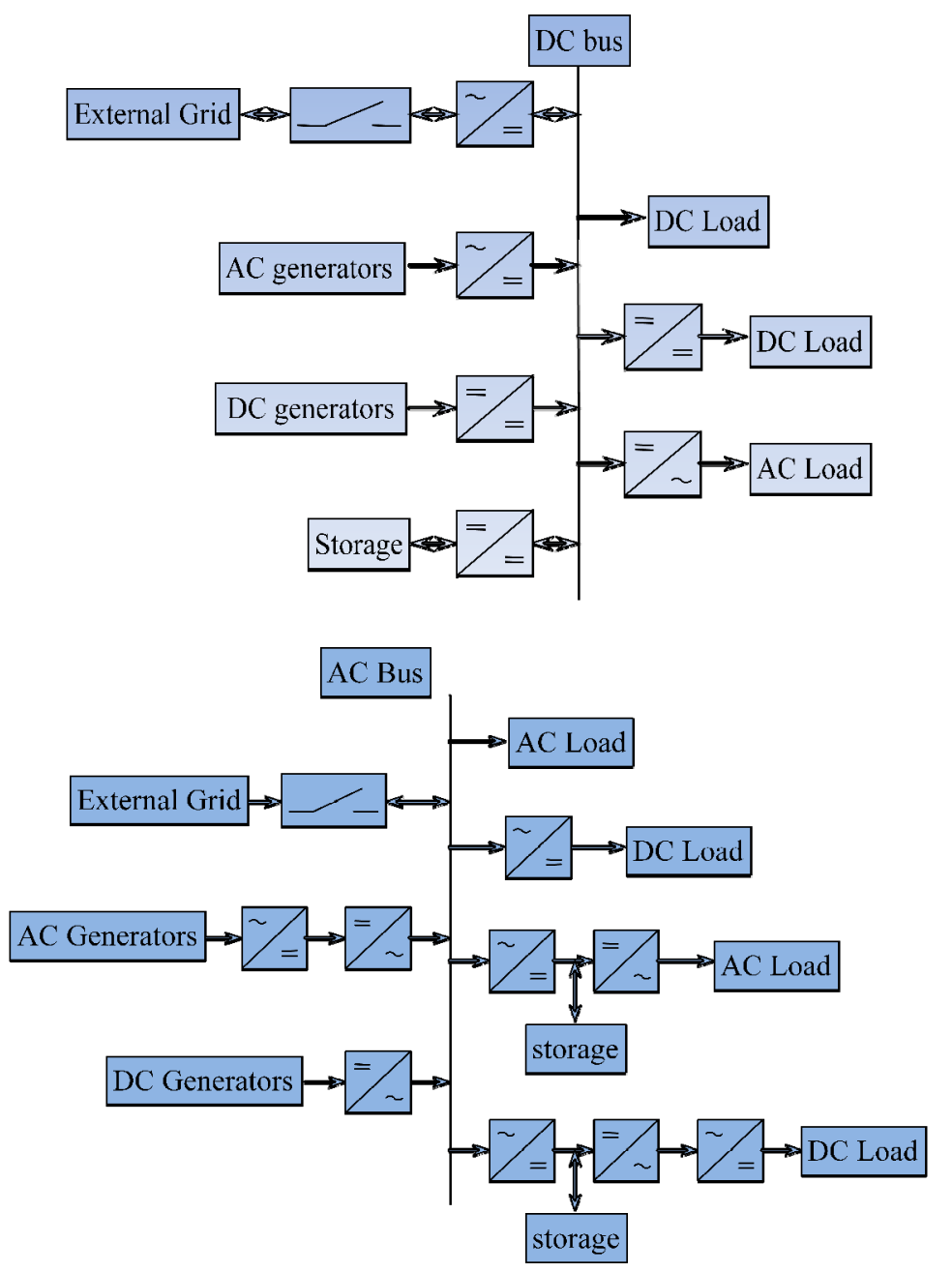

Figure 2.2: Comparison between AC and DC distribution systems

\subsection{Power System Operation Limits}

Power system operators are constantly monitoring the state of the transmission grid and dispatching the output of generating units. On the other hand, operators are also under considerable pressure to run the power system in the most economical manner. This means producing or buying electrical energy to meet the demand at the lowest possible cost and making many transactions feasible. These economic considerations invariably put more stress on the transmission system because more power ends up being 
transmitted over longer distances. Balancing the grid for profit and preventing of blackouts is thus the essence of operating a power system.

To resolve this conflict and help operators perform what is a complex technical task, special rules have been developed for transmission utilities and operators which should be followed at all times. However, the fundamental principle is to operate power system in such a way that no credible contingency could trigger cascading outages or another form of instability. This approach was chosen because all power systems are routinely affected by unpredictable faults and failures such as lightning strikes on transmission lines, mechanical failures in power plants, or fires in substations. Since events of this nature are unavoidable and relatively frequent, all power systems should be able to endure them without inconveniencing consumers. Avoiding blackouts and wide scale consumer disconnections is possible only if the system is operated with a sufficient security margin. There must be enough reserve generation capacity to make up for the loss of a generating unit and enough transmission capacity to handle the power flows displaced by the outage of a line. Note that it is always possible to dream-up a sequence of unfortunate events that would bring any power system to its knees. Since securing the system against all possible contingencies is clearly impossible, the fundamental principle only calls for securing against all credible contingencies. It is typically assumed that the probability of two or more independent faults or failures taking place simultaneously is too low to be considered credible. Most security rules therefore call for the system to be able to withstand the loss of any single component. When a power system satisfies this criterion, it is said to be "N-1 secure" because it could lose any one of its N components 
and continue operating. Similarly, in a system that is "N-2 secure", no consumer would be disconnected even if two components were suddenly disconnected.

System Operating Limit (SOL) is defined as a value (such as MW, MVar, Amperes, Frequency or Volts) that satisfies the most limiting of the prescribed operating criteria for a specified system configuration to ensure operation within acceptable reliability criteria. System Operating Limits are based upon certain operating criteria. These include, but are not limited to:

- Facility Ratings (Applicable pre- and post-contingency equipment or facility ratings)

- Transient Stability Ratings (Applicable pre- and post-contingency Stability Limits)

- Voltage Stability Ratings (Applicable pre- and post-contingency Voltage Stability)

- System Voltage Limits (Applicable pre- and post-contingency Voltage Limits) In the planning stage, the determination of SOL follows specific procedure. In order to apply reliability performance criteria or standard, all transmission lines facilities should be within their thermal ratings and system should be stable transiently and dynamically with adequate reactive margin to prevent post-transient voltage instability. Required studies include power flow, transient stability and post-transient voltage stability. In addition system should meet requirements for single contingencies and in some cases double contingencies may be required. Study models should be based on the most recent approved base cases available for the study time frame and conditions. 
By power flow calculations the performance of the system will be evaluated by calculation of thermal capacity limits which should be kept under $100 \%$ of its continuous rating under normal conditions. Following a single contingency or a credible double contingency, no transmission element will be loaded above its emergency rating. In addition, system voltage limits should be monitored by the system operators. Transient stability studies should be performed for all contingencies provided, reviewed and approved for the disturbances which will be initiated by a fault on the EHV bus adjacent to the major interconnected point and then to the related power plant. Evaluating faults should be cleared in accordance with the guidelines and also backup protection clearing time for breaker operation should be provided as well.

\subsection{Cascading Events and System Blackout}

A disruption in one infrastructure causes failure in a component of another infrastructure, causing a disruption in that one as well. This in turn may cause a failure in a third infrastructure and so on. Thus, the failure cascades through the infrastructures like a wave, sometimes revealing new interdependencies between seemingly independent critical infrastructures. For example, a failure in the natural gas infrastructure might result in a failure of a power generation unit, causing power blackouts.

Cascading failure is the usual mechanism for large blackouts of electric power transmission systems. For example, long, intricate cascades of events caused the August 1996 blackout in Northwestern America (NERC [84]) that disconnected 30,390 MW of power to 7.5 million customers [85]). An even more spectacular example is the August 2003 blackout in Northeastern America that disconnected 61,800 MW of power to an 
area spanning eight states and two provinces and containing 50 million people [86]. The vital importance of the electrical infrastructure to society motivates the understanding and analysis of large blackouts.

Power systems are complex systems that evolve over years in response to the economic growth of the country and to continuously increasing power demand. In spite of the reliability of these systems, there are widespread disturbances that have significant cost to society. The average frequency of blackouts in the United States is about one every 13 days [87]. This frequency has not changed over the last 30 years. Also the probability distribution of blackout sizes has a power tail; this dependence indicates that the probability of large blackouts is relatively high. Indeed, although large blackouts are rarer than small blackouts, it can be argued that their higher societal cost makes the risk of large blackouts comparable to or exceed the risk of small blackouts [87].

The operation of power transmission systems is studied from the perspective of complex dynamics in which a diversity of opposing forces regulate both the maximum capabilities of the system components and the loadings at which they operate. These forces enter in a nonlinear manner and may cause an evolution process to be ultimately responsible for the regulation of the system. This view of a power transmission system considers not only the engineering and physical aspects of the power system, but also the economic, regulatory and political responses to blackouts and increases in load power demand. From this perspective, the search for the cause of the blackouts must not be limited to the trigger of the blackout, which is normally a random event, but it must also consider the dynamical state of the power transmission system. A detailed incorporation of all these aspects of the dynamics into a single model is extremely complicated. 
Based on the blackout analysis, the operational conditions of a power system could be classified as follows:

- Normal condition, with fulfillment of the $\mathrm{N}-1$ criterion and power flow limitation

- Pre-emergency condition, involves the restoration of normal condition and prevention of cascading emergency development.

- Emergency condition, which begins with cascade process in wide area scale by tripping of generators or transmission lines.

- Frequency decline emergency, during which loss of thermal equipment stability is likely to occur.

Collapse of a power system can be triggered by the following emergency processes:

- Dynamic stability loss at short-circuit occurrence

- Dynamic stability loss at tripping a heavily loaded line

- Static stability loss due to voltage decrease

- Load node voltage drop avalanche

- Out-of-step generator running

- Accompanying voltage drop avalanche

- Transmission voltage drop avalanche

- Large-scale outage of generators due to overload at transmission voltage level

- Multiple tripping of lines as a response of protection to the voltage drop

- Multiple tripping of lines due to wire sagging and short-circuits

- Large-scale outages of power plants due to sudden load rejection 
- Large-scale outages of power plants due to stability loss of thermal equipment at under-frequency emergency

Postmortem analysis and other investigative reports have concluded that the power system must be provided with means and tools to avoid system-wide power failure under extreme contingencies. The system deterioration starts with alarming of steadystate violations and gradually evolves to faster dynamic phenomena with the system reacting quickly and defending itself with pre-determined protection schemes and relays setup. The analysis of short-term/mid-term states must be considered. Angular transients, voltage transient stability and security, frequency stability and small signal analysis tools would certainly help to devise dynamic actions and reconfiguration strategies. The process is expected to be highly automated and bounded by the latest communication technology and PMU allocation strategies. The following issues should be considered as part of any development plan to address catastrophic events:

- Early detection and monitoring of system security indices

- Analysis of critical real-time information to trigger the dynamic security assessment process

- Wide-area information, control and protection schemes

- Deployment of control actions for dynamic adjustment and reconfiguration In next section we will provide information to address new technologies for avoiding blackouts and catastrophic events. 


\subsection{Power System Monitoring with SCADA}

The current necessity for more and more energy in all the industrial sectors brings a variety of challenges for engineers involved in power system control. The requirements of a proper power system operation cannot be accomplished without a supervisory control and data acquisition system (SCADA). The main objective in power systems is maintaining the balance between power generation and production, assuring the reliability of the system. This purpose is becoming harder to achieve hence to the new renewable power sources that bring new uncertainties and parameters' variations into the power grid. Considering these aspects, the importance of monitoring systems is obvious.

The SCADA system supervises controls, optimizes and manages generation and transmission systems. The main component of these systems are RTUs (Remote Terminal Units) that collect data automatically and are connected directly to sensors, meters, loggers or process equipment. They are located near the monitored process and they transfer data to the controller unit when requested. They often include integral software, data logging capabilities, a real-time clock (RTC) and a battery backup. Most of the RTUs are time redundant. These devices are complete remote terminal units that contain all of the transceivers, encoders and processors needed for proper functioning in the event that a primary RTU stops working. Meter readings and equipment status reports can also be performed by PLCs (Programmable Logic Controllers).

A SCADA control center performs centralized monitoring and control for field sites over long-distance communications networks, including monitoring alarms and processing status data. Based on information received from remote stations, automated or operator-driven supervisory commands can be pushed to remote station control devices, 
which are often referred to as field devices. Field devices control local operations such as opening and closing valves and breakers, collecting data from sensor systems and monitoring the local environment for alarm conditions, [88]. Although SCADA is a widely used application in most industries, requirements within the electric utility industry for remote control of substations and generation facilities have probably been the driving force for modern SCADA systems.

The control center contains the SCADA Server (MTU) and the communications routers. Other control center components include the human machine interface (HMI), engineering workstations and the data historian, which are all connected by a LAN. The control center collects and logs information gathered by the field sites, displays information to the HMI and may generate actions based upon detected events. The control center is also responsible for centralized alarming, trend analyses and reporting. Field sites are often equipped with a remote access capability to allow field operators to perform remote diagnostics and repairs usually over a separate dial up modem or WAN connection. Standard and proprietary communication protocols running over serial communications are used to transport information between the control center and field sites using telemetry techniques such as telephone line, cable, fiber and radio frequency such as broadcast, microwave and satellite. The communication architectures are different depending on the implementation.

SCADA supervises the status or the changes of breakers, connectors and protective relays; induces of charged/uncharged status of lines and buses; supervises active/reactive power against operational/emergency limit; judges network faults. SCADA systems include several functions: State estimation and scheduling function 
estimates most likely numerical data set to represent current network. Load forecasting function anticipates hourly total loads for a few days ahead based on the weather forecast, type of the day, etc. utilizing historical data about weather and load. Power flow control function supports operators to provide effective power flow control by evaluating network reliability, considering anticipated total load, network configuration, load flow and contingencies. Data maintenance function enables operator to modify the database of power device status and network topology by defining parameters. Voltage/reliability monitoring function monitors present voltage reliability and transient stability and predicts future status some hours ahead.

\subsection{Wide Area Monitoring, Control and Protection}

The conventional power system monitoring, protection and control system are based on local measurements. However, it is quite difficult to maintain the stability and security of the system on the whole, if only local measurements are utilized in the monitoring, protection and control schemes. One promising way is to provide a system wide protection and control, complementary to the conventional local protection strategy. While it is not possible to predict or prevent all contingencies that may lead to power system collapse, a wide-area monitoring and control system that provides a reliable security prediction and optimized coordinated action is able to mitigate or prevent large area disturbances. The main tasks, which can be accomplished through wide-area based monitoring and control system, are early recognition of large and small scale instabilities, increased power system availability through well coordinated control actions, operation closer to the limit through flexible relaying schemes, fewer load shedding events and 
minimization of the amount of load shedding. The main disadvantage of the present conventional methods of system monitoring is the inappropriate system dynamic view, or the uncoordinated local actions, like those in decentralized protection devices. Solution to the above can be achieved through dynamic measurement system using synchronized phasor measurement units. A system comprising synchronized phasor measurements and performing the tasks of stability assessment with adaptive relaying is called a Wide-Area Monitoring, Protection and Control (WAMPAC) system [89].

Today, the application of synchronized phasor measurements is limited to the data collection for post event analysis and limited system monitoring. System wide area protection and control schemes have not been broadly applied by fast data acquisition from PMUs. Power System researchers are actively developing new applications of this technology, which can be generally categorized into wide area monitoring, control and protection. Therefore, the long-term development of wide area monitoring system is moving to utilization of the synchronized phasor data for real-time network protection and control. Figure 2.2 is an example of an achievable overview for wide area monitoring, protection and control. Here the PMU serves not only as a data acquisition system, but also as a wide area protection and control device. The PMU can provide local management of system functions like over/under-frequency or over/under-voltage, load shedding, overload protection, etc. In addition, the recent findings on system operation functions that can be managed locally with or without system operation permission can also be implemented by PMUs. Data that are sent to the PDCs can be utilized for specific system applications like state estimation (state measurement if the data are adequate) and voltage stability. Power flows can be monitored for system congestion. Data from 
terminals of the same line can be used for line thermal monitoring and power swing detection using fast data transferring capability with small latency. Applications can provide feedback control and protective actions through the PMU's or SCADA. The WAMPAC may also serve as backup to SCADA/EMS and may eventually merge with or replace it.

Researchers have shown numbers of examples of synchrophasors being used in the visualization, analysis and control of large power systems. As experience in this technology grows, more applications will be forthcoming:

- The visualization of power system conditions and dynamics is improved by the use of time-synchronized measurements. Operators with better information will make better decisions to maintain or achieve stable conditions.

- As power systems expand, dynamic conditions will arise that are beyond the ability of static tools to examine or determine appropriate corrective actions. Synchronized measurements, available at high message rates, provide an improved tool to determine system dynamic operating conditions.

- Both manual and automatic control systems can be improved by the application of synchrophasors. Real-time processing, either in relays or a vector processor, can take high-speed control action to maintain system stability.

- New methods have been developed for the stability assessment of transmission systems using measurements at either end of the transmission line.

- Enhancement in identification and prediction of system events and location of the disturbance(s) for real-time action. 
- Enhancement of decision and action making need to be undertaken to arrest the degradation of the system state, assure a safe return to the secure state and return to economical operating state.

\subsection{Phasor Measurements Units}

Synchronized phasor measurements (synchrophasors) provide a new method for improved visualization of system conditions and even wide-area-based control. The availability of streaming data in a standard format improves supervisory control and data acquisition (SCADA) data quality and provides automatic checks of state estimation calculations.

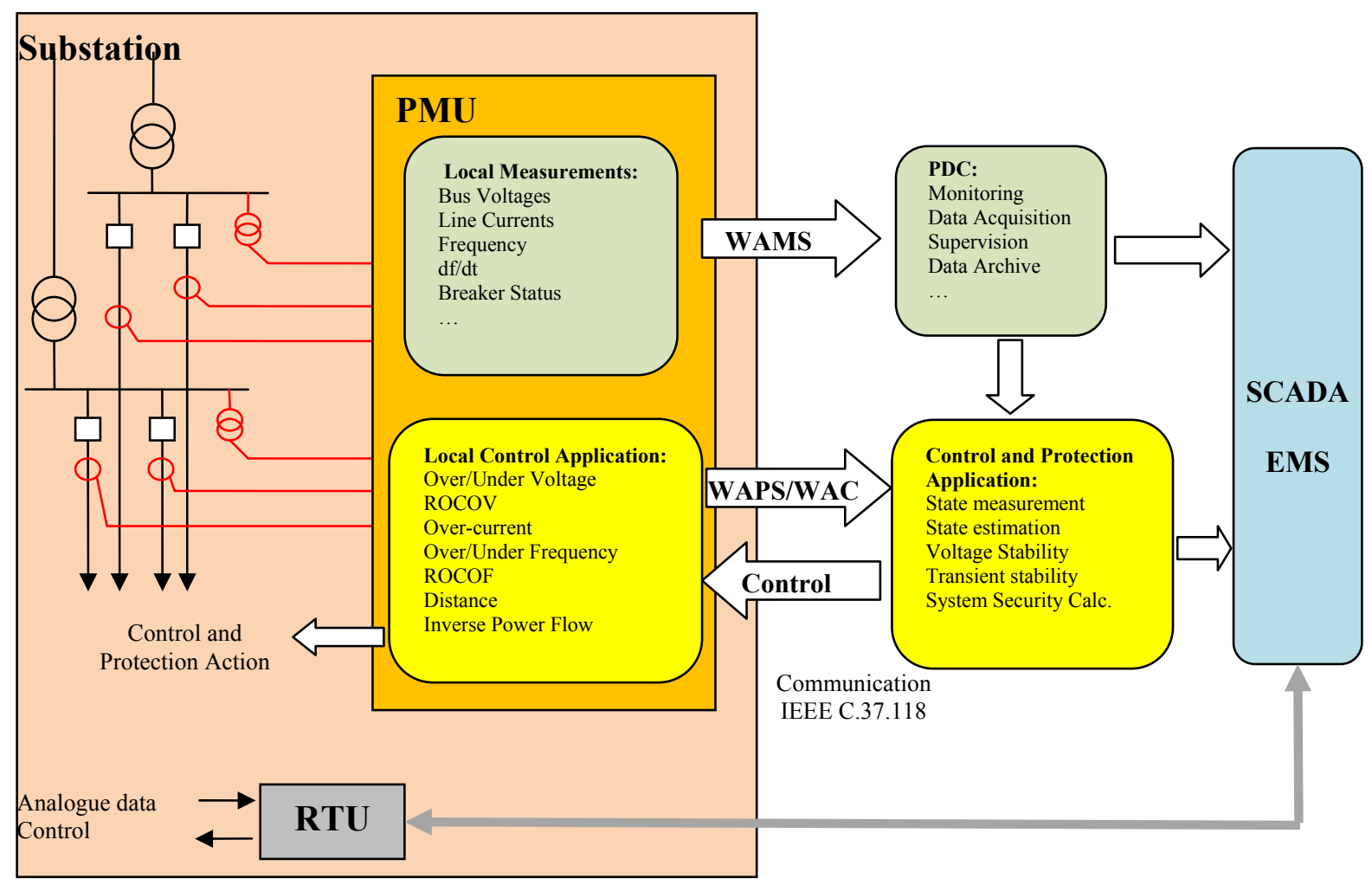

Figure 2.3: Wide Area Monitoring, Protection and Control System (WAMPAC)

The PMUs manufactured by different manufacturers differ from each other in many important aspects. It is therefore difficult to discuss the PMU hardware 
configuration in a way which is universally applicable. However, it is possible to discuss a generic PMU, which will capture the essence of its principal components. Figure 2.3 is based upon the configuration of the first PMUs built at Virginia Tech [90]. Remember that PMUs evolved out of the development of the "symmetrical component distance relay". Consequently the structure shown in this figure parallels that of a computer relay. The analog inputs are currents and voltages obtained from the transformers. All three phase currents and voltages are used so that positive-sequence measurement can be carried out. In contrast to a relay, a PMU may have currents in several feeders originating in the substation and voltages belonging to various buses in the substation.

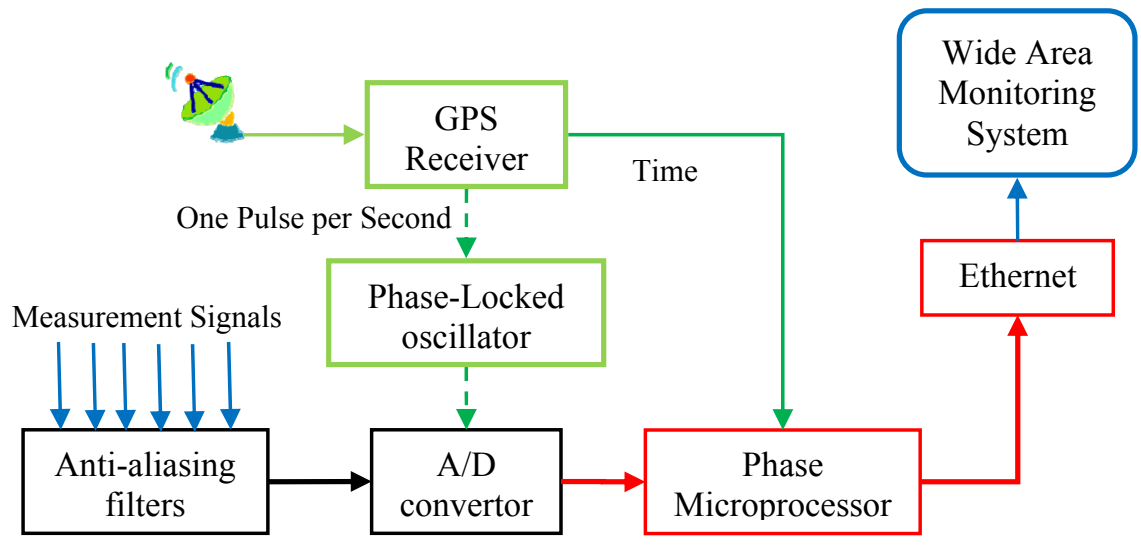

Figure 2.4: Major elements of the modern PMU [90]

The current and voltage signals are converted to voltages with appropriate shunts or instrument transformers (typically within the range of \pm 10 volts) so that they are matched with the requirements of the analog-to-digital converters.

The sampling rate chosen for the sampling process dictates the frequency response of the anti-aliasing filters. In most cases, these are analog-type filters with a cutoff frequency less than half of the sampling frequency in order to satisfy the Nyquist 
criterion. As in many relay designs one may use a high sampling rate (called oversampling) with corresponding high cut-off frequency of the analog anti-aliasing filters. This step is then followed by a digital 'decimation filter' which converts the sampled data to a lower sampling rate, thus providing a 'digital anti-aliasing filter' concatenated with the analog anti-aliasing filters. The advantage of such a scheme is that the effective anti-aliasing filters made up of an analog front end and a digital decimation filter are far more stable as far as aging and temperature variations are concerned. This ensures that all the analog signals have the same phase shift and attenuation, thus assuring that the phase angle differences and relative magnitudes of the different signals are unchanged. The sampling clock is phase-locked with the GPS clock pulse. Sampling rates have been going up steadily over the years - starting with a rate of 12 samples per cycle of the nominal power frequency in the first PMUs to as high as 96 or 128 samples per cycle in more modern devices, as faster analog-to-digital converters and microprocessors have become commonplace.

The most common use of the GPS system is in determining the coordinates of the receiver; although for the PMUs the signal which is most important is the one pulse persecond. This pulse as received by any receiver on earth is coincident with all other received pulses to within 1 microsecond. In practice much better accuracies of synchronization of the order of a few hundred nanoseconds have been realized.

The PMUs are installed in power system substations. The selection of substations where these installations take place depends upon the use to be made of the measurements they provide. The optimal placement of PMUs will be considered in some of the following chapters which discuss some of the applications of phasor 
measurements. In most applications, the phasor data is used at locations remote from the PMUs. Thus an architecture involving PMUs, communication links and data concentrators must exist in order to realize the full benefit of the PMU measurement system. A generally accepted architecture of such a system is shown in figure 2.4.

The PMUs are situated in power system substations and provide measurements of time-stamped positive-sequence voltages and currents of all monitored buses and feeders (as well as frequency and rate of change of frequency). The measurements are stored in local data storage devices, which can be accessed from remote locations for post-mortem or diagnostic purposes. The local storage capacity is necessarily limited and the stored data belonging to an interesting power system event must be flagged for permanent storage so that it is not overwritten when the local storage capacity is exhausted. The phasor data is also available for real-time applications in a steady stream as soon as the measurements are made. There may well be some local application tasks which require PMU data, in which case it can be made available locally for such tasks. However, the main use of the real-time data is at a higher level where data from several PMUs is available.

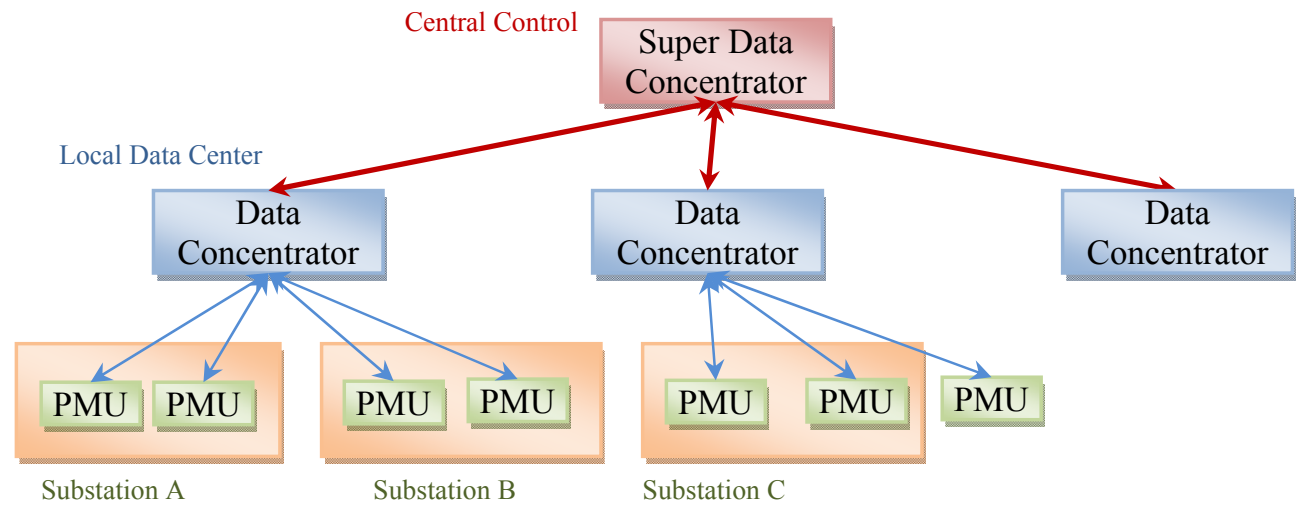

Figure 2.5: Phasor measurement systems and levels of phasor data concentrators 
The devices at next level of the hierarchy are commonly known as "phasor data concentrators" (PDCs). Typical function of the PDCs is to gather data from several PMUs, reject bad data, align the time-stamps and create a coherent record of simultaneously recorded data from a wider part of the power system. There are local storage facilities in the PDCs, as well as application functions which need the PMU data available at the PDC. This can be made available by the PDCs to the local applications in real-time. Clearly, the communication and data management delays at the PDCs will create greater latency in the real-time data, but all practical experience shows that this is not unmanageable. The question of data latency will be further considered when applications are discussed in later section.

For power system application, the common time frame is independent of the location of the measuring device. So, the phasor measurement considerably reduces the amount of analogue data of the power system to key characteristics of most interest to the power system operator. PMU devices are now installed all over the world to gather GPS time synchronized phasor information. Some major applications of PMU in power system are [91]:

- Automation of the power system in smart grids.

- Detecting isolated networks and preventing total system blackout.

- Load management and other load control techniques such as demand response mechanisms to maintain power system stability.

- Increase the reliability of the power system by fast detection of faults, isolation of healthy system and the prevention of power outages. 
- Increase power quality by precise analysis and automated correction of power sources of system.

- Wide area measurement, protection and control, in whole area of regional transmission networks and local distribution grids.

Figure 2.5 shows the conceptual diagram of a power system with PMUs available in important buses in different areas.

\subsection{Applications of PMUs in Power System}

Given a simplified power system model with application of PMUs in both buses is shown in figure 2.6. The left side bus is assumed to be sending end and then the power flow in the line will be from left bus to right bus. Without losing generality, this is just for simplicity in formulation of real-time parameters and one can consider the right bus as a sending end in any other situation.

If we neglect the parallel admittance of transmission line, $\mathrm{Y}$, the transmitted active power, $\mathrm{P}$ and reactive power, $\mathrm{Q}$, can be expressed by Eqs. 2.1 and 2.2, respectively.

$$
\begin{aligned}
& P=\left(\left(V_{s} \cos \left(\delta_{s}-\delta_{r}\right)-V_{r}\right) \frac{R}{R^{2}+X^{2}}+\left(V_{s} \sin \left(\delta_{s}-\delta_{r}\right)-V_{r}\right) \frac{X}{R^{2}+X^{2}}\right) \times V_{r} \\
& Q=\left(\left(V_{s} \cos \left(\delta_{s}-\delta_{r}\right)-V_{r}\right) \frac{X}{R^{2}+X^{2}}-\left(V_{s} \sin \left(\delta_{s}-\delta_{r}\right)-V_{r}\right) \frac{R}{R^{2}+X^{2}}\right) \times V_{r}
\end{aligned}
$$


Figure 2.6: Conceptual diagram of a power system with PMUs

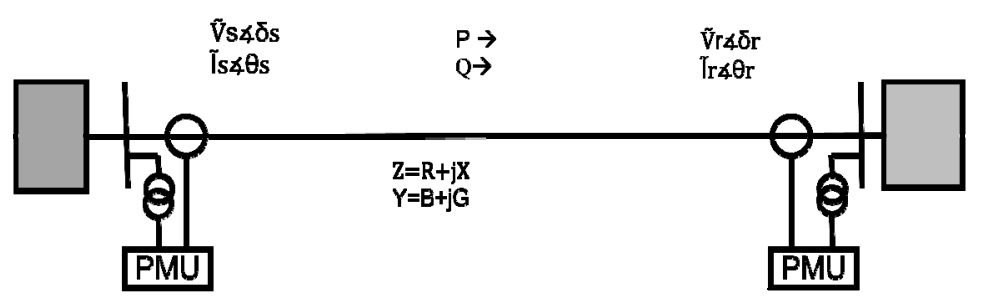

Figure 2.7: Two bus system with PMUs

In other example, PMUs deployed at both ends of a transmission line and measuring both currents and voltages facilitate online tracking of line parameters. Technically, we can calculate the line parameters by having all voltage and current data of both terminals. For this purpose we have Eq. 2.3 as voltage and current transfer matrix for nominal PI line model. Solving this equation for $\mathrm{Z}$ and Y leads to equations 2.4 and 2.5, which present line parameters according to measurement of voltages and currents in both sides of the line. In real-time calculation, this can provide real-time monitoring of 
line parameters to detect possible line faults, seasonal line condition and temperature changes as well as loading of transmission lines [92], [93].

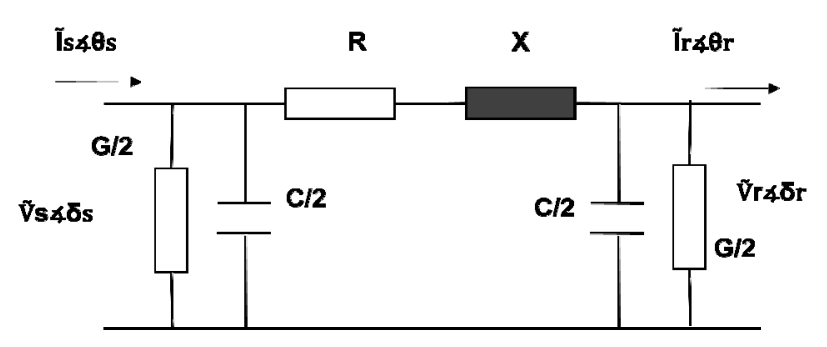

Figure 2.8: PI-Line Model

$$
\begin{aligned}
& {\left[\begin{array}{l}
V s \angle \delta s \\
I s \angle \theta s
\end{array}\right]=\left[\begin{array}{cc}
1+\frac{Y Z}{2} & Z \\
Y\left(1+\frac{Y Z}{4}\right) & 1+\frac{Y Z}{2}
\end{array}\right] \times\left[\begin{array}{c}
V r \angle \delta r \\
I r \angle \theta r
\end{array}\right]} \\
& Z=\frac{(V s \angle \delta s)^{2}-(V r \angle \delta r)^{2}}{(V s \angle \delta s)(I r \angle \theta r)+(V r \angle \delta r)(I s \angle \theta r)} \\
& Y=2 \frac{(I s \angle \theta s)-(I r \angle \theta r)}{(V s \angle \delta s)+(V r \angle \delta r)}
\end{aligned}
$$

By decoupling real and imaginary parts of $\mathrm{Z}$ and $\mathrm{Y}$, the resistive and reactance parts of the series impedance and capacitance and conductance part of the parallel impedance are captured in real-time format. This would be a great idea to calculate line parameters for realistic power line without measuring them directly, because most of the system studies like power flow, short circuit, contingency, long term transients etc, need line PI model parameters for analysis of power system.

Stability is one of the factors that play a significant role in modern power system operation. The instability conditions may have seen by system voltage and frequency quantity in online form. The phase angle differences across different nodes are measure of system proximity to instability. Real-time monitoring of phase angle by distributed 
PMUs around the grid is required to apply corrective control actions for stabilization of large power system [94].

Online voltage stability index (VSI) is proposed in [95]. This index can be calculated in real-time and predicts the power system steady-state voltage stability limit. The method to calculate this index starts by deriving three maximum transferrable powers including active, reactive and apparent power for each branch. So, the receiving end side voltage can be calculated from equations 1 and 2 and is presented in equation 2.6.

$$
V r=\sqrt{\frac{V s^{2}}{2}-(Q X+P R) \pm \sqrt{\frac{V S}{4}-(Q X+P R) V s^{2}-(P X-Q R)^{2}}}
$$

The maximum transferable power $\mathrm{S}_{\max }$ through the transmission line has been achieved when the internal root phrase equals to zero [94]. There is only one possible solution for $\mathrm{V}_{\mathrm{s}}$ and the receiving end voltage, $\mathrm{V}_{\mathrm{r}}$, is at the marginally stable operating point. The maximum transferred active power, $\mathrm{P}_{\max }$, maximum transmitted reactive power, $\mathrm{Q}_{\max }$ and the maximum transferable power, $\mathrm{S}_{\max }$, can be calculated by equations 2.8 and 2.9 where $\varphi=\arctan (Q / P)$ and $Z=\sqrt{R^{2}+X^{2}}$ and all right side parameters are assumed to be constant. These equations can be simplified by assuming high $\mathrm{X}$ to $\mathrm{R}$ ratio [96].

$$
\begin{aligned}
& \frac{V s}{4}-(Q X+P R) V s^{2}-(P X-Q R)^{2}=0 \\
& P_{\max }=\frac{Q R}{X}-\frac{V s^{2} R}{2 X^{2}}+\frac{|Z| V s \sqrt{V s^{2}-4 Q X}}{2 X^{2}} \\
& Q_{\text {max }}=\frac{P X}{R}-\frac{V s^{2} X}{2 R^{2}}+\frac{|Z| V s \sqrt{V s^{2}-4 P R}}{2 R^{2}}
\end{aligned}
$$




$$
S_{\max }=\frac{V s^{2}[|Z|-(X \times \sin \varphi+R \times \cos \varphi)]}{2(X \times \cos (\varphi)-R \times \sin (\varphi))^{2}}
$$

Therefore, according to these relations, the total Voltage stability Index (VSI) can be calculated as [97]:

$$
V S I=\min \left\{\frac{P_{\max }-P}{P_{\max }}, \frac{Q_{\max }-Q}{Q_{\max }}, \frac{S_{\max }-S}{S_{\max }}\right\}
$$

Smaller values of VSI indicate that the receiving end bus is close to its voltage stability margin and less power transferring margin is left and in its margin the VSI will be equal zero.

Based on PMUs, different types of wide area protection, emergency control and optimization systems can be designed. Synchrophasors provide an appropriate measurement for real-time wide area control and protection. Using modern communication protocols according $\mathrm{C} 37.118$, the measured data can be sent long distance to a control center between one and two or more cycles [91]. Phase angle difference between buses is considered for indicating power flow direction and topology changes that requires control actions.

Wide area protection system, may act upon transmission congestion, risky transient stability conditions, voltage and frequency degradation and thermal overloading. In order to implement an effective and intelligent protection system for the widespread power system condition, it is essential that a real-time and fast wide data collection system be available. This system can use PMUs to implement smarter and intelligent protection scheme over a wide area and as a backup supervision system for the existing SCADA system. This protection scheme may have different architecture, multilayered 
design and compatible by existing protection system to act in extensive system events. One application may be loss of synchronism that leads to a blackout of a part or whole power system. If proper actions are taken appropriately, the system could be protected from instability of a sub-network in first swing cycles and hence it prevents to complete grid frequency and voltage instability. In this situation, fault could not permeate by cascade events which cause whole system blackout or even an area outage. Figure 2.8 shows the concept of different protection schemes in different operation time scale versus the area which they are applicable. As it is obvious, the PMU is applicable for long-term wide area events protection as well as local and fast protection action.

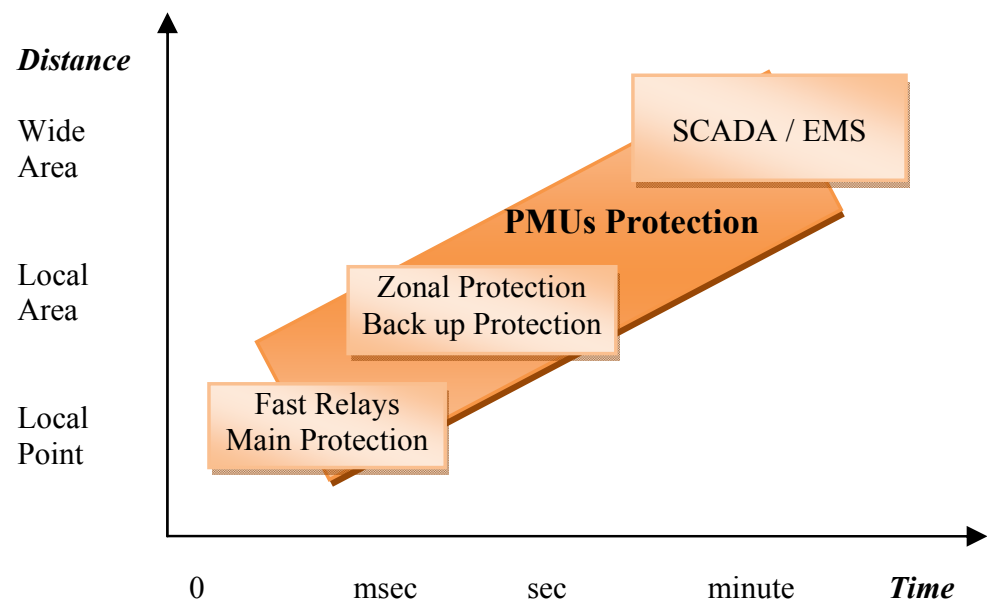

Figure 2.9: Time-Area Diagram of Different Protection Scheme 


\section{CHAPTER 3}

\section{DEVELOPMENT OF SMART POWER SYSTEM TEST-BED}

The smart grid is a power system that requires real-time communication and control between the consumer and utility allowing suppliers to optimize a consumer's energy usage based on environmental and price preferences. The smart grid operators need to create a test-bed which provides a structure that allows components and configurations to be tested in a flexible and dynamic way, enabling researchers to simulate real network conditions and study specific situations. Simulating different energy flows or fault conditions will help to understand which components to use and what choices to make to ensure the greatest accuracy possible when managing power grids. In addition the smart grid platform is required to integrate disparate applications and systems for real-time data flow and management. Using the specially designed laboratory, engineers will now be able to answer key questions such as how to determine the exact configuration of a system, what are the renewable energy interconnection issues and which is the proper way to design protection system and the tripping logic? How can

we increase the reliability of an electricity network, How to test the behavior of a communication device in the distribution network?

Smart Grid fundamentals include definitions, architecture, formulation of performance requirements, discussion of development of analytical and decision support tools, as well as renewable energy resources. The design of such a grid will be based on cross boundaries of knowledge in communication theory, optimization, control, social and environmental constraints as well as dynamic optimization techniques [99]. 
International standards such as IEC 61850 and 61968, define the overall architecture of a Smart Grid and its characteristics can be summarized as follows [100]:

- Self-healing by detecting and instantly responding to system problems and restoring

- Provision of high power quality to all consumers and industrial customers

- Accommodating a wide variety of generation options in local and regional scale

- Empowerment of the customer by allowing energy management

- Tolerance of attack by standing resilient to physical and cyber attacks

- Optimizing assets and operating efficiency

The advancement of the research and curriculum is necessary for preparing engineers to advance their skills in the new highly technical environment. This will require increased focus on coupling of power systems with communication systems, information technology, control technologies and embedded system. While many smart grid topics have been identified in the literature, the following are some illustrations of the topics that have not received adequate attention in the education so far and yet they are essential in understanding and implementing the smart grid developments [101]:

- Phasor measurements units and their application in power systems for improved monitoring, protection and control

- Integration of distributed sources including renewable resources (wind, solar, geothermal, etc) in power generation and their fully control structure

- The concept of digital protection in new protective digital relaying, monitoring, control, power quality and asset management applications 
- Intelligent protection schemes and their application in detecting, mitigating and preventing cascading outages, islanding situation and total grid blackout occurrences

- Impact of Plug-In Hybrid Electric Vehicles (PHEVs) and Electric Battery vehicles (EBVs) on electricity and transportation infrastructure

- Multifunctional uses of smart meters for revenue metering, demand side management, outage management and load control

- Integration of Hybrid AC-DC systems in creating new micro grid solutions for residential and industrial applications

- Enhancement of energy efficiency by the technologies that allow implementation of highly efficient and economical affordable solutions for the Smart Grid

- Green House Gas (GHG) emissions and means of lowering carbon footprint of the Smart grid solutions

The Energy System Research Laboratory at Florida International University is working on constructing and implementing of a small-scale power system test-bed which has different capabilities for experimental research and educational purposes. This setup uses laboratory scale of power system components in order to model the realistic behavior of a large power system. By having this type of power system, engineers and researchers are capable to implement their own idea about power system phenomenon in a practical way. It would be an excellent base not only for innovative research ideas, but also for teaching power system engineering concepts to students who are interested to get an overall idea of power system operation and the continual insertion of renewable resources. 


\subsection{Hardware Employed on the Test Bed}

The Test-bed power system grid in the Energy Systems Research Laboratory is being developed for different applications, as well as Smart Grid operation study of a power system. Most of the power system laboratories have some power system elements available for education and experimental tests and they don't provide the integrated power system test-bed for studying on interconnection, control and protection issues. Mostly, they use software emulation through Real-Time Digital Power System Simulators (RTDS). To our knowledge this is the first effort of its kind of involving both hardware and software interactions. The objective of this work is to integrate all power system components together for architecture more realistic laboratory scale power system

for research and education purpose. All the equipment used for this test-bed setup will be discussed and illustrated in the following sections with their control and communication capabilities.

\subsubsection{Generation Station Model}

The generators used in the test bed are $13.8 \mathrm{KVA}$ and $10.4 \mathrm{KVA}$, at $60 \mathrm{~Hz}, 230 \mathrm{~V}$, 1800 RPM synchronous machine. The prime mover of these generators is coupled to one of available motors (AC 230V: 1, 2, 3 and 4 HP; DC 180V 2 HP), which is derived by different frequency drives. All the generators are equipped with an Automatic Voltage Regulator (AVR) in order to maintain an output voltage magnitude.

The AVR model (SX460) is a half-wave phase-controlled thyristor type Automatic Voltage Regulator and forms part of excitation system for brush-less generators. In addition to regulating the generator voltage, the AVR circuitry includes under-speed and sensing loss protection features. Excitation power is derived directly 
from the generator terminals. Positive voltage build up from residual levels is ensured by the use of efficient semiconductors in the power circuitry of the AVR. The AVR is linked with the main stator windings and the exciter field windings to provide closed loop control of the output voltage with load regulation of $\pm 1.5 \%$. For control purposes, the only way to adjust the AVR output is to adjust its controller parameters by available potentiometers on this module. Hence, the output voltage of generators should be maintained by applying offline parameter settings.

The Frequency Drive is a Lenze AC Tech SMVector Series, which can be used with 3-phase AC induction motors rated from $1 / 3 \mathrm{HP}$ up to $25 \mathrm{HP}(0.25-18.5 \mathrm{~kW})$ on voltages from $120 \mathrm{~V}$ single-phase to $600 \mathrm{~V}$ three-phase. Programmable digital and analog $\mathrm{I} / \mathrm{O}$ allows the drive to be configured for many application specific tasks such as multiple preset speeds, electronic braking and motor jogging. Like all AC drives, they use Electric Programming Module (EPM) memory technology for fast and efficient programming. There are three ways to program the EPM: 1) use the intuitive SMVector integrated keypad, 2) program in a Microsoft Windows environment with Techlink, or 3) use with the lightweight portable EPM programmer. For implementing a smart grid with control, method two has been used in order to control the frequency and change the output active power of generators. The used control modes of this drive are:

- Vector Speed (for single-motor applications requiring higher starting torque and speed regulation)

- Vector Torque (for single-motor applications requiring torque control independent of speed) 
Figure 3.1 shows the generator station and its components. Figure 3.2 shows the implemented wireless remote control technique of generator frequency controllers. The generators parameters have been presented in Table 3.1.

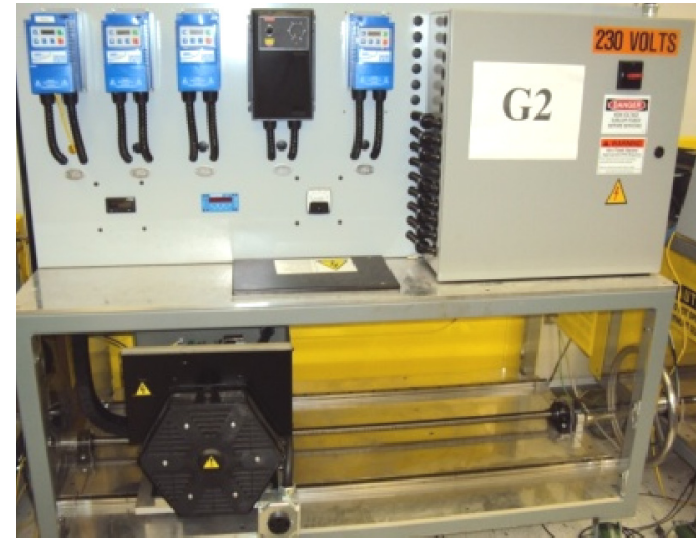

Generation Station

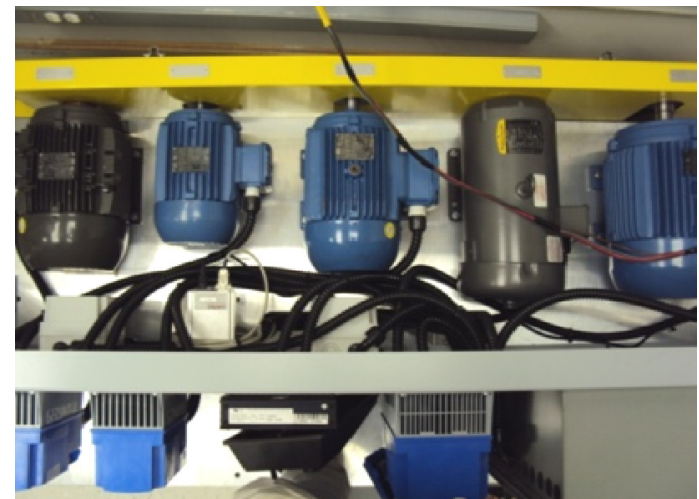

Prime Mover Motors

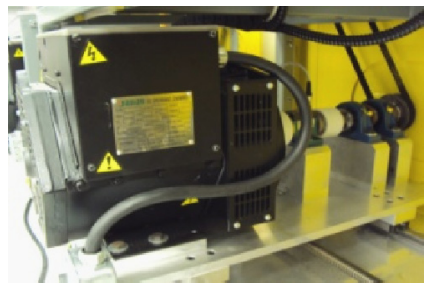

Synchronous Generator and Coupling Belt

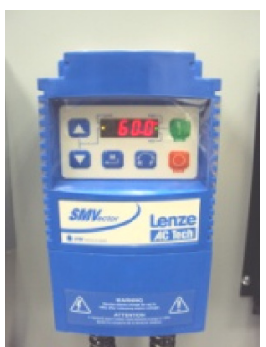

Frequency Drive

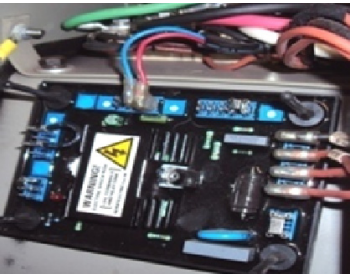

AVR Module

Figure 3.1: Generation station and its components

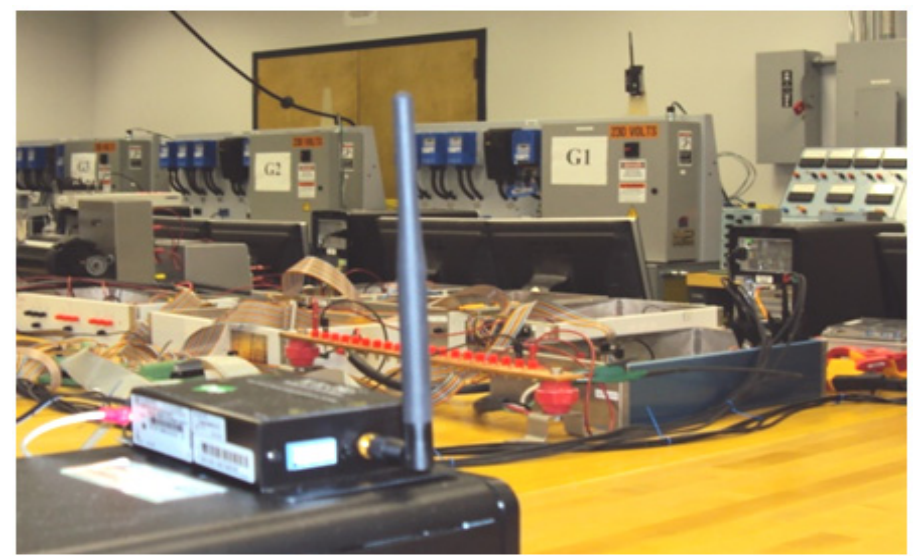

Figure 3.2: Wireless remote control of generators 


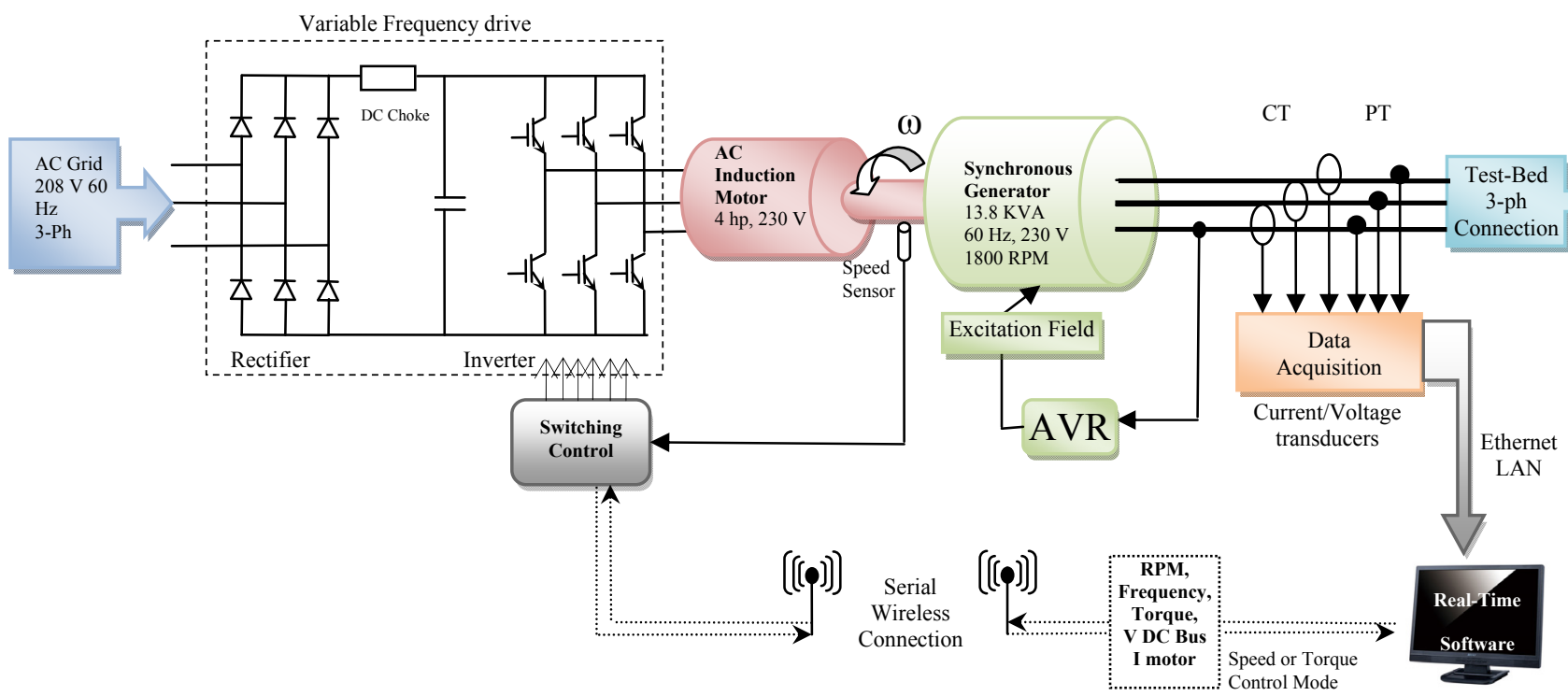

Figure 3.3: Overall schematic of a generator station and its measurements, communication and controller components

\subsubsection{Transmission Line Model}

All transmission lines were designed for emulating different lengths of typical $\pi$ model, in which every line has its own parameters. These are series inductor (which has an internal resistance) and two parallel capacitors (with internal resistor) for both sides in each phase as shown in figure 3.4 with single line diagram and one of developed boxes. In the case of fault or other type of event such as over-load, all lines are protected by fuses in order to prevent damaging hardware components in the test-bed setup.

The line components are designed for 250-V11, 15-A current per phase. The power transferring capacity for the three phase system in nominal voltage $(120-\mathrm{V}$ line to ground) is 5.4-kVA. The line designs allow for a five wire connection (three-phases, neutral and ground). The line parameters have been chosen in a way to scale down the emulation of real transmission line (i.e. $X / R>>1)$ and they are presented in Table 3.2. The developed test-bed is designed to represent a transmission system according to the selection of the pi-models emulating transmission lines as well as the ring configuration. 
However, this test-bed can be easily prepared to represent the distribution system by modifying the configuration and selecting the proper distribution line model.

Table 3.1: Synchronous Generators Parameters

\begin{tabular}{lcc}
\hline \hline \multicolumn{1}{c}{ Parameter Name } & G1, G3 & G2, G4 \\
\hline Apparent Power & $13.8 \mathrm{kVA}$ & $10.4 \mathrm{kVA}$ \\
Nominal Voltage & $230 \mathrm{~V}$ & $230 \mathrm{~V}$ \\
Nominal Power Factor & 0.8 & 0.8 \\
Acc. Time Constant & $0.005625 \mathrm{~s}$ & $0.004 \mathrm{~s}$ \\
Stator Leakage Reactance (xl) & $0.09 \mathrm{pu}$ & $0.09 \mathrm{pu}$ \\
d-axis Synchronous Reactance (Xd) & $2.21 \mathrm{pu}$ & $2.248 \mathrm{pu}$ \\
q-axis Synchronous Reactance (Xq) & $1.1 \mathrm{pu}$ & $1.117 \mathrm{pu}$ \\
d-axis Transient Time Constant (Td') & $0.014 \mathrm{~s}$ & $0.012 \mathrm{~s}$ \\
d-axis Transient Reactance (Xd') & $0.23 \mathrm{pu}$ & $0.23 \mathrm{pu}$ \\
d-axis Subtransient Time Constant (Td") & $0.0035 \mathrm{~s}$ & $0.003 \mathrm{~s}$ \\
q-axis Subtransient Time Constant (Tq") & $0.05 \mathrm{~s}$ & $0.05 \mathrm{~s}$ \\
d-axis Transient Reactance (Xd") & $0.14 \mathrm{pu}$ & $0.144 \mathrm{pu}$ \\
q-axis Transient Reactance (Xq") & $0.25 \mathrm{pu}$ & $0.258 \mathrm{pu}$ \\
\hline \hline
\end{tabular}

\subsubsection{Bus Model}

For switching and measurement purposes, special boxes were built to implement the bus model. Fig. 3.5 shows the design of one of the buses. This general design has three sets of three-phase inputs and outputs. Hence, we can connect an input to three different branches. Each input/output has a 530-V, 25-A solid-state relay, whose switching can be controlled by a 3-32 V dc voltage. This voltage can be applied by Data Acquisition Systems (DAQs) digital output and a computer controller. Each phase has its own Potential Transformer (PT) and Current Transformer (CT). The voltage and current ratios for the PT and CT are 20:1 and 3:1, respectively. The PTs are connected between phases and ground, so they can present line to ground voltage. The secondary side of these CTs and PTs are connected to communication terminal and then to the DAQs. 
Therefore, the real-time controller can switch any branch according to the overall system strategy and real-time monitored voltage and current data.

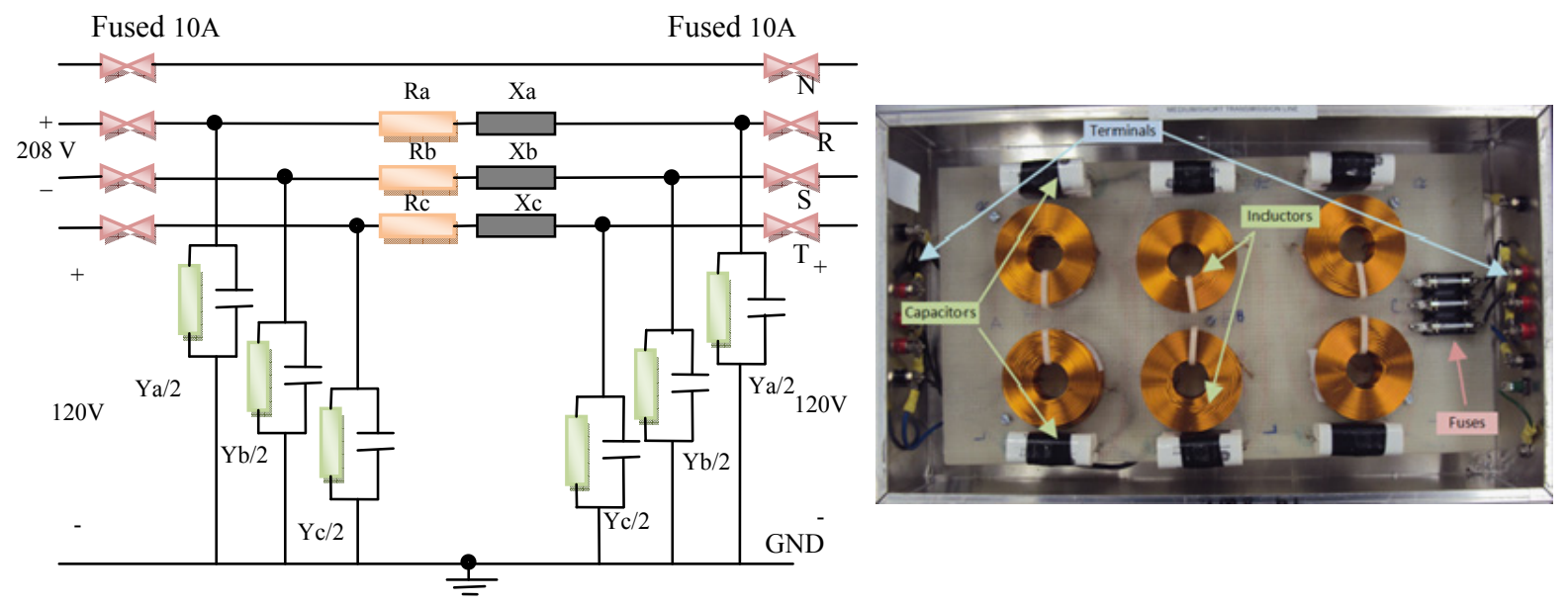

Figure 3.4: Single line diagram and developed box for power transmission line $\pi$-model with fuse protection

Table 3.2: Transmission Line Parameters

\begin{tabular}{lllll}
\hline \hline $\begin{array}{l}\text { Parameter } \\
\text { Name }\end{array}$ & $\begin{array}{l}\text { Maximum } \\
\text { Current }\end{array}$ & Resistance & Reactance & Suseptance \\
\hline Line 0170 & $15 \mathrm{~A}$ & $0.417 \Omega$ & $1.264 \Omega$ & $3200 \mu \mathrm{S}$ \\
Line 0250 & $15 \mathrm{~A}$ & $2.831 \Omega$ & $4.231 \Omega$ & $4350 \mu \mathrm{S}$ \\
Line 0510 & $15 \mathrm{~A}$ & $0.15 \Omega$ & $1.46 \Omega$ & $2558 \mu \mathrm{S}$ \\
Line 0520 & $15 \mathrm{~A}$ & $0.18 \Omega$ & $1.43 \Omega$ & $2571 \mu \mathrm{S}$ \\
Line 0530 & $15 \mathrm{~A}$ & $0.14 \Omega$ & $1.2 \Omega$ & $2581 \mu \mathrm{S}$ \\
Line 0540 & $15 \mathrm{~A}$ & $0.15 \Omega$ & $1.29 \Omega$ & $2574 \mu \mathrm{S}$ \\
Line 0550 & $15 \mathrm{~A}$ & $0.14 \Omega$ & $1.63 \Omega$ & $2590 \mu \mathrm{S}$ \\
Line 0560 & $15 \mathrm{~A}$ & $0.16 \Omega$ & $1.4 \Omega$ & $2582 \mu \mathrm{S}$ \\
\hline \hline
\end{tabular}

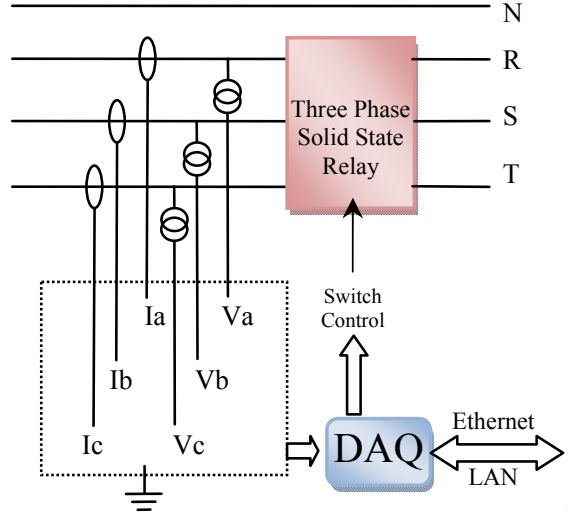

Figure 3.5: Implemented Bus Model with communication capability 


\subsubsection{Load Model}

Different load models were designed for the hardware smart grid test-bed. One of the passive loads built has a switching capacity of 10 levels parallel of resistive loads from $300-\mathrm{W}$ to $3-\mathrm{kW}$ power in steps of $300-\mathrm{W}$ at a nominal voltage $[2 \times 72-\Omega+4 \times 144-\Omega$ in each phase] that can be switched by the controller software to emulate various load patterns. Induction motors are used as dynamic load models. These loads have large reactive power demand and are coupled to variable mechanical load connected on their shaft. A real-time controllable load was also developed to control motors loading.

For studying system performance, the load level can be set by changing the reference signal. This type of load control makes the implementation of the smart grid concept in hardware more realistic and achievable. The motor load emulator system is shown in figure 3.6. As shown in this figure, this system consists of a $250-\mathrm{W}$ induction machine, operating as an induction motor load, a 250-W dc machine, operating as a separately excited DC generator, a resistive load connected to the DC generator, an IGBT switching circuit for controlling the field of the DC generator and finally a freewheeling diode for circulating the field current when the IGBT is open. An anti-windup PI controller provides the proper duty cycle for the IGBT operation. This duty cycle is applied to the IGBT via the Gate block which converts the duty cycle to the appropriate gating signals. The real-time controller is used to change load level according to system's overall status and implement the strategies such as load shedding and load side management. Performance of the system is exhibited in figure 3.7. The reference load pattern consists of 4 steps of $[90,250,150,200]$ Watts, with the step length of $1 \mathrm{sec}$. This pattern is fed into the control system as the reference. The output duty cycle provided by 
the PI controller is presented. Also, the reference power, $\mathrm{P}_{\text {ref }}$ and actual power, $\mathrm{P}(\mathrm{t})$, profiles have been shown together. Comparing these curves verifies the validity of the developed motor load emulator. As can be seen, the system can respond to the inputs in a range of almost $80 \mathrm{~W}$ (no-load power) to $250 \mathrm{~W}$ (rated power). It is witnessed that, the system is stable and the output follows the reference both fast and accurately. System output settles in less than $0.5 \mathrm{sec}$ which is a satisfactory response time for a mechanical system.

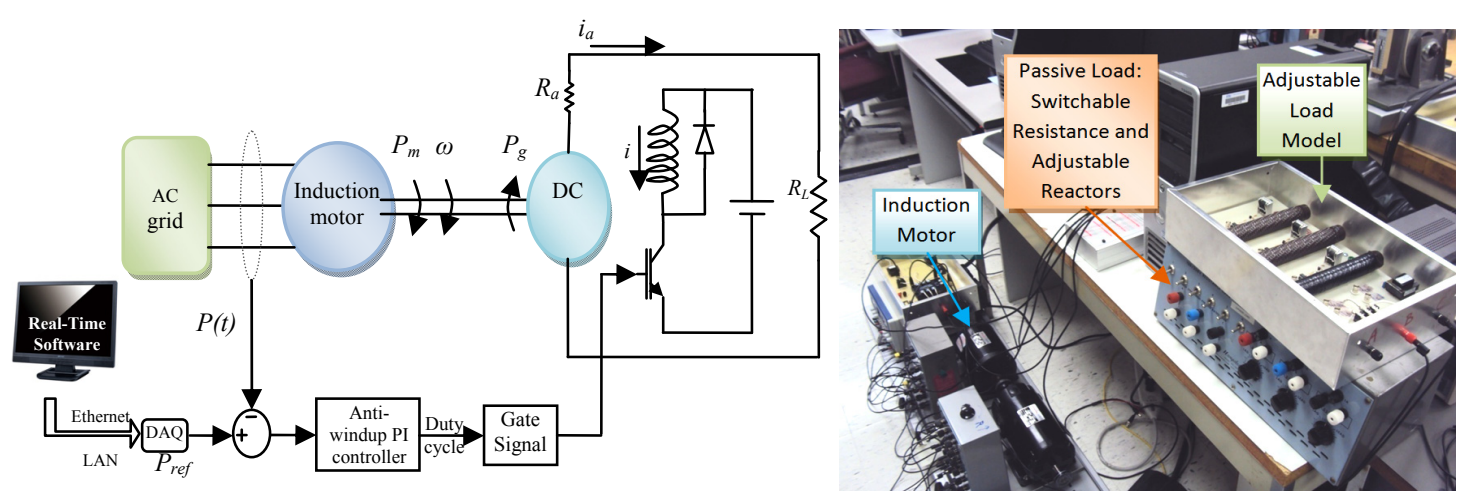

Figure 3.6: Motor load emulator model
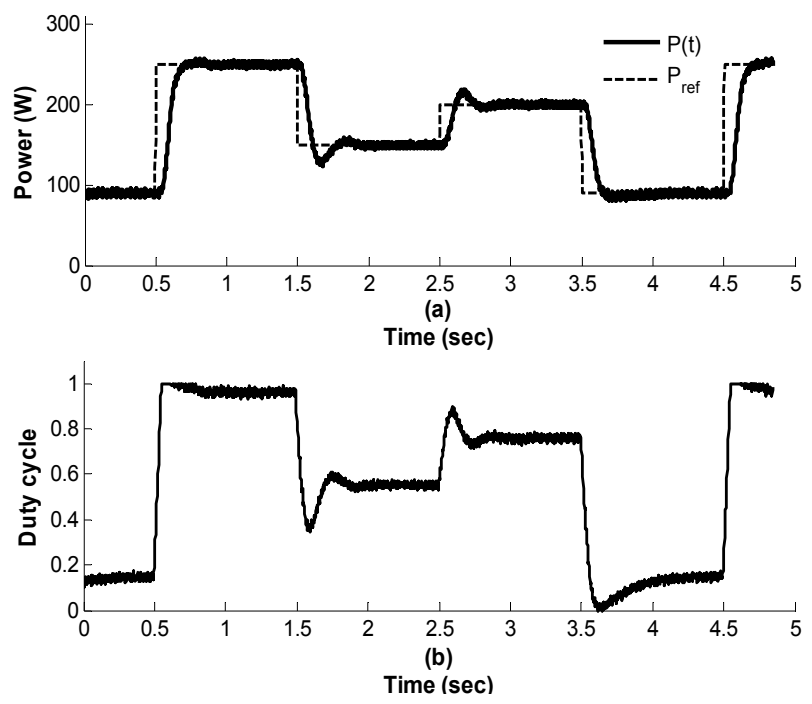

Figure 3.7: Motor load emulator model 


\subsubsection{Synchronizer Model}

Synchronizers assure that a generator attempting to connect in parallel with the electric system can do so without causing an electrical disturbance to the equipment connected to the same system. In addition, the synchronizer assures that the generator attempting to connect in parallel with the system will not be damaged due to an improper parallel action. Hence, the synchronizer model shown in figure 3.8 was developed. Each relay has PTs available on both sides for voltage measurements. The software environment of the synchronizer controller checks the synchronizer conditions. The left side is used for the generator side parameters and the right side is used for the system side parameters. These conditions are shown below:

$$
\begin{aligned}
& \left|\bar{V}_{\text {right }}-\bar{V}_{\text {left }}\right|<3 V \\
& \left|f_{\text {right }}-f_{\text {left }}\right|<0.1 \mathrm{~Hz} \\
& \left|\angle V_{R-\text { right }}-\angle V_{R-\text { left }}\right|<2 \mathrm{deg} \\
& \left|\angle V_{R S \text {-right }}-\angle V_{R S \text {-left }}\right|<5 \mathrm{deg}
\end{aligned}
$$

where $V_{\text {right }}$ and $V_{\text {left }}$ are the voltages of right and left sides of synchronizer, $f_{\text {right }}$ and $f_{\text {left }}$ are the frequencies measured in voltage waveform for the right and the left side of the synchronizer, respectively. Figure 3.9 shows the synchronization algorithm flowchart developed as part of the real-time software. 

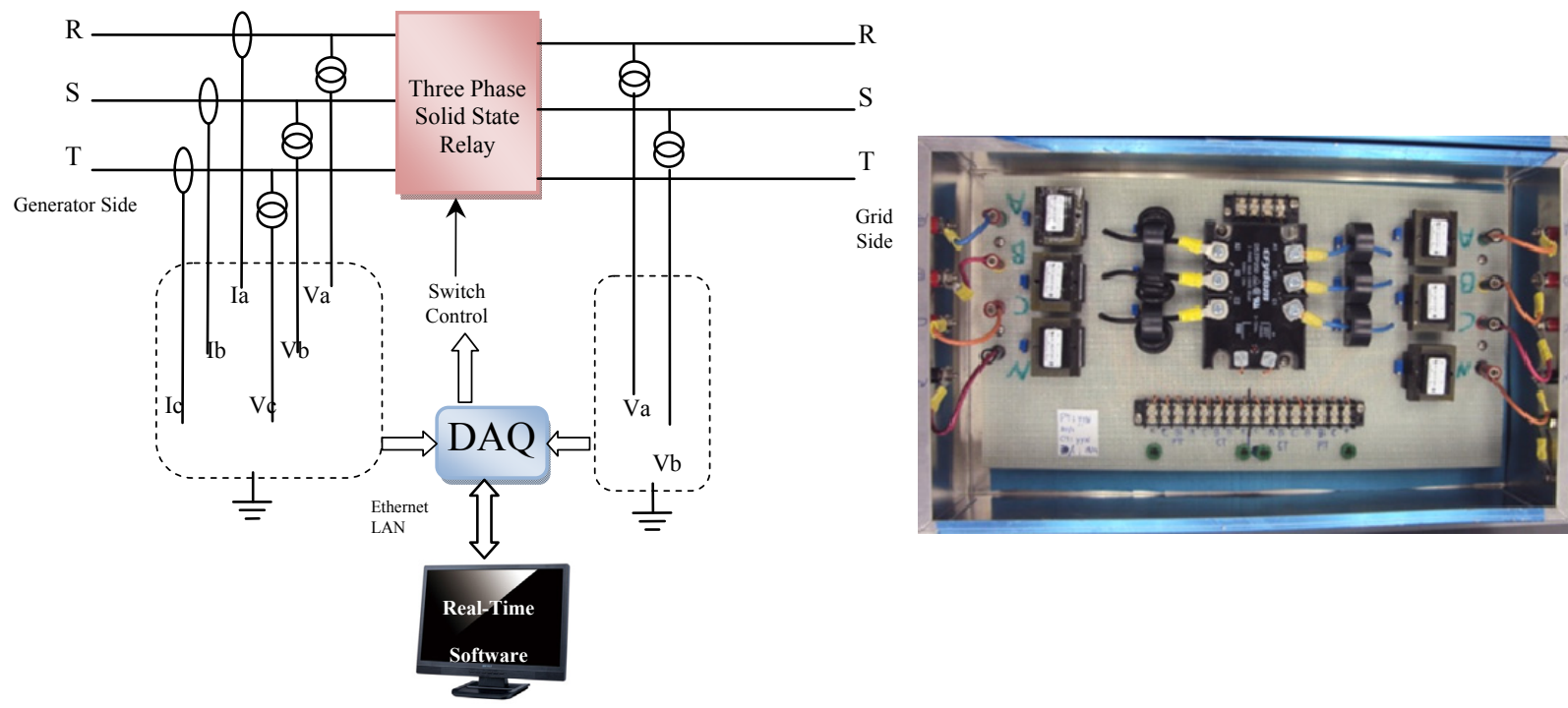

Figure 3.8: Synchronizer bus model with both side measurements

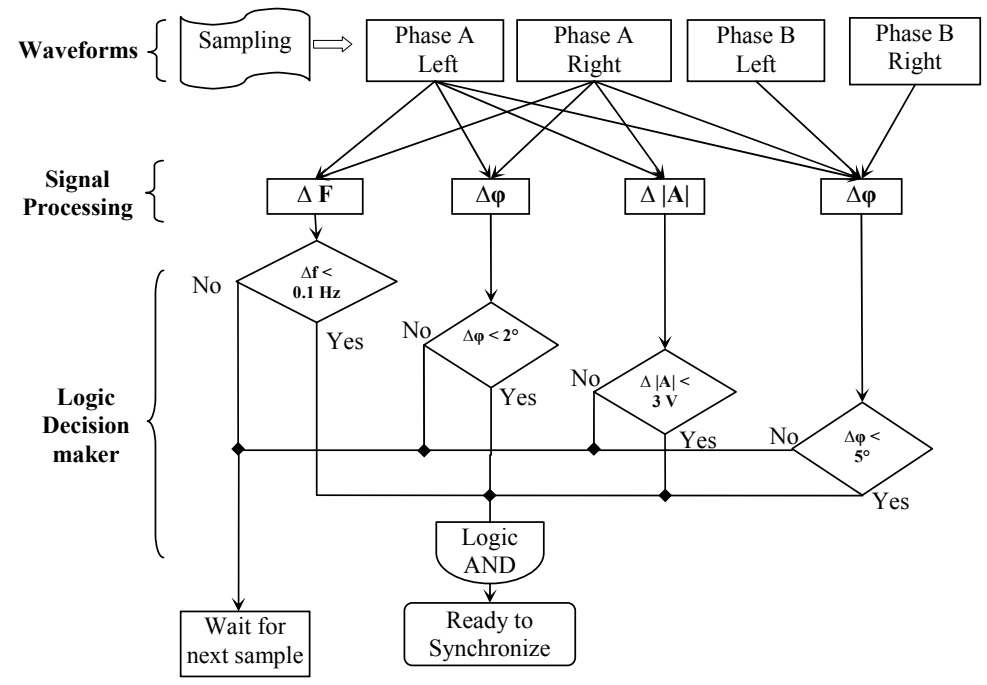

Figure 3.9: Flowchart algorithm of the synchronizer developed in real-time software

\subsection{Real-Time System Monitoring and Control Platform}

\subsubsection{Data Acquisition Devices}

There are several operating systems that have been implemented at power system laboratories; Programmable Logic Controller (PLC), Real-time Digital Simulator (RTDS) and Data Acquisition Devices (DAQ). The laboratory power system setup along with all 
related hardware was designed to use DAQs as real-time module. Using different DAQ types, the monitoring and control of all system components is possible. The main elements include several USB type (32 analog inputs, 16-bit; $1.25 \mathrm{MS} / \mathrm{s}$ single-channel and 4 analog outputs, 48 digital I/O; two 32-bit counters), PCI type (1.25 MS/s, 12-Bit, 64-Analog-Input Multifunction DAQ) and Wireless type (Isolated Wireless Voltage Input: 16-Ch, 16-Bit, $250 \mathrm{kS} / \mathrm{s})$. Finally, the compact-DAQ was also used to extend the reach of high-speed data acquisition to remote electrical measurement and controlling them from across the lab through Ethernet and computer connection. Figure 3.10 demonstrates the DAQs types and their connection to the Ethernet/LAN environment. Hence, all the computers connected to the same LAN can run real-time software in order to monitor and control the power system.

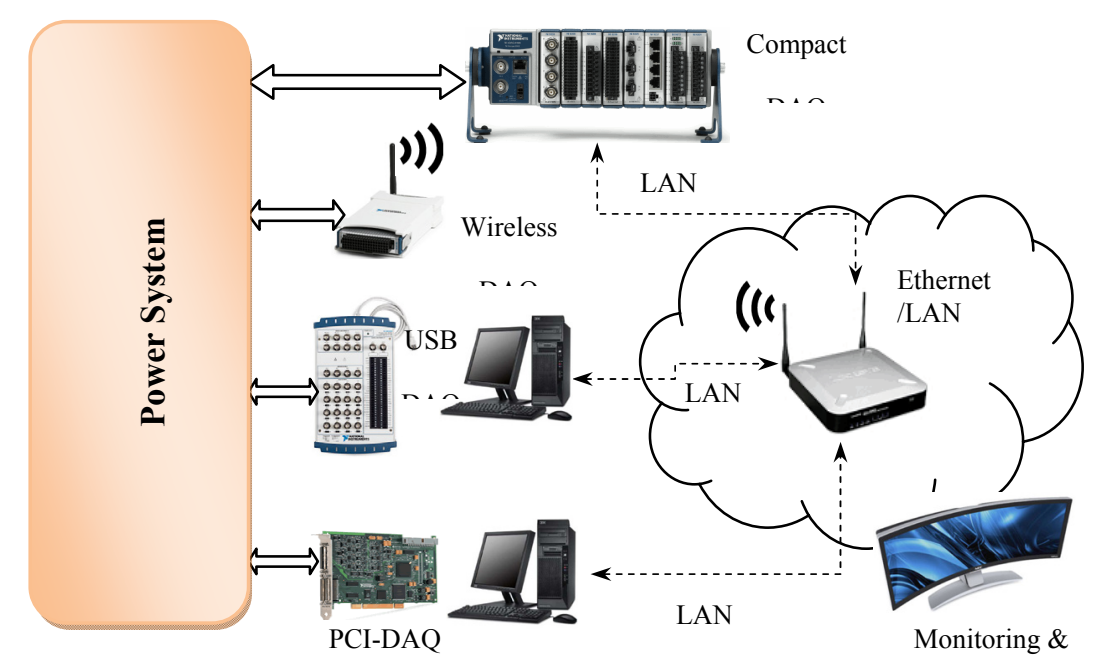

Figure 3.10: Different types of DAQs and their connection to the Ethernet/LAN

\subsubsection{Real Time LabVIEW Software}

LabVIEW graphical system design software has been selected because of its ease of use and seamless integration with NI DAQ hardware. LabVIEW is a graphical programming tool for test, measurement and automation and is widely used as a virtual 
instrumentation tool. It allows users to develop sophisticated measurement, test and control systems using intuitive graphical icons and wires that resemble a flowchart. One of the major advantages of LabVIEW, apart from being simple to use, is the ability to work with a number of hardware interfaces using real world analog and digital signals. LabVIEW programs work as simulation or data acquisition applications depending on the requirement using custom built hardware from National Instruments. This consists of two windows, a block diagram window where the actual graphical code is written and the front panel where the output can be visualized. The manner in which LabVIEW works, it ties the creation of user interfaces (called front panels) into the development cycle. The programs/subroutines are called virtual instruments (VIs) in which each VI has three components: a block diagram, a front panel and a connector panel. In the test-bed power system lab, we programmed the application using LabVIEW which provides a real-time view of the entire system on a primary screen and a live view of all analog values displayed as VIs. LabVIEW also provided the flexibility to display all analog signals in a graph and record them on-demand and on an external trigger.

\subsection{Test and Verification of Developed Techniques}

The overall AC part of the power system which is implemented in test bed by LabVIEW VI is shown in figure 3.11. The setup has four generators connected in ring combination and supply loads connected to the load buses in the end of lines which are

connected to generator buses. In addition, synchronizers placed after each generator in order to have the safe switching action for connecting generators to the grid. 
The overall system includes linking the communication infrastructure of our NI DAQs monitoring system to a common communication network in order to implement supervisory control and data acquisition (SCADA) power system control equipment and to have the ability to access all measurement data remotely via an Ethernet connection.

Figure 3.12 represents test-bed power system communication infrastructure for implementing real-time monitoring and control purposes. The wired and wireless communication platform for data transferring has been used in this setup. Figure 3.13 shows a part of an implemented block diagram of a sub VI for measurement of voltage phasors and sequences in LabVIEW environment in real time format. All the measurement points which have VTs and CTs available have been represented by a front panel similar to figure 3.14. In this figure the real time values of voltages and currents of the feeder in three phase format and sequences network are presented. In addition, by implementing proper block diagram including math functions, the active and reactive power as well as power factor characteristic of each branch, have been calculated and are shown in this page. The phasor diagram also show the phase difference between the voltages and the currents of each phase, which can be used as a synchronized phasor measurement by using reference time scale for all the measured signals. Overall, the monitoring system based on NI hardware and software proved to be a highly flexible and easy-to-use solution for our application.

The described power system hardware setup is implemented according to figure 3.15 at Energy Systems Research Lab. The real-time waveforms of the voltages and currents of every point have been captured by the LabVIEW environment. Figure 3.16 and 3.17 shows the test-bed setup voltages and currents of one generator, respectively. 


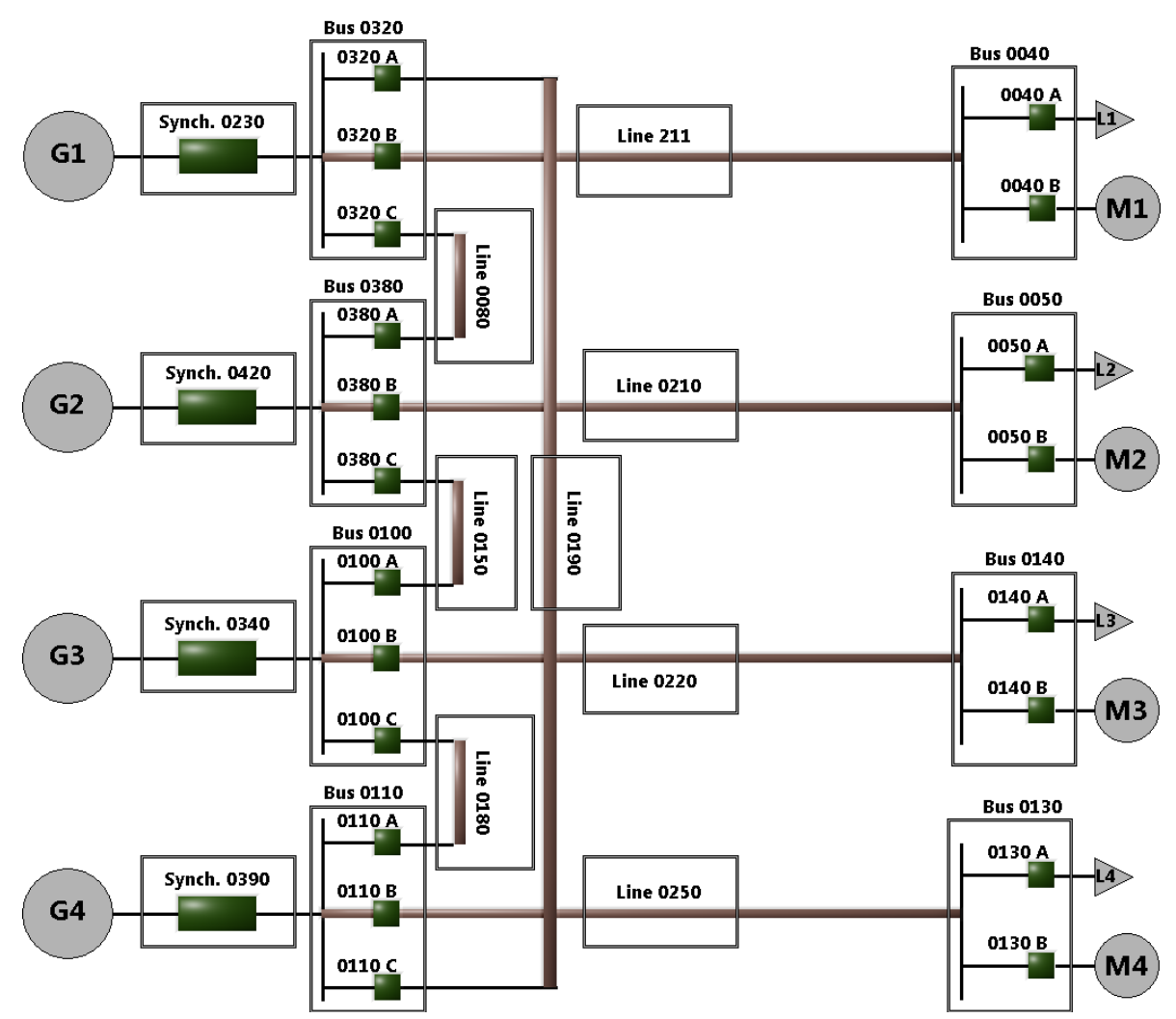

Figure 3.11: Overall power system schematic and single line

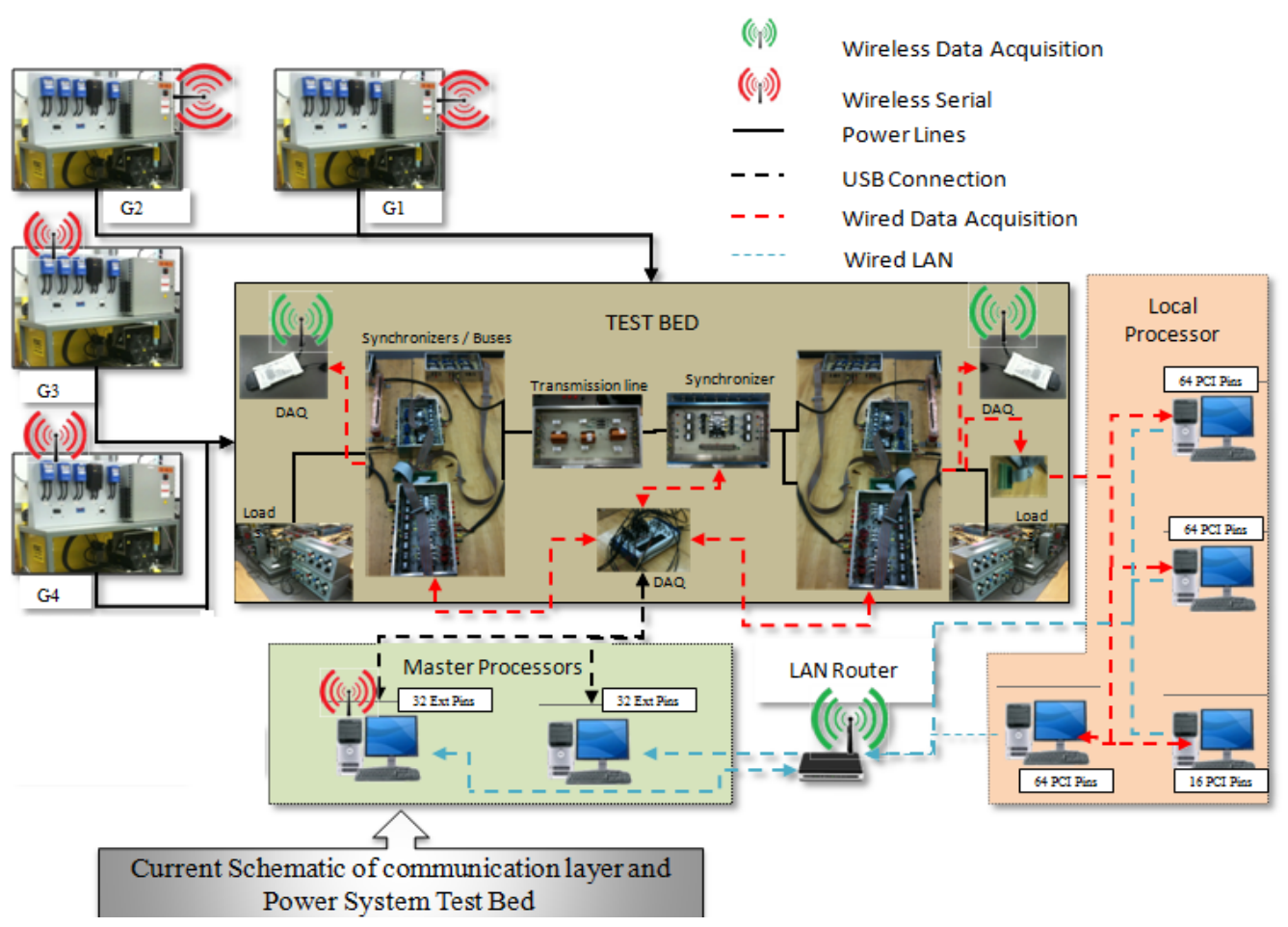

Figure 3.12: Test-bed power system communication infrastructure 


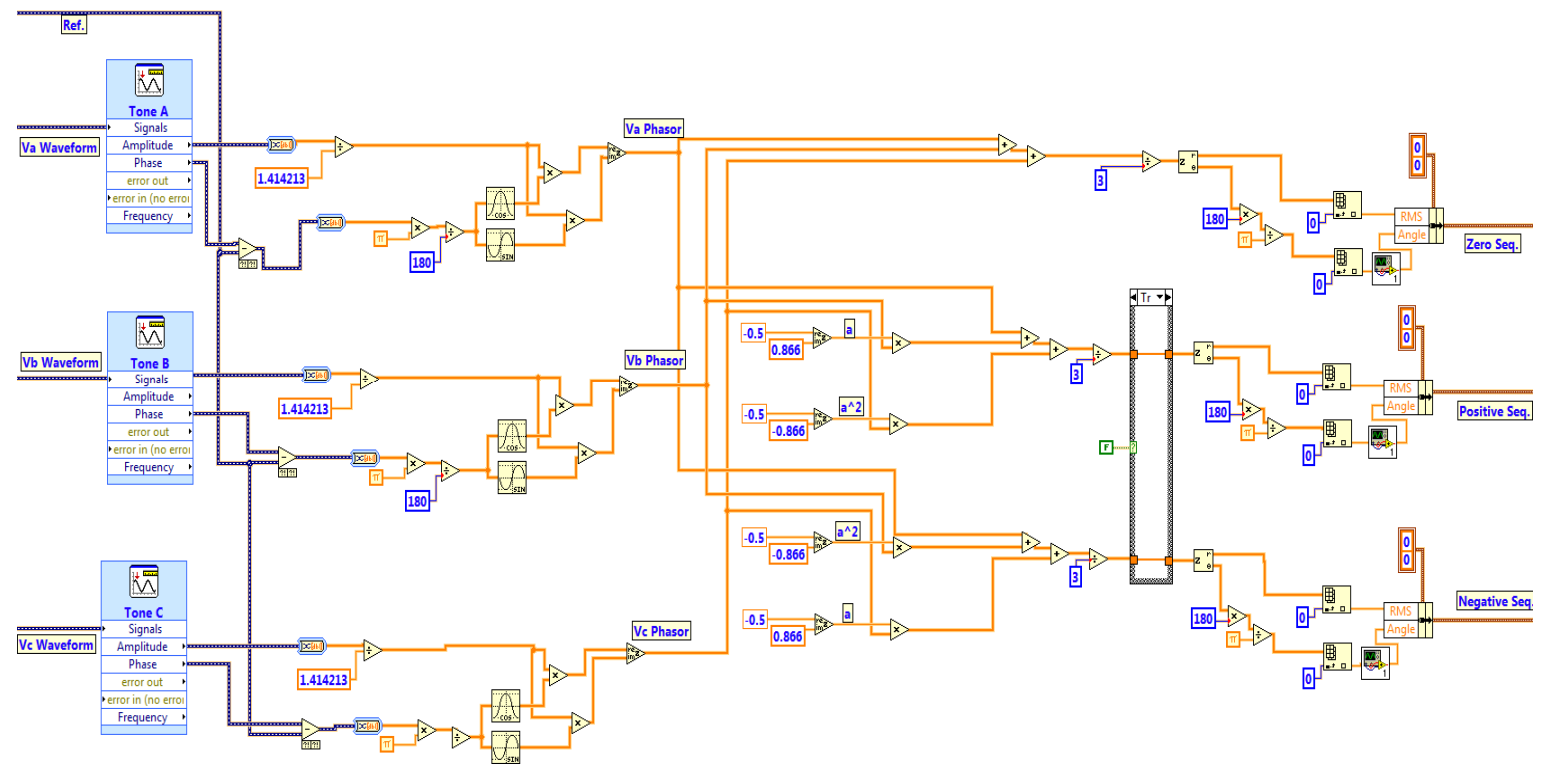

Figure 3.13: Block diagram of a phasor and sequences calculation sub VI in the LabVIEW

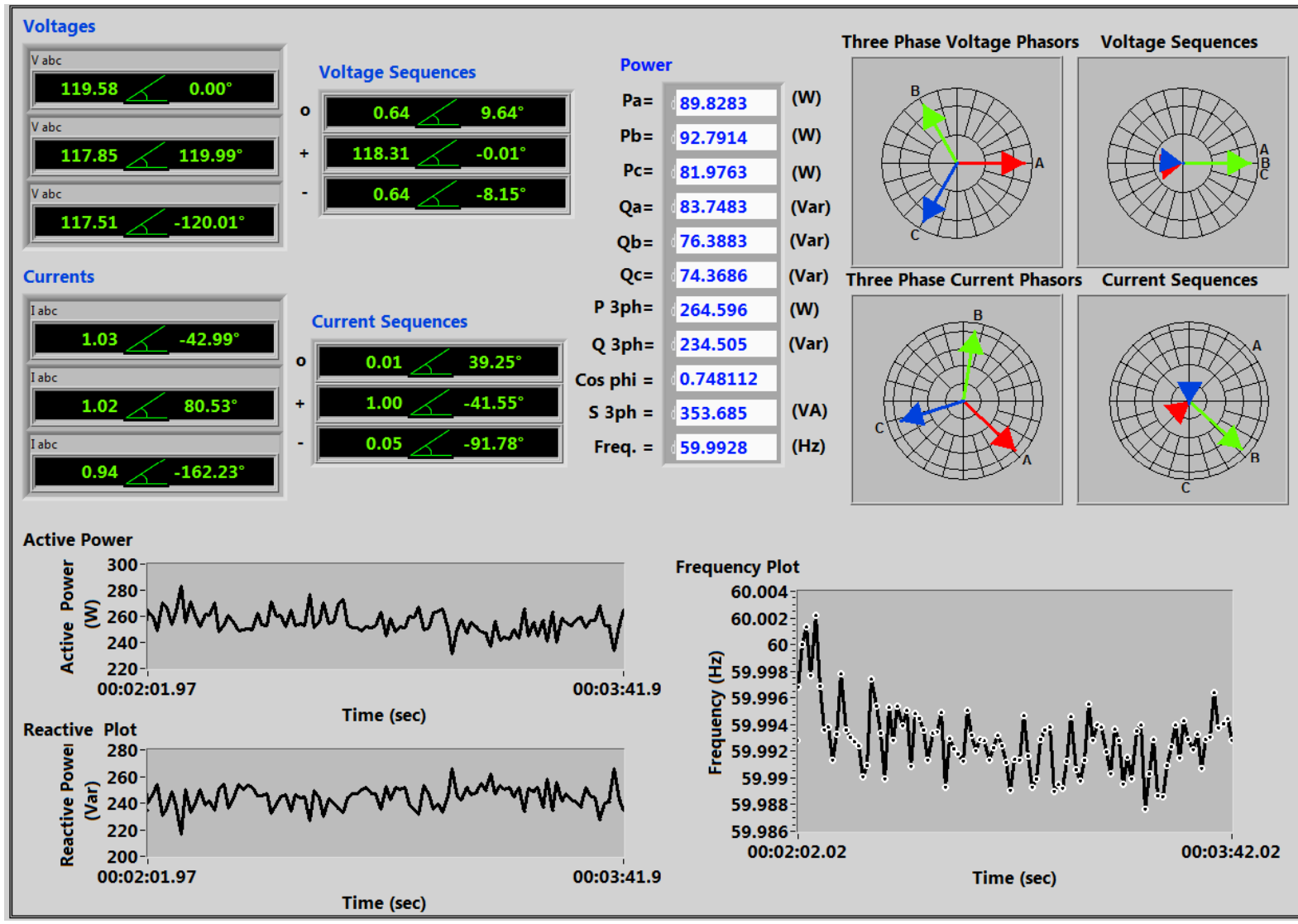

Figure 3.14: Front Panel of measurement sub VI in the LabVIEW 
. By using a measurement sub VI according to figure 3.14 one can also calculate the active and reactive power generated by this generator in real time format and present it in a time plot graph. Hence, we can measure all data available on the network, by using the DAQs to capture the waveforms of the PTs and CTs. The ON/OFF switching of the relays and the generator operation control are also done by the same DAQs and LabVIEW software.

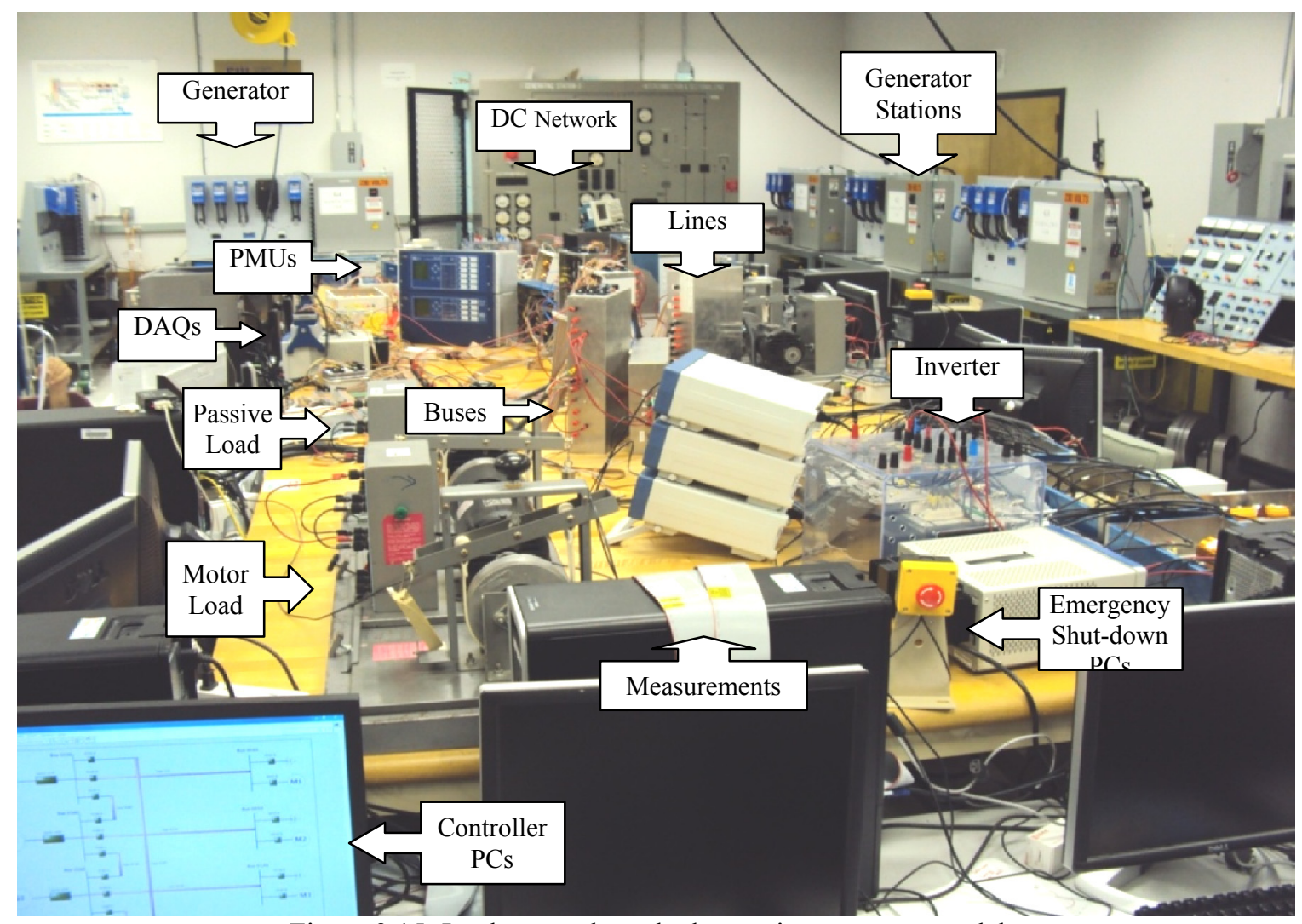

Figure 3.15: Implemented test-bed setup in power system lab

To verify our results, the same voltages, currents and phasor diagrams have been measured directly with a power quality analyzer (fluke 435). Figure 3.18 shows the voltages, currents and phasor diagram of generator G1. The measurement results obtained by the power quality analyzer verify the results for our implemented real time monitoring results. The laboratory experiment and analysis guarantee a high level of reliability when 


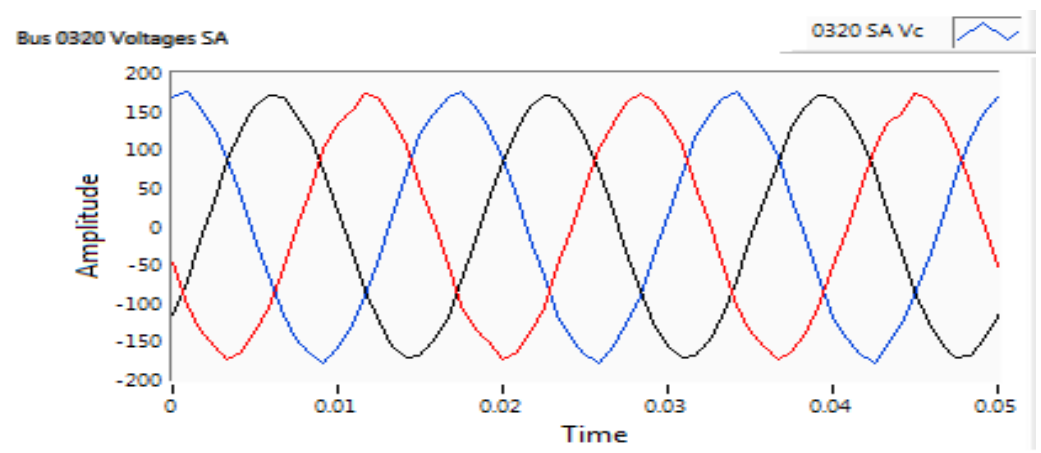

Figure 3.16: Real-Time voltages of generator G1 in LabVIEW

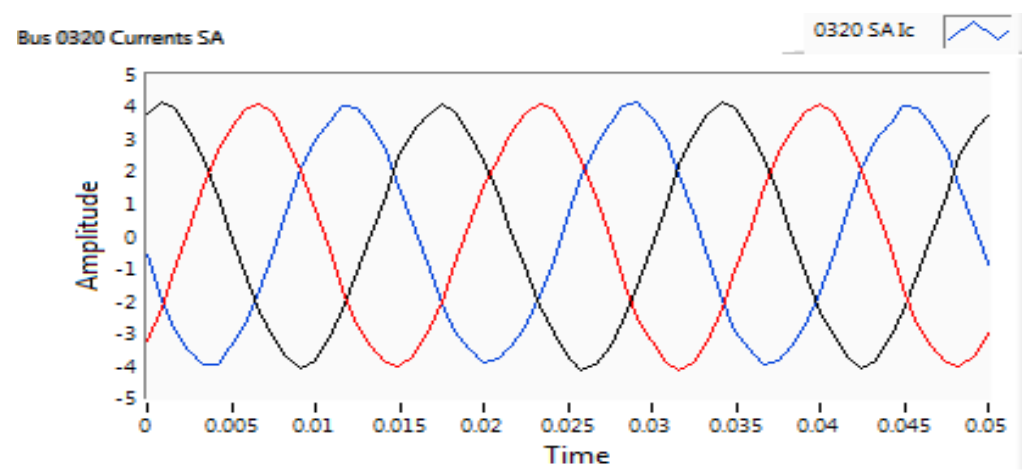

Figure 3.17: Real-Time currents of generator G1 in LabVIEW

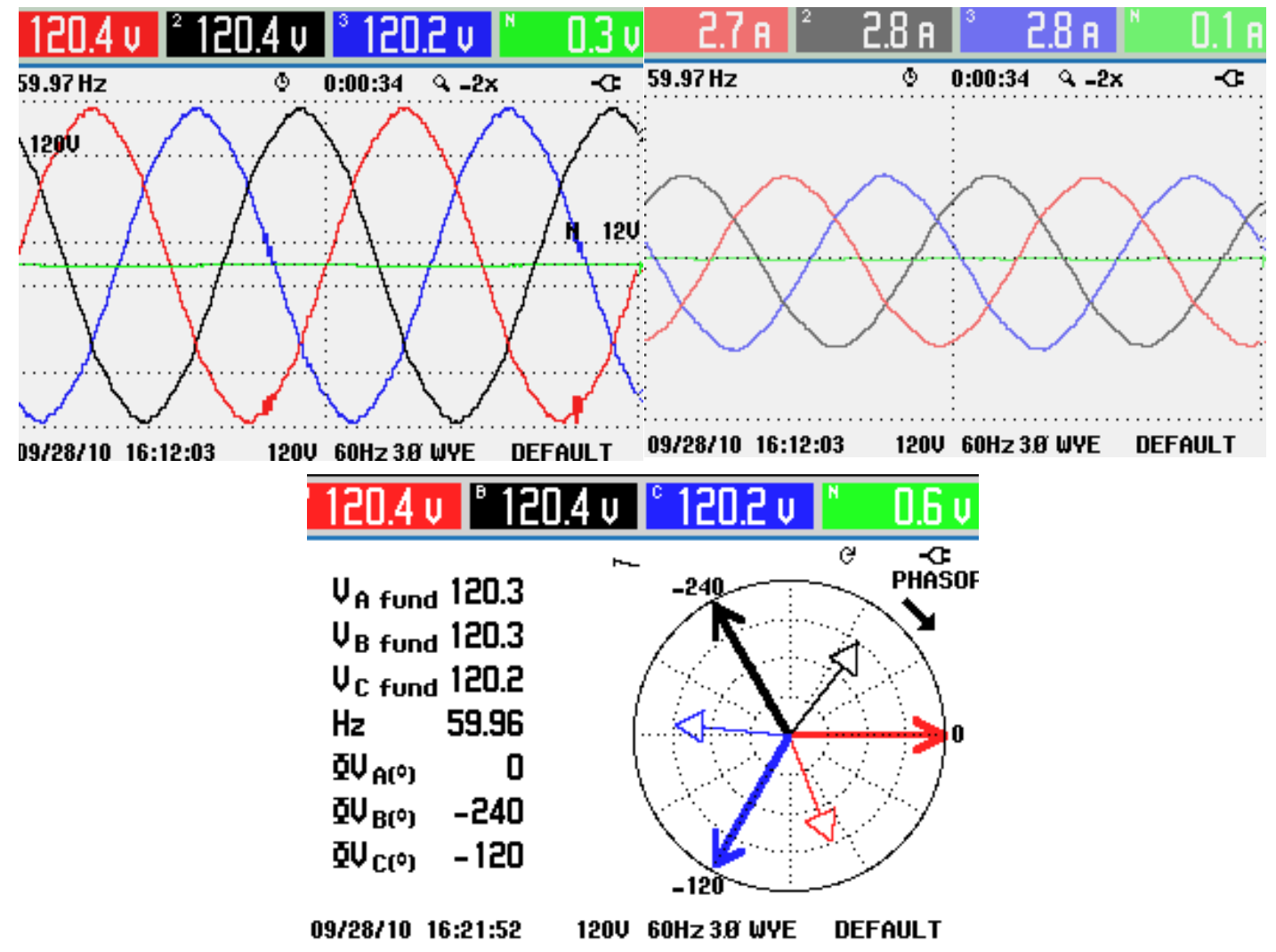

Figure 3.18: Voltages, currents and phasor diagram of generator G1 in measurement device (Fluke 435) 
the smart grid is implemented in the actual field. Educational application of laboratorybased smart grid and its real-time analysis capability provide the platform for investigation of the most challenging aspects of a real power system. 


\section{CHAPTER 4}

\section{PHASOR MEASUREMENT UNITS AND THEIR APPLICATIONS IN SMART GRID LABORATORY}

\subsection{Introduction}

The phase angle of voltage of the power system is great importance to engineers interested in calculating the well-known power-flow equation. The objective is for achieving transmitted active power between two buses. The earliest modern application of phase angle differences was reported in literature in 1980s in [106-108]. They used satellite and radio transmissions systems to obtain synchronization of reference time at different locations in a power system. The phase angle difference between voltages at two buses was implemented using the difference of measured angles on a common reference at different locations. Phasor measurement technology had major progress when the digital relaying of power system using microprocessor-based relays were developed and used in transmission lines. They are capable to calculate symmetrical components of voltage and current to detect different types of faults in power system. Various algorithms were introduced for computing the symmetrical components and the positive sequence voltage and the current of the three phase power systems. The first publication which implied the importance of phasor measurements and their application was published in 1983 [109]. This work appeared to have been the beginning of phasor measurement technology development. The Global Positioning System (GPS) [110] was beginning to be fully deployed around that time. Clearly, GPS system was the best solution to use effective way to synchronize power system measurements over a wide area. The primary 
prototype modern "Phasor Measurement Units" (PMUs) using GPS were built at Virginia Tech in early 1980s, which is shown in figure 4.1. The first commercial PMU devices are made by Macrodyne in 1991 [111]. Nowadays, there are a lot of PMU manufactures offers PMUs as a commercial product with different features which uses new hardware capabilities and modern communication systems. IEEE published a standard (IEEE C.37.118) in 1991 covering the data format for PMUs communication and the latest version was published in 2005 which added a method for evaluating a PMU measurement and requirements for steady-state measurement.

Currently, the developments of PMUs technology and their usage on power system as a measurement tools, provides the new challenges on applications of them in wide area monitoring, protection and control of power system. Therefore, the concentration of this research will be on implementation of PMUs in power system lab for testbed application and developing and verification of protection and control strategies using PMUs for wide area of power system.
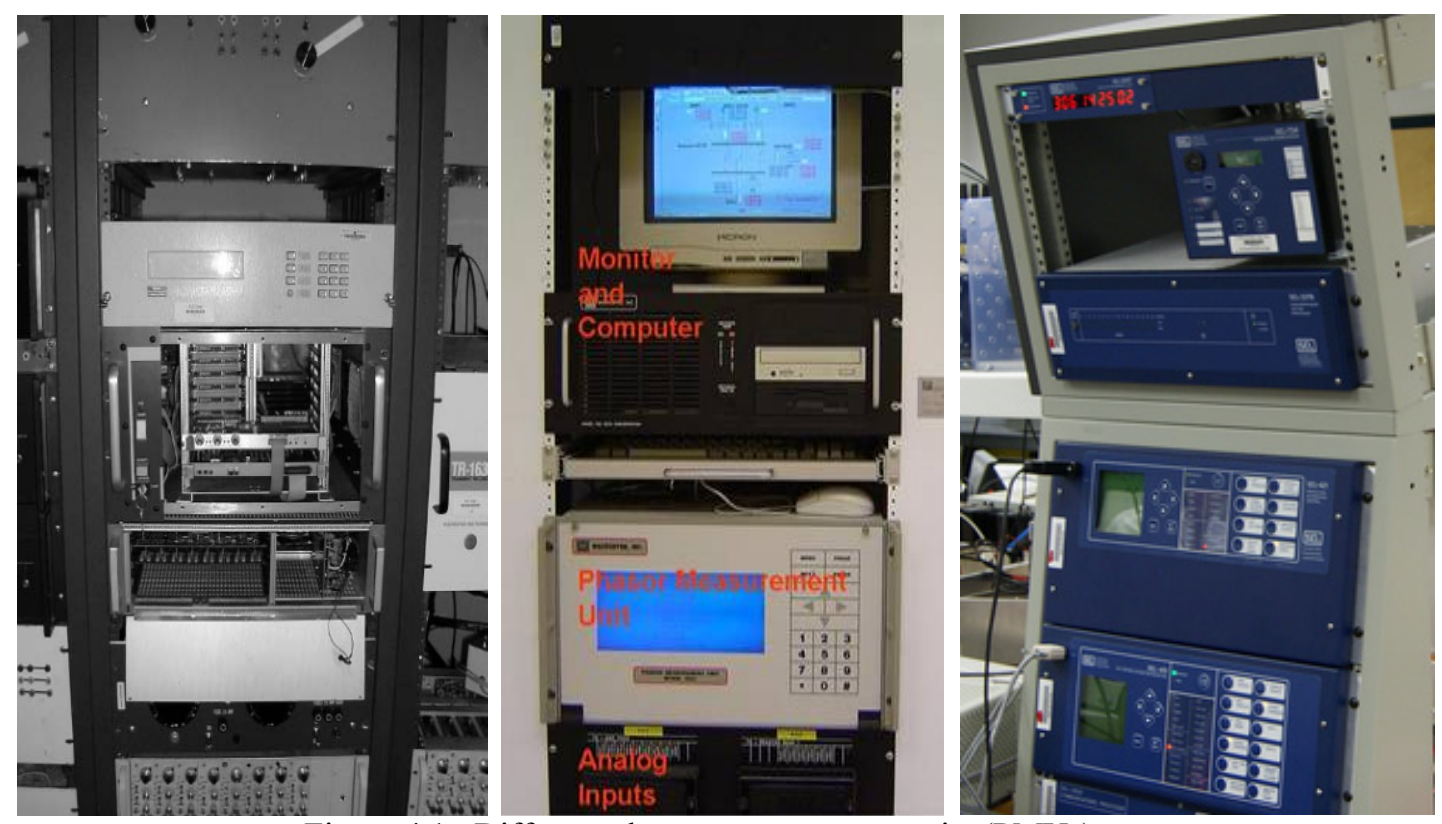

Figure 4.1: Different phasor measurement units (PMUs) 


\subsection{Measuring Synchronous Phasors}

Reference [90] provides the mathematical explanation for synchronous phasor measurement techniques which we used in this dissertation and will be presented here in summary.

\subsubsection{Phasor Representation of Sinusoids}

Consider a pure sinusoidal quantity given by:

$$
x(t)=X_{m} \cos (\omega t+\varphi)
$$

$\omega$ being the frequency of the signal in radians per second and $\varphi$ being the phase angle in radians. $\mathrm{X}_{\mathrm{m}}$ is the peak amplitude of the signal. The root mean square (RMS) value of the input signal is $\left(\mathrm{X}_{\mathrm{m}} / \sqrt{ } 2\right)$. Recall that RMS quantities are particularly useful in calculating active and reactive power in an AC circuit.

Equation (3.1) can also be written as:

$$
x(t)=\operatorname{Re}\left\{X_{m} e^{j(\omega+\varphi)}\right\}=\operatorname{Re}\left[\left\{e^{j \omega t}\right\} X_{m} e^{j \varphi}\right]
$$

It is customary to suppress the term $e^{j(\omega t)}$ in the expression above, with the understanding that the frequency is $\omega$. The sinusoid of equation 4.1 is represented by a complex number $\mathrm{X}$ known as its phasor representation:

$$
x(t) \leftrightarrow X=\left(X_{m} / \sqrt{2}\right) e^{j \varphi}=\left(X_{m} / \sqrt{2}\right)[\cos \varphi+j \sin \varphi]
$$

A sinusoid and its phasor representation are illustrated in figure 4.2.

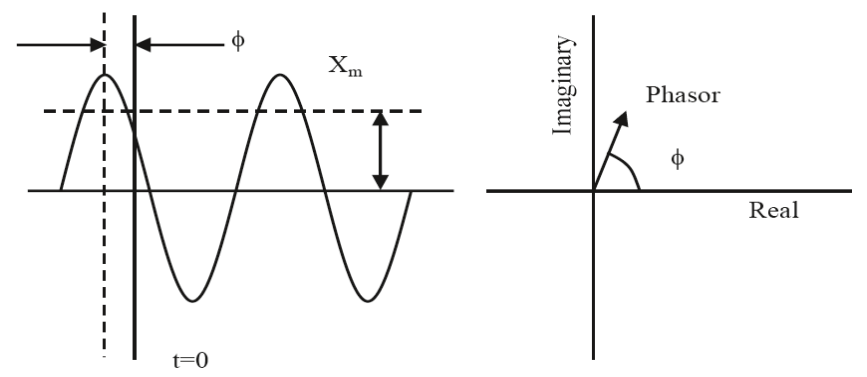

Figure 4.2: A sinusoid and its representation as a phasor 


\subsubsection{Techniques for Signal Analysis}

Discrete Fourier Transform (DFT) is a method of calculating the Fourier transform of a small number of samples taken from an input signal $\mathrm{x}(\mathrm{t})$. The Fourier transform is calculated at discrete steps in the frequency domain, just as the input signal is sampled at discrete instants in the time domain. Consider the process of selecting $\mathrm{N}$ samples: $\mathrm{x}(\mathrm{k} \Delta \mathrm{T})$ with $\{\mathrm{k}=0,1,2, \cdots, \mathrm{N}-1\}, \Delta \mathrm{T}$ being the sampling interval. This is equivalent to multiplying the sampled data train by a "windowing function" w(t), which is a rectangular function of time with unit magnitude and a span of $\mathrm{N} \Delta \mathrm{T}$. With the choice of samples ranging from 0 to $\mathrm{N}-1$, it is clear that the windowing function can be viewed as starting at $-\Delta \mathrm{T} / 2$ and ending at $(\mathrm{N}-1 / 2) \Delta \mathrm{T}$.

Consider the collection of signal samples which fall in the data window: $x(k \Delta T)$ with $\{\mathrm{k}=0,1,2, \cdots \cdot, \mathrm{N}-1\}$. These samples can be viewed as being obtained by the multiplication of the signal $\mathrm{x}(\mathrm{t})$, the sampling function $\delta(\mathrm{t})$ and the windowing function $\omega(\mathrm{t}):$

$$
y(t)=x(t) \delta(t) w(t)=\sum_{k=0}^{N-1} x(k \Delta T) \delta(t-k \Delta T)
$$

A sinusoid $\mathrm{x}(\mathrm{t})$ with frequency $\mathrm{kf}_{0}$ with a Fourier series:

$$
\begin{aligned}
x(t)= & a_{k} \cos \left(2 \pi k f_{o} t\right)+b_{k} \sin \left(2 \pi k f_{o} t\right) \\
x(t) & =\left\{\sqrt{\left(a_{k}^{2}+b_{k}^{2}\right)} \cos \left(2 \pi k f_{o} t+\varphi\right)\right\} \quad \text { where } \varphi=\arctan \left(\frac{-b_{k}}{a_{k}}\right)
\end{aligned}
$$

has a phasor representation:

$$
X_{k}=\frac{1}{\sqrt{2}}\left\{\sqrt{\left(a_{k}{ }^{2}+b_{k}{ }^{2}\right)}\right\}^{j \varphi}
$$


where the square-root of 2 in the denominator is to obtain the RMS value of the sinusoid. The phasor in complex form becomes:

$$
X_{k}=\frac{1}{\sqrt{2}}\left(a_{k}-j b_{k}\right)
$$

Using the relationship of the Fourier series coefficients with the DFT, the phasor representation of the $\mathrm{k}^{\text {th }}$ harmonic component is given by:

$$
X_{k}=\frac{1}{\sqrt{2}} \frac{2}{N} \sum_{n=0}^{N-1} x(n \Delta T) e^{\frac{-j 2 \pi k n}{N}}=\frac{1}{\sqrt{2}} \sum_{n=0}^{N-1} x(n \Delta T)\left\{\cos \left(\frac{2 \pi k n}{N}\right)-j \sin \left(\frac{2 \pi k n}{N}\right)\right\}
$$

Using the notation $x(n \Delta T)=x_{n}$ and $2 \pi / N=\theta(\theta$ is the sampling angle measured in terms of the period of the fundamental frequency component):

$$
X_{k}=X_{k c}-j X_{k s}
$$

Equations 4.6 through 4.9 will be used to represent the phasor in most of the computations in the rest of our discussion.

\subsubsection{Phasor of Nominal Frequency Signals}

Consider a constant input signal $\mathrm{x}(\mathrm{t})$ at the nominal frequency of the power system $\mathrm{f}_{0}$, which is sampled at a sampling frequency $\mathrm{Nf}_{0}$. The sampling angle $\theta$ is equal to $2 \pi / \mathrm{N}$ and the phasor estimation is performed using:

$$
x(t)=X_{m} \cos \left(2 \pi f_{o} t+\varphi\right)
$$

The $\mathrm{N}$ data samples of this input $\mathrm{x}_{\mathrm{n}}:\{\mathrm{n}=0,1,2, \cdots, \mathrm{N}-1)$ are:

$$
x_{n}=X_{m} \cos (n \theta+\varphi) \quad x_{n}:\{n=0,1,2, \ldots, N-1\}
$$

Since the principal interest in phasor measurements is to calculate the fundamental frequency component, we will set $\mathrm{k}=1$ in Eqs. $4.6-4.9$ to produce the fundamental frequency phasor obtained from the sample set $\mathrm{x}_{\mathrm{n}}$. The superscript $(\mathrm{N}-1)$ is 
used to identify the phasor as having the $(\mathrm{N}-1)^{\text {st }}$ sample as the last sample used in the phasor estimation.

$$
X_{c}^{N-1}=\frac{\sqrt{2}}{N} \sum_{n=0}^{N-1} x_{n} \cos (n \theta)=\frac{X_{m}}{\sqrt{2}} \cos (\varphi)
$$

The sine sum is calculated in a similar fashion:

$$
X_{s}^{N-1}=\frac{\sqrt{2}}{N} \sum_{n=0}^{N-1} x_{n} \sin (n \theta)=-\frac{X_{m}}{\sqrt{2}} \sin (\varphi)
$$

The phasor $\mathrm{X}^{\mathrm{N}-1}$ is given by:

$$
X^{N-1}=X_{c}^{N-1}-j X_{s}^{N-1}=\frac{X_{m}}{\sqrt{2}} e^{j \varphi}
$$

It is to be understood that equation 4.14 gives the fundamental frequency phasor estimate even though the subscript $\mathrm{k}=1$ has been dropped for the sake of simplicity. The result obtained in equation 4.14 conforms the phasor definition given in previous section and the phase angle $\varphi$ of the phasor is the angle between the time when the first sample is taken (corresponding to $\mathrm{n}=0$ ) and the peak of the input signal.

\subsubsection{Nonrecursive Updates of Phasors}

Considering that the phasor calculation is a continuous process, it is necessary to consider algorithms which will update the phasor estimate as newer data samples are acquired. When the $\mathrm{N}^{\text {th }}$ sample is acquired after the previous set of samples has led to the phasor estimate given by equation 4.14 , the simplest procedure would be to repeat the calculations implied in Eqs. 4.12-4.13 for the new data window which begins at $n=1$ and ends at $\mathrm{n}=\mathrm{N}$. 


$$
\begin{aligned}
& X^{N-1}=\frac{\sqrt{2}}{N} \sum_{n=0}^{N-1} x_{n}[\{\cos (n \theta)-j \sin (n \theta)\}] \\
& X^{N}=\frac{\sqrt{2}}{N} \sum_{n=0}^{N-1} x_{n+1}[\{\cos (n \theta)-j \sin (n \theta)\}]
\end{aligned}
$$

The two windows are shown in figure 4.3. Phasor 1 is the result of phasor estimation over window 1 , while phasor 2 is calculated with the data in window 2 . The first sample in window 1 is lagging the peak of the sinusoid by an angle $\varphi$, while the first sample of window $2(\mathrm{n}=1)$ lags the peak by an angle $(\varphi+\theta)$, with $\theta$ being the angle between samples.

It should be clear from figure 4.3 that in general the phasor obtained from a constant sinusoid of nominal power system frequency by this technique will have a constant magnitude and will rotate in the counterclockwise direction by angle $\theta$ as the data window advances by one sample. Since the phasor calculations are performed fresh for each window without using any data from the earlier estimates, this algorithm is known as a "Nonrecursive algorithm". Nonrecursive algorithms are numerically stable, but are somewhat wasteful of computation effort as will be seen in the following. Figure 4.4 is another view of the nonrecursive phasor estimation process. As newer samples are obtained, the table of sine and cosine multipliers is moved down to match the new data window. In this figure the multipliers are viewed as samples of unit-magnitude sine and cosine waves at the nominal power system frequency. The new data window has $\mathrm{N}-1$ samples in common with the old data window. In actual computation these are simply stored as tables of sine and cosine, which are used repeatedly on each window as needed. 

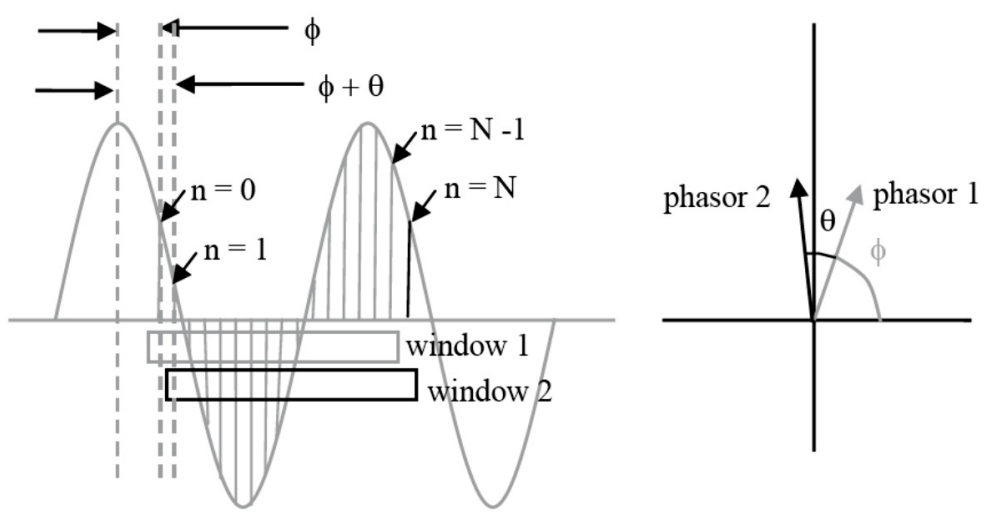

Figure 4.3: Update of phasor estimates with $\mathrm{N}$ sample windows

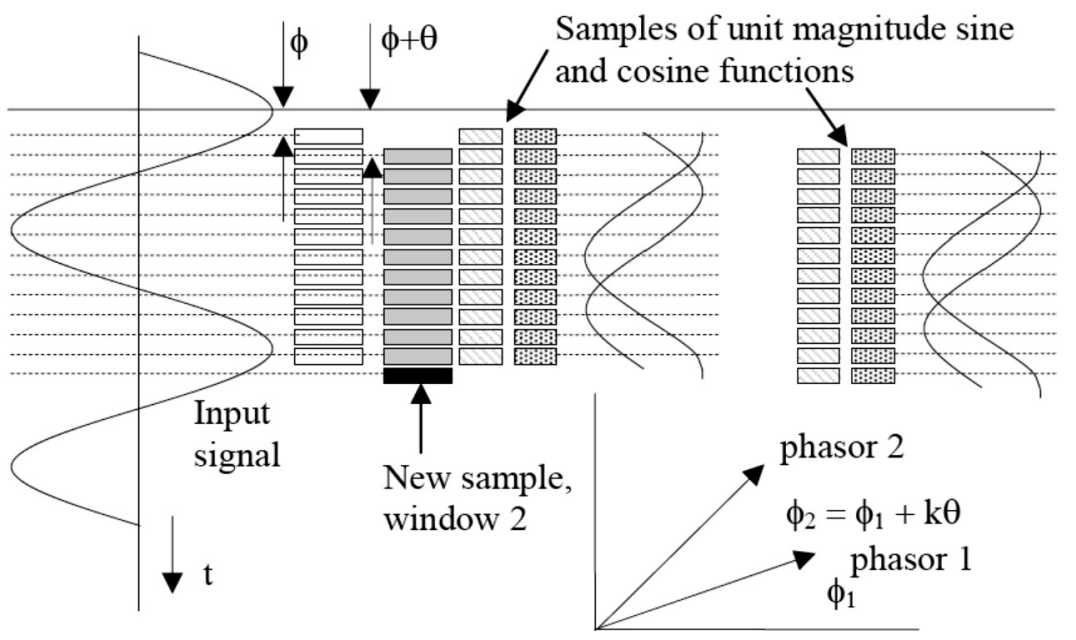

Figure 4.4: Nonrecursive phasor estimation

\subsubsection{Recursive Updates of Phasors}

The formulas for calculating the $(\mathrm{N}-1)^{\mathrm{st}}$ and $(\mathrm{N})^{\text {th }}$ phasors by the Nonrecursive algorithm are:

$$
\begin{aligned}
& X^{N-1}=\frac{\sqrt{2}}{N} \sum_{n=0}^{N-1} x_{n} e^{-j n \theta} \\
& X^{N}=\frac{\sqrt{2}}{N} \sum_{n=0}^{N-1} x_{n+1} e^{-j n \theta}
\end{aligned}
$$


The multipliers for a given sample are different in the two computations. For example, the multiplier for $(n=2)$ sample in the first sum is $\mathrm{e}^{-\mathrm{j} 2 \theta}$ while the multiplier for the same sample in the second sum is $\mathrm{e}^{-\mathrm{j} \theta}$. It should be noted that samples $\mathrm{x}_{\mathrm{n}}:\{\mathrm{n}=$ $1,2, \ldots, \mathrm{N}-1)$ are common to both windows. The second window has no $\mathrm{x}_{0}$, so that it begins with $\mathrm{x}_{1}$ and it ends with $\mathrm{x}_{\mathrm{N}}$, which did not exist in the first window. If one could arrange to keep the multipliers for the common samples the same in the two windows, one would save considerable computations in calculating $\mathrm{X}^{\mathrm{N}}$. If we multiply both sides of the second equation in 4.16 by $\mathrm{e}^{-\mathrm{j} \theta}$ we obtain the following result:

$$
\begin{aligned}
& \hat{X}^{N}=e^{-j \theta} X_{n}=\frac{\sqrt{2}}{N} \sum_{n=0}^{N-1} x_{n+1} e^{-j(n+1) \theta} \\
& \hat{X}^{N}=X^{N-1}+\frac{\sqrt{2}}{N}\left(x_{N}-x_{0}\right) e^{-j(0) \theta}
\end{aligned}
$$

where it has been made of the fact that $\mathrm{e}^{-\mathrm{j}(0) \theta}=\mathrm{e}^{-\mathrm{jN} \theta}$, since $\mathrm{N}$ samples span exactly one period of the fundamental frequency. The phasor defined by equation 4.18 differs from the nonrecursive estimate by an angular retardation of $\theta$. The advantage of using this alternative definition for the phasor from the new data window is that $(\mathrm{N}-1)$ multiplications by the Fourier coefficients in the new window are the same as those used in the first window. Only a recursive update on the old phasor needs to be made to determine the value of the new phasor. This algorithm is known as the "recursive algorithm" for estimating phasors. In general, when the last sample in the data window is $(\mathrm{N}+\mathrm{r})$, the recursive phasor estimate is given by:

$$
\hat{X}^{N+r}=\hat{X}^{N+r-1}+\frac{\sqrt{2}}{N}\left(x_{N+r}-x_{r}\right) e^{-j r \theta}
$$




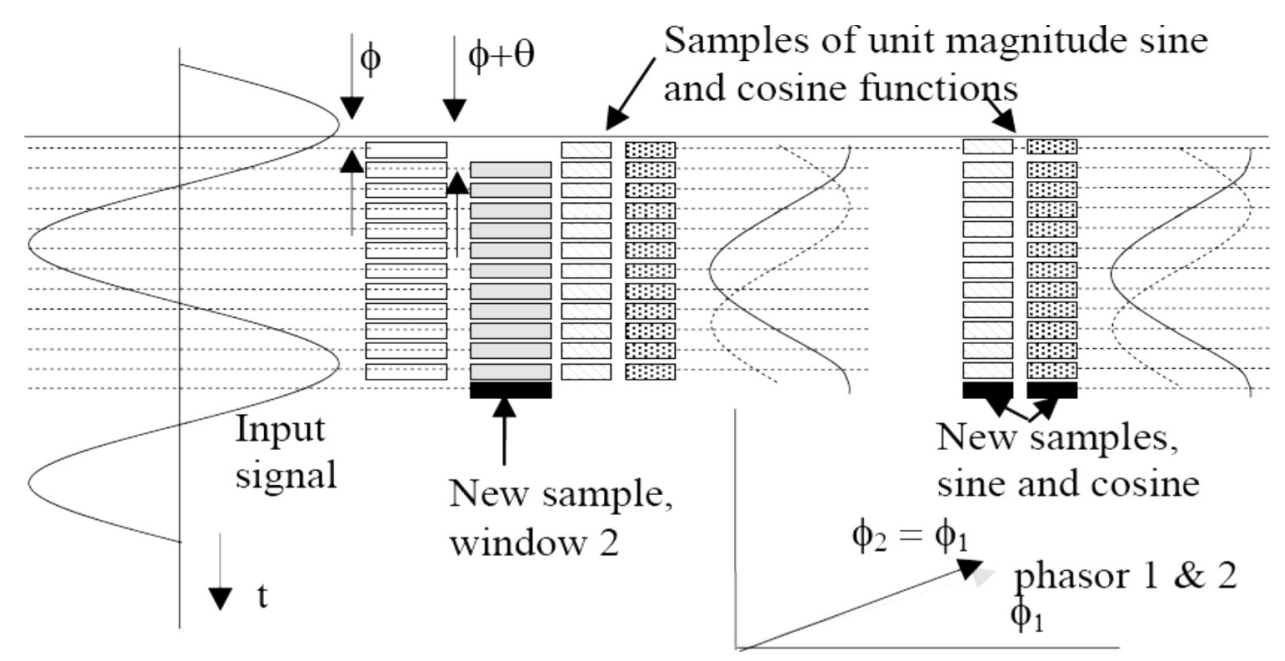

Figure 4.5: Recursive phasor estimation

When the input signal is a constant sinusoid, $\mathrm{x}_{\mathrm{N}+\mathrm{r}}$ is the same as $\mathrm{x}_{\mathrm{r}}$ and the second term in equation 4.8 disappears. The phasor estimate with data from the new window is the same as the phasor estimate with data from the old window when the input signal is a constant sinusoid. In general, the recursive algorithm is numerically unstable. Consider the effect of an error in the estimate from one window - for example caused by a roundoff error. This error is always present in all the phasor estimates from then on. This property of the recursive phasor algorithms must be kept in mind when practical implementation of these algorithms is performed [106]. Nevertheless, because of the great computational efficiency of the recursive algorithm, it is usually the algorithm of choice in many applications. Unless stated otherwise explicitly, we will assume that only the recursive form of the phasor estimation algorithm is in use.

\subsubsection{Effect of Noise Signal on Phasor}

The input signals are rarely free from noise. A spurious frequency component which is not a harmonic of the fundamental frequency signal may be considered to be noise. One may also have induced electrical noise picked up in the wiring of the input 
signal. Leakage effect caused by the windowing function contributes to an error in phasor estimation and should therefore be considered as a type of noise in the input. As an approximation, we will consider the noise in the input signal to be a zero-mean, Gaussian noise process. This should be a good approximation for the electrical noise picked up in the wiring and signal conditioning circuits. A phasor measurement system may be placed in an arbitrarily selected substation and will be exposed to input signals generated by the power system which is likely to change states all the time. Each of the power system states may lead to different non-harmonic frequencies and leakage effects and the entire ensemble of conditions to which the phasor measurement system is exposed may also be considered to be a pseudorandom Gaussian noise process.

Consider a set of noisy measurement samples:

$$
x_{n}=X_{m} \cos (n \theta+\varphi)+\varepsilon_{n} \quad x_{n}:\{n=0,1,2, \ldots, N-1\}
$$

Where $\varepsilon_{\mathrm{n}}$ is a zero-mean Gaussian noise process with a variance of $\sigma^{2}$. If we set $(X m / \sqrt{2}) \cos (\varphi)=X_{r}$ and $\left(X_{m} / \sqrt{2}\right) \sin (\varphi)=X_{i}$, the phasor representing the sinusoid is $X$ $=\mathrm{X}_{\mathrm{r}}+\mathrm{j} \mathrm{X}_{\mathrm{i}}$. We may pose the phasor estimation problem as one of finding the unknown phasor estimate from the sampled data through a set of $\mathrm{N}$ over determined equations:

$$
\left.\left[\begin{array}{c}
x_{0} \\
x_{1} \\
x_{2} \\
\vdots \\
x_{N-1}
\end{array}\right]=\sqrt{2} \times\left[\begin{array}{cc}
\cos (0) & -\sin (0) \\
\cos (\theta) & -\sin (\theta) \\
\cos (2 \theta) & -\sin (2 \theta) \\
\vdots & \vdots \\
\cos [(N-1) \theta] & -\sin [(N-1) \theta]
\end{array}\right]\left[X_{r}\right] X_{u}\right]+\left[\begin{array}{c}
\varepsilon_{1} \\
\varepsilon_{2} \\
\varepsilon_{3} \\
\vdots \\
\varepsilon_{N-1}
\end{array}\right]
$$

or, in matrix notation:

$$
[\mathrm{x}]=[\mathrm{S}][\mathrm{X}]+[\varepsilon]
$$


The weighted least-squares solution of equation 4.23 provides the estimate for the phasor:

$$
[\mathrm{W}]=\sigma^{2}[\mathrm{I}]
$$

The weighted least-squares solution of equation 4.24 provides the estimate for the phasor:

$$
\left[\mathrm{X}^{\prime}\right]=\left[\mathrm{S}^{\mathrm{T}} \mathrm{W}^{-1} \mathrm{~S}\right]^{-1} \mathrm{~S}^{\mathrm{T}} \mathrm{W}^{-1}[\mathrm{x}]
$$

Using 4.24 for $\mathrm{W}$ and calculating $\left[\mathrm{S}^{\mathrm{T}} \mathrm{S}\right]^{-1}$ for the $\mathrm{S}$ in Eq. 1.23:

$$
\left[X^{\prime}\right]=\left[S^{T} W^{-1} S\right]^{-1}\left[S^{T} W^{-1}\right][x]=\left[S^{T} S\right]^{-1}\left[S^{T}\right][x]=1 / N\left[S^{T}\right][x]
$$

Since the noise is a zero-mean process, the estimate given by equation 4.27 is unbiased and the expected value of the estimate is equal to the true value of the phasor. If $\mathrm{X}$ is the true value of the phasor, the covariance matrix of the error in the phasor estimate is:

$$
E\left(\left[X^{\prime}-X\right][X-X]^{T}\right)=\left[S^{T} W^{-1} S\right]^{-1}
$$

Substituting for [W] from equation 4.23, the covariance of the error in phasor estimate is $\left(\sigma^{2} / \mathrm{N}\right)$. The standard deviations of error in real and imaginary parts of the phasor estimate are $(\sigma / \sqrt{ } N)$. We may thus conclude that higher sampling rates will produce improvement in phasor estimates in inverse proportion of the square root of the number of samples per cycle. Alternatively, if longer data windows are used (multiple of cycles), then once again the errors in phasor estimate go down as the square root of the number of cycles used. Thus, a four-cycle phasor estimate is twice as accurate as a onecycle estimate in with noisy input. 


\subsubsection{Offet in Input Signal}

Fault currents in a power system often have an exponentially decaying DC component, which is generally known as the DC offset. Occasionally, voltage waveforms may also have a DC offset due to capacitive voltage transformer transients. In both cases, the DC offsets decay to negligible values in a few cycles. If the phasor estimate is performed while a DC offset is present in a waveform, one is likely to get significant errors in phasor estimate while the DC offset is non-zero. In computer relaying applications, powerful techniques have been developed to remove DC offsets before phasors are estimated and in very specific applications of phasors which require very high speed of response, it may be necessary to employ algorithms which will remove the DC offset from the signals. The earliest technique used in relays for removing the DC offset from fault currents is the one of using a "mimic" circuit in the secondary winding of a current transformer. For computer relays, there is a least-squares solution technique available for eliminating the DC offset, which is free from the noise amplification properties of the mimic circuit.

\subsubsection{Input Signal at Off-Nominal Frequency}

Now assume that the input signal is at a frequency:

$$
\omega=\omega+\Delta \omega
$$

Where $\omega_{0}$ is the nominal power system frequency. For a $60-\mathrm{Hz}$ system, $\omega_{0}$ is $120 \pi$ radians per second. The input signal is once again assumed to be:

$$
x=X_{m} \cos (\omega t+\varphi)
$$


The definition of a phasor representation of a sinusoid is independent of the frequency of the signal. Using the nominal frequency sinuses and cosines for phasor calculation will introduce an error in phasor estimation. Clearly, the error made in phasor estimation will depend upon the difference between the nominal and actual frequency. The input signal can be expressed as follows:

$$
x(t)=\sqrt{2} \operatorname{Re}\left\{\left(X_{m} / 2\right) e^{j \varphi} e^{j \omega t}\right\}=\sqrt{2} \operatorname{Re}\left\{X e^{j \omega t}\right\}
$$

Where $\mathrm{X}$ is the correct value of the phasor at the off-nominal frequency and the function "Re" is the real value function. Expressing the real value as the average of a complex number and its complex conjugate:

$$
x(t)=(\sqrt{2} / 2)\left\{X e^{j \omega t}+X^{*} e^{-j \omega t}\right\}
$$

The $\mathrm{k}^{\text {th }}$ sample of the signal represented by equation 4.32 is given by:

$$
x_{k}=(1 / \sqrt{2})\left\{X e^{j \alpha k \Delta t}+X^{*} e^{-j \omega k \Delta t}\right\}
$$

The phasor representation of $\mathrm{x}(\mathrm{t})$, that is, $\mathrm{X}^{\prime}$ (which is different from $\mathrm{X}$ unless the system frequency is equal to the nominal value $\omega_{0}$ ) - is calculated using equation 4.21 with $\mathrm{x}_{\mathrm{r}}$ as the first sample. Note that equation 4.21 uses sine and cosine terms at the nominal power system frequency $\omega_{0}$. Thus $X_{\mathrm{r}}{ }^{\prime}$ is given by:

$$
X_{r}^{\prime}=\frac{1}{N} \sum_{k=r}^{r+N-1}\left\{X e^{j \omega k \Delta t}+X^{*} e^{-j \omega k \Delta t}\right\} e^{-j k \omega_{0} \Delta t}
$$

by using geometric series and doing some calculations, the equation 4.34 can be expressed in closed form as: 


$$
\begin{gathered}
X_{r}^{\prime}=X e^{j r\left(\omega-\omega_{0}\right) \Delta t}\left\{\frac{\sin \frac{N\left(\omega-\omega_{0}\right) \Delta t}{2}}{N \sin \frac{\left(\omega-\omega_{0}\right) \Delta t}{2}}\right\} e^{j(N-1) \frac{\left(\omega+\omega_{0}\right) \Delta t}{2}}+ \\
X^{*} e^{-j r\left(\omega+\omega_{0}\right) \Delta t}\left\{\frac{\sin \frac{N\left(\omega+\omega_{0}\right) \Delta t}{2}}{N \sin \frac{\left(\omega+\omega_{0}\right) \Delta t}{2}}\right\} e^{-j(N-1) \frac{\left(\omega+\omega_{0}\right) \Delta t}{2}}
\end{gathered}
$$

\subsubsection{Frequency Estimated from Balance Three-Phase Inputs}

It is clear that the first and second derivatives of phase angle of the phasor estimate would provide an estimate of $\Delta \omega=\left(\omega-\omega_{0}\right)$ and the rate of change of frequency. Since there are estimation errors in phasor calculation, it is desirable to use a weighted least-squares approach over a reasonable data window for calculating the derivatives of the phase angle.

Assume that the positive-sequence phasors are estimated over one period of the nominal frequency and that the phasors calculated with several consecutive data windows over a span of 3-6 cycles are used for frequency and rate of change of frequency estimation.

Let $\left[\varphi_{\mathrm{k}}\right]\{\mathrm{k}=0,1, \ldots, \mathrm{N}-1\}$ be the vector of "N" samples of the phase angles of the positive-sequence measurement. The vector $\left[\varphi_{\mathrm{k}}\right]$ is assumed to be monotonically changing over the window of " $\mathrm{N}$ " samples. As the phase angles of the phasor estimate may be restricted to a range of $0-2 \pi$, it may be necessary to adjust the angles to make them monotonic over the entire spanning period by correcting any offsets of $2 \pi$ radians which may exist. If the frequency deviation from the nominal value and the rate of change of frequency at $\mathrm{t}=0$ are $\Delta \omega$ and $\omega^{\prime}$, respectively, the frequency at any time " $\mathrm{t}$ " is given by: 


$$
\omega(t)=\omega+\Delta \omega+t \omega^{\prime}
$$

The phase angle is the integral of the frequency:

$$
\phi(t)=\int \omega d t=\phi_{0}+t \omega_{0}+t \Delta \omega+\frac{1}{2} t^{2} \omega^{\prime}
$$

$\varphi_{0}$ being the initial value of the angle. Assuming that the recursive algorithm is used for estimating the phasors, the term "t $\omega_{0}$ " is suppressed from the estimated phase. Thus, the phase angle as a function of time becomes:

$$
\phi(t)=\phi_{0}+t \Delta \omega+\frac{1}{2} t^{2} \omega^{\prime}
$$

The vector of " $\mathrm{N}$ " angle measurements is given by:

$$
\left[\begin{array}{c}
\phi_{0} \\
\phi_{1} \\
\phi_{2} \\
\vdots \\
\phi_{N-1}
\end{array}\right]=\left[\begin{array}{ccc}
1 & 0 & 0 \\
1 & \Delta t & \Delta t^{2} \\
1 & 2 \Delta t & 2^{2} \Delta t^{2} \\
\vdots & \vdots & \vdots \\
1 & (N-1) \Delta t & (N-1)^{2} \Delta t^{t}
\end{array}\right] \times\left[\begin{array}{c}
\phi_{0} \\
\Delta \omega \\
\frac{\omega^{\prime}}{2}
\end{array}\right]
$$

In matrix notation:

$$
[\phi]=[B][A]
$$

where $[\mathrm{B}]$ is the coefficient matrix in equation 4.39 . The unknown vector $[\mathrm{A}]$ is calculated by the weighted least-squares (WLS) technique:

$$
[A]=\left[B^{T} B\right]^{-1} B^{T}[\phi]
$$

The matrix [A] in 4.41 is pre-calculated and stored for use in real time. It has " $\mathrm{N}$ " rows and three columns. The frequency and rate of change of frequency at any time $t$ (which is a multiple of $\Delta t$ ) can be calculated by equation 4.41 . 


\subsection{Description of Laboratory Implementation of PMUs Setup}

The most important issue in developing such research based power system testbed is precise measurement of system parameters in real-time format which is applicable by using PMUs. Therefore, this research will concentrate on developing PMUs by using analogue to digital DAQs and real-time software. The power system preview is shown in figure 4.6 as single line diagram which is implemented in LabVIEW, real-time software for measurement and control purposes. Figure 4.7 also shows the overall view of implemented test setup. The measurement system is built with National Instrument (NI) data acquisition module, NI 9206-Ethernet with 32 analogue input connected to secondary sides of all CTs and PTs. In addition, DAQ NI USB-6259 is used for switching control at this circuit.

In order to synchronize system voltage and current measured vectors, the industrial type of PMUs around the wide area of power system use GPS system to coordinate their time with global time reference. Hence, all PMUs receive a signal over a second to make time reference for whole measured parameters. For research application, this signal can be provided by one of the digital output ports of DAQs. Therefore, in this implementation we created a waveform for measurement devices to act as comparing waveform with any measured signal to achieve its phase angle in unique time reference for whole system. The phase voltage waveform that has been selected to be the reference for other measured signals and hence its phasor will have zero voltage angle and the others phasor will be referred to this voltage phasor angle.

The implemented software for PMU is illustrated in figure 4.8 as a block diagram. All PMU units shown as boxes in figure 4.6 use this structure to digitally measure whole 
system parameters and send data to the data center through the Ethernet to demonstrate system status and wide area monitoring, protection and control purposes. As shown, the local relay uses high sampling rate measurement of amplitude, phase and frequency of voltages and currents for fast fault detection in local application. For wide area system monitoring, the down-sampling technique is applied to measure power and sequences phasors and send them to data center via communication port.

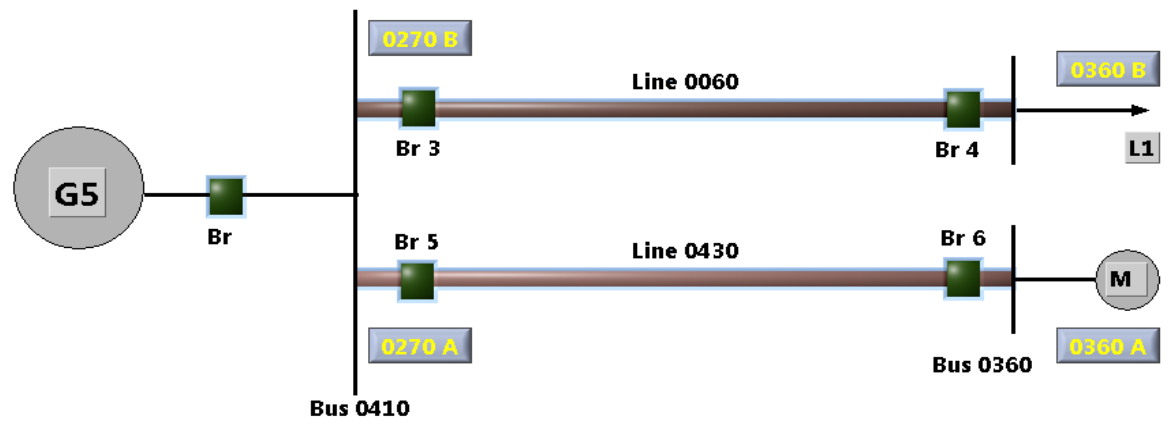

Figure 4.6: Single Line Diagram of Laboratory Test Setup in LabVIEW

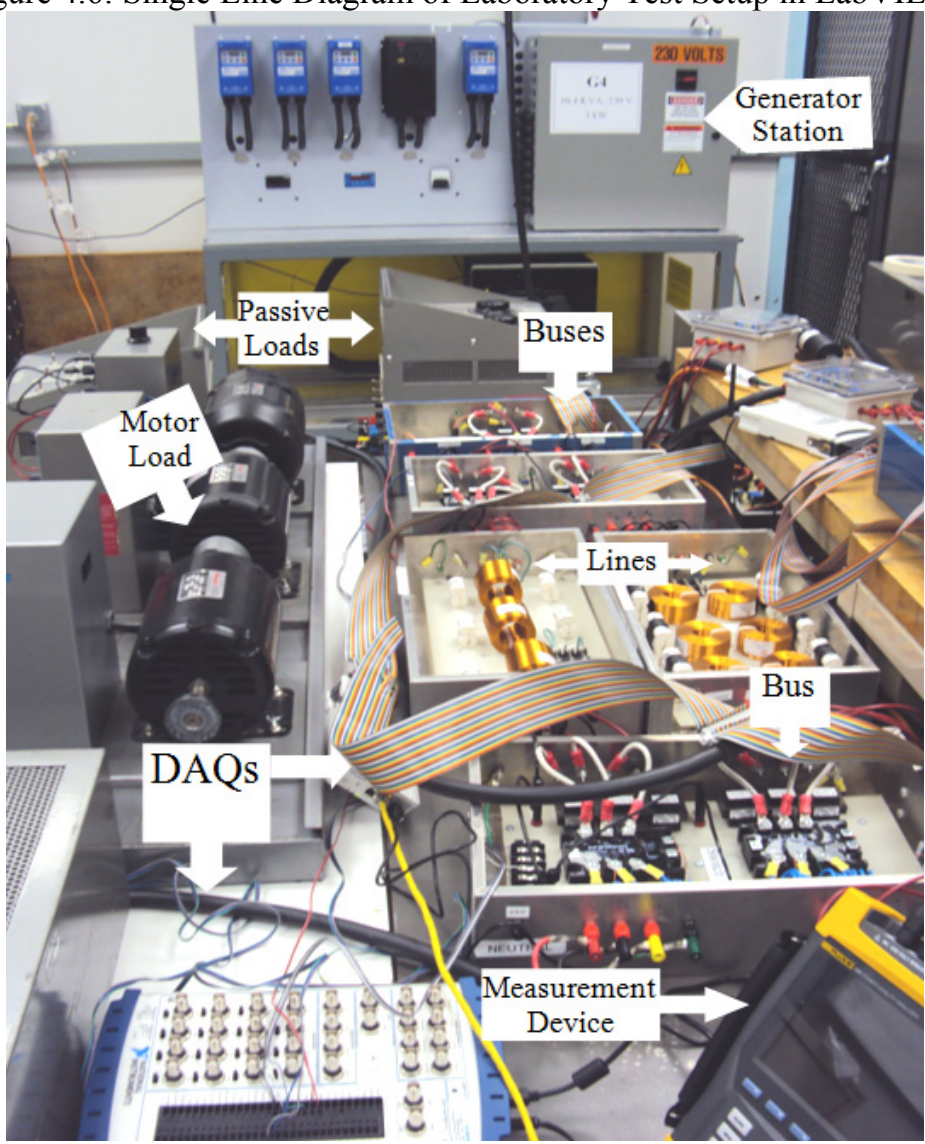

Figure 4.7: Overall View of Laboratory Test Setup 


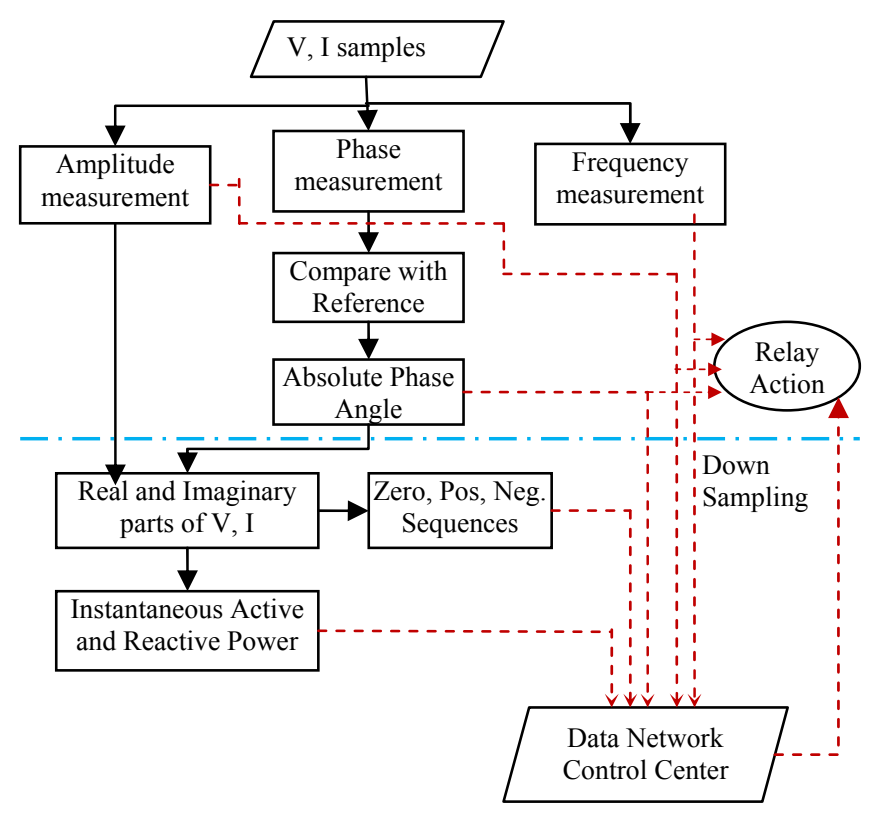

Figure 4.8: Block Diagram of Implemented PMU

\subsection{Real-Time Results of Developed PMUs}

According to figure 4.6, four PMUs have been implemented in the setup in load side of every solid state relay. By PMUs installed in 0270A and 0270B the voltages and currents of line inputs after the switches have been measured. Furthermore, the PMUs installed in 0360A and 0360B measures passive load and motor load parameters. Figure 4.9 show front panel of this digital PMU, which shows real-time values of voltages and currents in left and top side of window and calculated sequences for them. The phase A of voltage in this PMU is chosen as a reference angle for the whole system and then other phases are compared with this angle. As long as the measurements are related to individual DAQ, the phase angle difference for whole measurements can be achieved by using one signal of that DAQ as a reference for its other signals. So the positive sequence of voltage has a 0.01 degree phase angle because of unbalance condition in input voltages. Phase A of voltage at point $0270 \mathrm{~A}$ is chosen as a reference for other signal and 
hence its phase angle is zero according to figure 4.9. The phasor diagram for three phase system and sequences network for voltage and current have been shown in the right-top side of the front panel, too. Active and Reactive power, as well as frequency have been demonstrated for last 100 seconds. The real-time values of calculated powers for each phase, three phase, power factor and frequency have been presented by its real-time value. Figure 4.10 shows the similar front panel of PMU for point 0360A, which is the motor load connected point. The line components in this case, help to produce the amount of reactive power required by motor load and then the power factor is improved in source side of the line. Figure 4.11 depicts the passive load connected PMU parameters, as well.

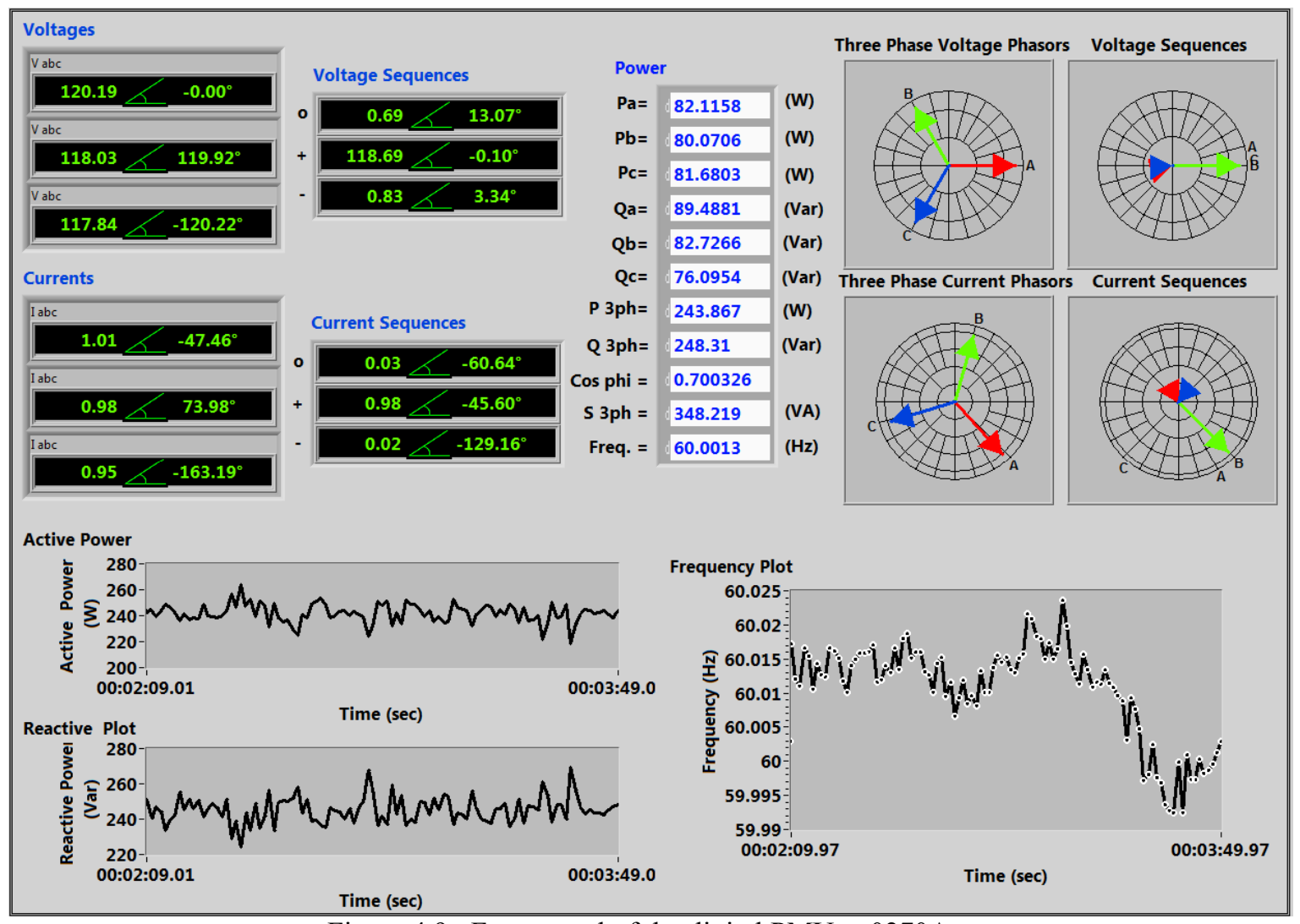

Figure 4.9: Front panel of the digital PMU at 0270A 


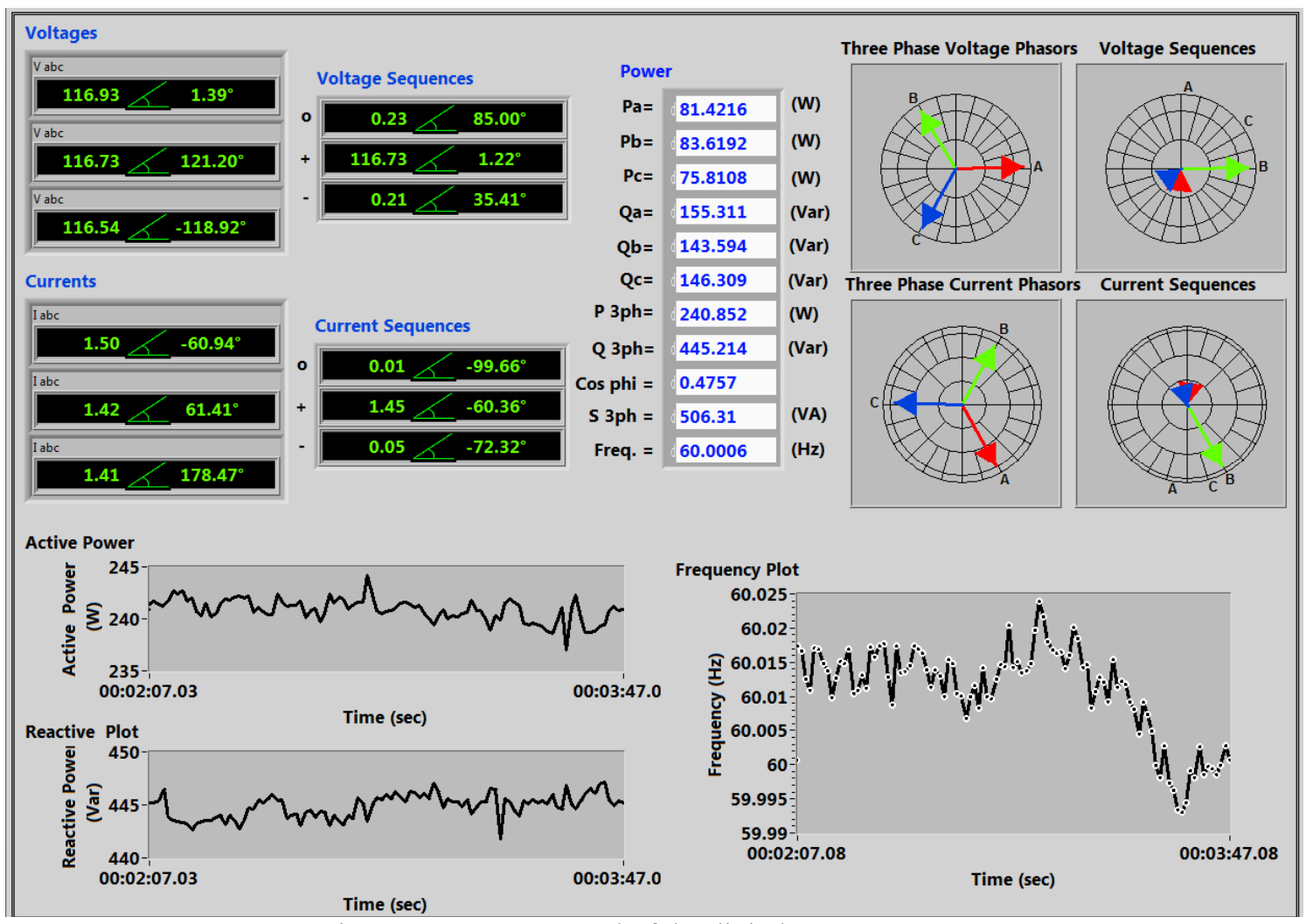

Figure 4.10: Front panel of the digital PMU at 0360A

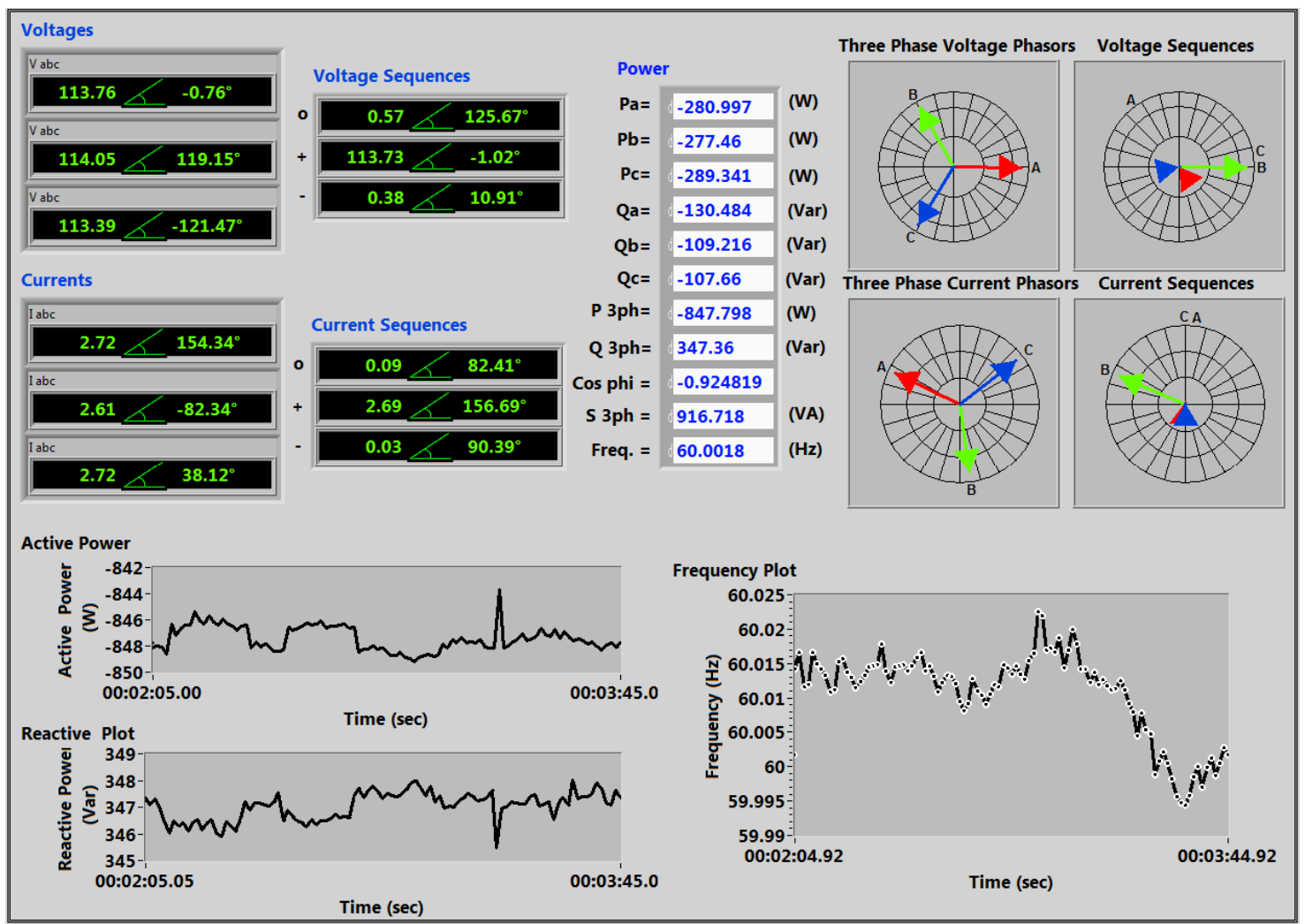

Figure 4.11: Front panel of the digital PMU at 0360B 


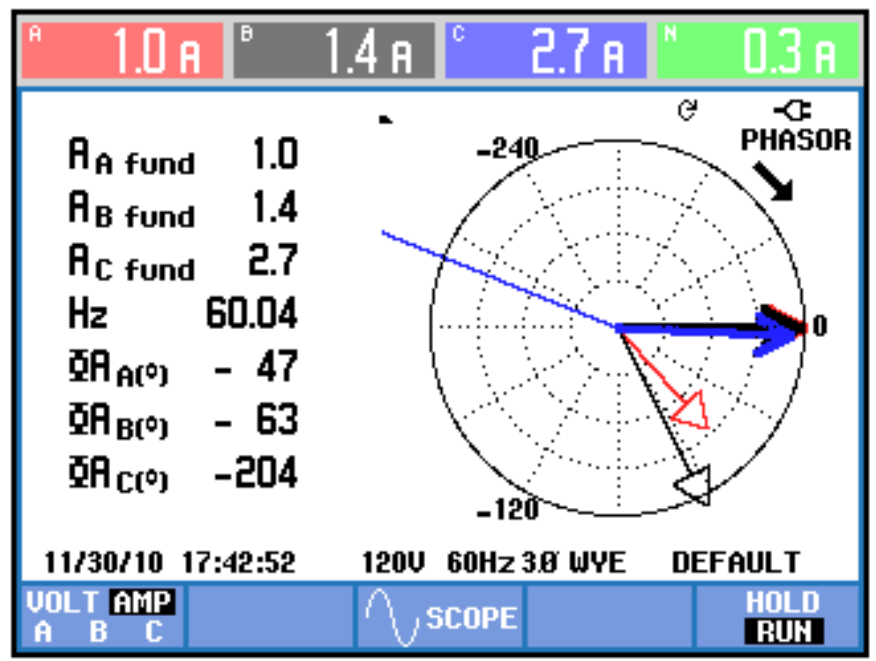

Figure 4.12: Result verifying by Fluke 43

As it is obvious from these figures, the voltage phase angle difference for buses connected to line 0430 is $1.39^{\circ}$ and for buses connected to line 0060 is $0.76^{\circ}$. These values are verified by power quality measurement device (Fluke 435), which figure 2.12 shows the screen of this monitoring device with voltage and current phasors of corresponding phase $\mathrm{A}$ in all three buses. This device has $1^{\circ}$ phase angle precision and hence it could not provide good accuracy in measurement. Therefore, the comparison between LabVIEW results and the fluke has $2^{\circ}$ phase angle difference due to error in measurement in both devices. Table 4.1 presents measurement comparison between PMU and Fluke 435 for results verification. By an acceptable accuracy we can conclude that power system parameters measurements by this PMU design will be explicit, inexpensive and achievable in any research laboratory and applicable for any real-time study.

Table 4.1: Measurement Verification

\begin{tabular}{c|c|c|c|c}
\hline \multirow{2}{*}{$\begin{array}{c}\text { Phase A of Voltage \& } \\
\text { Current }\end{array}$} & \multicolumn{2}{|c|}{ PMU } & \multicolumn{2}{c}{ Fluke 435 } \\
\cline { 2 - 5 } & Voltage (V) & Current (A) & Voltage (V) & Current (A) \\
\hline $0270 \mathrm{~A}$ & $120.19 \measuredangle 0$ & $1.01 \measuredangle-47.4$ & $120 \measuredangle 0$ & $1 \measuredangle-47$ \\
\hline $0270 \mathrm{~B}$ & $116.3 \measuredangle 1.39$ & $1.5 \measuredangle-60.94$ & $116 \measuredangle 1$ & $1.4 \measuredangle-63$ \\
\hline $0360 \mathrm{~A}$ & $113.76 \measuredangle-0.76$ & $2.72 \measuredangle 154$ & $114 \measuredangle-2$ & $2.7 \measuredangle-204$ \\
\hline
\end{tabular}




\subsection{Description of Developed Industrial PMUs Setup}

In order to test and modify the application of PMUs in Smart Power System, we tried to use new industrial level PMUs in test-bed power system lab. The mentioned setup is provided by Schweitzer Engineering Laboratories. Figure 4.13 shows the PMUs architecture to gather synchronized phasor Measurements from different points of power system and use Real-Time Automation Controller (RTAC) to monitor system in real-time format. The summarized explanation for each device will be presented here.

\subsubsection{SEL-421 Protection, Automation and Control System}

SEL-421 is a Protection, Automation and Control System Relay for high-speed distance and directional protection and complete control of a two-breaker bay. This relay protects any transmission line using a combination of five zones of phase- and grounddistance and directional over-current elements. The SEL-421 Relay records power system events with very high accuracy when high-accuracy clock input signals is provided, such as from a GPS receiver. SEL-421 relays placed at key substations can give you information on power system operating conditions in real time.

Based on the high-accuracy time input, the relay calculates synchrophasors for currents and line voltages (for each phase and for positive-sequence), as specified in C37.118, IEEE Standard for Synchrophasors for Power Systems. One can then perform detailed analysis and calculate load flow from the synchrophasors.

The value of synchrophasor data increases greatly when the data can be shared over a communications network in real time. Two synchrophasor protocols are available in the SEL-421 that allow for a centralized device to collect data efficiently from several 
phasor measurement units (PMUs). Some possible uses of a system-wide synchrophasor system include the following.

- Power-system state measurement

- Wide-area network protection and control schemes

- Small-signal analysis

- Power-system disturbance analysis

The PMU has 6 current channels and 6 voltage channels. Current Terminals W, X and voltage terminals $\mathrm{Y}, \mathrm{Z}$ are three-phase channels. The PMU combines channels $\mathrm{W}$ and $\mathrm{X}$ to create a pseudo Terminal S. From these 12 channels, the PMU can measure up to 20 synchrophasors; 15 phase synchrophasors and 5 positive-sequence synchrophasors. Synchrophasors are always in primary, so set the CT and PT ratios in the group settings appropriately. The PMU converts the synchrophasor data to primary units by multiplying them with the respective PT or CT ratios. The PMU calculates the positive sequence synchrophasor with the three-phase synchrophasors. The PMU then converts all synchrophasor data to polar and rectangular quantities. The data are available as analog quantities as well as for the synchrophasor data frames. The synchrophasor data are updated at the nominal power system frequency.

The PMU can be configured to process C37.118 synchrophasor data received from two remote PMUs over serial ports. The PMU processes the remote PMU data, time aligns them with the local data and makes them available as analogs and digitals. Use the local synchrophasor analogs and as many as two remote sets of synchrophasor analogs in SELOGIC equations to do real-time control (RTC) applications. 

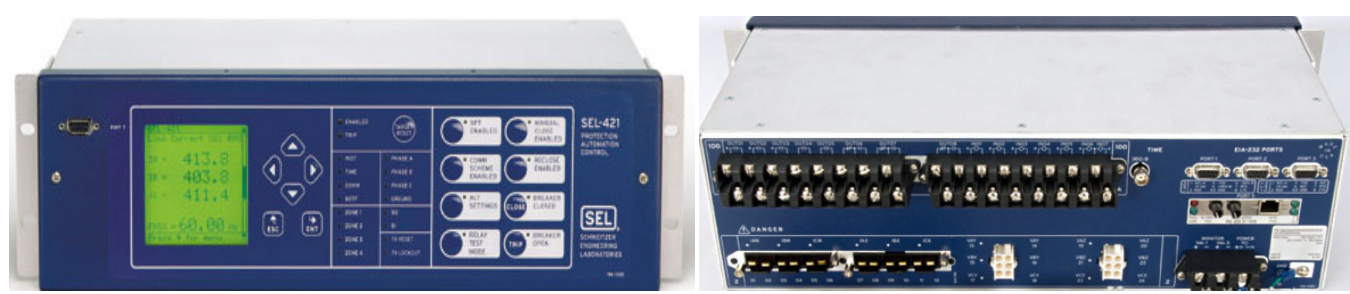

Figure 4.13: Front panel and rear view of SEL-421

\subsubsection{SEL-451 Protection, Automation and Control System}

SEL-451 is a complete stand-alone protection, automation and control system. The SEL-451 has speed, power and flexibility to combine complete substation bay control with high-speed breaker protection in one economical system. Use the SEL-451 as an integral part of a full substation protection, control and monitoring solution. Reduce Maintenance costs by accurately tracking breaker operation. Monitor breaker interruption times and accumulated breaker duty to easily determine the need for proactive maintenance. Integrate information with SCADA or automation systems through a communications processor or directly to the Ethernet port. Figure 4.14 shows the front panel of this relay.

This Relay records power system events with very high accuracy when highaccuracy clock input signals are provided, such as from a GPS receiver. SEL-451 relays placed at key substations can give information on power system operating conditions in real time. Based on the high-accuracy time input, the relay calculates synchrophasors for currents and line voltages (for each phase and for positive-sequence), as specified in C37.118, IEEE Standard for Synchrophasors for Power Systems. Then the operator can perform detailed analysis and calculate load flow from the synchrophasors.

The state of the power system is the set of all positive-sequence voltage phasors in the network. Typically, several seconds or minutes elapse from the time of the first 
measurement to the time of the first estimation. Therefore, state estimation is a steadystate representation of the power system. Consider using precise simultaneous positivesequence voltage measurements from the power system to verify your state estimation model. Take time synchronized high-resolution positive-sequence voltage measurements at all substations. SEL-451 Fast Messages can be sent to a central database to determine the power system state.

Power system contingency analysis models rely on state-estimation techniques and may have inaccuracies caused by incorrect present-state information, or errors in system characteristics, such as incorrect line and source impedance estimates. The simultaneous event-report triggering technique described earlier in this section can be used to verify present models.

With Phasor Measurement Units (PMUs) such as the SEL-451 installed in several substations, synchrophasor measurements can be transmitted to a central processor in near real-time, providing very accurate snapshots of the power system. This type of data processing system provides system-state measurements that are a few seconds old, rather than state estimates that may be several minutes old. In addition, the synchrophasor results are real measurements, rather than estimates.

Similar to SEL-421, this PMU has 6 current channels and 6 voltage channels. From these 12 channels, the PMU can measure up to 20 synchrophasors; 15 phase synchrophasors and 5 positive-sequence synchrophasors. Synchrophasors are always in primary, so set the $\mathrm{CT}$ and $\mathrm{PT}$ ratios in the group settings appropriately. 


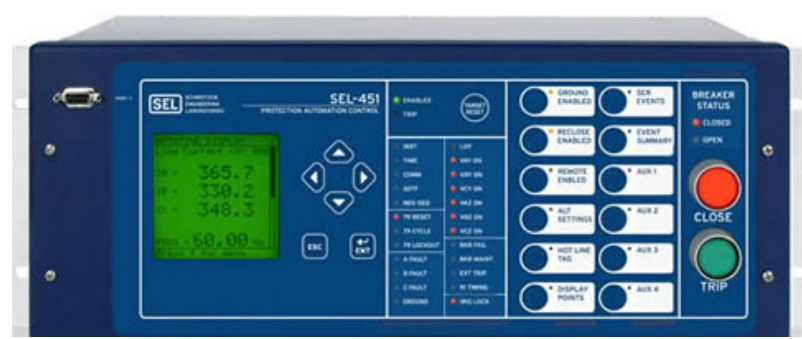

Figure 4.14: Front panel of SEL-451

\subsubsection{SEL-734 Meter}

SEL-734 Meter is designed with ANSI and IEC Class 0.2 accuracy for the purpose of energy and demand metering. Advanced load profile trending ensures integration with practically any billing system. Combine the SEL-734 with a SEL IRIG-B time source to measure the system angle in real time with a timing accuracy of $\pm 10 \mu \mathrm{s}$. The SEL-734 measures instantaneous voltage and current angles in real time to improve system operation with synchrophasor information.

The SEL-734 Meter records power system events with very high accuracy when it is provided with high-accuracy clock input signals, such as from a GPS receiver. Meters placed at key substations can give you information on power system operating conditions in real time or accumulate records when events occur.

Based on the high-accuracy time input, the meter calculates synchronized phasors for line currents and voltages (for each phase and for positive-sequence). Then detailed analysis and calculation of load flow from the synchrophasors can be performed. These measurements are used for following application areas:

- Meter Configuration for High-Accuracy Timekeeping

- Synchrophasor Measurements

- Power Flow Analysis 
- State Estimation Verification

Synchronized phasor measurement provides power system data referenced to the same instant in time from multiple SEL-734 meters at different locations on a power system. This feature reduces time for system state estimation, which results in improved system optimization and increased line loading with fewer safety margin requirements.

\subsubsection{SEL-3530 Real-Time Automation Controller (RTAC)}

SEL-3530-4 is the Real-Time Automation Controllers (RTACs) that combines the best features of the embedded microcomputer form factor, embedded real-time operating system and secure communications framework. It is designed and tested to provide service in substations and plant environments. This device can provide functionality from that of a simple intelligent port switch to the sophisticated communication and data handling required for advanced substation integration projects. The RTAC can act as a data concentrator by using protocols such as IEC 61850 .

The RTAC can integrate synchrophasor messages from the IEEE C37.118 protocol into SCADA protocols. Easily include the source PMU time stamps and time quality attributes in the SCADA message to allow for system wide usage of synchrophasor data. Within the RTAC logic engine, complex math and logic calculations on synchrophasor data from C37.118 compliant devices can be performed. The RTAC also synchronizes the time clocks in attached devices that accept a demodulated IRIG-B time signal. The RTAC regenerates the demodulated IRIG-B signal from an external modulated or demodulated source; this signal is precise enough for synchrophasor applications. 


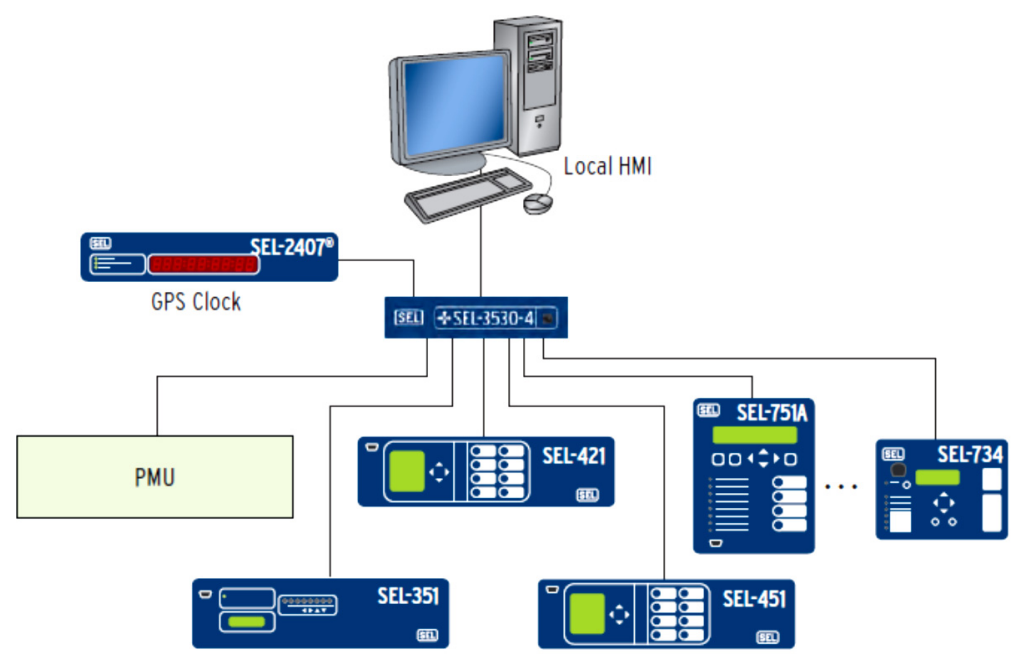

Figure 4.15: System Control and Synchrophasor Data Concentration

Figure 4.15 shows the architecture for system control and synchrophasor data concentration with RTAC SEL3530-4.

\subsubsection{SEL-2407 Satellite-Synchronized Clock}

The role of satellite clocks has grown from basic sequence-of-event and fault recorder time referencing to mission-critical roles such as synchrophasor measurement and detailed event analysis. These new applications require that satellite clocks meet the same environmental standards and be as reliable as the protective relays and other highreliability devices with which these clocks are used. Demodulated IRIG outputs with accuracy of $\pm 100 \mathrm{~ns}$ average ( $\pm 500 \mathrm{~ns}$ peak) meet requirements for real-time synchrophasor applications. This device provides demodulated IRIG-B time-code at six outputs for driving numerous point and distributed applications.

Using the mentioned devices the following architecture shows the hardware which is implemented in the Energy System Research Laboratory in order to measure synchrophasors by industrial level PMUs. 


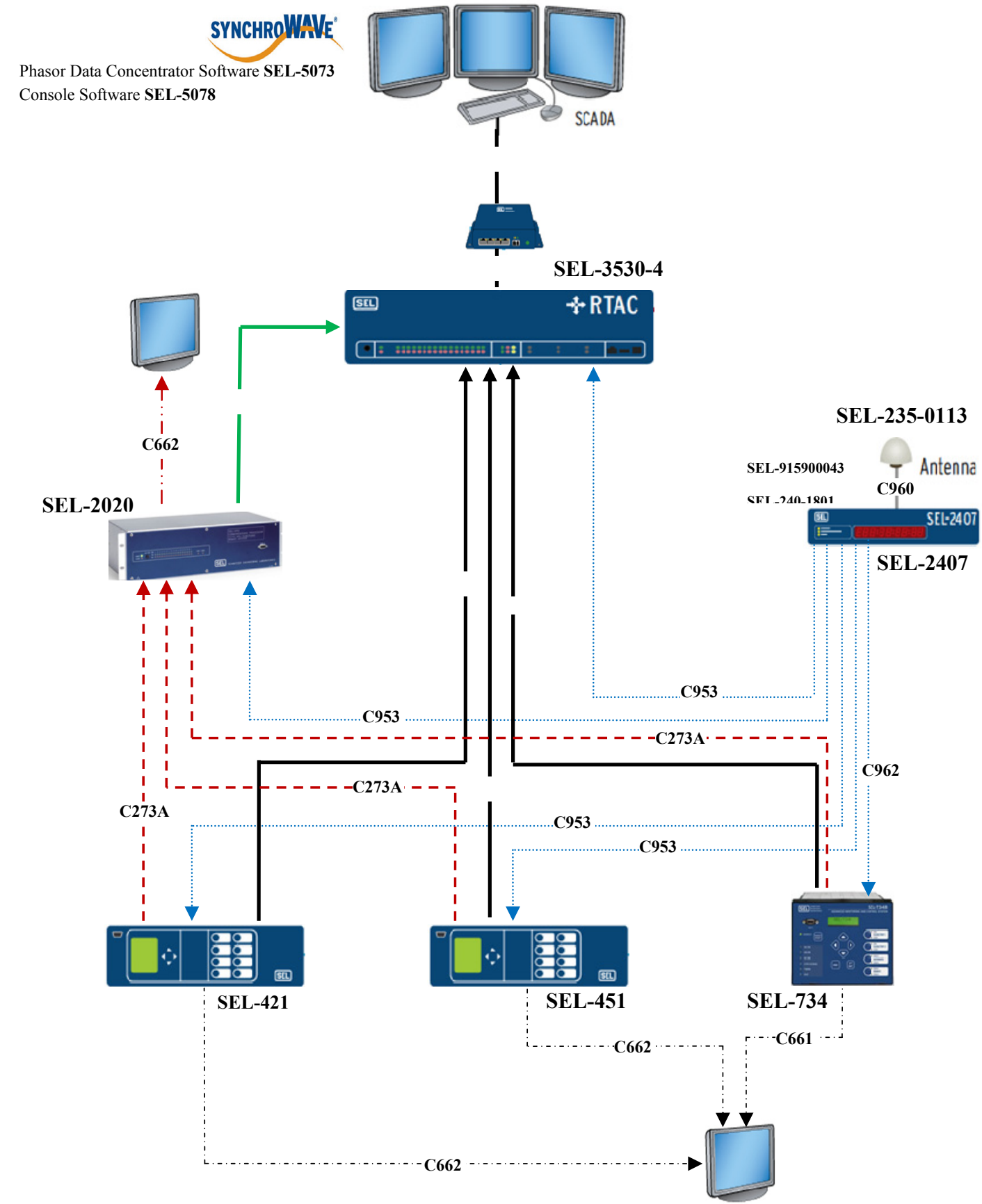

Figure 4.16: PMUs hardware architecture for implemented setup 


\section{CHAPTER 5}

\section{DESIGN AND IMPLEMENTATION OF AN INTEGRATED WIDE AREA MONITORING, CONTROL AND PROTECTION SYSTEM}

\subsection{Introduction,}

The possibility of measuring voltage and current phasors in a power system has created new control possibilities:

- Monitoring of operation of a large power system from the point of view of voltage angles and magnitudes and frequency. This is referred to as Wide Area Monitoring (WAM)

- Application of special power system protection based on measuring phasors in large parts of a power system. Such protection is referred to as Wide Area Protection (WAP)

- Application of control systems based on measuring phasors in large parts of a power system. Such control is referred to as Wide Area Control (WAC)

WAM integrated with WAC and WAP and is referred to as Wide Area

Measurement, Protection And Control (WAMPAC). Recent years have been seen a dynamic expansion of WAMPAC systems. Measurement techniques and telecommunication techniques have made rapid progress, but the main barrier for the expansion of WAMPAC systems is a lack of WAP and WAC control algorithms based on the use of phasors. There has been a lot of research devoted to that problem but the state of knowledge cannot be regarded as satisfactory. 
WAMPAC technology creates a platform to monitor power system dynamics in real time, identify system stability related weaknesses and helps to design and implement strategies in order to overcome problems of conventional power systems. Power system deterioration starts with alarming of steady-state violations and gradually evolves to faster dynamic phenomena with the system reacting quickly and defending itself with pre-determined protection schemes and relays setup. The analysis of short-term/mid-term states must be considered. Angular transients, voltage transient stability and security, frequency stability and small signal analysis tools would certainly help to take proper actions and apply remedies strategies. This process should be totally automated and be applicable by the latest communication technology. This technology is based on PMUs, which can deliver precisely time synchronized voltage and current phasors and other power system parameters like frequency, breaker positions etc which can be calculated from those values.

One basic application is Wide Area Real-time Monitoring and Fault Recording, which helps to understand the reason for power system dynamics followed by an outage or blackout after a very short analysis time. A significant higher challenge is the evolutionary replacement of voltage and current sensors of a SCADA/EMS system with PMUs to establish a highly dynamic "State Measurement System".

WAMPAC technology involves the use of system-wide information and the fast and modern communication of selected local information to a remote location to counteract the propagation of large disturbances. Probably one of the most exciting challenges of the new technology is the design and application of System Integrity 


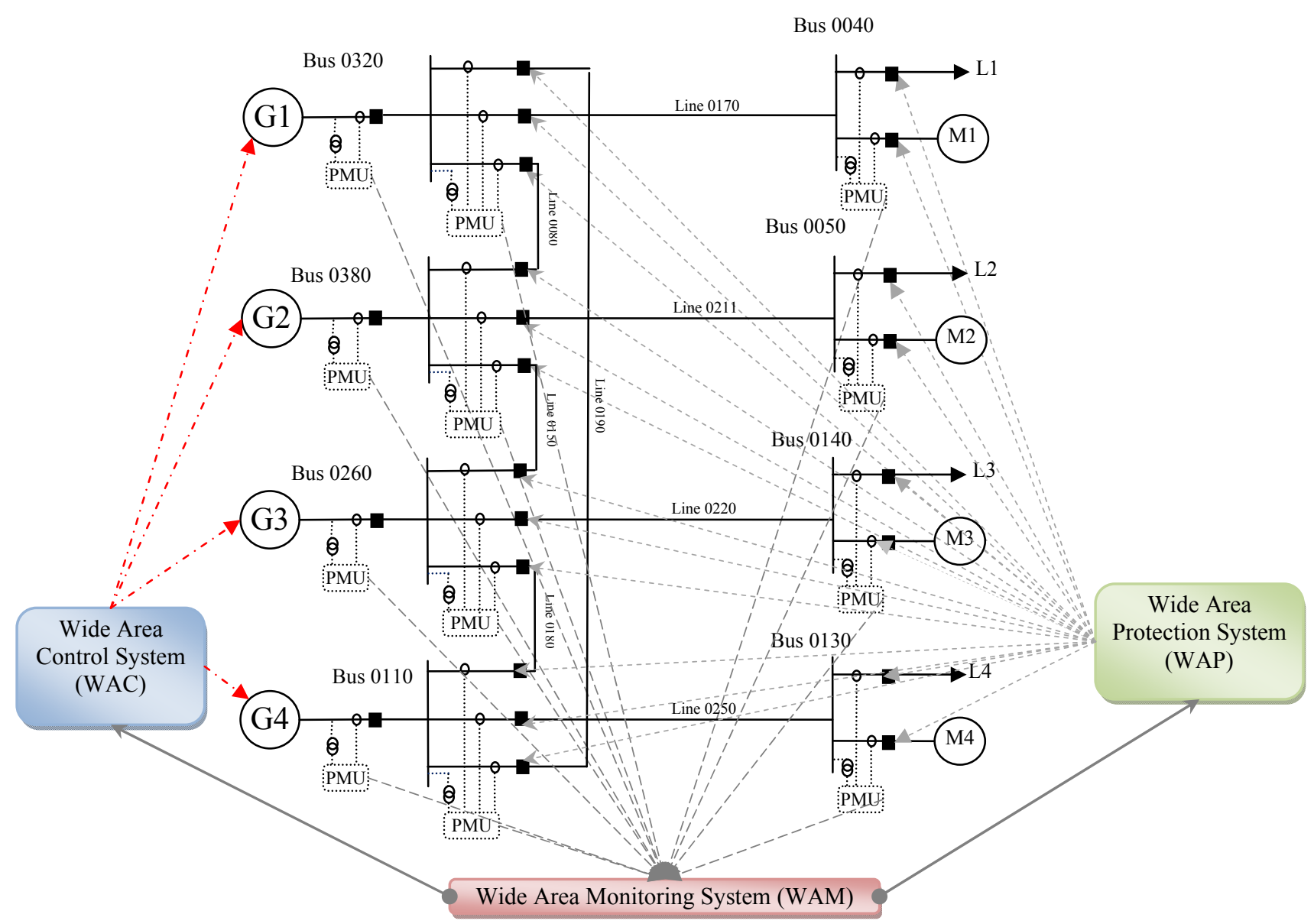

Figure 5.1: Overall implemented power system and developed wide area monitoring, protection and control system

Protection Schemes, which can automatically identify upcoming power system instabilities and minimize its effect with switching operations.

A successful novel monitoring, control and protection application requires a carefully designed WAMPAC architecture, including thoroughly considered communication delay time, cyber security and high system reliability including equipment (PMU, PDC) and communication channel health monitoring.

In this chapter, the focus is on the design of monitoring, control and protection systems and their integrated real-time operation as shown in figure 5.1. Various control scenarios for system startup and continuous operation are examined. We have developed 
a control system based on wide area measurements. The advanced measurement system based on synchrophasors was also implemented using DAQs real-time synchronous data.

The developed system features a wide variety of capabilities such as online system parameters calculation and online voltage stability monitoring which will be discussed in next chapter. These are implemented as an experimental case to enhance wide area monitoring systems. Moreover, the protection system was designed inside of the real-time software environment to monitor the real-time wide area data and make a comprehensive and reliable coordination for the whole system.

\subsection{Developed Wide Area Monitoring Systems}

The evolution of power systems creates major changes in their operational procedures, especially the monitoring networks in real time. We need to use advanced, smart monitoring tools to quickly and reliably estimate the real-time state of the systems. PMU which is presented in previous chapter is the modern technology in this field to monitor power system based on synchronized phasor information. They provide positive sequence voltage/current and frequency measurements synchronized within a microsecond. In addition, they may be customized to measure harmonics, negative and zero sequence quantities, as well as individual phase voltages and currents. Significant improvements in system operations can be achieved by utilizing the synchronized measurement technology in the enhancement of state estimation, real-time load management, real-time angular and voltage stability analysis and design and enhancement of an adaptive protection and control system. 
In this laboratory-based smart power system, the focus is on the real-time calculation of power system parameters using PMUs and their applications in power system studies. The PMUs implementation was carried out by the DAQs for research purposes. According to figure 3.7, different DAQs were used to get the whole system data. Every DAQ data are synchronized and hence can simulate different PMUs data concentrating in one system. For instance, all generators voltages and currents are measured in a DAQ and then the voltage phasors are presented in the same reference frame. In this case, the reference signal for this DAQ is phase A of generator G1 according to figure 5.2. As shown in this figure, the positive sequence of the voltages and currents are in the same reference and with a $12-\mathrm{kHz}$ sampling rate. It should be pointed out that 10 data packages can be sent per second. Other DAQs are connected to several

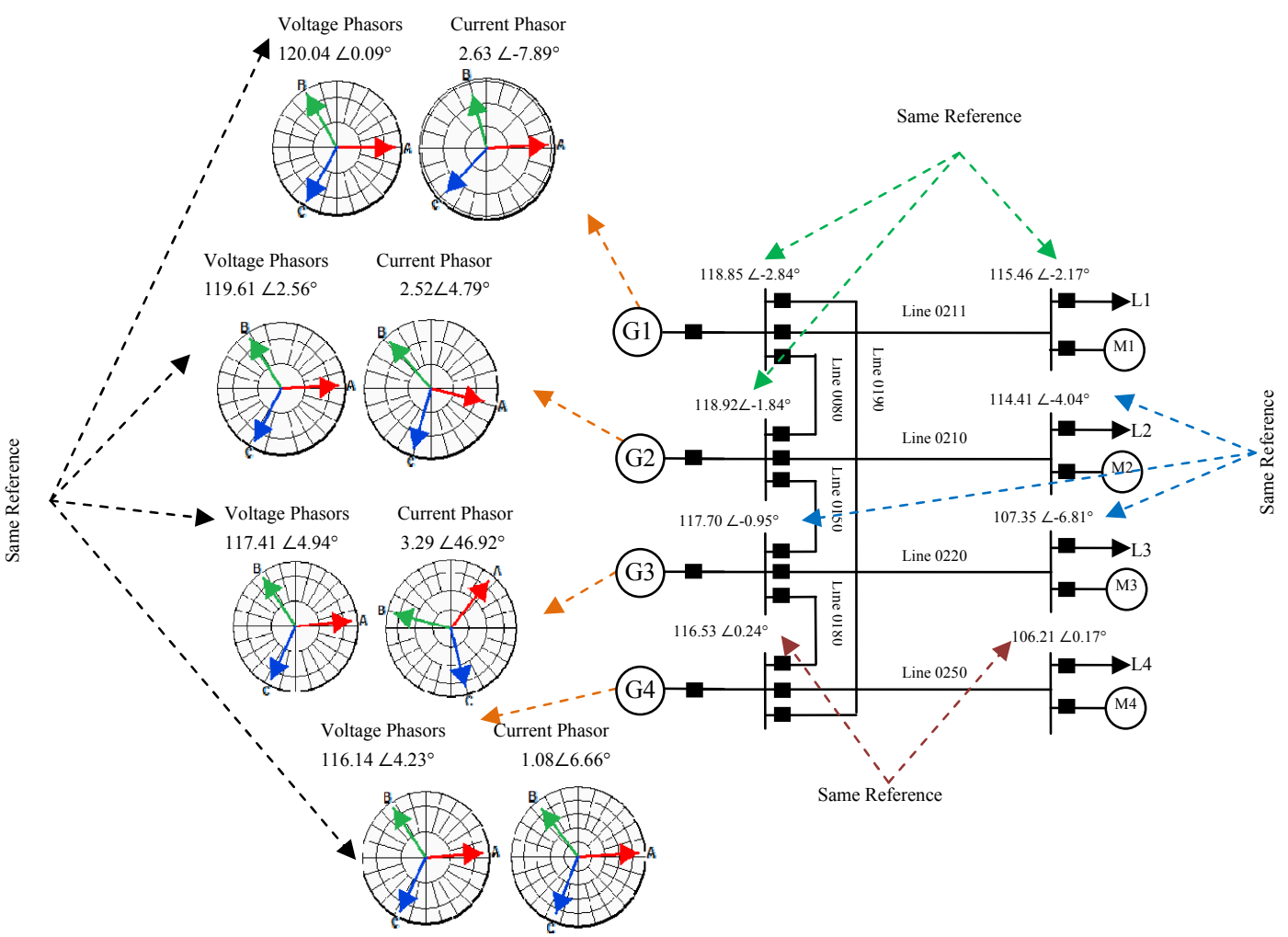

Figure 5.2: Synchronized phasor measurement system developed by real-time software 
buses which are shown with the same reference arrows. A challenge will be synchronizing all DAQs together. A possible solution would be the use of one DAQ in common with at least one measured signal with the other DAQs, similarly to the generators connected DAQ.

The implemented PMU concept is described in details in previous chapter. In addition, a comparison between the implemented real-time PMU and the available measurement devices also were discussed and the results were verified. Two different application cases were studied using the real-time monitoring system by developed PMUs.

\subsubsection{Real-Time Networked Model Parameters Measurement}

The implementation of PMUs meets the requirements for real-time estimation of unknown parameters or for updating network model parameters. For instant critical branches in the power system can be monitored if two PMUs are located at both sides of the line terminals as presented in figure 5.3. This configuration provides reliable information about the power angle differences between adjacent buses and hence determines the power transferred through the line. It is possible to directly calculate the actual line parameters if the voltages and currents at both side of the line are measured in a synchronized form. The following equations give the real-time line pi-model parameters calculation using synchrophasors:

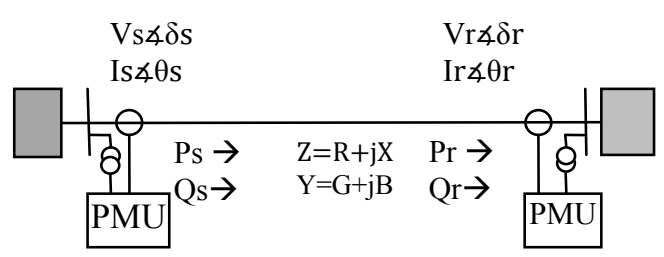

Figure 5.3: PMU placement for a transmission line and related pi-model 


$$
\begin{aligned}
& {\left[\begin{array}{c}
V{ }_{S} \angle \delta_{S} \\
I S_{S} \angle \theta_{S}
\end{array}\right]=\left[\begin{array}{cc}
1+\frac{Y Z}{2} & Z \\
Y\left(1+\frac{Y Z}{2}\right) & 1+\frac{Y Z}{2}
\end{array}\right] \times\left[\begin{array}{c}
V_{r} \angle \delta_{r} \\
I_{r} \angle \theta_{r}
\end{array}\right]} \\
& Z=R+j X=\frac{\left(V_{s} \angle \delta_{s}\right)^{2}-\left(V_{r} \angle \delta_{r}\right)^{2}}{\left(V_{s} \angle \delta_{s}\right)\left(I_{r} \angle \theta_{r}\right)+\left(V_{r} \angle \delta_{r}\right)\left(I_{s} \angle \theta_{r}\right)} \\
& Y=G+j B=2 \frac{\left(I_{s} \angle \theta_{s}\right)-\left(I_{r} \angle \theta_{r}\right)}{\left(V_{s} \angle \delta_{s}\right)+\left(V_{r} \angle \delta_{r}\right)}
\end{aligned}
$$

For measuring the line parameters, an experiment was designed to calculate pimodel line parameters. For one of the lines connected between the generation bus and the load bus, the measured real-time voltage and currents are identified for both the receiving and sending ends as in figure 5.4. The active and reactive power load starts to increase in different time steps according to figure 5.5 and lead to step changes in the values in this figure. Using the phasor calculation in real-time, and insertion into equations 5.2 and 5.3, the real line parameters can be achieved according to figure 5.6. For an ideal transmission line pi-model, circuit parameters should be constant during the load increase. This experiment however shows that the developed line has parameters which are dependent on the line current. For a healthy line, the calculated line parameter changes give some information about ambient condition and the line situation. With fast real-time PMU measurements, the parameter monitoring can indicate any kind of transmission line parameter changes due to the faults inside the line which may have considerable variations. 


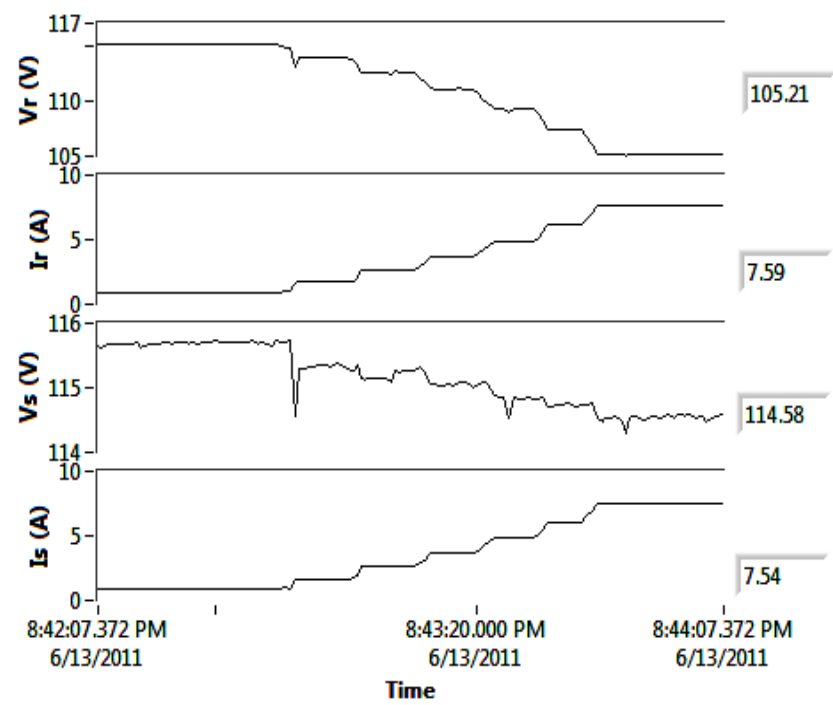

Figure 5.4: Voltages and Current magnitude of receiving and sending of the line during load increase
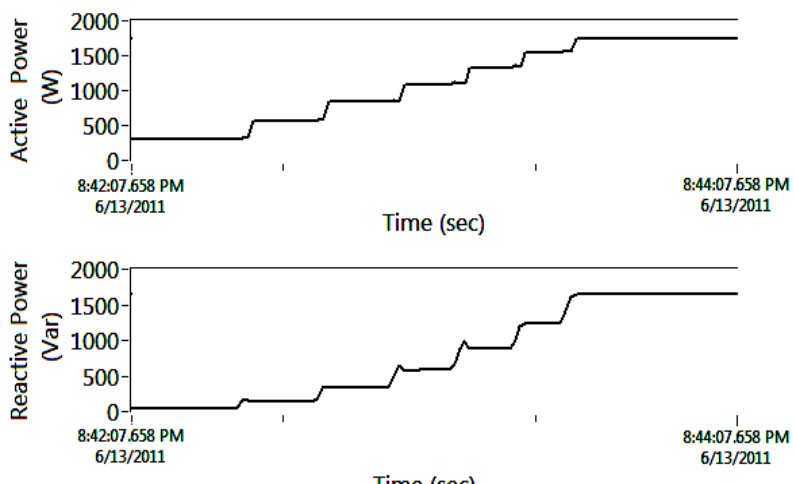

Figure 5.5: Active and reactive power of the load at receiving end side

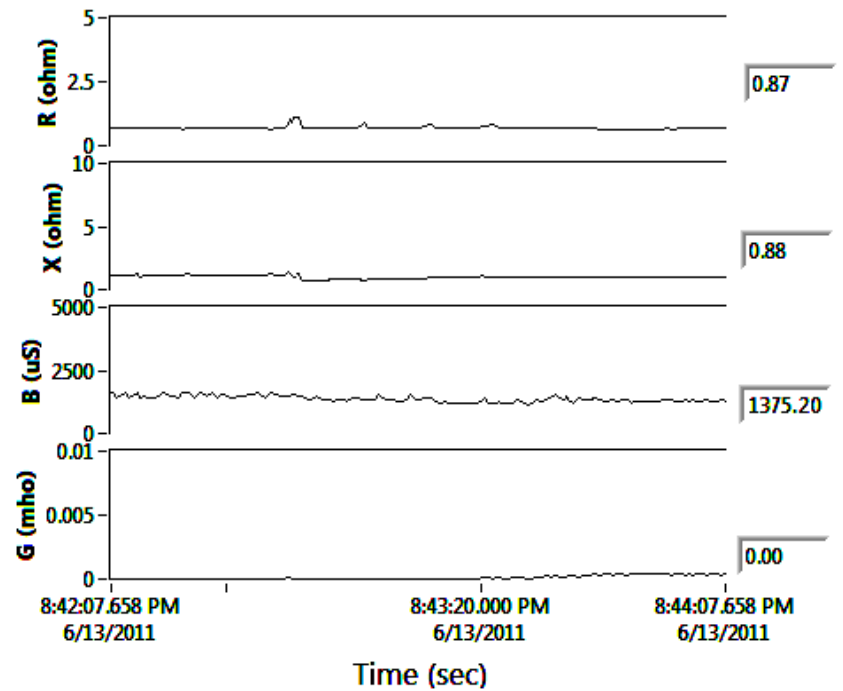

Figure 5.6: Measured line pi-model parameters during the experiment 


\subsubsection{Real-Time Voltage Stability Monitoring}

The voltage instability problem is always distinguished by the system voltage profile, heavily reactive line flows, inadequate reactive power support and heavily loaded power systems in general. Voltage collapse typically occurs right after an event which causes one of the conditions and it may last in time frames of a few seconds to several minutes, but rarely hours [119]. Many indices were developed to detect the voltage stability in a static security-stability assessment manner [94]-[97], [114], [120]-[126]. Many of these indices are based on the proximity of the power system operating state to its collapse point which can be achieved by well-known PV curve. A voltage stability margin, as an index, determines the distance of the power system operating point to the instability point. Furthermore, it can be used to recognize weak buses and the highly risky areas involved in the voltage instability. In this study, several voltage stability indices which are developed in literature [94]-[97], [120]-[126] are presented in Table 5.1. They are used to monitor online voltage stability margins in power system using PMUs. The real-time application was developed to monitor the behavior of these indices under system dynamic changes. By comparison and verification of these indices in a real power system, a better view can be achieved to a specific security margin and therefore a proper remedial action is to be taken. These indices were achieved for a transmission line with two end bus power system using the PMUs data. This is because all indices need the data from both sides of the line in synchronous form.

Figure 5.7 presents the indices variation under the active load increase at different time steps similar to the previous experiment in section 5.2.1. All the indices show negative changes in voltage stability margins expected during load increasing with 
different sensitivities. Here, $P_{R \max }$ and $V_{R \min }$ present the knee point of well-known PV curve for voltage stability and hence the available power margin can be achieved after subtracting $P_{R \max }$ from the load active power.

Table 5.1: Real-time Voltage Stability Indices

\begin{tabular}{|c|c|c|}
\hline Factor & Equation & $\begin{array}{l}\text { Stable } \\
\text { Range }\end{array}$ \\
\hline Line Stability Index & $L_{m n}=\frac{4 X Q_{r}}{\left[V_{S} \sin (\theta-\delta)\right]^{2}}$ & $L_{m n} \leq 1$ \\
\hline Line Stability Factor & $L Q P=4\left(\frac{X}{V_{S}^{2}}\right)\left(\frac{X}{V_{S}^{2}} P_{S}^{2}+Q_{r}\right)$ & $L Q P \leq 1$ \\
\hline Fast Voltage Stability Index & $F S V I=\frac{4 Z^{2} Q_{r}}{V_{S}^{2} X}$ & $F S V I \leq 1$ \\
\hline $\begin{array}{l}\text { Online Voltage Stability } \\
\text { Index }\end{array}$ & $L S V I=\frac{\sin \theta\left(1-\sqrt{1-\frac{4 P R}{V_{S}^{2}}}\right)+\cos \theta\left(1-\sqrt{1-\frac{4 Q X}{V_{S}^{2}}}\right.}{\sin \theta+\cos \theta-1}$ & $L V S I \leq 1$ \\
\hline $\begin{array}{l}\text { Voltage Sensitivity } \\
\text { Stability Margin }\end{array}$ & $d S / d Y=\frac{V_{s \cdot p u}^{2} \times\left(1-Y^{2} Z^{2}\right)}{\left(1+Y^{2} Z^{2}+2 Y Z \cos \alpha\right)^{2}}$ & $d S / d Y \geq 0$ \\
\hline $\begin{array}{l}\text { Transmission Line } \\
\text { Marginal Loading }\end{array}$ & $\begin{array}{l}P_{R \max }=\frac{V_{S}^{2} \cos \left(\delta_{r}-\theta_{r}\right)}{2 K\left[R \cos \left(\varphi_{Z V}+\varphi_{K}\right)+X \sin \left(\varphi_{Z V}+\varphi_{K}\right)+Z\right.} \\
V_{R \min }=\sqrt{\left.\frac{P_{R \max }}{\left.K \cos \varphi_{Z V}\right)}\left[R \cos \varphi_{Z V^{+}} \varphi_{K}\right)+X \sin \left(\varphi_{Z V^{+}}+\varphi_{K}\right)\right]-\frac{V_{s}^{2}}{2 K^{2}}} \\
\vec{K}=1+\frac{1}{2}\{R G-B X+j(R B+X G)\}\end{array}$ & $\begin{array}{l}P_{R \max } \geq P \\
V_{R \min } \leq V\end{array}$ \\
\hline $\begin{array}{l}\text { Online Voltage Stability } \\
\text { Index }\end{array}$ & $\begin{array}{l}V S I=\min \left\{\frac{P_{\max }-P}{P_{\max }}, \frac{Q_{\max }-Q}{Q_{\max }}, \frac{S_{\max }-S}{S_{\max }}\right\} \\
P_{\max }=\frac{Q_{r} R}{X}-\frac{V_{S}^{2} R}{2 X^{2}}+\frac{|Z| V_{S} \sqrt{V_{S}^{2}-4 Q_{r} X}}{2 X^{2}} \\
Q_{\max }=\frac{P_{r} X}{R}-\frac{V_{S}^{2} X}{2 R^{2}}+\frac{|Z| V_{S} \sqrt{V_{S}^{2}-4 P_{r} R}}{2 R^{2}} \\
S_{\max }=\frac{V_{S}^{2}\{|Z|-(X \sin \varphi+R \cos \varphi)\}}{2(X \cos \varphi-R \sin \varphi)^{2}}\end{array}$ & $V S I \leq 1$ \\
\hline
\end{tabular}

$V_{s}, V_{r}:$ Voltage on sending and receiving buses; $P_{s}, Q_{s}$ : Active and reactive power on the sending bus; $P_{r}, Q_{r}$ : Active and reactive power on the receiving bus; $\delta_{s}, \delta_{i}$ : Voltage angle on sending and receiving buses; $R, X$ : Line resistance and reactance; $B, G$ : Line suceptance and conductance; $\delta=\delta_{s}-\delta_{i}$ : angle difference between sending and receiving buses; $\theta=\arctan (X / R)$ : line series impedance angle. 


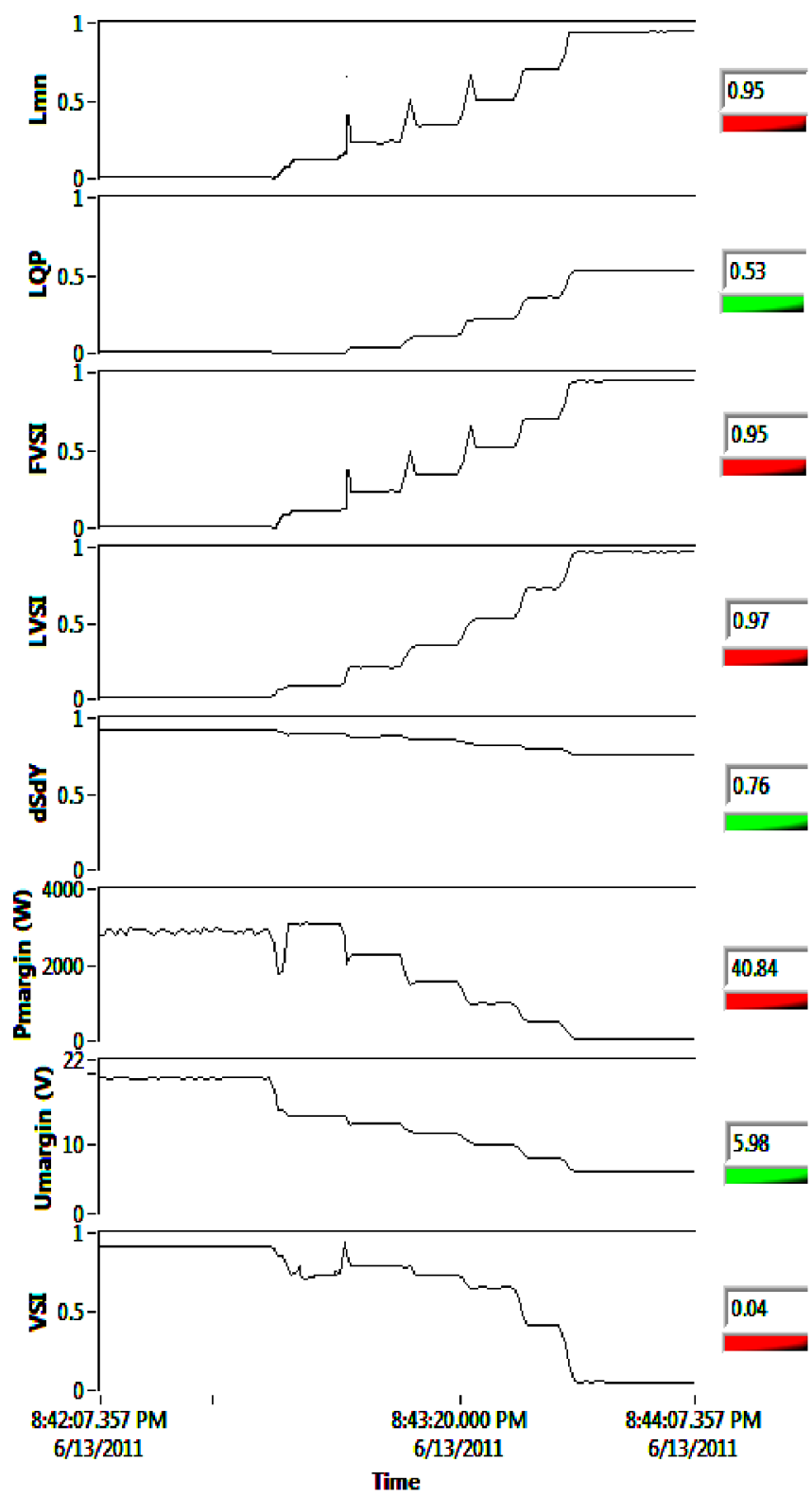

Figure 5.7: Experimental results of online voltage stability indices monitoring

\subsection{Developed Generation Control Scheme in Smart Grid Test-Bed}

\subsubsection{System Start-up}

The generators are controlled solely by the real-time software by sending the control commands to their prime mover drive controllers whenever needed. They keep the last commands as their operating setting until they receive a new one. These 
commands are the prime mover start/stop and change the output frequency or torque to control the generators frequency and output active power. Hence, the generators can be set as a slack generator to maintain frequency, or as a constant output voltage-active power, i.e. in PV control mode. Figure 5.8 shows the control Virtual Instrument (VI) inside the LabVIEW environment to control and visualize the input/output information as data, curves, or indicators. Here, G1 is used as a slack generator to control the micro grid frequency whereas the other generators are in the PV control mode. While the grid is energized by G1, generator G2 should be synchronized with the grid in order to be connected to it safely. This should be done by checking synchronizing conditions and then use the implemented automatic controller to increase/decrease G2's torque until all the synchronizing conditions are satisfied. Figure 5.9 shows the automatic controller attempt to synchronize and connect the generators to the grid. The main VI also presents the used capacity $(\mathrm{kVA})$ of each generator and the torque change buttons to change the generator's active power during the system operation. In addition, a dynamic break has been designed in order to synchronize generators to the grid faster than the case when the synchronization is approached by just changing torque commands. The front panel of control VI for synchronizing generators by dynamic break is shown in figure 5.10. Different methods for soft synchronizing of micro generators by design procedure and experimental results are provided in [127]. 


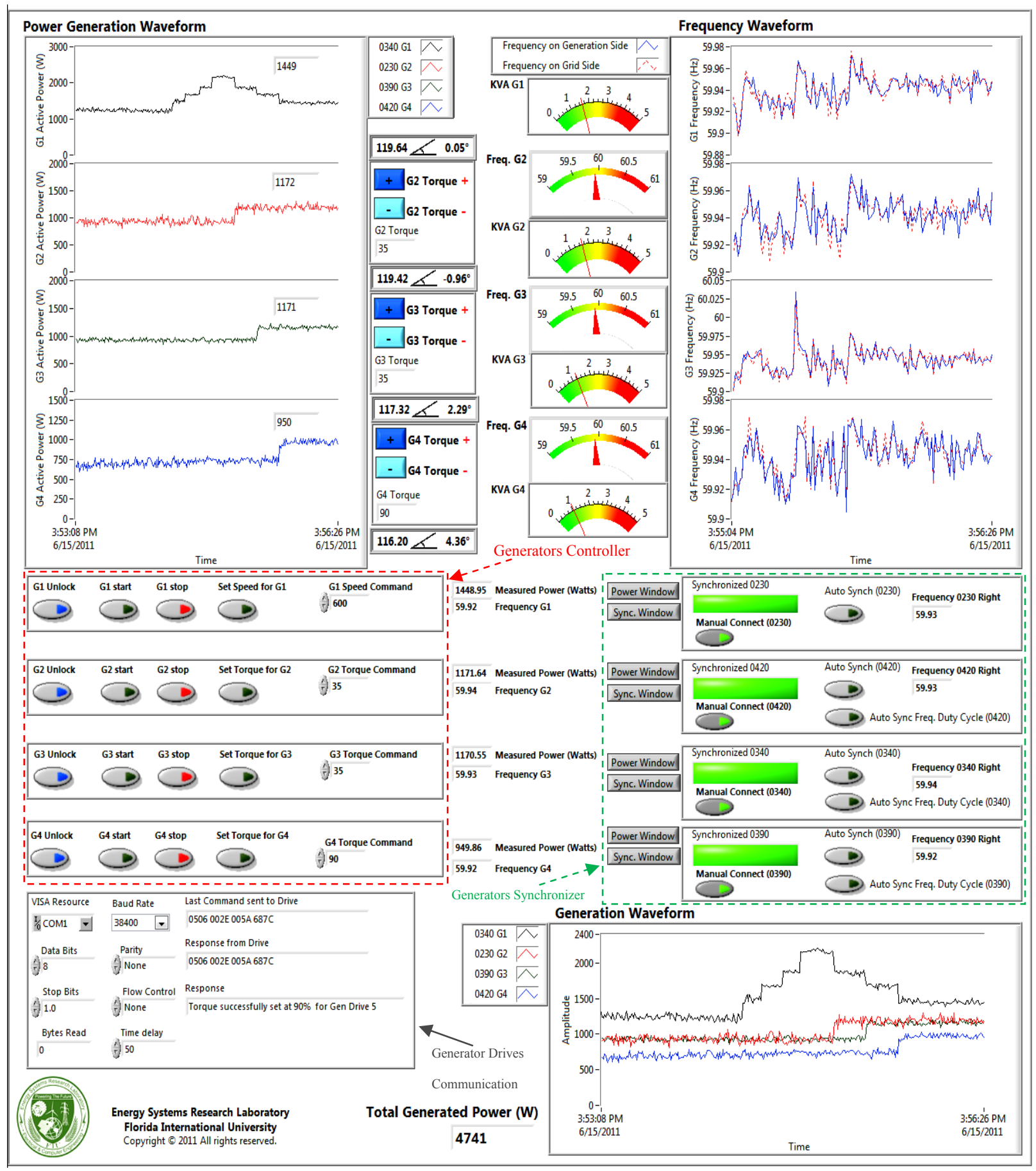

Figure 5.8: Overall view of real-time generation monitoring, communication and controller VI 

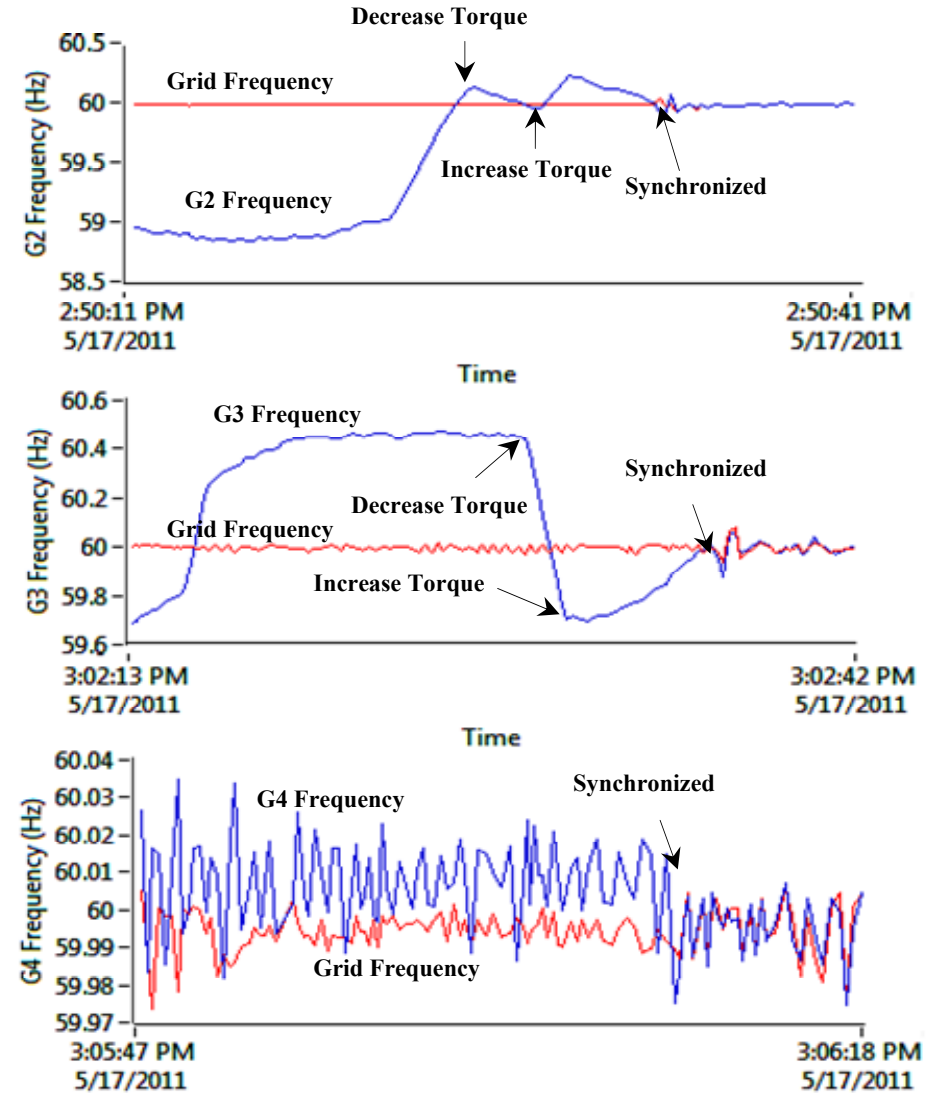

Figure 5.9: Synchronizers controller actions in order to connect generators to grid

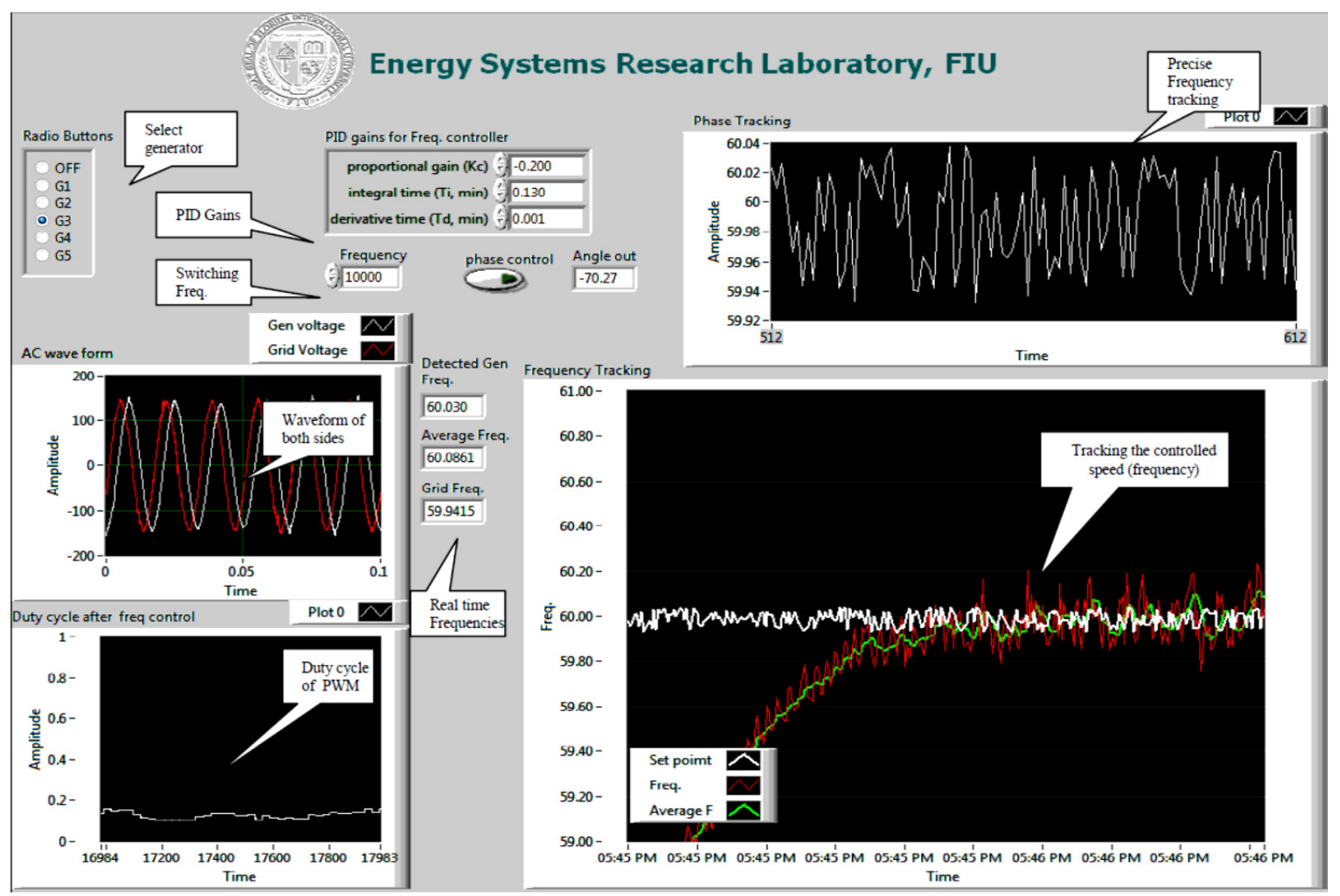

Figure 5.10: Front panel of dynamic load to match the frequency of the generator side to Grid side 


\subsubsection{System Continuous Operation}

The steady state parameters in the real-time monitoring software determine the system components loading, over/under voltage situation, frequency drop, active and reactive power flow, losses and so on. The main generator control VI also presents the generator's loadings in order to share the generation level optimally when one of them encounters an overload situation. The constant active power-voltage magnitude generators known as PV generators, G2, G3 and G4, should participate in generation according to the control commands after the system start-up. Hence, their active power is increased to a reasonable value according to the system total load and the slack generator loading. This procedure was implemented manually by entering a proper torque command to the prime mover as shown in figure 5.11. The system's active power load is increased in steps of $300 \mathrm{~W}$ for each load in different times (L1, L2, L3 and L4). Without any change in generation, the slack generator is responsible to maintain the system frequency at $60 \mathrm{~Hz}$. Therefore, the total load change leads to an increase in the active power generation of the slack generator, G1. Increasing the active power of the generators will alleviate the total generation of G1. The active and reactive power changes of all four generators are shown in figure 5.11. Practically, the reactive powers return back to their initial values since the total reactive load was constant during the experiment.

A real-time power controller was implemented to monitor the active power of the load buses and specify the same amount of power to the nearest generator. This is the automatic power sharing process in order to show the effectiveness of the integrated realtime monitoring and control in wide area system. Figure 5.12 shows the active and 
reactive power change of every generator which was controlled automatically by the realtime software. Similar to previous experiments, the same increase in load pattern was considered in this case and test results of this controller were compared with the manual generation control. Whenever a load is increased, the monitoring and control system recognized it. The system also changes the torque control command of the generator connected close to that load to change the amount of generated power. This procedure is clear as presented in figure 5.12. Since the monitoring system has a time latency of about $1 \mathrm{sec}$, the control system will follow the load variation by a step change within a same or twice time delay. The automatic control scheme is fast enough to operate the system in a smart manner and to share the load among the generation stations. The range of variation of the active and reactive power of each generator in the automatic mode is less than manual control because of the fast response of the control system. Figure 5.13 shows the benefits of automatic generation control system versus manual control through the voltage and frequency changes for the slack generator.

The automatic control system for the generators should be comprehensive considering system conditions such as loadings of equipments, voltage and generation limitations as well as stability issues. The use of the real-time software makes it possible to monitor all system conditions and create a proper power sharing algorithm such as Optimal Power Flow (OPF). Figure 5.14 shows the developed integrated wide-area system on the laboratory-scale Smart Grid test-bed with including monitoring, control and protection systems with fast communication features. The implementation of this system in the real-time software creates an environment for studying and verifying new control and protection schemes for the whole power system. 


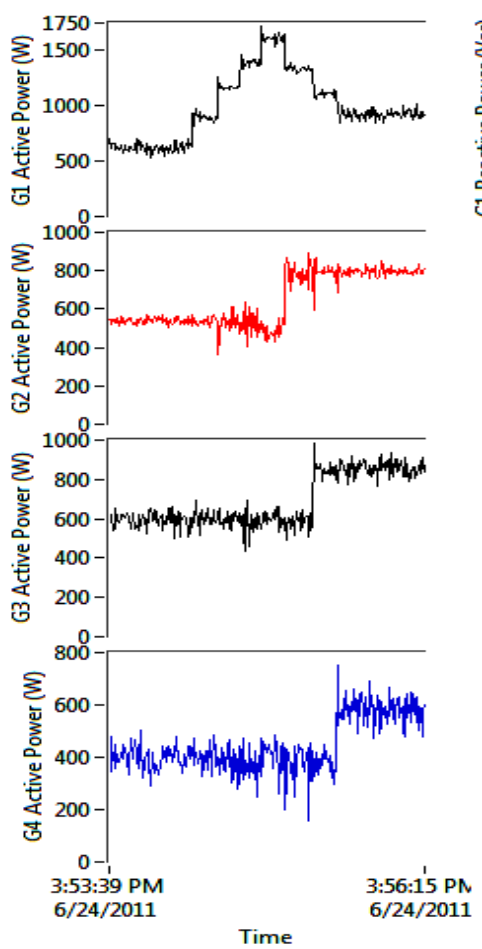

(a)

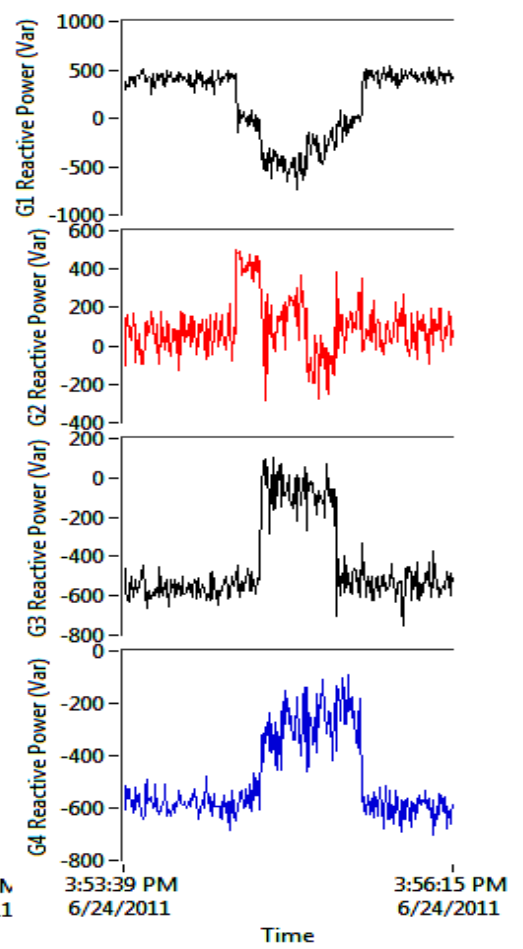

(b)

Figure 5.11: (a) Active power and (b) Reactive power of generators during load increase in manual control

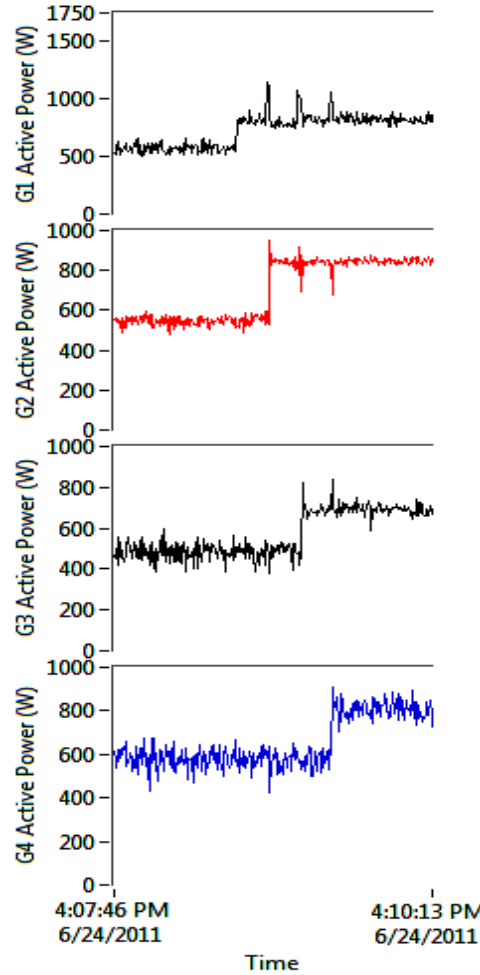

(a)

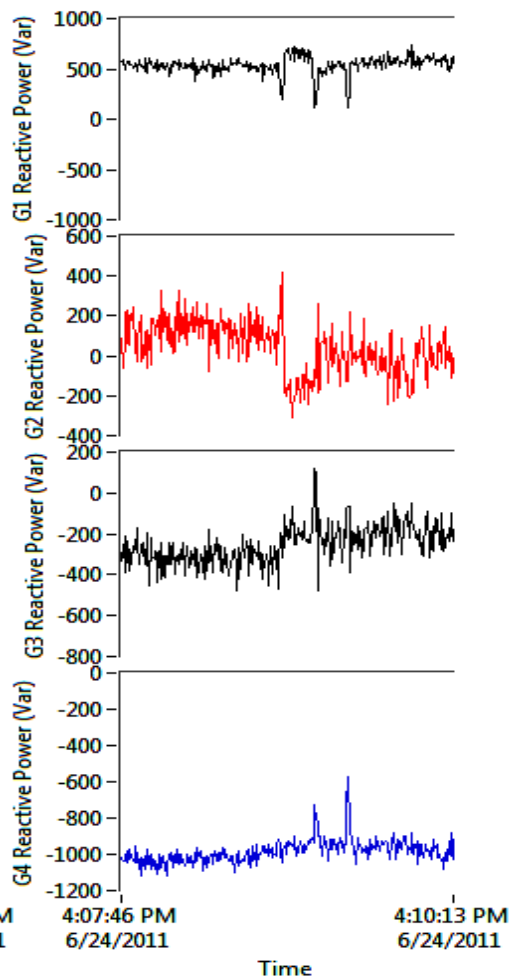

(b)

Figure 5.12: (a) Active power and (b) Reactive power of generators during load increase in automatic control 

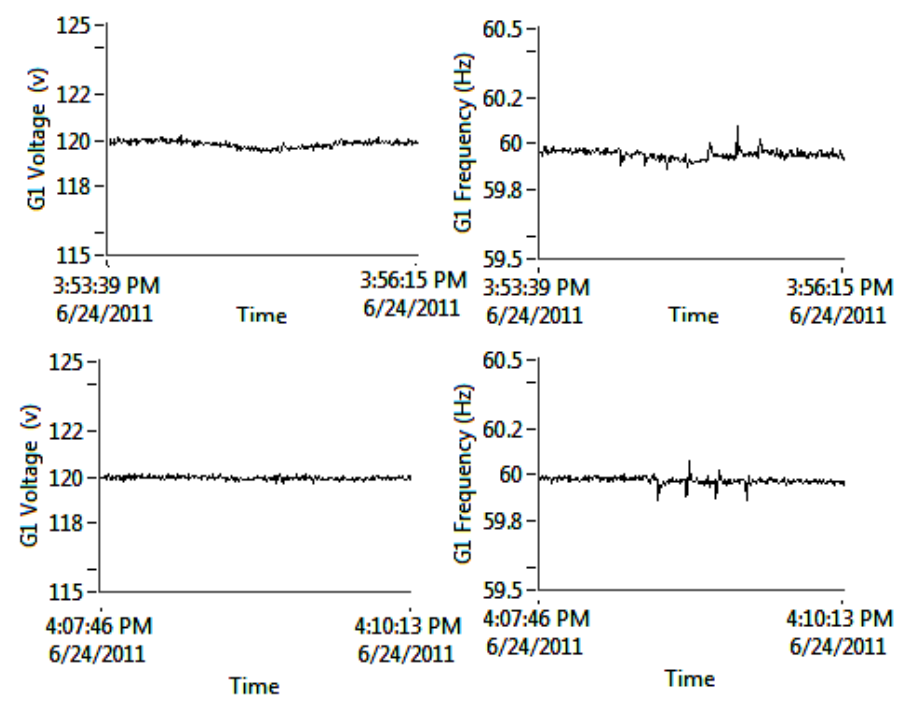

(a)

(b)

Figure 5.13: (a) Voltage and (b) frequency of generators during load increase in manual (upper plot) and automatic (lower plot) control mode

This system was used for studying integrated wide area control and protection system to monitor the system status for abnormalities such as over/under voltage, overloads of equipments and any other conditions. This system was also used to monitor real-time system stability and security margin. The indices formulation and their implementation on wide area monitoring and control centers were presented. This provides an applicable view of system stability and security margin using PMUs in wide area networks. The voltage stability indices were measured during the operation of the power system when the load changes take places. A wide area monitoring system with high data resolution rate was developed. This system was designed to have capabilities such as maintaining system normal operation and take a proper remedial action when encountered by unexpected circumstances by monitoring critical states in wide area system. As a result, the system operator will have proper knowledge and visualization about the power system's current situation and the distance of stability margin. 


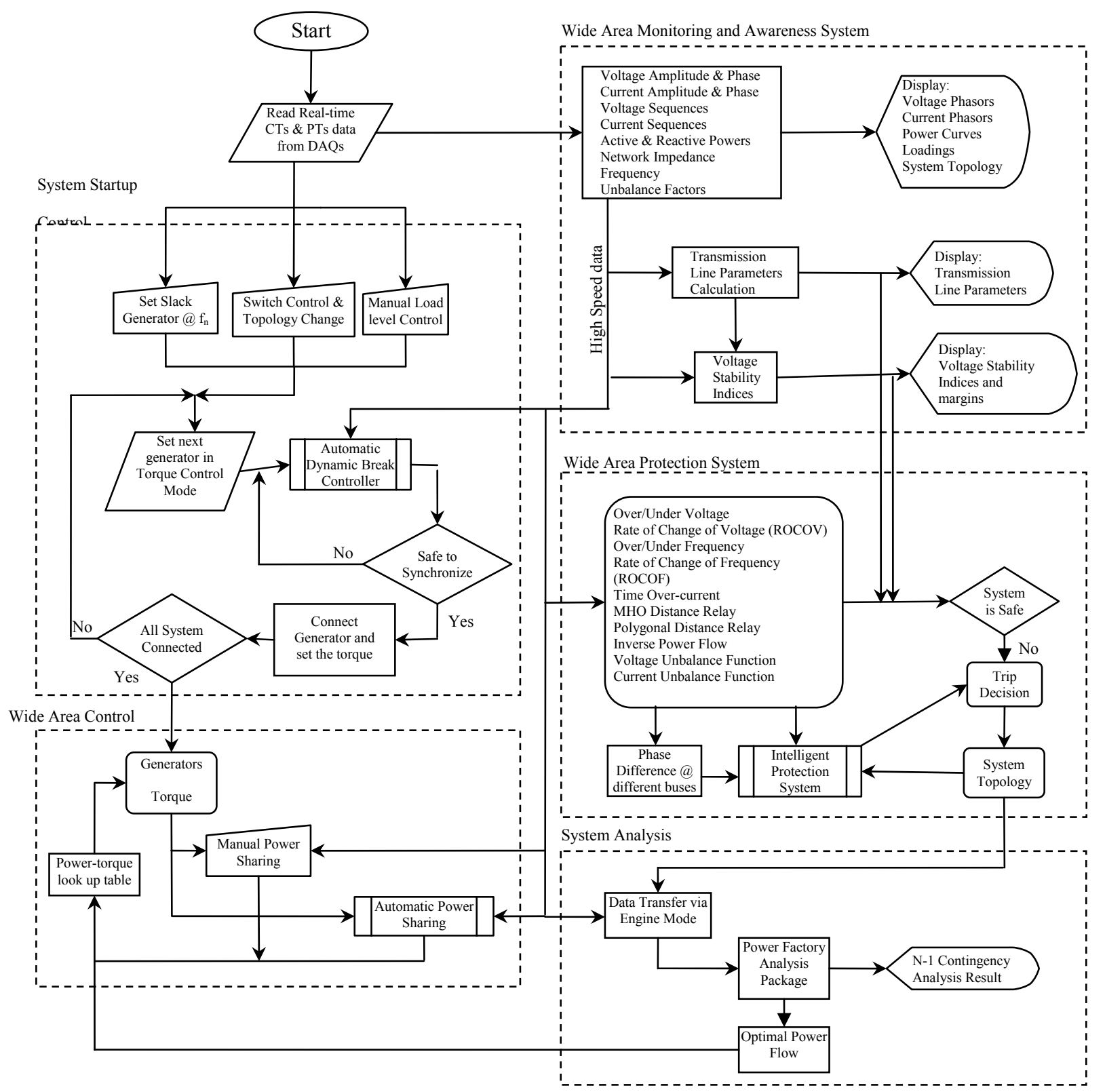

Figure 5.14: Overall implemented real-time system for integrated wide area monitoring, control and protection systems 


\subsection{Virtual Protection System}

In a self-healed power system, unplanned outages and abnormalities could be prevented through better prediction, analysis and control. Recognition and diagnosis of fault conditions has a significant role that may prevent disturbances extension and spreading to other healthy parts of the power system [128]. Such capability requires timely based coordination between monitoring, analysis and control of power system in different scales. Recently, the high performance IT infrastructure has been deployed in power system communication platform in order to respond to transient and steady-state operating conditions in real-time format [129]. This feature can encompass the whole power system control levels and consist of all functions implemented for power system wide area monitoring and control system. Figure 5.15 shows the conventional power system real-time monitoring, analysis and control procedure. Each function operates at different timescale from milliseconds to an hour corresponding to the physical phenomena of the power systems [130]. Recent technologies enhanced the communication structure of power system by its high accuracy, security and data transfer speed. Hence, smart grid, the most challenging subject in power system uses modern communication infrastructure at wide area power grid to improve grid reliability, reduce the price of electricity, improve operational efficiency, improve security and safety and promote environmental quality [72].

Protection devices are the first and major devices in defense line against faults and other disturbances that may cause extensive damage to power system equipments. They are the most important self-healing mechanisms of a power system. Modern protective relays have evolved into a highly reliable system over many years [131]. 
Today relays and meters also include time-synchronized measurement abilities. Modern communication technology provides fiber-optic and low cost radio links and makes precise time available over wide areas [132]. A phasor measurement unit (PMU) measures the electrical waves on an electricity grid to determine the health of the system. In power engineering, these are considered one of the most important measuring devices in the future of power systems. By application of these devices, the typical minute scale SCADA times will decrease to a second or less [133].

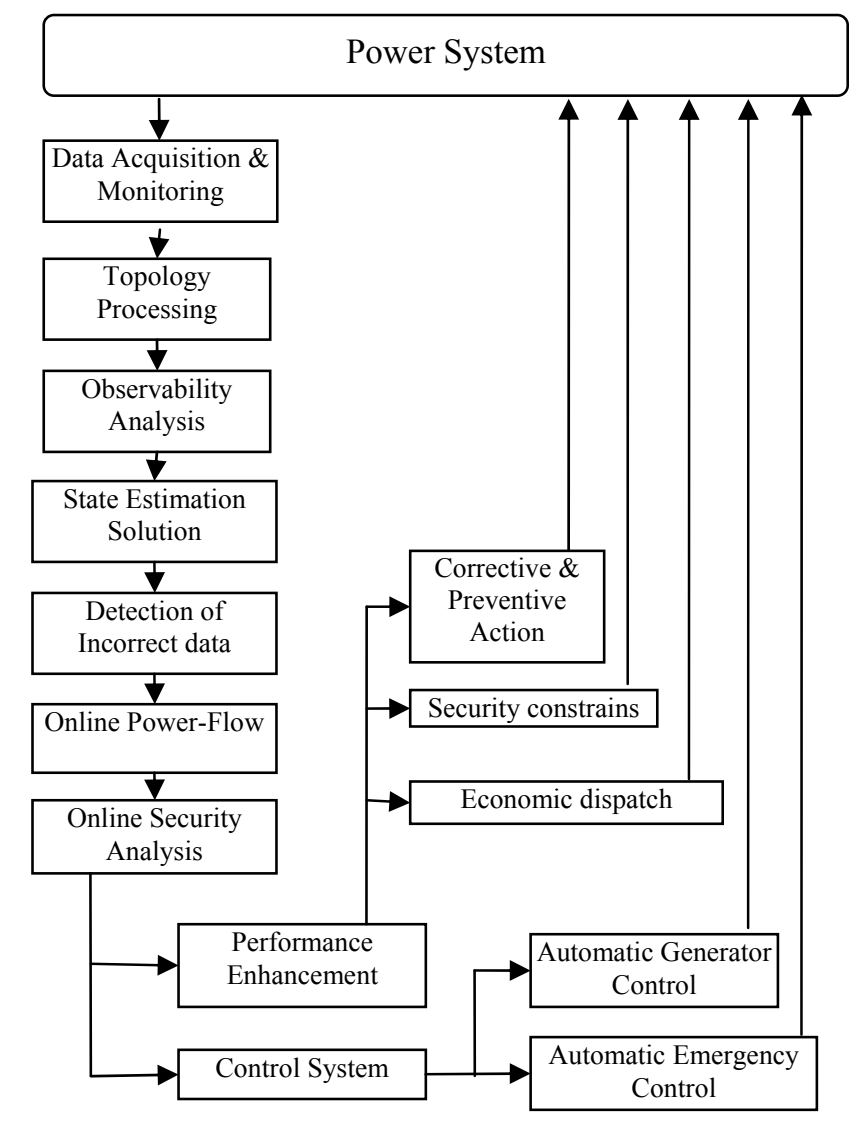

Figure 5.15: Conventional power system real-time monitoring, analysis and control procedure

The objective of this part of the research is to implement a real-time protection system which can offer various types of advantages for controlling and self-healing in power system. We name this system by Virtual Protection System (VPS) which can be implementation of present protection system in a real-time format in a wide area control 
centers. Most real-time softwares have the capabilities to implement mathematical, logical and controlling build in functions to model available relay functions and settings inside their environment. This will be modifying the same operated relay in digital form in order to use in wide area monitoring and control system. The PMU feature of modern digital relays which are all over the grid and communication aspects, give us better chance to run VPS by realistic network data. The speed of communication guarantees the accuracy of operation of this real-time system to predict relays operation in real power system and simulate the system behavior as fast as system topology and parameters variations. In this chapter, the VPS system will be introduced by its implemented functions and features that presents. The application of this system to control and have self-healing strategies in a power system will be demonstrated by a practical example.

In order to build an intelligent and adaptive protection system, this research proposes Virtual Protection System (VPS), applicable in power system control centers, not only to investigate coordination between realistic relays, but also for prediction of cascade events in power system and self-healing purposes. Figure 5.16 demonstrates the whole VPS idea and its role in modern wide area monitoring and control system. This system will use PMUs for monitoring very fast real-time data with rate of at least 10 data package per second for $60 \mathrm{~Hz}$ system according to Std C37.118. The PMUs are particularly effective in improving protection functions which have relatively slow response times. For such protection functions, the latency of remote measurements was not a significant issue. For example, back-up protection functions of distance relays and protection functions concerned with managing angular or voltage stability of networks 
can benefit from remote measurements with propagation delays with latencies of up to several hundred milliseconds [90].

The virtual environment for protective relaying evaluation has been discussed in reference [134]. But in this environment authors used simulators for power system, instrumentation and protective relays. The most important issue in developing such research based power system test-bed is accurate measurement of the system parameters in real-time format which is applicable using PTs and CTs. Then, these data will be transferred to the Digital PMUs developed in Real-time software LabVIEW by using analogue to digital DAQ.

\subsection{Design and Developing of Virtual Protection System}

In this research, the generic form of digital relay with different capability for protecting power system elements have been developed in real time software environment and for using as virtual protection system; it has following capabilities:

- Over/Under Voltage

- Rate of Change of Voltage (ROCOV)

- Over/Under Frequency

- Rate of Change of Frequency (ROCOF)

- Time Over-current

- MHO Distance Relay

- Polygonal Distance Relay

- Inverse Power Flow

- Voltage Unbalance Function

- Current Unbalance Function

The whole functions are developed in LabVIEW software which can receive all voltages and currents from PMUs and then make a decision for breaker action. In the following sections the functionality programming of this relay in digital form will be explained by detail and test verification. 


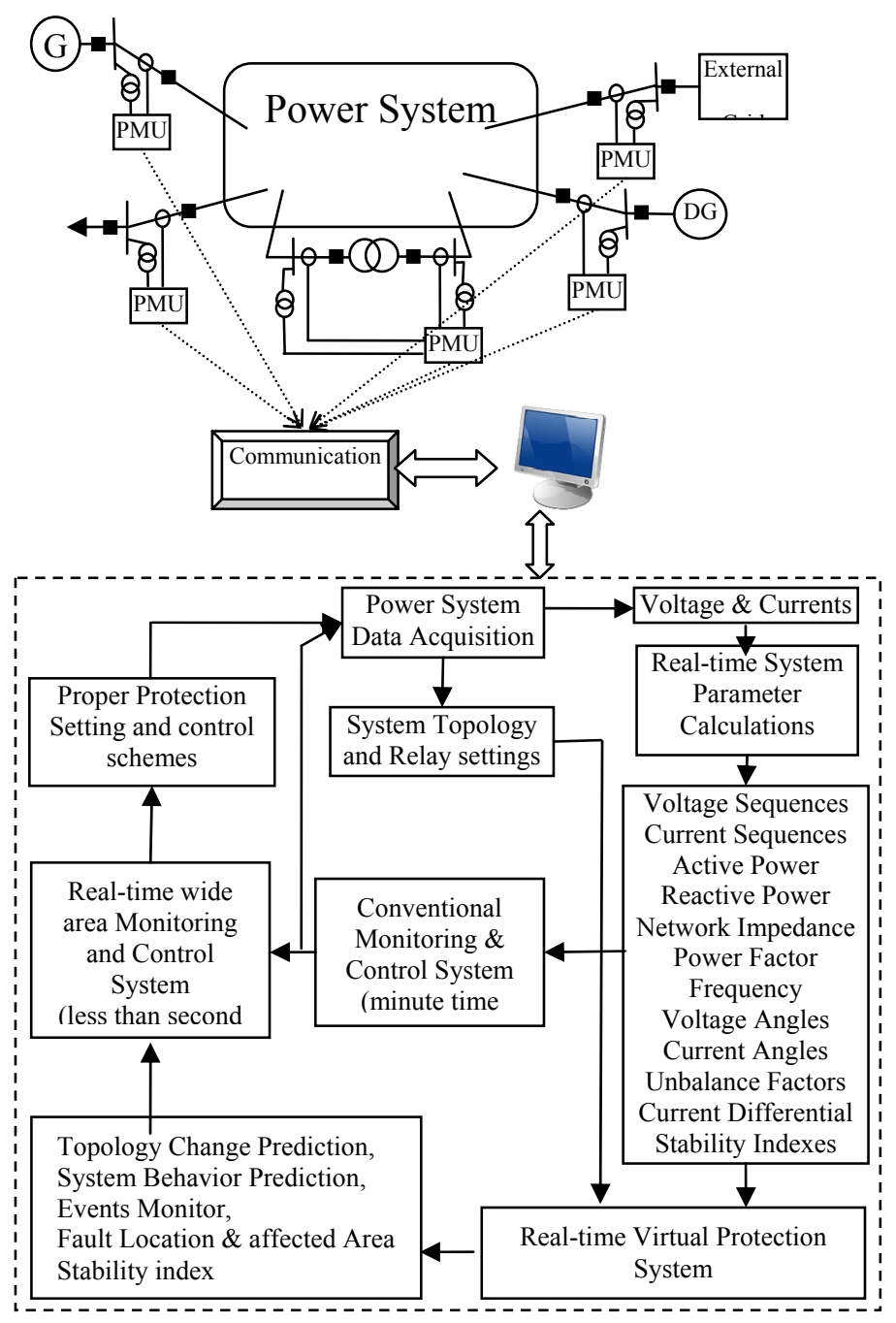

Figure 5.16: Modern power system real-time monitoring, analysis and control procedure

\subsubsection{Over/Under Voltage and ROCOV Protection}

In an electrical power system overvoltage may be classified into two categories: temporary over/under voltage and permanent over/under voltages. Protection relay settings were applied according to criteria developed by the power equipment owner or manufacturer. The operating voltage must normally be within the limits of 0.95 p.u and 1.05 p.u, in order to have a good quality of voltage for the customers or clients. These limits are known as the "Voltage Quality Limits". During a contingency or emergency 
the quality limits can be exceeded. In such cases the criteria to control the overvoltage are those required to protect power equipment such as transformers and generators from damage. The limits of overvoltage in this case are defined by the capability limits of the power system equipments such as the transformers and the generators.

Figure 5.17 shows the implementation of the over/under voltage function in LabVIEW environment. This function has three settings consist of maximum voltage setting $\left(V_{\max }\right)$, minimum voltage setting $\left(V_{\min }\right)$ and rate of change of voltage during the step size of time of system running. So, by activating this function the positive sequence RMS voltage was compared with $\min / \max$ voltage value and with its previous sample in order to observe its change of rate and finally the decision for tripping have been made instantaneously.
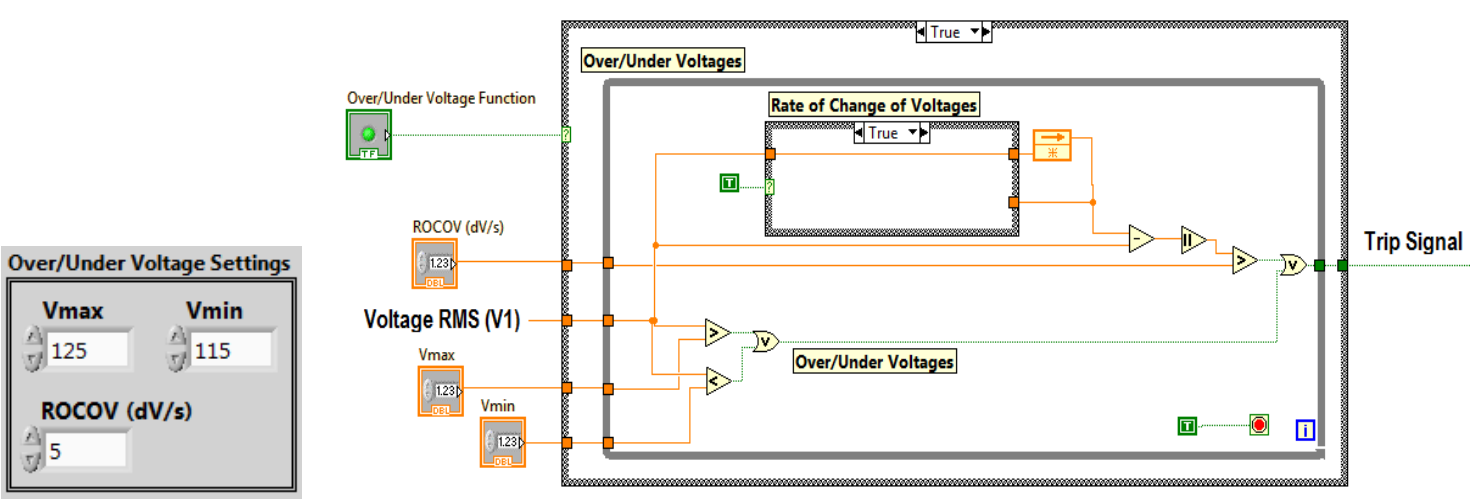

Figure 5.17: Over/Under Voltage Function in LabVIEW real-time software

\subsubsection{Over/Under Frequency and ROCOF Protection}

Frequency relays are used whenever deviations from nominal system frequency need to be detected. Frequency deviations can be harmful to connected objects, such as generators and motors, or when abnormal frequency creates inconvenience for power 
consumers and may cause failures of electrical apparatuses. Frequency relays are also used where detection of high or low frequency indicates system abnormalities, such as faults in speed regulation units or system overload. An over/under frequency relay is generally used as a backup protection to mechanical over speed devices to avoid damages to prime mover. These relays are used to protect the turbine generator system from making up over-speed during start up operation and under-speed during heavy load condition. Apart from these applications, other real-time control loops have processed measurements of power system frequency with a degree of speed, precision and stability, not available with conventional electromechanical instruments.

Under-frequency relays should be considered for applications where the detection of under-speed conditions for synchronous motors and condensers is required. On lines where reclosing of the source breaker was utilized, damage to large synchronous motors can be avoided by disconnecting the motors from the system. Likewise, disconnection of synchronous condensers can be initiated upon loss of power supply.

The over-frequency relay was generally utilized for the protection of ac machines from possible damage due to over-speed conditions. These conditions can occur, for example, on machines with no mechanical governor or on those with the machine shaft linked to a prime mover or to another machine, either one of which could accelerate the combination to a hazardous over-speed condition (e.g. a hydro generator).

Under-frequency relay applied to protect field winding from excessive current, voltage regulated from over load. For load shedding applications df/dt attachment was normally provided with the under-frequency relay. 
Figure 5.18 shows the implemented logic for over/under frequency relay as well as Rate of Change of Frequency (ROCOF) function. The settings are minimum frequency $\left(\mathrm{F}_{\min }\right)$, maximum frequency $\left(\mathrm{F}_{\max }\right)$ in $\mathrm{Hz}$ and Rate of Change of Frequency in $\mathrm{dF} / \mathrm{dt}$. The trip signal will be send to breaker whenever the one of the condition didn't maintain according to inserted settings.

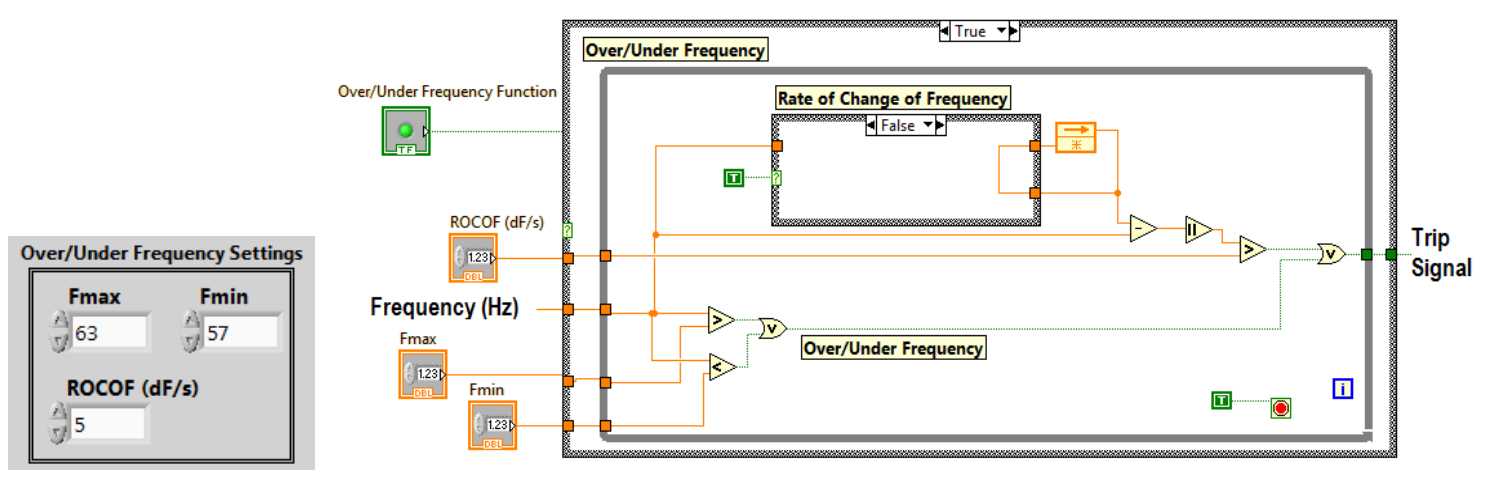

Figure 5.18: Over/Under Frequency Function in LabVIEW real-time software

\subsubsection{Over-current Function}

The protective relaying which responds to a rise in current flowing through the protected element over a pre-determined value is called over-current protection and the relays used for this purpose are known as over-current relays. Earth fault protection can be provided with normal over-current relays, if the minimum earth fault current is sufficient in magnitude. The design of a comprehensive protection scheme in a power system requires the detailed study of time-current characteristics of the various relays used in the scheme. Thus it is necessary to obtain the time-current characteristics of these relays. 
The over-current relay is designed to be used for over-current protection of distribution feeders, motors, medium-sized and large generators and power transformers. The relay can be used both as main protection relay and back-up protection relay.

Over-current relay function monitors the general balanced overloading and has current/time settings. This is determined by the overall protective discrimination scheme. Their advantage over definite time relays is that they can have much shorter tripping times can be obtained without any risk to the protection selection process. These are classified in accordance with their characteristic curves, this indicates the speed of the operation. Based on this they are defined as being inverse, very inverse or extremely inverse.

These relays always have two units: Instantaneous Over-current unit and Time Over-current unit. Instantaneous relays operate without intentional time delay. They are used for faults close to the source when the fault current is very high. The operating time is approximately $10 \mathrm{~ms}$. Time Over-current relays operate with a time delay. The time delay is adjustable. For a given setting, the actual time delay depends on the current through the relay coil. In general, higher current will cause a faster operation of the relay. The minimum current at which the relay operates (pick-up current) is also adjustable.

Time over-current relays come in different versions that are defined by the steepness of the time over-current characteristic according IEEE/ANSI:

- I squared $\mathrm{T} \rightarrow \mathrm{T}=\frac{\mathrm{T}_{\mathrm{p}} * 50.7+10.14}{\left(\frac{1}{\mathrm{I}_{\mathrm{p}}}\right)^{2}}$

- Definite inverse $\rightarrow \mathrm{T}=\mathrm{T}_{\mathrm{p}} \times\left(\frac{0.479}{\left(\frac{\mathrm{I}}{\mathrm{I}_{\mathrm{p}}}\right)^{1.562}-1}+0.21\right)$

- Extremely inverse $\rightarrow \mathrm{T}=\mathrm{T}_{\mathrm{p}} \times\left(\frac{5.64}{\left(\frac{\mathrm{I}}{\mathrm{I}}\right)^{2}-1}+0.0243\right)$ 
- $\quad$ Inverse $\rightarrow \mathrm{T}=\mathrm{T}_{\mathrm{p}} \times\left(\frac{8.934}{\left(\frac{\mathrm{I}}{\mathrm{I}_{\mathrm{p}}}\right)^{2}-1}+0.179\right)$

- Long inverse $\rightarrow \mathrm{T}=\mathrm{T}_{\mathrm{p}} \times\left(\frac{5.614}{\left(\frac{\mathrm{I}}{\mathrm{I}}\right)-1}+2.185\right)$

- Moderately inverse $\rightarrow \mathrm{T}=\mathrm{T}_{\mathrm{p}} \times\left(\frac{0.0103}{\left(\frac{\mathrm{I}}{\mathrm{I}}\right)^{0.02}-1}+0.0228\right)$

- Short inverse $\rightarrow \mathrm{T}=\mathrm{T}_{\mathrm{p}} \times\left(\frac{0.266}{\left(\frac{\mathrm{I}}{\mathrm{I}_{\mathrm{p}}}\right)^{1.296}-1}+0.0339\right)$

- Very inverse $\rightarrow \mathrm{T}=\mathrm{T}_{\mathrm{p}} \times\left(\frac{3.922}{\left(\frac{\mathrm{I}}{\mathrm{I}}\right)^{2}-1}+0.098\right)$

Where $T_{p}$ is the time dial setting and $I_{p}$ is the pick-up current setting for the relay which user can set after choosing the relay characteristic type. Therefore, the relay will calculate $T(s)$, time delay for tripping, by having the network current level $I(\mathrm{~A})$. Figure 5.19 shows the ANSI/IEEE time inverse over-current characteristic for different Tp. The minimum current level for activating relay (I/Ip) is 1.1 and the maximum current level is $I / I p=20$. If fault current exceed more than 20 times of pick-up current then the instantaneous part will be operated. Time counter has been developed in the real-time software which is shown in figure 5.20. After the detection of fault the time is locked until the calculated time was compared with the present time and locked one. Whenever relay reaches to the time provided by calculation the trip signal will be sent to the breaker. Figure 5.21 shows implementation of over-current relay setting in LabVIEW real-time software. 

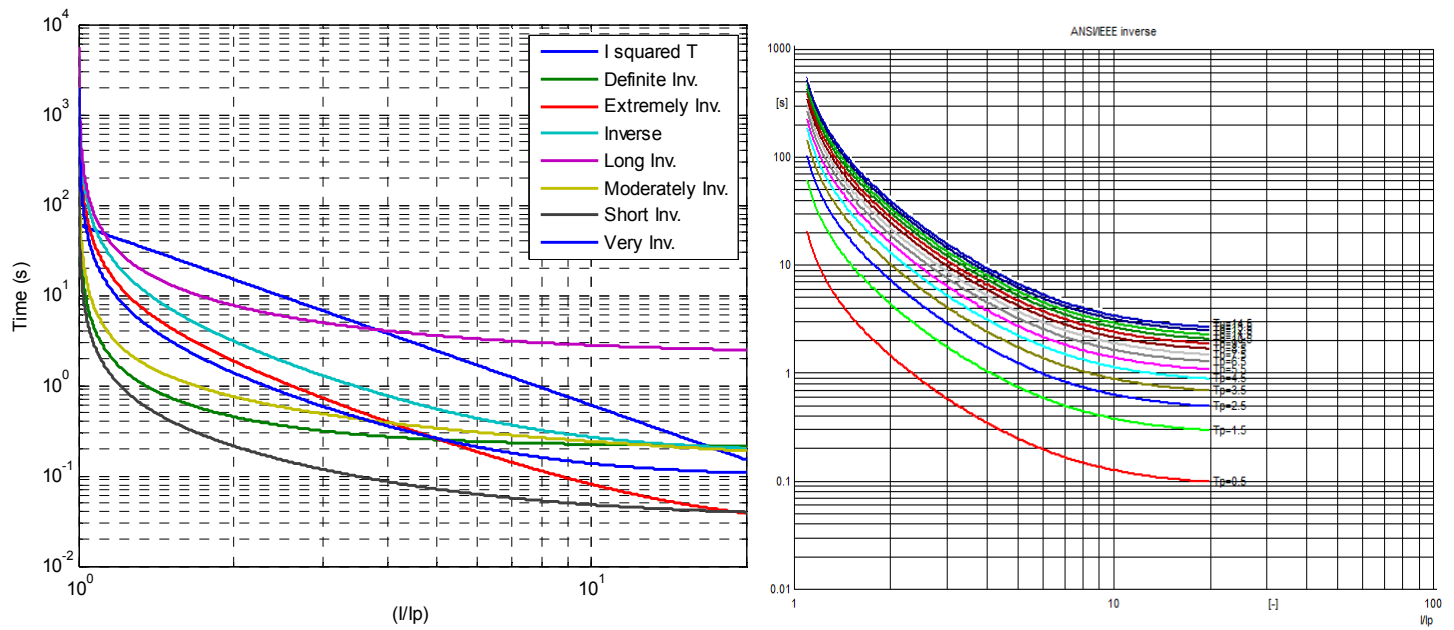

Figure 5.19: ANSI/IEEE characteristics for $\mathrm{Tp}=1$ and time inverse overcurrent characteristic for different $\mathrm{Tp}$

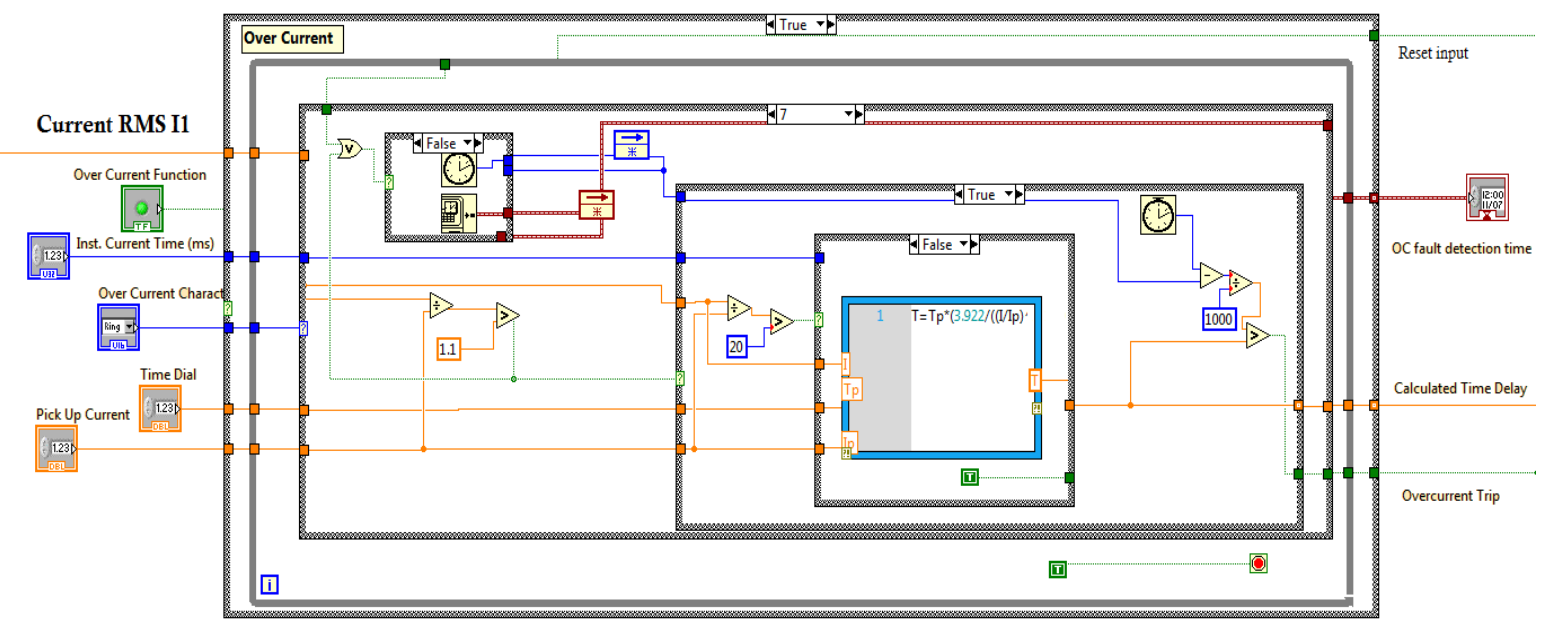

Figure 5.20: Over-current Function in LabVIEW real-time software

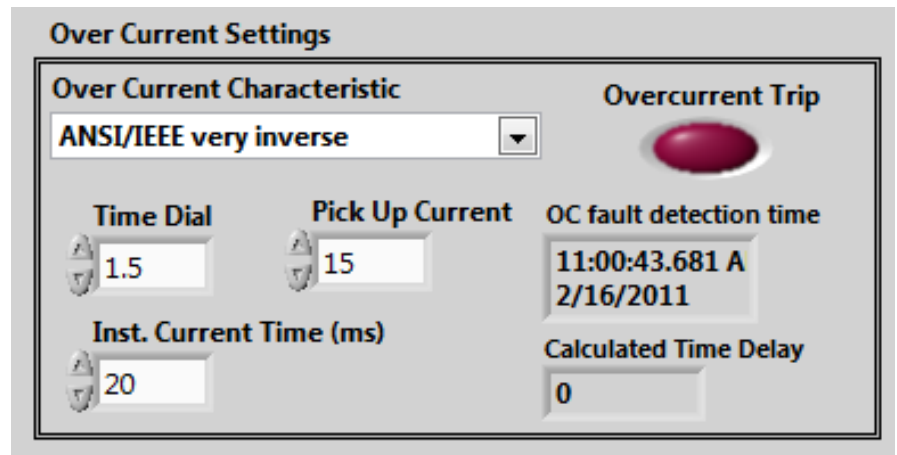

Figure 5.21: Over-current relay setting in LabVIEW real-time software 


\subsubsection{Distance Protection, MHO Characteristic}

Distance relaying should be considered when over-current relaying is too slow or is not selective. Distance relays are generally used for phase-fault primary and back-up protection on sub-transmission lines and on transmission lines where high-speed automatic reclosing is not necessary to maintain stability and where the short time delay for end-zone faults can be tolerated. Distance functions have been in use for many years and have progressed from the original electromechanical types through analog types and now up to digital types of functions. A simple mho distance function, with a reach of $\mathrm{Z}$ ohms, is shown in figure 5.22. This diagram is exactly equal to an R-X diagram except that all of the impedance vectors have been operated on by the current I. The mho function uses the current and voltage measured at the relay to determine if the apparent impedance plots within the mho characteristic. The determination was made by comparing the angle between the operating quantity $(\mathrm{IZ}-\mathrm{V})$ and the polarizing quantity $\left(V\right.$, where $\left.V=I Z_{f}\right)$. If the angle is less than or equal to $90^{\circ}$, then the fault impedance $Z_{f}$ plots within the characteristic and the function will produce an output. If the angle is greater than $90^{\circ}$, then $\mathrm{Z}_{\mathrm{f}}$ falls outside of the characteristic and no output will be produced. Assume that the angle of maximum reach $(\theta)$ and the angle of $Z L(\Phi)$ are equal. The key point to note in this phasor analysis (a convenient way to view relay performance) is the magnitude of the $\mathrm{IZ}-\mathrm{V}\left(\mathrm{V}_{\mathrm{op}}\right)$ phasor and its relationship to the $\mathrm{V}\left(\mathrm{V}_{\mathrm{pol}}\right)$ phasor. Operation will occur whenever $\mathrm{V}_{\text {op }}$ and $\mathrm{V}_{\text {pol }}$ phasors are within $90^{\circ}$ of each other and provided both $\mathrm{V}_{\text {op }}$ and $\mathrm{V}_{\text {pol }}$ are greater than the minimum values established by the sensitivity of the relay design. For the balance point fault, IZ-V is zero, therefore no operation occurs, which was expected. For an internal fault, IZ-V and V are in phase, therefore the function 
operates as expected. For the external fault, operation does not occur because IZ-V and V are $180^{\circ}$ out of phase. Observe that for the balance point fault, the $\mathrm{V}$ is exactly equal to IZ.

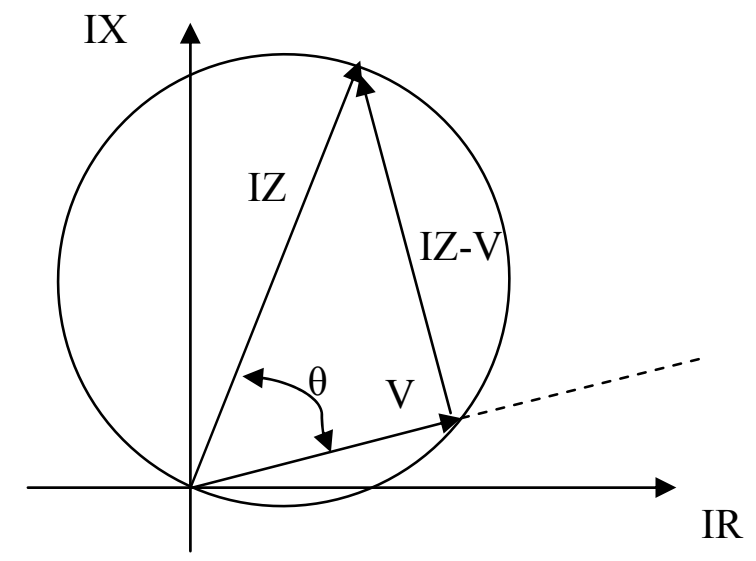

Figure 5.22: MHO impedance relay characteristic

The MHO distance relay has been implemented in LabVIEW environment by defining three different zones and the settings for zones according to figure 5.23 are the relay angle and each zone reach impedance, as well as time settings for each zone tripping. Figure 5.24 shows the block diagram of the VI (Virtual Instrument) which is developed in LabVIEW. The circle equation has been used for each zone and impedance location was compared with this equation for fault detection criteria. Hence, by settings all zones impedance and coordinate time between the zones, when fault occurs, the impedance seen by relay start to move inside the third zone and depends on fault impedance it may be placed in second or first zone. Therefore, time settings for each zone should be the faster tripping action for internal zones. The time-locking technique has been used for real-time implementation of decision making process in this relay function. 
Fault detection time for each zone is the output of this function for studying swing problem in the power system.

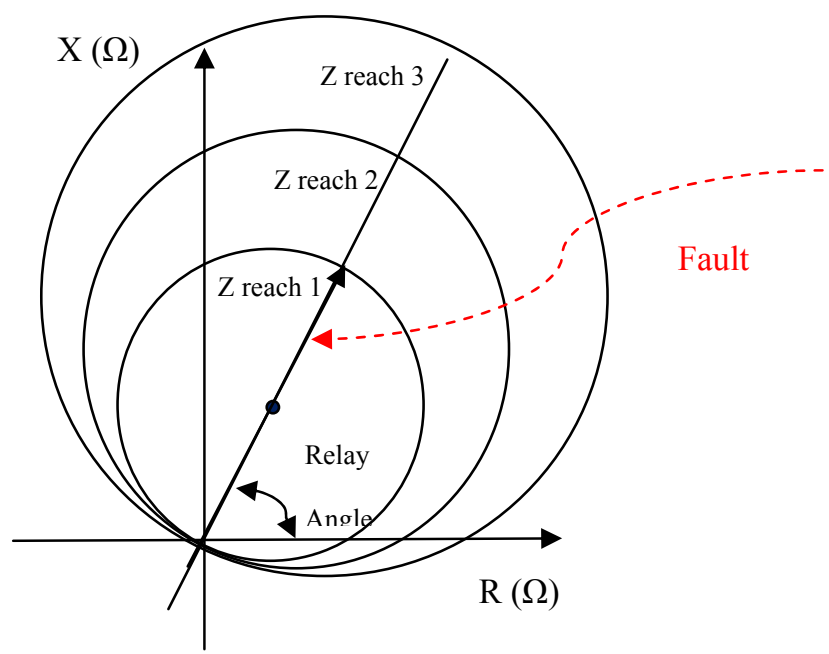

Figure 5.23: MHO zone definition and settings parameters

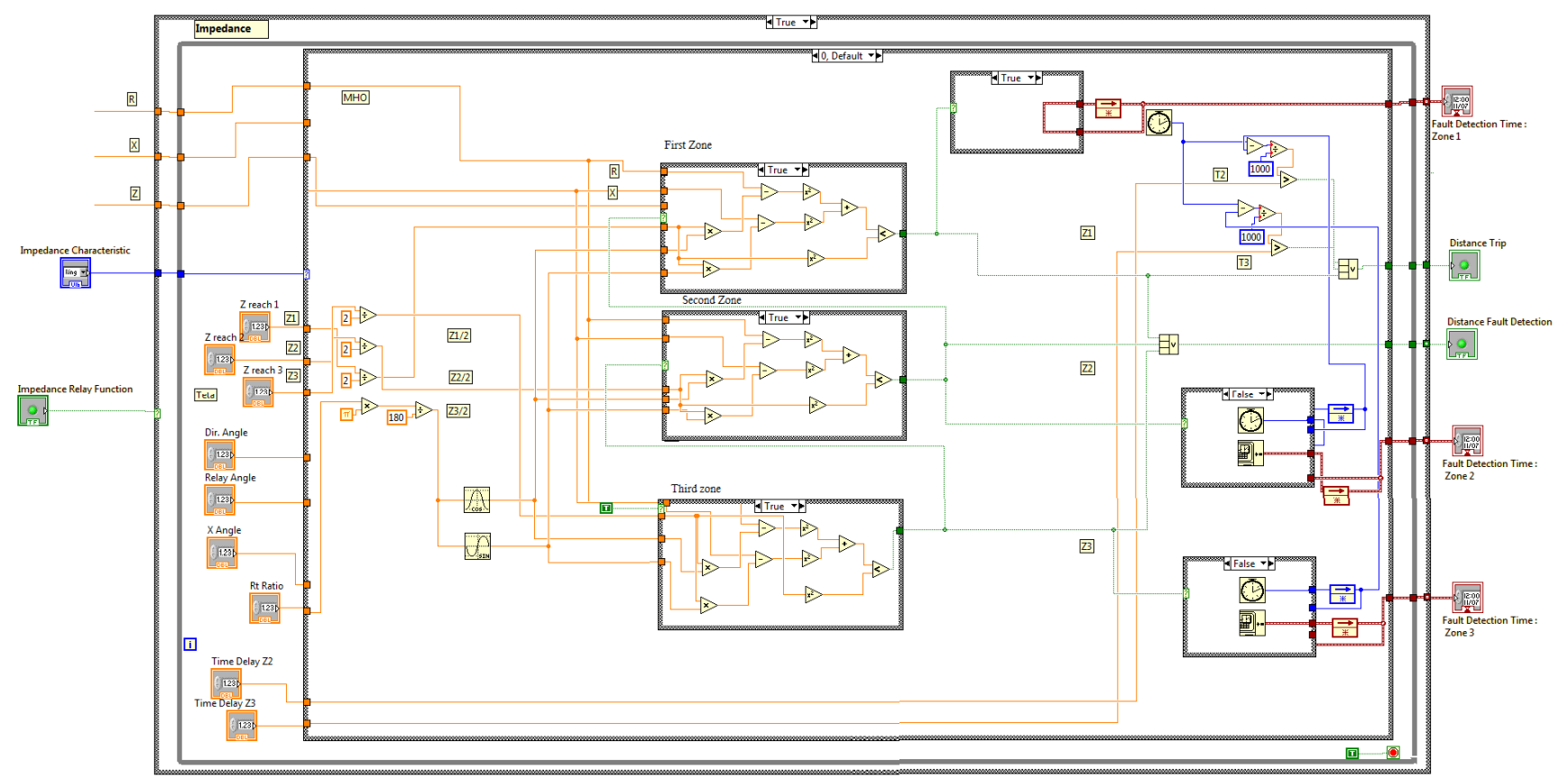

Figure 5.24: MHO Distance relay in LabVIEW real-time software

\subsubsection{Distance Protection, Polygonal Characteristic}

This form of polygonal impedance characteristic is shown in figure 5.25. The characteristic is provided with forward reach and resistive reach settings that are 
independently adjustable. It therefore provides better resistive coverage than any mhotype characteristic for short lines. This is especially true for earth fault impedance measurement, where the arc resistances and fault resistance to earth contribute to the highest values of fault resistance. To avoid excessive errors in the zone reach accuracy, it is common to impose a maximum resistive reach in terms of the zone impedance reach. Recommendations in this respect can usually be found in the appropriate relay manuals. Quadrilateral elements with plain reactance reach lines can introduce reach error problems for resistive earth faults where the angle of total fault current differs from the angle of the current measured by the relay. This will be the case where the local and remote source voltage vectors are phase shifted with respect to each other due to pre-fault power flow. This can be overcome by selecting an alternative to use of a phase current for polarization of the reactance reach line. Polygonal impedance characteristics are highly flexible in terms of fault impedance coverage for both phase and earth faults. For this reason, most digital and numerical distance relays now offer this form of characteristic. For this purpose, the polygonal characteristic is defined according to figure 5.26 by shown setting for each zone definition. The real-time software uses these settings to compare real-time impedance point with line equations which are defined by those setting in order to check placing in to zone. As same as MHO distance relay, time settings are available for second and thirds zone. First zone will trip as soon as fault inserted inside that region.

The whole front panel for distance relay is shown in figure 5.27. In left-top side user can select the MHO or polygonal characteristic for distance relay. Some indicators are used to show the fault detection and fault tripping for relay as well as time for placing 
fault inside the every zone. The middle part was used for inserting settings for MHO or Polygonal characteristic separately. The right side was for representing calculated impedance (magnitude and angle) and to plot it.

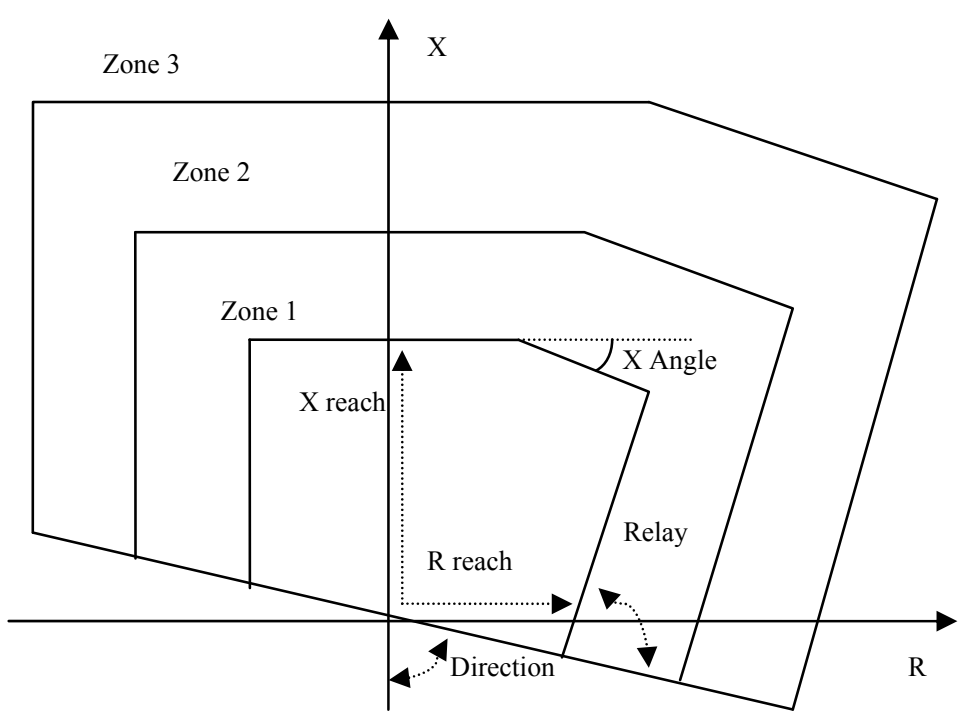

Figure 5.25: Polygonal zone definition and settings parameters

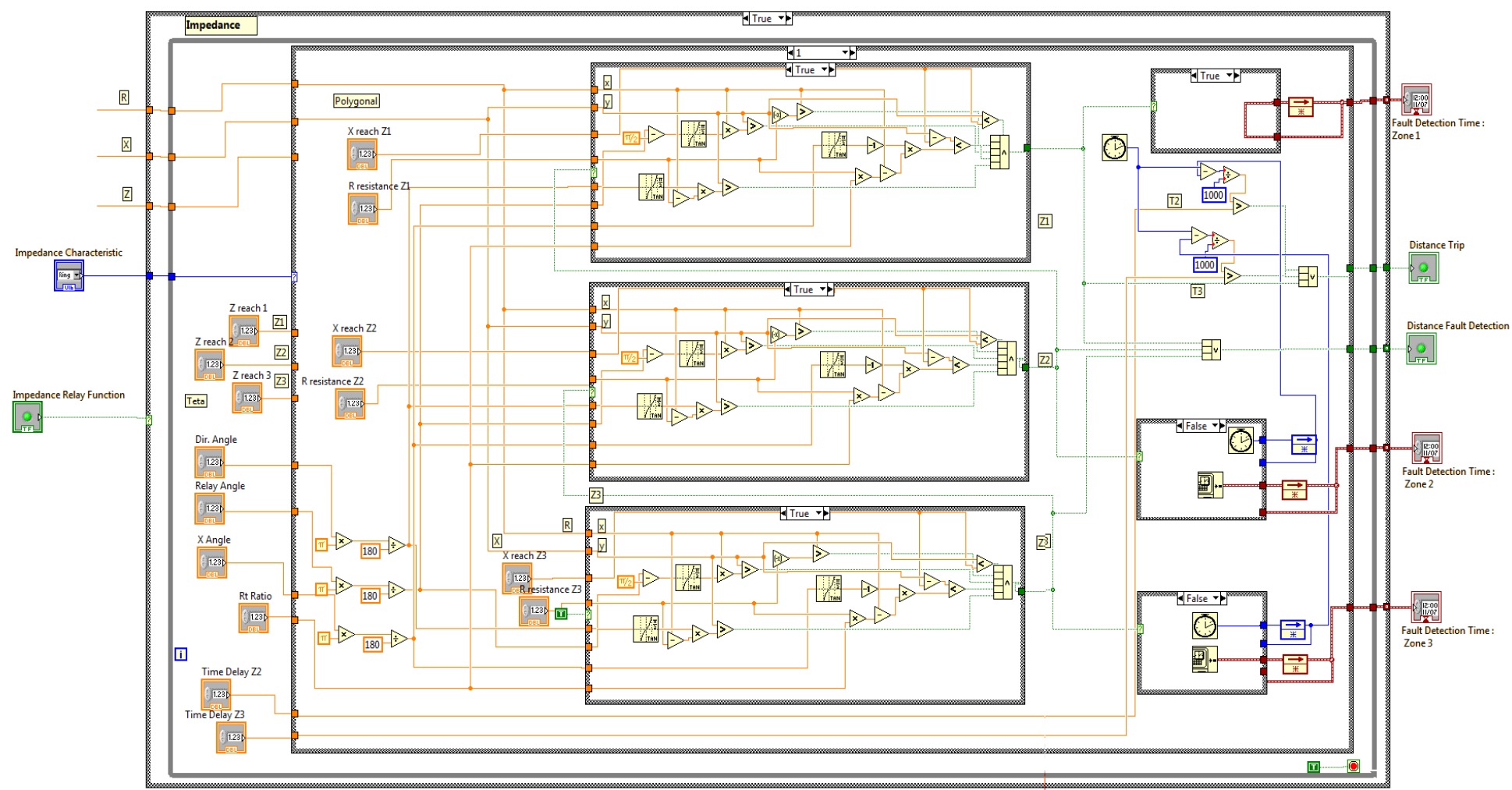

Figure 5.26: Polygonal Distance relay in LabVIEW real-time software 


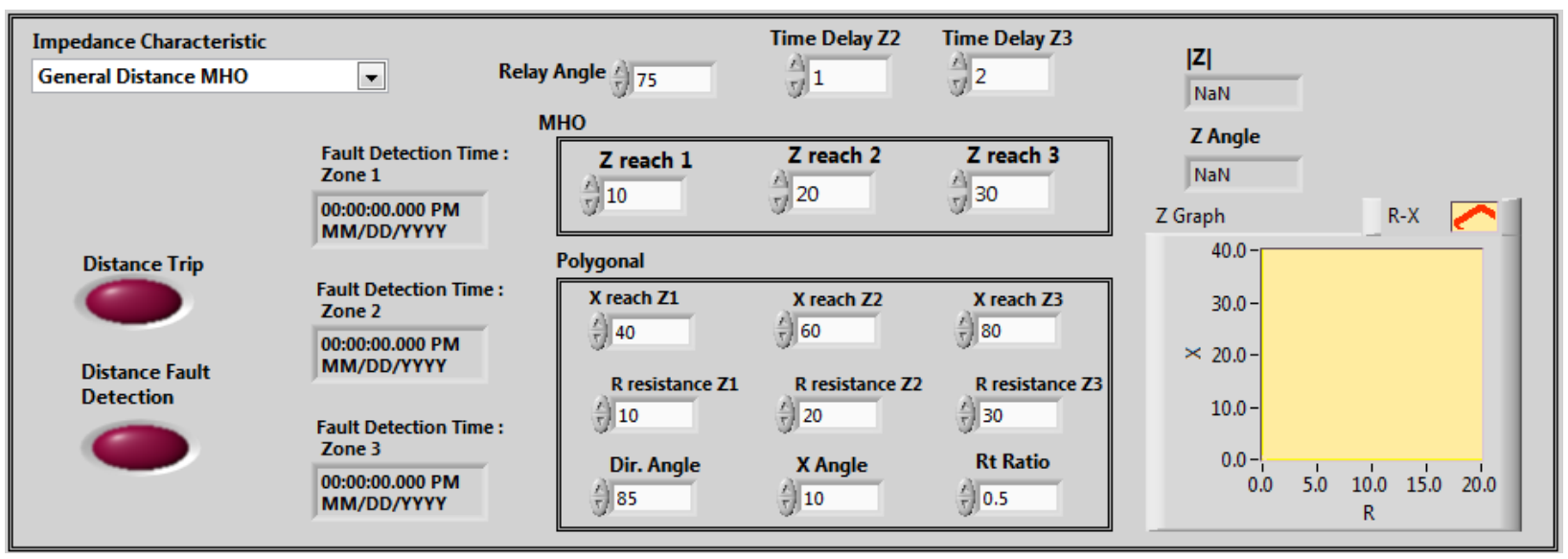

Figure 5.27: Distance relay setting in LabVIEW real-time software

\subsubsection{Voltage Unbalance Function}

In a three-phase system, voltage unbalance takes place when the magnitudes of phase or line voltages are different and the phase angles differ from the balanced conditions, or both. Voltage unbalance is defined as the ratio of the negative sequence voltage component to the positive sequence voltage component. The percentage voltage unbalance factor (\% VUF), or the true definition is given by:

$$
\% \text { Voltage Unbalance Factor }=\frac{\text { Negative Sequence Voltage Component }}{\text { Positive Sequence Voltage Component }} \times 100
$$

In addition we can check the zero sequence voltage magnitude for any ground fault which may cause increases on zero components.

$$
\% \text { Voltage Unbalance Factor }=\frac{\text { Zero Sequence Voltage Component }}{\text { Positive Sequence Voltage Component }} \times 100
$$

Application of the voltage unbalance relay is to protect three phase motors from the damage which may be caused by single phase operation. When one fuse blows in a three-phase supply to a group of motors, these motors will continue to run on single phase power. At this time the voltage unbalance will be small since the motors will maintain nearly full voltage across the open phase and a sensitive voltage unbalance relay 
was required to detect the single phase power so the relay was used to trip the supply breaker or to indicate an alarm.

Since the relay operates on a percentage of the unbalance in line voltages, it will detect a single-phase condition on light load as well as on heavy load. The unbalance voltage relay is usually connected to a bus and it will effectively protect a group of many motors when the open circuit is between source and the bus. Figure 5.28 shows the implementation of this function on LabVIEW. This Block takes the sequences components of voltage and after calculating unbalance factor compares it by inserted setting by user and makes decision for tripping action with considering time setting.

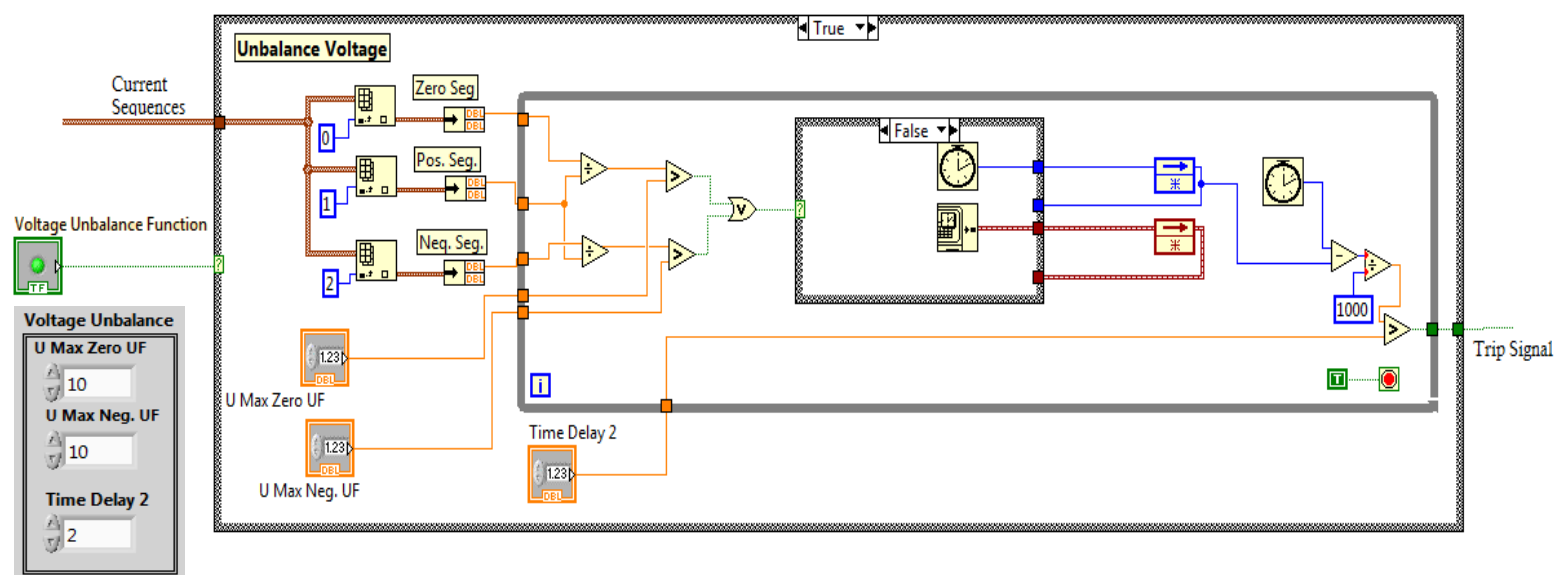

Figure 5.28: Voltage Unbalance Function in LabVIEW real-time software

\subsubsection{Current Unbalance Function}

The Current Unbalance Function monitors three-phase lines to detect an unbalanced current. It can also monitor three separate single phase lines for equal current levels. Line-voltage unbalances usually cause these line-current unbalances. In fact, current unbalance can be 20 times as high as the voltage unbalance. Unevenly distributed single-phase loads can cause the latter across a three-phase power system. In other words, instead of balancing phase-to-neutral loads evenly across all three phases, more are 
distributed onto one or two phases. The same goes for phase-to-phase single-phase loads. Instead of balancing the loads on an A-B, B-C, A-C basis, they are distributed unevenly on two pair of phases. Voltage and current unbalance can also result when using some types of transformer connections such as the open-delta connection, where two transformers are used to make a three-phase system. Any new digital motor control protection relays use a current unbalance trip and in some cases, the trip limit can be set as low as $5 \%$. The line-current unbalance also increases current harmonic distortion, which can overload building wiring and transformers. It may be seen excessive 3rd harmonics (normally associated with phase-to-neutral nonlinear loads) as one of the resulting consequences of line-current imbalance (a phase-to-phase nonlinear load).

Similar to unbalance voltage factor, the current unbalance factor is defined by following equations. This factor can detect any unbalance situation caused by unbalance faults to prevent any kind of damages.

$$
\% \text { Current Unbalance Factor }=\frac{\text { Negative Sequence Current Component }}{\text { Positive Sequence Current Component }} \times 100
$$

or

$$
\% \text { Current Unbalance Factor }=\frac{\text { Zero Sequence Current Component }}{\text { Positive Sequence Current Component }} \times 100
$$

Figure 5.29 shows the current unbalance function implemented in LabVIEW and the settings of acceptable limits for unbalance factors. The time delay is also available for users. 


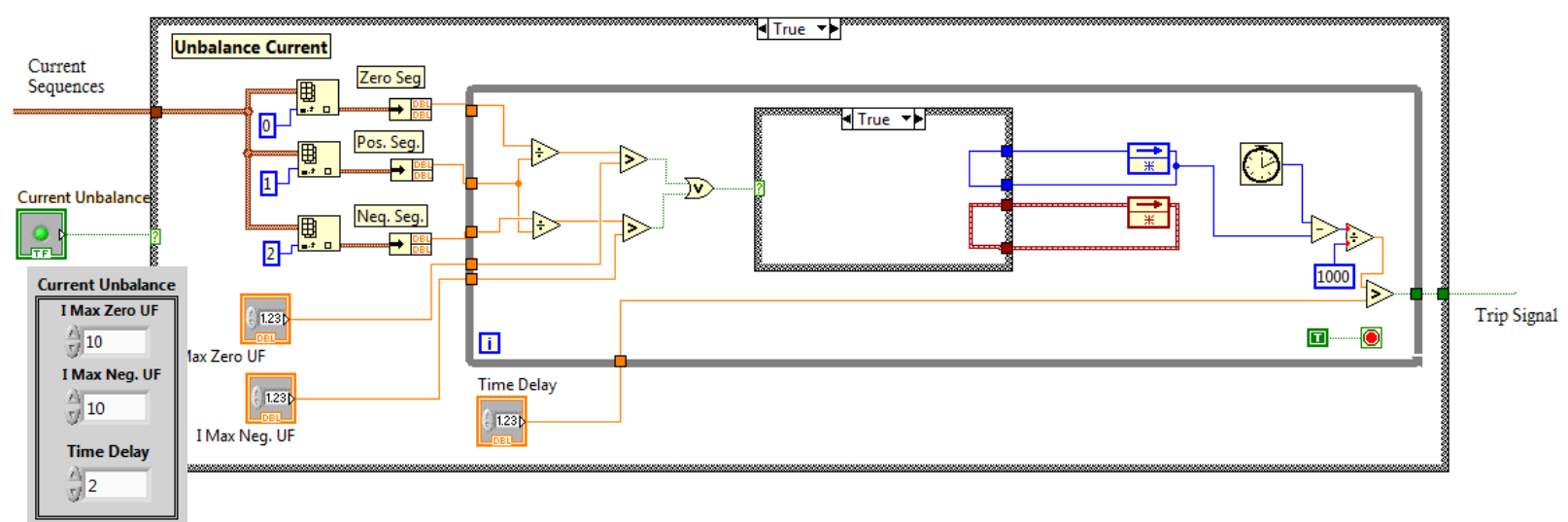

Figure 5.29: Current Unbalance Function in LabVIEW real-time software

\subsubsection{Inverse Power Flow Function}

This function calculates actual power flow and protects branches against reverse power flow (under power flow), the measurement are implemented for active power with a specific time setting. The minimum active power and time delay, are the settings for this relay function according to figure 5.30. The applications of this function are for unwanted power flow direction which system couldn't tolerate and also for reverse power flow which may occur under fault conditions.

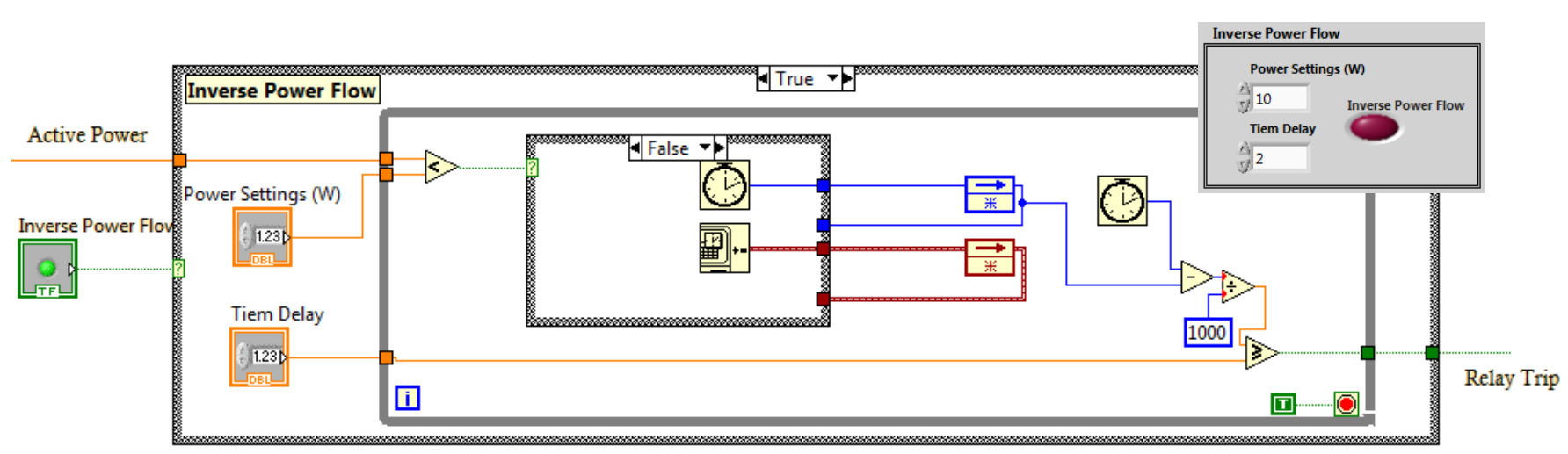

Figure 5.30: Inverse Power Flow Function in LabVIEW real-time software 


\subsection{Test and Verification of Virtual Protection System in Test-bed}

All mentioned functions are implemented in LabVIEW environment as a generic relay model. This relay can be operated by real-time data and at the speed of communication system. In the laboratory test system, the relay speed will be as fast as DAQ's speed used to gather data from the secondary side of PTs and CTs. Figure 5.31 represents the front panel of this digital generic relay for testing the idea of virtual protection system. Technically, all available relays model can be implemented by their functions and characteristics as a library inside the VPS database system.

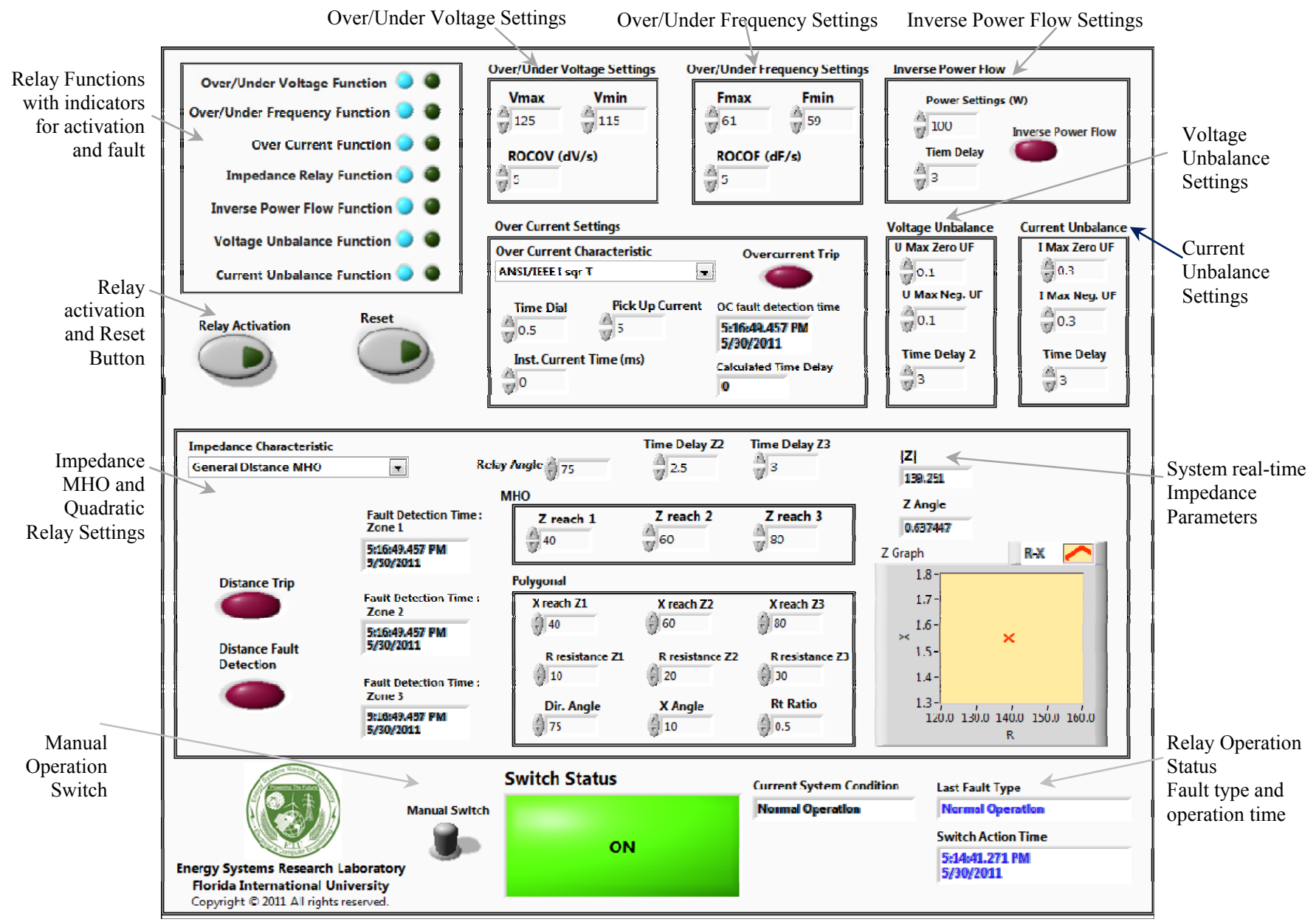

Figure 5.31: Digital generic relay model implemented at real-time software 
Hence, the system designer can add the VPS model of any realistic relay and monitor protection system behavior by applying PMUs data in the virtual environment.

In order to test this relay two different experiment including single phase short circuit and single phase outage has been carried out on the system which is described at section 4.12 and has been shown in figure 4.6. All fault conditions are placed on motor load terminal. In one case the single phase fault had been occurred in motor terminal and the PMU at that terminal sends the fault data to virtual relay at the control center. The active and reactive powers as well as frequency changes are plotted in figure 5.32 respectively. The currents and voltages are changing during this fault and cause the considerable changes in active and reactive powers. The generic relay will trip this situation by its own available functions which are visible in figure 5.33 .

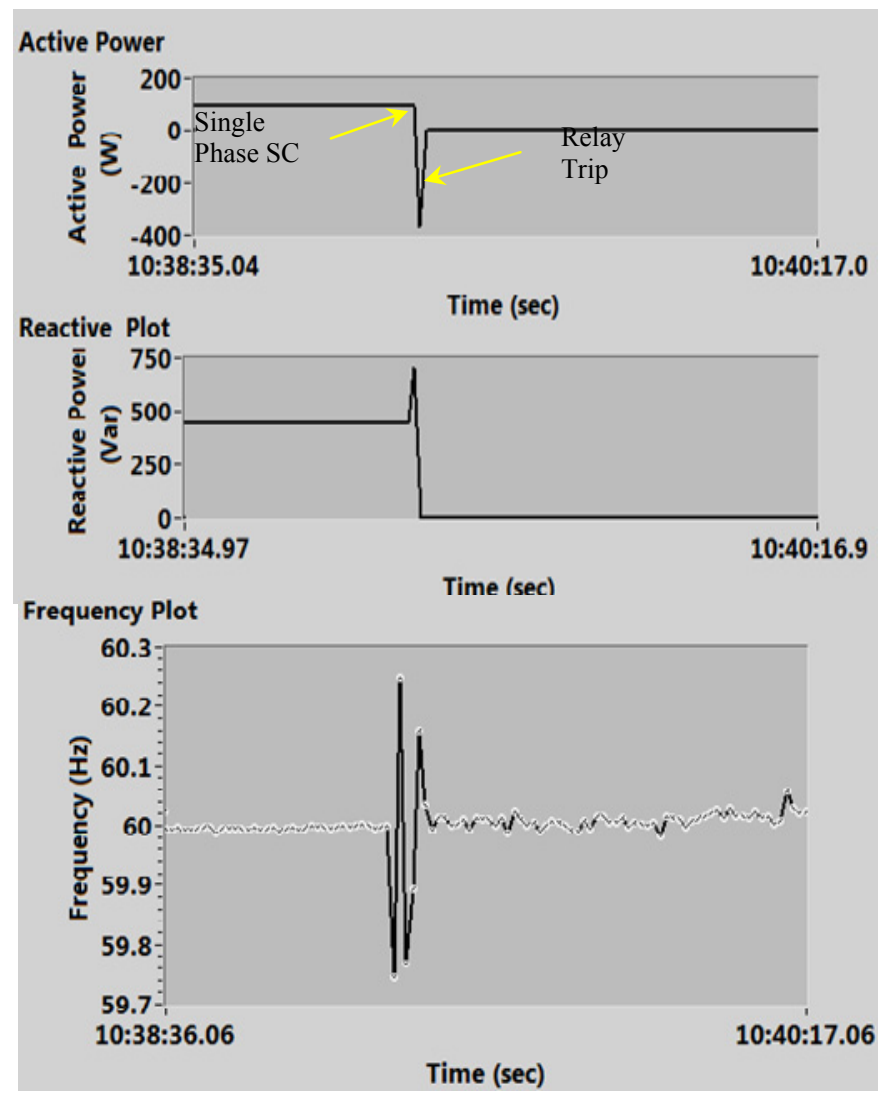

Figure 5.32: Single-phase fault at motor terminal and relay tripping action 
In another case one of the phases of the induction motor has been disconnected in two stages: relay is inactive and when it is active. Figure 5.34 shows this situation at active and reactive powers and frequency calculated in PMU. The unbalance current function will be the first function which will disconnect the relay.

These two cases are two applicable tests that we can carry out at lab scale for testing the generic relay model which was developed for monitoring and protection schemes. Technically, in the laboratory environment we just can perform experiments involving protection devices which are not harmful for human health and equipment safety and reliability. Therefore, the short circuits and other risky faults are not experimentally tested and they are just verified by simulated signals in design procedure.

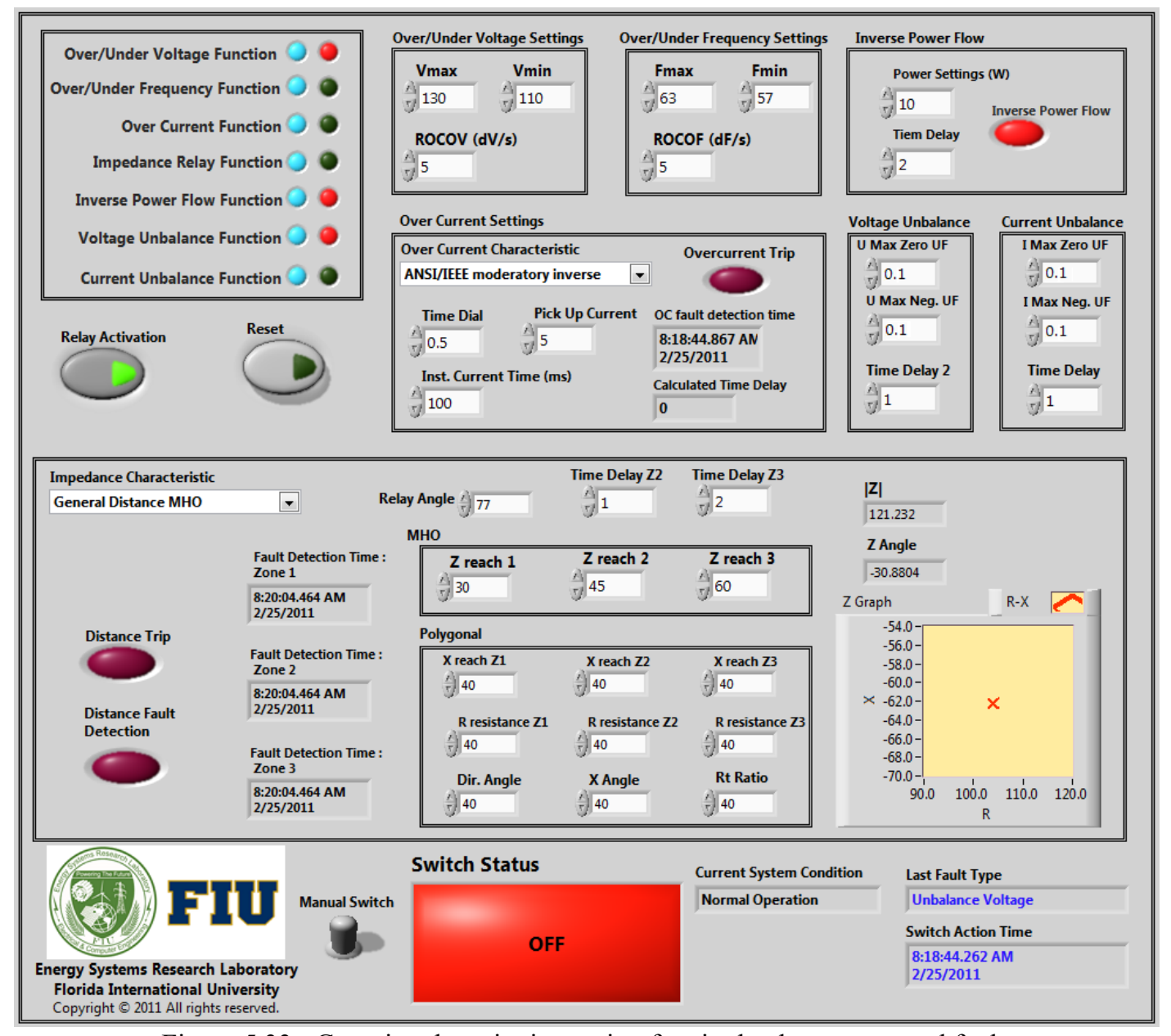

Figure 5.33: Generic relay tripping action for single phase to ground fault 


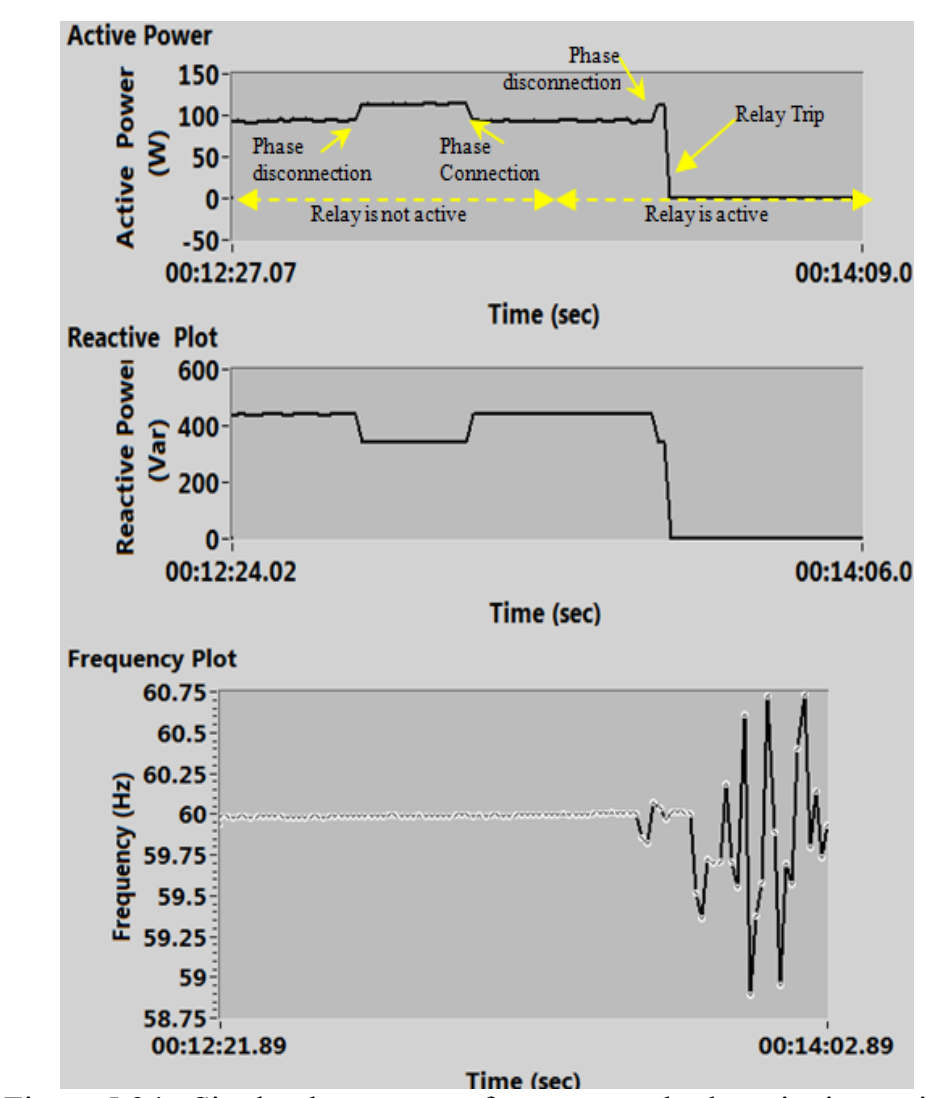

Figure 5.34: Single-phase outage for motor and relay tripping action

Figure 5.35 shows the application of this VPS for power system wide area monitoring, protection and control system.

This system is capable for applying online setting of protection devices. The protection coordination settings in a large area network are completely dependent on the topology and system status which are varying frequently. For example the application of distributed generation, whose generation depends on energy availability such as photovoltaic and wind power, may cause different operational settings for relays connected to the same network. This system could detect the grid status and will run protection coordination software to achieve proper settings for relays. Finally, it will apply new settings by real-time software and communication networks to the selected system protection devices. 


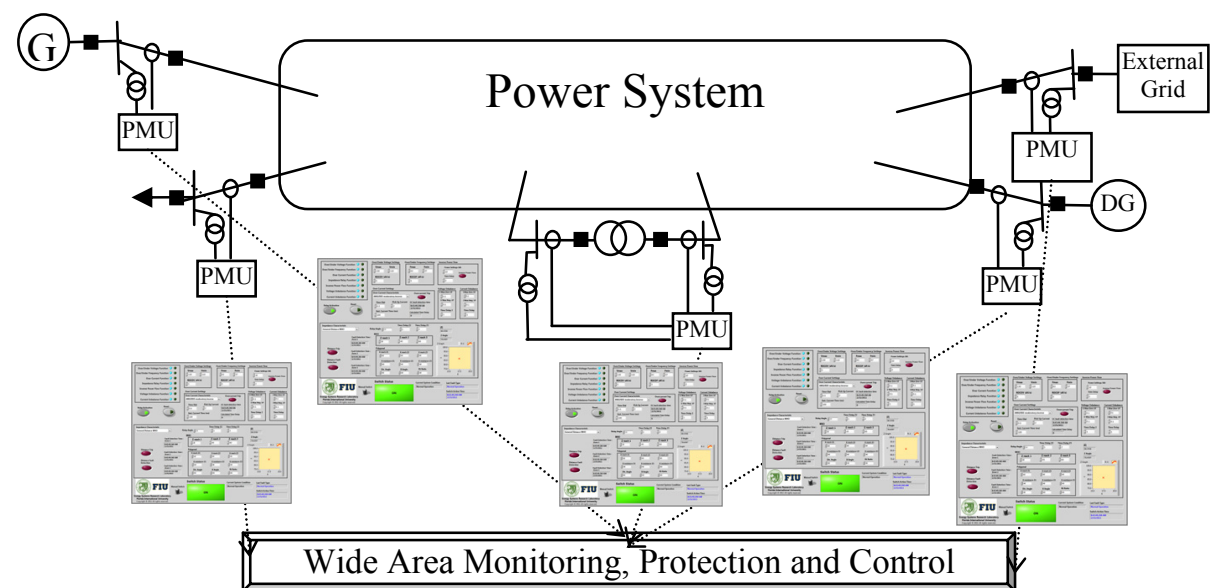

Figure 5.35: Using generic relay for system wide area monitoring, protection and control

In this research the Virtual Protection System was proposed for wide area system protection. This system was used to control power system in a manner to heal any abnormal conditions that may cause damage, outage and perhaps blackout. The VPS was developed as a generic digital relay with variety of protection functions that uses PMUs current and voltage outputs to simulate power system behavior in a virtual environment. This gives us some information about upcoming faults or system states moving to abnormal situation. Proper control action for self-healing can be developed in a control system to maintain system stable conditions with minimum outages. A laboratory based power system test-bed was developed to test events and verify the VPS idea in a large power system. Applications of VPS was discussed for modern power system for predicting upcoming events, detecting blackout and cascade events and applying online settings to protective relays.

\subsection{Experiments for test of Wide Area Protection System in Test-bed}

Since all the switching control and relay models were implemented inside the real-time environment, the wide area protection system was designed to test the new protection schemes for wide area systems. 
For the test-bed which has been introduced in figure 5.1, an experiment was performed involving the outage of the slack generator G1. The event was the disconnection of the slack generator, G1, which will cause frequency drop of the whole system since generation and demand balance is no longer maintained. The frequency functions of all generators relays are activated. For G2 and G3 frequency settings considered $59 \mathrm{~Hz}$ and for G4 is $58 \mathrm{~Hz}$. So, whenever the frequency drops, the monitoring system will calculate the frequency and the relay function will detect the situation and will send the trip signal to the breaker right away. Figure 5.36 shows this procedure with all generators' relay trip signals and breaker one, respectively in left and right plots. Here, G1 was disconnected at 2:26':22”.245 and G2, G3 and G4 relays are detected at $2: 26^{\prime}: 23^{\prime \prime} .032,2: 26^{\prime}: 23^{\prime \prime} .012$ and $2: 26^{\prime}: 23^{\prime} .567$, respectively. Because of the frequency setting for G4, it detects the event with a larger delay. Figure 5.37 presents the active power change as well as frequency drop for this event for all generators. The generator's G1 frequency was returned to the no load frequency after disconnection from the grid and the other generators' frequency started to drop until the relays trip them from the grid. The remaining generators take the load share of the disconnected generators. For instance, after disconnection of G1 and then G2 and G3, suddenly, G4 which was producing $250-\mathrm{W}$ in normal condition takes the active power load of whole system (1300 Watt) and leads to large drop of frequency $(48-\mathrm{Hz})$. A wide area protection algorithm was also implemented for this case, which is the disconnection of all generators when G1 is disconnected from the grid right away. In this case we don't need to worry about the frequency relays settings and their operation delay. Consequently, all the power system data are available by the implemented PMUs for monitoring the system and hence any 
kind of intelligent and smart wide area protection system integrated with system controllers can be developed in real-time to enhance power system operation.

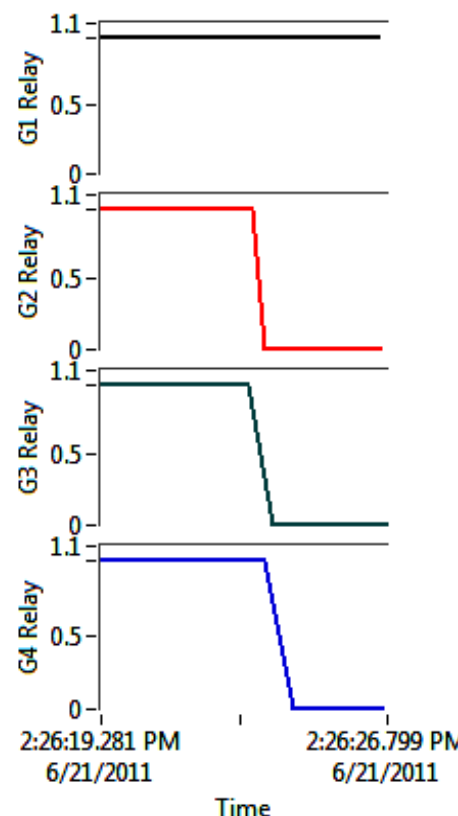

(a)

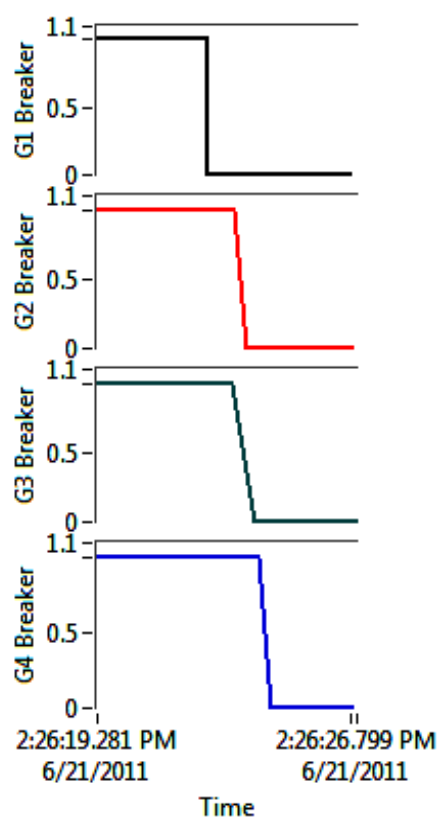

(b)

Figure 5.36: (a) Relays trip signal and (b) breaker status of all generators during G1 outage

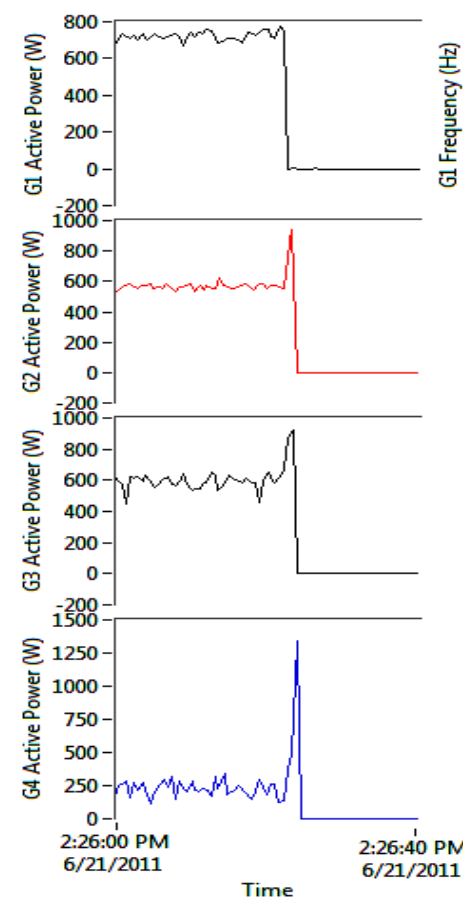

(a)

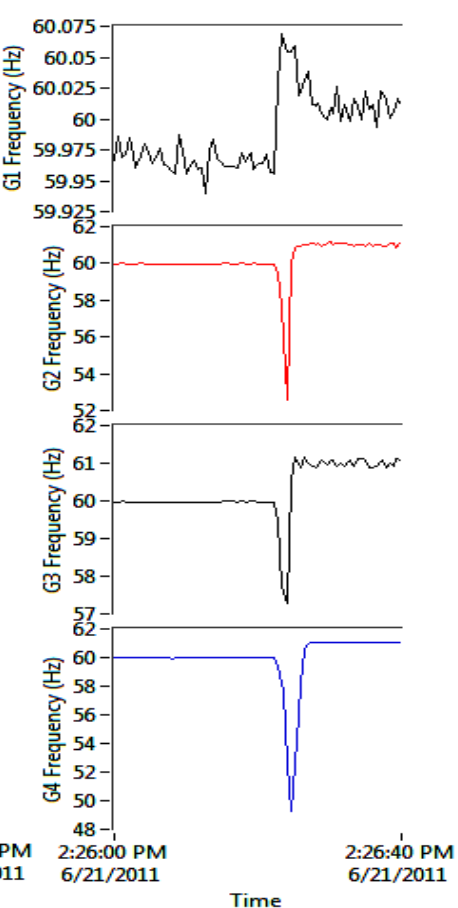

(b)

Figure 5.37: (a) Active power and (b) frequency of all generators during G1 outage 
This system can be used for studying integrated wide area control and protection system to monitor the system status for abnormalities such as over/under voltage, overloads of equipments and any other conditions. In the implemented example, when the loadings of a power system line increases, the relay indicated the system moving condition to the fault situation which may be disconnected by available protection devices. Hence by setting the protection relay under the settings of the physical relay, the control scheme can retrieve the normal status by proper controlling action. This case may be the changing of system topology or power dispatching in alarms in a control center, self healing strategies can maintain the system continuous operation with appropriate control scheme.

The developed system can be used for cascaded failures detection and applying proper remedies on the power system. Following a disturbance, one or more components overload and hence fail. The equilibrium of the load flow will consequently change and the load will then be redistributed to other normal components and this makes additional load transfer to other elements. Thus, a cascading failure is triggered by the overload failures and it cause network's collapse resulting in a blackout. The PMUs data can be used to follow the phase angle of each bus to detect the system failures which may cause cascaded events. In this process, we need to detect upcoming faults by network data and analyze the network reaction to this outage by some algorithms such N-1 Contingency, Fault calculation, OPF, etc. Therefore, the online calculation software is used to analyze system situation and will be presented in next chapters. 


\section{CHAPTER 6}

\section{DESIGN AND CONTROL OF HYBRID AC/DC SYSTEMS}

\subsection{Introduction}

DC distribution systems have been proposed to address the challenges of increased energy demand. These systems can also be used to improve or maintain system reliability. DC microgrids and DC distribution systems succeeded to gain popularity and reacquire the interest of several researchers and industry entities. High penetration of stationary renewable energy sources yielding DC output, increased importance of storage elements and increased number of electronic loads, machine drives and other loads operating with DC input are all essential reasons for this reconsideration of DC networks. Moreover, several systems are currently employing DC distribution systems already. For instance, in systems that require high reliability and are involving a big number of electronic loads such as data centers, DC provides a more efficient solution for electric power distribution. Another important example is shipboard power systems with implemented medium voltage DC distribution, zonal DC distribution or other types of DC distributed architectures for power delivery on board. This increased importance of DC distribution systems have encouraged researchers to investigate issues related to them. The hybrid system was discussed in chapter II of this dissertation and its advantages and disadvantages were presented as well. In this chapter, we will focus on the implementation of a DC microgrid setup in test-bed as a Hybrid AC/DC microgrid. This system was implemented and presented in details in [135]-[140] including the results of test and verification for the operation of DC microgrid. 


\subsection{Bus and DC Microgrid}

The AC side of the system was presented in chapter 3 including the AC sources as synchronous generators and several types of loads including passive impedance and motor loads. The DC side of the grid was used to connect the various renewable energy sources in addition to the battery storage system and other dc loads. The energy available from renewable sources on the dc side of the network should serve not only local loads on the de grid, but also loads demands on the AC side. Power sharing can be used to mitigate fast load fluctuations and maintain high power and voltage stability levels on the AC side. Therefore, a bi-directional AC-DC/DC-AC converter was used to allow bidirectional power flow between the AC and DC sides. Hence, the dc micro grid shown in figure 6.1 in detail consists of the components which will be present here.

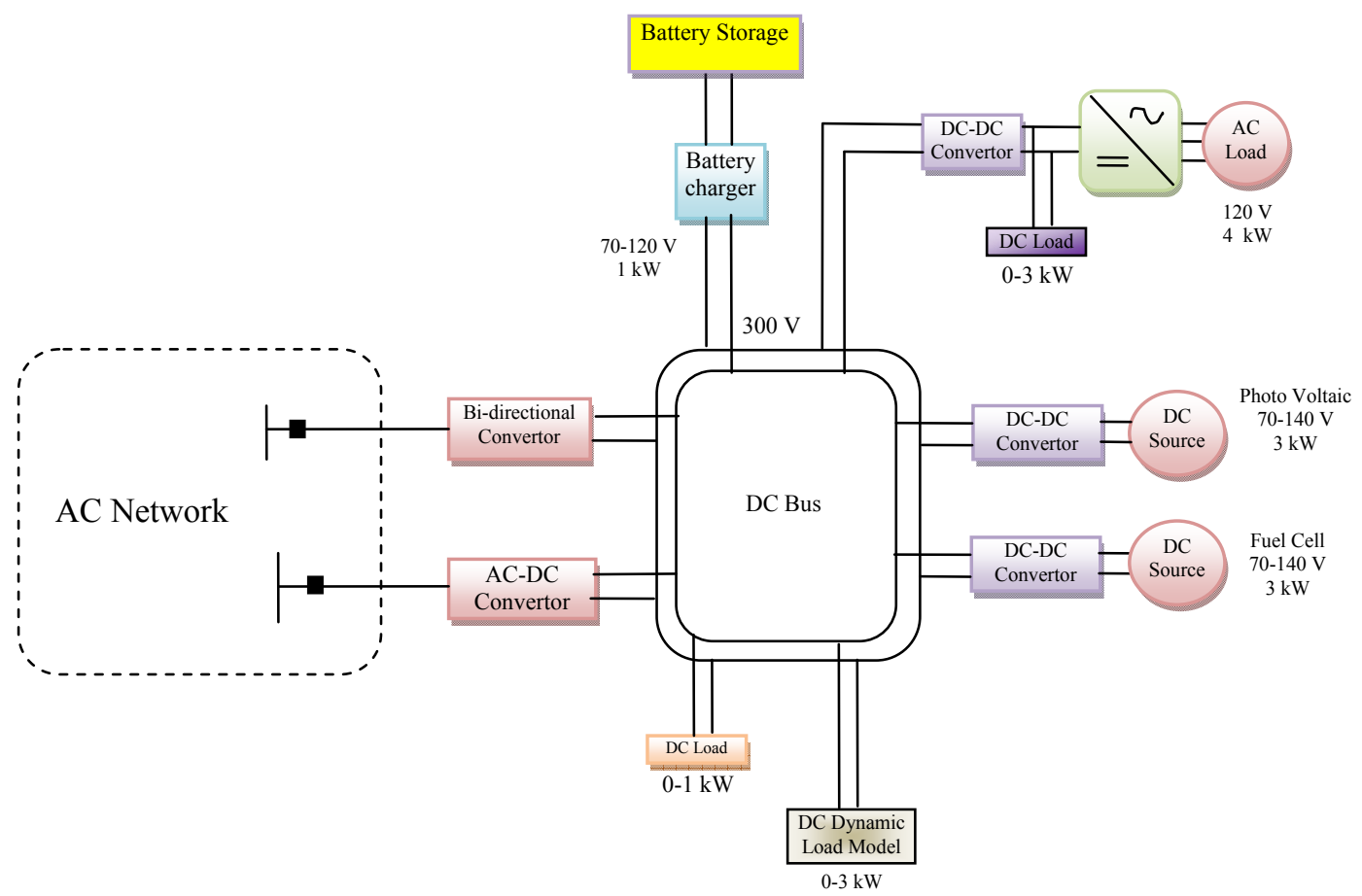

Figure 6.1: DC network schematic interconnected to AC side and DC source and load emulators 


\subsubsection{Photovoltaic (PV) Emulator}

A 5-kW programmable DC Power supply was used to emulate a typical I-V characteristics curve. The programmable power supply allows emulating different I-V characteristic curves in the form of lookup tables. Moreover, it allows including the effect of other parameters such as environmental conditions i.e. ambient temperature and solar insolation.

The V-I characteristics of a PV cell is modeled by the formula given in (6.1),

$$
I=I_{L G}-I_{O S}\left(e^{\frac{q}{A K T}\left(V+I R_{s}\right)}-1\right)-\frac{V+I R_{s}}{R_{s h}}
$$

Where:

$\mathrm{I}_{\mathrm{LG}} \quad$ Light generated current $[\mathrm{A}]$

I $\quad$ Reverse saturation current [A]

q Electronic charge [C]

A Dimensionless factor

K Boltzmann's constant [m2kg/ks2]

$\mathrm{R}_{\mathrm{s}} \quad$ Series resistance of the cell [ $\left.\Omega\right]$

$\mathrm{R}_{\mathrm{sh}} \quad$ Shunt resistance of the cell $[\Omega]$

$\mathrm{T} \quad$ Ambient temperature in Kelvin [ $\left.{ }^{\circ} \mathrm{k}\right]$

\subsubsection{Fuel Cell (FC) Emulator}

A programmable DC power supply is programmed to emulate Solid Oxide Fuel Cells (SOFC) characteristics. The DC power supply can be programmed with a lookup tables representing the relation between the voltage and current or voltage versus time. For instance, in order to examine the robustness of the DC-DC boost converter interfacing fuel cells energy into the grid, the voltage profile shown in Figure 6.2 was programmed. The purpose of sharp and sudden changes in the voltage value is to examine the system and its components under severe conditions. The results of the experiment are published by Ahmed, et. al [138]. 


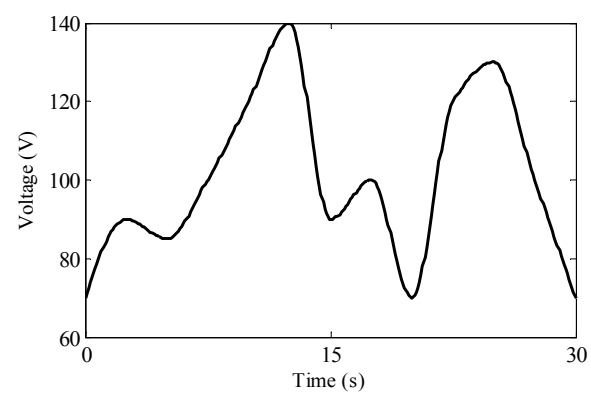

Figure 6.2: Output voltage from the fuel cell emulator

\subsubsection{Controlled DC-DC Boost Converter}

The system contains two controlled DC-DC boost converters operating as interfaces between different renewable energy sources and the DC grid. The first DC-DC boost converter is used to integrate the power generated by the PV emulator to the system. This converter is operating in the maximum power point tracking (MPPT) mode. The other converter is used to integrate the FC energy to the system and is operating in a current control mode. These converters are designed to receive a variable input voltage ranging from 70 to $140-\mathrm{V}$ and integrate its power into the DC bus at $300-\mathrm{V}$. The power rating of each is $3 \mathrm{~kW}$.

In the case of the DC-DC boost converter connected to the PV source, the voltage to be controlled is on the input side of the converter. The PV can be equivalent to a current source in parallel with a resistor using Norton's theorem. Hence, the small signal input voltage to duty cycle transfer function used to control this converter is given by:

$$
G_{p v}=\frac{\widetilde{v}_{p v}}{\widetilde{d}}=-\frac{\left(\frac{R_{g} V_{d c}}{L_{p v}}\right)\left[s+\frac{1}{C_{p v} R_{p v}}\right]}{s^{2}+\left(\frac{R_{p v}}{L_{p v}}\right) s+\left(\frac{1}{L_{p v} C_{p v}}\right)}
$$

$\widetilde{v}_{p v} \quad$ The small signal of the converter input voltage [V] 
$\widetilde{d}$

The small signal of the duty cycle

$V_{d c} \quad$ The large signal of the DC bus voltage [V]

$R_{p v} \quad$ Photovoltaic equivalent resistance [ $\left.\Omega\right]$

$C_{p v} \quad$ Photovoltaic array equivalent capacitance [F]

$L_{p v} \quad$ Converter inductance $[\mathrm{H}]$

This transfer function has been used to tune the parameters of the PI controller which is used to control the converter as shown in figure 6.3.

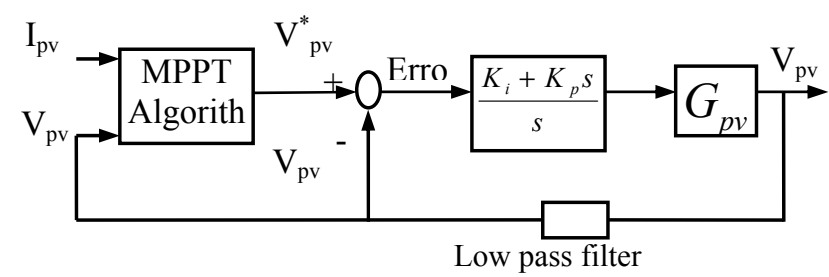

Figure 6.3: A block diagram of the DC-DC boost converter controller

\subsubsection{Bi-directional DC-DC battery charger/discharger}

The smart power system presented here is also equipped with a battery bank consisting of 12 lead acid batteries connected in series with a 12-V output each. The battery bank is used as the main energy storage of the system.

The output voltage of the battery storage system is not constant depending on the state of the charge of the battery. Moreover, the amount of charge or discharge from the battery should be controlled depending on the energy management algorithm used for system operation. Thus, a 1-kW controlled bi-directional DC-DC converter was designed and implemented in the system. In the charging mode, the transfer function of the output voltage to the duty cycle (GBC) is as given by (6.3): 


$$
G_{B C}=\frac{\widetilde{v}_{o}}{\widetilde{d}}=\frac{\frac{V_{d c}}{D}}{L_{b a t t} C_{\text {in }}\left[s^{2}+\left(\frac{1}{R C_{\text {in }}}\right) s+\left(\frac{1}{L_{\text {batt }} C_{\text {in }}}\right)\right]}
$$

Whereas, the ratio of the output voltage to duty cycle transfer function in the discharging mode (GBD) is given by,

$$
G_{B D}=\frac{\widetilde{v}_{o}}{\widetilde{d}}=\frac{V_{d c}\left[\frac{L_{\text {batt }}}{R(1-D)^{2}}\right] s}{L_{\text {batt }} C_{\text {out }}\left[s^{2}+\left(\frac{1}{R C_{\text {out }}}\right) s+\left(\frac{(1-D)^{2}}{L_{\text {batt }} C_{\text {out }}}\right)\right]}
$$

Where:

$\widetilde{v}_{o} \quad$ The small signal of the converter output voltage [V]

$\tilde{d} \quad$ The small signal of the duty cycle

$\mathrm{V}_{\mathrm{dc}} \quad$ The large signal of the DC bus voltage [V]

D The large signal of the duty cycle

$\mathrm{L}_{\text {batt }}$ Converter inductance $[\mathrm{H}]$

$\mathrm{C}_{\text {in }} \quad$ Converter capacitance on the DC bus side $[\mathrm{F}]$

$\mathrm{C}_{\text {out }} \quad$ Converter capacitance on the battery side $[\mathrm{F}]$

$\mathrm{R} \quad$ DC Load Resistance $[\Omega]$

These two transfer functions for the converter in the charging and discharging modes were used to design the PI controller used to control the charge/discharge process of the battery as shown in figure 6.4. The PI controller parameters were set as follows, $\mathrm{K}_{\mathrm{pC}}=0.0035, \mathrm{~K}_{\mathrm{iC}}=0.3, \mathrm{~K}_{\mathrm{pD}}=0.0035$ and $\mathrm{K}_{\mathrm{iD}}=0.0035$. Moreover, the pulse width modulation technique at a switching frequency of 5-kHz was used as a switching strategy for the bi-directional charger/discharger. More details of the design process and the maximum power point tracking algorithm are presented in [135]. 


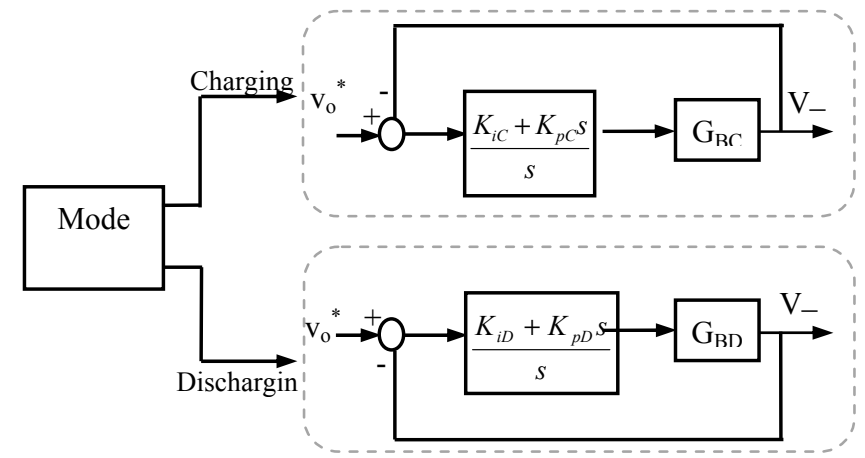

Figure 6.4: A block diagram of the Bi-directional battery charger controller

\subsubsection{Three-phase controlled AC-DC rectifier and bi-directional AC-DC/DC-}

\section{AC converter}

The voltage controlled rectifier was used to regulate the voltage level on the DC side of the network. This rectifier allows power flow from the AC side to the DC. The converter was controlled using a voltage decoupling control technique that allows full control of active and reactive power independently. Hence, the converter is operating at a unity PF. Sinusoidal pulse width modulation (SPWM) technique is used for switching. The complete details of the design and implementation of this converter can be found in [136].

Moreover, a current-controlled bi-directional converter was also designed. The same voltage decoupling control technique was utilized for this converter. Hence, any desired amount of active or reactive power can be transferred from the DC microgrid to the main AC grid, which allows the DC microgrid to supply active power, act as a reactive power compensator or supply active and reactive power simultaneously if required by the wide area control technique implemented. The design and implementation procedure of this converter are given in [137] and the focus is on presenting the operation of the developed bi-directional converter at unity power factor. However, it is proved to 
operate effectively at any power factor. Figure 6.5 shows the response of the bidirectional converter to a step change in the reactive power from $0.3-\mathrm{kVAR}$ to $1-\mathrm{kVAR}$. It can be seen that the converter succeeds in transfering the desired amount of reactive power and that the transient period does not exceed $1 \mathrm{~ms}$. The phase difference between the voltage and current waveforms is 90 degrees corresponding to the setting of the active power to zero. The DC voltage regulated by the controlled rectifier is hardly affected by this change in the reactive power flow. The values of the parameters for all the components in the DC microgrid are given in table 6.1.

\subsubsection{Single phase DC-AC inverter}

This voltage-controlled inverter is used to connect different sensitive AC loads to the DC bus. This inverter has a power rating of $4-\mathrm{kW}$ and is designed to output a constant 120-V. The SPWM technique at 4-kHz switching frequency was used as a switching strategy. Moreover, the PI controller was used to control the output voltage.

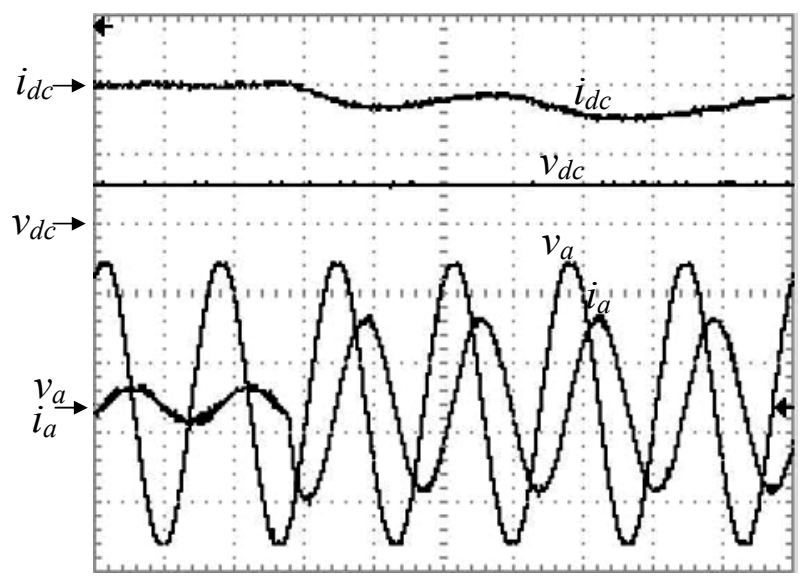

Figure 6.5: Operation of the bi-directional converter as a reactive power compensator, a step change in the reactive power reference from $0.3 \mathrm{KV}$ ar to $1 \mathrm{KVar}$ is applied (a) DC current, idc(4 A/div, $10 \mathrm{~ms}$ ). (b) DC voltage, vdc(1000 V/div, $10 \mathrm{~ms})$. (c) AC phase voltage, ea (30 V/div, $10 \mathrm{~ms})$. (d) AC current, $\mathrm{ia}(2 \mathrm{~A} / \mathrm{div}, 10 \mathrm{~ms})$. 
Table 6.1: DC Microgrid Parameters

\begin{tabular}{|c|c|c|}
\hline "Component & Parameter & Specification \\
\hline \multirow{6}{*}{$\begin{array}{l}\text { Controlled } A C-D C \\
\text { Rectifier }\end{array}$} & Power Rating & $5 \mathrm{~kW}$ \\
\hline & IGBT Module & SKM50GB123D \\
\hline & Switching Freq. & $8 \mathrm{kHz}$ \\
\hline & $L_{r}$ & $12 \mathrm{mH}$ \\
\hline & $r_{r}$ & $0.98 \Omega$ \\
\hline & $C_{r}$ & $1200 \mu F$ \\
\hline \multirow{7}{*}{$\begin{array}{l}\text { Bi-directional } A C \text { - } \\
D C / D C-A C \text { Converter }\end{array}$} & Power Rating & $5 \mathrm{~kW}$ \\
\hline & IGBT Module & SKM50GB123D \\
\hline & Switching Freq. & $8 \mathrm{kHz}$ \\
\hline & $L_{b}$ & $24 m H$ \\
\hline & $r_{b}$ & $1.96 \Omega$ \\
\hline & $C_{b}$ & $1200 \mu F$ \\
\hline & $L_{b d c}$ & $12 m H+0.98 \Omega$ \\
\hline \multirow{4}{*}{$\begin{array}{l}D C \text {-DC Boost } \\
\text { Converter }\end{array}$} & Power Rating & $3 \mathrm{~kW}$ \\
\hline & IGBT Module & SKM150GAL12V \\
\hline & Switching Freq. & $5 \mathrm{kHz}$ \\
\hline & $L_{P V}, L_{F C}$ & $3 m H+0.4 \Omega$ \\
\hline \multirow{5}{*}{$\begin{array}{l}\text { Bi-directional DC-DC } \\
\text { Battery Charger }\end{array}$} & Power Rating & $1 \mathrm{~kW}$ \\
\hline & IGBT Module & SKM800GA126D \\
\hline & Switching Freq. & $5 \mathrm{kHz}$ \\
\hline & $L_{\text {batt }}$ & $2.7 m H+0.22 \Omega$ \\
\hline & $C_{\text {in }}=C_{\text {batt }}$ & $1200 \mu F$ \\
\hline \multirow{4}{*}{$\begin{array}{l}\text { DC Dynamic Load } \\
\text { Model }\end{array}$} & Power Rating & $3 \mathrm{~kW}$ \\
\hline & IGBT Module & SKM800GA126D \\
\hline & Switching Freq. & $5 \mathrm{kHz}$ \\
\hline & $L_{D L}$ & $2.7 m H+0.22 \Omega$ \\
\hline \multirow{9}{*}{$\begin{array}{l}\text { Single phase } D C-A C \\
\text { Inverter }\end{array}$} & Power Rating & $3 \mathrm{~kW}$ \\
\hline & IGBT Module & SKM50GB123D \\
\hline & Switching Freq. & $6 \mathrm{kHz}$ \\
\hline & $L_{i d c}$ & $3 m H$ \\
\hline & $R_{i d c}$ & $0.4 \Omega$ \\
\hline & $C_{i d c}$ & $2400 \mu F$ \\
\hline & $L_{i a c}$ & $12 \mathrm{mH}$ \\
\hline & $R_{i a c}$ & $0.98 \Omega$ \\
\hline & $C_{i a c}$ & $196 \mu F$ \\
\hline
\end{tabular}

\subsubsection{Dynamic DC Load emulator}

The dynamic DC load consists of a voltage-controlled buck converter connected to a constant resistive load. The converter was designed to respond very quickly to changes in the reference. Hence, by changing the reference of the output voltage, the output power changes and the dynamic load emulator can follow a certain load pattern. An example of the load curves programmed in the dynamic load model is given in figure 7.6. The load power varies from 0 to $3 \mathrm{~kW}$ iteratively in six-minute intervals. 


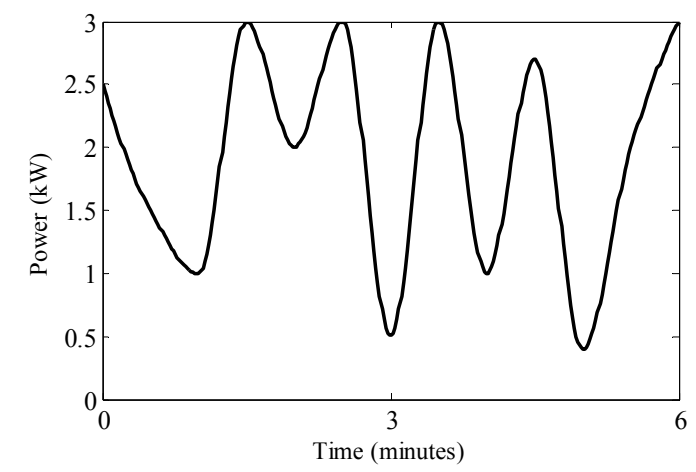

Figure 6.6: Output power of the dynamic load emulator

\subsection{Hybrid AC/DC System Architecture in Smart Grid Test-bed}

The DC bus is connected to the AC system via a bi-directional converter. This allows bi-directional power flow between AC and DC. The system described in figure 7.1 consists of two DC-DC converters integrating two sustainable energy sources into a common DC bus. They have the ability to control the power injected to the DC network. In normal operation, the voltage on the DC bus is regulated via a controlled rectifier connecting both sides. However, if the system is working in an islanded mode, i.e. isolated from the $\mathrm{AC}$ side, one of the DC-DC converters interfacing the sustainable energy sources to the DC system must take this responsibility. A fully controlled rectifier was designed to tie the $\mathrm{DC}$ grid with the $\mathrm{AC}$ one. A vector decoupling controlled sinusoidal pulse width modulation (SPWM) technique was used to allow the designed rectifier to maintain a constant output voltage while being able to control the active and reactive power drawn from the grid independently. Moreover, a bi-directional ACDC/DC-AC converter was used to allow bi-directional power flow between both sides. The amount of power flowing in either direction can be set to a certain pre-set value while the controlled rectifier working as a voltage rectifier maintains the power balance. This is because it is free to supply the power needed in the DC grid. In order to increase 
the operating range of the rectifier, an adaptive controller having the ability to dynamically change its parameters corresponding to the condition of the system was proposed in [138]. The emphasis of this chapter is mostly on the overall design of AC/DC parts of the test-bed. It should be pointed out that [135]-[137] describe the DC grid connectivity in further details. Figure 6.7 shows the overall power system schematic and single line diagram of implemented hybrid AC/DC setup.

\subsection{Microgrid Operation; Experimental Results}

In the DC microgrid we used a DC supervisor controller which is the master controller and is responsible for sending the dc bus voltage reference to the controlled rectifier and distributing power references to the local (slave) controllers of the other source converters. Moreover, this supervisor controller communicates with the main AC grid and is the place to execute various real-time energy management algorithms in order to manage the power sharing among the various sustainable energy sources, the interaction with the main $\mathrm{AC}$ grid and the battery charging and discharging.

For instance, the real-time battery charging/discharging algorithm presented in figure 6.8 was implemented. This algorithm aims mainly at shifting the power demanded from the main $\mathrm{AC}$ grid to off-peak time by managing the charging/discharging process of the battery such that:

- If there is a surplus in the total power generated locally from the PV and fuel cells and it exceeds the demand on the DC, the charging rate of the battery is increased during off-peak time since the energy tariff is relatively low so it is more worthy to charge the battery than to sell power to the grid. On the other hand, the charging rate 
was decreased during peak time to charge the battery later on when the energy tariff drops.

- If there is a deficiency in power meaning that the renewable energy sources are not capable of satisfying the load demanded on the microgrid, the discharging rate of the battery is increased during peak time to enhance the saving by reducing the energy bought from the main grid with the high tariff. Moreover, the discharging rate is decreased during off-peak time since the tariff is low and the available energy in the battery can be more effectively utilized during the coming peak period. The developed laboratory-based smart power system can be used to test more complex real time energy management algorithms involving online prediction and modeling of renewable energy sources output power uncertainty, load forecasting and pulsed load mitigation such as the algorithms developed in [139], [140].

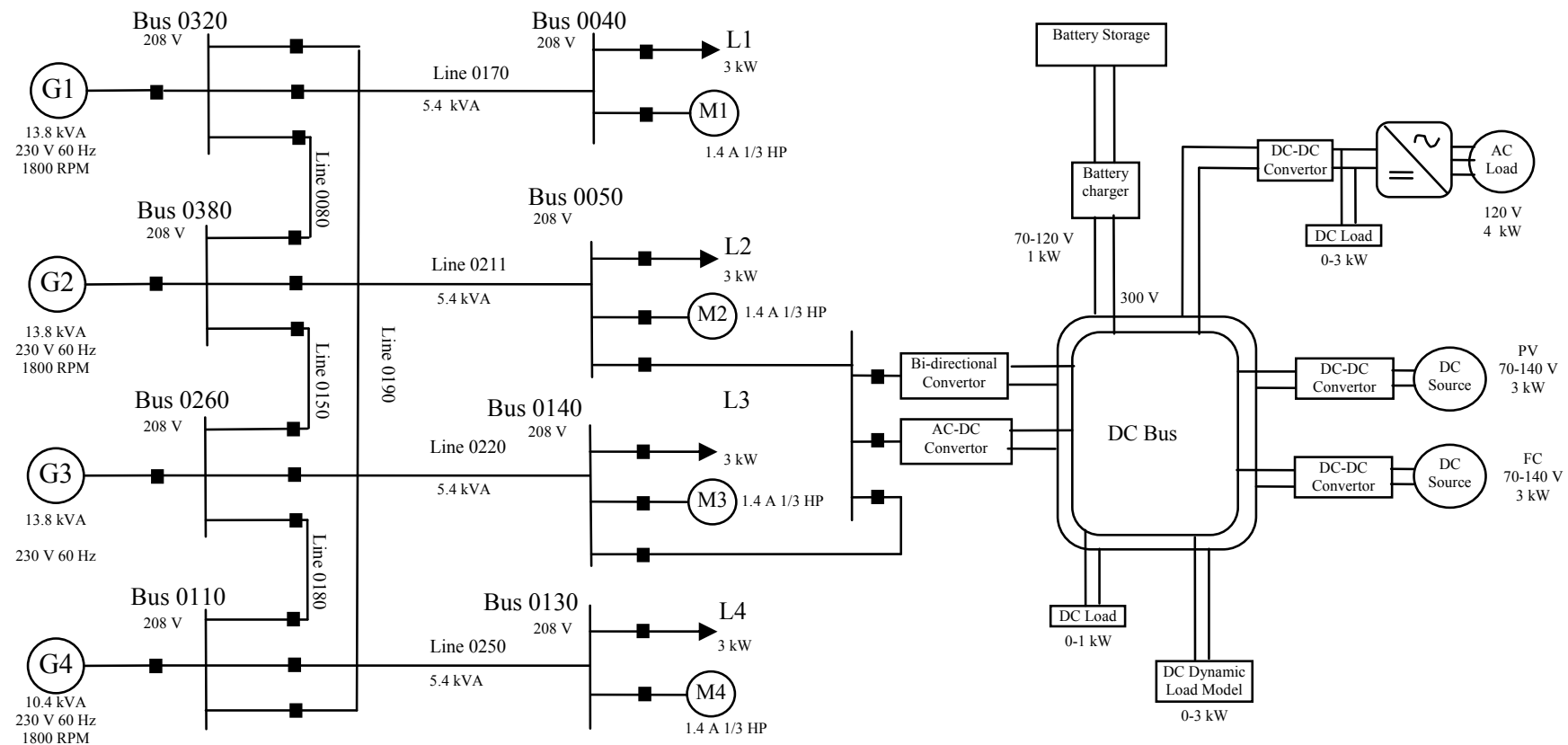

Figure 6.7: Overall power system schematic and single line diagram of implemented hybrid AC/DC setup 


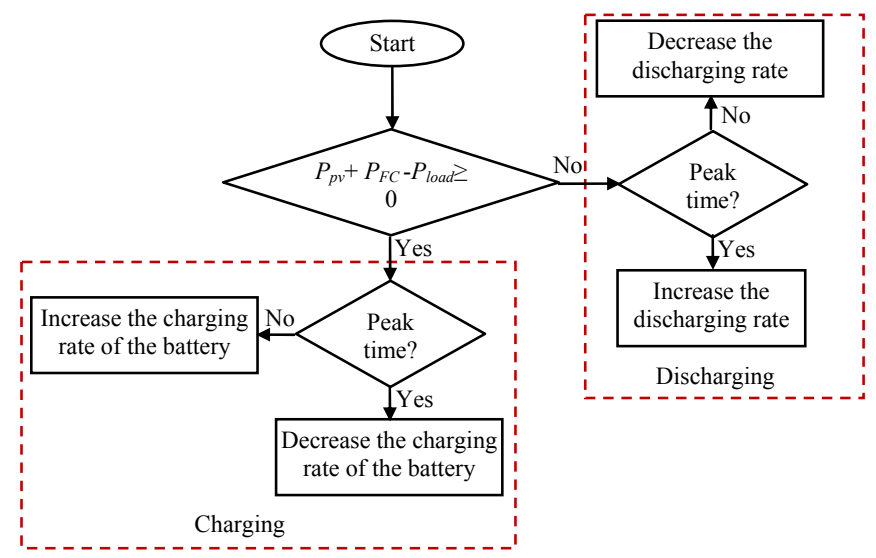

Figure 6.8: A flow chart of the real-time algorithm to manage the charge/discharge process of batteries

In order to investigate the real-time operation of the DC microgrid, figure 7.9 shows the performance of the various components of the DC side corresponding to several step changes in the reference power. The curves shown (from top to bottom) are corresponding to the DC bus voltage $\left(v_{d c}\right)$, the rectifier current $\left(i_{r}\right)$, the bi-directional current $\left(i_{b d c}\right)$, the photovoltaic $\left(P_{V}\right)$ output current $\left(i_{p v}\right)$, the fuel cells output current $\left(i_{F C}\right)$ and the battery current $\left(i_{\text {batt }}\right)$, respectively. The case study is described as follows:

- The supervisor controller sends reference signals to the various converters in the DC microgrid.

- The bi-directional converter is set to transfer $1 \mathrm{~A}$ from the main $\mathrm{AC}$ grid to the $\mathrm{DC}$ microgrid.

- $i_{P V}$ is initially set to $2 \mathrm{~A}$, then a step change from 2 to $0.5 \mathrm{~A}$ was applied after $0.1 \mathrm{sec}$

- $i_{F C}$ is set to $0.25 \mathrm{~A}$ along the whole interval shown in figure 6.9

- The battery is operating in the discharging mode, $i_{\text {batt }}$ was initially set to $0.2 \mathrm{~A}$ then the discharging current reference was changed to $0.7 \mathrm{~A}$ after $1.1 \mathrm{sec}$

- The total loads connected to the DC bus are increased by $75 \%$ after $1.6 \mathrm{sec}$ 

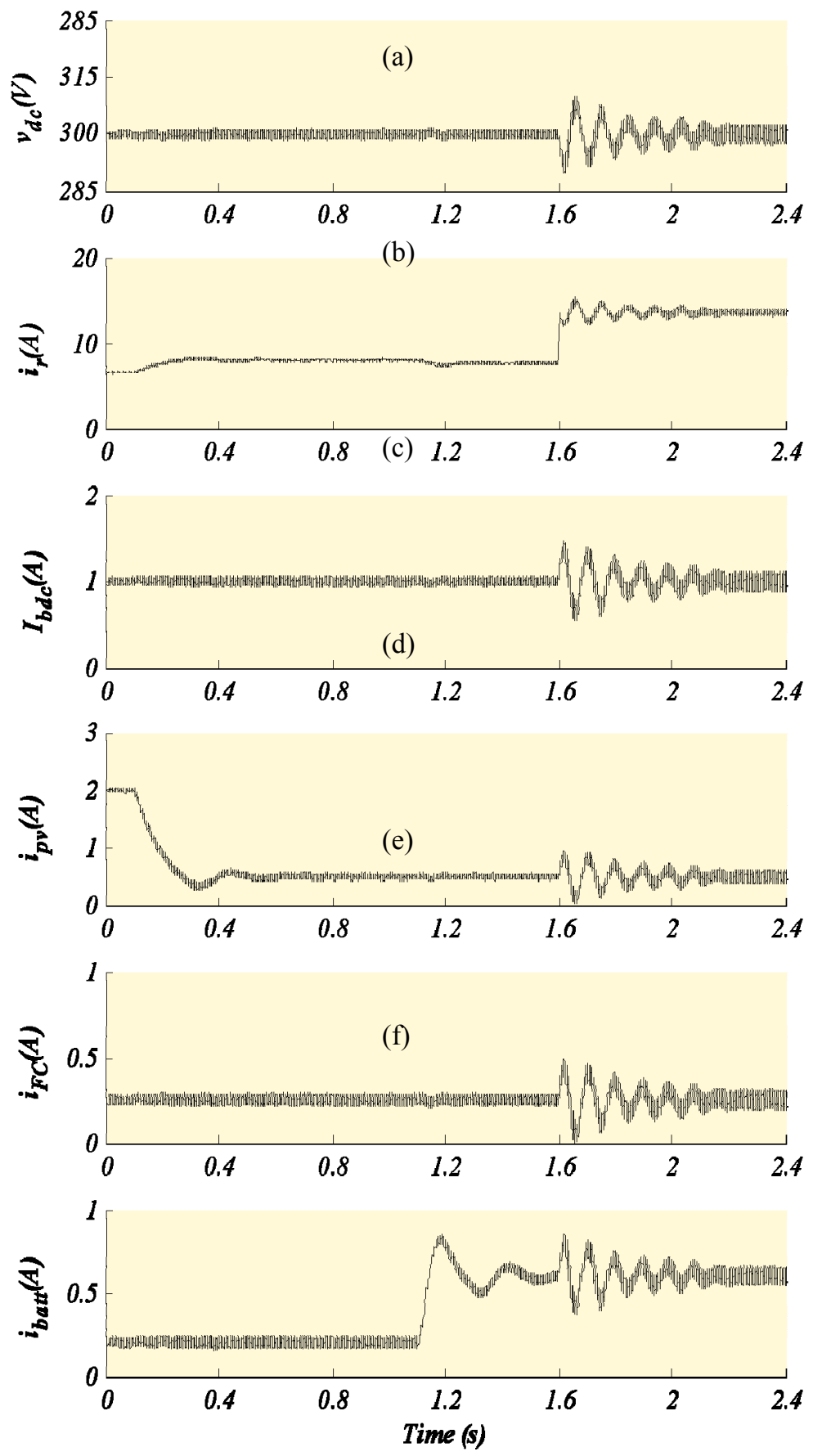

Figure 6.9: Performance of the various components of the DC microgrid corresponding to step change in the power reference. (a) shows the DC bus voltage $\left(v_{d c}\right)$, (b) the rectifier current $\left(i_{r}\right)$, (c) the bi-directional current $\left(i_{b d c}\right),(\mathrm{d})$ the PV output current $\left(i_{p v}\right)$, (e) the fuel cells output current $\left(i_{F C}\right)$ and (f) the battery current $\left(i_{\text {batt }}\right)$.

As can be seen in the figure, the DC bus voltage is hardly affected by the step changes in the PV or battery power. However, the big step change in the load creates 
some fluctuation in the bus voltage of around $1 \%$, which is definitely below all the standard limits. This transient period lasts for about $0.5 \mathrm{sec}$.

The rectifier current is free to maintain the power balance in the system. When the $\mathrm{PV}$ power decreases from $2 \mathrm{~A}$ to $0.5 \mathrm{~A}$, the rectifier current increases by the same amount. Moreover, when the battery current increases after $1.1 \mathrm{sec}$, the rectifier current decreases to compensate for this increase.

The bi-directional converter is capable of maintaining its reference current of $1 \mathrm{~A}$ during the step changes of other converters. However, a fluctuation of the output current can be seen at $1.6 \mathrm{~s}$ when the sudden change in the load takes place. This is due to the fluctuation in the output voltage.

The DC-DC converter interfacing the PV successfully supplies the desired current with the some fluctuation during the sudden load change. In addition, the DC-DC converter interfacing the fuel cell was able to maintain the desired output current during the various states of the system with some ripple in the output when the load is suddenly changed.

Finally, the battery charger/discharger successfully supplies the desired amount of current from the battery, which helps implementing any kind of real-time operation algorithms involving the battery. The supervisor controller sends reference signals to the various converters in the DC microgrid.

\subsection{Integrated Hybrid AC/DC Operation; Experimental Results}

In order to evaluate the integration of the $\mathrm{AC} / \mathrm{DC}$ system and study the system performance during load variations, an experiment was conducted to show the system 
behavior and $\mathrm{AC} / \mathrm{DC}$ power sharing control while the load variations take place in active and reactive power. In this experiment, the DC microgrid quantifies its DC power availability during the upcoming interval of time. Hence, it will inject any amount of active/reactive power demanded by the AC side, through the WAMPAC considering all constraints.

The DC system is connected to Bus-0050 through a bi-directional converter as shown in figure 6.7 and injects any specified amount of active and/or reactive power to the AC point of common coupling (PCC). In this experiment the DC microgrid was used to regulate the voltage at the PCC.

Figure 6.10 shows the experimental results which a unity power factor load of $700-\mathrm{W}$ was initially connected to Bus- 0050 . The DC microgrid was commanded to receive $100 \mathrm{~W}$ and zero Vars. Hence, AC grid takes the responsibility of supplying both AC load and DC microgrid demand. The steady state voltage at PCC in this situation was 0.94 p.u. whereas the voltage on the DC bus was 1 p.u. After $20 \mathrm{sec}$, the DC microgrid was commanded by WAMPAC system to inject the total amount of demanded power on the AC side. Therefore, the voltage amplitude was increased to 1.02 p.u. The controlled rectifier regulating the voltage on the DC bus maintains a voltage of 1 p.u. after a transient period of around $6 \mathrm{sec}$ with an overshoot of 0.02 p.u.

A reactive load of 450 VARs was increased to PCC after $43 \mathrm{sec}$. Consequently the voltage amplitude drops to around 0.95 p.u. The DC microgrid was then commanded by WAMPAC to inject 300 -VARs to the AC grid. Hence, the voltage at the PCC increases to 0.98 p.u. The DC bus voltage is hardly affected by this change in its reactive 
power reference. The frequency variations are also shown in this figure. A maximum of $0.2 \mathrm{~Hz}$ deviation from $60 \mathrm{~Hz}$ was depicted in the measured frequency.
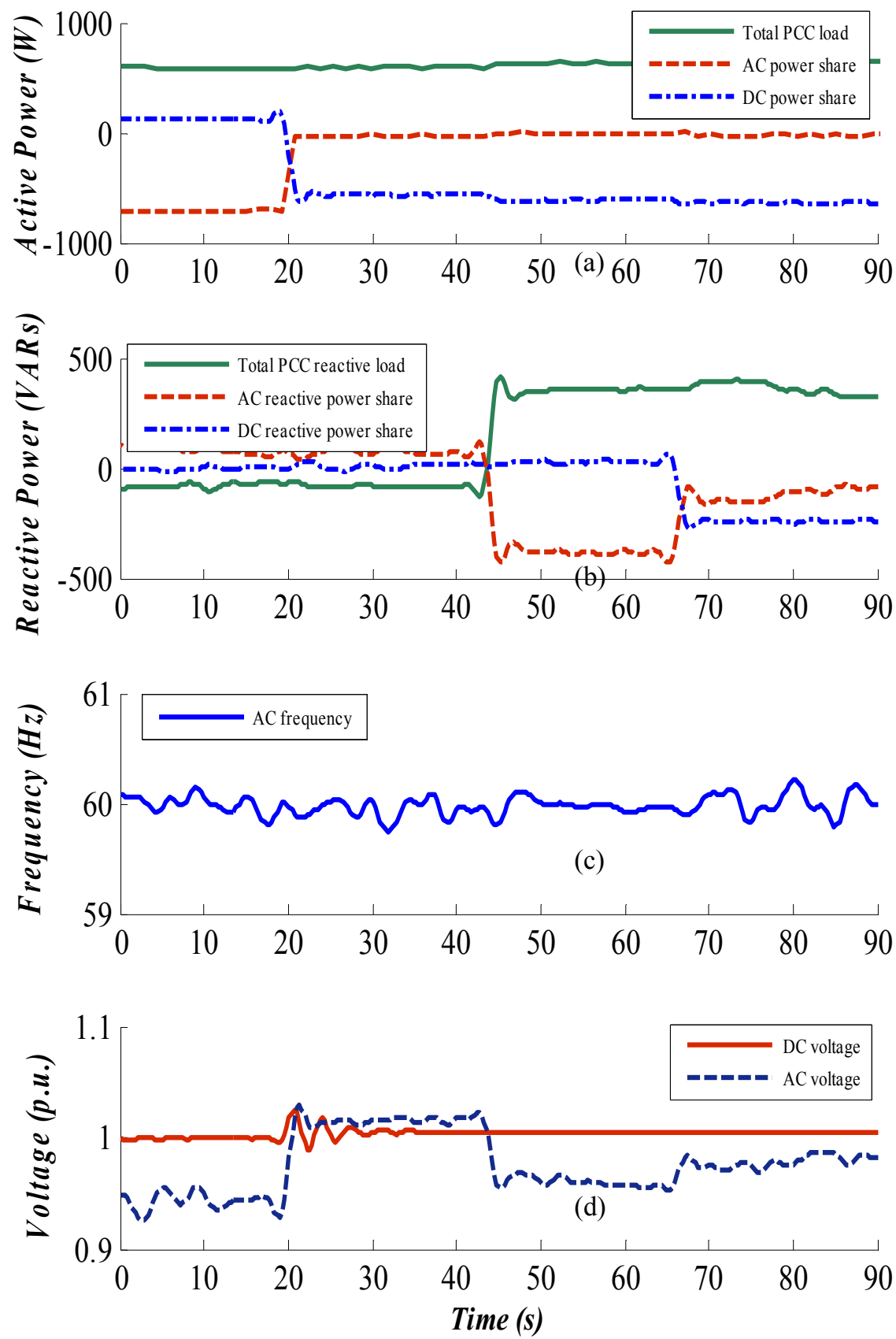

Figure 6.10: Performance of the integrated hybrid AC/DC microgrid corresponding to step change in the load demand reference. (a) the load, DC and AC active power share, (b) the load, DC and AC reactive power share, (c) the frequency of the AC bus, (d) the voltage of the AC and DC buses. 
Figure 6.11 shows details about the quality of the active and reactive power transferred between the $\mathrm{AC}$ and $\mathrm{DC}$ grids after $10 \mathrm{sec}$. This figure also shows the current and voltage waveforms, as well as the THD at the PCC and the inverter terminals.

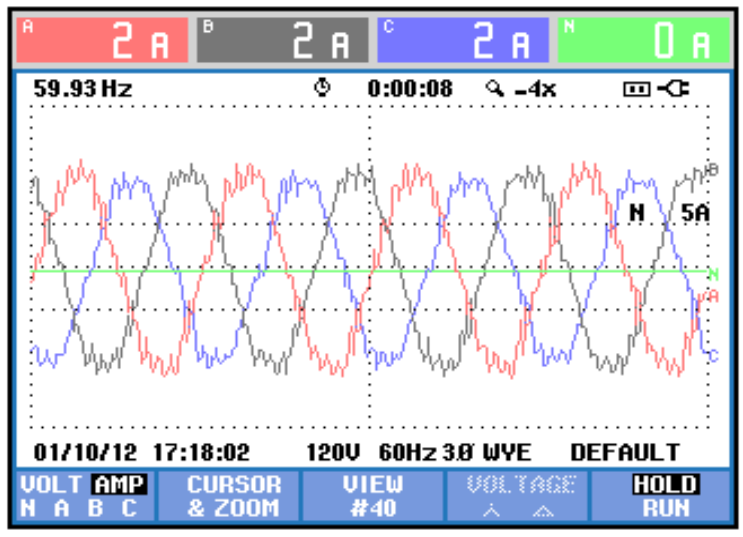

(a)

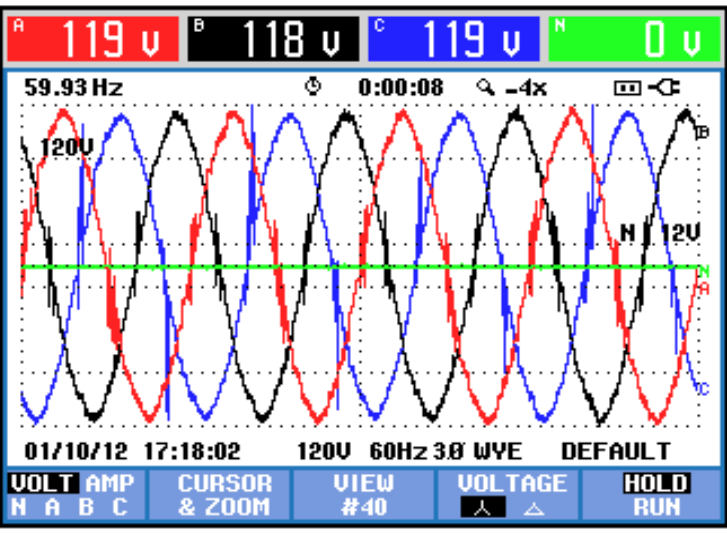

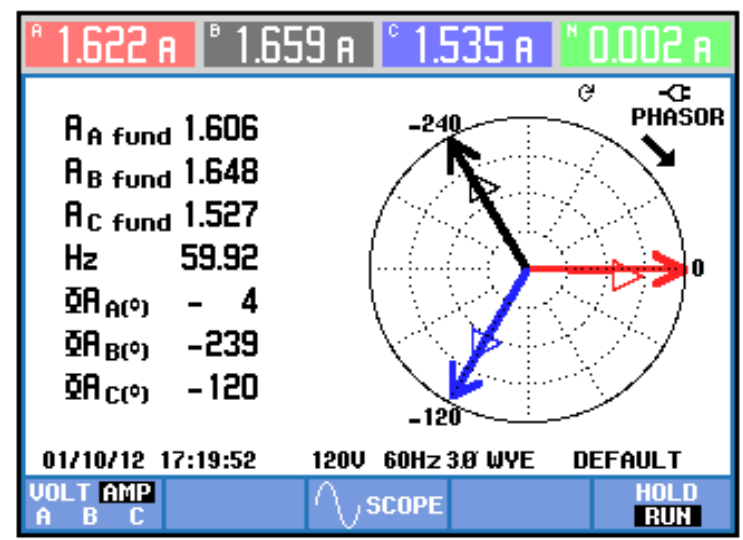

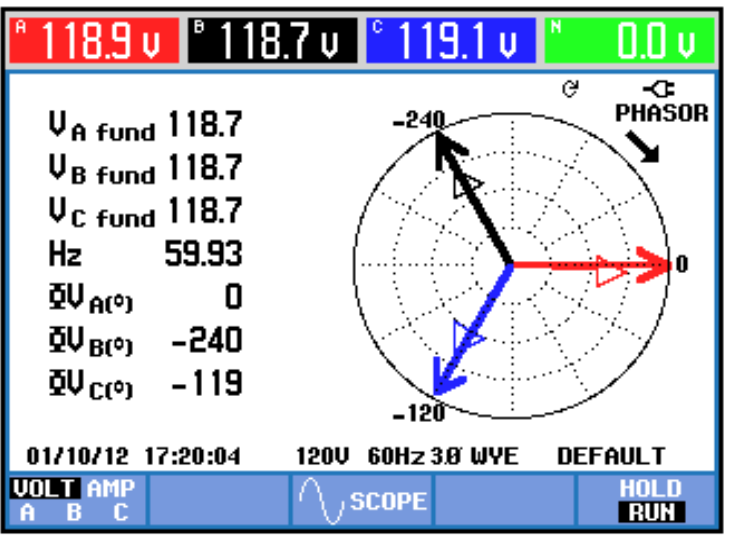

(b)
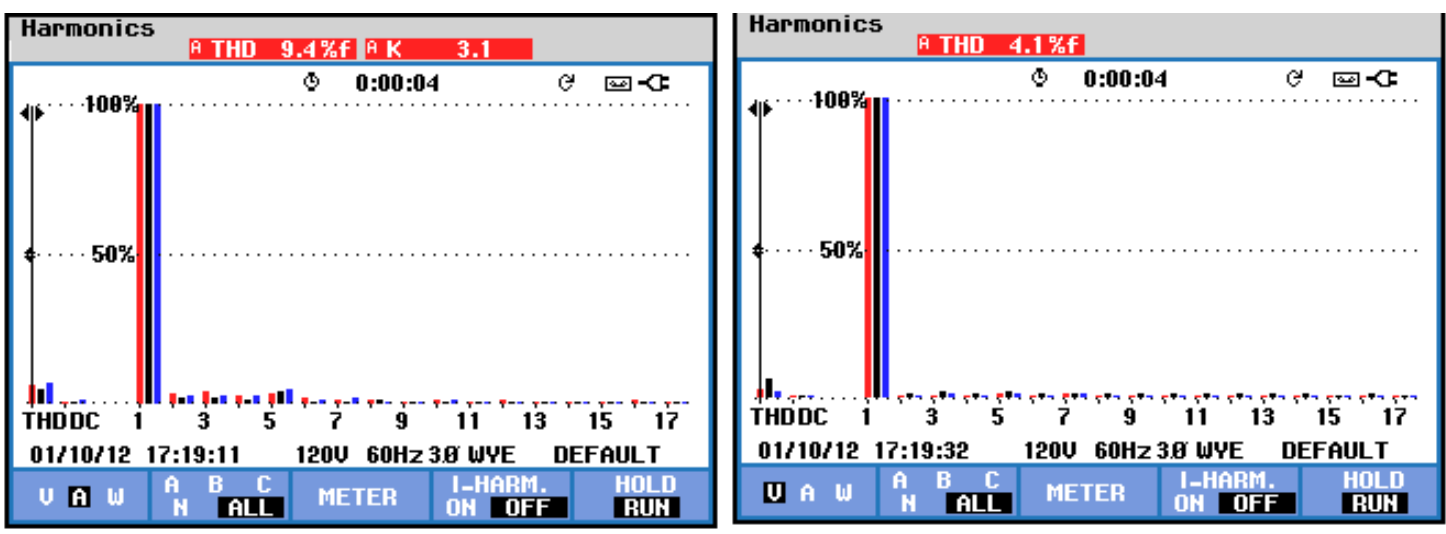

(c)

Figure 6.11: Current and voltage waveforms, their THD after $10 \mathrm{~s}$. (a) shows the PCC current and voltage waveforms, (b) the inverter side current and voltage phasors, (c) the current and voltage THD 
Figure 6.12 depicts also same details about the quality of the active and reactive power transferred between the AC and DC grids this time after $70 \mathrm{sec}$. This figure also presents the current and voltage and THD at the PCC at 70 seconds. Using this integrated controller for hybrid AC/DC microgrids will enhance the performance of the system.
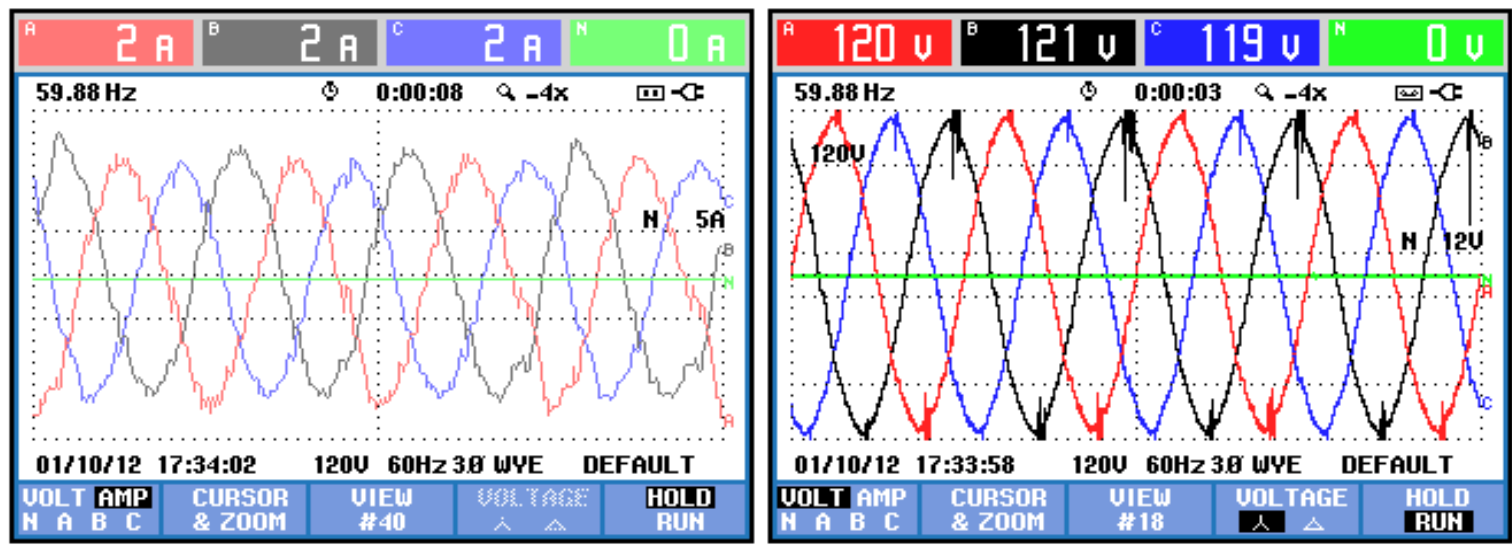

(a)
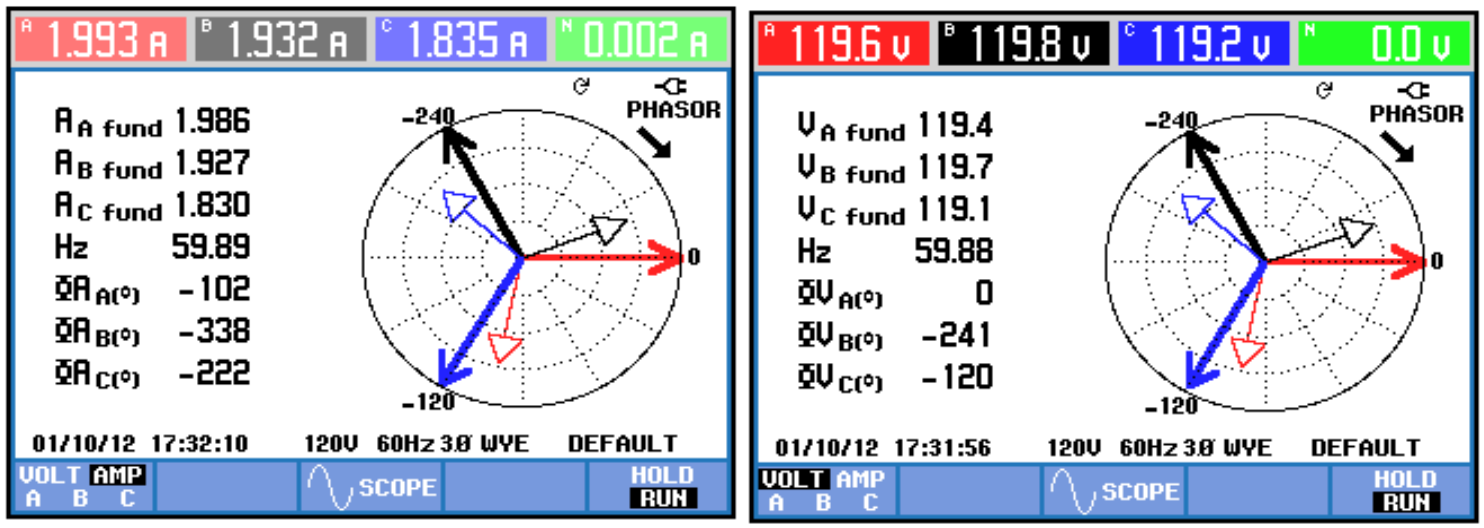

(b)
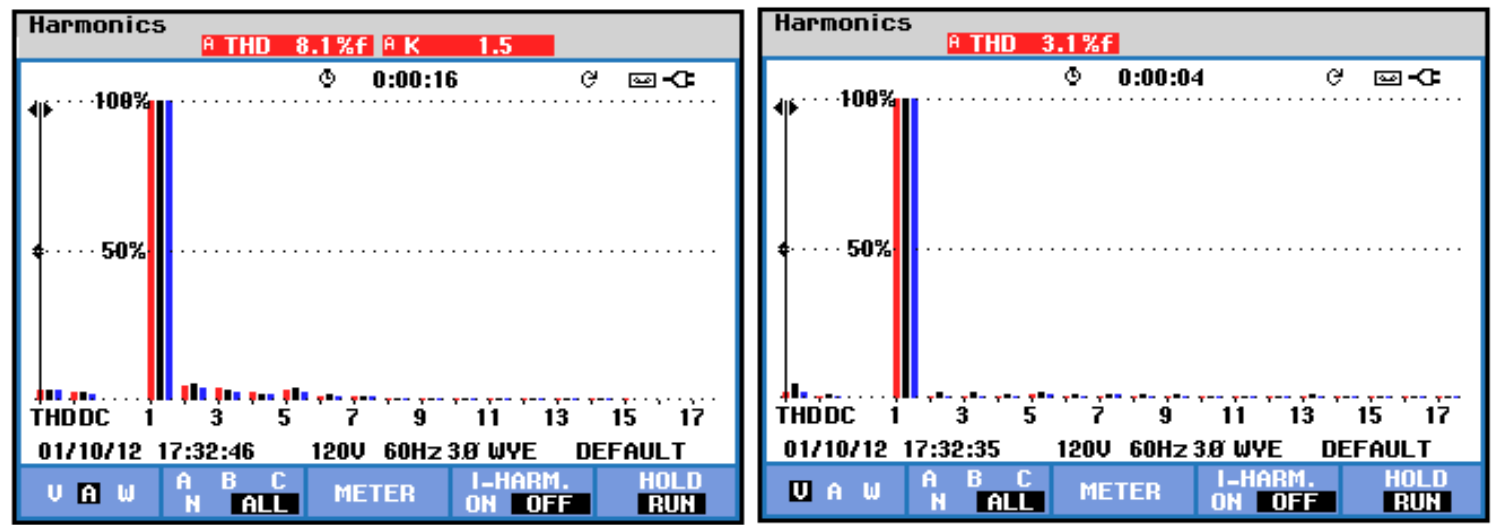

(c)

Figure 6.12: Current and voltage waveforms, their THD after $70 \mathrm{~s}$. (a) shows the PCC current and voltage waveforms, (b) the inverter side current and voltage phasors, (c) the current and voltage THD. 


\section{CHAPTER 7}

\section{IMPLEMENTATION OF REAL-TIME POWER SYSTEM SECURITY AND VOLTAGE STABILITY MONITORING SYSTEM}

\subsection{Introduction}

This chapter explores the challenges and opportunities to implement online system analysis capability in order to monitor the system security and stability through the establishment of operational indices. Therefore, the implemented hardware/software setup for monitoring and analyzing system in real-time format was presented by the online results available from real-time software. Online load flow and contingency results aim to help provide proper security monitoring of the power system in wide area which is useful for most applications including system remedial actions.

In real-time operation of power system, the reliability is always referred to as security [156]. Power system security is the ability of the system to withstand contingencies which are subjected to it by wide variety of disturbances. For example, sudden change in load demand, loss of transmission line, system configuration change, equipment outage and generator failures are typical power system circumstances. The smart grid uses modern communication infrastructure at wide area power system to improve grid reliability, reduce the price of electricity, improve operational efficiency, security and safety and promote environmental quality [21], [22].

System state monitoring is technically can be achieved using fast wide area measurements and hence it enhances WAM system in order to demonstrate system current status which is known as normal state, restorative state and emergency state [156]. At normal operation, if one or more operational limit are violated the system state 
changes to emergency and if it leads to load disconnection such as partial or total blackout from which the rest of the system remained in a normal state, the system moves to the restorative state. A normal state is secure if all contingencies result in secure normal operation. Therefore, the secure power system is one that has low probability of blackout or equipment damage. The WAMPAC system is responsible to maintain a secure system state in order to prevent system state transition to emergency state. The wide range of operational control and protection strategies in smart and intelligent form should be implemented in WAMPAC system. Power system dynamics bring the new challenges for online changing at control scenarios. These control actions are deployed to ensure that the supply-demand balance is met reliably and securely [157]-[159].

The objective of this research is to use real-time measurements at smart grid to identify the power system state. Online power system analysis feature are developed to deploy real-time data in order to monitor system state with online load flow and security assessment results. The implemented laboratory setup for a micro smart power grid has been presented previously with the developed software environment to monitor real-time data as a SCADA center. The real-time software has the capability to run analysis software in engine mode and hence the system online analysis can be implemented by real-time data monitoring speed. In this setup, the monitoring system updates the SCADA system approximately every one second and then the analysis software can update the data immediately after and calculates security indices. Consequently, this system can analyze power system less than 3 seconds snapshot and returns the real-time calculation results. Figure 7.1 shows the power system phenomena time frame as well as control 
actions in their time frame. Studies related to the time range of phenomena and control actions has also shown as well as developed security assessment.

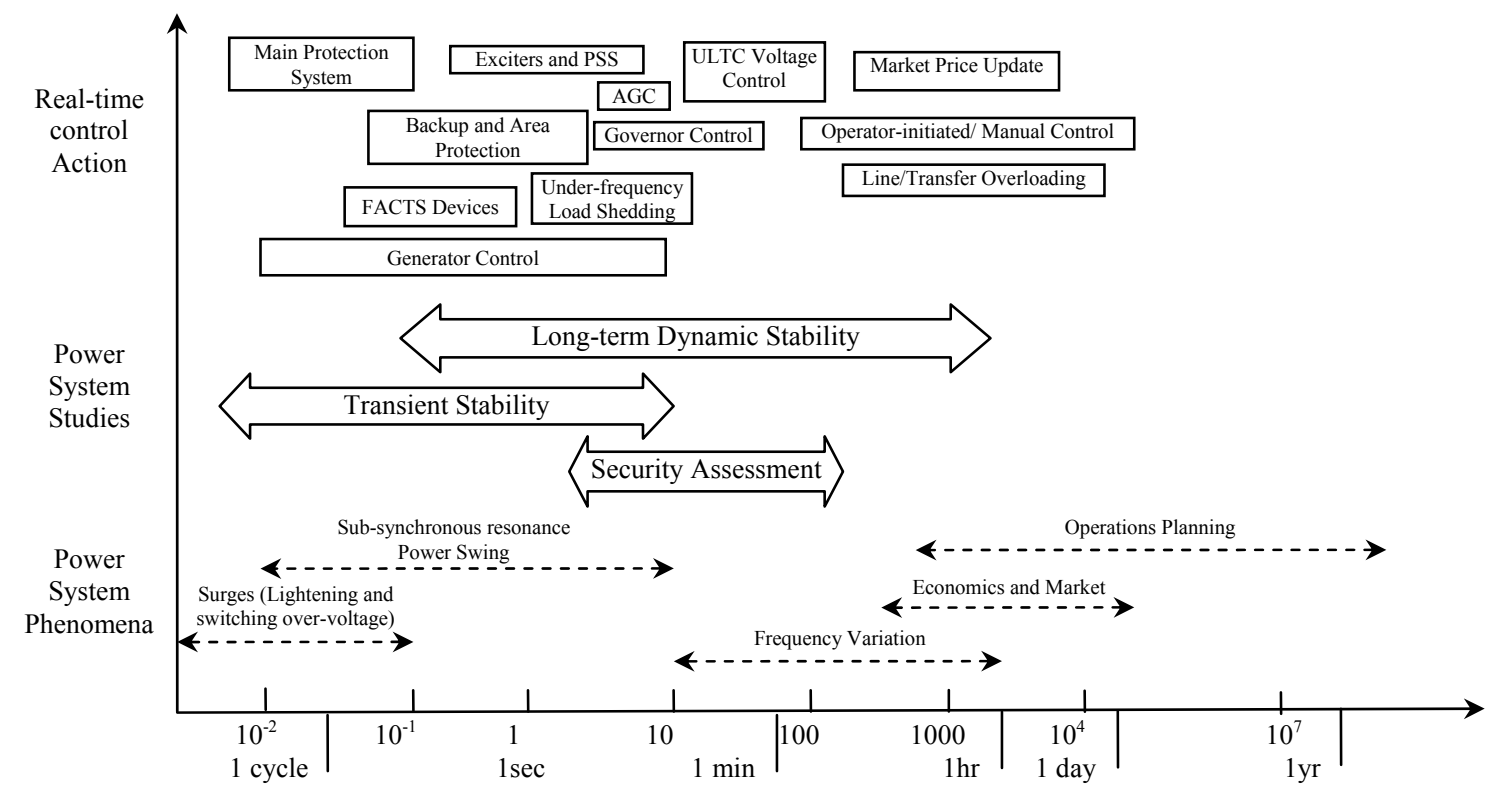

Figure 7.1: Power system time frame for phenomena, control actions and studies

\subsection{Modern Monitoring and Control Systems in Power Systems}

With today's current technology, understanding data using analytical tools and software has made the data overwhelm problem small if the set up is done correctly. With advancements in computer processing power, fiber optic network speeds and the improvement on software, reading data is now more than just a simple tabular displays and one-line diagrams. The large amounts of data received is dispersed in a program to be read simultaneously to read bus voltage magnitude/angles, active and reactive power to and create power flow calculations and diagrams. For an operator to properly understand the data that is being obtained, a virtual environment (VE) or human user interface (HMI) is used to simplify the complex environment of the power system to the operator so that one can directly interact with the real-time data. With the ability to view real-time data with the VE or HMI, an effective alarm management can be created. To avoid 
overwhelming an operator with alarms, the analytical tools and software can be set up in such a manner that allows the user to easily view the location of the alarm in the system and from where the fault/problem is originating from. One major concern that still exists today is the mismatch between actual system performance and simulation predictions. One solution to this problem is the use of state estimation. The state estimator can provide the most up-to-date snapshot of the system. Modern measurements and communications technology impact the power system by a new capability to make phasor measurement. Phasors, represent voltages and currents at various buses in a power system which defines the state of the system. They should be measured in a common reference. In order to achieve a common reference for the phasors, the sampling at different points should be synchronized with a high precision. The Global positioning System (GPS) which provides a 1 pulse per second (1 pps) with an accuracy of $1 \mu$ sec has been used at any location to synchronize phasor measurement devices [12]. With the early stages being done on state estimation for the power system, the use of high sampling PMU helps adding measurements to the set available for state estimation. With the advancements, steady state and dynamic response, operations are presently being read as real-time data which accurately reflects the actual system constraints.

In the laboratory-based smart power system, the focus is on real-time calculation of power system parameters using PMUs which has been introduced in chapter IV with implementation details. Figure 7.2 shows the single line of the setup for implementing online security assessment. The PMUs functional implementation was carried out by the Data Acquisition System (DAQ) for research purposes. 


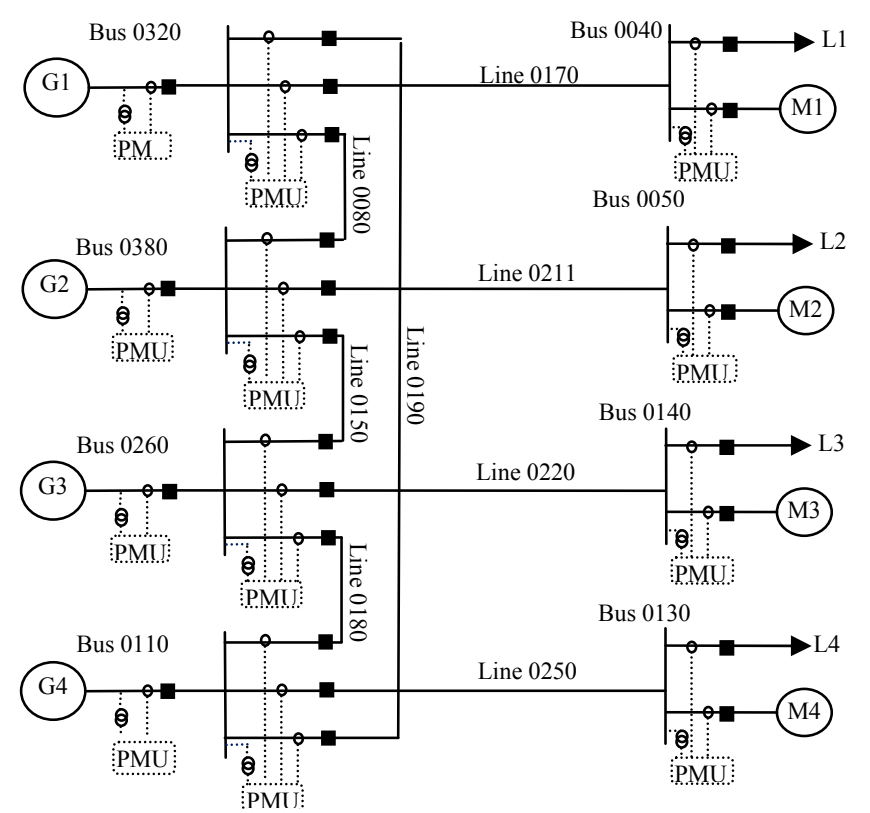

Figure 7.2: single line of the setup for implementing online security assessment

Different DAQs were used to get the whole system data. Every DAQ system data were synchronized and hence can simulate different PMUs data concentrator in one system. For instance, all generators voltages and currents are measured in a DAQ and the voltage phasors are in the same reference frame. The positive sequence of voltages and currents in the same reference are calculated using measured signals by $12 \mathrm{kHz}$ sampling rate and sent 10 data packets per second. Figure 7.3 shows the front panel of measurement VI in which all system parameters are displayed in synchronized phasor form. Moreover, the active and reactive power measurements are shown for all three phases and are plotted. This VI is available for all branches in the setup and they are subVIs for an overall real-time monitoring system as shown in figure 7.4. This VI in contrast to conventional SCADA systems is very fast and the update time is around one second. The update time is good enough to be observed and followed by a SCADA user to monitor system and control it manually. Hence, the real-time wide area protection and control system has around one-second time interval in order to apply new settings and 
strategies in the entire power system. The ability to show the systems fast dynamic oneline display can provide visually real and reactive power flows, line loadings to point out overloads, bus voltage magnitude/angles. Using the contours and animation features, the operator could immediately recognize the system state which may be changed over a specific time period. The challenging work is the construction of appropriate pattern for the interactive display of large amount of power system data and the development of effective human factors for such applications. The real-time SCADA system should be equipped with proper alarms. Without effective alarm management, the system operator is easily overwhelmed by alarms during the system disturbance and hence the initiation of an event will be complicated. The visual real-time application can be effectively exploited to indicate to the operator the key initiating event and the extent of the system problems.

With the current and ever rapidly increasing technology, the success for reading real-time data and reliable operations and control of the power system is widely based on software using the DAQs, communications and computer processing power. The ability to duplicate the software and use it throughout the system is very cost effective. However, this exposes the system to produce possible risks due to common programming error which in turn may cause system failure. A properly calibrated model of the system using the new metering technology can provide data for improved modeling of the equipment in use, which is a requirement for an effective control system design and implementation. 


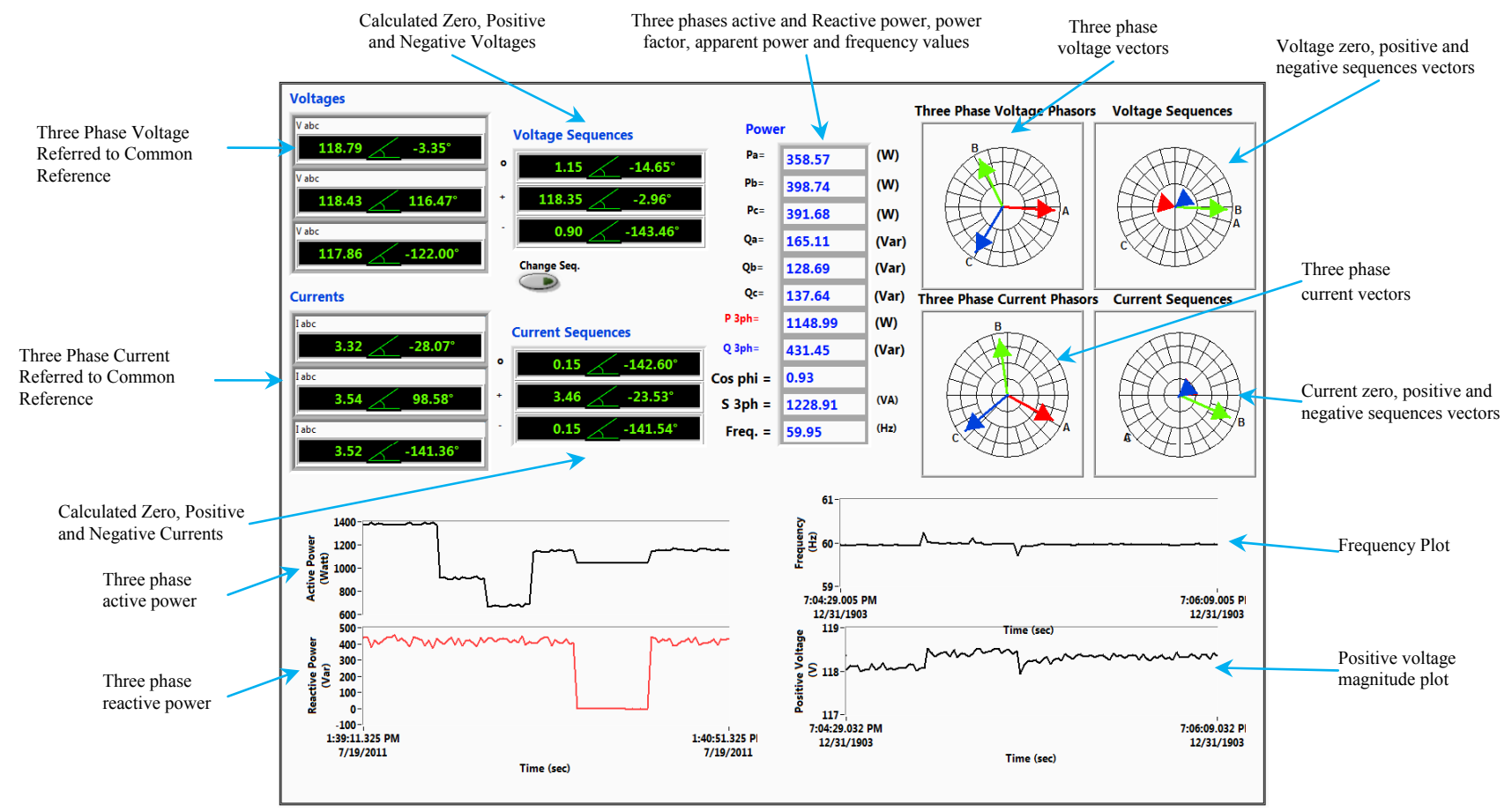

Figure 7.3: Front panel of measurement VI with all system parameters

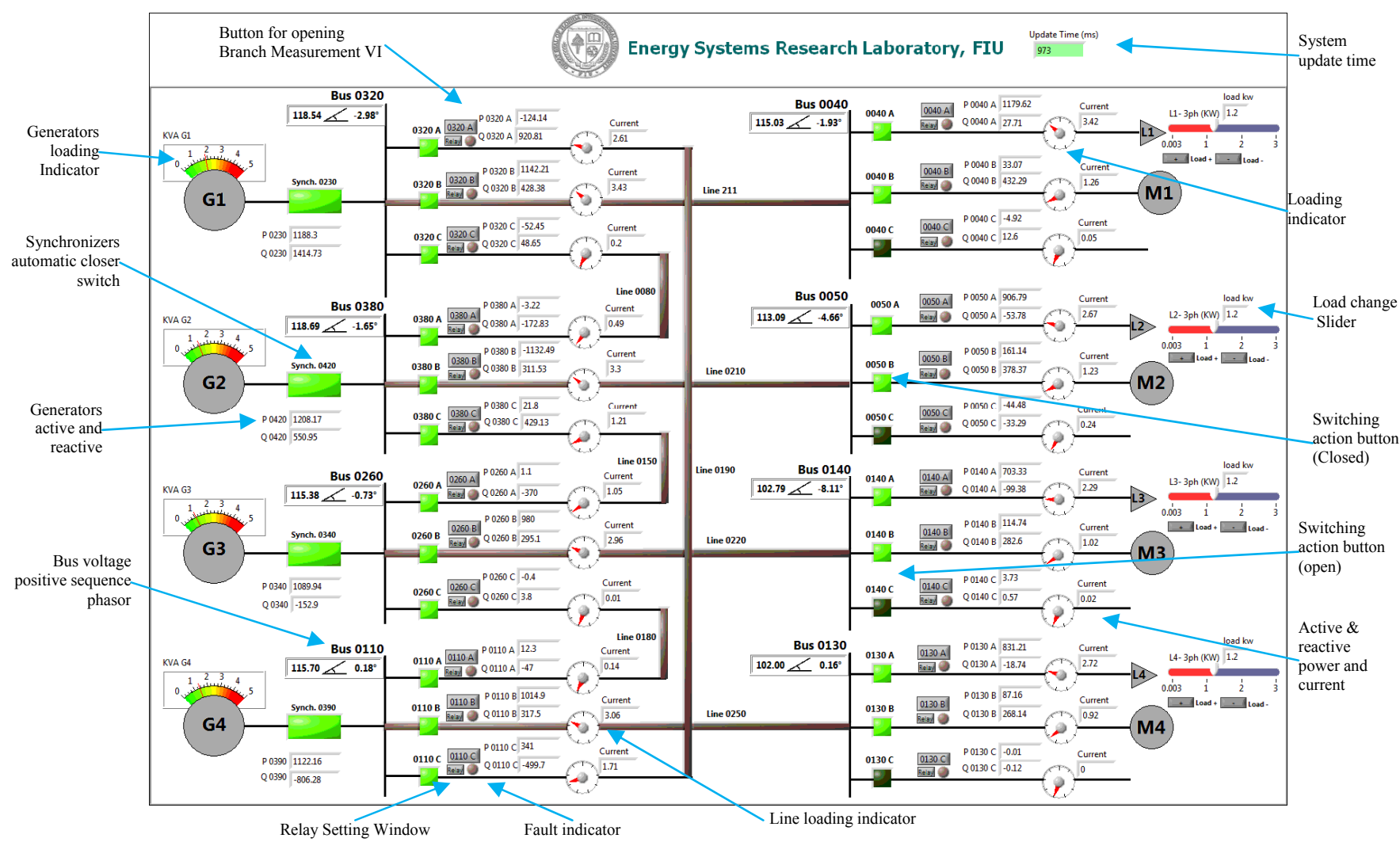

Figure 7.4: Implemented fast real-time SCADA system with WAMPAC capability in test-bed setup 


\subsection{Developed Online Security Monitoring System; Experimental Results}

The management of system situation alarms and emergency state has become a major issue in system operation and control. Without effective security monitoring, the system operator or intelligent controller couldn't recognize system circumstances properly in response to a disturbance. The aim is to distinguish the initiating event from the onslaught of alarms which may due to a specific initiating incident. For example, the opening of a heavily loaded transmission line could create numerous low bus voltages, line overload on other transmission lines and even generator VAR loading, alarms. The real-time system should indicate the system situation to the operator by developing appropriate system security monitoring system which can predict the scope of the local interruptions or major blackouts.

A lot of challenges have been introduced on describing steady state system limits and collapse. However, approaches for detecting or preventing dynamic voltage collapse, cascade events and the ability of the real-time controllers to mitigate those circumstances are not implemented or even investigated intensely. Most of these events happen on a time scale of several minutes. Hence, the integration of local and wide area controllers utilizing fast security monitoring system can effectively prevent system from changing to emergency state by intelligent and optimal operations. The conventional approach to design such control elements in an integrated power network would involve construction of complete dynamic models for the interconnected grid, generator units and their controllers. The generator dynamic data may be more difficult to obtain and is less reliable. Therefore, the modern estimation techniques based on real-time WAMPAC data could enhance reliability and real-time control performance. 
The developed security monitoring system uses power factory DIgSILENT software as an analytical tool for calculating real-time security in engine mode. This software has the online data transfer capability with the real-time applications. Hence, by implementing an interface between real-time environment and the power factory engine, we can use it in on-line form for running system analysis features such as on-line power flow, on-line contingency assessment, on-line optimal power flow, on-line fault and stability calculation, simulation of grid disturbance scenario, sensitivity analysis on grid operating conditions, tuning optimization of controllers, investigation of control structure, etc. Hardware-in-the-loop testing is also required to develop, analyze and tune control systems for any kind of turbine-generator or superimposed control systems such as a Smart Grid Controllers are the other application of the integrated fast real-time SCADA systems with on-line analysis engines [155].

When referring to security assessment, we are essentially referring to the analysis of abnormal system conditions resulted from contingency cases. Contingency analysis is defined as the evaluation of the violations in system operating states (if any) that certain contingencies can pose to the electrical power system. In other words, it is the execution and evaluation (loading and voltage-wise) of post-fault load flows, each of which reflects the outage of a single or group of elements (such as transformers, busbars, transmission lines, etc.). Figure 7.5 illustrates the general sequence of contingency assessment. The results of both pre- and post-fault load flows are compared to the specified loading and voltage limits which results are generated based on them. For online application, the computation time required to perform a contingency analysis largely depends on two factors: the size of the power system and the number of contingencies considered. 


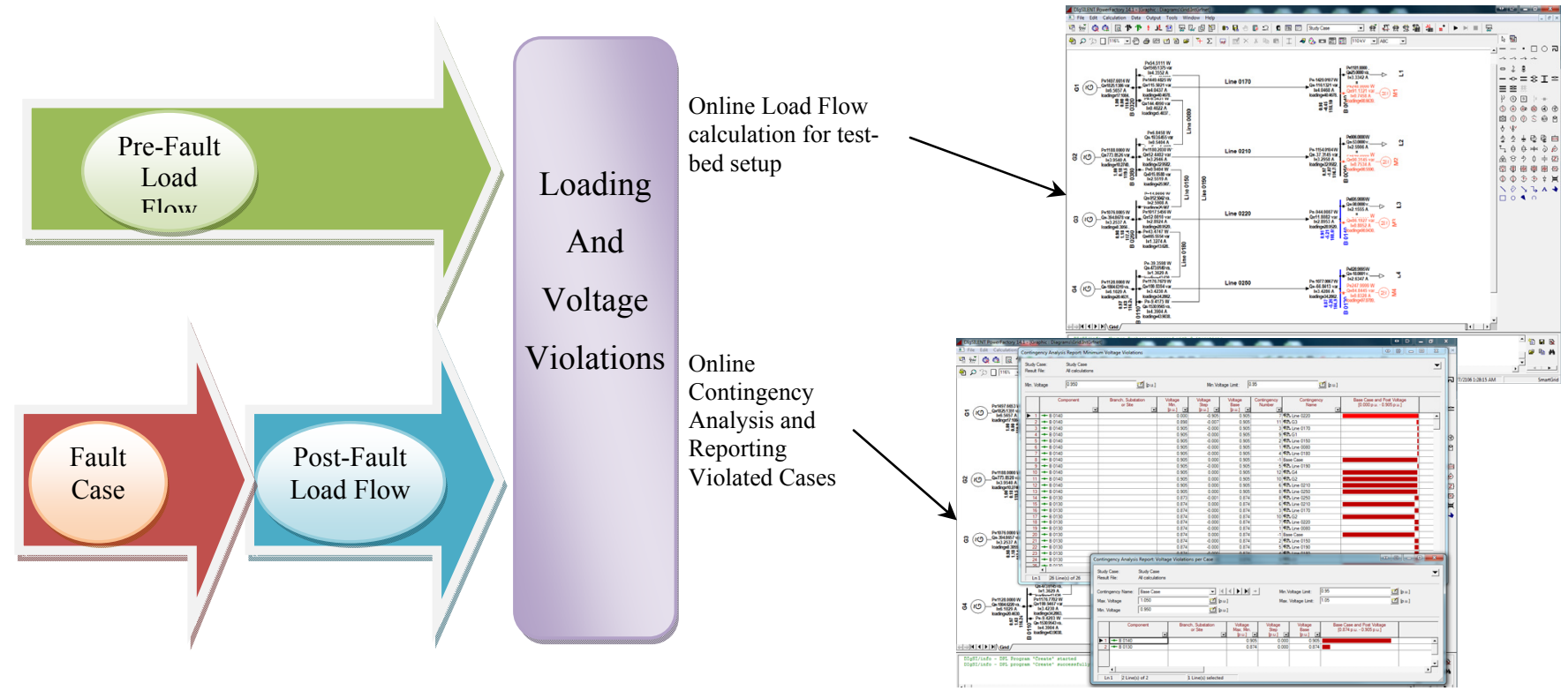

Figure 7.5: General sequence for contingency assessment and provided results for both pre- and post-fault load flows

Depending on these factors, the computation time could take from a couple of seconds (or less) up to several minutes. For this setup, all the contingency results are provided within two seconds. Therefore, by adding the real-time data update time (around a second), the system security state will be updated around three seconds.

For each security assessment analysis, the typical type of report can be generated in real-time form. Figure 7.6 shows the typical report in a tabular format. Here, Table I shows the maximum loaded component (according to the specified loading limit) for each contingency. It shows a worth case for four lines that have loading problem in different contingency cases. Table II presents all overloaded components (according to the specified loading limit) for each contingency. Table III demonstrate all voltage deviations of terminals (between the base case and the contingency case) for each contingency in a single list. 
Table I. Maximum Loading:

\begin{tabular}{|l|l|r|r|r|r|r|l|l|}
\hline & Component & $\begin{array}{c}\text { Branch, Substation } \\
\text { or Site }\end{array}$ & $\begin{array}{c}\text { Loading } \\
\text { Continuous } \\
{[\%]}\end{array}$ & $\begin{array}{c}\text { Loading } \\
\text { Short-Term } \\
{[\%]}\end{array}$ & $\begin{array}{c}\text { Loading } \\
\text { Base Case } \\
{[\%]}\end{array}$ & $\begin{array}{c}\text { Contingency } \\
\text { Number }\end{array}$ & $\begin{array}{c}\text { Contingency } \\
\text { Name }\end{array}$ & $\begin{array}{c}\text { Base Case and Continuous Loading } \\
{[\mathbf{0 . 0} \%-\mathbf{1 1 2 . 2} \% \mathbf{0}}\end{array}$ \\
\hline 1 & Line 0080 & 112.2 & 112.2 & 45.8 & 6 & Line 0210 & \\
\hline 2 & Line 0190 & & 102.9 & 102.9 & 62.1 & 2 & Line 0150 & \\
\hline 3 & Line 0150 & & 92.0 & 92.0 & 42.6 & 5 & Line 0190 & \\
\hline 4 & Line 0170 & & 81.7 & 81.7 & 78.5 & 9 & G1 & \\
\hline
\end{tabular}

Table II. Loading Violations:

\begin{tabular}{|c|c|c|c|c|c|c|c|c|}
\hline & Component & $\begin{array}{c}\text { Branch, Substation } \\
\text { or Site }\end{array}$ & \begin{tabular}{|c|} 
Loading \\
Continuous \\
{$[\%]$} \\
\end{tabular} & \begin{tabular}{|c|} 
Loading \\
Short-Term \\
{$[\%]$} \\
\end{tabular} & \begin{tabular}{|c|} 
Loading \\
Base Case \\
{$[\%]$} \\
\end{tabular} & $\begin{array}{c}\text { Contingency } \\
\text { Number }\end{array}$ & $\begin{array}{c}\text { Contingency } \\
\text { Name }\end{array}$ & $\begin{array}{c}\text { Base Case and Continuous Loading } \\
{[0.0 \%-112.2 \%]}\end{array}$ \\
\hline 1 & Line 0080 & & 112.2 & 112.2 & 45.8 & 6 & Line 0210 & \\
\hline 2 & Line 0080 & & 89.7 & 89.7 & 45.8 & 5 & Line 0190 & \\
\hline 3 & Line 0080 & & 70.6 & 70.6 & 45.8 & 4 & Line 0180 & \\
\hline 4 & Line 0190 & & 102.9 & 102.9 & 62.1 & 2 & Line 0150 & \\
\hline 5 & Line 0190 & & 99.4 & 99.4 & 62.1 & 8 & Line 0250 & \\
\hline 6 & Line 0190 & & 91.6 & 91.6 & 62.1 & 1 & Line 0080 & \\
\hline 7 & Line 0190 & & 81.7 & 81.7 & 62.1 & 6 & Line 0210 & \\
\hline 8 & Line 0190 & & 79.4 & 79.4 & 62.1 & 7 & Line 0220 & \\
\hline 9 & Line 0150 & & 92.0 & 92.0 & 42.6 & 5 & Line 0190 & \\
\hline 10 & Line 0150 & & 70.2 & 70.2 & 42.6 & 4 & Line 0180 & \\
\hline
\end{tabular}

Table III. Voltage Steps:

\begin{tabular}{|l|l|r|r|r|r|r|r|r|}
\hline & Component & $\begin{array}{c}\text { Branch, Substation } \\
\text { or Site }\end{array}$ & $\begin{array}{c}\text { Voltage } \\
\text { Step } \\
\text { [p.u.] }\end{array}$ & $\begin{array}{c}\text { Voltage } \\
\text { Base } \\
\text { [p.u.] }\end{array}$ & $\begin{array}{c}\text { Voltage } \\
\text { Min./Max. } \\
\text { [p.u.] }\end{array}$ & $\begin{array}{c}\text { Contingency } \\
\text { Number }\end{array}$ & $\begin{array}{c}\text { Contingency } \\
\text { Name }\end{array}$ & $\begin{array}{c}\text { Voltage Step } \\
\text { [0.000 p.u. - 0.038 p.u.] }\end{array}$ \\
\hline 1 & B 0040 & & 0.038 & 0.972 & 0.934 & 9 & $G 1$ & \\
\hline 2 & B 0320 & & 0.037 & 1.000 & 0.963 & 9 & $G 1$ & \\
\hline
\end{tabular}

Table IV. Maximum/Minimum Voltage

\begin{tabular}{|l|l|r|r|r|r|r|r|r|}
\hline & Component & $\begin{array}{c}\text { Branch, Substation } \\
\text { or Site }\end{array}$ & $\begin{array}{c}\text { Voltage } \\
\text { Min. } \\
\text { [p.u.] }\end{array}$ & $\begin{array}{c}\text { Voltage } \\
\text { Step } \\
\text { [p.u.] }\end{array}$ & $\begin{array}{c}\text { Voltage } \\
\text { Base } \\
\text { [p.u.] }\end{array}$ & $\begin{array}{c}\text { Contingency } \\
\text { Number }\end{array}$ & $\begin{array}{c}\text { Contingency } \\
\text { Name }\end{array}$ & $\begin{array}{c}\text { Base Case and Post Voltage } \\
\text { [0.871 p.u. - 0.972 p.u.] }\end{array}$ \\
\hline 1 & B 0130 & & 0.871 & -0.009 & 0.880 & 12 & G4 & \\
\hline 2 & B 0140 & & 0.895 & -0.018 & 0.913 & 11 & G3 & \\
\hline 3 & B 0050 & & 0.926 & -0.017 & 0.943 & 10 & G2 & \\
\hline 4 & B 0040 & & 0.934 & -0.038 & 0.972 & 9 & G1 & \\
\hline
\end{tabular}

Table V. Loading Violation per Case

\begin{tabular}{|l|l|c|c|c|c|c|}
\hline Component & $\begin{array}{c}\text { Branch, Substation } \\
\text { or Site }\end{array}$ & $\begin{array}{c}\text { Loading } \\
\text { Continuous } \\
{[\%]}\end{array}$ & $\begin{array}{c}\text { Loading } \\
\text { Short-Term } \\
{[\%]}\end{array}$ & $\begin{array}{c}\text { Loading } \\
\text { Base Case } \\
{[\%]}\end{array}$ & $\begin{array}{c}\text { Base Case and Continuous Loading } \\
{[\mathbf{0 . 0} \%-\mathbf{7 8 . 5} \%]}\end{array}$ \\
\hline 1 & Line 0170 & & 78.5 & 78.5 & 78.5 & \\
\hline
\end{tabular}

Table.VI. Maximum/Minimum Voltage Violation

\begin{tabular}{|l|l|r|r|r|r|r|r|r|}
\hline & Component & $\begin{array}{c}\text { Branch, Substation } \\
\text { or Site }\end{array}$ & $\begin{array}{c}\text { Voltage } \\
\text { Min. } \\
\text { [p.u.] }\end{array}$ & $\begin{array}{c}\text { Voltage } \\
\text { Step } \\
\text { [p.u.] }\end{array}$ & $\begin{array}{c}\text { Voltage } \\
\text { Base } \\
\text { [p.u.] }\end{array}$ & $\begin{array}{c}\text { Contingency } \\
\text { Number }\end{array}$ & $\begin{array}{c}\text { Contingency } \\
\text { Name }\end{array}$ & $\begin{array}{c}\text { Base Case and Post Voltage } \\
\text { [0.000 p.u. - 0.972 p.u.] }\end{array}$ \\
\hline 1 & B 0040 & & 0.000 & -0.972 & 0.972 & 3 & Line 0170 & \\
\hline 2 & B 0040 & & 0.934 & -0.038 & 0.972 & 9 & G1 & \\
\hline
\end{tabular}

Table.VII. Voltage Violation per Case

\begin{tabular}{|l|l|c|r|r|r|r|}
\hline & Component & $\begin{array}{c}\text { Branch, Substation } \\
\text { or Site }\end{array}$ & $\begin{array}{c}\text { Voltage } \\
\text { Max. Min. } \\
\text { [p.u.] }\end{array}$ & $\begin{array}{c}\text { Voltage } \\
\text { Step } \\
\text { [p.u.] }\end{array}$ & $\begin{array}{c}\text { Voltage } \\
\text { Base } \\
\text { [p.u.] }\end{array}$ & $\begin{array}{c}\text { Base Case and Post Voltage } \\
\text { [0.880 p.u. - 0.943 p.u.] }\end{array}$ \\
\hline 1 & B 0050 & & 0.943 & 0.000 & 0.943 & \\
\hline 2 & B 0140 & & 0.913 & 0.000 & 0.913 & \\
\hline 3 & B 0130 & & 0.880 & 0.000 & 0.880 & \\
\hline
\end{tabular}

Figure 7.6: Contingency analysis typical reports 
Table IV reports the greatest voltage violation of a terminal (greater/less than or equal to the specified voltage limits) considering all contingencies. Any such terminal is reported only once (i.e. it is reported for the contingency causing this violation). Table $\mathrm{V}$ demonstrate all voltage violations of a terminal (greater/less than or equal to the specified upper/lower voltage limit) considering all contingencies. Table VI reports all voltage violations of a terminal (greater than or equal to the specified upper voltage limit) considering all contingencies. Finally, table VII presents all bus-bars with exceeding voltage (maximum or minimum) in separate lists at.

This chapter presented a brief summary of the critical research needs to meet the vast scope of challenges in real-time security analysis to ensure reliable and secure grid operation for the futuristic smart grid. A related issue is the availability of real-time data from PMUs all over the power system; any inaccessibility of fast real-time data may affect the feasibility of WAMPAC for fast data monitoring and analysis. The purpose of automatic control is to automatically keep the power system within normal operating conditions. The purpose of security monitoring is to look ahead for possible contingencies that may transition the power system from normal operation state to the emergency state. Fast computation is intensively effective for monitoring the security of the large interconnected system in order to apply remedies in proper time. Since the problems caused by a contingency can range from the simple overloading of a line to the various complicated instability phenomena, identifying, categorizing and ranking the security problems on a continuing basis will require considerable attempts. 


\subsection{Voltage Stability Monitoring System on Smart Grid}

The voltage stability refers to the ability of the system to maintain load bus voltages within acceptable limit, following some disturbances or power demand changes [120]. The voltage stability problem normally starts as a local phenomenon and then extends to power system if no remedies are taken into action. Preventing the system voltage at every load center from passing the marginally stable point is an effective way to avoid voltage collapse. This margin, which is called critical point, is reached by maximum deliverable power by the transmission systems and hence is mostly characterized by transmission line parameters and can be achieved by obtaining the maximum deliverable power to each load center.

They are presenting the proximity of the system to voltage collapse. By comparison and verification of those indices in real power system the better sight of those indices will be achieved in order to specify security margin and take proper remedial action. Hence, here some online voltage stability indices will be formulated for a simple two bus power system and analytical results by simulation and real-time implementation will be provided.

\subsection{Voltage Stability Indices}

Voltage stability indices have been developed in order to determine the point of voltage instability and describe how the system is close to voltage instability. Furthermore, it can used to recognize weak buses and the high risky areas involved for voltage instability. There are several methods to calculate these indices in real-time format by using PMUs data. 


\subsubsection{Line Stability Index}

Reference [121] proposes an overall system stability index based on power flow concept. According to figure 5.3, for a transmission line which is equipped with two PMUs at both sides in order to measure voltage and current synchrophasors in sending and receiving ends, the parameters have been shown. By neglecting the line admittance we can write:

$$
I=\frac{V_{s} \angle \delta_{s}-V_{r} \angle \delta_{r}}{R+j X}=\frac{V_{s} \angle 0-V_{r} \angle \delta}{R+j X}
$$

Where $\delta=\delta_{S}-\delta_{r}$ and if $\delta_{S}=0$ then $\delta=-\delta_{r}$ so:

$$
S_{r}=V_{r} I^{*} \Rightarrow I=\left(\frac{S_{r}}{V_{r}}\right)^{*}=\frac{P_{r}-j Q_{r}}{V_{r} \angle \delta}=\frac{V_{s} \angle 0-V_{r} \angle-\delta}{R+j X}
$$

equalizing real and imaginary part in this equation leads to:

$$
\left\{\begin{array}{l}
V_{r} V_{S} \cos (\delta)-V_{r}^{2}=R P_{r}+X Q_{r} \\
V_{r} V_{S} \sin (\delta)=X P_{r}-R Q_{r}
\end{array}\right.
$$

solving this equations results to:

$$
V_{r}^{2}-\left(\frac{R}{X} \sin (\delta)-\cos (\delta)\right) V_{S} V_{r}+\left(\frac{X^{2}+R^{2}}{X}\right) Q_{r}=0
$$

considering line series impedance angle as $\theta=\arctan \left(\frac{X}{R}\right)$ in (10.4) we will reach to:

$$
\sin (\theta) V_{r}^{2}+\sin (\theta-\delta) V_{s} V_{r}+\frac{X}{\sin (\theta)} Q_{r}=0
$$

In order to have the real values for $V_{r}$ the discriminant of the quadratic equation should be greater or equal to zero and hence: 


$$
V_{s}^{2} \sin ^{2}(\theta-\delta)-4 X Q_{r} \geq 0
$$

Finally, the line stability index can be defined as equation 8.7 and the value of this index should remain under 1 for the stable system and whenever it exceed from that value system will lose the stability and the voltage collapse will occur.

$$
L_{m n}=\frac{4 X Q_{r}}{\left[V_{S} \sin (\theta-\delta)\right]^{2}}
$$

\subsubsection{Fast Voltage Stability Index}

This index is proposed in [122], to detect voltage instability situation in power transmission system. The mathematical formulation is very simple that could speed up the computation and hence referred as fast index. Finding positive discriminant of the quadratic equation in (8.4) results to:

$$
\left[\left(\frac{R}{X} \sin (\delta)-\cos (\delta)\right) V_{s}\right]^{2}-4\left(\frac{X^{2}+R^{2}}{X}\right) Q_{r} \geq 0
$$

If $\delta \rightarrow 0$, then $\sin \delta \approx 0, \cos \delta \approx 1$, hence:

$$
F V S I=\frac{4 Z^{2} Q_{r}}{{V_{S}}^{2} X}
$$

The measured value of FVSI which is close to 1 indicates that the particular line is close to its instability point which may lead to voltage collapse in the entire system. To keep the system under the normal condition this value should be maintained less than 1 .

\subsubsection{Line Stability Factor}

According to Ref. [123], the line stability factor is based on load flow concept and system topology. This factor begins by modeling transmission lines just with series 
reactance and hence, it is proper for systems with $R / X<1$. The sending end active power and receiving can be achieved by:

$$
\left\{\begin{array}{l}
P_{s}=\frac{V_{s} V_{r}}{X} \sin (\delta) \\
Q_{r}=-\frac{V_{r}^{2}}{X}+\frac{V_{s} V_{r}}{X} \cos (\delta)
\end{array}\right.
$$

eliminating $\delta$ in both equations leads to:

$$
\left(\frac{X P_{s}}{V_{s} V_{r}}\right)^{2}+\left(\frac{V_{r}^{2}+X Q_{r}}{V_{s} V_{r}}\right)^{2}=1
$$

solving this equation versus $V_{r}^{2}$ results to a new quadratic equation:

$$
\left(V_{r}^{2}\right)^{2}-\left(V_{s}^{2}-2 X Q_{r}\right)\left(V_{r}^{2}\right)+X^{2}\left(Q_{r}^{2}+P_{s}^{2}\right)=0
$$

the discriminant of this quadratic equation should be positive:

$$
\left(V_{s}^{2}-2 X Q_{r}\right)^{2}-4 X^{2}\left(Q_{r}^{2}+P_{s}^{2}\right) \geq 0
$$

solution for this inequality is:

$$
4\left(\frac{X}{V_{s}^{2}}\right)\left(Q_{r}+\frac{X}{V_{s}^{2}} P_{s}^{2}\right) \leq 1
$$

Therefore, this index can be defined as equation 7.15 and should be kept under 1 during the system normal and stable operation.

$$
L Q P=4\left(\frac{X}{V_{s}^{2}}\right)\left(\frac{X}{V_{s}^{2}} P_{s}^{2}+Q_{r}\right)
$$




\subsubsection{Line Voltage Stability Index}

An on-line voltage stability index is based on pi equivalent branch model for power transmission line and the relationship between voltage and active power and it is formulated by using equation 7.3 and elimination $Q_{r}$ concludes:

$$
V_{r}^{2}-\left(\cos \delta+\frac{X}{R} \sin \delta\right) V_{s} V_{r}+\left(\frac{X^{2}+R^{2}}{R}\right) P_{r}=0
$$

considering line series impedance angle as $\theta=\arctan \left(\frac{X}{R}\right)$ in equation 7.16 we will reach to:

$$
V_{r}^{2}-\left(\cos \delta+\frac{\sin \theta}{\cos \theta} \sin \delta\right) V_{s} V_{r}+\left(\frac{X^{2}+R^{2}}{R}\right) P_{r}=0
$$

and then:

$$
\cos (\theta) V_{r}^{2}-\cos (\theta-\delta) V_{s} V_{r}+\left(\frac{R}{\cos (\theta)}\right) P_{r}=0
$$

the discriminant of this quadratic equation should be positive:

$$
\left[\cos (\theta-\delta) V_{s}\right]^{2}-4 R P_{r} \geq 0
$$

Hence the on-line voltage stability can be defined as:

$$
L_{V S I}=\frac{4 P_{r} R}{\left[V_{s} \cos (\theta-\delta)\right]^{2}}
$$

This index presents the ratio of the current branch receiving active power and the limited transmit power and its value should be under 1 [123].

\subsubsection{Linear Voltage Stability Indicator}

Reference [124] proposed a new index for monitoring and assessment of static voltage stability using a unique technique based on underlying tool for evaluation of 
voltage stability margins. The mathematical procedure can be followed by this paper to reach this index:

$$
L V S I=\frac{\sin \theta\left(1-\sqrt{1-\frac{4 P_{r} R}{V_{s}^{2}}}\right)+\cos \theta\left(1-\sqrt{1-\frac{4 Q_{r} X}{V_{s}^{2}}}\right)}{\sin \theta+\cos \theta-1}
$$

Voltage collapse will occur when LVSI approaches unity.

\subsubsection{The dS/dY Voltage Stability Sensitivity Factor}

This index in reference [125] is based on the same two-bus equivalent system.

The power margin in this case will be achieved by calculating $\Delta S=I Z_{\text {load }}^{2}-I Z_{\text {line when we }}^{2}$ neglect the shunt admittance for transmission line then $I=I_{s}=I_{r}$ and representing the extra apparent power that can be delivered to the load prior voltage collapse occurrence. The ratio of power supplied by the source to the power consumed at the load rapidly increases as the system approach voltage collapse. The apparent power of load side can be archived by equation 7.22 :

$$
S=V_{r}^{2} Y_{\text {load }}=\frac{V_{s p u}^{2} Z_{\text {load }}}{Z_{\text {line }}^{2}+Z_{\text {load }}^{2}+2 Z_{\text {line }} Z_{\text {load }} \cos (\theta-\varphi)}
$$

where $\varphi$ is the phase angle of the load impedance. Hence, this index is defined by equation 7.23:

$$
d S / d Y=\frac{V_{s \text { pu }}^{2} \times\left(1-Y_{\text {load }}^{2} Z_{\text {line }}^{2}\right)}{\left[1+Y_{\text {load }}^{2} Z_{\text {line }}^{2}+2 Y_{\text {load }} Z_{\text {line }} \cos (\theta-\varphi)\right]^{2}}
$$


If $Z_{\text {load }}=Z_{\text {line }}$ then $d S / d Y=0$, it confirms the well-known critical point of voltage instability. At no load when $Y=0$, the $d S / d Y=1$. Therefore, maximum loading point can be monitored accurately.

\subsubsection{Online Voltage Stability Index}

The online voltage stability index (VSI) is proposed in [126]. This index can be calculated in real-time and predicts the power system steady-state voltage stability limit. The method to calculate this index starts by deriving three maximum transferrable powers including active, reactive and apparent power for each branch. So, the receiving end side voltage is derived from equation 8.3 and presented in equation 8.24.

$$
V_{r}=\sqrt{\frac{V_{s}^{2}}{2}-\left(Q_{r} X+P_{r} R\right) \pm \sqrt{\frac{V_{s}^{4}}{4}-\left(Q_{r} X+P_{r} R\right) V_{s}^{2}-\left(P_{r} X-Q_{r} R\right)^{2}}}
$$

The maximum transferable power, $S_{\max }$, through the transmission line has been achieved when the internal root phrase equals to zero [94]. There is only one possible solution for $V_{s}$ and the receiving end voltage, $V_{r}$, is at the marginally stable operating point. The maximum transferred active power, $P_{\max }$, maximum transmitted reactive power, $Q_{\max }$ and the maximum transferable power, $S_{\max }$, can be calculated by Eqns. (8.26)-(8.28) where $\varphi=\arctan (X / R)$ and all right side parameters are assumed to be constant. These equations can be simplified by assuming high $\mathrm{X}$ to $\mathrm{R}$ ratio.

$$
\begin{aligned}
& \frac{V s^{4}}{4}-\left(Q_{r} X+P_{r} R\right) V s^{2}-(P X-Q R)^{2}=0 \\
& P_{\max }=\frac{Q_{r} R}{X}-\frac{V_{s}^{2} R}{2 X^{2}}+\frac{|Z| V_{s} \sqrt{V_{s}^{2}-4 Q_{r} X}}{2 X^{2}}
\end{aligned}
$$




$$
\begin{aligned}
& Q_{\max }=\frac{P_{r} X}{R}-\frac{V_{s}^{2} X}{2 R^{2}}+\frac{|Z| V_{s} \sqrt{V_{s}^{2}-4 P_{r} R}}{2 R^{2}} \\
& S_{\max }=\frac{V_{s}^{2}\{|Z|-(X \sin \varphi+R \cos \varphi)\}}{2(X \cos \varphi-R \sin \varphi)^{2}}
\end{aligned}
$$

Therefore, according to these relations, the total Voltage stability Index (VSI) can be calculated as [97]:

$V S I=\min \left\{\frac{P_{\max }-P}{P_{\max }}, \frac{Q_{\max }-Q}{Q_{\max }}, \frac{S_{\max }-S}{S_{\max }}\right\}$

Smaller values of VSI indicates that the receiving end bus is close to its voltage stability margin and less power transferring margin is left and in its margin the VSI will be equal zero.

\subsubsection{Transmission Line Marginal Loading}

The line operation curve is the well-known PV-curve which shows voltage of the receiving end bus versus transferred active power and it is characterizes by an extreme point as a vertex of the parabola. For a specific load impedance angle, this point will be achieved by increasing the load in continuous load flow calculation and monitor the load bus voltage. According to [114] and figure 8.1 for a pi model line, the receiving end voltage can be achieved by (7.30) and be summarized by (7.31).

$$
\begin{aligned}
\vec{V}_{r}=\vec{V}_{s} \frac{\frac{1}{\frac{1}{\vec{Z}_{l}}+j \omega \frac{C}{2}+\frac{G}{2}}}{R+j \omega L+\frac{1}{\frac{1}{\vec{Z}_{l}}+j \omega \frac{C}{2}+\frac{G}{2}}} \\
\vec{V}_{r}=\vec{V}_{s} \frac{\vec{Z}_{l}}{\vec{Z}_{l}\left[1+\frac{R G}{2}+j \omega\left(\frac{R C}{2}+\frac{L G}{2}\right)-\omega^{2} \frac{L C}{2}\right]+R+j \omega L}
\end{aligned}
$$


Here $\vec{Z}_{l}$ is the load impedance. Simplification can be applied by defining a new phasor, $\vec{K}$ :

$$
\vec{K}=1+\frac{1}{2}\left(R G-\omega^{2} L C+j \omega(R C+L G)\right)
$$

The resulting magnitude of the voltage at the line receiving end is calculated as:

$$
V_{r}=\frac{Z_{l} V_{s}}{\sqrt{\left(Z_{l} K \cos \left(\varphi_{Z l}+\varphi_{k}\right)+R\right)^{2}+\left(Z_{l} K \sin \left(\varphi_{Z l}+\varphi_{k}\right)+\omega L\right)^{2}}}
$$

Which $\varphi_{Z l}=\delta_{r}-\theta_{r}$ is the load angle and $K$ is the magnitude of $\vec{K}$ and $\varphi_{k}$ is the phase angle of $\vec{K}$. The definition of active power is made through the definition of apparent power at the receiving end by:

$$
\vec{S}_{r}=\vec{V}_{r} \cdot \vec{I}_{r}^{*}, \vec{I}_{r}=-\frac{\vec{V}_{r}}{\vec{Z}_{l}} \Rightarrow S_{r}=-\frac{V_{r}^{2}}{Z_{l}} \cdot e^{j \varphi_{Z l}}=\frac{V_{r}^{2}}{Z_{l}} \cdot e^{j\left(\varphi_{Z l}+\pi\right)}
$$

Insertion of (10.33) to (10.34) yields:

$$
S_{r}=\frac{Z_{l} V_{s}^{2}}{\left(Z_{l} K \cos \left(\varphi_{Z l}+\varphi_{k}\right)+R\right)^{2}+\left(Z_{l} K \sin \left(\varphi_{Z l}+\varphi_{k}\right)+\omega L\right)^{2}} \cdot e^{j\left(\varphi_{Z l}+\pi\right)}
$$

The real part of $(10.35)$ is the active power flow at the receiving end, expressed as a function of the load impedance.

$$
P_{r}=\frac{Z_{l} V_{s}^{2}}{\left(Z_{l} K \cos \left(\varphi_{Z l}+\varphi_{k}\right)+R\right)^{2}+\left(Z_{l} K \sin \left(\varphi_{Z l}+\varphi_{k}\right)+\omega L\right)^{2}} \cos \left(\varphi_{Z l}+\pi\right)
$$

Replacing load impedance with $Z_{l}=-\frac{V_{r}^{2} \cos \varphi_{Z l}}{P_{r}}$ and solving for $V_{r}$ leads to:

$$
V_{r}^{4}-2 V_{r}^{2}\left[-\frac{V_{s}^{2}}{2 K^{2}}+\frac{P_{r}}{\cos \varphi_{Z l} K}\left[R \cos \left(\varphi_{Z l}+\varphi_{k}\right)+\omega L \sin \left(\varphi_{Z l}+\varphi_{k}\right)\right]\right]+\frac{P_{r}^{2} Z_{\text {line }}^{2}}{\cos ^{2} \varphi_{Z l} K^{2}}=0
$$

The solution of (8.37) after re-substitution for the receiving end voltage which is a 
absolute value is found:

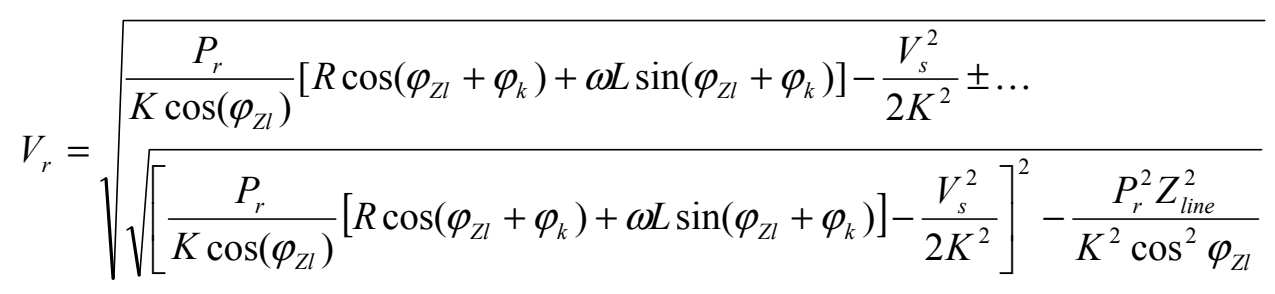

In order to have a real value for $V_{r}$ the internal square-root term should be positive and hence:

$$
\left[\frac{P_{r}}{K \cos \left(\varphi_{Z l}\right)}\left[R \cos \left(\varphi_{Z l}+\varphi_{k}\right)+\omega L \sin \left(\varphi_{Z l}+\varphi_{k}\right)\right]-\frac{V_{s}^{2}}{2 K^{2}}\right]^{2} \geq \frac{P_{r}^{2} Z_{\text {line }}^{2}}{K^{2} \cos ^{2} \varphi_{z l}}
$$

Therefore, the voltage stability index in this case can be defined as (8.40) and for stability purpose it should be kept under a unity.

$$
T V S I=\frac{Z_{\text {line }}}{R \cos \left(\varphi_{Z l}+\varphi_{k}\right)+X \sin \left(\varphi_{Z l}+\varphi_{k}\right)-\frac{V_{s}^{2} \cos \varphi_{Z l}}{2 K P_{r}}} \leq 1
$$

At the vertex point the stability index is 1 and we can calculate the vertex point coordination for voltage which can be termed as $V_{r \min }$ in (7.38). Hence, regard to the stability of the transfer mode the maximum transferable active power and reactive power by a corresponding minimal acceptable voltage value, for a stable transmission of the electric energy can be calculated by equations 7.42 and 7.43 .

$$
\begin{aligned}
& V_{r \text { min }}=\sqrt{\frac{P_{r \max }}{K \cos \left(\varphi_{Z l}\right)}\left[R \cos \left(\varphi_{Z l}+\varphi_{k}\right)+\omega L \sin \left(\varphi_{Z l}+\varphi_{k}\right)\right]-\frac{V_{s}^{2}}{2 K^{2}}} \\
& P_{r \text { max }}=\frac{V_{s}^{2} \cos \varphi_{Z l}}{2 K\left(R \cos \left(\varphi_{Z l}+\varphi_{k}\right)+\omega L \sin \left(\varphi_{Z l}+\varphi_{k}\right)+Z_{\text {line }}\right)} \\
& Q_{r \text { max }}=\frac{V_{s}^{2} \sin \varphi_{Z l}}{2 K\left(R \cos \left(\varphi_{Z l}+\varphi_{k}\right)+\omega L \sin \left(\varphi_{Z l}+\varphi_{k}\right)+Z_{\text {line }}\right)}
\end{aligned}
$$




\subsection{Comparing Voltage Stability Indices By Simulation and Experiment}

In this research the mentioned indices are measured in laboratory scale test-bed power system which is developed in Smart Grid Test-bed. Figure 7.7 shows the single line diagram of a small test setup in order to implement voltage stability indices in realtime platform. This figure also demonstrate overall view of laboratory test setup consist of a generation station, three buses with five switches, two line model and passive and motor loads. The whole voltage stability indices defined at previous section are developed in LabVIEW software which has all voltages and currents available from PMUs. Hence, for transmission line the voltages and currents phasors at both side of the line are available and for the pi-model of the line the line parameters can be achieved by using Eqns. (2.3)-(2.5). Therefore, not only we can track line parameters during the operation of power system in real-time to monitor the line situation, but also we can use more realistic line parameters to calculate voltage stability indices which is not possible by simulation software. For the line models that we used at laboratory, the line parameters are varying by current changes through the line.

\subsubsection{Voltage Stability Indices in Steady State}

The first experiment is to see stability indices behavior in steady state situation and verify them by simulation result. For this purpose, the fix amount of load has been used at the end of the line and has been shown in figure 7.8. The load model is constant impedance and the power factor is 0.87 by this load level. The PMUs provide the phasors of the sending end side positive sequence voltage which is $114.86 \angle 0.65^{\circ} \mathrm{V}$ and the current which is $3.45 \angle-10.3^{\circ} \mathrm{A}$ in steady state. Similarly, the positive voltage and current in receiving end side are $112.02 \angle 0.7^{\circ} \mathrm{V}$ and $3.66 \angle-28.24^{\circ} \mathrm{A}$. 

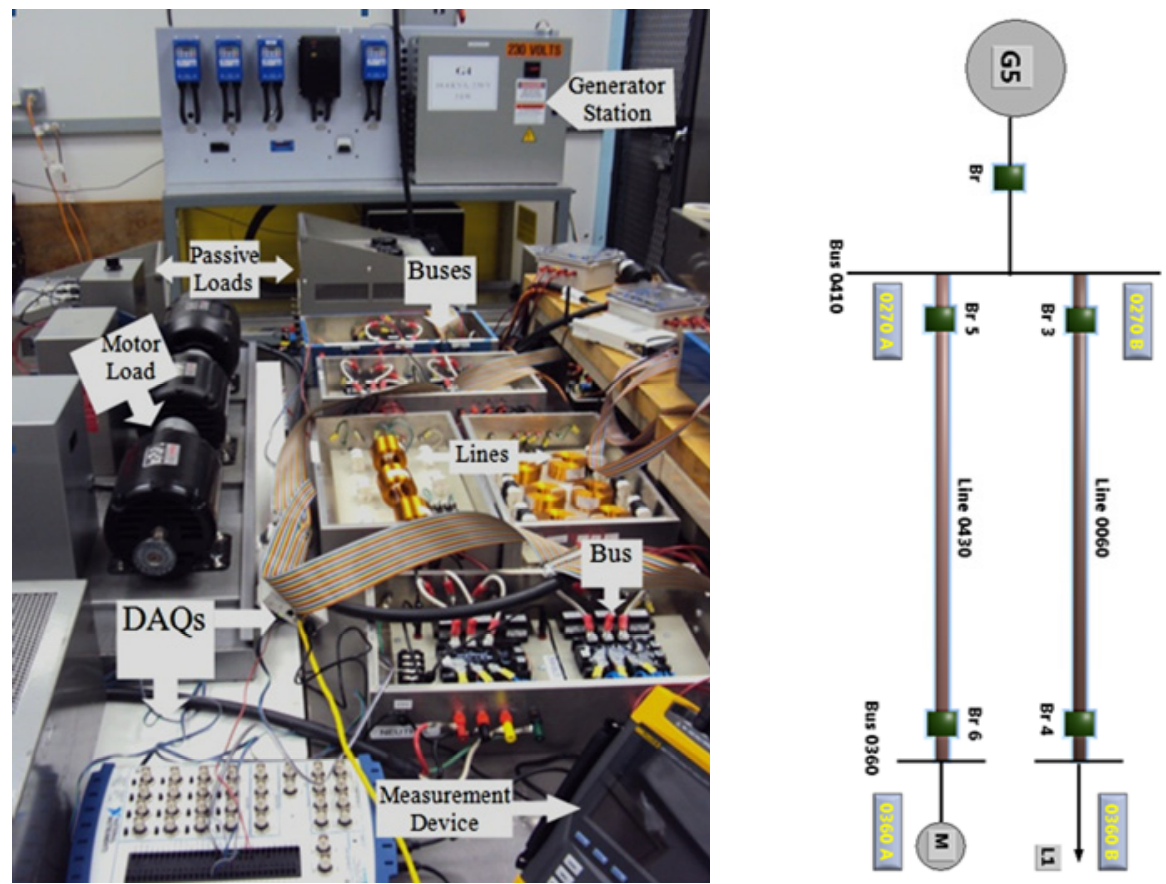

Figure 7.7: Single Line Diagram and the test Setup for implementing Voltage Stability Indices

Figure 7.9 shows the calculated indices for voltage stability presented at first section. For verification of these results, the simulation of this system has been done on power factory DIgSILENT environment and steady state results for the same amount of load during the first two second of the simulation verifies the experiment results.
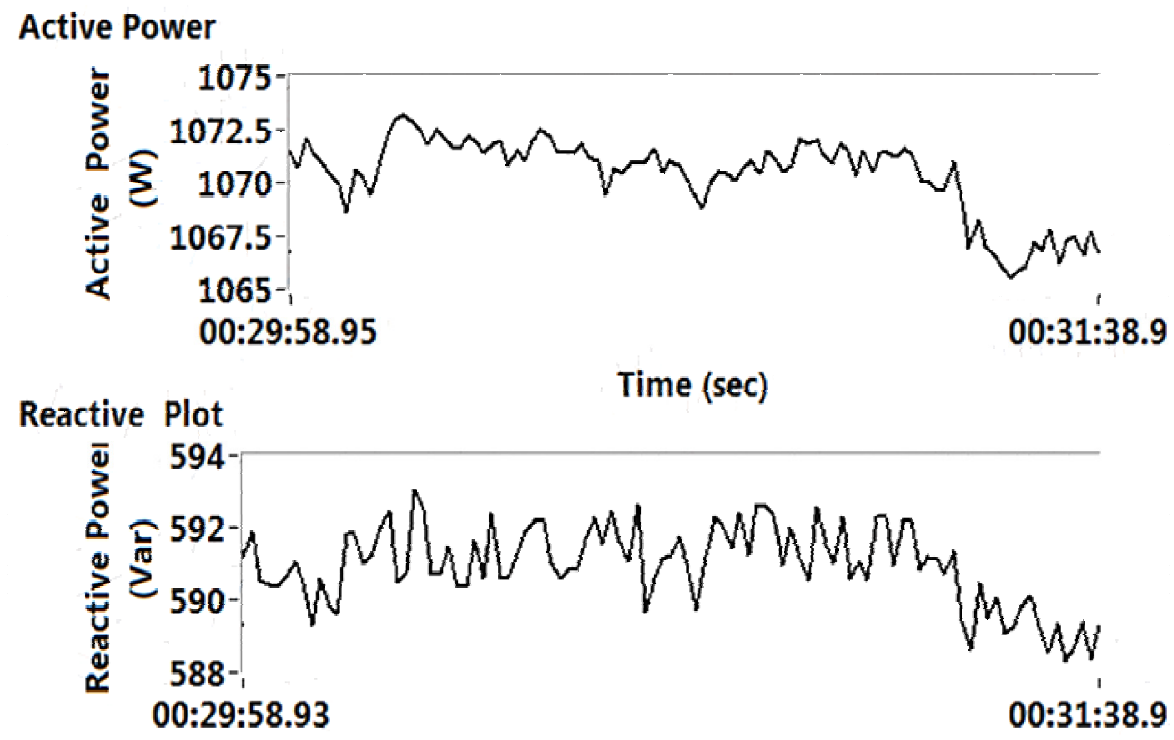

Time (sec)

Time (sec)

Figure 7.8: Reciving end active and reactive load power 


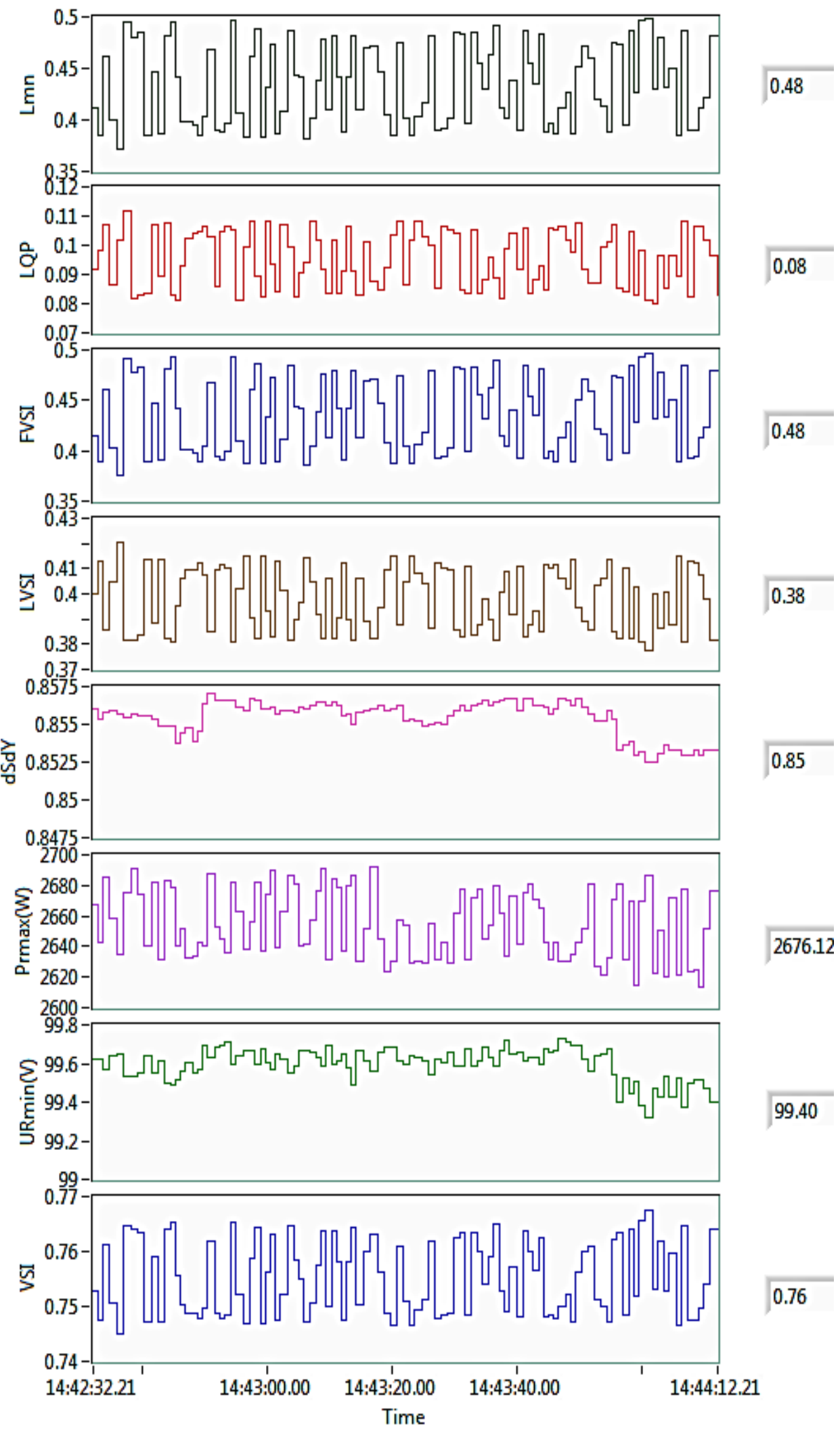

Figure 7.9: Voltage stability indices in steady state 


\subsubsection{Voltage Stability Indices during Dynamic Load Change}

The experiment of load increasing in several steps has also been accomplished and the more realistic results have been achieved. Figure 7.10 presents the active and reactive power changes during the experiment. Technically, the source side voltage which is supposed to be constant in simulation, now in the experiment is varying because of the current increment and the source side series impedance, we will have voltage drop in source side, which is shown at figure 7.11. In addition, the line parameters at the Laboratory built-in model are varying by current increase and then the exact line parameters in online format are calculated in order to measure stability indices. The line parameter results which developed by Eqns. (2.4) and (2.5) are shown in figure 7.12 and it shows the considerable change in series impedance during current increasing through the line.

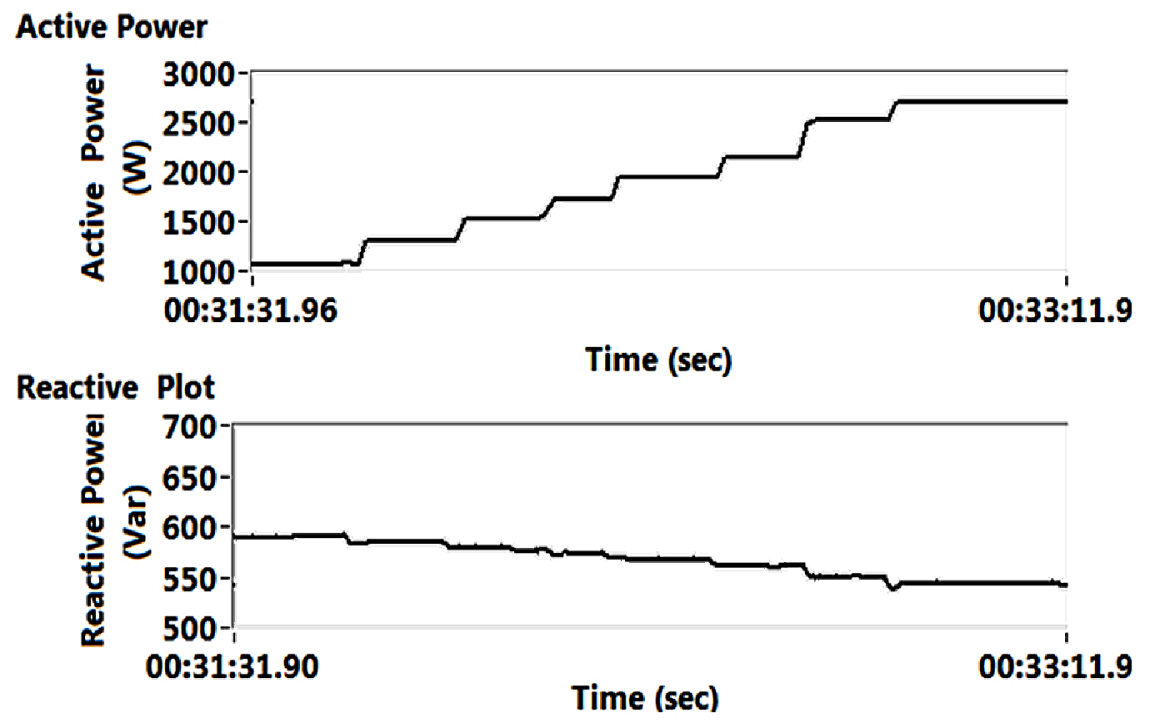

Figure 7.10: Experimental results of load active and reactive power 


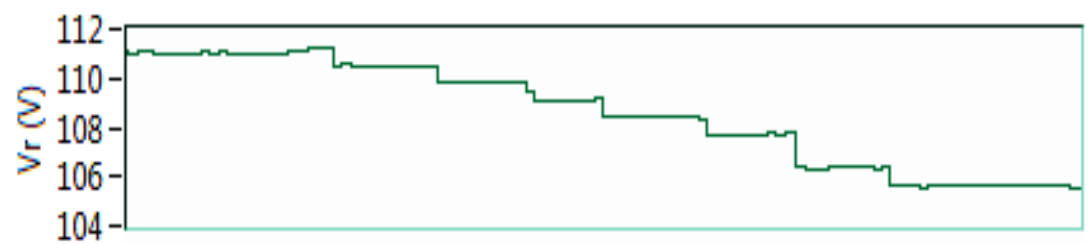

105.53

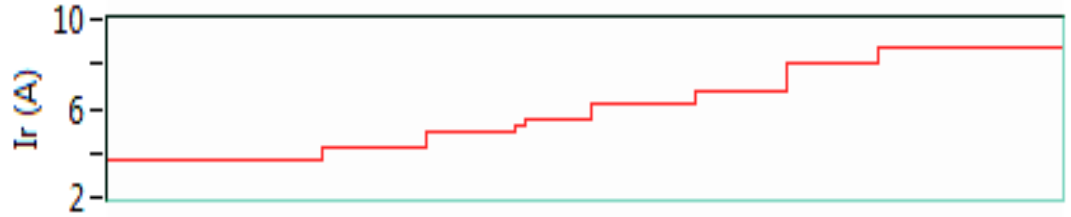

8.68
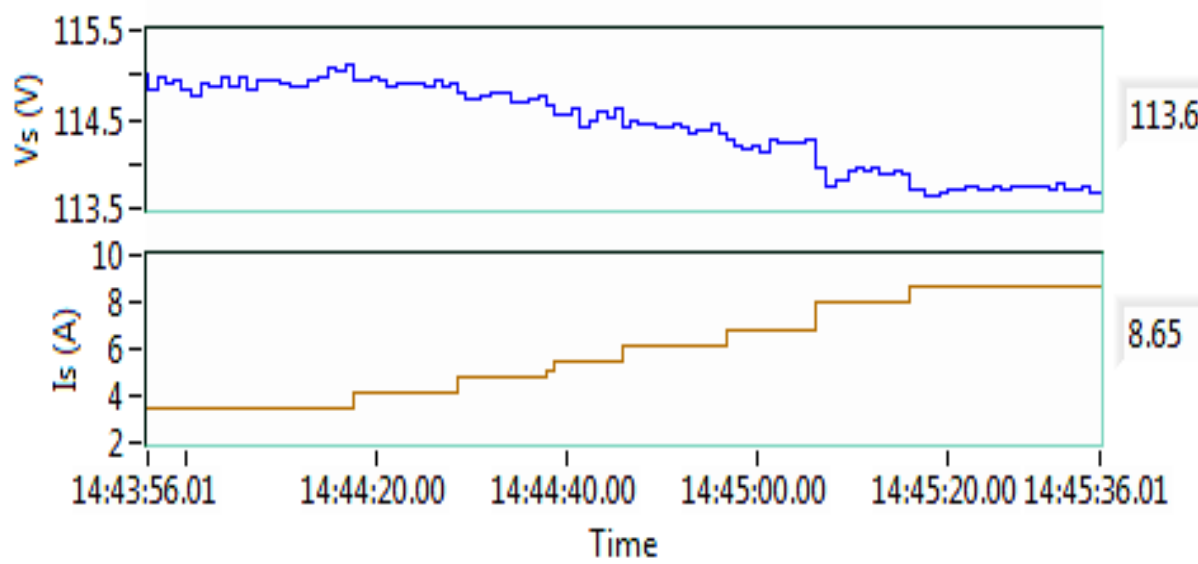

Figure 7.11: Experimental results of two sides voltage and current amplitude
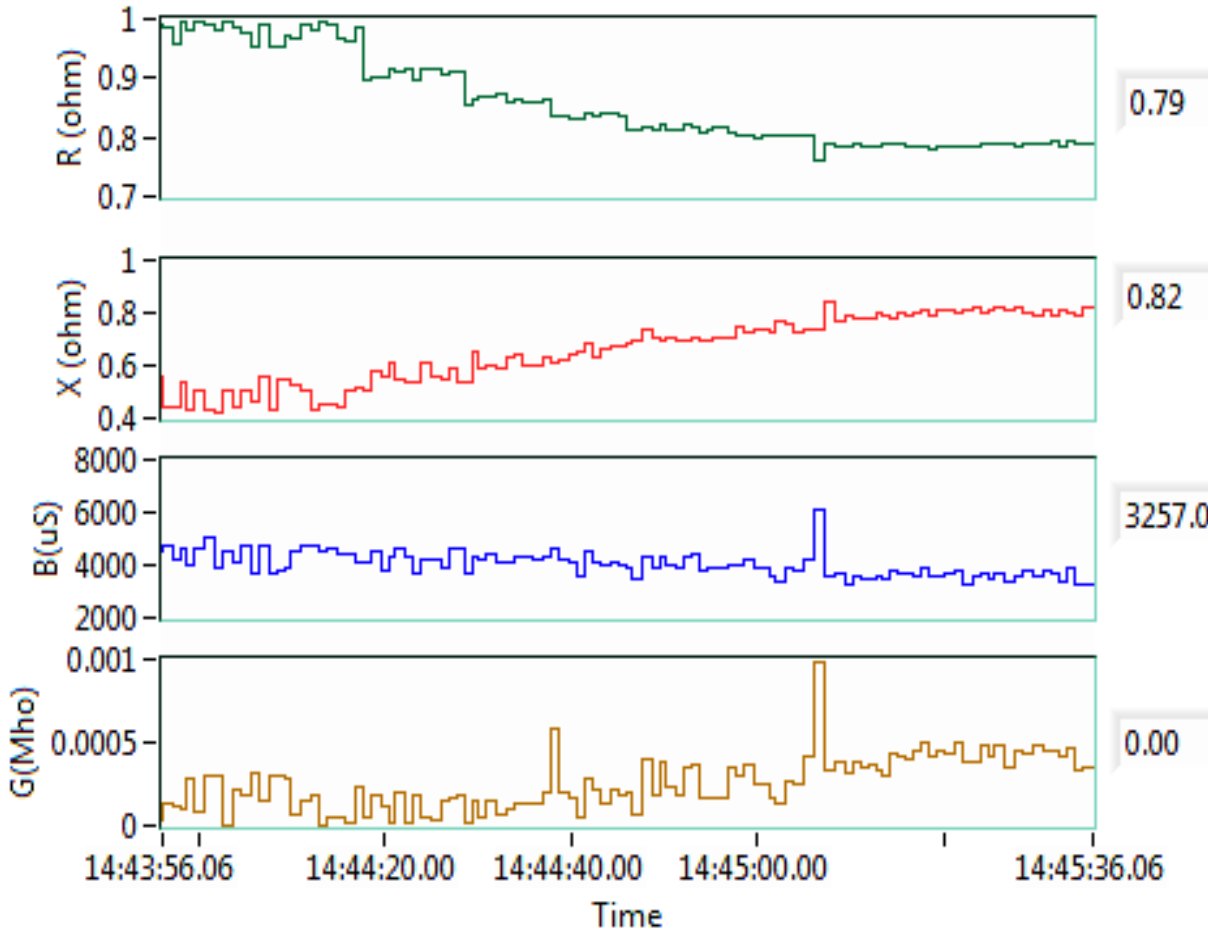

Figure 7.12: Experimental results of line parameters 
Consequently, because of the mentioned points it is expected that voltage stability indices be different from the simulation in values, but they should present the same behavior which is demonstrated at figure 7.13 for experimental circuit. By monitoring these indices, one can conclude about the proper index in order to monitor system stability state and prevent the instability situation by making proper decision whenever the calculated security margin indicates the voltage instability. In addition figure 7.14 shows the simulation results for the same load increment by using constant line parameters. The comparison between two figures indicates the same variation behavior and verifies the results.

The active power load increasing has the inverse effect on voltage stability in tie line system. Hence, all the indices should indicate the decrease in stability margin. By reviewing the experimental results, one can conclude:

- The index $L_{m n}$ is mostly depends on reactive power of the receiving end, so its variation shows the stability improvement because of the decreasing in reactive power.

- The index $L Q P$ increased from 0.1 to 0.27 . Then it doesn't present the good stability margin in this case and we don't know how far the system is from the instability point.

- The Index FVSI, similar to $L_{m n}$ is depended to reactive power and therefore it shows positive changes in stability margin.

- The LVSI shows the increment from 0.4 to 1.54 which means we lost voltage stability around $2 \mathrm{~kW}$ load which is not correct and doesn't match to the simulation results. Hence, this factor couldn't be a proper index for practical measurement. 


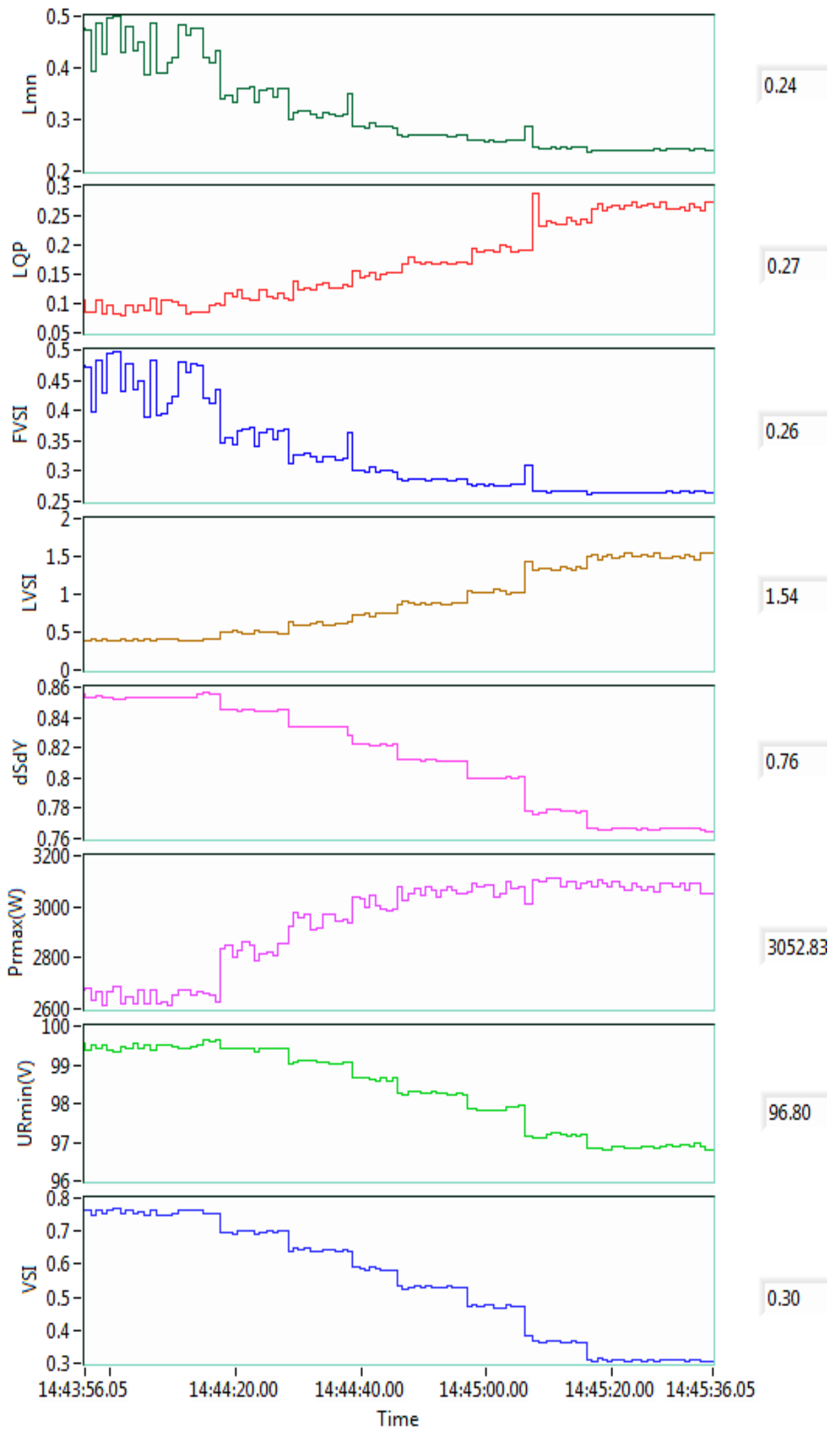

Figure 7.13: Experimental results of monitored voltage stability indices 

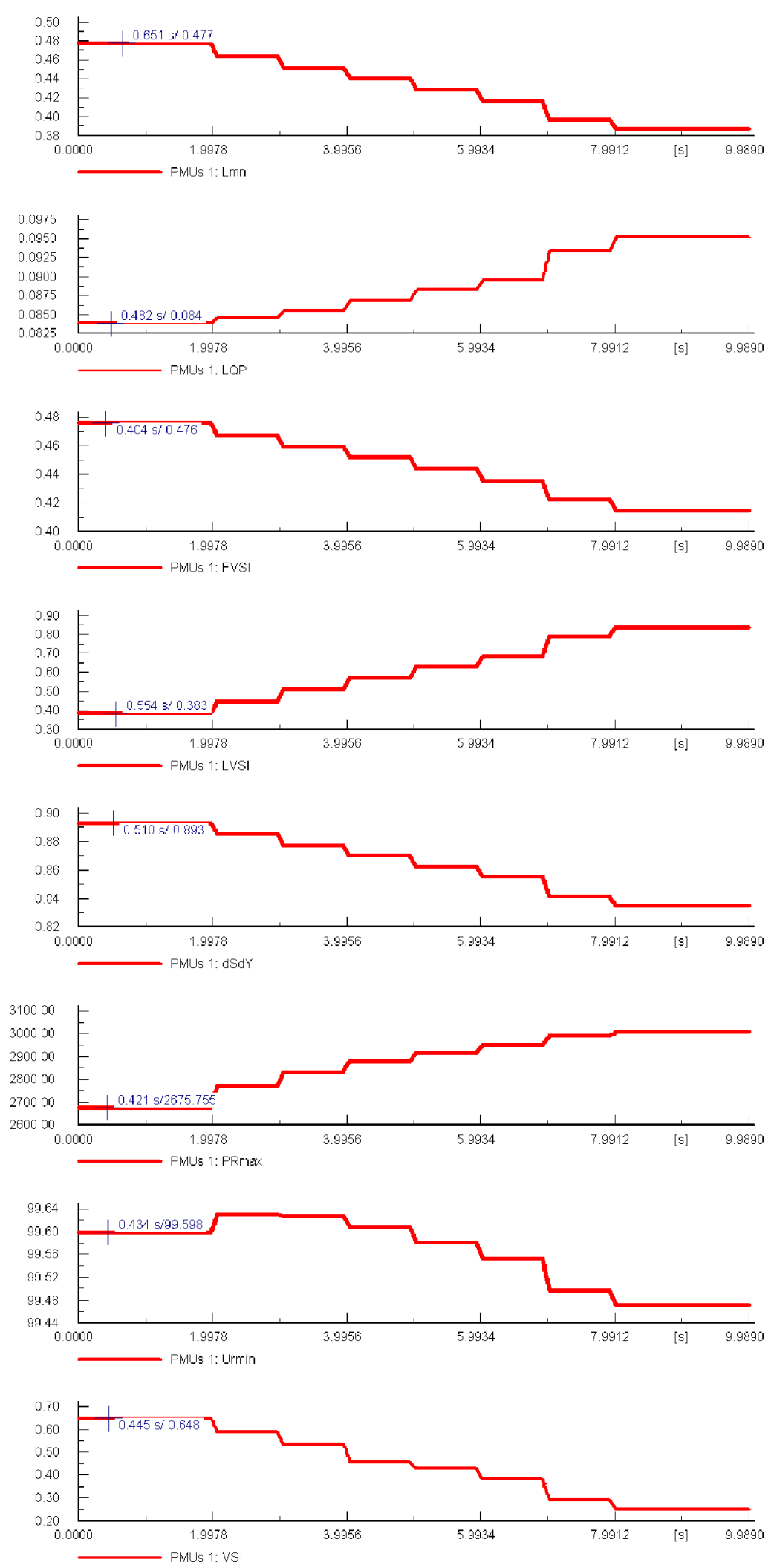

Figure 7.14: Simulation results of voltage stability indices 
- The $d S / d Y$ index shows the expected variation during load increasing, but the sensitivity of this margin is not good enough to follow critical stability point and it dropped from 0.85 to 0.76 at worst load situation.

- The $P_{r \max }$ and $U_{r \min }$ are the two useful parameters which really presents the max available margin for the voltage security after subtracting from load active power. The reason for increment of $P_{r \max }$ from 2676 to 3052 Watt is the decreasing of the reactive power because of the voltage drop at constant reactance and therefore power factor enhances. So, we can track maximum point at PV-curve in online format in the system and consequently find the available margin by subtracting it from the load active power. The margin will be decreased during the load increase.

- The VSI index is the best real-time index because of the formulation and considering all system margins including active, reactive and apparent power margins. In addition the change of this index during load increases very sensitive to system behavior and it prepares proper factor for decision making about system stability and security margin. Using PMUs in both side of the line prepares a chance to monitor real-time system stability and security margin and hence the simulation and experimental results presented in this paper, consequent that the indices formulation and implementation on wide area monitoring and control centers will provide a vary applicable view of system stability and security margin by using PMUs in wide area networks. 


\section{CHAPTER 8}

\section{ENERGY MANAGEMENT SCHEME FOR STABILITY AND SECURITY ENHANCEMENT OF HYBRID MICROGRIDS}

\subsection{Introduction}

It is expected that the rapidly growing implementation of smart grids and microgrids will continue to change current systems in terms of design and operation. New designs may include much larger local generation, storage elements, hybrid AC/DC distribution systems and more extensive involvement of power electronic converters and loads with special characteristic and time duration (pulsed loads) [160]-[163]. An example would be a shipboard power system, which resembles the concept of a microgrid operating in a smart grid system where the system is capable of self-diagnosing, selfhealing and self-reconfiguring [164], [165]. In these systems, some particular loads draw very high short time current in an intermittent fashion such as electromagnetic launch systems and free electron lasers. In future power grid architectures may include plugin electric cars in large numbers in a car park or a city and collectively may present a pulse load demand on the system. Henceforth, all these types of loads will be referred to collectively as pulsed loads [166]. Such current behavior can potentially cause the system voltage and frequency to drop in the entire microgrid, momentarily. This disturbance can trip other sensitive control loads off-line.

In the shipboard example, when a large magnitude and prolonged voltage/frequency sag occurs, the propulsion system may shut down, or perhaps the fighting loads themselves may be thrown off-line. Therefore, there is a great concern about how these loads and their existence in the same electrical environment and share 
the same energy storage systems while allowing a diverse range of operational scenarios [167], [168]. However, pulse loads are not limited to shipboard power systems as explained above. For instance, a plug-in hybrid electric vehicle (PHEV), or a group of PHEVs during their charging process, or even a large machine during its starting can be considered as pulse load in residential and industrial applications.

In this chapter, the performance of a fully-integrated microgrids power system with a pulsed power load is analyzed. It is expected that simulation may play a large role in the design process for future system architectures. Although simulation can potentially lend itself as an effective design tool, there are a number of challenges in the practical application to the design of these systems. Accurately capturing the behavior of the electrical systems often requires the use of computationally intensive transient simulations. Due to the closely coupled nature of microgrids and the potential for interactions between the many components and subsystems of which they are composed, it may be further necessary to model the entire system in great detail. Microgrids loads are characterized in a wide manner. While the majority of these loads are still in the research and development stage, it is important to understand how these loads can be integrated in the microgrid system and how they will dynamically interact with each other and with the interconnected electrical system.

The impact of pulsed power loads on microgrids power system need to be properly determined to prevent pulsed loads from causing unacceptable power deficiency, interference with other loads and degradation of overall system performance. Some pulse-loads in microgrid systems are fed directly from the prime power system. In order to evaluate the impact of these types of pulsed loads, the detailed models for prime 
mover system in the power system should be used and the existing power system standards (related to voltage transients and frequency variations) are applied.

\subsection{Stability Transient Simulation of Microgrids; Electric Ship Integrated Power System (IPS)}

The concept of an electric ship power system in many ways resembles that of a smart micro-grid where the system is capable of self-diagnosing, self-healing and selfreconfiguring. The electric ship board power system typically consists of two to four main and auxiliary generators as shown in figure 8.1. This system is described in detail in [183] and [184], in which a ship power system is modeled. The ship power plant includes two $36 \mathrm{MW}$ main generators (MTG) and two $4 \mathrm{MW}$ auxiliary generators (ATG) connected in a ring bus configuration. The bulk of the load consists of two 36.5 MW propulsion motors, modeled as induction and permanent magnet machines supplied by PWM drives, with hydrodynamic propeller models as the mechanical load. There are also ship service loads, which absorb a maximum of $4 \mathrm{MW}$. Each rectifier supplies one of two $1 \mathrm{kV}$ DC busses. DC distribution zone are simultaneously supplied by the two other rectifiers, which supply loads with different DC voltage levels by using buck DC/DC converters. Although various models for the ship service loads may be used, for this work, lumped resistive models were used in the DC network.

The simulation model employs dq-axis models for electrical machines and switching models for all converters and motor drives, along with associated controls. Generator gas turbine engines follow the model described in [185] which is proper model for system dynamic performance. In addition, a rudimentary load sharing controls were 
implemented for the generators. The gas turbine model was used for all generators governors to control the active power output of each generator. A diode rectifier pulseload charging circuit, described in [183], was connected to the DC bus by the MTG1 Bus connected rectifier and the effects of the charging event were studied across the ship. Of particular interest, as pointed out in [183], was the propagation of the effects through the DC distribution system to ATG2, which would typically be considered to be decoupled through the DC system. This propagation through the DC system was observed for a similar configuration, although relatively small. In this chapter, we concentrate on worst case effects of pulse-loads on the stability of the power system. Simulation results of pulse-load effects on shipboard power systems were considered in order to attempt to study the possible practical significance of such propagations of effects. Among all the loads, the propulsion load is of critical importance to the functioning of the ship and demands reliable power. Other loads include computers, control equipment general lighting, heating and cooling loads. Pulse-loads are the most interesting from system perspective as they have a high transient power demand during their operation. Since power quality phenomena are dominated by electromagnetic transients, harmonics and voltage sags due to short-circuit conditions or starting of large loads, they are best analyzed using a time-domain simulation model. Therefore, the time-domain model can be used to perform load flow, short-circuit, electromechanical transient stability, electromagnetic transient and harmonic analyses. 


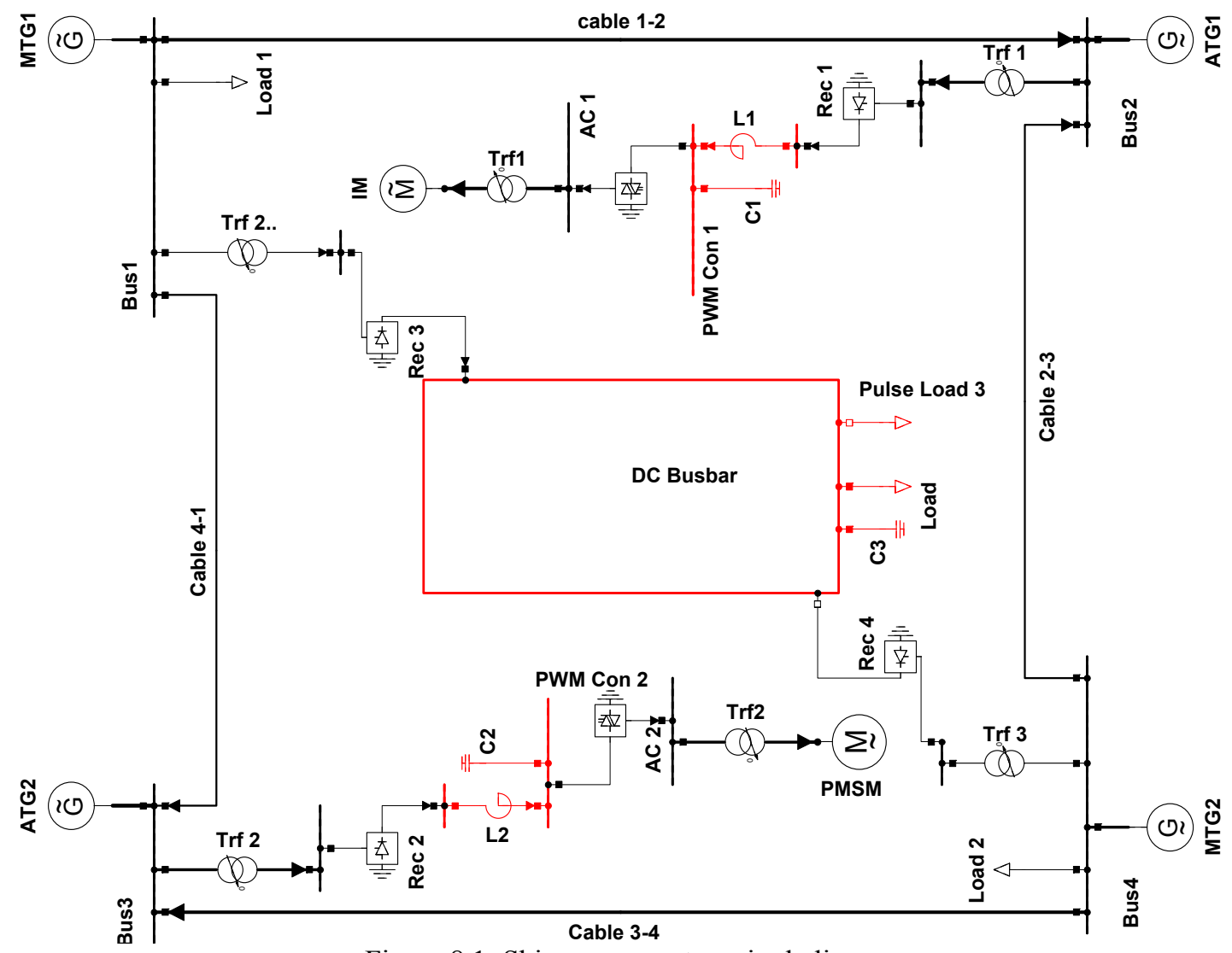

Figure 8.1: Ship power system single line

\subsubsection{Single Pulse-load Simulation}

When the pulse-load is in operation, it offers a very small resistance and looks like a 'short circuit' or a huge load connection to the power system. The operation of the pulse-load can be controlled by changing the rate of triggering of the switch. A number of studies have been performed to investigate the requirements and challenges posed to the electric ship power system. In this chapter we will study the effect of magnitude and duration of the pulse-load as well as a number of iterative pulses with some delay (impulse train) on system stability. The dynamic behavior of system is the main concern when encountering a pulse-load. The system parameters which affect the system stability under pulse-load conditions are also studied. 
The first simulation is for the case in which a $30 \mathrm{MW}$ DC pulse load on the DC bus is switched with a 0.1 second duration. The results show that the system is stable during this pulse-load. Figure 8.2 shows the pulse-load and main and auxiliary generators an active power output for 15 seconds of the simulation. All parameters come back to their steady state initial values. MTG1 produces a large amount of the power for the pulsed load, around $64 \%$ and MTG2 produces around 30\% the remaining demand. The remaining load amount is shared between the two auxiliary generators. The percentage is dependent upon the location of pulse-load they supply. In the other case the DC bus rectifier is connected to bus 4 , which is supplied directly by MTG2. In this situation, MTG2 is responsible for a large amount of the power supplied. The power sharing of the generators for pulse-load will be varied depending on the pulse-load location and topology of the ship power system. The voltages of the buses during the presence of the pulse-load are presented in figure 8.3 for the main AC buses as well as the DC buses.

The voltage changes in the buses depend on the pulse-load parameters like the magnitude of the pulse, its duration and the number of iterations. The voltage controller parameters, such as the AVRs settings of the generators also affect the system behavior during pulse-load conditions and after its departure. One of the key factors of transient stability is the rotor angle of each generator during and after an event like a pulse-load. During the pulse-load, the power and angle jump to a new operating range for both generators. After the passing of the pulse-load, the power and rotor angle return to their normal values with some oscillations around the steady state values. The magnitude and duration of these oscillations depend on the system inertia, the generator voltages and the power controller parameters. Figure 8.4 shows the transmitted power versus rotor angle 
of the generator that is connected to the infinite bus during the pulse-load. In addition, the simulated powers versus rotor angle are plotted for the two main generators in figure 9.5. The power and angle of generator increase suddenly and after the pulse-load passage they return to normal load values with some oscillation around the stability point. Herein, $\delta_{o}$ is the rotor angle in the normal load situation and $\delta_{p}$ is calculated from the pulse-load duration. We can apply the equal area criterion to determine the transient stability margin for one machine connected to the infinite bus.
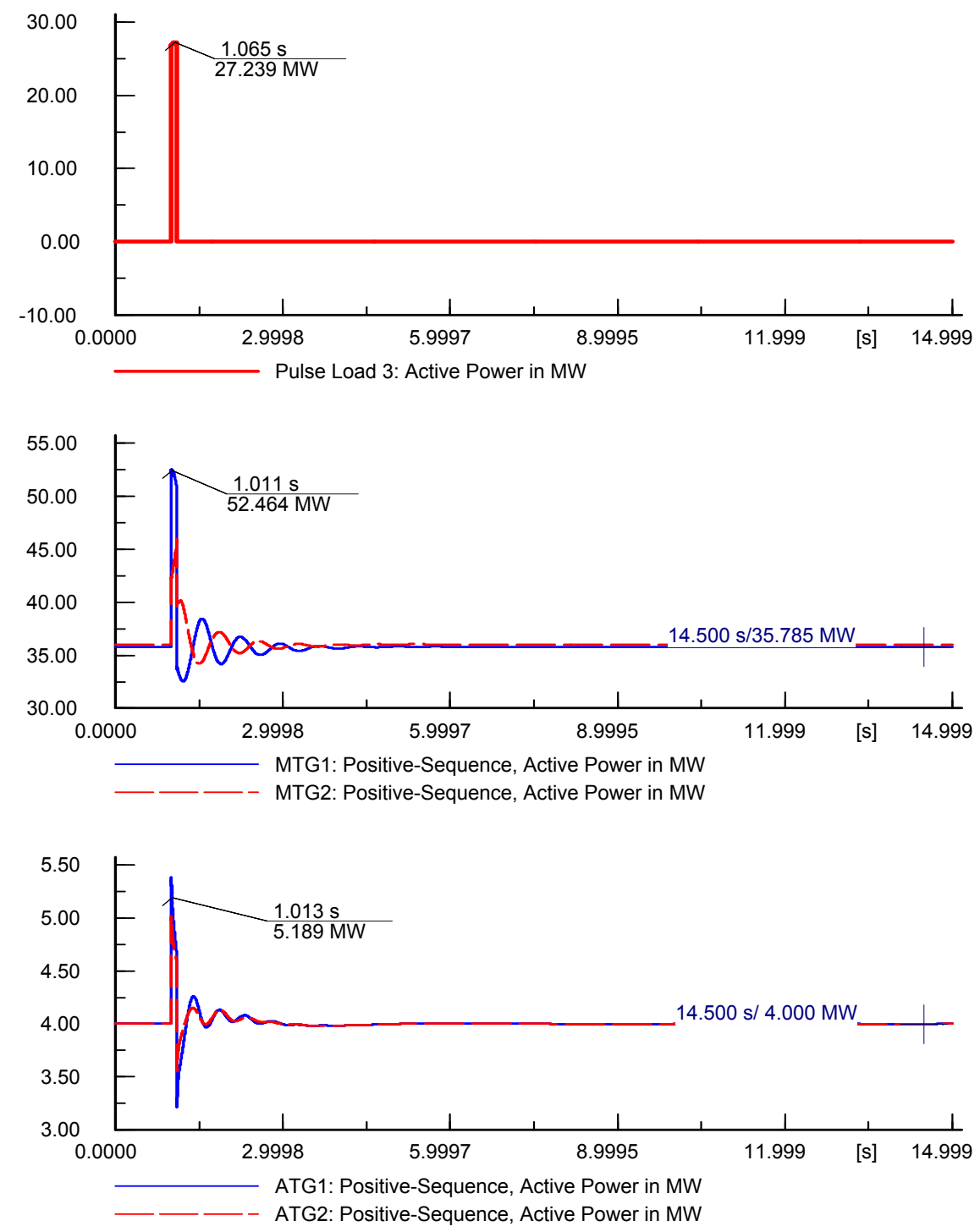

Figure 8.2: Pulsed Load power and generator active power behavior 

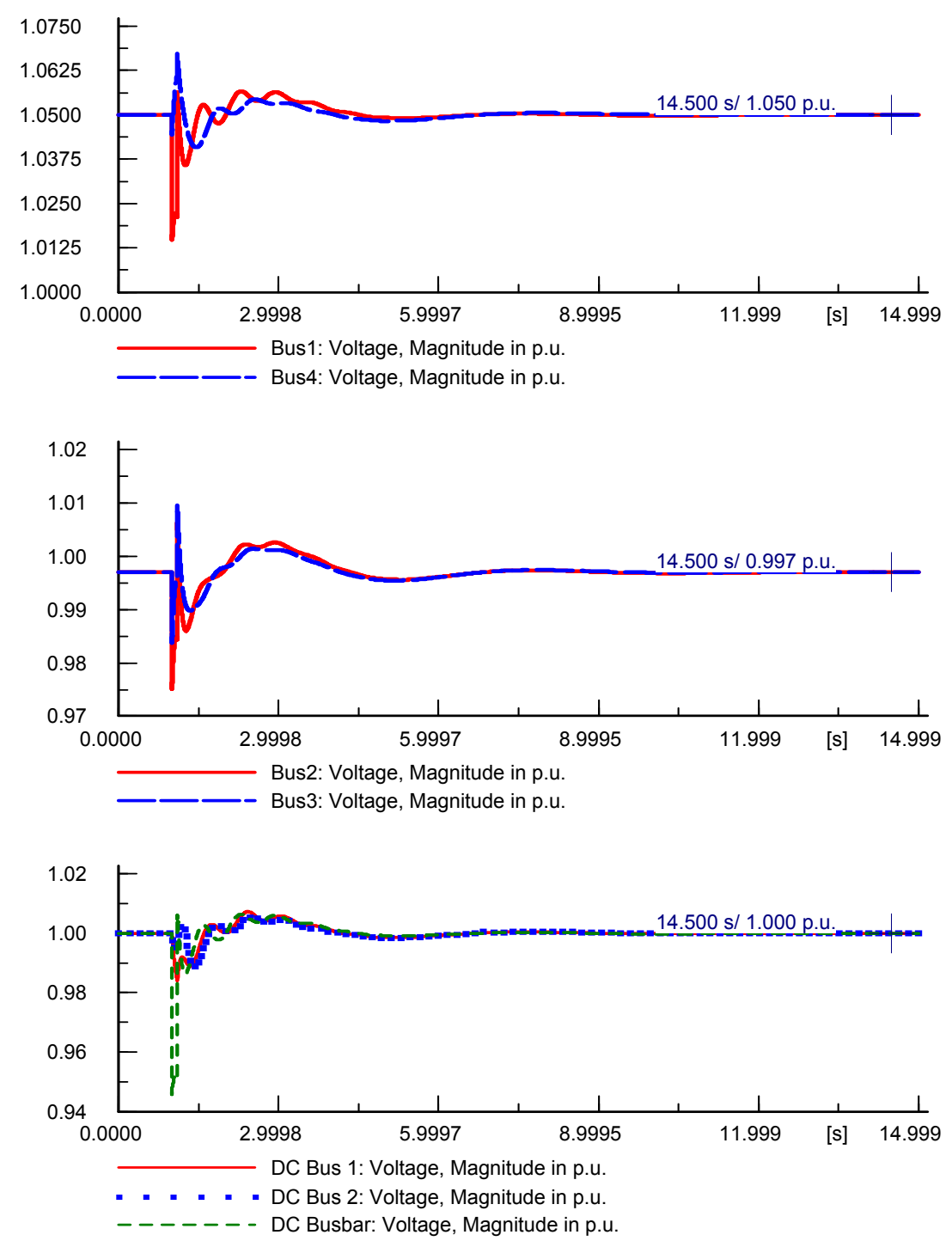

Figure 8.3: Main AC buses and DC buses voltage during pulse-load

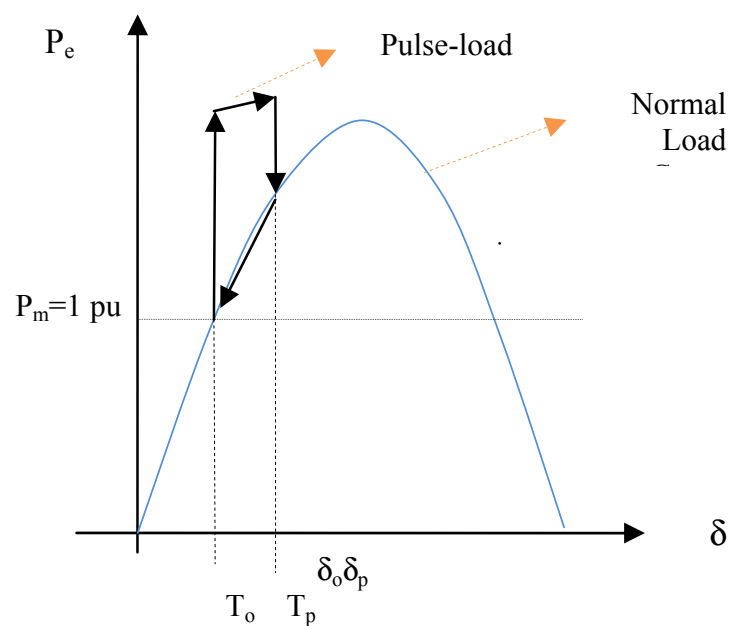

Figure 8.4: Power-Delta curve behavior during Pulsed Load 
Hence, in the multi machine system, the transient stability of the power system is not only characterized by the system topology and the controller parameters when pulseload occurs, but also the pulse magnitude and its duration are other factors which affect the transient stability.

The setting of the AVR and "Governor" parameters are very important. If the system is modeled without using these controllers or with improper parameters then the system will be unstable according to the frequency and voltage waveforms which are shown in figure 8.6. In this figure the controller time constant increased by 0.5 seconds.
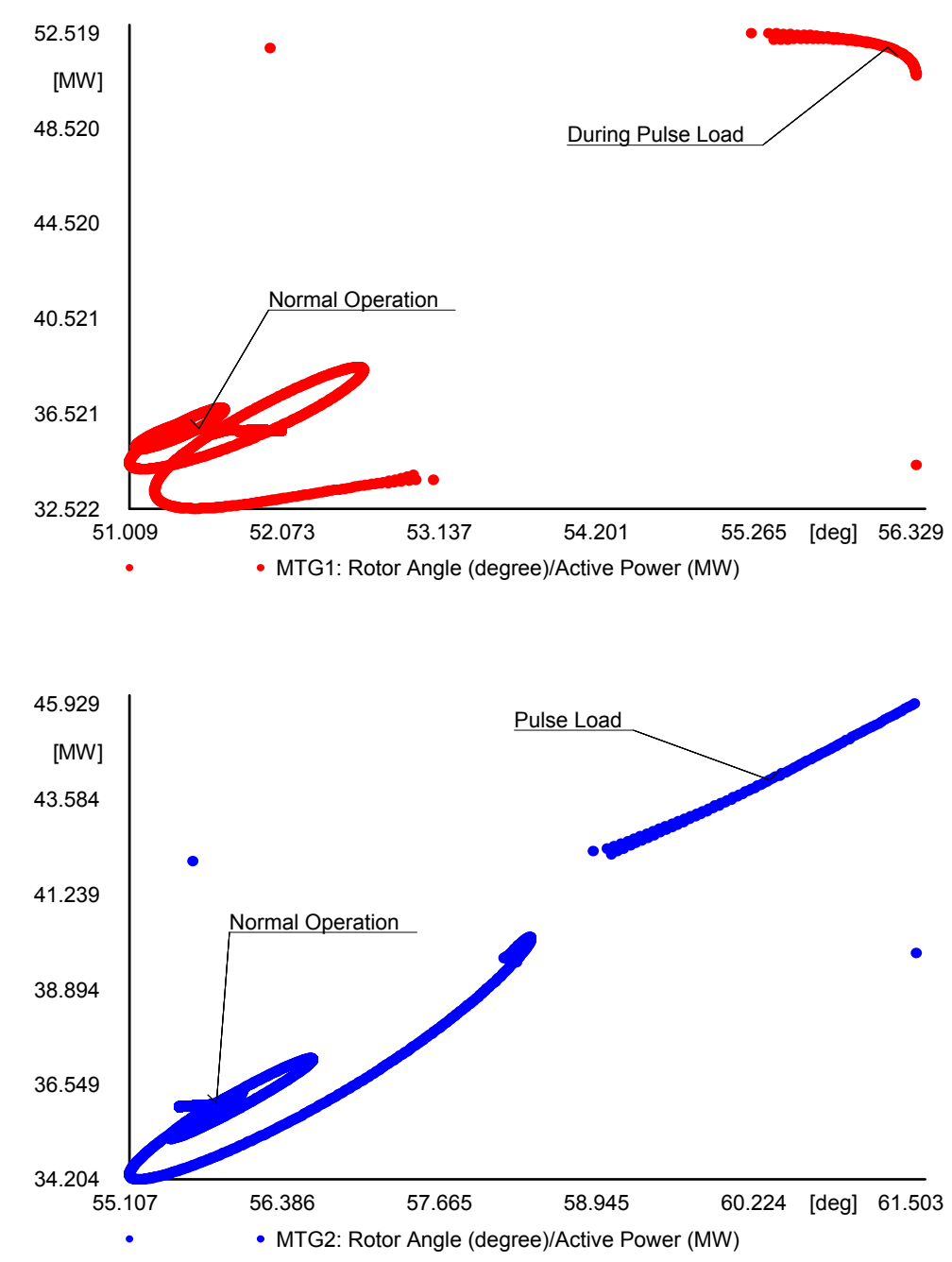

Figure 8.5: Power-Delta Curve for main generators during Pulsed Load 

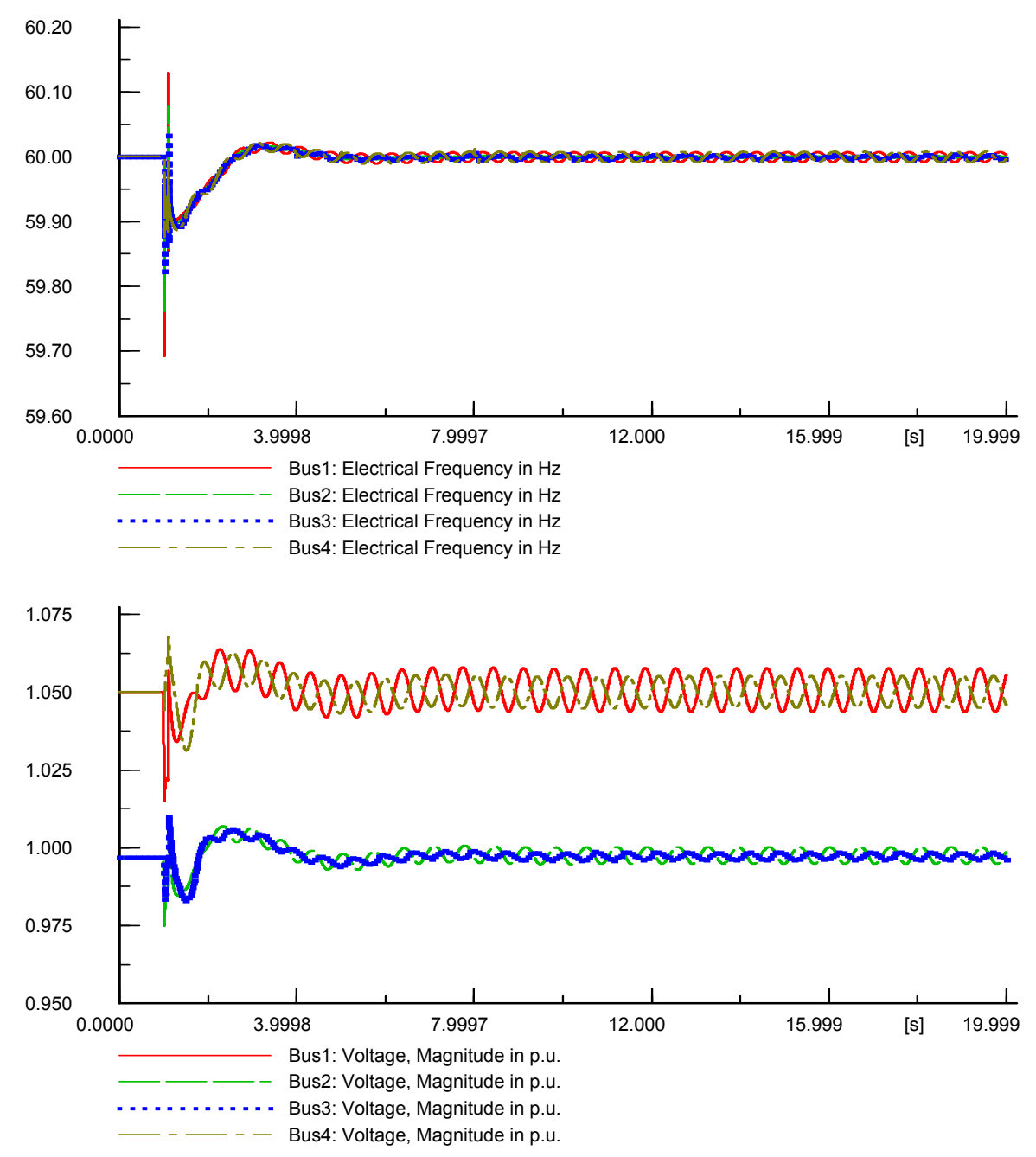

Figure 8.6: Main buses voltages and frequencies during pulsed Load

For investigating the voltage and frequency behavior under unstable conditions, the simulation was performed with one second $50 \mathrm{MW}$ pulse-load duration from 1 second to 2 seconds. Figure 8.7 shows the frequency and voltage changes during the aforementioned pulse-load.

In this condition, the AVR and governor operation set to maintain the voltage and output power of the generators. However, from figure 8.8 , one can observe that the induction motor used as propulsion load in ship power system causes the voltage to collapse and therefore leads to instability. Consequently, the pulse-load has an adverse 
effect on the system stability from the perspective of voltage and frequency. In addition to the rotor angle stability, the voltage and frequency variation should be considered when the pulse-load causes a large drop in those parameters.

Protection devices may disconnect the system under large pulse-loads with a long duration because of a large drop in voltage or frequency. Accordingly, there should be a compromise between the protection device and the power system, which includes a pulse-load feasibility study on them. The coordination of these protection devices should include detection methods for pulse-load high current situations and other abnormal conditions, such as short circuits. It should be noted that faults and safe application of pulse-loads must be distinguished from each other. In addition, the equipment loading under this condition and their rated short time (1 second) current should be investigated for equipment safety and damage prevention.

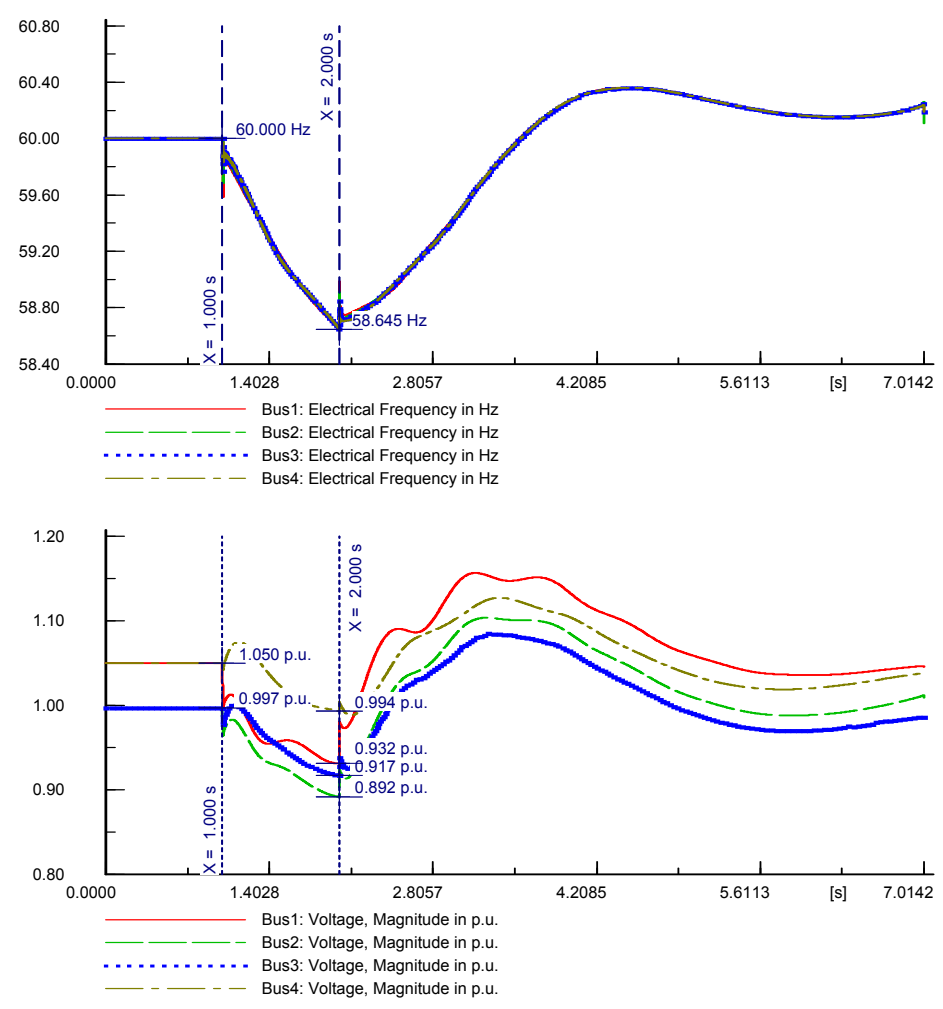

Figure 8.7: Frequency and voltage variation for 1 second Pulsed load 

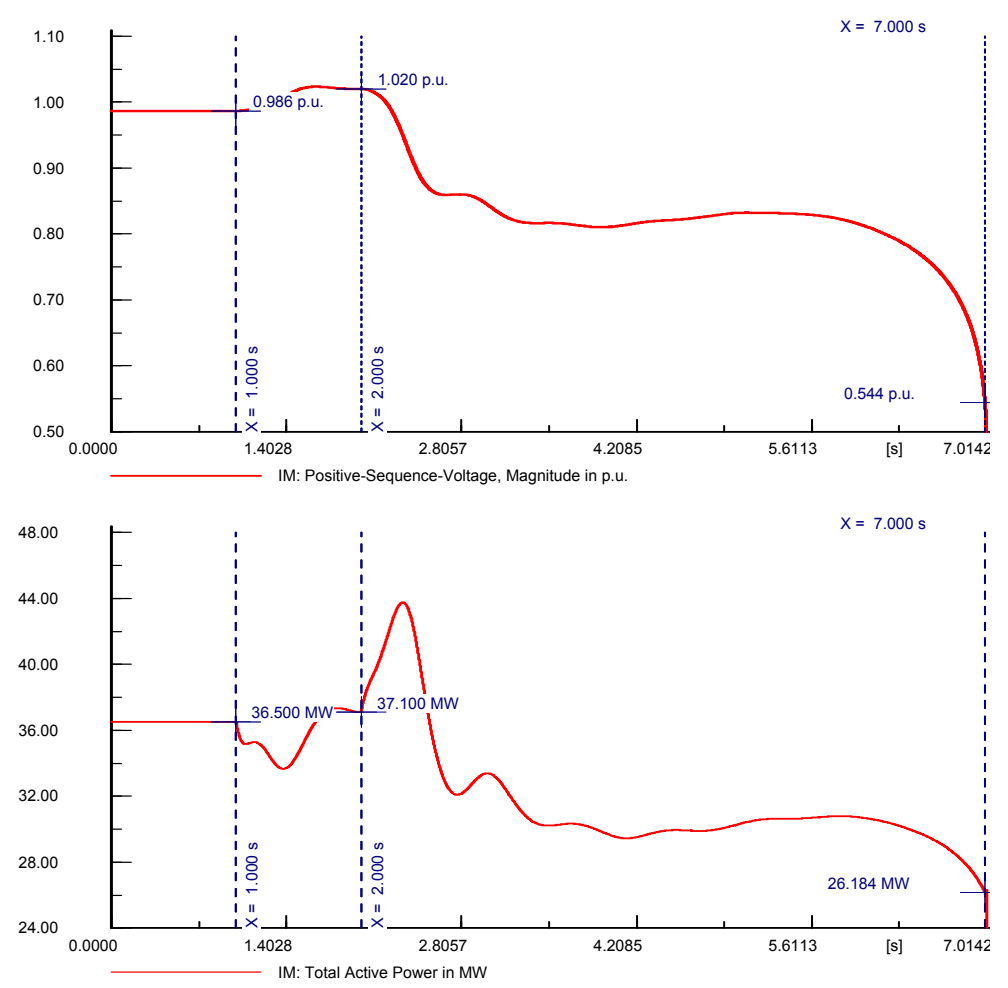

Figure 8.8: Voltage and active power of Induction generator for 1 second pulsed Load

\subsubsection{Repeated Pulses (Train of Pulsed-load) Simulation}

Another case in the simulation is the pulse train load in which several pulses occur periodically in the system. This situation occurs on some weapons used in the electric ship power system. Here, ten pulses with 0.1 second duration and a repetition period of 1 second are simulated to investigate the system behavior under these pulse train loads. Figure 9.9 shows the pulse train applied to the system and the generators output active powers are shown in this figure. Figure 8.10 depicts the voltages and the frequencies of the main buses. Figure 8.11 also presents the power-delta curve for main generators which show that the system will be stable after pulsed train departs and that the system will return to its initial set-point curve. The iteration or period of these pulses is one of the factors which affect stability issues in the power system. The frequency and 
voltages changes should be observed during these pulses. Several pulse-loads may cause a large frequency drop, because the system damping ratio or large inertia will not allow the frequency to return to its normal band after a pulse and the next pulse occurs when the system frequency is down which results in more frequency drop. Figure 9.12 shows this situation which frequency drop is near $2 \mathrm{~Hz}$ and frequency stability is not satisfied in the system.
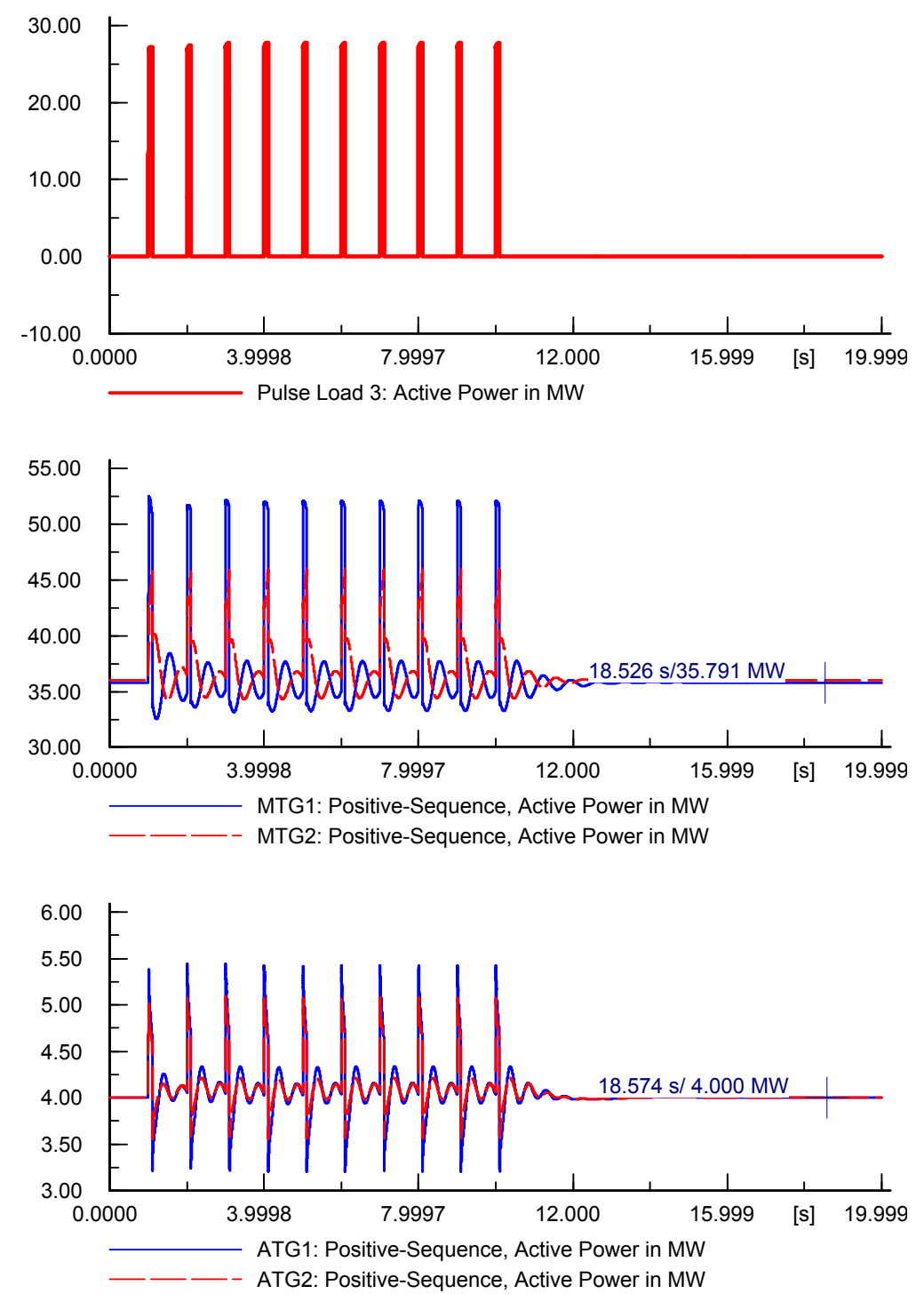

Figure 8.9: Pulsed train load and generators active power 

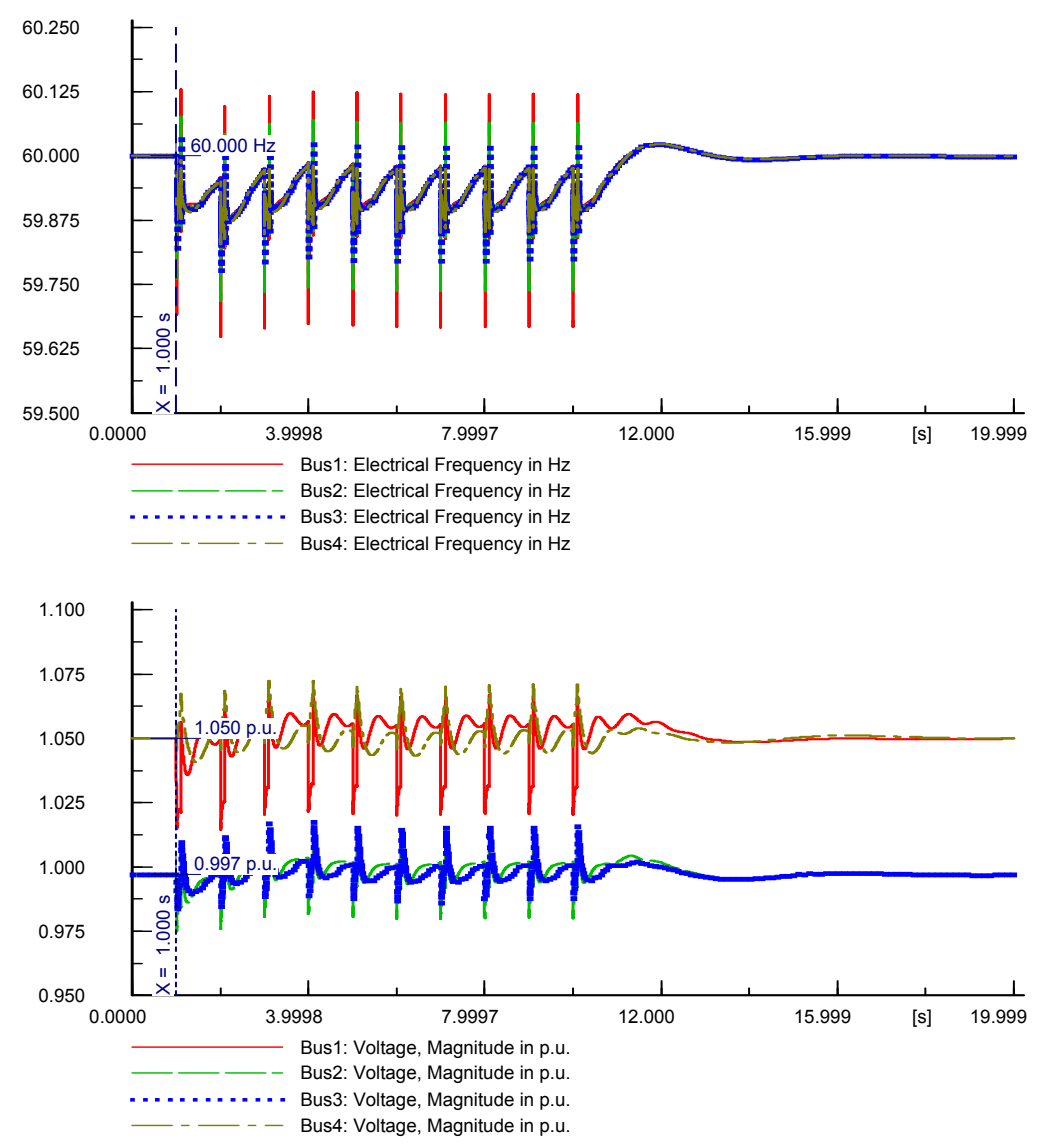

Figure 8.10: Main buses frequency and voltage behavior for train pulse-load
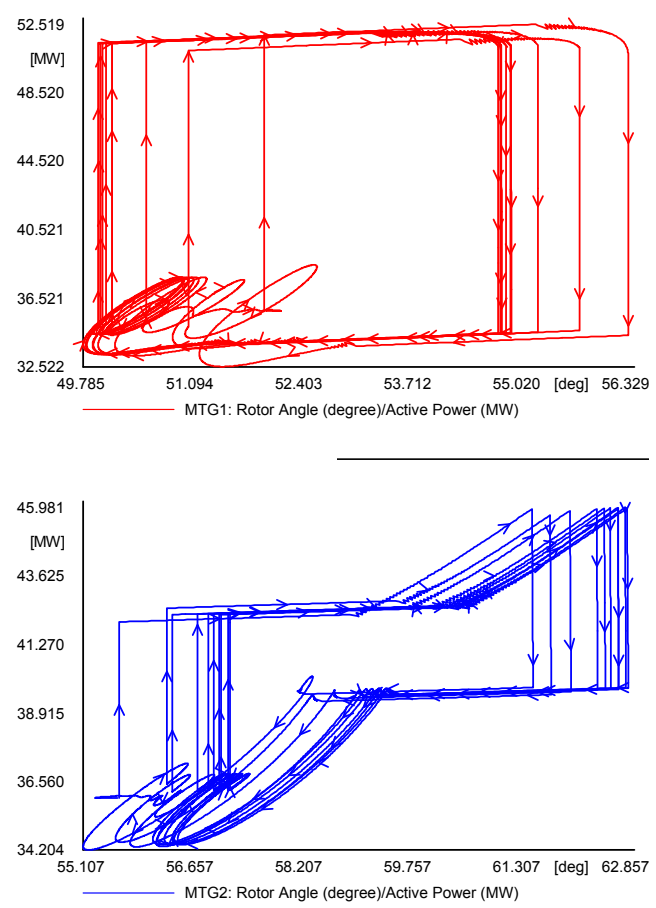

Figure 8.11: Power-Delta curve for train pulse-load for two main generators 

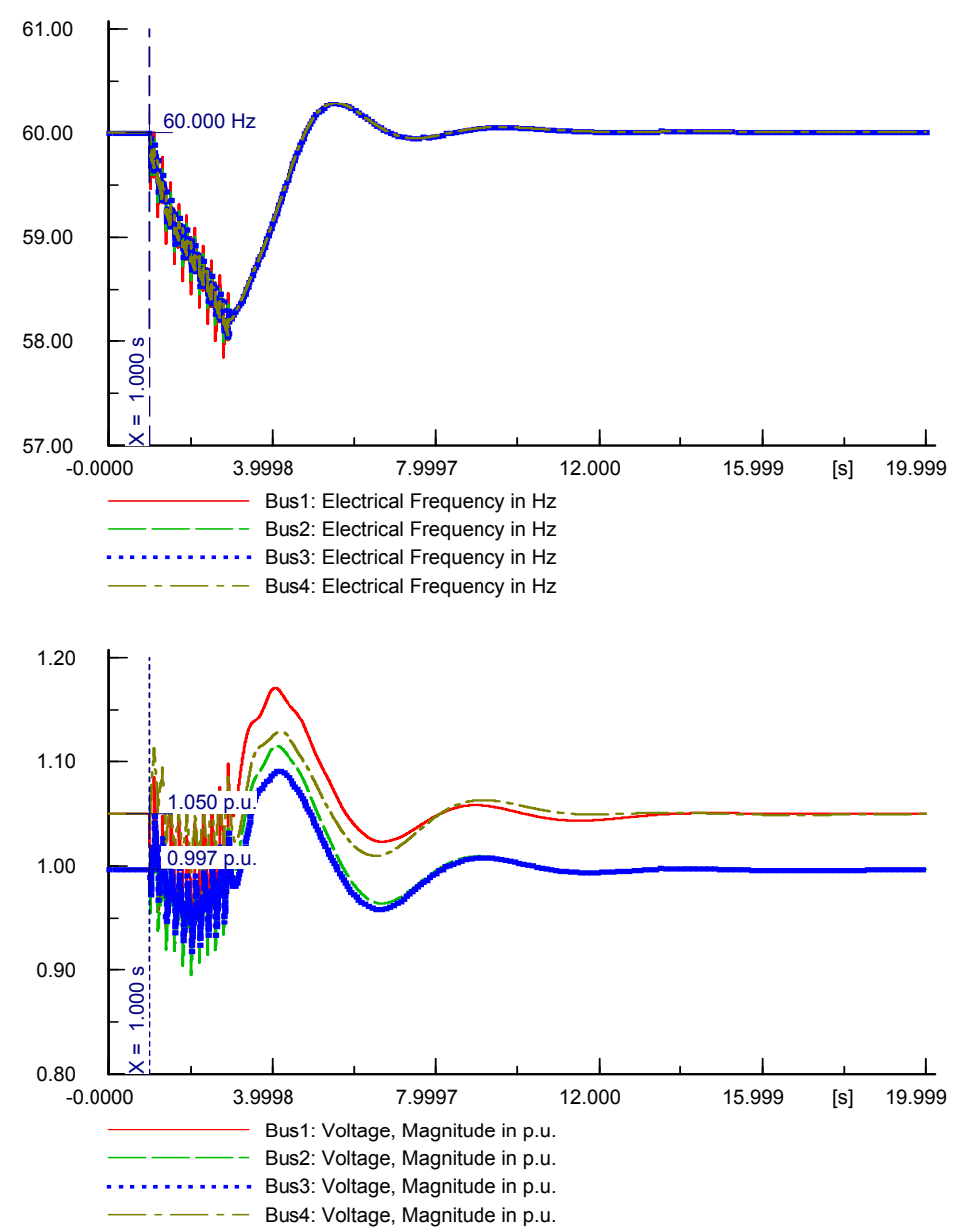

Figure 8.12: Main buses frequencies and voltages during fast pulse train load

At the system level, modeling and simulation are an integral part of the power system architecture design and evaluation. They can determine the size and the efficiency of the overall system. In addition, by using a developed simulation, one can predict the behavior of the ship power system under the prescribed conditions, determine the effects of pulse-load on the power system, assess the performance of new load types, study the integration of new components and the interaction among components, as well as provide a platform to analyze reconfiguration schemes.

The impacts of pulse-load magnitude and duration have been investigated on the system stability and performance. This study was achieved through a sophisticated ship 
power system models which include the transient and dynamic behaviors. To the best of this author's knowledge such complete system is absent in the literature.

The simulation results show that the pulse magnitude, duration and its iteration the affect power system stability. The system topology and the controller parameters also have a direct impact on the system performance.

\subsection{Dynamic Security Analysis of the Various Shipboard IPS Architectures Involving Pulse Loads_-Effect of Contingencies}

Shipboard integrated power systems (IPS) like any microgrid have some challenging unique features from a power system point of view. First of all, IPS is an isolated power system, not supported with a relatively stronger grid. Therefore, the system encounters faster dynamics due to the finite generation inertia and is more susceptible to disturbances. Secondary, transients on the load side may cause considerable transients in the system since load/generation ratio is close to 1 . Moreover, the system is physically small, hence the connecting cables have negligible impedance, which may cause instability issues among the different components of the system. Finally, the IPS is more subjected to severe damages, especially during battle conditions, which must be taken into account while operating security analysis.

With the existence of pulse loads on such systems, dynamic security analysis must be performed during the various contingency conditions and corresponding to different storage distribution techniques. The main security constrains are consist of voltage limits in AC and DC buses in steady state and transient condition during pulse load occurrences. In addition, the AC voltage frequency may oscillate only within 
acceptable limit. Finally none of the system components such as cables and transformers should be overloaded. Table 8.1 shows the security constrains considered in this study.

In this research the IPS system configuration is changed to zonal DC loads in 4 different buses in order to study the load distribution effects on system security under contingencies. Hence, figure 8.13 (a) shows the single line diagram of IPS with zonal load distribution. The system resembles a shipboard power system with scaled down ratings. Each rectifier supplies one of two $0.318 \mathrm{kV}$ DC busses. Furthermore, a DC generation system of $10 \mathrm{~kW}$ rated capacity, lithium-ion batteries with 3000 Ah rated capacity and super capacitors with $200 \mathrm{~F}$ are included in the DC microgrid. A PWM controlled DC-DC converter is used as an interface between the PV system and the DC bus. Moreover, a vector decoupling PWM controlled AC-DC/DC-AC bidirectional converter was used for connectivity between the AC and DC sides. In the steady state case, the system voltages and loadings are within the normal limits. For transient simulations, we considered a pulse train of four pulses with a rate of $0.2 \mathrm{~Hz}$, a duty ratio of $10 \%$ and amplitude of $20 \mathrm{~kW}$.

Table 8.1: System Security Constrains

\begin{tabular}{|c|c|}
\hline Parameter & Secure Range \\
\hline AC Voltage Amplitude [p.u.] & $0.9<|\mathrm{Vac}|<1.1$ \\
\hline AC Voltage Frequency [Hz] & $59.5<f<60.5$ \\
\hline Loading of System Components [\%] & Loading $<100 \%$ \\
\hline DC Voltage Level [p.u.] & $0.9<V_{d c}<1.1$ \\
\hline
\end{tabular}


Each of the four DC zones may include, a pulse load, battery, SC, a normal load or a combination of these elements based on the event studied.

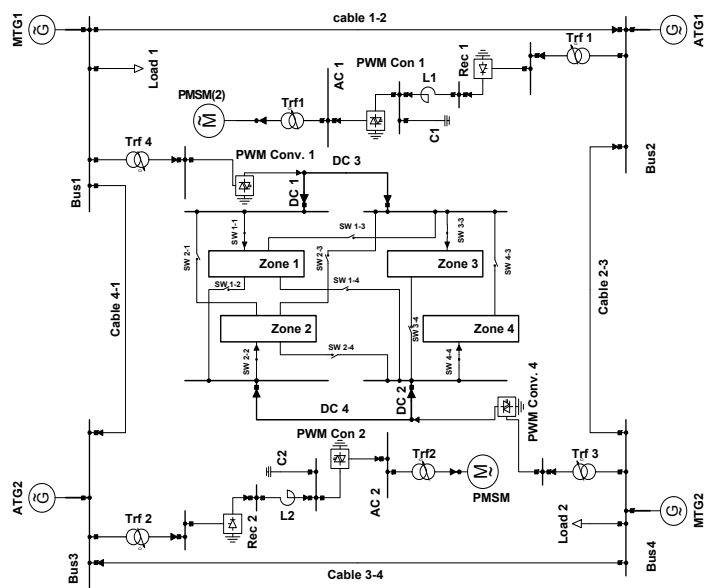

(a) Single Line Diagram
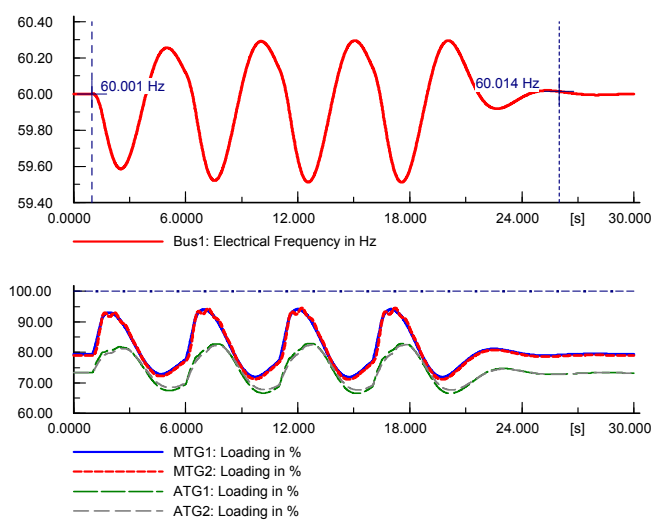

(c) Frequency of the system and loading of generators

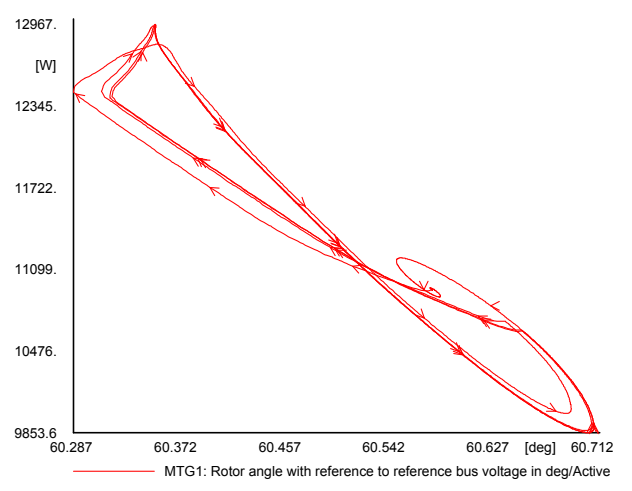

(e) Active power versus rotor angle

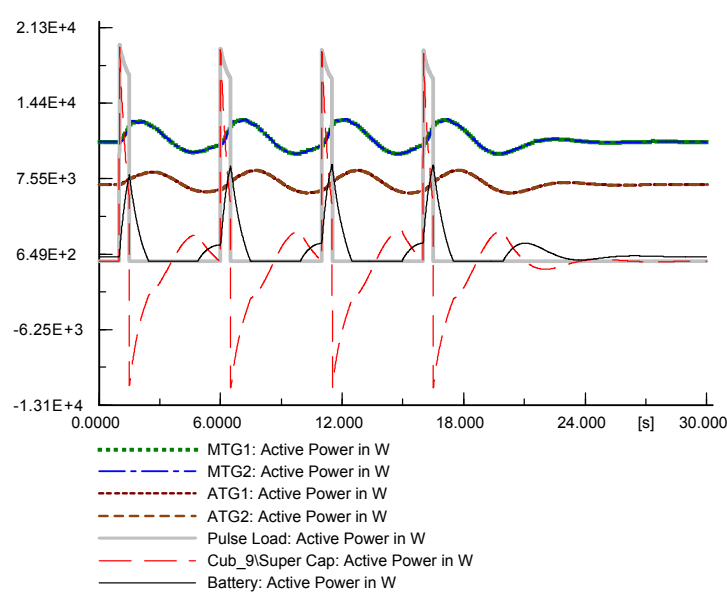

(b) Power sharing corresponding to the Pulse Load
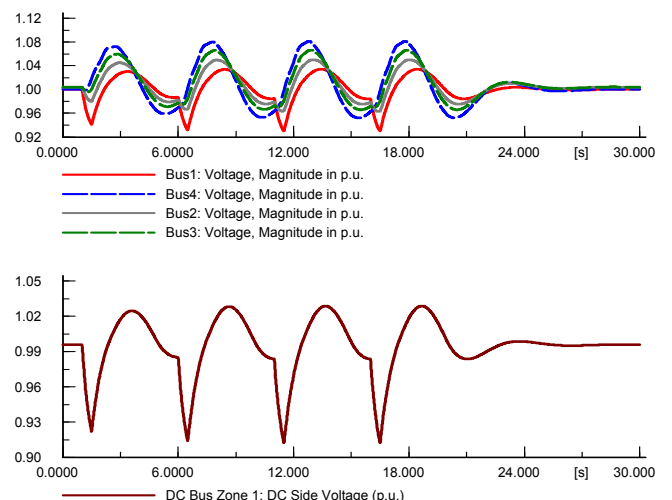

(d) Voltage of AC and DC buses
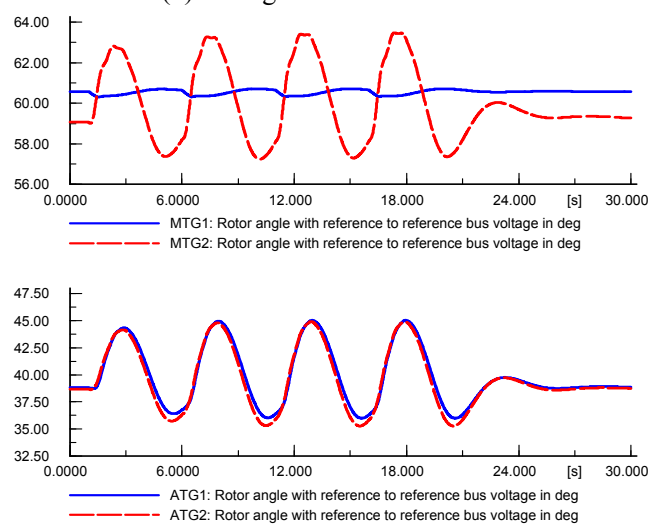

(f) Rotor Angle of the generators

Figure 8.13: The IPS system with zonal load distribution in Normal Condition and Pulse Loadccurance 
As shown in figure 8.13 (b) Super Capacitors quickly responds at the beginning of the pulse load due to its high power density, however its power share drops and the battery takes over due to its higher energy density. Main and auxiliary generators encounter some swinging and oscillation. Super Capacitor and battery, are capable of riding the system through the disturbance caused by the pulse load.

The system frequency oscillations are within $\pm 0.5 \mathrm{~Hz}$ limits and we have no overloading at any generator according to figure 8.13 (c). Transient values of voltages are within limits according to figure 8.13 (d) and in addition the steady state values of voltages are within limits. Generators rotor angle and MTG1's power-delta curve have been shown in (e) and (f) of figure 8.13.

The security analysis was performed aiming at studying the effects of

- System contingencies: in which the system security will be examined under several outages conditions along with the pulsed load.

- Storage distribution: in which the effect of distributing the battery storage system (for thermal management, increased reliability, etc) was examined during the occurrence of pulse loads.

The event list and descriptions are shown in table 8.2. The first simulations are related to contingencies of main elements during the pulse load happening. The pulse load is included of 4 pulses and the contingency of the equipment take place after second pulse, i.e. 9 sec. Pulse load is placed at zone 1 and this zone includes a super capacitor and the battery storage system. We will study the behavior of the system by power, voltage, frequency and power-delta curves during the contingency and occurrence of pulse loads. In addition the storages distribution in different zones are studied in order to 
evaluate the system performance while we have enough storages connected to system DC sides.

Table 8.2: Event List for Contingency Analysis

\begin{tabular}{|c|l|c|}
\hline$\#$ & Event Description & $\begin{array}{c}\text { Event } \\
\text { Type }\end{array}$ \\
\hline 1 & Cable 1-2 Outage & \\
\hline 2 & Cable 1-4 Outage & \\
\hline 3 & MTG2 1-2 Outage & \\
\hline 4 & MTG2 Outage with Distributed Energy Storages & \\
\hline 5 & ATG2 1-2 Outage & \\
\hline 6 & PMSM Outage & \\
\hline 7 & Pulse Load and Storage at Zone 1 & \\
\hline 8 & Pulse Load at Zone 1 and Storage at Zone 4 & \\
\hline 9 & Pulse Load at Zone 1 and Storage distributed between Zones 1\&4 \\
\hline 10 & Pulse Load at Zone 1 and Storage distributed between Zones 1, 2,3 \& 4 & \\
\cline { 1 - 2 } 11 & Pulse Load at Zone 1 without storage & \\
\hline
\end{tabular}

\subsubsection{Outage of Main Components during Pulse Load}

Figure 8.14 shows the system simulation results for the case of cable 1-2 outage.

In this figure (a) shows the single line diagram of the system with steady state results after the contingency and pulse load occurrences (i.e. $\mathrm{t}=30 \mathrm{sec}$ ). Portions in blue encounter an under voltage after steady state is reached. Figure 8.14 (b) shows the power sharing between generators, super capacitors and the battery storage. The system frequency and generators loading are shown in (c) of this figure and shows that oscillation are within $\pm 0.5 \mathrm{~Hz}$ limits and there is no overloading reported. According to figure 8.14 (d) there is under voltage violation during transient. Rotor angle changes during the system transients are shown in (e) and (f). All rotor angles are returned to previous angle or like MTG2 reach to the new steady state condition. 
Figure 8.15 shows the results for contingency of cable 1-4. As previous cases Super Capacitor (SC) is the fastest source to supply the pulses. Batteries contribute in all four pulses to mitigate the worse effect on the generators. Under voltage violation happens during transient only at AC buses 3 and 4. No voltage violation occurred on the DC bus. Frequency oscillations are within $\pm 0.5 \mathrm{~Hz}$ limits and there is no overloading reported.

Figure 8.16 presents the results for outage of MTG2, one of the main units in this system. As it shown in single line diagram of this figure, over voltage (red lines) and under voltage (blue lines) are reported at different parts of the system at steady state of post events. The loss of MTG2 causes instability in the system after few seconds of outage and SC oscillates significantly during the instability region due to the voltage oscillations. A severe voltage collapse is reported due to the outage of MTG2. Frequency oscillations are out of $\pm 0.5 \mathrm{~Hz}$ limits. Overloading is reported before the instability occurrence; hence it is very essential to monitor the loading of the generators dynamically to ensure the system security. This contingency does not lead to the system stable operation.

Therefore, the role of using energy storage system on MTG2 outage was studied with energy storage at the four zones. The energy stored in the batteries utilized to delay the instability of the system and give more time for a remedial action. In this case the battery storage is distributed among all four zones, and the oscillations of all the sources are within limits according to figure 8.17. No voltage violation or loading problem occurred and frequency is almost at its limit range. 
The contingency of ATG2 is studied by figure 8.18 and it shows that the steady state values of power sharing from MTG1, ATG1 and ATG2 are higher than their initial values because of losing this generator. Under voltage violation during transient only at AC buses 3 and 4. Under frequency violation and overloading of MTG1, ATG1 and ATG2 are the problems in this case. The rotor angle curves for main generators show the system instability for this case.

Last contingency study is related to outage of one of propulsion motors, PMSM and is shown in figure 8.19. Steady state values of power sharing from all generators are less than their initial values. Bus 3 (where the PMSM was connected) and an adjacent bus (Bus 4) encounter over voltage violation. TheDC Bus voltage has no voltage violation.

There is over frequency violation and over loading at MTG2, where the PMSM was connected, happens for a very short time due to the oscillations in its rotor angle and frequency. Rotor angle depicts no stability issue.

\subsubsection{Effects of Storages Distribution during Pulse Load}

In this section we will study the effect of distributing storages in different zone while the pulse load occurs in first zone. The aim of this study is to analyze the performance of distributed voltage control in different zones of microgrids. Hence the pulse load and super capacitor is connected to bus 1 and we placed storages at different zones.

At first case the only storage is connected to the same zone of occurrence pulse load (i.e. zone 1) and figure 8.20 shows the results for this simulation. According to this figure SC contributes to supply the pulse load and the battery contributes to supplying the 
pulse load, too. No voltage violation reported on AC or DC buses and frequency of all buses are within the limits and no overloading is reported.

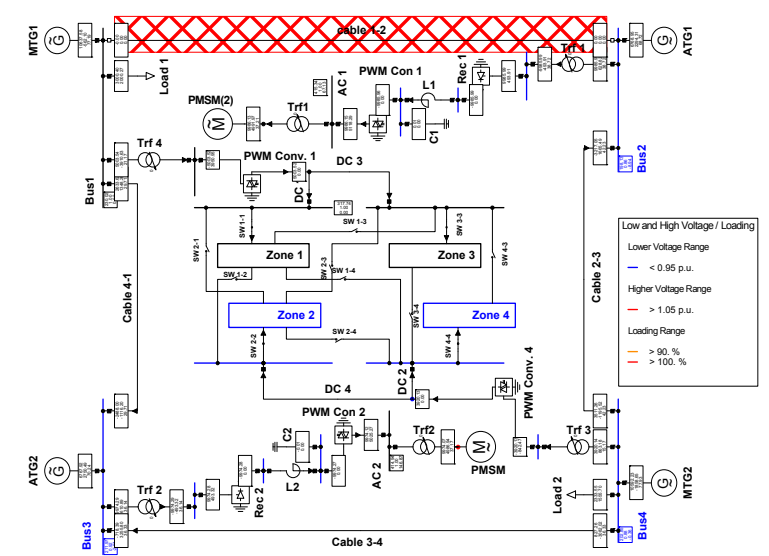

(a) Single Line Diagram
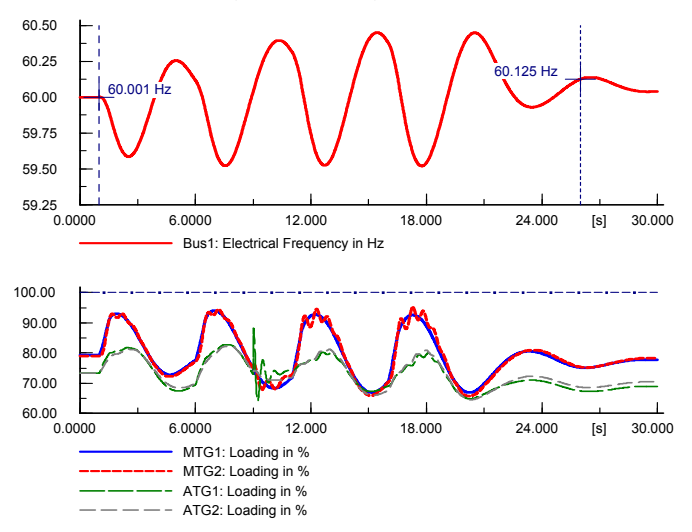

(c) Frequency of the system and loading of generators

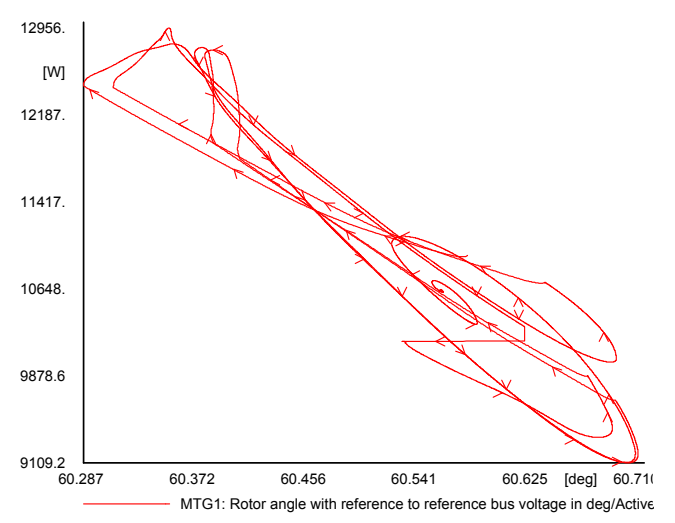

(e) Active power versus rotor angle

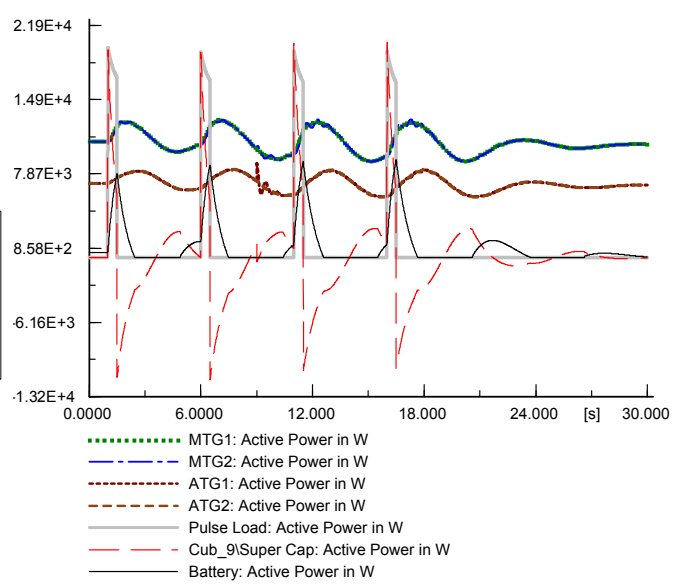

(b) Power sharing corresponding to the Pulse
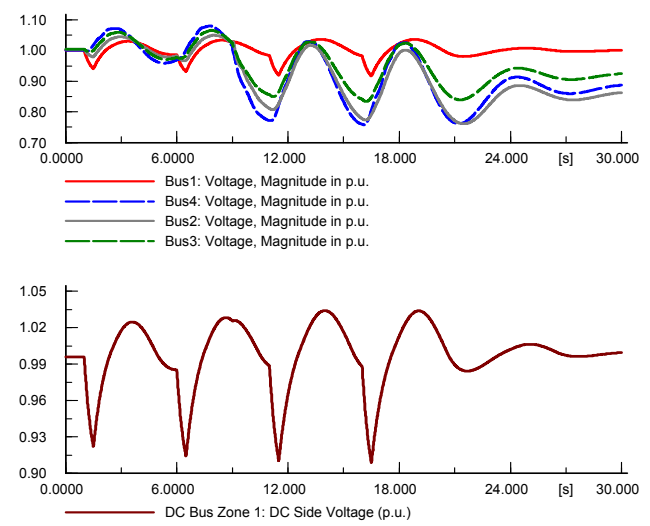

(d) Voltage of AC and DC buses

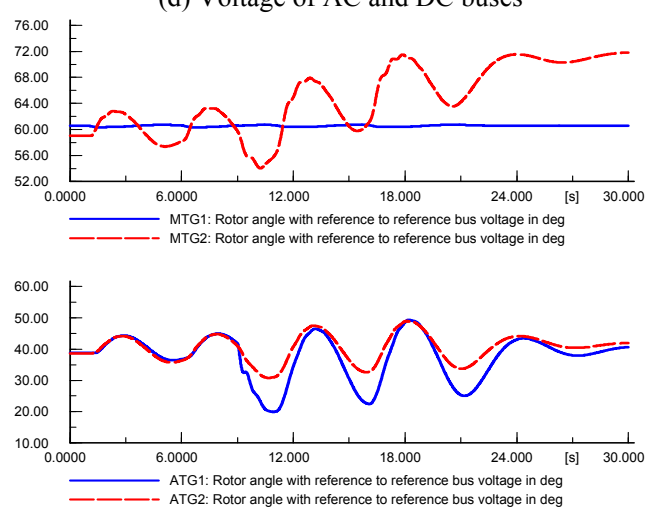

(f) Rotor Angle of the generators

Figure 8.14: Contingency events, Cable 1-2 outage (an open ring, close to radial, architecture) 


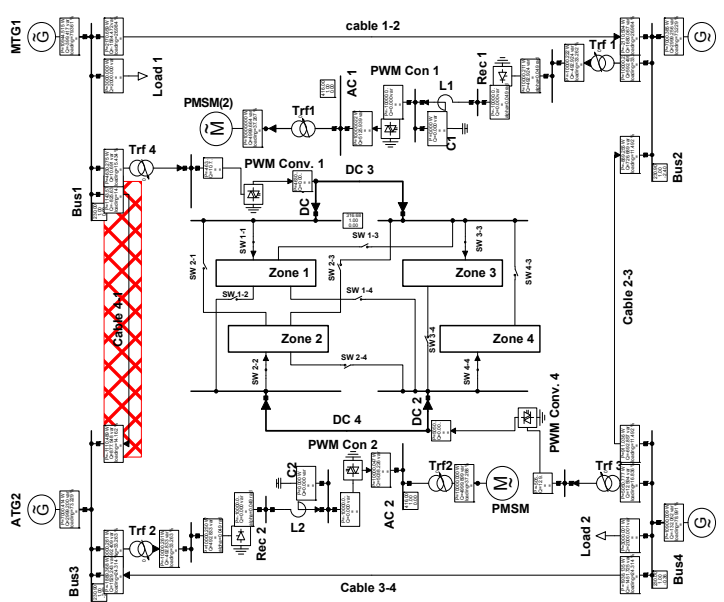

(a) Single Line Diagram
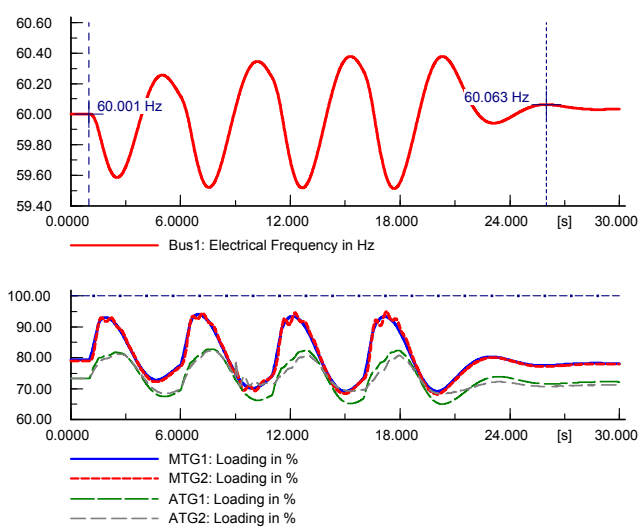

(c) Frequency of the system and loading of generators

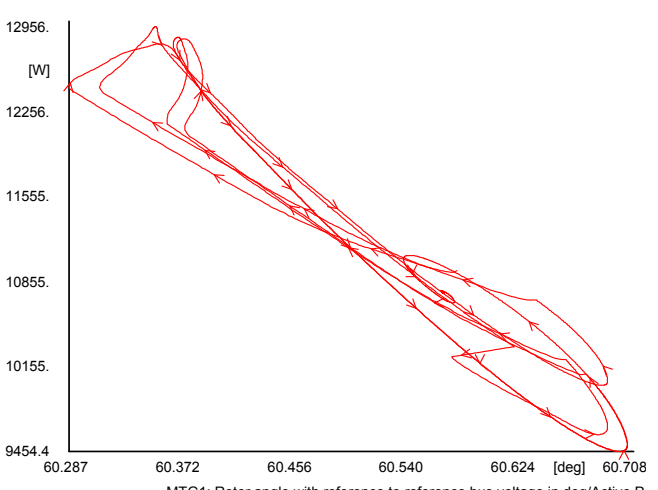

(e) Active power versus rotor angle

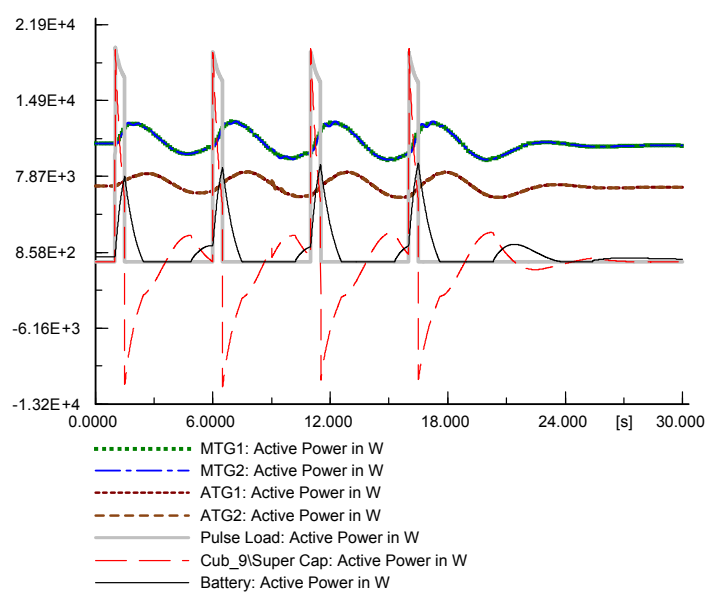

(b) Power sharing corresponding to the Pulse Load
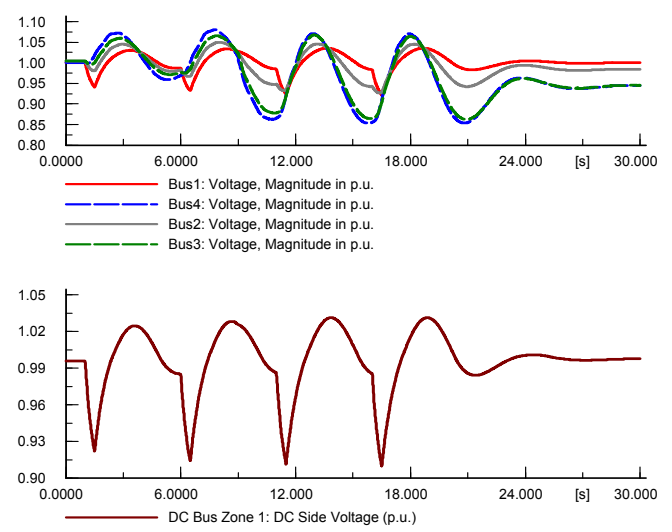

(d) Voltage of AC and DC buses
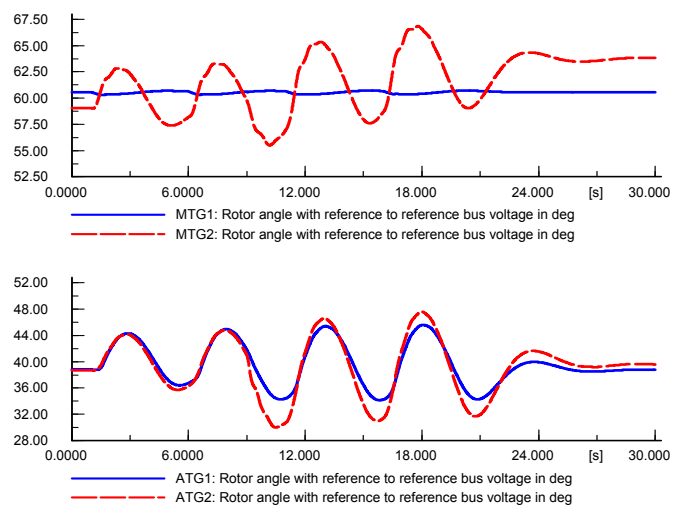

(f) Rotor Angle of the generators

Figure 8.15: Contingency events, Cable 1-4outage (an open ring, close to radial, architecture) 


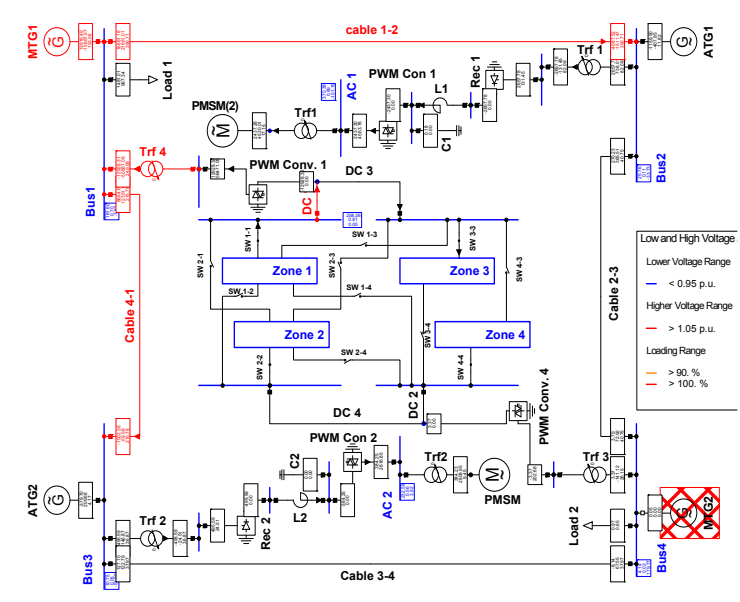

(a) Single Line Diagram
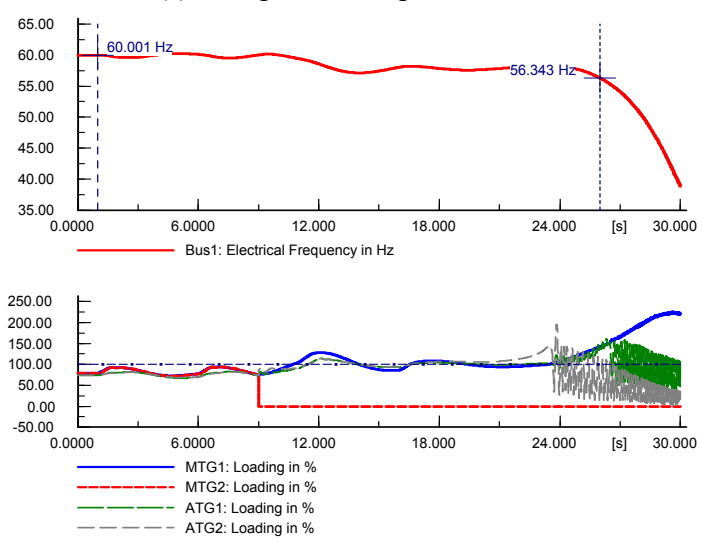

(c) Frequency of the system and loading of generators

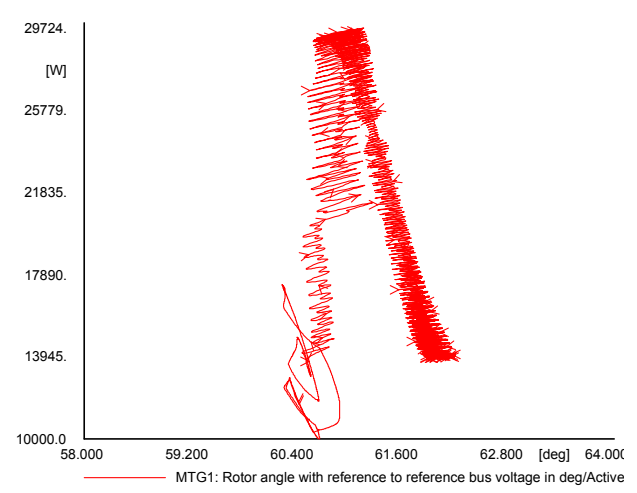

(e) Active power versus rotor angle

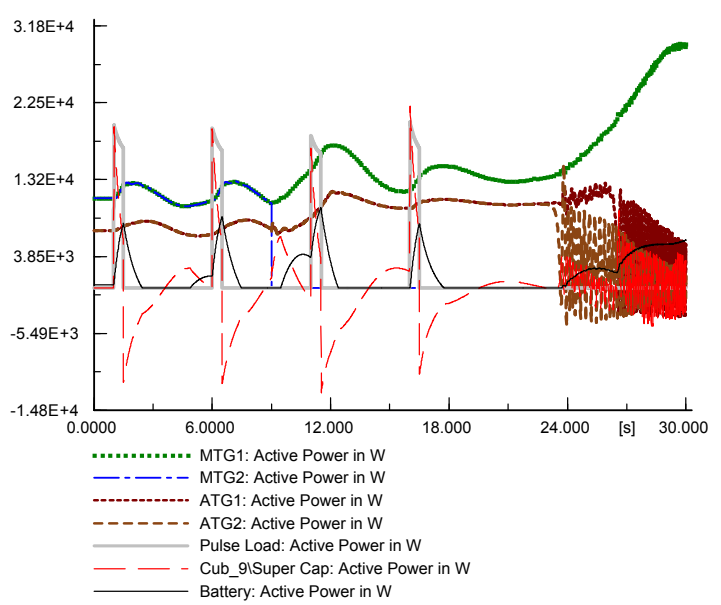

(b) Power sharing corresponding to the Pulse Load
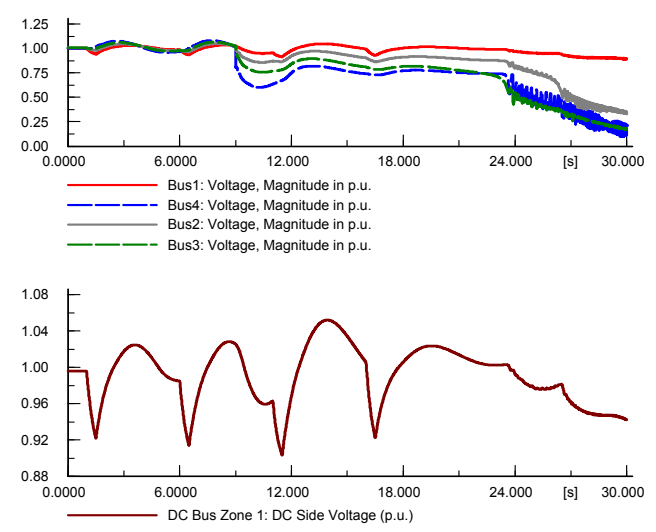

(d) Voltage of AC and DC buses
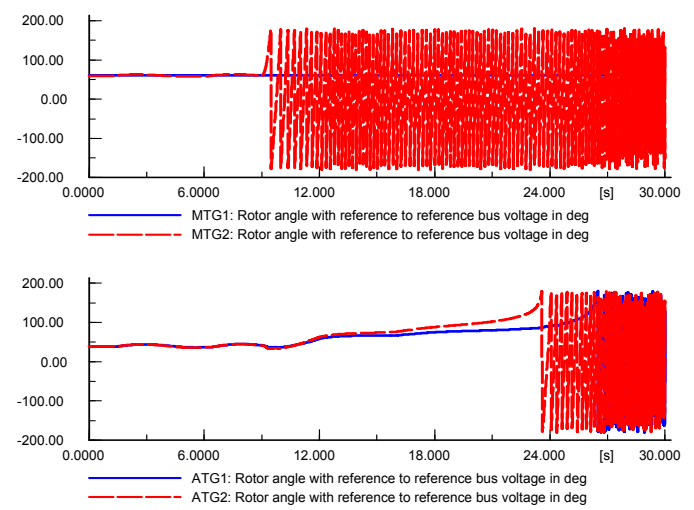

(f) Rotor Angle of the generators

Figure 8.16: Contingency events of MTG2 outage 

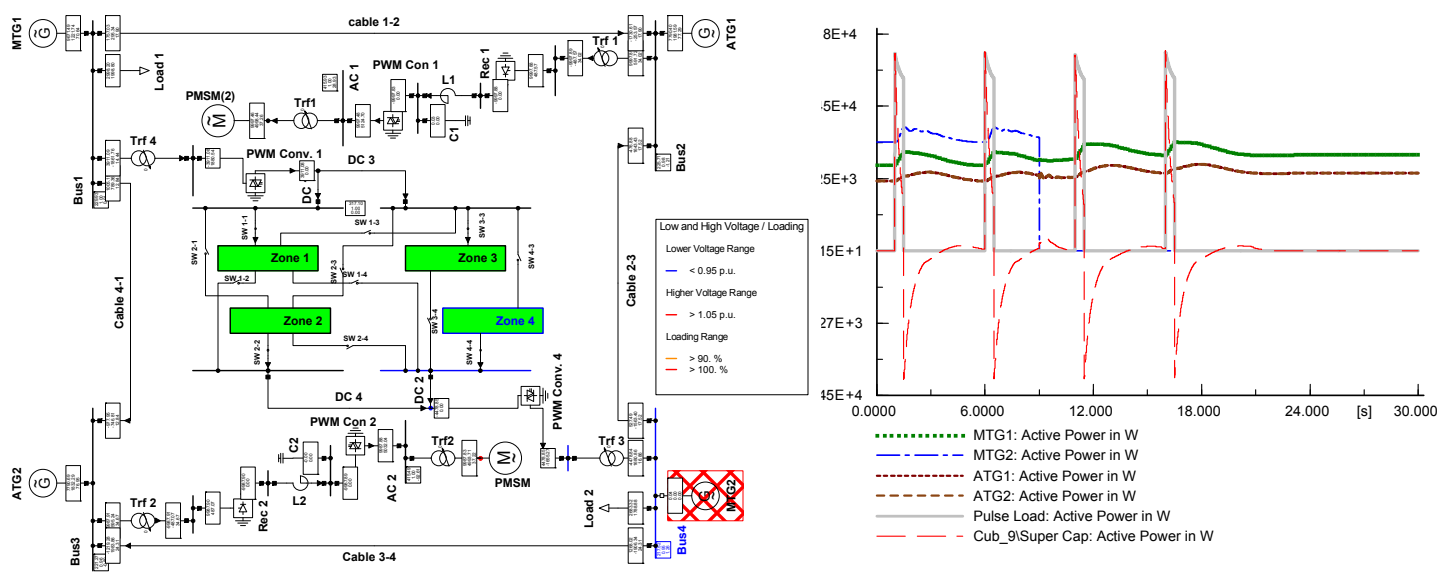

(a) Single Line Diagram
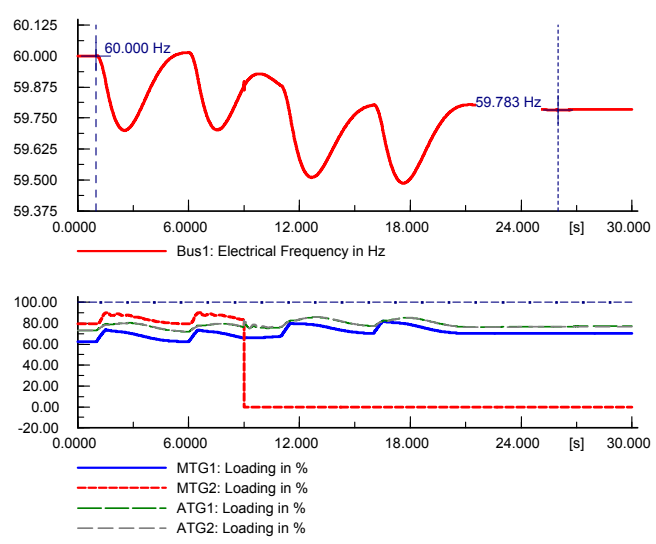

(c) Frequency of the system and loading of generators

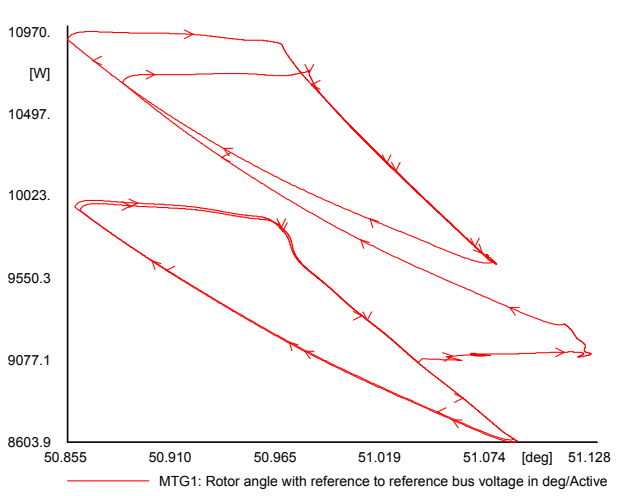

(e) Active power versus rotor angle (b) Power sharing of AC Generators during Pulse Load
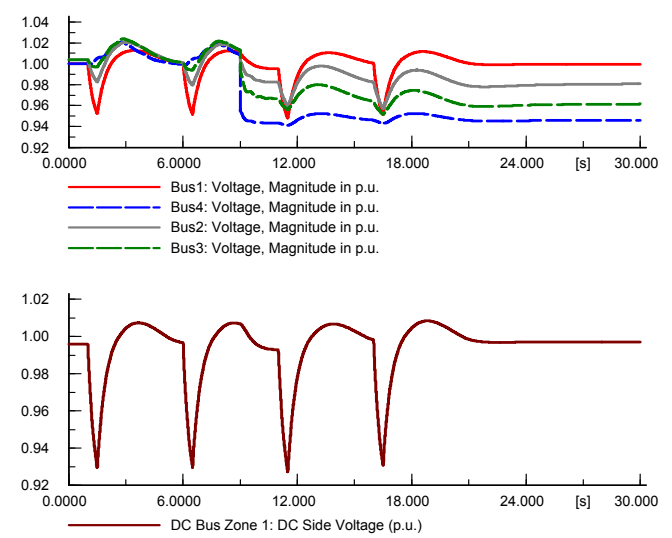

(d) Voltage of AC and DC buses

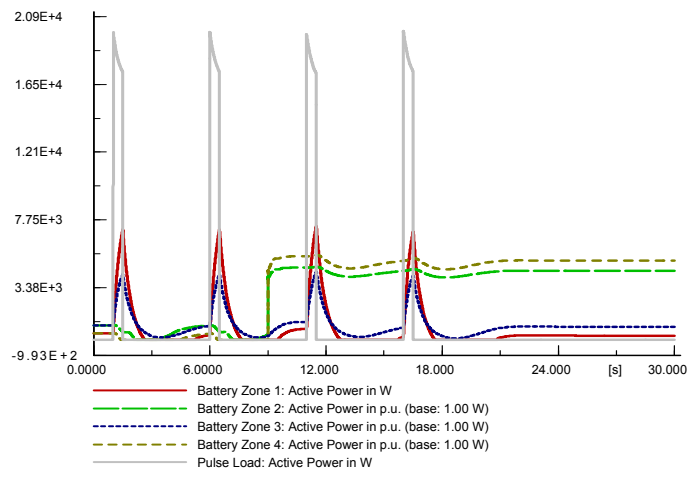

(f) Power sharing of storages during Pulse Load

Figure 8.17: Contingency events of MTG2 outage with distributed storages in all zones 


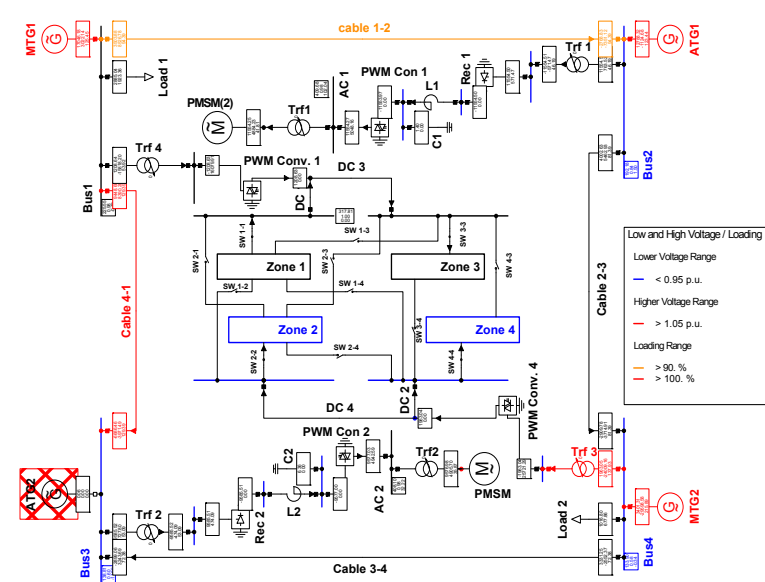

(a) Single Line Diagram
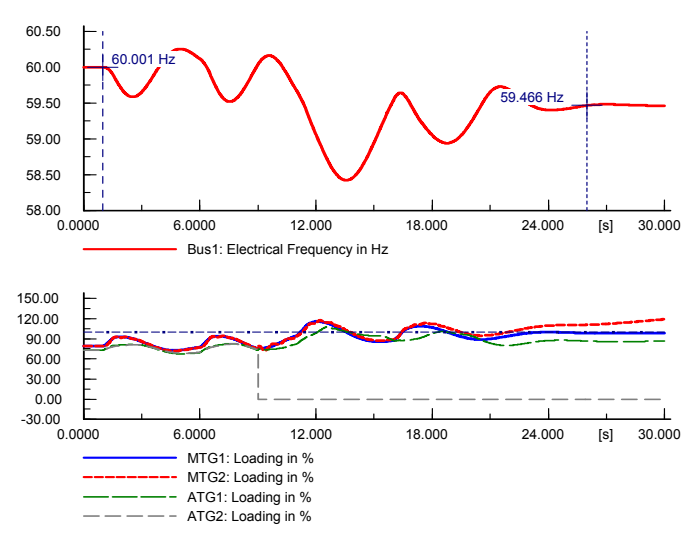

(c) Frequency of the system and loading of generators

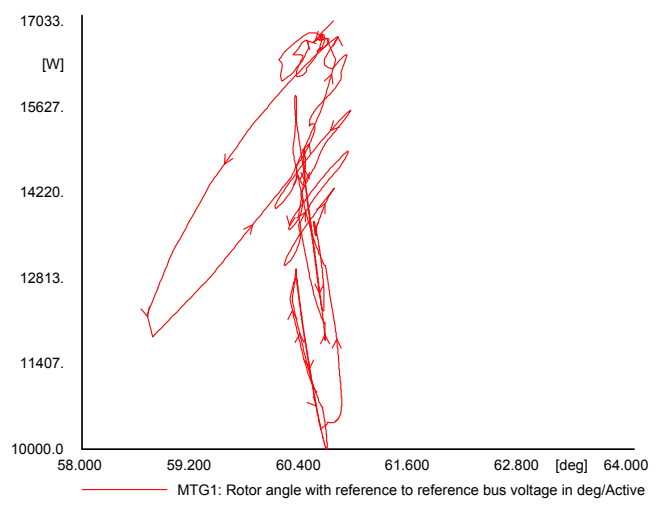

(e) Active power versus rotor angle

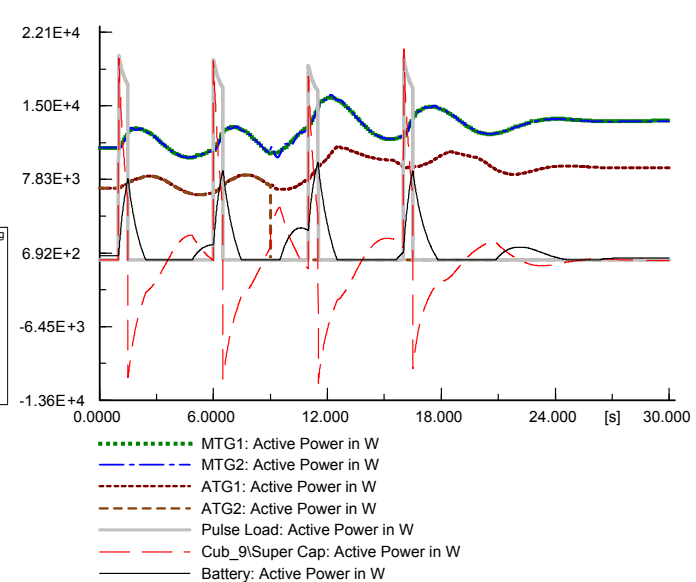

(b) Power sharing corresponding to the Pulse Load
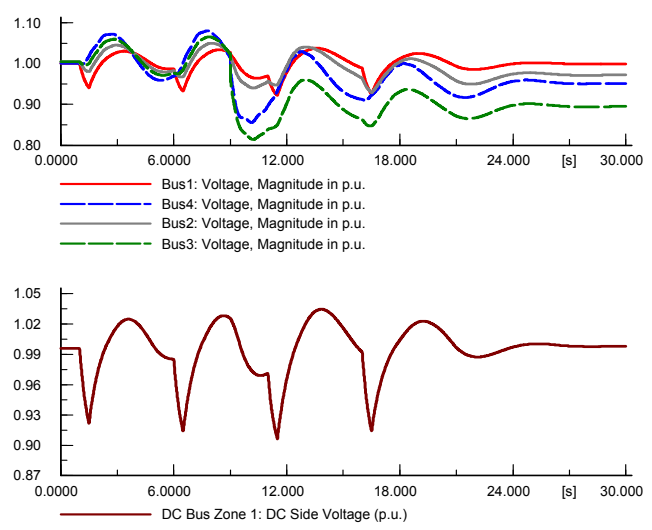

(d) Voltage of AC and DC buses
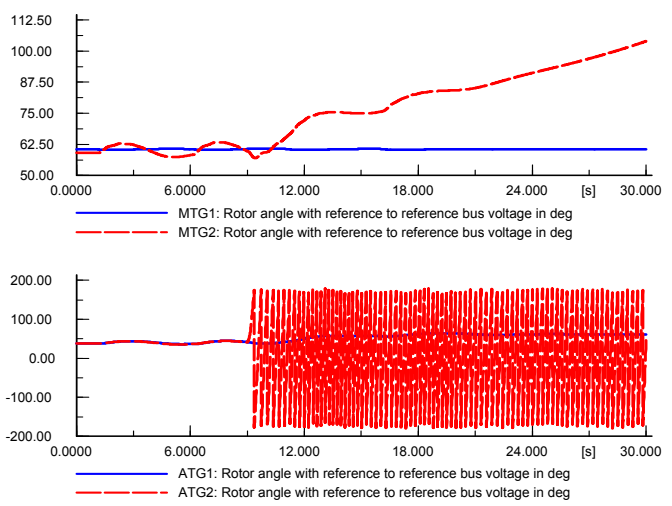

(f) Rotor Angle of the generators

Figure 8.18: Contingency events of ATG2 outage 


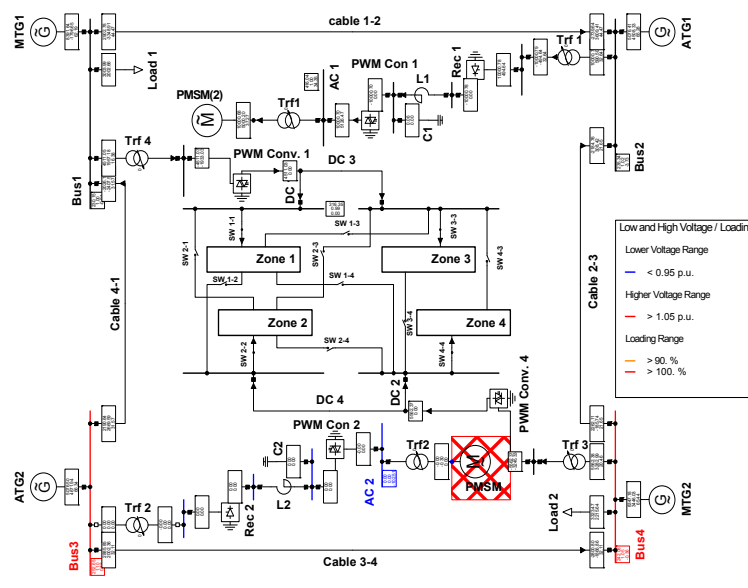

(a) Single Line Diagram
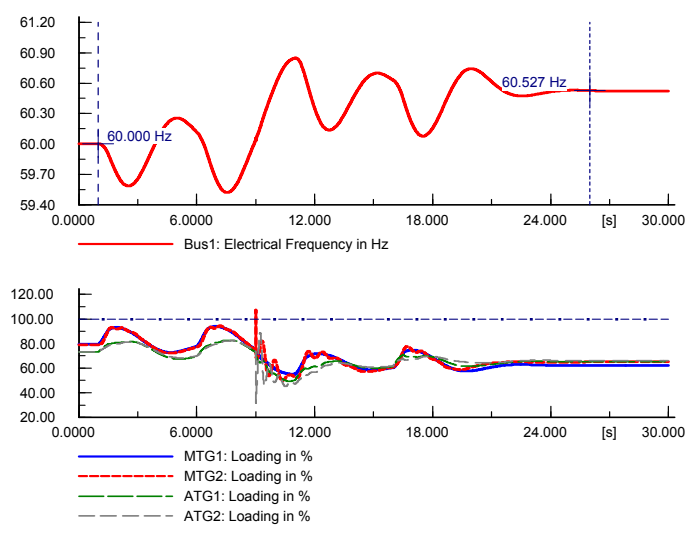

(c) Frequency of the system and loading of generators

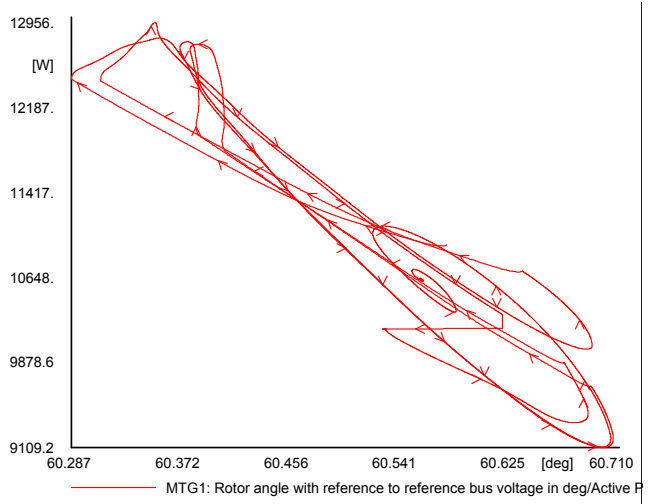

(e) Active power versus rotor angle

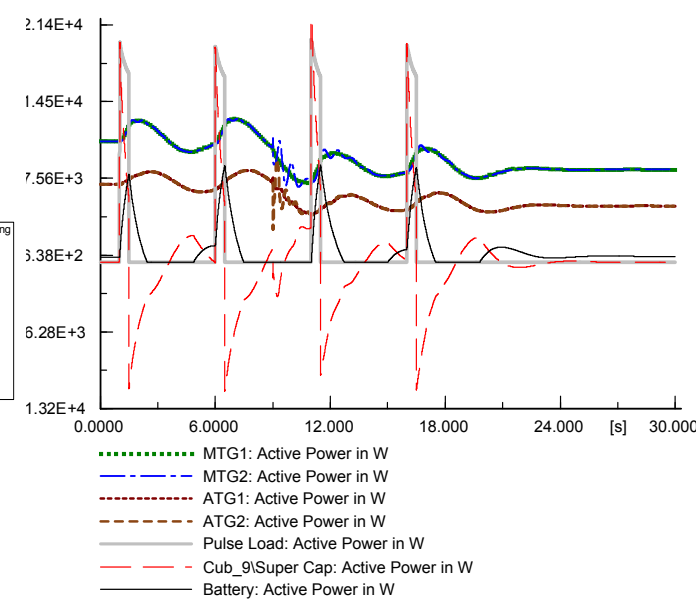

(b) Power sharing corresponding to the Pulse Load
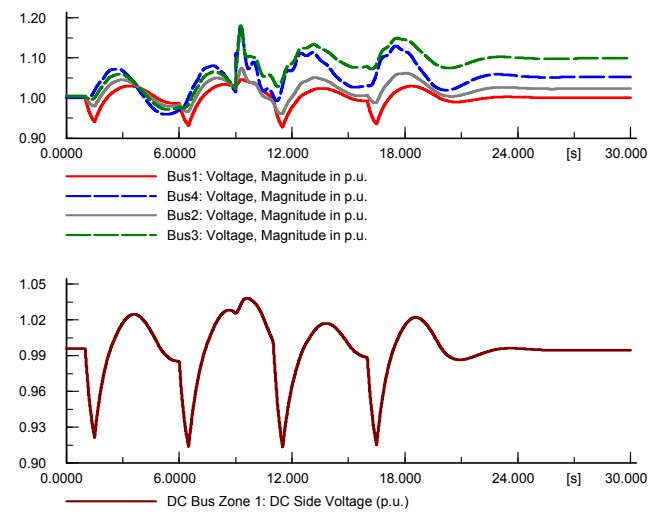

(d) Voltage of AC and DC buses
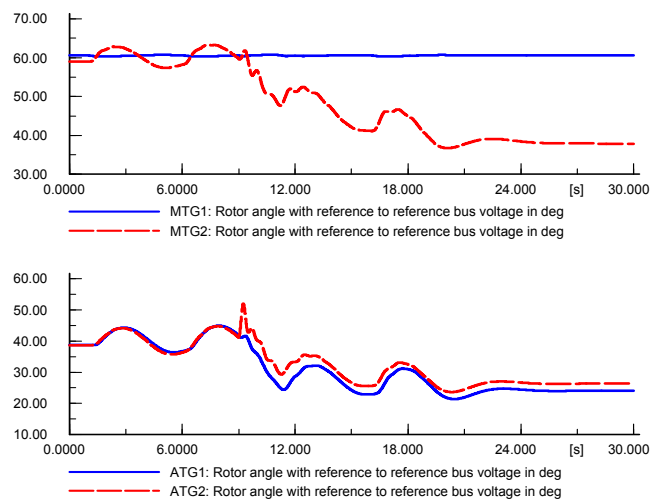

(f) Rotor Angle of the generators

Figure 8.19: Contingency events of PMSM outage 
Figure 8.21 shows the result for the case that we placed the storage at the further place of pulse load, i.e. Zone 4. According to this figure at this case SC power oscillates more significantly and it takes more time be charged (batteries are on another zone). Batteries start discharging not in synchronous with the pulse load. In addition they do not supply the same amount of energy while connected at the same bus where the pulse load is occurred. Overvoltage violation at buses 3 and 4 and under-voltage violation at bus 1 and the DC bus are reported.

In another case storages are distributed in two zones: zone 1 and 4; and the simulation results are shown in figure 8.22. In this case, oscillations are generally less than the case when pulse load occurs at zone 1 and battery storage is at bus 4 . Similar to previous case, battery at the far bus (Bus 4) starts discharging not in synchronous with the pulse load. Battery (or the portion of battery storage) at Bus 1 significantly assists the SC in mitigating the effect of the pulse load. No voltage, frequency or loading violation reported when the energy storage is divided between two zones.

The most reliable distribution of battery storage during sever conditions is when that the storage is divided into all four zones. The results for this case are shown in figure 8.23. According to this figure, all the generators encounter significantly less oscillations when the battery storage is distributed to different zones. Batteries contribute to mitigating the pulse loads and the voltage oscillations are within the $\pm 10 \%$ limit. No frequency violation is reported and the loading of all generators is within limits and is relatively low. 

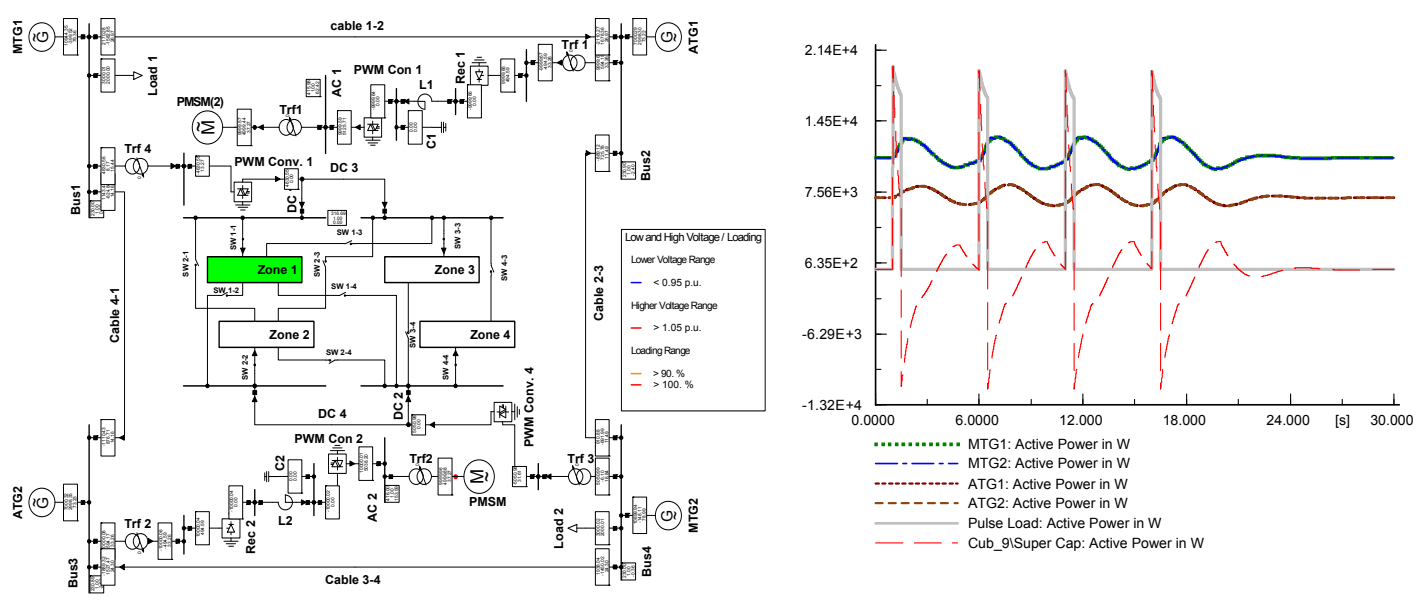

(a) Single Line Diagram

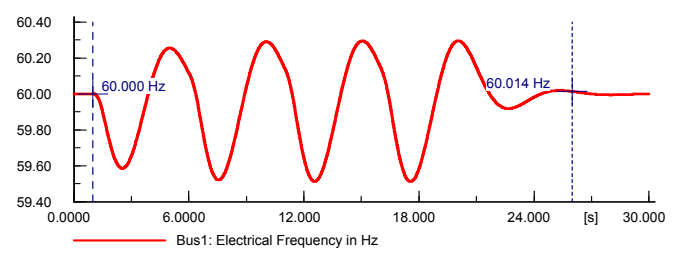

(b) Power sharing corresponding to the Pulse Load
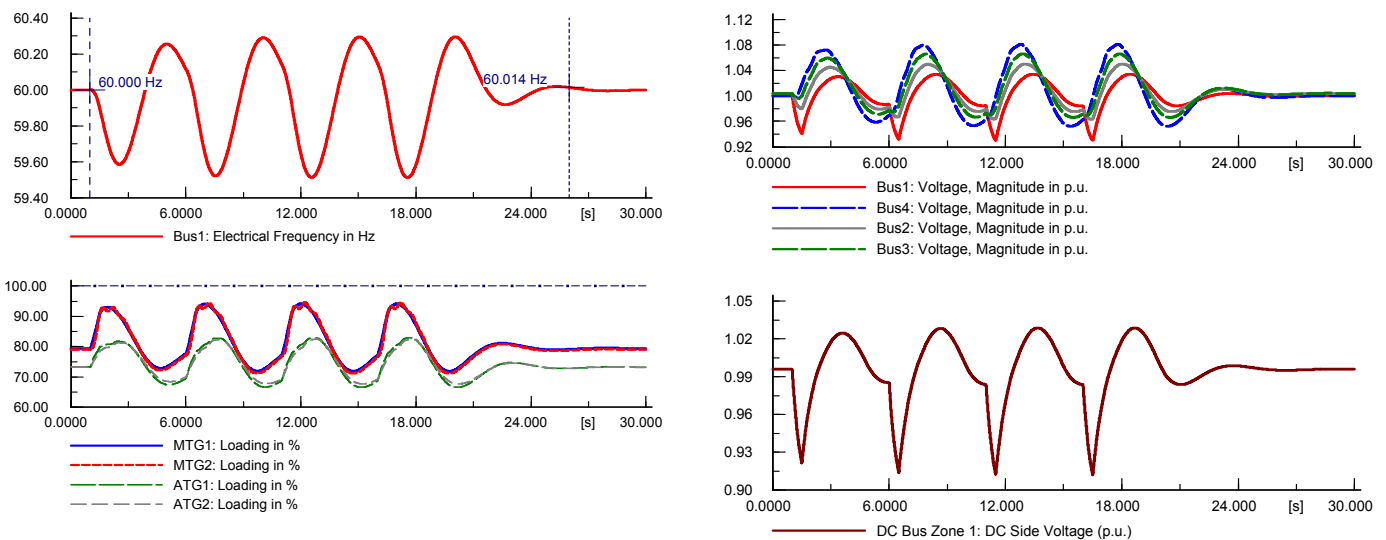

(c) Frequency of the system and loading of generators

(d) Voltage of AC and DC buses

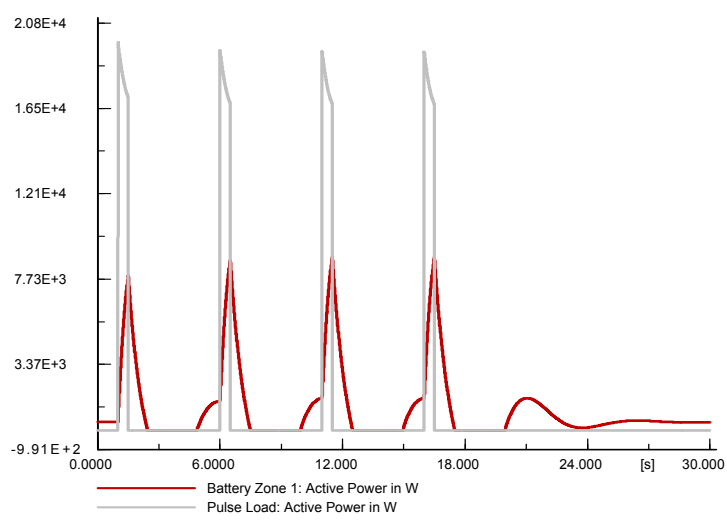

(e) Power sharing of storages during Pulse Load

Figure 8.20: Pulse Load and Storage at Zone 1 


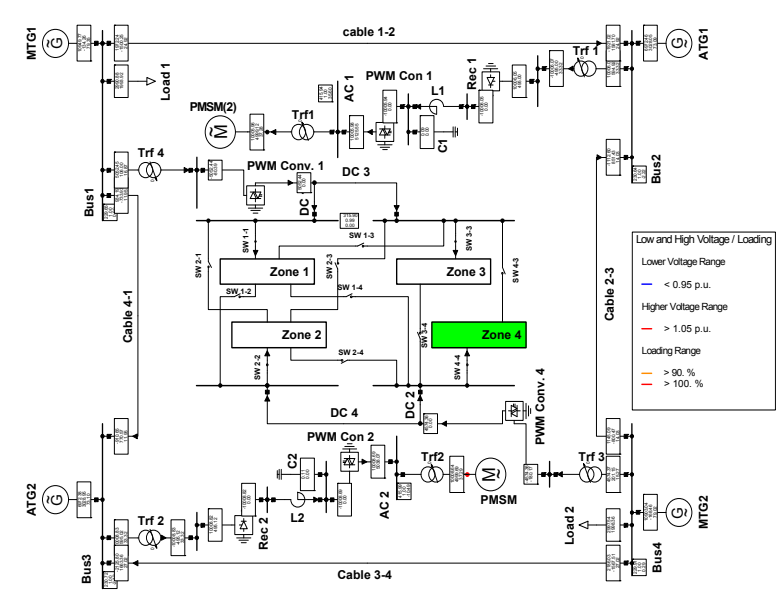

(a) Single Line Diagram
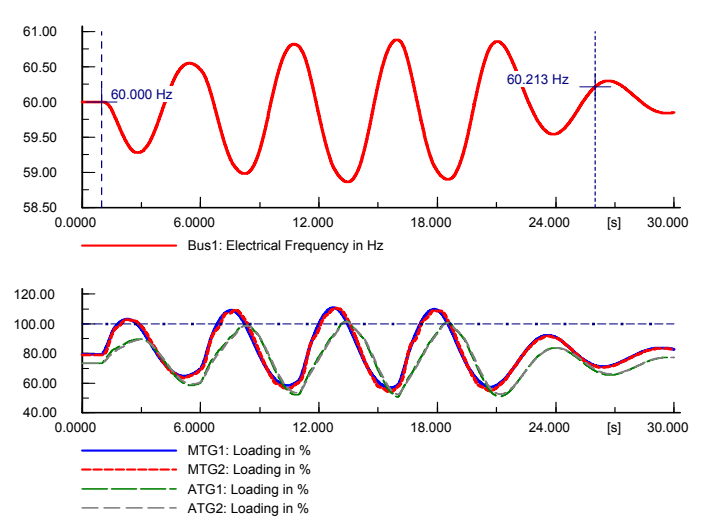

(c) Frequency of the system and loading of generators

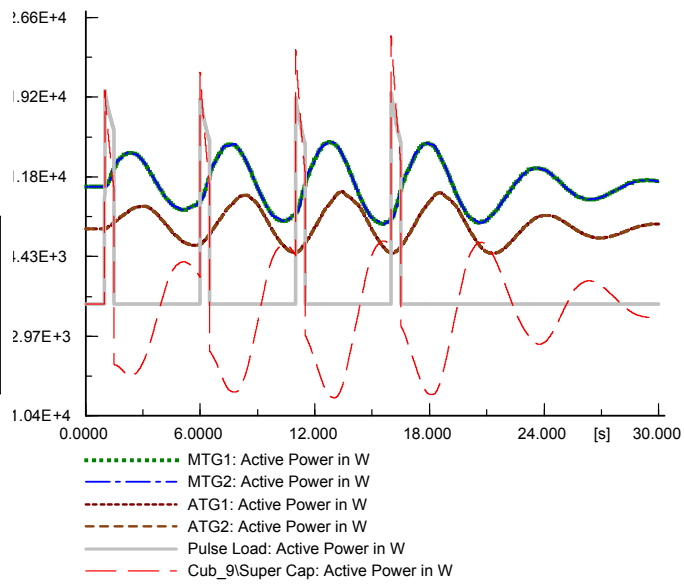

(b) Power sharing corresponding to the Pulse Load
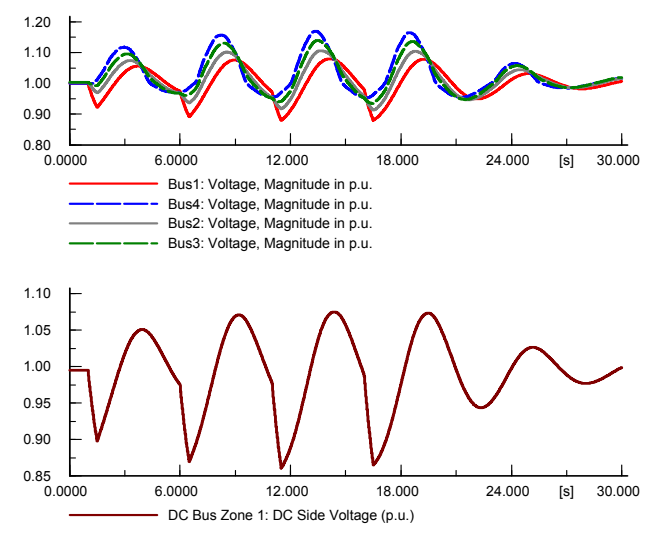

(d) Voltage of AC and DC buses

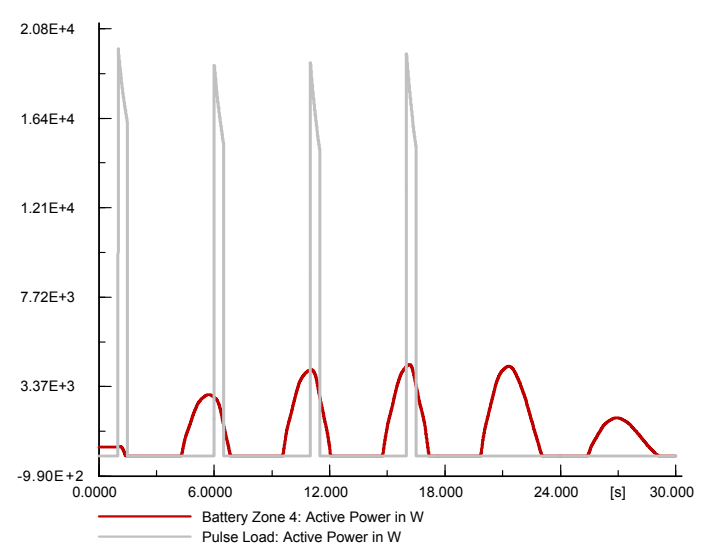

(e) Power sharing of storages during Pulse Load

Figure 8.21: Pulse Load at Zone 1 and Storage at zone 1 

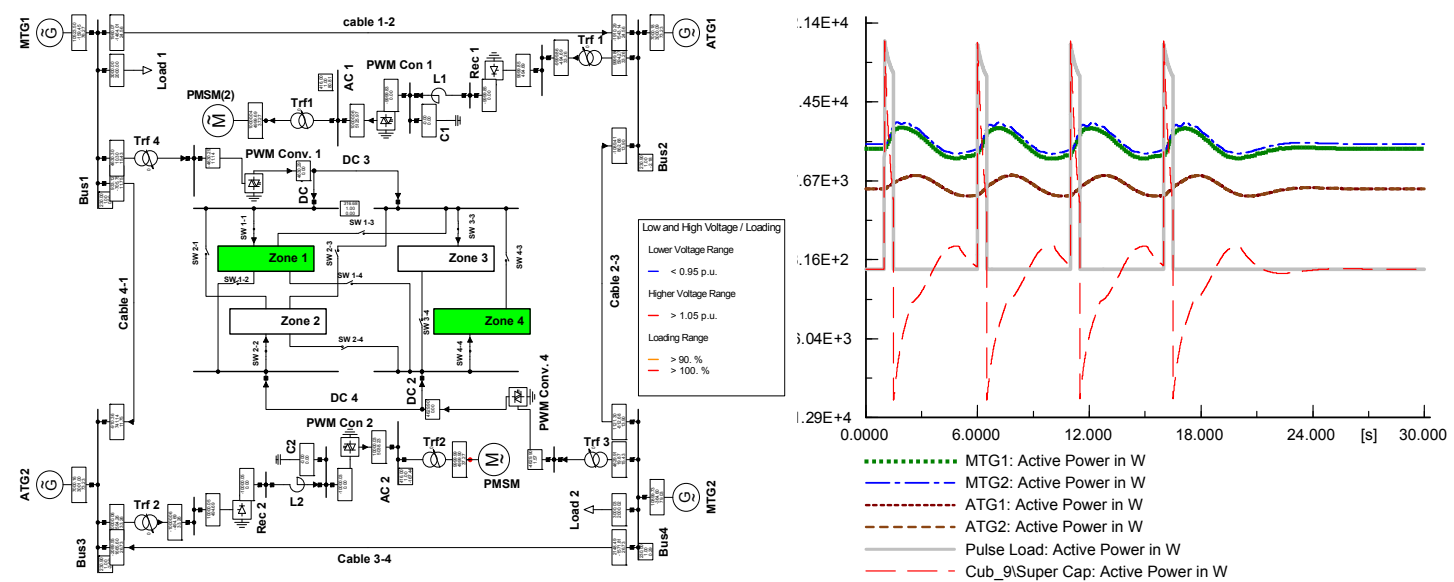

(a) Single Line Diagram

(b) Power sharing corresponding to the Pulse Load
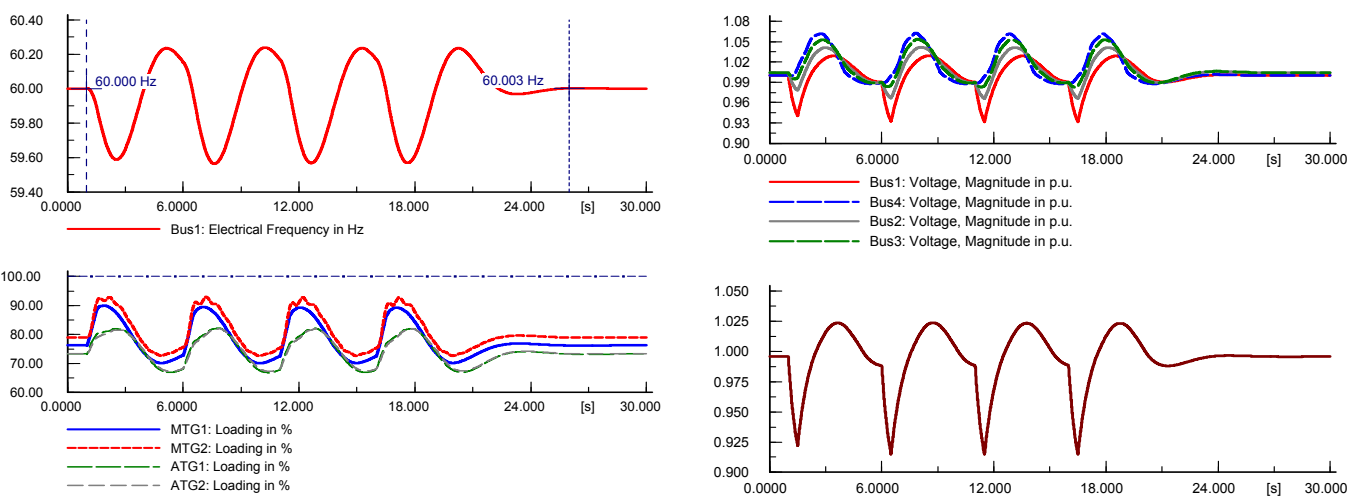

(c) Frequency of the system and loading of generators

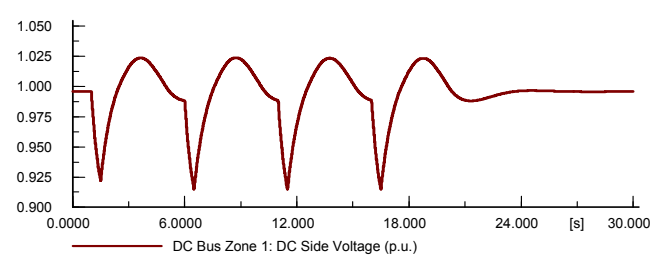

(d) Voltage of AC and DC buses

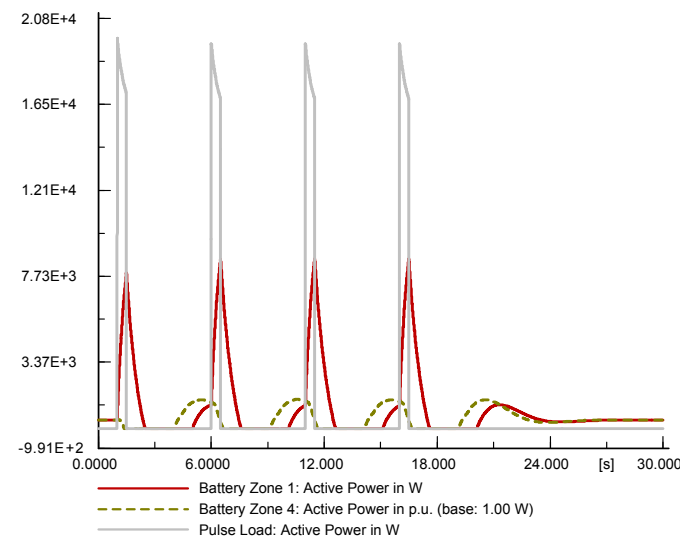

(e) Power sharing of storages during Pulse Load

Figure 8.22: Pulse Load at Zone 1 and storage distributed between Zones 1,4 


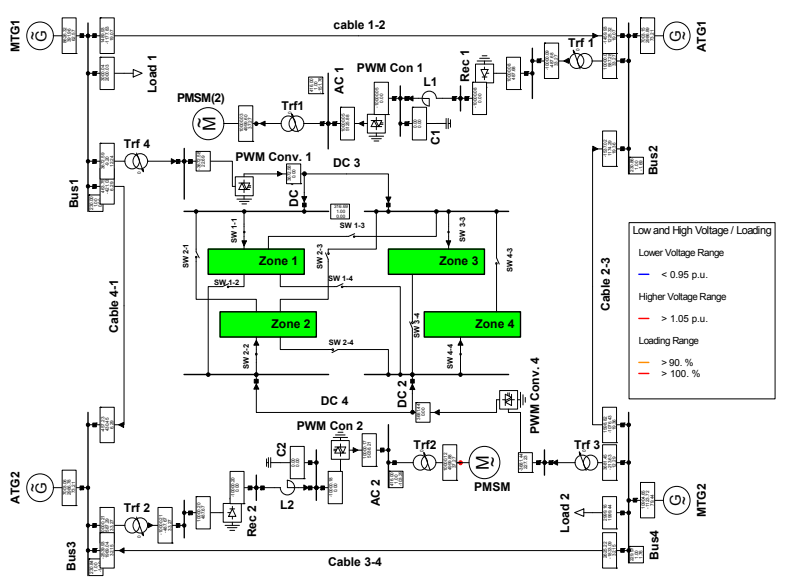

(a) Single Line Diagram
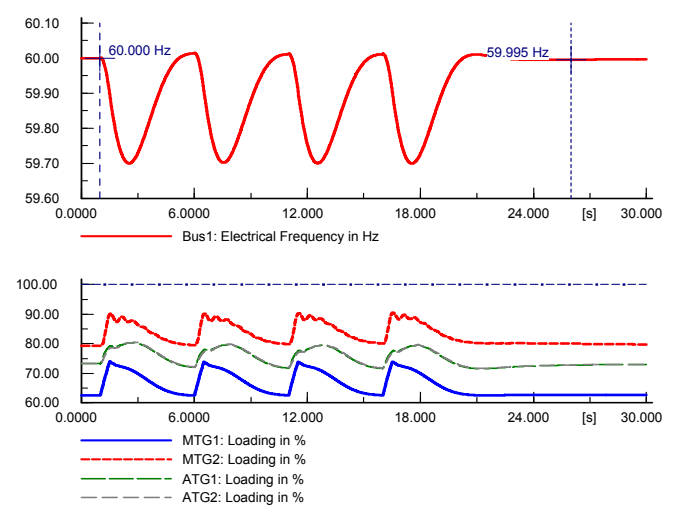

(c) Frequency of the system and loading of generators

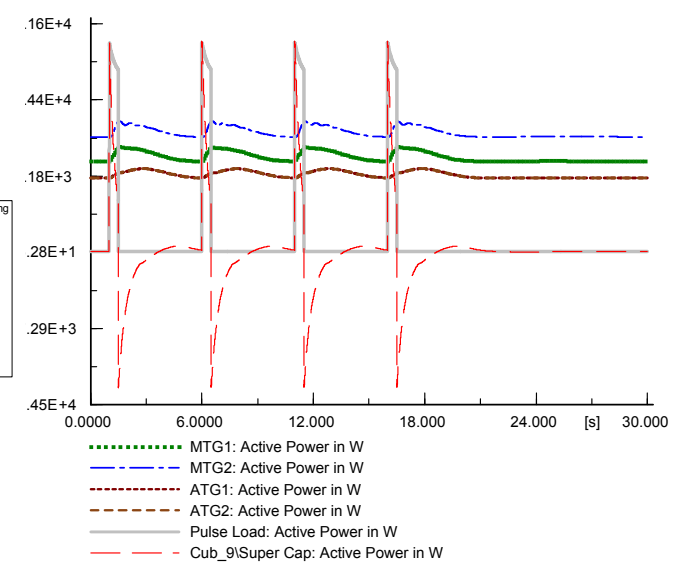

(b) Power sharing corresponding to the Pulse
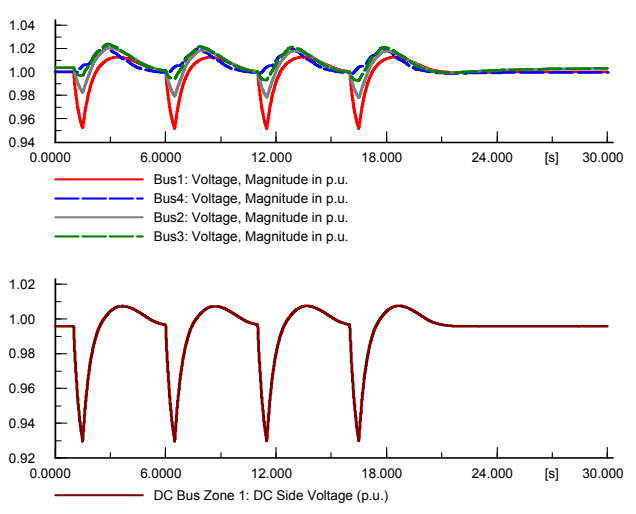

(d) Voltage of AC and DC buses

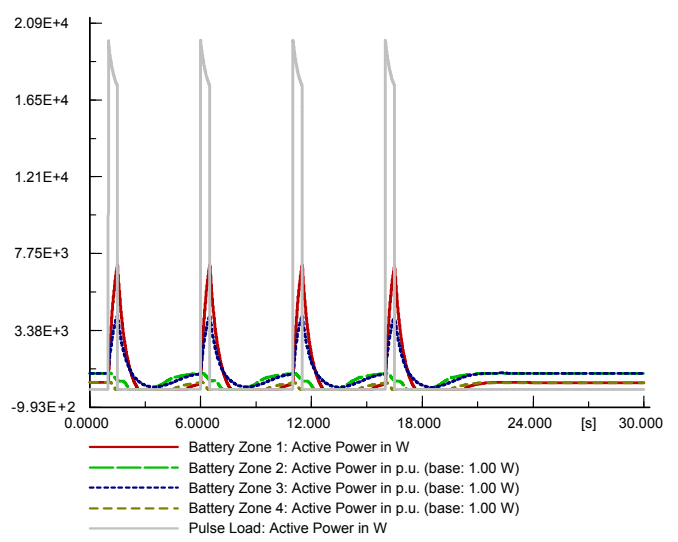

(e) Power sharing of storages during Pulse Load

Figure 8.23: Pulse Load at Zone 1 and Storage distributed between Zones 1,2,3\&4 
As the last case, the simulation has done for the case that we don't have any storage device in this microgrid. Figure 8.24 shows the results for this case and it illustrates that all the sources in the system including the generators and the SC encounter big oscillations corresponding the pulse load. Voltage violation is reported on the AC buses and on the DC bus and frequency violation and overloading are reported too.

Table 8.3 shows the summary of results for security and stability assessment of the system studied cases for better comparison. The system violations in different cases also have been shown in terms of voltage, frequency and loading of equipment.

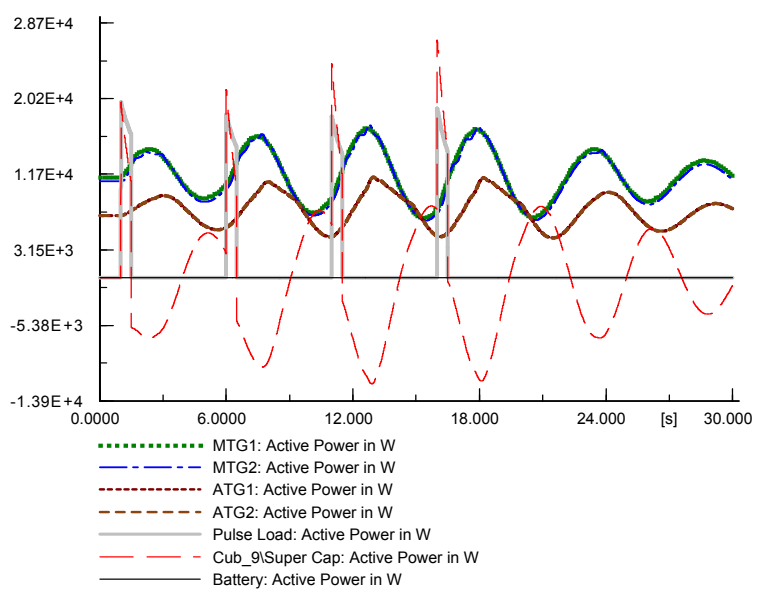

(a) Power sharing corresponding to the Pulse Load
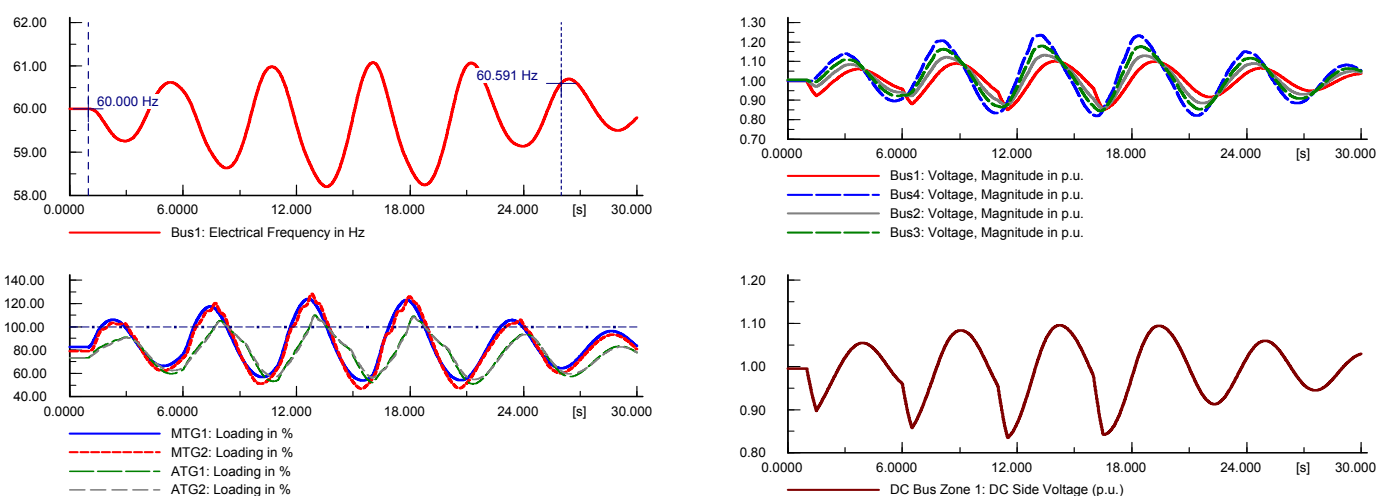

(b) Frequency of the system and loading of generators

(c) Voltage of AC and DC buses

Figure 8.24: Pulse Load at Zone 1 without any storage 
Table 8.3: System security and stability assessment for studied cases

\begin{tabular}{|c|c|c|c|c|c|c|c|}
\hline$\#$ & Event Description & Voltage & Frequency & Loading & Security & Stability & $\begin{array}{l}\text { Event } \\
\text { Type }\end{array}$ \\
\hline 1 & Cable 1-2 Outage & $x$ & $\checkmark$ & $\checkmark$ & Unsecure & Stable & \multirow{6}{*}{ 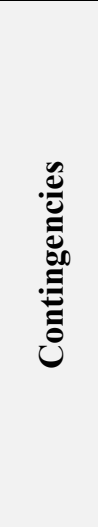 } \\
\hline 2 & Cable 1-4 Outage & $x$ & $\checkmark$ & $\checkmark$ & Unsecure & Stable & \\
\hline 3 & MTG2 Outage & $x$ & $x$ & $x$ & Unsecure & Instable & \\
\hline 4 & $\begin{array}{l}\text { MTG2 Outage with } \\
\text { Distributed Energy Storages }\end{array}$ & $\checkmark$ & $\checkmark$ & $\checkmark$ & Secure & Stable & \\
\hline 5 & ATG2 Outage & $x$ & $x$ & $x$ & Unsecure & Instable & \\
\hline 6 & PMSM Outage & $x$ & $x$ & $\begin{array}{l}x \text { (on } \\
\text { limit) }\end{array}$ & Unsecure & Stable & \\
\hline 7 & $\begin{array}{l}\text { Pulse Load and Storage at } \\
\text { Zone } 1\end{array}$ & $\checkmark$ & $\checkmark$ & $\checkmark$ & Secure & Stable & \multirow{5}{*}{ 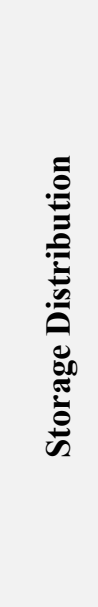 } \\
\hline 8 & $\begin{array}{l}\text { Pulse Load at Zone } 1 \text { and } \\
\text { Storage at Zone } 4\end{array}$ & $x$ & $x$ & $x$ & Unsecure & Stable & \\
\hline 9 & $\begin{array}{l}\text { Pulse Load at Zone } 1 \text { and } \\
\text { Storage distributed between } \\
\text { Zones } 1 \& 4\end{array}$ & $\checkmark$ & $\checkmark$ & $\checkmark$ & Secure & Stable & \\
\hline 10 & $\begin{array}{l}\text { Pulse Load at Zone } 1 \text { and } \\
\text { Storage distributed between } \\
\text { Zones } 1,2,3 \& 4\end{array}$ & $\checkmark$ & $\checkmark$ & $\checkmark$ & Secure & Stable & \\
\hline 11 & $\begin{array}{l}\text { Pulse Load at Zone 1 } \\
\text { without storage }\end{array}$ & $x$ & $x$ & $x$ & Unsecure & Stable & \\
\hline
\end{tabular}

\subsection{Energy Management in Hybrid Microgrids}

In previous section, a ship board power system was introduced as an example of a microgrid case study to identify the stability and security of the system. Other challenges include the situation of heavy loads with repeated short or long duration. Examples of such situations include large scale charging stations for plug-in electric vehicles and short duration load spikes. For these types of conditions in this chapter, we will give an example of an energy management system for mitigation of these special load conditions in a hybrid microgrid. These hybrid $\mathrm{AC} / \mathrm{DC}$ microgrids involve sustainable energy 
sources and hybrid energy storage. The hybrid storage system may consist of super capacitors (SC) for ultra-fast load matching beside lithium-ion batteries for relatively long term load buffering. For the energy management, an algorithm needs to be developed to manage the energy within the system such that the effect of these special heavy loads on the power system stability is minimized. The expected energy needed during a future peak, the time of its occurrence and the current state of charge of both elements of the hybrid storage system are all examples of the inputs required by such an algorithm. This author, along with others has developed a real time energy management to mitigate such conditions as published by Ahmed, Salehi, et. al [186]. In this work, the system loads are based on hourly average variations and can be considered as lowfrequency variation, whereas power transients, which is sustained for minutes, seconds, or milliseconds come under the high-frequency segment. To buffer these low-frequency oscillations and to compensate for the intermittency of the renewable energy sources on the microgrid in addition to the energy storage with high energy density is required. To provide the high-frequency component of power and also to supply or absorb the highpower transients, energy storage with high power density is required [169]. Recently, high-power capability of super capacitors hybrid energy with batteries or fuel cells are exploited in pulsed operating modes for portable power systems, electric vehicle and digital telecommunication systems [170]-[172]. Advanced storage technologies now allow extraordinary energy densities where the load draws large power impulses. This deficiency can be solved by using more batteries in parallel. Technically, hybrid power sources that utilize batteries with advanced charge/discharge strategies in parallel with 
super capacitors can overcome the power deficiency problems and increase the operating time [173], [174], [186 ].

Dynamic energy management in a smart grid whether directed towards the microgrid or electric vehicle applications was investigated in several publications [169], [174]-[177]. The effort here aims at handling renewable energy and its uncertainty, managing the demand side in an intelligent manner to enhance the performance of the microgrid and the connected main grid. Additionally, this is also done to achieve optimal economic operation of the system while operating, sometimes under special conditions such as occurrence of a pulsed load condition.

\subsection{Hybrid Storage System}

According to Ragone Plot [178], which is usually employed to classify the available energy sources according to their power/energy density, high energy Li-ion rechargeable batteries have the highest energy density of all modern batteries 50-500 $\mathrm{Wh} / \mathrm{kg}$ and low power density of 10-500 W/kg. On the other hand, super capacitors have a high power density of $1000-5000 \mathrm{~W} / \mathrm{kg}$ and a very low energy density of $1-10 \mathrm{Wh} / \mathrm{kg}$. Moreover, the internal resistance of super capacitors is much lower than that of a battery resistance. Therefore, it has much higher charging/discharging efficiency [179], [180]. In

order to retain the benefits of high specific power and energy, a hybrid Li-ion/super capacitor storage system was utilized in this research to mitigate these special load conditions and use the batteries for relatively longer time to supply normal load conditions as well. 
The hybrid storage system is modeled as shown in figure 9.25 (a). The battery is modeled by an ideal voltage source in series with its internal resistance $R_{b}$ whereas the super capacitors are modeled by the nominal capacitance $\mathrm{C}_{\mathrm{c}}$ in series with an equivalent series resistance $R_{c}$. A hybrid storage system with battery and super capacitors only, without utilizing any DC-DC converters as shown in figure 9.25 (b), is called a passive hybrid. However, if a DC-DC converter is used, as an interface between the batteries and the super capacitor, significant improvement can be achieved. This was experimentally verified in [181] in which the battery current controlled to a constant value.

Moreover, there will be no need for voltage matching between the battery and the load. The active hybrid yields a peak power that is 3.2 times that of a passive hybrid and a specific power of 2.7 times that of a passive hybrid [181]. These features allow the active hybrid storage systems to mitigate these special loads conditions efficiently if properly designed.

\subsection{Real-Time Energy Management Algorithm}

The main objective of the energy management algorithm developed by Ahmed, Salehi, et al in [186] is to mitigate pulse loads. In addition, the total cost of energy is to be reduced using this algorithm by minimizing the energy drawn from the main grid and/or shifting it to off-peak hours. Therefore, we can define two main modes of operation namely; large heavy load mitigation mode and the normal operation or cost minimization mode. The priority setting of the algorithm can be listed as follows: 


\subsubsection{Fully Charged Super Capacitors}

The super capacitors are fully charged to mitigate possible heavy short duration loads. To achieve this in real time, the amount of energy available in the super capacitor $\left(E_{S C}\right)$ must be monitored and compared to the energy that would be available in fully charged super capacitors $\left(E_{F S C}\right)$ to assure having all the super capacitors initially charged and ready to operate. If the super capacitors are not fully charged, in case they are connected to the DC bus through a DC-DC converter [180], they are immediately charged using the batteries and/or the grid power according to the availability of energy in the battery.

\subsubsection{Li-ion Batteries Support Super Capacitors}

The Li-ion batteries have enough energy to help super capacitors mitigate pulsed loads. Li-ion battery is used along with super capacitors to mitigate the effect of pulsed loads. Therefore, the batteries are controlled such that they are 100\% charged, according to the design of the storage system -, before the moment of occurrence of a heavy short duration load. However, if the future heavy load pulse is predicted to take place after time $\left(T_{P L}\right)$ that is more than the time needed to fully charge the battery $\left(T_{F B}\right)$, the battery is used in the normal manner for the cost minimization mode.

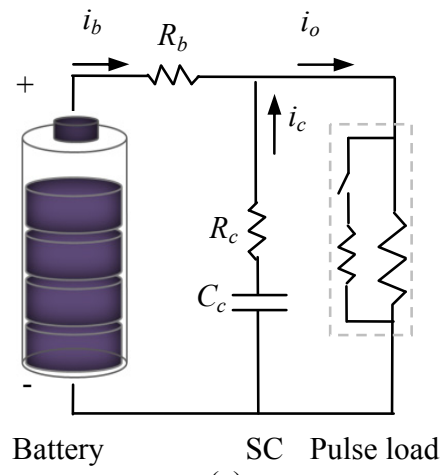

(a)

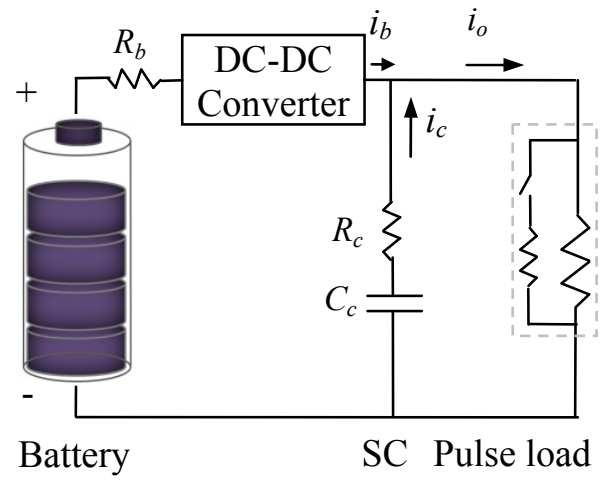

(b)

Figure 8.25: Battery/SC hybrid storage system: (a) Passive hybrid, (b) Active hybrid. 


\subsubsection{Supplying Normal Loads by PVs}

Normal loads on the smart microgrid are supplied using PV system operating at its Maximum Power Point (MPP). The loads on the DC microgrid are supplied by the PV generation. If there is a surplus in power, which means that the difference $P_{\text {diff }}>0$, where

$$
P_{\text {diff }}=P_{P V}-P_{\text {Load }}
$$

There will be two options; either to charge the battery or to sell this surplus power to the grid. In order to minimize the annual overall cost of energy and maximize the saving, the decision is made based on the energy tariff. Utility grids increase the tariff during peak hours. Hence, if it is a peak period, which is defined as two hours around the peak of the daily load curve, priority is given to selling the power to the utility grid. Otherwise, batteries are charged. However, this all depends on the state of charge $(\mathrm{SoC})$ of the batteries. If there is a surplus power during a peak period, the percentage of the surplus power that goes to the battery $\left(P_{\text {batt }}\right)$ follows the formula proposed in [139] as:

$$
P_{\text {batt }}=\left(100 \times e^{-0.05 \times \mathrm{SoC}}\right) \times P_{\text {diff }}
$$

However, if the surplus power is available during an off-peak period, the percentage of the surplus power that goes to the battery $\left(P_{b a t t}\right)$ follows the following formula [139]:

$$
P_{\text {batt }}=\left(-1.9287 \times 10^{-20} \times e^{0.5 \times \mathrm{SoC}}+100\right) \times P_{\text {diff }}
$$

The batteries' SoC can be estimated as follows:

$$
\operatorname{SoC}(t)=\frac{E_{F B}-\int_{0}^{t} i_{b}(\tau) d \tau}{E_{F B}} \times 100 \%
$$

where, $E_{F B}(\mathrm{Ah})$ is the full charge or total energy capacity of the battery. 
The hour at which the next peak load is taking place $\left(T_{P K}\right)$ can be calculated using the predicted load curve of the day. At peak instance, we have:

$$
\left.\frac{\partial P_{\text {Load }}}{\partial H}\right|_{D=D 1}=0, i=1: 365
$$

where, $D 1$ is the day in which we are calculating. The solution of Eqn. (8.5) yields the peak hour TPK. The peak period is defined as

$$
T_{P K}-1<t \leq T_{P K}+1
$$

\subsubsection{Supply Loads with Battery during Peak Period}

Any load deficiency on the microgrid is supplied from the battery if it is during a peak period or from the grid if it is an off-peak Period. If there is a deficiency in power defined by, $P_{\text {diff }}<0$, priority is given to satisfy this deficiency using the energy stored in the batteries. However, the energy tariff is again taken into consideration. If ' $t$ ' lies within the peak period, the energy stored in the battery system is used to satisfy the biggest possible share of the load demand. The rest is satisfied from the grid. The percentage of the power deficiency that will be shared by the battery system is implemented mathematically using the following exponential curve [139]:

$$
P_{\text {batt }}=\left(36.7879 \times e^{0.01 S o C}\right) \times P_{\text {diff }}
$$

If ' $t$ ' lies within an off-peak period, $P_{\text {diff }}$ is covered partially by the battery system based on $T_{P K}$. If there is enough time to recharge the batteries and have them ready during the future peak period, the batteries are more involved in satisfying the load deficiency. However, if the time needed to charge the batteries is less than $T_{P K}$, the batteries are carefully discharged. This algorithm is implemented using a fuzzy agent within the algorithm. Inputs to the fuzzy agent are $T_{P K}$ and $R$, which is the ratio between the energy 
available in the batteries to the energy needed during the future peak period. The fuzzy system is explained in details in the following subsection by the battery system. This fuzzy system was based on the forecasted data of the expected next peak period and the energy needed within it. The energy demand during the peak period $E_{P K}$ can be calculated by:

$$
E_{P K}=\int_{T_{P K}-1}^{T_{P K}+1} P_{\text {load }} \cdot d t
$$

\subsection{Testing the Real-Time Energy Management Algorithm}

In order to evaluate the performance of the algorithm developed by Ahmed, Salehi, et. al. presented in [186] under special heavy load conditions, an example hybrid AC/DC system was simulated. The example system resembles a ship power system with scaled down ratings. This system includes two $13.8 \mathrm{~kW}$ main generators (MTG) and two $10.4 \mathrm{~kW}$ auxiliary generators (ATG) connected in a ring bus configuration. The bulk of the load consists of two $50 \mathrm{~kW}$ propulsion motors, modeled as permanent magnet machines supplied by PWM drives, with hydrodynamic propeller models as the mechanical load. Each rectifier supplies one of two $0.318 \mathrm{kV}$ DC busses. The DC distribution zone is supplied by one of the two available rectifiers. Although various models for the loads may be used, constant impedance models were used in this example.

Furthermore, a photovoltaic (PV) system of $10 \mathrm{~kW}$ rated capacity, lithium-ion batteries with $3000 \mathrm{Ah}$ rated capacity and super capacitors with $200 \mathrm{~F}$ are included in the DC microgrid. A PWM controlled DC-DC converter is used as an interface between the PV system and the DC bus. 
A vector decoupling PWM controlled AC-DC/DC-AC bidirectional converter was used for connectivity between the AC and DC sides [186]. In the steady state case, the system voltages and loadings are within the normal limits.

In order to evaluate the performance of the developed optimal algorithm designated (Algorithm 1), its operation was compared with the operation of a standard algorithm designated (Algorithm 2) [186]. The latter does not take into consideration any variation in the power tariff during the day and its operation can be summarized as follows; if there is a surplus in power $\left(P_{\text {diff }}>0\right)$, the batteries will be charged until they are fully charged then the extra power will be sold to the grid. However, if there is a deficiency in power, the batteries will be discharged until they reach their lower discharge limit then the power will be drawn from the grid.

In normal operation with no heavy short duration loads predicted or taking place in the system in the near future, the developed algorithm aims at minimizing the total cost of energy. This is achieved by handling the battery and controlling the power flow between the $\mathrm{AC}$ and $\mathrm{DC}$ sides based on the changes of the power tariff. Figure 8.26 shows the PV and load data of the 24 hours at a given day $(\mathrm{D} 1=200)$ of the year. As can be seen, there is a deficiency $\left(P_{\text {diff }}<0\right)$ on that day until around hour $7(7: 00 \mathrm{am})$, then there is a surplus in power until around hour $14.67(2: 40 \mathrm{pm})$ and finally there is a deficiency in the power until the end of the day. The peak load of that day takes place at hour 18 , hence $T_{P K}=18$. Moreover, $E_{P K}$ can be found by integrating the area under the load curve within the interval 17-19. The second subplot of figure 8.26 shows the power surplus/deficiency during the day, the power shares from the battery and the grid using both algorithms. The sampling time $T_{s}$ is $0.1 \mathrm{~ms}$, whereas the initial $\mathrm{SoC}$ is $90 \%$. During 
the power deficiency taking place at the beginning of the day, both algorithms tend to satisfy the load using the energy stored in the battery. Moreover, when there is a surplus in power, the battery is charged to its full capacity and the extra power is injected back to the grid. However, during the second deficiency interval starting at 2:40 pm, there is a significant difference between the operations of both algorithms. Since the future peak period is approaching soon, Algorithm 1 saves the energy stored in the battery and satisfies the load demanded using the grid power. This stored energy can be used to minimize the power drawn from the grid during the peak period when the power tariff is the highest during the day. On the other hand, Algorithm 2 tends to satisfy the load deficiency using the energy stored in the battery without considering the future peak period.

Consequently, when the peak period starts, there is no means to supply the load other than drawing the power from the main AC grid with the high tariff. The difference in the energy supply is the highlighted area between the two curves; $P_{\text {battl }}$ and $P_{\text {batt } 2}$. Hence, a significant saving in the total energy cost during the year can be achieved by applying Algorithm 1. The saving during a day can be calculated as

$$
S=\left(T A R_{P K}-T A R_{O F F-P K}\right) \times \int_{T_{P K}-1}^{T_{P K}+1}\left(P_{b a t t 2}-P_{b a t t 1}\right) \cdot d t
$$

where $T A R_{P K}$ and $T A R_{O F F-P K}$ are the power tariffs during the peak and off-peak periods, respectively. Assuming that $T_{A R}$ is $100 \%$ higher than $T_{A R_{O F F-P K}}$ [29], a total annual saving of $7-9 \%$ can be achieved. It should be noticed here that the developed algorithm was based on forecasted data of PV generation and load demand, which will definitely include some prediction error, hence the total annual saving is dependent on 
that error. For instance if the PV and load models used are replaced with models having MAPE values of $10 \%$ and $13 \%$, respectively, the total saving drops to $5.8-7.2 \%$.

This operation can be also seen in the third subplot of figure 8.26 by noticing the state of charge (SoC) of the battery using both algorithms. It can be seen that at the beginning of the peak period, Algorithm 1 enables the battery to have a SoC of almost 98\% whereas Algorithm 2 depletes the battery to almost its lower limit.

These findings are important in managing the energy in microgrid systems and enabling the utilization of the energy available on the microgrid to be utilized on the AC side of the grid. Furthermore, the economic functions verified above can be utilized for allowing proper microgrid energy management. The above developed ideas can be implemented in smart meters or as economic functions in the real time operation of hybrid AC/DC power systems. Additional details on this microgrid energy management system and additional further utilization of this algorithm on the AC side of the grid are detailed in reference [186] by Ahmed, Salehi, et. al.
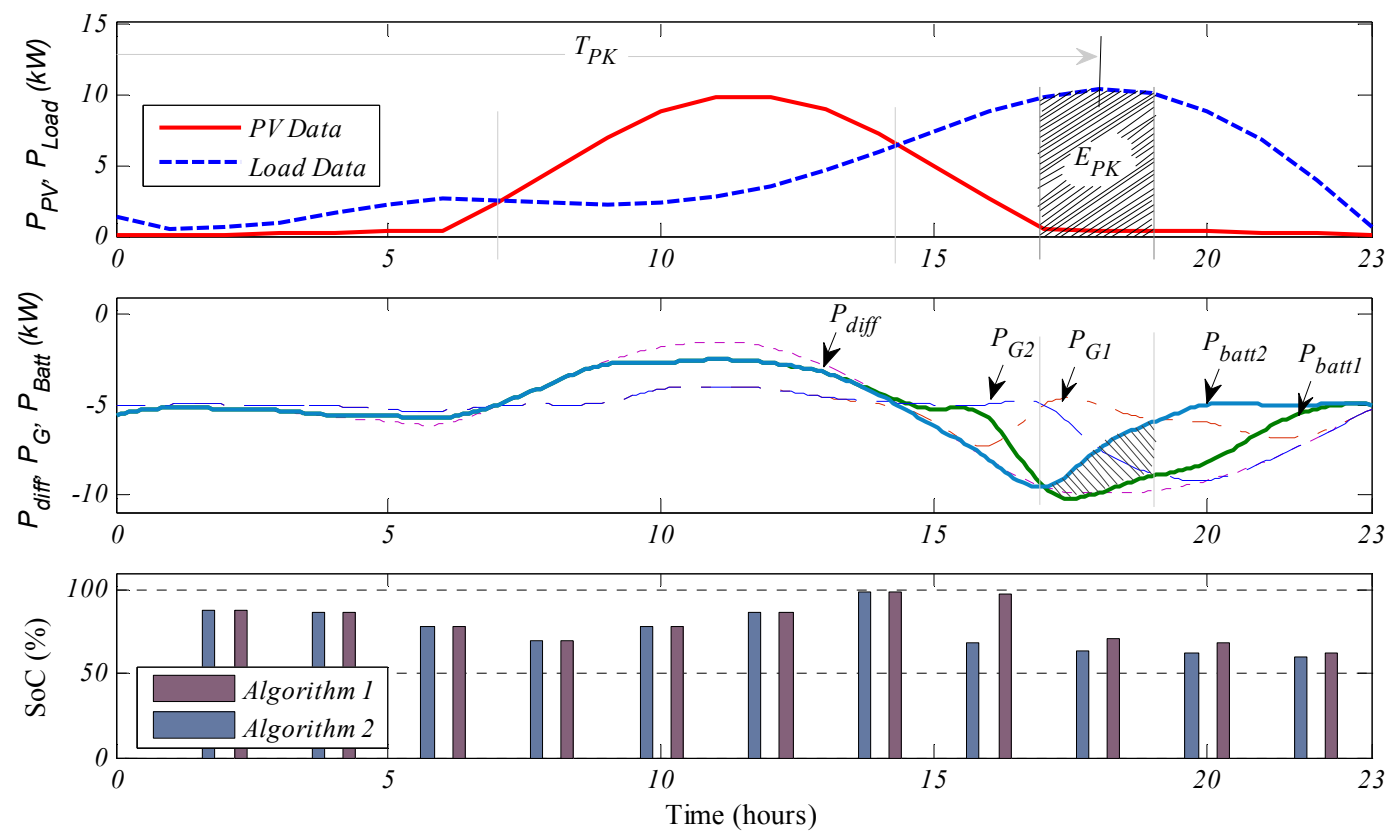

Figure 8.26: Operation of the system for a 24 hours interval, while applying Algorithms 1 and 2 


\subsection{Power Sharing Control of Hybrid System during Generation Outage}

As long as we implemented a distributed control concept on the testbed which involves separated AC and DC control systems, various power sharing control scenarios can be implemented. The communication link between the $\mathrm{AC}$ and $\mathrm{DC}$ controllers give the system the capability to facilitate the power sharing between the AC and DC parts. As it was discussed in chapter 6 , it can be used for optimal power sharing between the AC generators and the DC side power injected power. It was shown that by proper reactive power injection we can optimize AC side problems such as overloading of equipment and under voltage of buses. Hence the communication link between the AC and DC controller systems may enhance the system performance under different circumstances, particularly in outage of generators.

Since G1 generator has the responsibility to maintain the system frequency, during the outage of other generators it will take the share of the power deficit to keep the loads supplied. Because of the large amount of disconnected power, the slack generator may experience overloading for a while until the setting of other generators change in order to alleviate the loading of the slack generator. This scenario was studied in section 5.3.2 by switching large loads and using manual and automatic power sharing experiments.

Another scenario will be using the DC microgrid power to keep the loads in service by injecting the required power requested from the $\mathrm{AC}$ side and Wide Area Monitoring and Control system. An experiment was designed with three AC generators and connected the DC side which is ready to inject active and reactive power when it is requested from the real-time controller. The outage of generator G3 with $750 \mathrm{~W}$ 
generation level is intended. According to figure 8.27 which shows the active and reactive power of all $\mathrm{AC}$ generators, after the outage of large $\mathrm{AC}$ generator, $\mathrm{G} 3$, first the slack generator, G1, will increase active and reactive power to keep the system frequency maintained at nominal value rapidly (see the figure 8.28 ). This already occurs because the frequency drives is set in this way to respond quickly to system fast dynamic changes. Meanwhile, when the WAMPAC system detects the generator over-loading, it will request the extra compensation power from DC microgrid to alleviate system overloaded equipment (in this case G1 generator). In this experiment the requested power is $750 \mathrm{~W}$ which is send to DC microgrid controller after 20 seconds. This delay can be decreased to 2-3 seconds which is the data resolution for developed WAMPAC system.

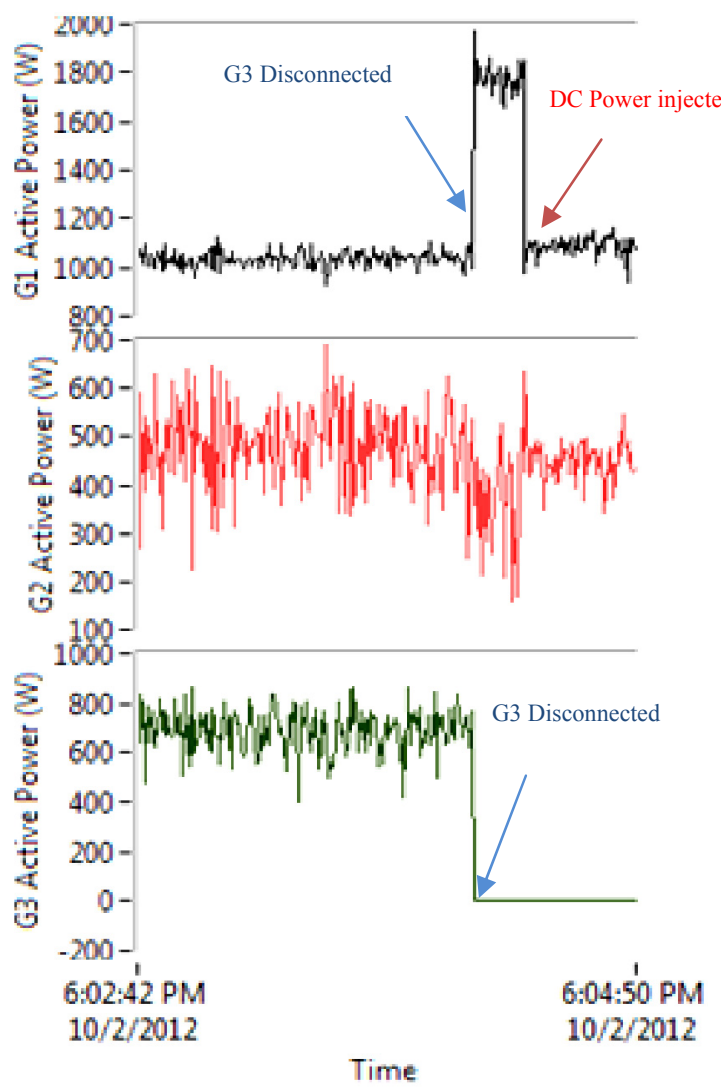

(a) Active Power

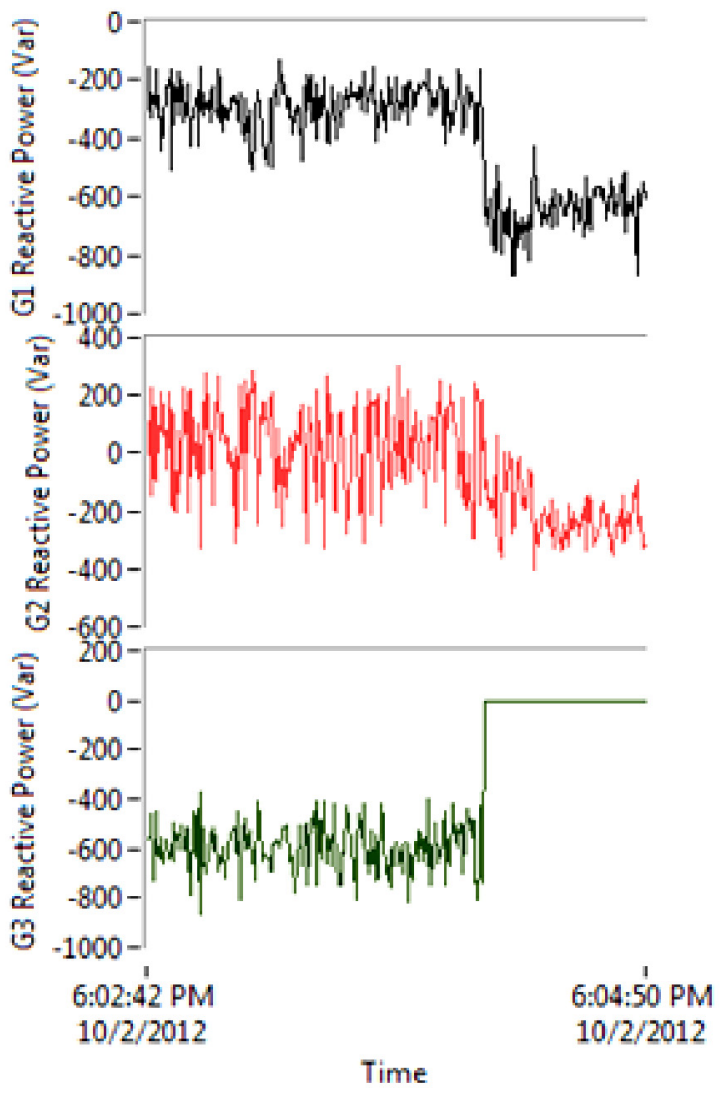

(b) Reactive Power

Figure 8.27: Generators active and reactive power during G3 outage and DC side power injection 


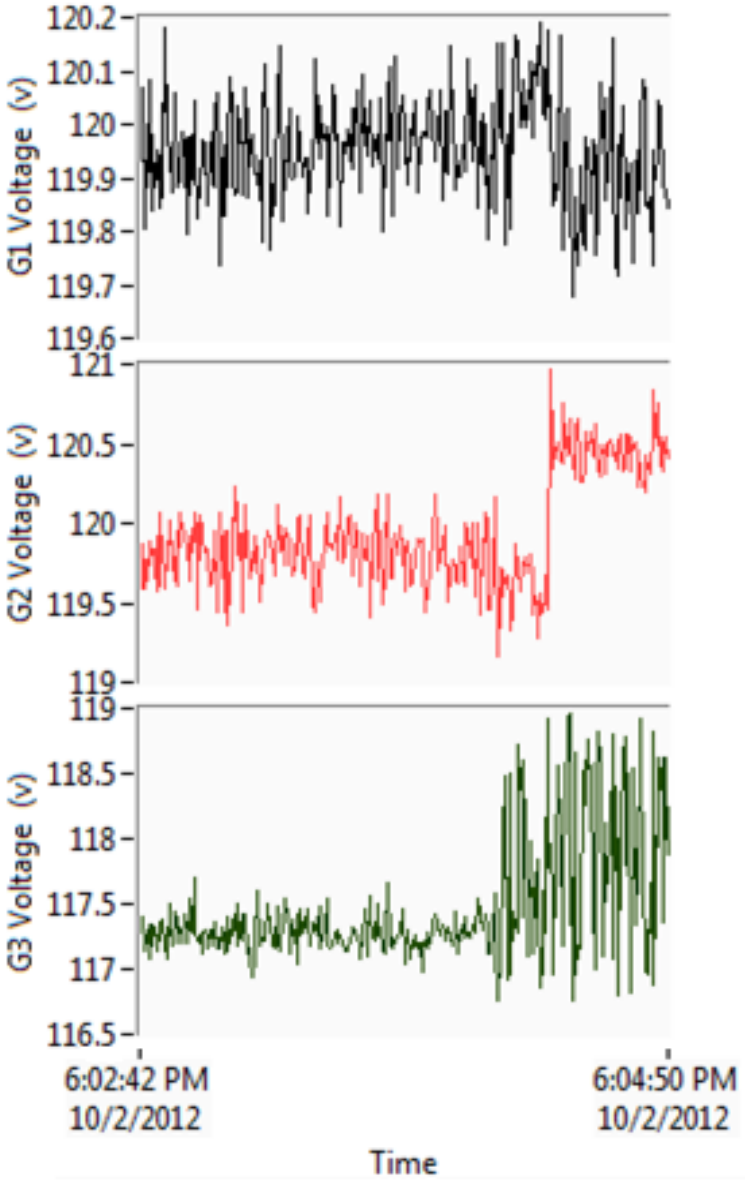

(a) Voltage

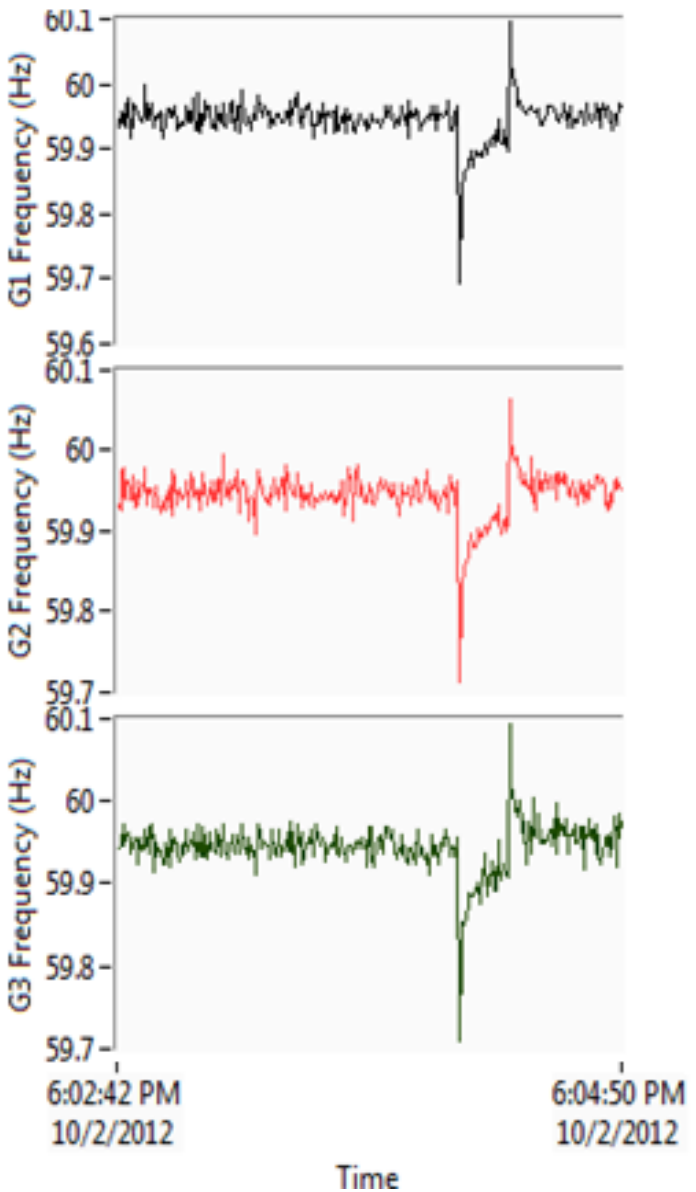

(b) Frequency

Figure 8.28: Generators voltage and frequency during G3 outage and DC side power injection

Figure 8.28 also shows the voltage and frequency of the generators terminal during the defined scenario. If the test-bed system doesn't include reactive loads, the lines generated reactive power is consumed in generators and hence they are sinking the extra reactive power of the system. Therefore, their reactive powers are in negative side during the operation. The generator G3 reactive power share after disconnection is shared between G1 and G2. This is since the DC side doesn't provide any reactive power changes to system. Hence, the voltage changes take place for G1 and G2 because the AVR tries to mitigate the extra reactive power and this occurs considerably for G2 
because it is smaller than G1. The frequency changes remained in limited scale and they returned to initial value after the DC side alleviated the active power deficit.

The DC microgrid is also capable of injecting the requested reactive power. This will enable solving any problems caused by reactive power deficit or surplus for voltage support the voltage. This implementation is the proof of feasibility of using distributed control system ideas and communication features of system controllers to solve operational problems.

\subsection{Real-time Optimal Power Flow Management System}

One of the main technical issues to be addressed in Smart Grid is the integration of control and management systems for optimal operation of the system and sources allocation. Renewable sources such as wind and solar energy sources feature unpredictable and intermittent behavior because of their uncertainty. Hence, the fluctuating power of renewable energy might degrade power quality, security and reliability [141], [142]. For instance, solar and wind renewable energy's power scale is growing, so the management of the operation mechanism of renewable energy sources has a significant role in power generation planning, specifically including forecast of data, supporting the management associated with renewable energy such as demand, energy storage and AC/DC transferred power [143], [144].

A microgrid can be described as an integration of AC/DC micro-sources, energy storage systems (ESS) and loads. Special protection, control and management system should be designed for microgrid operation in either grid-connected or stand alone mode [141]. Technically, this system should have a high update rate of sensing, analyzing and 
sending unit dispatch command in order to follow sudden changes of load and highlyvariable energy sources. The electric system is monitored by the Supervisory Control and Data Acquisition (SCADA) Systems, which periodically acquire system voltage and current measurements and status of switching devices from the network. It also provides the remote control capability on switching status and generation dispatches [145].

Modern control centers of electrical power systems are equipped with computational tools to help the system planners and operators to achieve a high quality service with minimum power interruptions and cost. In addition, energy management system (EMS) is also used to implement sophisticated functions of diagnoses, analysis and advising available to operators. Network topology configuration, state measurement and estimator, contingency analysis, emergency control and optimal power flow are examples of such system functions [146].

Some authors in recent literatures have addressed the real-time OPF (RTOPF) and formulated it as a real-time problem where the proposed algorithm is able to keep track of the optimal operating points of the power system and adjust the control variables in realtime [147]-[150]. However, these references didn't provide a real-time implementation of the OPF in different system scenarios and AC/DC system. Therefore, this chapter will focus on implementing a real-time system in a real lab-scaled hybrid AC/DC microgrid and related issues for rapid calculation, convergence and the adoption of the OPF with renewable resources uncertainties.

Initially the OPF problem and their solution techniques will be investigated to define and solve the objective function. The implementation of real-time system to control laboratory microgrid with its communication and control layers will be 
demonstrated. Finally, the real-time system designed to communicate with the OPF solver and then the results were interpreted for application in real-time system for different load cases.

\subsection{Optimal Power Flow Techniques}

Optimal Power Flow (OPF) algorithms are applied for optimizing the operation of electric power networks by satisfying all system constrains. Their formulation is based on solving power flow equations to minimize an objective function by considering system quality and security constrains. The objective function may be the total generation cost or the system loss. There are other objective functions such as minimization of load shedding. Several approaches have been illustrated to solve OPF problem. In this chapter we will focus on interior point methods [151], [152] and linear programming method [153], [154]. Mostly, these techniques are available in analyses software packages and can be run in discrete instant of time for given sets of system conditions. This procedure requires that $\mathrm{OPF}$ is run so frequently that the system topology and loading conditions do not vary much between two different runs and hence, the control variables are close enough to the previous and optimal settings. The general concept of these two methods is presented as follows:

\subsubsection{AC Optimization (Interior Point Method)}

This non-linear optimization method uses an iterative interior-point algorithm based on the Newton-Lagrange method. The goal of the optimization is to minimize an objective function L subject to the equality constraints imposed by the load flow equations and also to the inequality constraints defined for various power system elements. This is summarized mathematically as follows: 
$\min L(\vec{x}, \vec{s}, \vec{\delta})=f(\vec{x})-\mu \sum_{i} \log \left(s_{i}\right)+(\vec{\lambda})^{T} \cdot[g(\vec{x})+h(\vec{x})+\vec{s}]$

Where $L(\vec{x}, \vec{s}, \vec{\delta})$ is the Lagrange function, $\vec{x}$ is the system parameters vector, $f(\vec{x})$ is the objective function (cost or loss), subject to the equality constraints, $g(\vec{x})=0$, imposed by the load flow equations and also to the inequality constraints, $h(\vec{x}) \leq 0$, defined for various power system elements and introduces a slack variable for each inequality constraint. It can also be formulated by $h(\vec{x})+\vec{s}=0$, where $\vec{s} \geq 0$. $\mu$ is the penalty weighting factor in order to change the contribution of the penalty function, $\sum_{i} \log \left(s_{i}\right)$. Finally, $\vec{\lambda}$ is the Lagrange multiplier which may be defined separately for equality and inequality constrains. For system security and quality purpose some selective system parameters should be kept within the specific limits, hence:

$$
\forall i: \quad h_{\text {min }} \leq h\left(x_{i}\right) \leq h_{\text {max }}
$$

When the loss minimization objective function is selected, the goal of the optimization is to find a power dispatch which minimizes the overall active power losses. Similarly, when the cost objective function is considered, the goal of the optimization is to supply the system under optimal operating costs. More specifically, the aim is to minimize the cost of power dispatch based on non-linear operating cost functions for each generator and on tariff systems for each external grid.

\subsubsection{Optimization (Linear Programming)}

Linear programming methods gained much attention because of its speed and flexibility for application in the nonlinear world of OPF. Linear programming 
formulation requires linearization of objective function as well as constraints with nonnegative variables. In this method the load flow is calculated using the linear DC load flow method.

This technique uses piecewise-linear approximation of the generators cost function subject to an incremental linearization of the network constraints. The general form of this problem is:

$$
\begin{gathered}
\min \quad c_{x}^{T} \Delta x+c_{u}^{T} \Delta u \\
\text { subject to: } \quad G_{x} \Delta x+G_{u} \Delta u=-g(x, u) \\
H_{x} \Delta x+H_{u} \Delta u=-h(x, u)
\end{gathered}
$$

Where $\vec{x}$ is the vector of state variables and $\vec{u}$ is the vector of controllable variables and $c_{x}$ and $c_{u}$ are vectors of cost coefficients. $G_{x}$ and $G_{u}$ are the equality function gradient of $\mathrm{x}$ and $\mathrm{u}$, respectively. Similarly, $H_{x}$ and $H_{u}$ are the inequality function gradient of $\mathrm{x}$ and $\mathrm{u}$, as well.

The result of the linear optimization tool includes calculated results for control variables, such that all imposed constraints are fulfilled and the objective function is optimized.

The algorithm can check the feasibility of the network considering the specified controls and constraints (i.e. performs a constrained load flow). The objective for minimization of costs is used to minimize generation costs. To perform a cost minimization calculation for each generator, a cost factor needs to be entered as a cost curve $[\$ / \mathrm{MWh}]$ for each generator. The (linear) algorithm uses a fixed cost-factor per 
generator. This cost factor is the average cost considering the costs at the generator's active power limits.

\subsection{Real-time Control and Monitoring System of Hybrid AC/DC Microgrid}

Figure 8.29 shows a single line diagram of implemented hybrid AC/DC setup which all was presented at previous chapters. One of the larger generators (G1) runs at a constant frequency of $60 \mathrm{~Hz}$ to maintain the frequency of the system, while the other three generators operate under torque control.

The DC bus is connected to the AC system via a bi-directional converter. This allows bi-directional power flow between AC and DC. The system as shown in figure 8.29 consists of a DC-DC converter integrating a Photo Voltaic (PV) energy sources into a common DC bus. They have the ability to implement the Maximum Power Point Tracking (MPPT) on the injected power to the DC network. The bi-directional ACDC/DC-AC converter is used to allow bi-directional power flow between both sides. The amount of power flowing in either direction can be set to a certain pre-set value. An AC load with an inverter and also two DC loads are applied in different DC Voltage.

The experimental results of output active power versus input torque command for these generators have been shown in figure 8.30. These curves allow us to enter a right torque comment to achieve a specific amount of power at the generators terminals by using their linear equation with a good approximation as presented in the figures and can be implemented inside the real-time software. Once all the generating stations are synchronized, the VI allows the user to control the amount of output power of the generator by controlling the input torque command of each generator, individually. This 
method is performed incrementally to increase or decrease the output power of the corresponding generator.

In addition, the active and reactive power measurements can be demonstrated for all three phases and plotted. This VI is available for all branches in the setup and they are sub-VIs for an overall real-time monitoring system shown in figure 8.31. This overall VI as a SCADA system represents all measurement in general in its front panel and all measurement VIs can appear by clicking on their icon.

This VI in contrast to conventional SCADA systems is very fast and the update time is around one second. The update time is good enough to be observed and followed by a SCADA user to monitor system and control it manually. Hence, the real-time wide area protection and control system has few seconds' latency in order to apply new settings and strategies in the entire power system. The ability to show the systems fast dynamic single line display can provide visually real and reactive power flows, line loadings to point out overloads, bus voltage magnitude/angles or even derived values such as voltage stability indices.

Using the contours and animation features, the operator could immediately recognize the system state which may be changed over a specific time period. The challenging work is the construction of appropriate pattern for the interactive display of large amount of power system data and the development of effective human factors for such applications. The real-time SCADA system should be equipped with proper alarms. Without effective alarm management, the system operator can be easily overwhelmed by alarms during the system disturbance and hence the initiation of an event will be complicated. 


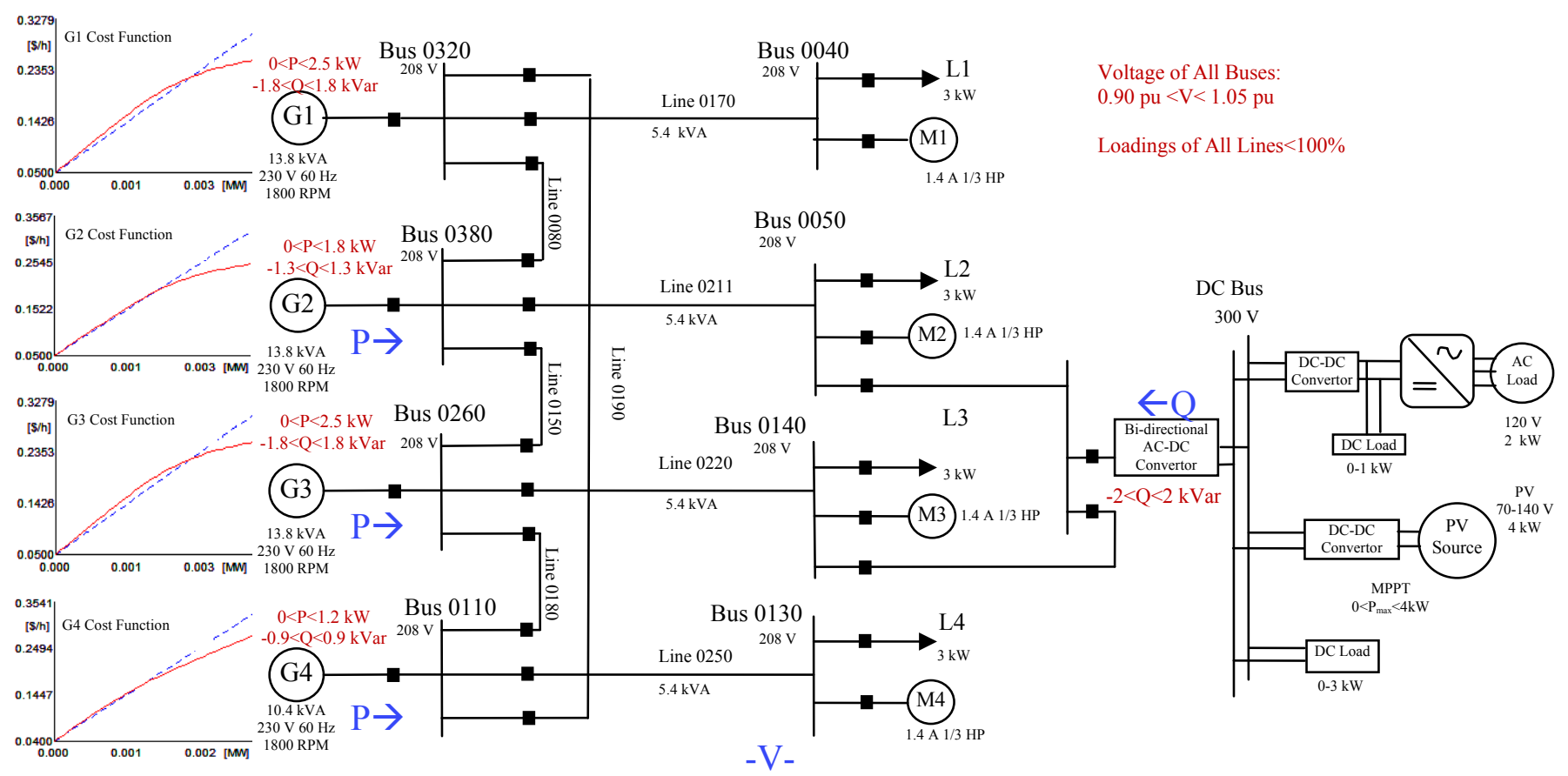

Figure 8.29: Overall power system single line diagram of implemented setup in the real-time software
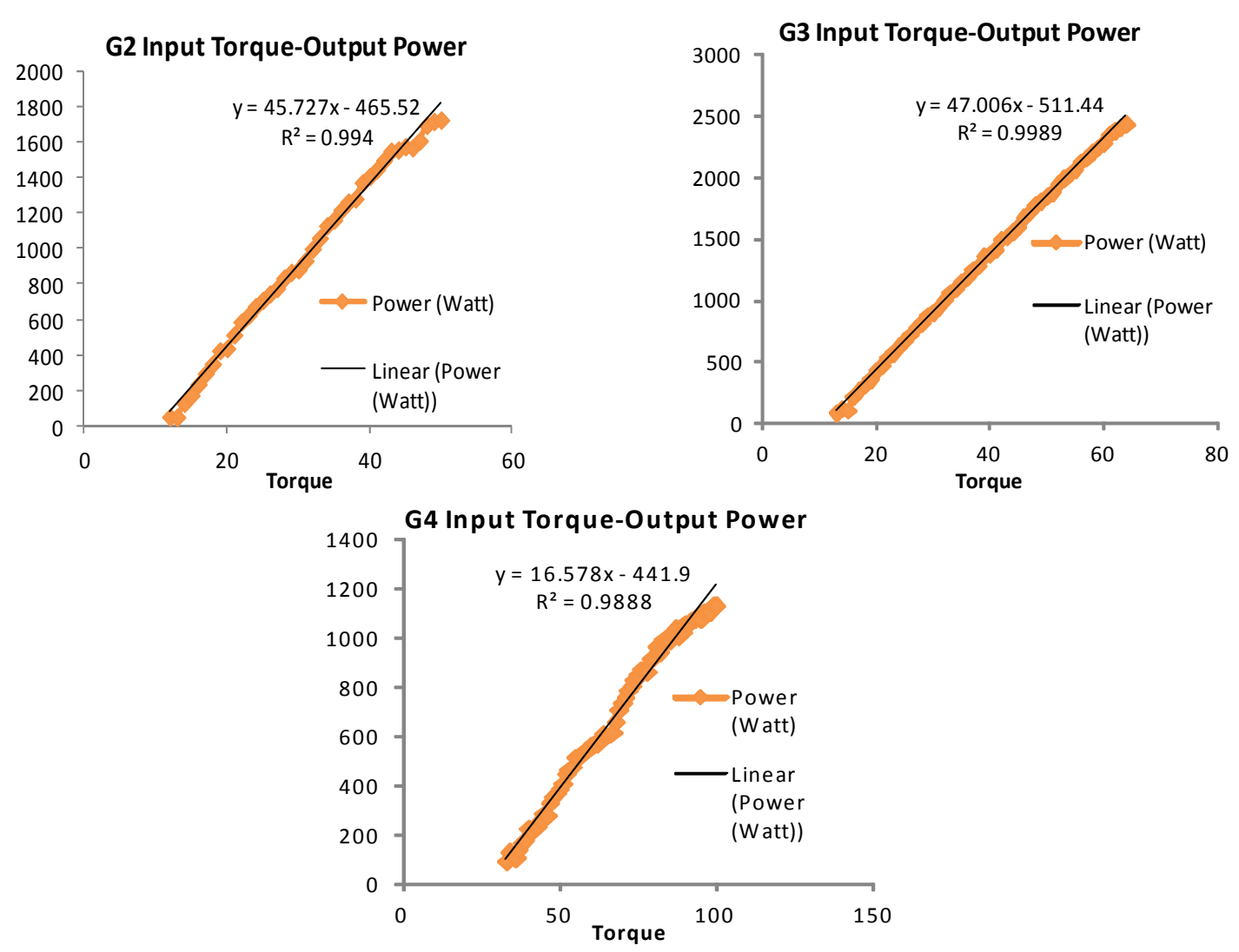

Figure 8.30: Experimental input torque-output power curves for generators 


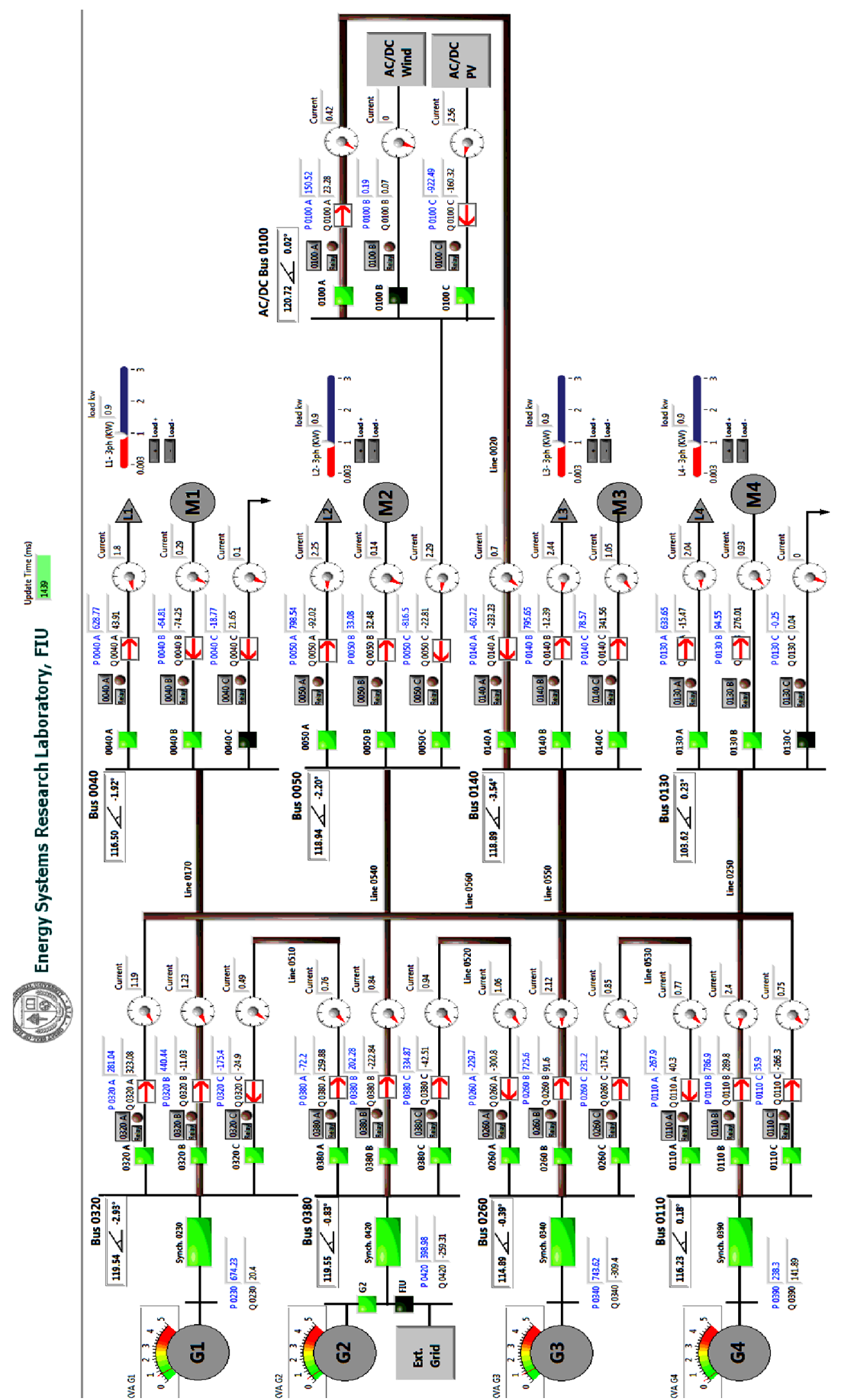

Figure 8.31: Implemented overall real-time SCADA system with WAMPAC capability in test-bed setup 


\subsection{Implementation of Real-time Optimal Power Flow}

In this section, the implementation of OPF in real-time format with a few seconds (2-3 Sec) will be presented. The developed monitoring system uses power factory DIgSILENT software as an analytical tool for calculating real-time OPF in engine mode. This software has the online data transfer capability with the real-time applications. Hence, by implementing an interface between real-time environment and the power factory engine, we can use it in on-line form for running system analysis features such as on-line power flow, on-line contingency assessment, on-line optimal power flow, on-line fault and stability calculation, simulation of grid disturbance scenario, sensitivity analysis on grid operating conditions, tuning optimization of controllers, investigation of control structure, etc. Hardware-in-the-loop testing is also required to develop, analyze and tune control systems for any kind of turbine-generator or superimposed control systems such as a Smart Grid Controllers are the other application of the integrated fast real-time SCADA systems with on-line analysis engines [155].

The implemented procedure for running OPF algorithm is shown in figure 8.32 which plays as an algorithm inside real-time application. The Power Factory DIgSILENT software receives data from the real-time LabVIEW software for switches status (topology data) and system voltage, power data. The generators cost functions, system constraints and controllable parameters for OPF as shown in figure 8.29 are the parameters inside analytical software. It is feasible to enter those data dynamically from real-time application too. Hence, the cost function for different generators, active and reactive power constrains for generators are shown in figure 8.29 , too. Other constrains involve buses voltages and branches flow. 


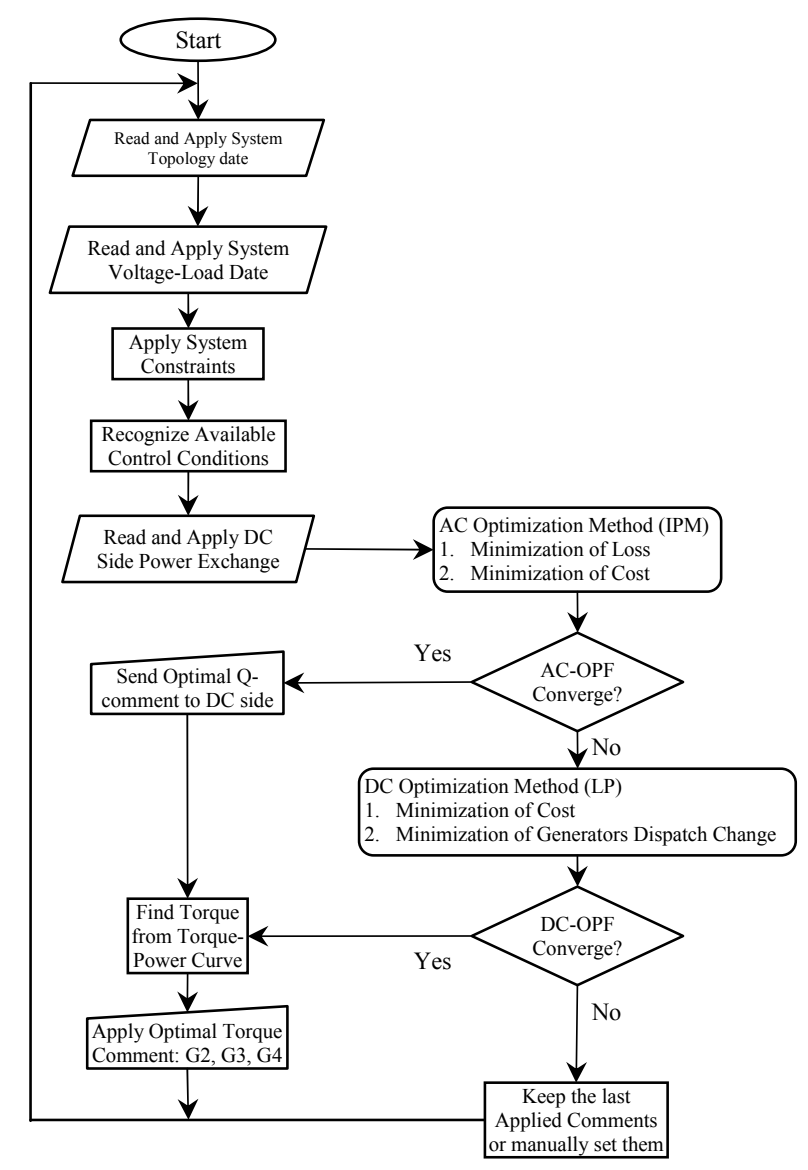

Figure 8.32: Overall power system single line diagram of implemented setup

For the DC side connection the reactive power exchange is the main constrain which can be controlled via OPF results and sending the reactive power set-point to the DC controller. This communication link is also implemented to set the injected/consumed reactive power flow from DC side inside real-time application while the injected active power is intended to be Maximum by MPPT algorithm. After importing the system real data automatically, the real-time software will analyze conventional load flow analysis in a case of the initializing of OPF from load flow analysis was activated. Then AC optimization Method (Interior Point method) was used to minimize objective function which may be system loss or the generation cost. If the system converges then the optimal setting for the available controlled parameters are applied. These settings consist 
of active powers for G2, G3 and G4 and the reactive power request from DC side. If this method doesn't converge then the DC optimization method is utilized to better convergence. The DC optimization method simply neglects the reactive power flow from the system and solves the optimization problem just by considering the active power flow. Hence, this method is not accurate as AC-IPM but it converges faster than AC optimization method and makes it suitable for real-time applications.

\subsection{Experimental Results of Real-Time OPF Management System}

In this section, the results of implementing OPF are investigated for different methods applied in real-time software. The system data are imported to analytical software as a snapshot. Because of measuring all system data, the state measurement of the system is feasible and therefore it is possible to perform a classic load flow calculation. The results of load flow are validated by system measurements and has negligible mismatch in generation-load level. Hence, the generation of the slack bus is left free in order to compensate generation-load level balanced. Figure 8.33 shows the system measurements in a snapshot and the load flow calculation results for all equipments. The results depicts that the Generator G1 is going to reach its operational limit which is $2.5 \mathrm{~kW}$. In addition, Bus-0130 experiences an under voltage situation in which its voltage is $0.94 \mathrm{pu}$. To mitigate these problems proper constraints are applied in optimal power flow methods to alleviate loadings of equipments and maintain the whole system voltage in the acceptable range. The OPF calculation with AC Interior Point Method was done and the results are demonstrated in figure 8.34. The system loading and voltage problems are mitigated by applying new generators control. 


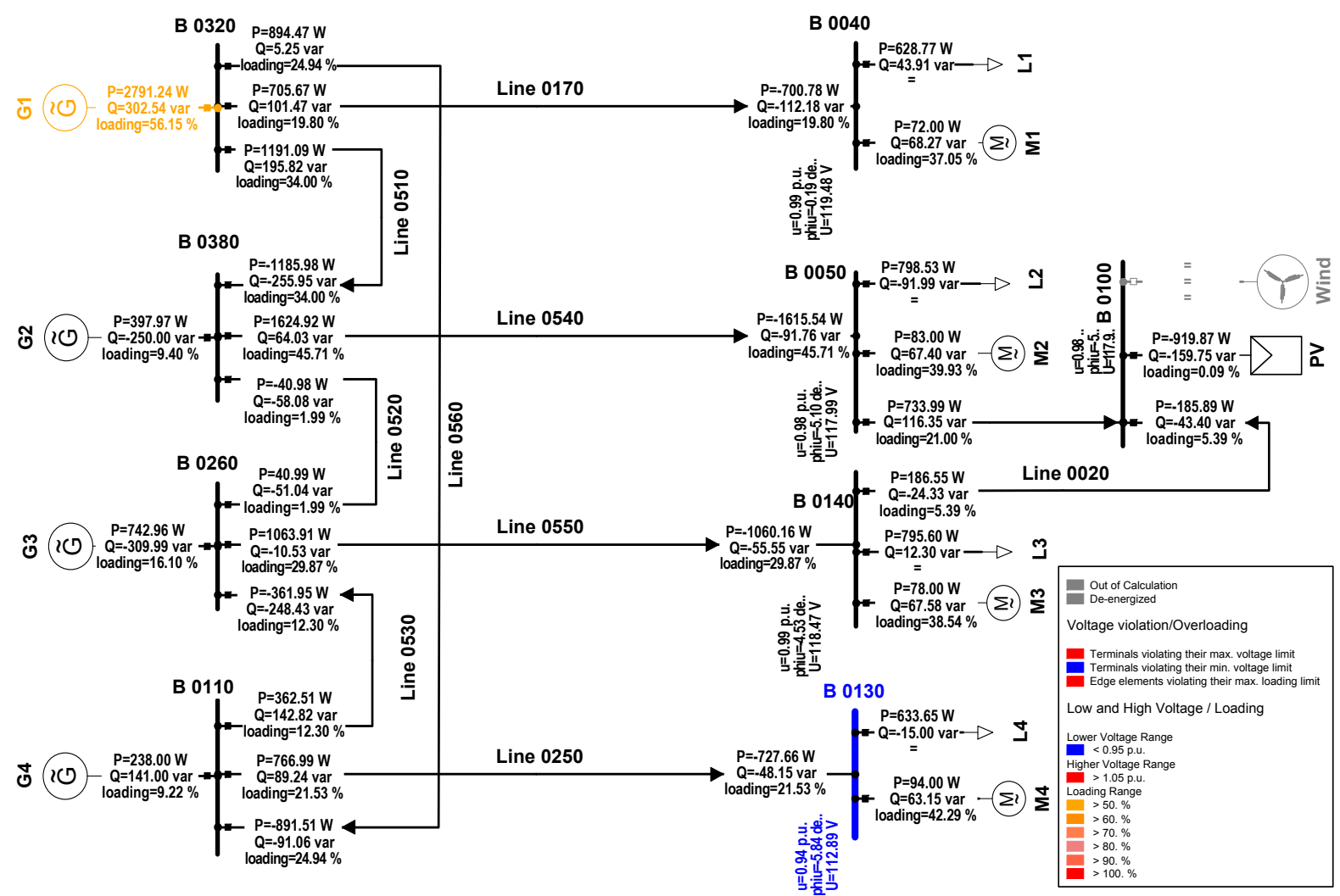

Figure 8.33: Real-time system load flow results for a specific system snapshot

Table 8.4 presents OPF results under different methods and objective functions.

Following the total generation cost and total system losses in the results for AC OPF, the objective function is properly minimized and we need to just reschedule generators and request the specific amount of reactive power from DC side. As long as DC OPF does not consider the reactive power, the reactive power will be vanished from calculation and hence, the DC side reactive power will not be scheduled as it is obvious in this table. Figure 8.35 show the implemented real time monitoring and control system of AC sides with OPF core engine. Figure 8.36 also show the implemented DC side equipment and its real-time controller desktop. 


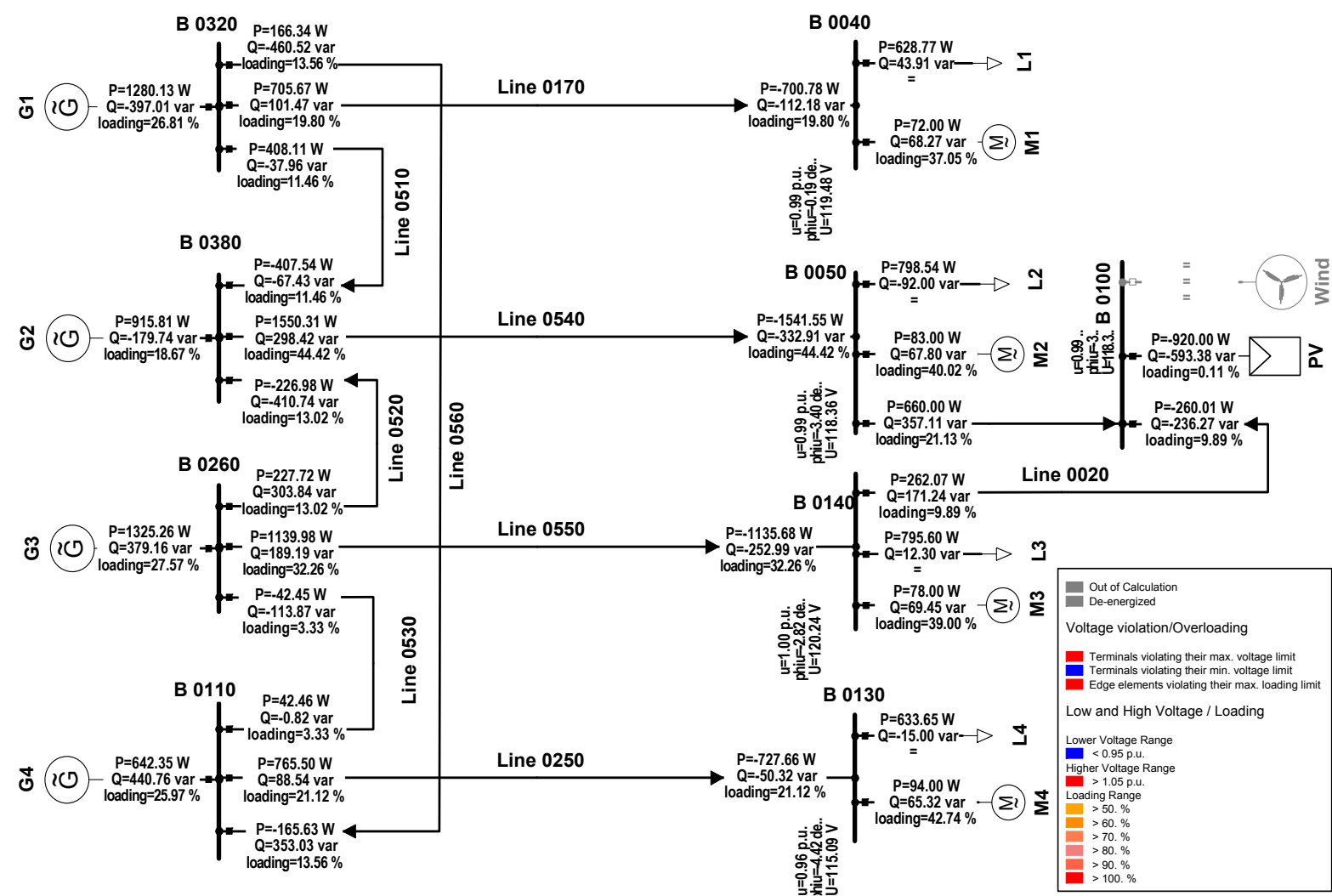

Figure 8.34: Real-time system optimal power results for a same system snapshot using AC-IPM

Table 8.4: Different OPF Results for Real-time System Snapshot data

\begin{tabular}{|c|c|c|c|c|c|}
\hline & \multirow{2}{*}{$\begin{array}{c}\text { Classic } \\
\text { Load-Flow }\end{array}$} & \multicolumn{2}{|c|}{$\begin{array}{c}\text { AC Optimization } \\
\text { (Interior Point Method) }\end{array}$} & \multicolumn{2}{|c|}{$\begin{array}{c}\text { DC Optimization } \\
\text { (Linear Programming) }\end{array}$} \\
\hline & & $\begin{array}{c}\text { Minimize } \\
\text { Losses }\end{array}$ & $\begin{array}{c}\text { Minimize } \\
\text { Cost }\end{array}$ & $\begin{array}{c}\text { Minimize } \\
\text { Cost }\end{array}$ & $\begin{array}{c}\begin{array}{c}\text { Minimization of } \\
\text { Generators } \\
\text { Dispatch Change }\end{array} \\
\end{array}$ \\
\hline G1's Active Power (W) & 2791.24 & 1280.12 & 2156.74 & 2500 & 2500. \\
\hline G2's Active Power (W) & 397.97 & 915.80 & 724.47 & 0. & 398. \\
\hline G3's Active Power (W) & 742.95 & 1325.26 & 1081.97 & 1603.56 & 967.56 \\
\hline G4's Active Power (W) & 237.99 & 642.34 & 203.19 & $\mathbf{0 .}$ & 238. \\
\hline G1's Costs $(\$ / h)$ & 0.23393 & 0.1515 & 0.2083 & 0.0981 & 0.0981 \\
\hline G2's Costs (\$/h) & 0.08213 & 0.1233 & 0.1082 & o. & 0.0320 \\
\hline G3's Costs $(\$ / h)$ & 0.10973 & 0.1549 & 0.1363 & 0.1105 & 0.0758 \\
\hline G4's Costs (\$/h) & 0.06782 & 0.1134 & 0.0637 & 0. & 0.0276 \\
\hline Total Generation Cost $(\$ / \mathbf{h})$ & 0.49363 & 0.5433 & 0.5167 & 0.4886 & 0.5011 \\
\hline System Losses (W) & 66.62 & 59.87 & 62.71 & 0. & 0. \\
\hline DC Side Active Power (W) & 919.87 & 920. & 920. & 920 & 920 \\
\hline DC Side Reactive Power (W) & 159.750 & 593.38 & 561.01 & o. & 0. \\
\hline Loading Problem & G1 (56.15\%) & - & - & G1(50\%) & G1(50 \%) \\
\hline Voltage Problem & В $0130(0.94$ pu $)$ & - & - & - & - \\
\hline
\end{tabular}




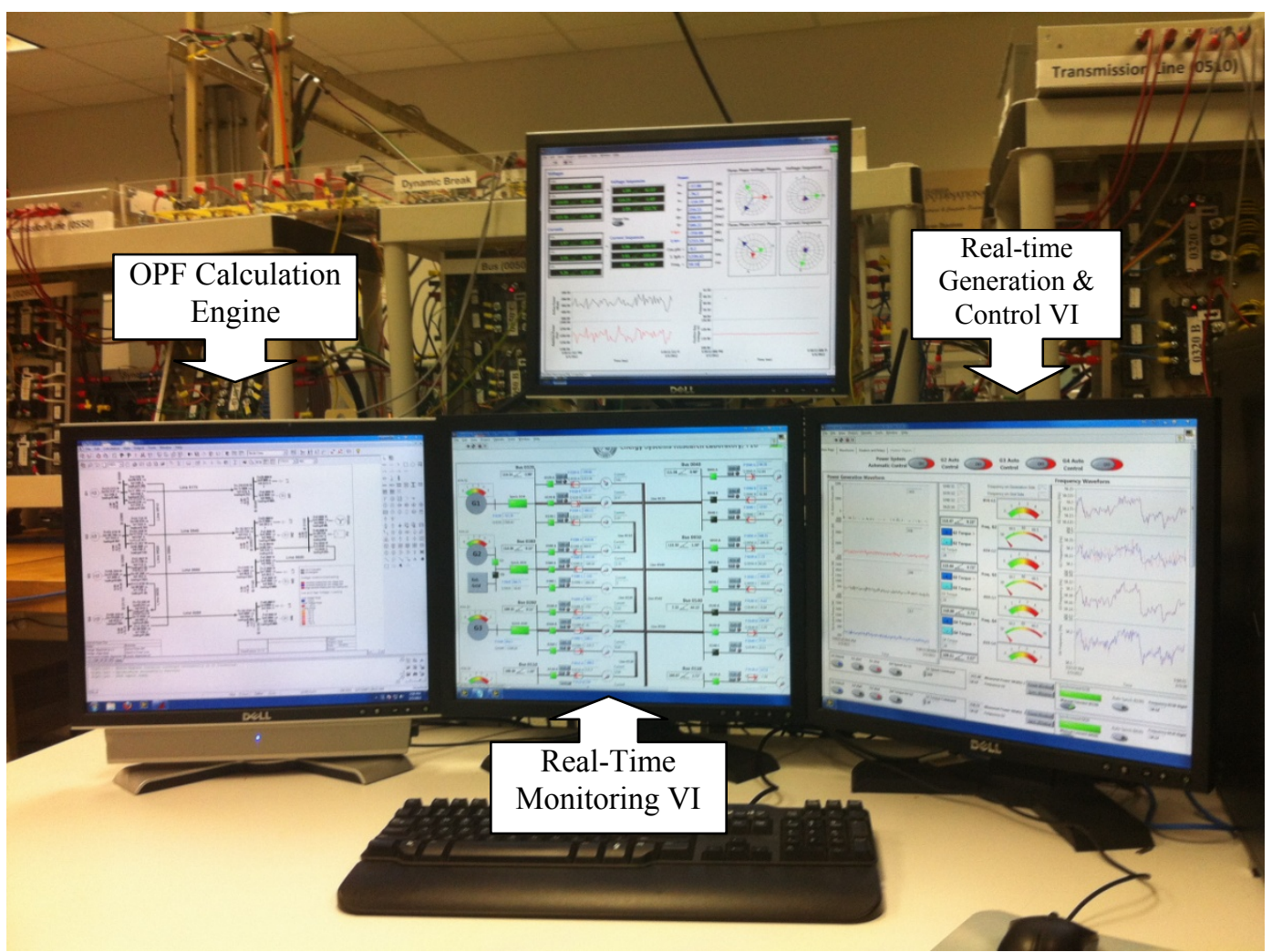

Figure 8.35: Real-time Monitoring and Control System of AC-Side

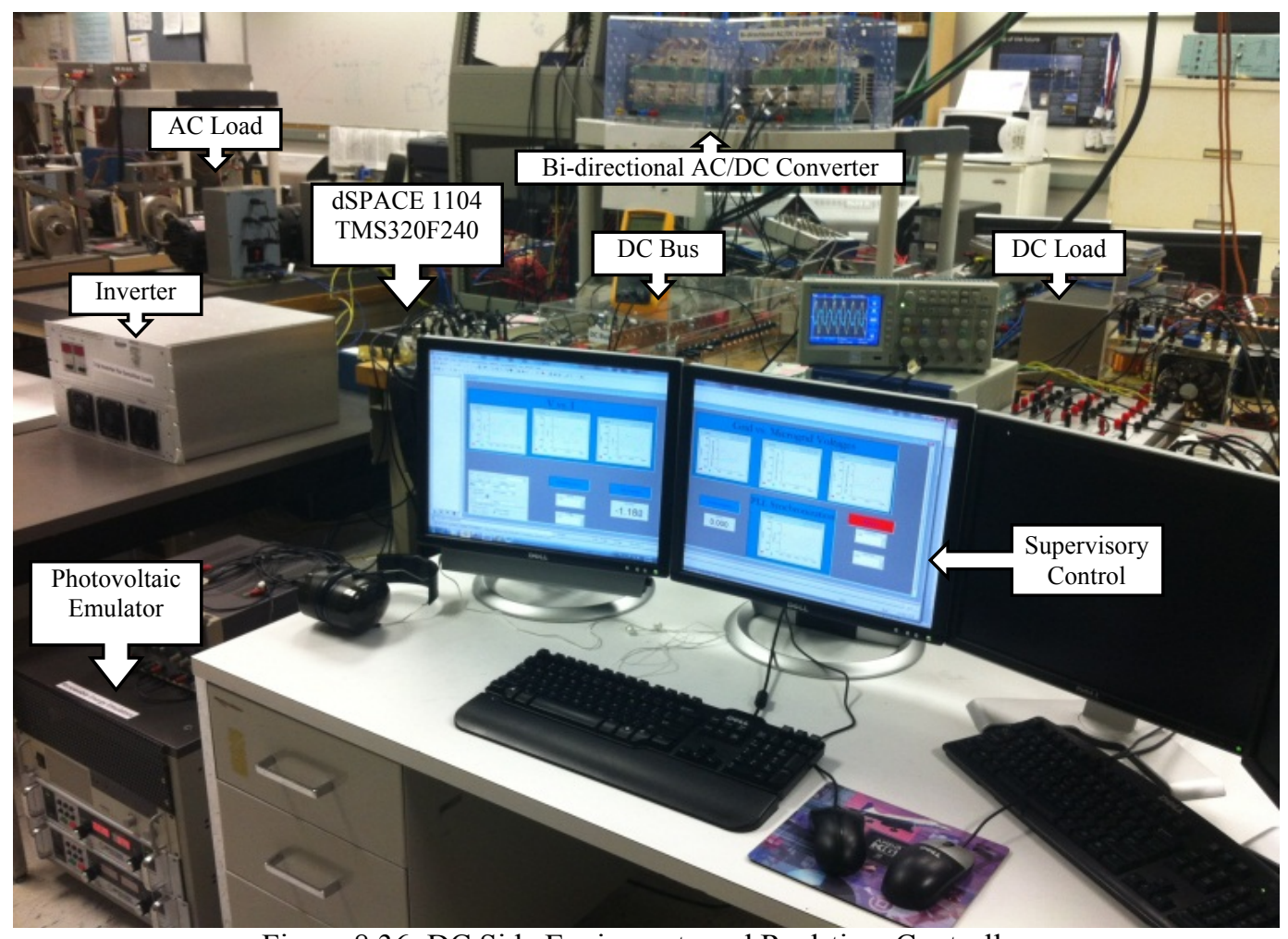

Figure 8.36: DC Side Equipments and Real-time Controller 
For the snapshot data shown in table 8.4, classic load flow is resulted from real time data and shows normal operation of power system. In second column the results for AC optimization using interior point method are presented. The generator dispatches are changed in a way to keep the system losses minimized at $59.87 \mathrm{~W}$. The G1 active power is also alleviated. The reactive power injection from DC side is also increased from 159.75 VAR to 593.38 VAR. This also eliminates the under voltage problem at Bus 0130 which was $0.94 \mathrm{pu}$. If we change the objective function to minimize the generation cost according their power-cost curves, unexpectedly the generation cost is increased from $0.493 \$ / \mathrm{h}$ to $0.516 \$ / \mathrm{h}$. This increase is resulted from forcing constrains to loadings and voltage problem of system equipment. However, this objective function results decrease of generation cost from $0.543 \$ / \mathrm{h}$ to $0.516 \$ / \mathrm{h}$ regard to the case of minimizing of losses.

As stated before, the DC optimization was used while the AC optimization method does not converge and hence just the generations active power can be reschedule in order to minimize the objective function. Applying these setting in real time cause generation consistency in case of not to be able to optimize generation levels quickly and accurately in time base OPF run.

The developed OPF real time system in testbed is capable to run OPF every 10 seconds and reschedule the generators at that time. But for keep the system safety we inserting the generators torque command manually to maintain system safe and continuous operation.

In summary, in this chapter, we presented the development and implementation of a real-time Optimal Power Flow Management for the developed laboratory Smart Micro Grid. The commercial Power Factory Software Package was used to communicate with 
the real-time LabVIEW software to receive system data and utilize real-time data in the online engine mode to calculate OPF and return the optimal schedule of generators and reactive power demand from the DC side by the available communication link. The results were presented for different OPF methods. An objective function was used to apply proper optimal management solution in the Hybrid AC/DC microgrids on the testbed. 


\section{CHAPTER 9}

\section{CONCLUSIONS AND RECOMMENDATIONS FOR FUTURE RESEARCH}

\subsection{Conclusions}

This dissertation presented a unique hardware/software/communication based Smart Grid test-bed facility in a laboratory environment. We developed an infrastructure for studying and developing real-time automated power system network operation and control strategies. In addition, the developed system can be used as a platform to study, test and implement grid connectivity issues of alternate energy sources and validate power sharing and energy storage ideas. The developed system was achieved by the integration of different hardware devices and emulators. The communication interfaces between these devices and their operation in a wide area network infrastructure. The size of the system is significant enough reaching a $72 \mathrm{~kW}$ level of $\mathrm{AC}$, alternate generation and energy storage. Research applications and examples of experiments of this laboratory-based smart grid test-bed are presented. The real-time analysis capability of the developed system provides a platform for investigation of the most challenging aspects of operating a real power system.

The developed setup is an integrated wide area system with capabilities for WAMS, WACS and WAPS. The implementation of this system in real-time creates an environment for studying and verifying new control and protection schemes for the whole power system. This was accomplished through modeling and software development which were implemented in the hardware environment. 
In this dissertation, one of the focal issues was on developing and implementing a PMU in a laboratory scale power system test-bed in digital form by using real-time analysis software. A simple power system was implemented in order to test the real-time application of the developed PMUs. This system uses different DAQs to gather voltage and current data and measure the power system parameters such as the voltage and current phasors, positive, negative and zero sequences as well as power and frequency of each component. Verification experiments were presented to show the performance and capabilities of the implemented system.

This system was used for studying an integrated wide area control and protection system to monitor the system status for abnormalities such as over/under voltage, overloads of system components and any other conditions. In the implemented example, when the loadings of a power system line increases, the relay indicated that the system tends to reach the fault condition and may need to be disconnected by available protection devices. Hence by setting the protection relay below the settings of the physical relay, the control scheme can retrieve the normal status by appropriate control actions. This may be achieved by changing the system topology, proper power dispatching or by self healing strategies in the control center.

In this research the Virtual Protection System (VPS) was proposed for the wide area system protection implemented on the test-bed. This system was used to control power systems in a manner to heal any abnormal conditions that may cause damage, outage and perhaps blackout. The VPS was developed as a generic digital relay with variety of protection functions that uses the PMUs current and voltage outputs to simulate power systems behavior in a virtual environment. This gives us some information about 
upcoming faults or system states moving forward an abnormal situation. Proper control action for self-healing can be developed in a control system to maintain the system stable conditions with minimum outages. A laboratory based power system test-bed was utilized to test events and verify the VPS idea in a large power system. Applications of VPS were discussed for modern power system for predicting upcoming events, detecting blackout and cascade events and applying online settings to protective relays.

This real-time system that was developed was is also used to monitor real-time system stability and security margin. The indices formulation and their implementation on wide area monitoring and control center example on the test-bed were presented. This provides an applicable view of the system voltage stability and security margin using PMUs in wide area networks. The voltage stability indices were measured during the operation of the power system when the load changes take place. A wide area monitoring system with high data resolution rate (around one second) was developed. As a result, the system operator will have proper knowledge and visualization about the power system's current situation and the distance of stability margin.

This dissertation also presented a brief summary of the critical research needs to meet the enormous challenges in real-time security analysis to ensure reliable and secure grid operation for the future grid. A related issue was the availability of the real-time data from the PMUs all over the power system; any inaccessibility of fast real-time data may affect the feasibility of WAMPAC for fast data monitoring and analysis. The purpose of security monitoring is to look ahead for possible contingencies that may transition the power system from normal operation state to the emergency state. Fast computation is intensively effective for monitoring the security of the large interconnected system in 
order to apply remedies in proper time. Since the problems caused by a contingency can range from the simple overloading of a line to the various complicated instability phenomena, identifying, categorizing and ranking the security problems on a continuing basis will require considerable attempts.

Moreover, we concentrated on the development and implementation of real-time Optimal Power Flow Management for the developed smart microgrid. The power factory analysis software was used by getting its data through communication with the real-time LabVIEW software. This interface received system data and utilized such real-time data in the online engine mode to calculate OPF and return the optimal schedule of generators and reactive power demand from the $\mathrm{DC}$ side by the available communication link. The results were presented for different OPF methods and objective function in order to apply proper Optimal Management solution in Hybrid AC/DC Microgrids.

Finally, we developed real-time energy management algorithm in order to mitigate pulse loads effects on system performance in smart microgrids. The main objective of the algorithm is to manage the energy storage devices in real-time in order to maintain system stability and performance in the short term operation and minimize the energy cost in the long term operation particularly for peak shaving purposes. The algorithm involves non-linear mathematical models for PV and load data as well as smart techniques including fuzzy logic and adaptive control concepts. An investigation on the system performance under pulse loads shows that when the battery's state of charge is managed by the developed algorithm, the system has better stability margin and the battery is sustained to share all its stored energy to pulse loads. The comparison with the system performance without fully charged battery results in more system parameters 
changes, which may cause stability problem or protection reaction due to high drop in frequency/voltage and hence the system may be thrown off-line.

\subsection{Recommendations for Future Work}

The topics discussed in this dissertation demonstrate the difficulties encountered in monitoring, control and protection of power system applying the new modern technologies such as PMUs and effort strategies for determining the state of the power system. In view of the urgent need for better analyzing and determining the system planning and operation conditions and constrains in wide area, it is recommend that the following topics be expanded by others:

- There is a need to develop novel smart control concept in wide area power system that can be implemented using PMUs. There are already some applications which have been developed for PMUs, but more needs to be done. Since the number of power utility installed PMUs is increasing all over the grid, there is a need to use their information in a SCADA center to monitor the system with very fast and real-time data. Hence, there is a gap in the research for developing such algorithm for wide area applications using PMUs.

- There is a need for research centers to develop the real-time monitoring and control features for implementing the developed strategies in smart control of the power system. Application and verification of such algorithms need a platform for real power system setup and system realtime control infrastructure. 
- There is a need to continue the applications of Virtual Protection Systems for applying online setting of protection devices. This should be studied further. The protection coordination settings in a large area network are completely dependent on the topology and system status which are varying frequently. The developed system could detect the grid status and will run protection coordination software to achieve proper settings for relays. Then, it will apply new settings by real-time software and communication networks to the selected system protection devices. This should be verified by developing new protection algorithms and experiment them in the testbed setup as well as in utility environments.

- The developed voltage stability indices should be implemented in commercial PMU package and should be applicable for any control center to monitor system situation in terms of marginal transmission loading and its closeness to the instability point. This can create an opportunity for preventive alarming system in power system control centers.

- The hybrid systems which are in the development stage are almost using $\mathrm{AC}$ and DC renewable generations. In most microgrids the load and generation behavior needs further study in terms of stability and security. We studied the system behavior under the presence of pulse load and developed a novel algorithm to mitigate their adverse effects on microgrid operation. Hence, the real-time energy management algorithm which is applicable for generation control and storages should be studied in more details and be implemented in related microgrids. 
- Further focus needs to be paid to integrate monitoring, protection and control system in real-time format to enhance the operation of smart power system.

- New and modern control centers should use the real-time PMUs data to make the power system much smarter to increase the system stability, security and reliability.

- Real-time energy management algorithm for hybrid AC/DC microgrids should be implemented and tested on a hybrid AC/DC smart power system test-bed to design and test the performance of such algorithm on system stability in the presence of different events, load patterns including heavy and pulsed loads. 


\section{References}

[1] P. Zhang, F. Li, N. Bhatt, "Next-Generation Monitoring, Analysis, and Control for the Future Smart Control Center"; IEEE Transactions on Smart Grid; Vol. 1, Issue: 2 pp. 186 - 192, 2010

[2] P.P. Varaiya, F.F. Wu, J.W. Bialek, "Smart Operation of Smart Grid: RiskLimiting Dispatch," Proceedings of the IEEE, vol.99, no.1, pp.40-57, Jan. 2011

[3] A. Molderink, V. Bakker, M. G. C. Bosman, J.L. Hurink, G. J. M. Smit, "Management and Control of Domestic Smart Grid Technology"; IEEE Transactions on Smart Grid, Vol. 1, Issue: 2, pp. 109 - 119, 2010

[4] K. Moslehi, R. Kumar, "A Reliability Perspective of the Smart Grid," Smart Grid, IEEE Transactions on , vol.1, no.1, pp.57-64, June 2010

[5] A. Bose, 'Smart Transmission Grid Applications and Their Supporting Infrastructure,” IEEE Transactions on Smart Grid, vol.1, no.1, pp.11-19, June 2010

[6] M. Shahraeini, M.H. Javidi, M.S. Ghazizadeh, M.S. , "Comparison Between Communication Infrastructures of Centralized and Decentralized Wide Area Measurement Systems," IEEE Transactions on Smart Grid, vol.2, no.1, pp.206211, March 2011

[7] K. Vu, M.M. Begouic, D. Novosel, D.; "Grids get smart protection and control", Computer Applications in Power, IEEE, Volume: 10, Issue: 4, pp. 40 - 44, 1997

[8] M. Liserre, T. Sauter, J.Y. Hung, "Future Energy Systems: Integrating Renewable Energy Sources into the Smart Power Grid Through Industrial Electronics," Industrial Electronics Magazine, IEEE, vol.4, no.1, pp.18-37, March 2010

[9] T. Kim, S. Park, Kyung, B. G. Lee, "What Is Appropriate Strategy for Smart Grid Business?: A Case Study of Test-bed in Korea", Proceedings of the 5th International Conference on Information Technologies and Applications (CUTE), pp. $1-5,2010$

[10] J.A. Momoh, "Fundamentals of analysis and computation for the Smart Grid"; Power and Energy Society General Meeting, 2010 IEEE, pp. 1 - 5, 2010

[11] J.A. Momoh, "Smart grid design for efficient and flexible power networks operation and control", In Proc. IEEE Power Systems Conference and Exposition, pp 1-8, 2009.

[12] De La Ree, J.; Centeno, V.; Thorp, J.S.; Phadke, A.G.; , "Synchronized Phasor Measurement Applications in Power Systems," IEEE Transactions on Smart Grid, vol.1, no.1, pp.20-27, June 2010

[13] R. Abe, H. Taoka, D. McQuilkin, "Digital Grid: Communicative Electrical Grids of the Future," IEEE Transactions on Smart Grid, vol.PP, no.99, pp.1, 0

[14] Fangxing Li; Wei Qiao; Hongbin Sun; Hui Wan; Jianhui Wang; Yan Xia; Zhao $\mathrm{Xu}$; Pei Zhang; , "Smart Transmission Grid: Vision and Framework," IEEE Transactions onSmartGrid, vol.1, no.2, pp.168-177, Sept. 2010 
[15] J. Medina, N. Muller, I. Roytelman, "Demand Response and Distribution Grid Operations: Opportunities and Challenges," IEEE Transactions on Smart Grid, vol.1, no.2, pp.193-198, Sept. 2010

[16] A. Mohsenian-Rad, V.W.S. Wong, J. Jatskevich, R. Schober, A. Leon-Garcia, "Autonomous Demand-Side Management Based on Game-Theoretic Energy Consumption Scheduling for the Future Smart Grid," IEEE Transactions onSmart Grid, vol.1, no.3, pp.320-331, Dec. 2010

[17] K. Kurohane, T. Senjyu, A. Yona, N. Urasaki, T. Goya, T. Funabashi, "A Hybrid Smart AC/DC Power System," IEEE Transactions on Smart Grid, vol.1, no.2, pp.199-204, Sept. 2010

[18] A. Molderink, V. Bakker, M.G.C. Bosman, J.L. Hurink, G.J.M. Smit, "Management and Control of Domestic Smart Grid Technology," IEEE Transactions on, vol.1, no.2, pp.109-119, Sept. 2010

[19] K.M. Rogers, R. Klump, H. Khurana, A.A. Aquino-Lugo, T.J. Overbye, “An Authenticated Control Framework for Distributed Voltage Support on the Smart Grid," IEEE Transactions onSmart Grid, vol.1, no.1, pp.40-47, June 2010

[20] W. Juanjuan, F. Chuang, Z. Yao, "Design of WAMS-Based Multiple HVDC Damping Control System," IEEE Transactions onSmart Grid, vol.2, no.2, pp.363374, June 2011

[21] K. Seethalekshmi, S.N. Singh, S.C. Srivastava, "A Synchrophasor Assisted Frequency and Voltage Stability Based Load Shedding Scheme for Self-Healing of Power System," IEEE Trans. on Smart Grid, vol.2, no.2, pp.221-230, June 2011

[22] M. McGranaghan, D. Von Dollen, P. Myrda, E. Gunther, "Utility experience with developing a smart grid roadmap," Power and Energy Society General Meeting Conversion and Delivery of Electrical Energy in the 21st Century, 2008 IEEE , pp.1-5, 20-24 July 2008

[23] Z. Yingchen, P. Markham, X. Tao, C. Lang,Y. Yanzhu, W. Zhongyu, Y. Zhiyong, W. Lei, J. Bank, J. Burgett, R.W. Conners, L. Yilu, "Wide-Area Frequency Monitoring Network (FNET) Architecture and Applications," IEEE Trans. on Smart Grid, vol.1, no.2, pp.159-167, Sept. 2010

[24] A. Armenia, J.H. Chow, "A Flexible Phasor Data Concentrator Design Leveraging Existing Software Technologies," IEEE Trans. on Smart Grid, vol.1, no.1, pp.73-81, June 2010

[25] V. Terzija, G. Valverde, C. Deyu, P. Regulski, V. Madani, J. Fitch, S. Skok, M.M. Begovic, A. Phadke, "Wide-Area Monitoring, Protection, and Control of Future Electric Power Networks," Proceedings of the IEEE, vol.99, no.1, pp.80-93, Jan. 2011

[26] Kawano, F.; Baber, G.P.; Beaumont, P.G.; Fukushima, K.; Miyoshi, T.; Shono, T.; Ookubo, M.; Tanaka, T.; Abe, K.; Umeda, S.; "Intelligent protection relay system for Smart Grid", Developments in Power System Protection (DPSP 2010). Managing the Change, 10th IET International Conference on Digital Object Identifier: 10.1049/cp.2010.0211, Publication Year: 2010 , Page(s): 1 - 5 
[27] K. Seethalekshmi, S.N. Singh, S.C. Srivastava, "A Synchrophasor Assisted Frequency and Voltage Stability Based Load Shedding Scheme for Self-Healing of Power System," IEEE Trans. on Smart Grid, vol.2, no.2, pp.221-230, June 2011

[28] M. McGranaghan, D. Von Dollen, P. Myrda, E. Gunther, "Utility experience with developing a smart grid roadmap," Power and Energy Society General Meeting Conversion and Delivery of Electrical Energy in the 21st Century, 2008 IEEE , pp.1-5, 20-24 July 2008

[29] Smart grid policy U.S. Federal Energy Regulatory Commission (FERC), Docket PL09-4-000, 2009.

[30] Zhifang Wang; Scaglione, A.; Thomas, R.J.; "Generating Statistically Correct Random Topologies for Testing Smart Grid Communication and Control Networks" Smart Grid, IEEE Transactions on Volume: 1, Issue: 1, Digital Object Identifier: 10.1109/TSG.2010.2044814 Publication Year: 2010 , Page(s): 28 - 39

[31] Modern grid initiative U.S. Dept. Energy, Natl. Energy Technol. Lab. [Online]. Available:

http://www.netl.doe.gov/moderngrid/opportunity/vision_technologies.html

[32] EPRI Intelligrid [Online]. Available: http://intelligrid.epri.com/

[33] M. McGranaghan, D. Von Dollen, P. Myrda, and E. Gunther, "Utility experience with developing a smart grid roadmap," Power and Energy Society General Meeting - Conversion and Delivery of Electrical Energy in the 21st Century, 2008 IEEE Digital Object Identifier: 10.1109/PES.2008.4596927 Publication Year: 2008 , Page(s): 1 - 5

[34] T. F. Garrity, "Getting smart," Power and Energy Magazine, IEEE Volume: 6 , Issue: 2 Digital Object Identifier: 10.1109/MPE.2007.915181 Publication Year: 2008 , Page(s): $38-45$

[35] S. M. Amin and B. F. Wollenberg, "Toward a smart grid: Power delivery for the 21st century," Power and Energy Magazine, IEEE Volume: 3 , Issue: 5 Digital Object Identifier: 10.1109/MPAE.2005.1507024 Publication Year: 2005 , Page(s): $34-41$

[36] J. R. Roncero, "Integration is key to smart grid management," in Proc. IET-CIRED Seminar Smart Grids Distribution., Jun. 23-34, 2008, pp. 1-4.

[37] L. H. Tsoukalas and R. Gao, "From smart grids to an energy internet: Assumptions, architectures and requirements," in Proc. 3rd Int. Conf. Electr. Util. Deregulation and Restructuring and Power Technol., Apr. 6-9, 2008, pp. 94-98.

[38] D. G. Hart, "Using AMI to realize the smart grid," Power and Energy Society General Meeting - Conversion and Delivery of Electrical Energy in the 21st Century, 2008 IEEE Digital Object Identifier: 10.1109/PES.2008.4596961 Publication Year: 2008 , Page(s): 1 - 2

[39] M. G. Lauby and W. P. Malcolm, "North American industry trends supporting intelligent grids," in Proc. Int. Conf. Intelligent. Syst. Appl. Power Syst., Nov. 5-8, 2007, pp. 1-6. 
[40] Pacific Northwest GridWise, Testbed Demonstration Projects, Available Online at: http://www.pnl.gov/main/publications/external/technical_reports/PNNL17176.pdf

[41] Advanced Architecture and Control Concepts for more Microgrids: Available Online at: http://www.smartgrids.eu/documents/projects/ESR.pdf

[42] Available Online at: http://canmetenergy-canmetenergie.nrcanrncan.gc.ca/

[43] Choi, J. Y.; Kim, J.; "An integrated simulation framework of a virtual test-bed for Smart Grid"; 40th International Conference on Computers and Industrial Engineering (CIE), pp. 1 - 5, 2010

[44] k.H. Kang, D. J. Won, "Power management strategy of stand-alone hybrid system to reduce the operation mode changes" Transmission \& Distribution Conference \& Exposition: Asia and Pacific, 2009, 26-30 Oct. 2009 Page(s):1-4

[45] Z. Jiang, "Power management of hybrid photovoltaic - fuel cell power systems", Power Engineering Society General Meeting, 2006. IEEE, 0-0 0 Page(s):6 pp.

[46] Kurohane, K.; Senjyu, T.; Yona, A.; Urasaki, N.; Goya, T.; Funabashi, T.; “A Hybrid Smart AC/DC Power System",Smart Grid, IEEE Transactions on , Volume: 1 , Issue: 2 Digital Object Identifier: 10.1109/TSG.2010.2053392 Publication Year: 2010 , Page(s): 199 - 204

[47] A. P. Johnson, "The history of the smart grid evolution at Southern California Edison," presented at the IEEE PES Innovative Smart Grid Technologies Conf., Gaithersburg, MD, 2010.

[48] V. K. Sood, D. Fischer, J. M. Eklund, and T. Brown, "Developing a communication infrastructure for the smart grid," in Proc. Electr. Power Energy Conf., 2009, pp. 1-7.

[49] M. A. Masoum, P. S. Moses, and S. Deilami, "Load management in smart grids considering harmonic distortion and transformer derating," presented at the IEEE PES Innovative Smart Grid Technologies Conf., Gaithersburg, MD, 2010.

[50] Kawano, F.; Baber, G.P.; Beaumont, P.G.; Fukushima, K.; Miyoshi, T.; Shono, T.; Ookubo, M.; Tanaka, T.; Abe, K.; Umeda, S.; "Intelligent protection relay system for Smart Grid",Developments in Power System Protection (DPSP 2010). Managing the Change, 10th IET,International Conference on Digital Object Identifier: 10.1049/cp.2010.0211 Publication Year: 2010 , Page(s): 1 - 5

[51] Yi Zhang; Prica, M.; Ilic, M.D.; Tonguz, O.K.; "Toward smarter current relays for power

Power Engineering Society General Meeting, 2006. IEEE Digital Object Identifier: 10.1109/PES.2006.1709580

Publication Year: 2006

[52] Terzija, V.; Valverde, G.; DeyuCai; Regulski, P.; Madani, V.; Fitch, J.; Skok, S.; Begovic, M.M.; Phadke, A.; , "Wide-Area Monitoring, Protection, and Control of Future Electric Power Networks," Proceedings of the IEEE, vol.99, no.1, pp.8093, Jan. 2011 
[53] Kamwa, I.; Pradhan, A.K.; Joos, G.; , "Adaptive Phasor and Frequency-Tracking Schemes for Wide-Area Protection and Control," Power Delivery, IEEE Transactions on , vol.26, no.2, pp.744-753, April 2011

[54] QunGao; Rovnyak, S.M.; , "Decision Trees Using Synchronized Phasor Measurements for Wide-Area Response-Based Control," Power Systems, IEEE Transactions on, vol.26, no.2, pp.855-861, May 2011

[55] Elkhatib, M.E.; El-Shatshat, R.; Salama, M.M.A.; , "Novel Coordinated Voltage Control for Smart Distribution Networks With DG," Smart Grid, IEEE Transactions on , vol.2, no.4, pp.598-605, Dec. 2011

[56] Husheng Li; Lifeng Lai; Weiyi Zhang; , "Communication Requirement for Reliable and Secure State Estimation and Control in Smart Grid," Smart Grid, IEEE Transactions on, vol.2, no.3, pp.476-486, Sept. 2011

[57] Seethalekshmi, K.; Singh, S.N.; Srivastava, S.C.; , "A Synchrophasor Assisted Frequency and Voltage Stability Based Load Shedding Scheme for Self-Healing of Power System," Smart Grid, IEEE Transactions on , vol.2, no.2, pp.221-230, June 2011

[58] Meliopoulos, A.P.S.; Cokkinides, G.; Renke Huang; Farantatos, E.; Sungyun Choi; Yonghee Lee; Xuebei Yu; , "Smart Grid Technologies for Autonomous Operation and Control," Smart Grid, IEEE Transactions on, vol.2, no.1, pp.1-10, March 2011

[59] Yan Li; Yun Wei Li; , "Power Management of Inverter Interfaced Autonomous Microgrid Based on Virtual Frequency-Voltage Frame," Smart Grid, IEEE Transactions on , vol.2, no.1, pp.30-40, March 2011

[60] Wang, S.; Meng, X.; Chen, T.; , "Wide-Area Control of Power Systems Through Delayed Network Communication," Control Systems Technology, IEEE Transactions on , vol.20, no.2, pp.495-503, March 2012

[61] Vandoorn, T.L.; Meersman, B.; Degroote, L.; Renders, B.; Vandevelde, L.; , "A Control Strategy for Islanded Microgrids With DC-Link Voltage Control," Power Delivery, IEEE Transactions on, vol.26, no.2, pp.703-713, April 2011

[62] Jing Ma; Jinlong Li; Thorp, J.S.; Arana, A.J.; Qixun Yang; Phadke, A.G.; , "A Fault Steady State Component-Based Wide Area Backup Protection Algorithm," Smart Grid, IEEE Transactions on , vol.2, no.3, pp.468-475, Sept. 2011

[63] Borowski, J.F.; Hopkinson, K.M.; Humphries, J.W.; Borghetti, B.J.; , "ReputationBased Trust for a Cooperative Agent-Based Backup Protection Scheme," Smart Grid, IEEE Transactions on, vol.2, no.2, pp.287-301, June 2011

[64] Kezunovic, M.; , "Smart Fault Location for Smart Grids," Smart Grid, IEEE Transactions on, vol.2, no.1, pp.11-22, March 2011

[65] Nian Liu; Jianhua Zhang; Wenxia Liu; , "Toward Key Management for Communications of Wide Area Primary and Backup Protection," Power Delivery, IEEE Transactions on , vol.25, no.3, pp.2030-2032, July 2010 
[66] Zhi-Hui Dai; Zeng-Ping Wang; Yan-Jun Jiao; , "Reliability Evaluation of the Communication Network in Wide-Area Protection," Power Delivery, IEEE Transactions on, vol.26, no.4, pp.2523-2530, Oct. 2011

[67] Zhiqin He; Zhe Zhang; Wei Chen; Malik, O.P.; Xianggen Yin; , "Wide-Area Backup Protection Algorithm Based on Fault Component Voltage Distribution," Power Delivery, IEEE Transactions on , vol.26, no.4, pp.2752-2760, Oct. 2011

[68] Bernabeu, E.E.; Thorp, J.S.; Centeno, V.; , "Methodology for a Security/Dependability Adaptive Protection Scheme Based on Data Mining," Power Delivery, IEEE Transactions on , vol.27, no.1, pp.104-111, Jan. 2012

[69] MeikangQiu; WenzhongGao; Min Chen; Jian-Wei Niu; Lei Zhang; , "Energy Efficient Security Algorithm for Power Grid Wide Area Monitoring System," Smart Grid, IEEE Transactions on, vol.2, no.4, pp.715-723, Dec. 2011

[70] Chakrabortty, A.; Chow, J.H.; Salazar, A.; , "A Measurement-Based Framework for Dynamic Equivalencing of Large Power Systems Using Wide-Area Phasor Measurements," Smart Grid, IEEE Transactions on , vol.2, no.1, pp.68-81, March 2011

[71] Borghetti, A.; Nucci, C.A.; Paolone, M.; Ciappi, G.; Solari, A.; , "Synchronized Phasors Monitoring During the Islanding Maneuver of an Active Distribution Network," Smart Grid, IEEE Transactions on , vol.2, no.1, pp.82-91, March 2011

[72] Shahraeini, M.; Javidi, M.H.; Ghazizadeh, M.S.; , "Comparison Between Communication Infrastructures of Centralized and Decentralized Wide Area Measurement Systems," Smart Grid, IEEE Transactions on , vol.2, no.1, pp.206211, March 2011

[73] Kun Zhu; Chenine, M.; Nordstrom, L.; , "ICT Architecture Impact on Wide Area Monitoring and Control Systems' Reliability," Power Delivery, IEEE Transactions on , vol.26, no.4, pp.2801-2808, Oct. 2011

[74] Chenine, M.; Nordstrom, L.; , "Modeling and Simulation of Wide-Area Communication for Centralized PMU-Based Applications," Power Delivery, IEEE Transactions on , vol.26, no.3, pp.1372-1380, July 2011

[75] Xiong Liu; Peng Wang; Poh Chiang Loh; , "A Hybrid AC/DC Microgrid and Its Coordination Control," Smart Grid, IEEE Transactions on , vol.2, no.2, pp.278286, June 2011

[76] Vandoorn, T.L.; Renders, B.; Degroote, L.; Meersman, B.; Vandevelde, L.; , "Active Load Control in Islanded Microgrids Based on the Grid Voltage," Smart Grid, IEEE Transactions on , vol.2, no.1, pp.139-151, March 2011

[77] Laaksonen, H.J.; , "Protection Principles for Future Microgrids," Power Electronics, IEEE Transactions on , vol.25, no.12, pp.2910-2918, Dec. 2010

[78] Kwasinski, A.; , "Quantitative Evaluation of DC Microgrids Availability: Effects of System Architecture and Converter Topology Design Choices," Power Electronics, IEEE Transactions on, vol.26, no.3, pp.835-851, March 2011 
[79] Lasseter, R.H.; , "Smart Distribution: Coupled Microgrids," Proceedings of the IEEE , vol.99, no.6, pp.1074-1082, June 2011

[80] Vasquez, J.C.; Guerrero, J.M.; Miret, J.; Castilla, M.; de Vicuña, L.G.; , "Hierarchical Control of Intelligent Microgrids," Industrial Electronics Magazine, IEEE , vol.4, no.4, pp.23-29, Dec. 2010

[81] Zamani, M.A.; Sidhu, T.S.; Yazdani, A.; , "A Protection Strategy and Microprocessor-Based Relay for Low-Voltage Microgrids," Power Delivery, IEEE Transactions on , vol.26, no.3, pp.1873-1883, July 2011

[82] Changhee Cho; Jin-Hong Jeon; Jong-Yul Kim; Soonman Kwon; Kyongyop Park; Sungshin Kim; , "Active Synchronizing Control of a Microgrid," Power Electronics, IEEE Transactions on , vol.26, no.12, pp.3707-3719, Dec. 2011

[83] Sortomme, E.; Venkata, S.S.; Mitra, J.; , "Microgrid Protection Using Communication-Assisted Digital Relays," Power Delivery, IEEE Transactions on , vol.25, no.4, pp.2789-2796, Oct. 2010

[84] NERC (North American Electric Reliability Council), 1996 system disturbances, (Available from NERC, Princeton Forrestal Village, 116-390 Village Boulevard, Princeton, New Jersey 08540-5731), 2002.

[85] D.N. Kosterev, C.W. Taylor, W.A. Mittelstadt, Model validation for the August 10, 1996 WSCC system outage, IEEE Transactions on Power Systems, vol. 13, no. 3, pp. 967-979, 1999.

[86] M.D. Stubna, J. Fowler, An application of the highly optimized tolerance model to electrical blackouts, International Journal of Bifurcation and Chaos, vol. 13, no. 1, 2003, pp. 237-242.

[87] B. A. Carreras, V. E. Lynch, I. Dobson, D. E. Newman, "Complex Dynamics of Blackouts in Power Transmission Systems", available online at: http://www.ece.wisc.edu/ dobson/PAPERS/carrerasCHAOS04.pdf

[88] K. Stouffer, J. Falco, K. Scarfone, Guide to Industrial Control Systems (ICS) Security (Final Public Draft), USA, 2008

[89] R.Rosales, J.Gir, "Wide-Area Monitoring and Control For Power System Grid Security" 15th PSCC, Liege, 22-26 August 2005

[90] A.G. Phadke and J.S. Thorp, "Synchronized Phasor Measurements and Their Applications", Springer, 2008, p. 197

[91] J. De La Ree, V. Centeno, J.S Thorp, A.G Phadke, "Synchronized Phasor Measurement Applications in Power Systems", IEEE Transactions on Smart Grid, Volume: 1 , Issue: 1 Digital Object Identifier: 10.1109/TSG.2010.2044815, 2010 , Page(s): $20-27$

[92] K.E. Martin, D. Hamai, M.G. Adamiak, S. Anderson, M. Begovic, G. Benmouyal, G. Brunello, J. Burger, J.Y. Cai, B. Dickerson, V. Gharpure, B. Kennedy, D. Karlsson, A.G. Phadke, J. Salj, V. Skendzic, J. Sperr, Y. Song, C. Huntley, B. Kasztenny, E. Price, "Exploring the IEEE Standard C37.118-2005 Synchrophasors for Power Systems ", Power Delivery, IEEE Transactions on 
Volume: 23 , Issue: 4 Digital Object Identifier: 10.1109/TPWRD.2007.916092 Publication Year: 2008 , Page(s): 1805 - 1811

[93] Nguegan, Y.; Claudi, A.; Strunge, C.; "Online Monitoring of the Electrical Power Transfer Stability and Voltage Profile Stability Margins in Electric Power Transmission Systems Using Phasor Measurement Units Data Sets ", Digital Object Identifier: 10.1109/APPEEC.2009.4918561 Publication Year: 2009 , Page(s): $1-9$

[94] D. Thukaram, B. Ravikumr, V. SeshadriSravan Kumar, Y. Prasad Rao, S. Surendra, S.R. Kolla, "Real-time monitoring of critical nodes with minimal number of Phasor Measurement Units", ICPS '09. International Conference on Power Systems, Publication Year: 2009 , Page(s): $1-6$

[95] G. Yanfeng; N. Schulz, A. Guzman, "Synchrophasor-Based Real-Time Voltage Stability Index", Power Systems Conference and Exposition, 2006. PSCE '06. 2006 IEEE PES, Digital Object Identifier: 10.1109/PSCE.2006.296452 Publication Year: 2006 , Page(s): 1029 - 1036

[96] G. Yanfeng, "Development Of an Improved On-Line Voltage Stability Index Using Synchronized Phasor Measurement", A Dissertation for the Degree of Doctor of Philosophy in Electrical Engineering Mississippi State University Department of Electrical and Computer Engineering, Mississippi State, Mississippi, December 2005.

[97] Y. G. Zeng, A. Berizzi, and P. Marannino, "Voltage Stability Analysis Considering Dynamic Load Model," in Proceedings of the 4th International Conference on Advanced Power System Control, Operation and Management, Hong Kong, pp. 396-401, 1997.

[98] Kim, T.; Park, S. Kyung; Lee, B. G.; "What Is Appropriate Strategy for Smart Grid Business?: A Case Study of Test Bed in Korea", Proceedings of the 5th International Conference on Information Technologies and Applications (CUTE), pp. $1-5,2010$

[99] Momoh, J.A.; "Fundamentals of analysis and computation for the Smart Grid"; Power and Energy Society General Meeting, 2010 IEEE, pp. 1 - 5, 2010

[100]Momoh, J.A.; "Smart grid design for efficient and flexible power networks operation and control", In Proc. IEEE Power Systems Conference and Exposition, pp 1-8, 2009.

[101]Kezunovic, M.; "Teaching the smart grid fundamentals using modeling, simulation, and hands-on laboratory experiments"; Power and Energy Society General Meeting, pp. $1-6,2010$

[102] Pacific Northwest GridWise, Testbed Demonstration Projects, Available Online at: $\quad$ http://www.pnl.gov/main/publications/external/technical_reports/PNNL17176.pdf

[103] Advanced Architecture and Control Concepts for more Microgrids: Available Online at: http://www.smartgrids.eu/documents/projects/ESR.pdf 
[104] Available Online at: http://canmetenergy-canmetenergie.nrcanrncan.gc.ca/

[105]Choi, J. Y.; Kim, J.; “An integrated simulation framework of a virtual test-bed for Smart Grid"; 40th International Conference on Computers and Industrial Engineering (CIE), pp. $1-5,2010$

[106] Missout,G. and Girard, P., "Measurement of bus voltage angle between Montreal and Sept-Iles", IEEE Transactions on PAS. Vol. 99, No. 2, March/April 1980, pp $536-539$.

[107] Missout, G., Beland,J., and Bedard, G., "Dynamic measurement of the absolute voltage angle on long transmission Lines", IEEE Transactions on PAS. Vol. 100, No. 11, November 1981, pp 4428-4434.

[108]Bonanomi,P., "Phase angle measurements with synchronized clocks - principles and applications", IEEE Transactions on PAS. Vol. 100, No. 11, November 1981, pp 5036-5043.

[109]Phadke, A.G., Thorp, J.S, and Adamiak,M.G., "A new measurement technique for tracking voltage phasors, local system frequency, and rate of change of frequency", IEEE Transactions on PAS. Vol. 102, No. 5, May 1983, pp 1025-1038.

[110] Available at the web-site: http://en.wikipedia.org/wiki/Gps

[111] "Macrodyne Model 1690 PMU Disturbance Recorder", Macrodyne Inc. 4 Chelsea Place, Clifton Park, NY, 12065.

[112]J. De La Ree, V. Centeno, J.S Thorp, A.G Phadke, "Synchronized Phasor Measurement Applications in Power Systems", IEEE Transactions on Smart Grid, Volume: 1 Issue: 1 Digital Object Identifier: 10.1109/TSG.2010.2044815, 2010 , Page(s): $20-27$

[113]K.E. Martin, D. Hamai, M.G. Adamiak, S. Anderson, M. Begovic, G. Benmouyal, G. Brunello, J. Burger, J.Y. Cai, B. Dickerson, V. Gharpure, B. Kennedy, D. Karlsson, A.G. Phadke, J. Salj, V. Skendzic, J. Sperr, Y. Song, C. Huntley, B. Kasztenny, E. Price, "Exploring the IEEE Standard C37.118-2005 Synchrophasors for Power Systems ", Power Delivery, IEEE Transactions on Volume: 23 , Issue: 4 Digital Object Identifier: 10.1109/TPWRD.2007.916092 Publication Year: 2008 , Page(s): $1805-1811$

[114]Y. Nguegan, "Real-time identification and monitoring of the voltage stability margin in electric power transmission systems using synchronized phasor measurements," Ph.D. thesis, dept. Elect. Eng., Kassel Univ., Germany, 2009.

[115]D. Thukaram, B. Ravikumr, V. SeshadriSravan Kumar, Y. Prasad Rao, S. Surendra, S.R. Kolla, "Real-time monitoring of critical nodes with minimal number of Phasor Measurement Units ", Power Systems, 2009. ICPS '09. International Conference on Digital Object Identifier: 10.1109/ICPWS.2009.5442648, Publication Year: 2009, Page(s): 1 - 6

[116]G. Yanfeng; N. Schulz, A. Guzman, "Synchrophasor-Based Real-Time Voltage Stability Index ”, Power Systems Conference and Exposition, 2006. PSCE '06. 
2006 IEEE PES Digital Object Identifier: 10.1109/PSCE.2006.296452 Publication Year: 2006 , Page(s): 1029 - 1036

[117]G. Yanfeng, "Development Of an Improved On-Line Voltage Stability Index Using Synchronized Phasor Measurement", A Dissertation Submitted to the Faculty of Mississippi State University in Partial fulfillment of the Requirements for the Degree of Doctor of Philosophy in Electrical Engineering in the Department of Electrical and Computer Engineering, Mississippi State, Mississippi, December 2005

[118]Y. G. Zeng, A. Berizzi, and P. Marannino, "Voltage Stability Analysis Considering Dynamic Load Model," in Proceedings of the 4th International Conference on Advanced Power System Control, Operation and Management, Hong Kong, pp. 396-401, 1997

[119]J.A. Momoh, X. Yan, G. Boswell, "Voltage stability enhancement using Phasor Measurement Unit (PMU) technology," Power Symposium, 2008. NAPS '08. 40th North American, pp.1-6, 28-30 Sept. 2008

[120]P. Kundur, J. Paserba, V. Ajjarapu, G. Andersson, A. Bose, C. Canizares, N. Hatziargyriou, D. Hill, A. Stankovic, C. Taylor, T.VanCustem, V. Vittal, "Definition and classification of power system stability IEEE/CIGRE joint task force on stability terms and definitions," IEEE Trans. on Power Systems, Vol.19, Issue 3, pp. 1387-1410, Aug.2004

[121]M. Moghavvami, F.M. Omar, "Technique for contingency monitoring and voltage collapse prediction," IEEE Proceeding on Generation, Transmission and Distribution, Vol. 145, N6, pp. 634-640, Nov.1998

[122]I. Musirin, T.K. Abdul Rahman, "Online voltage stability based contingency ranking using fast voltage stability index (FVSI)," Transmissionaand Distribution Conference and Exhibition 2002: Asia Pacific. IEEE/PES, Vol. 2, pp. 1118-1123, Oct. 2002

[123] M.V. Suganyadevia, C.K. Babulal "Estimating of loadability margin of a power system by comparing Voltage Stability Indices," International Conference on Control, Automation, Communication and Energy Conservation, INCACEC, pp.14, 4-6 June 2009

[124]M.M. El-Kateb, S. Abdelkadar, M.S. Kandil, "Linear indicator for voltage collapse in power systems," IEEE Proceedings in Generation, Transmission and distribution, vol. 144, no.2, pp. 139-146, Mer 1997

[125]N. Sudipta, P. Shrabani, "A novel technique for determination of voltage stability margin," 5th IET International Conference on System Safety 2010, pp.1-5, 18-20 Oct. 2010

[126]Y. Gong, N. Schulz, A. Guzman, "Synchrophasor-Based Real-Time Voltage Stability Index," Power Systems Conference and Exposition, 2006. PSCE '06. 2006 IEEE PES, pp.1029-1036, Oct. 29 2006-Nov. 12006 
[127] A. Mazloomzadeh, V. Salehi, O. Mohammed, "Soft synchronization of dispersed generators to micro grids for smart grid applications," Innovative Smart Grid Technologies (ISGT), 2012 IEEE PES , vol., no., pp.1-7, 16-20 Jan. 2012

[128]Y. Haibo, V. Vittal and Y. Zhong, "Self-healing in power systems: an approach using islanding and rate of frequency decline-based load shedding," IEEE Trans. on Power System, vol. 18, pp. 174 - 181, Feb. 2003.

[129]C. Chuang, Y. Wang, C. Lee, M. Liu, Y. Hsiao, and J. Jiang. "An Adaptive Routing Algorithm Over Packet Switching Networks for Operation Monitoring of Power Transmission Systems," IEEE Trans. Power Delivery, vol. 25, pp. 882 890, Apr. 2010.

[130] G. Zhang, S. Lee, R. Carroll, Z. Jian, L. Beard, L. and L. Yilu, "Wide area power system visualization using real-time synchrophasor measurements," IEEE Power and Energy Society General Meeting, pp. 1-7, 2010

[131]F. Li, Z. Chen, L. Fan and P. Zhang, "Toward a self-healing protection and control system, " 40th North American Power Symposium (NAPS), pp. 1-5, 2008

[132]Q. Huang, C. Zhang, Q. Liu, Y. Ning and Y. Cao, "New type of fiber optic sensor network for smart grid interface of transmission system," Power and Energy Society General Meeting, pp. 1-5, 2010

[133]R. F. Nuqui, "State Estimation and Voltage Security Monitoring Using Synchronized Phasor Measurements" Ph.D. dissertation, Dept. Elec. Eng., Univ. Virginia, Blacksburg, 2001.

[134] A.P.S Meliopoulos and G.J. Cokkinides, "A virtual environment for protective relaying evaluation and testing," IEEE Trans. On Power Systems, vol. 19, pp. 104111,2004

[135]M. Elshaer, A. Mohamed and O. Mohammed, "Integration of Sustainable Energy Sources into DC Zonal Electric Distribution Systems," Power and Energy Society General Meeting, 2011 IEEE, vol., no., pp.1-7, 24-29 July 2011

[136] A. Mohamed, M. Elshaer and O. Mohammed, "Grid Connected DC Distribution System for Efficient Integration of Sustainable Energy Sources," in Proc. of IEEE Power Systems Conference and Exposition, PSCE 2011, Phoenix, Arizona, USA 20 -23 May 2011

[137] A. Mohamed, M. Elshaer and Osama Mohammed, "Bi-Directional AC-DC/DCAC converter for Power Sharing of Hybrid AC/DC Systems," Power Systems Conference and Exposition (PSCE), 2011 IEEE/PES , vol., no., pp.1-9, 20-23 March 2011

[138]A. Mohamed, M. Elshaer and O. Mohammed, "Control Enhancement of Power Conditioning Units for High Quality PV Systems," Electric Power System Research, ELSEVIER, Volume 90, September 2012, Pages 30-41

[139]A. Mohamed, M. Elshaer and O. Mohammed, "Dynamic Energy Commitment Scheme for Hybrid Renewable Energy Systems in Smart Grid Applications," Energy Systems (ENSY), SPRINGER, manuscript \# ENSY-D-11-00038, 2011 
[140]A. Mohamed. V. Salehi and O. Mohammed, "Real-Time Energy Management Algorithm for Mitigation of Pulse Loads in Smart Microgrids," Accepted to IEEE Trans. on Smart Grid, 2012

[141] Olivares, D.E.; Canizares, C.A.; Kazerani, M.; , "A centralized optimal energy management system for microgrids," Power and Energy Society General Meeting, 2011 IEEE , vol., no., pp.1-6, 24-29 July 2011

[142]Bando, S.; Sasaki, Y.; Asano, H.; Tagami, S.; , "Balancing control method of a microgrid with intermittent renewable energy generators and small battery storage," Power and Energy Society General Meeting - Conversion and Delivery of Electrical Energy in the 21st Century, 2008 IEEE , vol., no., pp.1-6, 20-24 July 2008

[143]Zeng Ming; Huang Lixin; Yan Fan; Jiang Danwei; , "Research of the Problems of Renewable Energy Orderly Combined to the Grid in Smart Grid," Power and Energy Engineering Conference (APPEEC), 2010 Asia-Pacific, vol., no., pp.1-4, 28-31 March 2010

[144]Dagdougui, H., Minciardi, R., Ouammi, A., Sacile, R., "Optimal control of a regional power microgrid network driven by wind and solar energy", Systems Conference (SysCon), 2011 IEEE International, On page(s): 86 - 90, Volume: Issue: , 4-7 April 2011

[145]Borges, C.L.T.; Alves, J.M.T.; , "Power System Real Time Operation based on Security Constrained Optimal Power Flow and Distributed Processing," Power Tech, 2007 IEEE Lausanne, vol., no., pp.960-965, 1-5 July 2007

[146]Ling Su; Jianhua Zhang; Ziping Wu; , "Energy Management Strategy for Lab Microgrid," Power and Energy Engineering Conference (APPEEC), 2011 AsiaPacific , vol., no., pp.1-4, 25-28 March 2011

[147]Al-Awami, A.T.; El-Sharkawi, M.A.; "Feedback-control-based optimal power flow for real-time operation," Power Systems Conference and Exposition, 2009. PSCE '09. IEEE/PES , vol., no., pp.1-6, 15-18 March 2009

[148]R. Bacher, H.P. Van Meeteren, "Real-time optimal power flow in automatic generation control", IEEE trans. Power syst., vol. 3, no. 4, November 1988, pp. 1518-1529.

[149] S.K. Joshi, K.N. Patel, "Real time economic dispatch", Proc. intl. conf. on power syst. technology, vol. 3, December 2000, pp.1263-1268.

[150]C.W. Sanders, C.A. Monroe, "An algorithm for real-time security constrained economic dispatch", IEEE trans. power syst., vol. PWRS-2, no. 2, November 1987, pp. 1068-1074

[151]Xie, K.; Song, Y.H.; "Dynamic optimal power flow by interior point methods," Generation, Transmission andDistribution, IEEE Proceedings-, vol.148, no.1, pp.76-84, Jan 2001 
[152]Xie, K.; Song, Y.H.; "Power market oriented optimal power flow via an interior point method," Generation, Transmission and Distribution, IEE Proceedings-, vol.148, no.6, pp.549-556, Nov 2001

[153]Tao, Ye; Meliopoulos, A. P. Sakis; , "An sequential linear programming algorithm for security-constrained optimal power flow," North American Power Symposium (NAPS), 2009 , vol., no., pp.1-6, 4-6 Oct. 2009

[154]Momoh, J.A.; El-Hawary, M.E.; Adapa, R.; , “A review of selected optimal power flow literature to 1993. II. Newton, linear programming and interior point methods," Power Systems, IEEE Transactions on, vol.14, no.1, pp.105-111, Feb 1999

[155] Available Online at: www.digsilent.de

[156] G. Gross, A. Bose, C. DeMarco, M. Pai, J. Thorp and P. Varaiya, "White Paper on Real-Time Security Monitoring and Control of Power Systems," prepared for Transmission Reliability Program, Office of Power Technologies, Assistant Secretary for Energy Efficiency and Renewable Energy, Department of Energy, August 30, 1999.

[157]M.M. Begovic, A.R. Messina, "Editorial: Wide Area Monitoring, Protection and Control," Generation, Transmission \& Distribution, IET , vol.4, no.10, pp.10831085, October 2010

[158] S. Mohagheghi, G.K. Venayagamoorthy, R.G. Harley, "Optimal Wide Area Controller and State Predictor for a Power System," Power Systems, IEEE Transactions on, vol.22, no.2, pp.693-705, May 2007

[159]V. Salehi, O. Mohammed, "Developing Virtual Protection System for Control and Self-healing of Power System", Industry Applications Society Annual Meeting (IAS), 2011 IEEE , Oct. 2011

[160]A.G. Tsikalakis, N.D. Hatziargyriou, "Centralized Control for Optimizing Microgrids Operation," IEEE Transactions on Energy Conversion, vol. 23, no. 1, pp. 241-248, March 2008.

[161] N. Amjady, F. Keynia, H. Zareipour, "Short-Term Load Forecast of Microgrids by a New Bilevel Prediction Strategy," IEEE Transactions on Smart Grid, vol. 1, no. 3, pp. 286-294, Dec. 2010.

[162]M. Zhixin, A. Domijan, F. Lingling, "Investigation of Microgrids with Both Inverter Interfaced and Direct AC-Connected Distributed Energy Resources," IEEE Transactions on Power Delivery, vol. 26, no. 3, pp. 1634-1642, July 2011.

[163]P. Tenti, H.K.M Paredes, P. Mattavelli, "Conservative Power Theory, a Framework to Approach Control and Accountability Issues in Smart Microgrids," IEEE Transactions on Power Electronics, vol. 26, no. 3, pp. 664-673, March 2011.

[164]V. Salehi, B. Mirafzal, O. Mohammed, "Pulse-load effects on ship power system stability," IECON 2010 - 36th Annual Conference on IEEE Industrial Electronics Society, pp.3353-3358, 7-10 Nov. 2010. 
[165]J.M. Crider, S.D. Sudhoff, "Reducing Impact of Pulsed Power Loads on Microgrid Power Systems," IEEE Transactions on Smart Grid, vol. 1, no. 3, pp. 270-277, Dec. 2010.

[166] S. Kulkarni, S. Santoso, "Impact of pulse loads on electric ship power system: With and without flywheel energy storage systems," IEEE Electric Ship Technologies Symposium, pp.568-573, 20-22 April 2009.

[167]J.J.A. Burgt, P. Gelder, E. Dijk, "Pulsed power requirements for future naval ships," 12th IEEE International Pulsed Power Conference, vol. 2, pp. 1357-1360, 1999.

[168]E.L. Zivi, "Integrated shipboard power and automation control challenge problem," IEEE Power Engineering Society Summer Meeting, vol. 1, pp.325-330 vol.1, July 2002.

[169]Z. Haihua, T. Bhattacharya, D. Tran, T.S. Terence and A.M. Khambadkone, "Composite Energy Storage System Involving Battery and Ultracapacitor With Dynamic Energy Management in Microgrid Applications, " IEEE Transaction on Power Electronics, vol. 26, no. 3, pp. 923-930, March 2011.

[170]I. Aharon, A. Kuperman, "Topological Overview of Powertrains for BatteryPowered Vehicles With Range Extenders," IEEE Transactions on Power Electronics, vol. 26, no. 3, pp. 868-876, March 2011.

[171]A. Khaligh, L. Zhihao, "Battery, Ultracapacitor, Fuel Cell, and Hybrid Energy Storage Systems for Electric, Hybrid Electric, Fuel Cell, and Plug-In Hybrid Electric Vehicles: State of the Art," IEEE Transactions on Vehicular technology, vol. 59, no. 6, pp. 2806-2814, July 2010.

[172]A.H. Eghbali, B. Asaei, P. Nader, "Fuel efficient control strategy, based on battery-ultracapacitor energy storage system, in parallel hybrid electric vehicles," IEEE Vehicle Power and Propulsion Conference (VPPC), pp. 1-5, 1-3 Sept. 2010.

[173]L.P. Jarvis, T.B. Atwater, P.J. Cygan, "Hybrid power sources for Land Warrior scenario," IEEE Aerospace and Electronic Systems Magazine, vol. 15, no. 9, pp. 37-41, Sep 2000.

[174] M. Zandi, A. Payman, J.P. Martin, S. Pierfederici, B. Davat, F. Meibody-Tabar, "Energy Management of a Fuel Cell Supercapacitor Battery Power Source for Electric Vehicular Applications," IEEE Transactions on Vehicular Technology, vol. 60, no. 2, pp. 433-443, Feb. 2011.

[175]J. Byun, I. Hong, B. Kang, S. Park, "A smart energy distribution and management system for renewable energy distribution and context-aware services based on user patterns and load forecasting," IEEE Transactions on Consumer Electronics, vol. 57, no. 2, pp. 436-444, May 2011.

[176]H. Kanchev, D. Lu, F. Colas, V. Lazarov, B. Francois, "Energy Management and Operational Planning of a Microgrid With a PV-Based Active Generator for Smart Grid Applications," IEEE Transactions on Industrial Electronics, vol. 58, no. 10, pp. 4583-4592, Oct. 2011. 
[177]P. Palensky, D. Dietrich, "Demand Side Management: Demand Response, Intelligent Energy Systems, and Smart Loads," IEEE Transactions on Industrial Informatics, vol. 7, no. 3, pp. 381-388, Aug. 2011.

[178]T. Christen and M.W. Carlen, "Theory of Ragone plots," Journal of Power Sources, September, 2000, vol. 91, Issue 2, pp. 210-216, Dec. 2000.

[179]G. Sikha, B.N. Popov, "Performance optimization of a battery-capacitor hybrid system," Journal of Power Sources, vol. 134, pp. 130-138, 2000.

[180] A. Kuperman, I. Aharon, "Battery-ultracapacitor hybrids for pulsed current loads: A review, " Renewable and Sustainable Energy Reviews, vol. 15, Issue 2, pp. 981992, Feb. 2011.

[181]L. Gao, R.A. Dougal, L. Shengyi, "Power Enhancement of an Actively Controlled Battery/Ultracapacitor Hybrid," IEEE Trans. on Power Electronics, vol. 20, no. 1, Jan. 2005.

[182] Kevin M. Passino and Stephen Yurkovich, Fuzzy Control, Addison-Wesley Longman, Inc., California (1998).

[183] Steurer, M.; Andrus, M.; Langston, J.; Qi, L.; Suryanarayanan, S.; Woodruff, S.; Ribeiro, P.F., "Investigating the Impact of Pulsed Power Charging Demands on Shipboard Power Quality", Electric Ship Technologies Symposium, 2007. ESTS' 07. IEEE, Publication Year: 2007 , Page(s): 315 - 321

[184]J. Langston, S. Suryanarayanan, M. Steurer, M. Andrus, S. Woodruff, and P. F. Ribeiro, "Experiences with the simulation of a notional allelectric ship integrated power system on a large-scale high-speed electromagnetic transient simulator," in Proc. 2006 IEEE Power Engineering Society General Meeting, 18-22 June, 2006.

[185]L. N. Hannet, G. Jee, and B. Fardanesh, "A governor/turbine model for a twinshaft combustion turbine," IEEE Trans. On Power Systems, vol.10, no. 1, February 1995.

[186]Mohamed, A.; Salehi, V.; Mohammed, O.; , "Real-Time Energy Management Algorithm for Mitigation of Pulse Loads in Hybrid Microgrids," Smart Grid, IEEE Transactions on , vol.PP, no.99, pp.1-12, 0 doi: 10.1109/TSG.2012.2200702 
VITA

Vahid Salehi Pour Mehr

13 Sep 1980

$1998-2003$

2003-2006

2006-2009

2009-2011

2011-2012
Born, Tabriz, Iran

B.Sc., University of Tabriz, Tabriz, Iran

M.Sc., University of Tehran, Tehran, Iran

Principal Power System Engineer, MATN International Co., Tehran, Iran

Principal Power System Engineer, MATN International Co., Tehran, Iran

Teaching Assistant, Florida International University, Miami, USA

Doctoral Evidence Acquisition Fellowship (DEA), Florida International University, Miami, USA

Research Assistant, Florida International University, Miami, USA

\section{SELECTED PUBLICATIONS}

Salehi, V.; Mohamed, A.; Mazloomzadeh, A.; Mohammed, O. A.; , "Laboratory-Based Smart Power System, Part I: Design and System Development," Smart Grid, IEEE Transactions on , vol.PP, no.99, pp.1-11, 0 doi: 10.1109/TSG.2012.2194518

Salehi, V.; Mohamed, A.; Mazloomzadeh, A.; Mohammed, O. A.; , "Laboratory-Based Smart Power System, Part II: Control, Monitoring, and Protection," Smart Grid, IEEE Transactions on , vol.PP, no.99, pp.1-13, 0 doi: 10.1109/TSG.2012.2194519

Mohamed, A.; Salehi, V.; Mohammed, O.; , "Real-Time Energy Management Algorithm for Mitigation of Pulse Loads in Hybrid Microgrids," Smart Grid, IEEE Transactions on, vol.PP, no.99, pp.1-12, 0 doi: 10.1109/TSG.2012.2200702

Salehi V., Mohamed A., Mohammed O., "Implementation of Real-time Optimal Power Flow Management System on Hybrid AC/DC Smart Microgrid," IEEE-IAS Industrial Application Society Annual Meeting, Las Vegas, USA, 7-11 Oct. 2012

Mohamed A., Salehi V., Mohammed O., "Reactive Power Compensation in Hybrid AC/DC Networks for Smart Grid Applications", 2012 3rd IEEE PES Innovative Smart Grid Technologies Europe (ISGT Europe) 
Salehi V., Mazloomzadeh A., Fernandez J.F., Mohammed, O.A., "Real-time power system analysis and security monitoring by WAMPAC systems," Innovative Smart Grid Technologies (ISGT), 2012 IEEE PES , pp.1-8, 16-20 Jan. 2012

Mazloomzadeh A., Salehi V., Mohammed O., "Soft synchronization of dispersed generators to micro grids for smart grid applications," Innovative Smart Grid Technologies (ISGT), 2012 IEEE PES , pp.1-7, 16-20 Jan. 2012

Salehi V., Mazloomzadeh A., Mohammed O.A.; "Development and Implementation of a Phasor Measurement Unit for Real-Time Monitoring, Control and Protection of Power Systems", IEEE Power \& Energy Society General Meeting, Detroit Michigan, USA- July 24-28, 2011

Salehi V., Mazloomzadeh A., Mohammed O.A.; "Real-Time Analysis for Developed Laboratory-Based Smart Micro Grid", IEEE Power \& Energy Society General Meeting, Detroit Michigan, USA- July 24-28, 2011

Salehi V., Mazloomzadeh A., Fernandez J., Parra J., Mohammed O.A.; "Design and Implementation of Laboratory-Based Smart Power System", ASEE, $118^{\text {th }}$ Annual Conference and Exposition, Vancouver, B.C. CANADA, June 26-29, 2011

Salehi V., Mohammed O.A.; "Developing Virtual Protection System for Control and Self-healing of Power System", IEEE Industry Applications Society (IAS) Annual Meeting, Orlando, FL USA, October 9-13, 2011

Salehi V., Mohammed O.A.; "Real-Time Voltage Stability Monitoring and Evaluation Using Synchorophasors", $43^{\text {rd }}$ North American Power Symposium (NAPS),Boston, MA, USA, August 4-6, 2011

Salehi, V., Mirafzal, B. and Mohammed, O.; "Pulse-load effects on ship power system stability", IECON, $36^{\text {th }}$ Annual Conference on IEEE Industrial Electronics Society, Phoenix , AZ, November 7-10, 2010

\section{Awards}

- 1st Rank of Engineering Department, Florida International University, Scholarly Forum Prize, Graduate Student Appreciation Week, March 2012

- 2012 DEA Fellowship, Florida International University, Graduate School for completion of Dissertation Work, 2012

- Three Travel Awards Grant from GSA (2011 and 2012)

\section{Organization and Memberships}

- Graduate Student Member, PES-IAS IEEE since 2009

- Chapter Vice Chair of Power \& Energy Society IEEE, Miami Section, 2011

- Peer Reviewer:

- IEEE Transaction on Smart Grid

- IEEE Transaction on Industry Applications

- IET Generation, Transmission \& Distribution 